\title{
Origin of the Immiscibility of Alkanes and Perfluoroalkanes
}

\author{
Journal Article
}

Author(s):

Pollice, Robert (i); Chen, Peter

Publication date:

2019-02-27

Permanent link:

https://doi.org/10.3929/ethz-b-000336839

Rights / license:

In Copyright - Non-Commercial Use Permitted

Originally published in:

Journal of the American Chemical Society 141(8), https://doi.org/10.1021/jacs.8b10745 


\title{
Origin of the Immiscibility of Alkanes and Perfluoroalkanes
}

\author{
Robert Pollice, Peter Chen*
}

\begin{abstract}
Perfluoroalkanes are considered generally to have weak inter- and intramolecular forces compared to alkanes explaining their relatively low boiling points, low surface tensions and poor solvent properties. However, currently accepted models do not satisfactorily explain several trends in their properties, for instance boiling point trends as size increases. Herein, we report a comprehensive computational study of the intermolecular interaction of alkanes and perfluoroalkanes demonstrating that perfluoroalkanes have a higher intrinsic ability for dispersive interactions than their alkane counterparts and that dispersion in perfluoroalkane dimers mainly stems from fluorine-fluorine interactions. In addition, the reasons for relatively weak intermolecular forces in perfluoroalkanes compared to alkanes are their ground-state geometries, which are increasingly unsuitable for intermolecular interactions as the carbon chain length increases, and their rigidity, which makes deformation from the ground-state geometries unfavorable. Overall, these trends are reflected in a dependence of the bulk properties of perfluoroalkanes on the carbon chain length as the fluorine content decreases and the interaction geometries become increasingly unsuitable.
\end{abstract}

\section{Introduction}

Perfluoroalkanes differ from alkanes on many accounts: ${ }^{1-5}$ One of the most fundamental difference is the larger size of perfluoroalkanes compared to their counterparts. ${ }^{1,6}$ For comparison, looking at various steric parameters, it was shown that a trifluoromethyl group is at least as large as an isopropyl group. ${ }^{4}$ In addition, the electronegative fluorine atom depletes the carbon chain of electrons and results in a negatively polarized shell in perfluoroalkanes, whereas in alkanes the hydrogen atoms are slightly positively polarized. Furthermore, perfluoroalkanes are significantly more rigid than alkanes ${ }^{7}$ and the low miscibility of perfluoroalkanes with alkanes is generally known as fluorophobic effect, ${ }^{8-11}$ sometimes referred to as fluorous effect. Perfluoroalkanes are generally assumed to have weak intermolecular interaction energies compared to alkanes, both in bulk media and in molecular complexes, explaining their relatively low boiling points, small cohesive energies as well as the fluorophobic effect..$^{2-4,8,12,13}$ It has been argued that, due to the low atomic polarizability of the fluorine atom, perfluoroalkanes have a low propensity to interact with any other molecule (by London dispersion ${ }^{14}$ ) and therefore segregate to minimize intermolecular contacts with non-fluorous matter thereby maximizing the contact of non-fluorous matter with itself. ${ }^{3,8}$ However, several trends in their properties are not readily explained by that model, for instance the boiling point inversion of linear alkanes and perfluoroalkanes as a function of chain length. ${ }^{4,12}$ Additionally, high-level ab initio calculations of molecular dimers of methane and perfluoromethane found $\mathrm{CF}_{4}$-dimer to be significantly stronger bound than the $\mathrm{CH}_{4}$-dimer suggesting that the ubiquitous and general assumption that perfluoroalkanes show weaker intermolecular forces needs to be reassessed. ${ }^{15-17}$

We herein report on a computational study of the interaction energies of homo- and heterodimers of alkanes and perfluoroalkanes of increasing carbon chain length in which:

i. We observe that several state-of-the-art computational methods have problems to estimate accurately the interaction energies of alkane-perfluoroalkane and perfluoroalkaneperfluoroalkane complexes compared to benchmark results; 
ii. We find that dispersion in interacting perfluoroalkanes originates mainly from contacting fluorine atoms;

iii. We show that perfluoroalkanes have a higher intrinsic ability for intermolecular dispersion than alkanes with the same carbon chain length;

iv. We establish that the preferred geometries of perfluoroalkanes get increasingly unsuitable for interactions with either alkanes or perfluoroalkanes as the carbon chain length increases, which is the principal reason for the interaction energies being weaker than expected from shorter analogues.

v. We demonstrate that trends in interaction energies and various experimental properties are determined by the concomitant decreasing fluorine content and the increasingly unsuitable interaction geometries.

\section{Test Systems}

In this work, we study the interactions of alkane-alkane (HH), alkane-perfluoroalkane (HF) and perfluoroalkane-perfluoroalkane (FF) complexes wherein the interacting molecules have the same number of carbons. The number of carbons is varied systematically from one to six. The abbreviations used to refer to particular molecules and molecular complexes are explained in Table 1.

Table 1. Explanation of abbreviations used in this work with two examples.

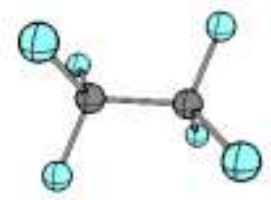

$2-\mathrm{F}$

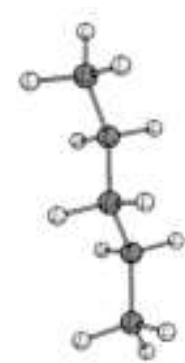

(1) $\mathbf{H}$
(1) $\mathbf{~} \mathbf{F}$

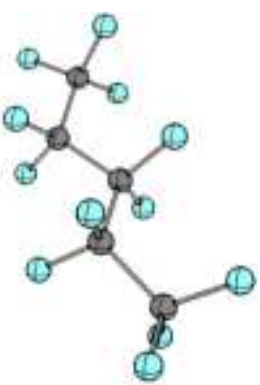

5-HF3
Number of carbon atoms in the molecule(s)
One or two-letter code describing the molecule(s) ( $\mathrm{H}=$ alkane, $\mathrm{F}=$ perfluoroalkane)
Optional number to distinguish between several conformers

Additionally, a consistent color-coding will be used in Figures and Tables when a particular subclass of the molecular complexes is investigated. Magenta will be used for $\mathrm{HH}$, cyan for HF and yellow for FF.

\section{Computational Details and Definitions}

Computed energies discussed in this work are either bond association energies (BAEs), i.e. the relaxation of the interacting fragments to their respective ground-state geometries is included, or bond interaction energies (BIEs), i.e. the corresponding relaxation is not included. Various programs were employed in this study including (but not limited to) Orca (versions 3.0.3 and 4.0.1), ${ }^{18,19}$ PSI4 (versions 1.1 and 1.2), ${ }^{20,21}$ Molpro (version 2015.1.18), ${ }^{22}$ Gaussian (version 09, revision D.01), ${ }^{23}$ ADF (version 2016) ${ }^{24}$ and Q-Chem (version 4.3). ${ }^{25} \mathrm{~A}$ more detailed description of all the programs and methods used is included in the Supporting Information. 
For benchmarking of computational methods, we look at the following statistical measures describing the deviation of a dataset with values $x_{i}$ from a reference dataset with values $x_{i}^{\text {ref }}$ (Equations $1-4$ ). It should be noted that the relative mean absolute deviation $\left(M A D_{\text {rel }}\right)$ is sometimes also referred to as mean absolute relative deviation (MARE) in the literature. Notably, the MAD from the mean is always smaller than the corresponding standard deviation, i.e. the MAD gives a lower estimate of the deviation in the data.

$$
\begin{aligned}
& \text { Mean Absolute Deviation: } M A D=\sum_{i}\left|x_{i}-x_{i}^{r e f}\right| \\
& \text { Relative Mean Absolute Deviation: } M A D_{r e l}=\sum_{i}\left|\frac{x_{i}-x_{i}^{r e f}}{x_{i}^{r e f}}\right| \\
& \text { Mean Signed Deviation: } M S D=\sum_{i}\left(x_{i}-x_{i}^{r e f}\right) \\
& \text { Relative Mean Signed Deviation: } M S D_{r e l}=\sum_{i} \frac{x_{i}-x_{i}^{r e f}}{x_{i}^{r e f}}
\end{aligned}
$$

One central measure of interest is the relative molar content of a certain atom type $\mathrm{X}$ in a molecule (Equation 5) describing the atomic composition.

$$
\text { Relative Molar Content of X: } n_{\text {rel }}(X)=\frac{\text { Number of } X \text { Atoms }}{\text { Total Number of Atoms }}
$$

\section{Results}

Benchmarking. We set out this study by looking for literature of computational benchmark data of complexes of alkanes $(\mathbf{H})$ and perfluoroalkanes $(\mathbf{F})$ in order to select suitable computational methods for geometry optimizations and subsequent single point calculations. We realized that the available benchmark data for perfluoroalkanes are extremely sparse ${ }^{15-17,26,27}$ and therefore decided to begin by benchmarking computational methods against interaction energies from high-level ab initio methods. We chose DSD-PBEP86/def2-TZVP(spd) as density functional theory (DFT) method for geometry optimizations because of its good performance over a wide range of benchmarks, which indicates its robustness. ${ }^{28}$ To assess the performance of DSD-PBEP86 in geometry optimizations for our test systems we performed rigid geometry scans of the intermolecular separation of interacting molecules using MP2/CBS and DLPNO-CCSD(T)/QZ. ${ }^{29-31}$ These results suggest that DSD-PBEP86 geometries are appropriate for all the molecular complexes under study (Details in the Supporting Information).

On the basis of general literature benchmarks for noncovalent interactions and dispersion ${ }^{32,33}$ we selected CCSD(T)/CBS and CCSD(T)-F12/VTZ as reference methods for single point energies; we looked for a cheaper method that reproduces these interaction energies best. CCSD(T)-F12/VDZ, which has a significantly lower computational cost, shows only minor deviations (Details in the Supporting Information). Using CCSD(T)-F12/VDZ, we were able to perform benchmark calculations for most of our complexes under study. To do that, we determined DSD-PBEP86 geometries and single point energies to find the lowest energy conformers for each complex. These conformers were then used to benchmark interaction energies using various methods (Details in the Supporting Information). The performance of all the DFT and wavefunction theory (WFT) approaches tested against the CCSD(T)F12/VDZ reference values is illustrated in Table 2.

Table 2. Benchmark of various WFT and DFT methods against CCSD(T)-F12/VDZ energies of most of the molecular complexes studied. Numbers in parentheses indicate the corresponding standard 
deviations. B97M-V34/def2-QZVP, $\omega B$ B97M-V35/def2-QZVP, DLPNO-CCSD(T)/CBS beyond TightPNO and B3LYP-XDM ${ }^{36-39} / \mathrm{aTZ}$ perform best across the three subclasses.

\begin{tabular}{|c|c|c|c|c|c|c|c|c|}
\hline \multirow[b]{2}{*}{ Method } & \multicolumn{2}{|c|}{$\mathrm{HH}$} & \multicolumn{2}{|c|}{ HF } & \multicolumn{2}{|c|}{ FF } & \multicolumn{2}{|c|}{ Overall } \\
\hline & $\begin{array}{c}\text { MAD }_{\text {rel }} \\
{[\%]}\end{array}$ & $\begin{array}{c}\text { MSD }_{\text {rel }} \\
{[\%]}\end{array}$ & $\begin{array}{c}\text { MAD }_{\text {rel }} \\
{[\%]}\end{array}$ & $\begin{array}{c}\mathrm{MSD}_{\text {rel }} \\
{[\%]}\end{array}$ & $\begin{array}{c}\text { MAD }_{\text {rel }} \\
{[\%]}\end{array}$ & $\begin{array}{c}\text { MSD }_{\text {rel }} \\
{[\%]}\end{array}$ & $\begin{array}{c}\text { MAD }_{\text {rel }} \\
{[\%]}\end{array}$ & $\begin{array}{c}\mathrm{MSD}_{\text {rel }} \\
{[\%]}\end{array}$ \\
\hline B97M-V & $7(5)$ & $5(7)$ & $3(2)$ & $3(3)$ & $3(1)$ & $0(3)$ & $4(3)$ & $3(5)$ \\
\hline ExtremePNO & $2(1)$ & $-1(2)$ & $3(4)$ & $3(4)$ & $9(4)$ & $9(4)$ & $4(4)$ & $3(4)$ \\
\hline$\omega B 97 M-V$ & $5(3)$ & $5(3)$ & $4(2)$ & $-4(2)$ & $7(4)$ & $-7(4)$ & $5(3)$ & $-2(6)$ \\
\hline B3LYP-XDM & $3(1)$ & $1(4)$ & $2(2)$ & $2(2)$ & $14(3)$ & $14(3)$ & $5(5)$ & $4(6)$ \\
\hline VeryTightPNO & $2(1)$ & $0(2)$ & $5(2)$ & $5(2)$ & $13(4)$ & $13(4)$ & $6(5)$ & $5(5)$ \\
\hline TightPNO & $1(1)$ & $1(1)$ & $6(2)$ & $6(2)$ & $17(5)$ & $17(5)$ & $7(6)$ & $7(6)$ \\
\hline $\begin{array}{c}\mathrm{CCSD}(\mathrm{T}) / \mathrm{CBS}(\mathrm{LPNO}- \\
\mathrm{CEPA} / 1)\end{array}$ & $3(3)$ & $1(5)$ & $11(2)$ & $-11(2)$ & $7(3)$ & $-7(3)$ & $8(4)$ & $-7(6)$ \\
\hline RI-MP2 & $4(3)$ & $-3(4)$ & $5(5)$ & $5(5)$ & $17(7)$ & $17(7)$ & $8(7)$ & $5(9)$ \\
\hline PBE-XDM & $10(13)$ & $-8(15)$ & $7(8)$ & $-6(9)$ & $16(8)$ & $12(14)$ & $10(10)$ & $-3(13)$ \\
\hline SAPT2+ & $4(2)$ & $4(3)$ & $9(3)$ & $9(3)$ & $20(5)$ & $20(5)$ & $10(7)$ & $10(7)$ \\
\hline DSD-PBEP86 & $5(5)$ & $-5(5)$ & $12(2)$ & $12(2)$ & $28(1)$ & $28(1)$ & $13(9)$ & $10(12)$ \\
\hline BLYP-XDM & $9(7)$ & $5(10)$ & $10(4)$ & $10(4)$ & $26(3)$ & $26(3)$ & $13(8)$ & $12(10)$ \\
\hline M06L & $9(12)$ & $8(13)$ & $13(13)$ & $13(13)$ & 19(15) & 19(15) & $13(13)$ & $13(13)$ \\
\hline B3LYP-NL & $22(10)$ & $22(10)$ & $7(4)$ & $-5(6)$ & $20(4)$ & $-20(4)$ & 14(9) & $-1(17)$ \\
\hline $\operatorname{CCSD}(\mathrm{T}) / \mathrm{CBS}(\mathrm{MP} 2)$ & $3(2)$ & $-2(3)$ & $19(5)$ & $-19(5)$ & 19(3) & $-19(3)$ & 14(9) & $-14(9)$ \\
\hline PBE-D3 & $22(16)$ & $-22(16)$ & $8(8)$ & $1(11)$ & $23(14)$ & $22(15)$ & $15(14)$ & $-1(21)$ \\
\hline GFN2-xTB & $24(13)$ & $23(14)$ & $9(8)$ & $-6(10)$ & 19(12) & $2(24)$ & $15(12)$ & $4(19)$ \\
\hline M06-2X-D3 & $6(4)$ & $-4(6)$ & $15(5)$ & $15(6)$ & $27(5)$ & $27(5)$ & $15(9)$ & $12(13)$ \\
\hline BLYP-NL & $28(18)$ & $28(18)$ & $9(6)$ & $-5(10)$ & $20(6)$ & $-20(6)$ & $17(13)$ & $1(22)$ \\
\hline B3LYP-dDsC & $22(15)$ & $-20(18)$ & $8(7)$ & $7(8)$ & $29(8)$ & $29(8)$ & $17(13)$ & $4(21)$ \\
\hline NormalPNO & $4(4)$ & $4(4)$ & $18(4)$ & $18(4)$ & $33(5)$ & $33(5)$ & $17(11)$ & $17(11)$ \\
\hline M06L-D3 & $18(9)$ & $-15(14)$ & $19(6)$ & $-17(11)$ & $14(4)$ & $-3(15)$ & $18(7)$ & $-14(14)$ \\
\hline B2PLYP-D3 & $11(4)$ & $11(4)$ & $18(3)$ & $18(3)$ & $30(2)$ & $30(2)$ & $18(7)$ & $18(7)$ \\
\hline TPSS-D3 & $10(10)$ & $-3(14)$ & $20(8)$ & 19(9) & $39(18)$ & $39(18)$ & $21(15)$ & $17(19)$ \\
\hline B3LYP-D3 & $3(1)$ & $1(3)$ & $23(3)$ & $23(3)$ & $40(3)$ & $40(3)$ & $21(14)$ & $20(15)$ \\
\hline GFN1-xTB & $27(18)$ & $27(18)$ & $14(5)$ & $14(5)$ & $37(5)$ & $37(5)$ & $23(14)$ & $23(14)$ \\
\hline$\omega B 97 X-D 3$ & $13(7)$ & $-13(7)$ & $20(5)$ & $20(5)$ & $65(13)$ & $65(13)$ & $28(21)$ & $20(29)$ \\
\hline vdW-DF10 & $14(9)$ & $-14(9)$ & $36(7)$ & $-36(7)$ & $32(12)$ & $-32(12)$ & $29(13)$ & $-29(13)$ \\
\hline PBE-dDsC & $37(6)$ & $-37(6)$ & $34(6)$ & $-34(6)$ & $22(6)$ & $-22(6)$ & $32(8)$ & $-32(8)$ \\
\hline BLYP-D3 & $12(9)$ & $12(9)$ & $36(6)$ & $36(6)$ & $58(5)$ & $58(5)$ & $34(18)$ & $34(18)$ \\
\hline M06-2X & $16(6)$ & $16(6)$ & $42(10)$ & $42(10)$ & $46(9)$ & $46(9)$ & $35(15)$ & $35(15)$ \\
\hline B97-D3 & $18(9)$ & $-18(9)$ & $33(8)$ & $33(8)$ & $67(22)$ & $67(22)$ & $36(21)$ & $26(33)$ \\
\hline SCS-MP2 & $38(3)$ & $38(3)$ & $41(6)$ & $41(6)$ & $50(6)$ & $50(6)$ & $42(7)$ & $42(7)$ \\
\hline sSAPTO & $53(13)$ & $53(13)$ & $64(11)$ & $64(11)$ & 79(14) & $79(14)$ & $64(15)$ & $64(15)$ \\
\hline
\end{tabular}

Table 2 shows that B97M-V performs best across all methods together with DLPNO-CCSD(T)/CBS in conjunction with ExtremePNO parameters (Supporting Information). In general, DLPNO-CCSD(T)/CBS methods with TightPNO parameters and beyond provide good results. However, MP2 ${ }^{40} / C B S$ energies are not considerably worse than DLPNO-CCSD(T)/CBS numbers using TightPNO parameters, at a significantly smaller computational cost (especially when RI-MP2 $2^{41,42}$ is used). Additionally, B97M-V and $\omega B 97 M-V$ are very considerably computationally less expensive compared to the DLPNO-CCSD(T) methods but perform equally well. It is also worth to mention the relatively good performance of all 
the XDM-corrected ${ }^{36-39}$ methods using various functionals (i.e. B3LYP, PBE and BLYP, cf. Table 2). B3LYP-XDM is only outperformed by DLPNO-CCSD(T)/CBS in conjunction with ExtremePNO parameters, which is very considerably more expensive, as well as B97M-V and $\omega B 97 \mathrm{M}-\mathrm{V}$. Overall, $B 97 \mathrm{M}-\mathrm{V}$ is the most efficient approach to compute accurate interaction energies across all the classes of complexes, especially for HF and FF, with B3LYP-XDM being slightly better for HF and B3LYP-D3 being most efficient for $\mathbf{H H}$.

Energy Decomposition Analysis. After benchmarking, we moved on to carry out energy decomposition analysis (EDA) using symmetry adapted perturbation theory (SAPT). ${ }^{43-46}$ The corresponding results are illustrated in Figure 1.

a) All energy components.

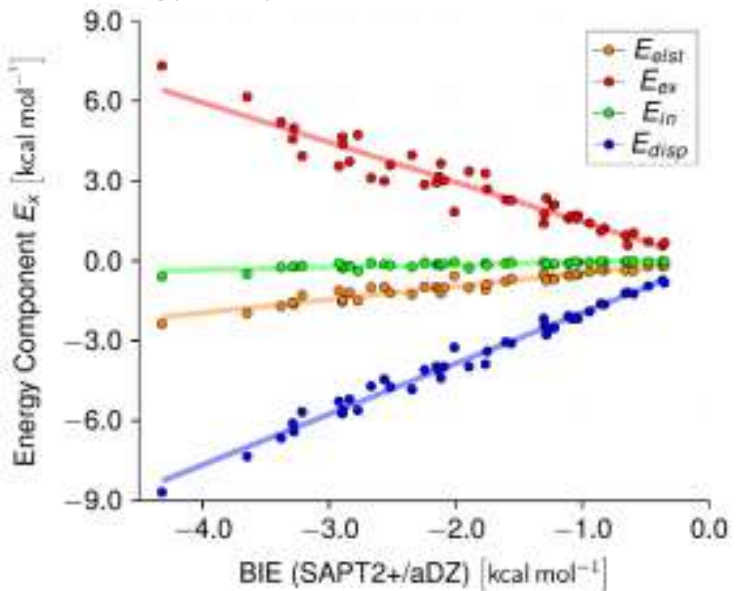

b) Only exchange, dispersion and their sum.

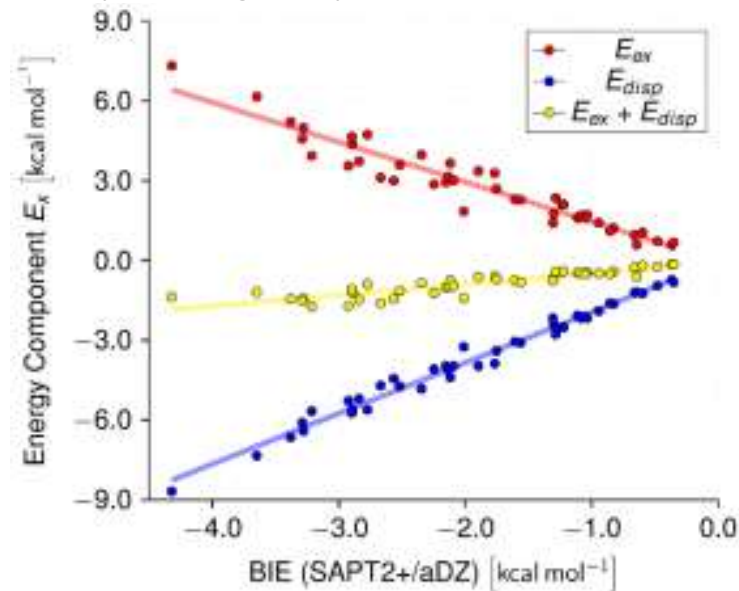

Figure 1. Interaction energy decomposition of the molecular complexes using SAPT2+ illustrated by plotting the energy components against the corresponding overall BIEs. a) Pauli repulsion and dispersion show the biggest change with interaction energy. b) The sum of Pauli repulsion and dispersion is overall still attractive.

When plotting the SAPT2+ energy components against the corresponding BIEs for all the test systems (Figure 1a) the relative importance of the energy components is essentially invariant, irrespective of whether the system belongs to $\mathbf{H H}, \mathbf{H F}$ or FF. Figure $1 \mathrm{a}$ also shows that induction is almost zero across all the complexes whereas the electrostatic component does show a small but significant contribution. Exchange and dispersion are much larger in magnitude and steeper in slope but are of opposite sign in both energy and slope. Additionally, the sum of exchange and dispersion is overall attractive for all complexes showing that dispersion outweighs repulsive exchange (Figure 1b). Looking into the sum of exchange and dispersion in the three subsystems $\mathbf{H H}, \mathbf{H F}$ and $\mathbf{F F}$ separately, it can be seen that $\mathbf{F F}$ provide overall more stabilization due to dispersion than the corresponding $\mathbf{H F}$ or $\mathbf{H H}$ (Details in the Supporting Information), at least in the SAPT partitioning.

Next, we compared dispersion from SAPT and LED ${ }^{47,48}$ and found an excellent correlation (Supporting Information). Additionally, we used LED-DLPNO-CCSD(T)/QZ to gain insight into the contributions of specific groups and atoms towards dispersion by dissecting the dimers into fragments containing only one atom type (Scheme 1, Details in the Supporting Information). 
Scheme 1. Decomposition of dispersion energy of complex A-B into contributions of atoms and bonds.

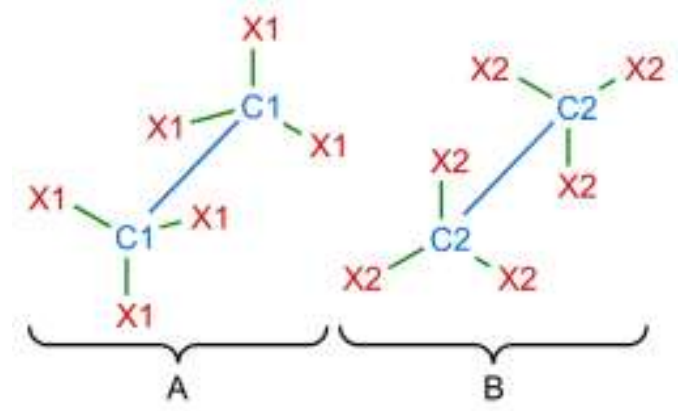

$$
\begin{aligned}
E_{\text {disp }}(A-B)= & E(C 1-C 2)+E(C 1-X 2)+E(X 1-C 2)+E(X 1-X 2) \\
& +E(C 1-C X 2)+E(C X 1-C 2)+E(X 1-C X 2) \\
& +E(C X 1-X 2)+E(C X 1-C X 2)
\end{aligned}
$$

The overall intermolecular dispersion is dissected into the interactions of the carbon chains, the $\mathrm{C}-\mathrm{X}$ bonds and the $X$ atoms with each other (where $X=H, F$ ) resulting in nine terms overall (Scheme 1 ). Looking into the relative importance of these terms for each of the three complex subclasses revealed differing main contributors (Figure 2).

a) 1-HH

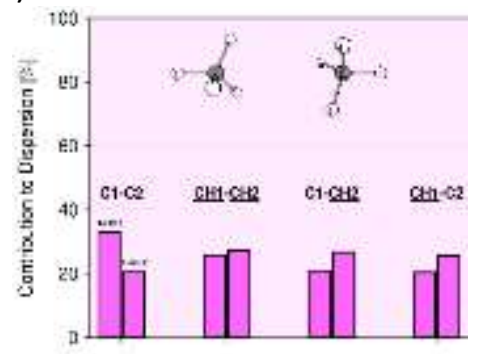

b) 1-HF

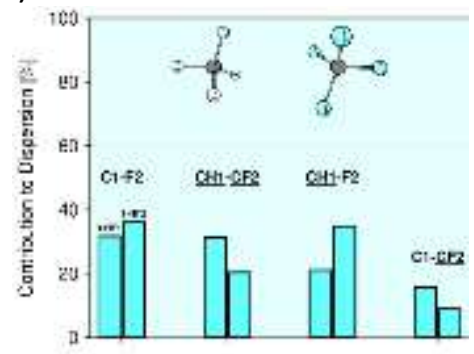

c) 1-FF

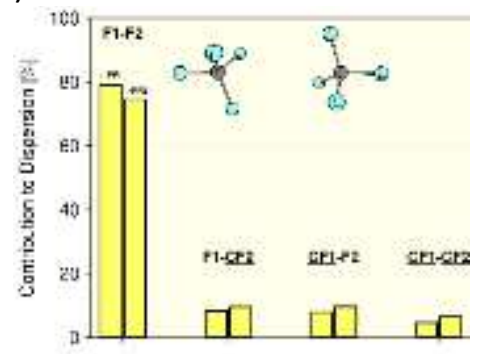

Figure 2. Decomposition of dispersion using LED into contributions from atoms and bonds for methane complexes divided into the subclasses a) HH, b) HF and c) FF. Only non-zero contributions are depicted.

Figure 2 shows that while in $\mathbf{1 - H}$ fragments the dispersion originates from the carbon atoms and the $\mathrm{C}-\mathrm{H}$ bonds, the dispersion in 1-F fragments originates from the fluorine atoms and the $\mathrm{C}-\mathrm{F}$ bonds. Consequently, almost all of the intermolecular dispersion in 1-FF originates from interactions between fluorine atoms, while in $\mathbf{1 - H H}$ and $\mathbf{1 - H F}$ it is distributed more equally between different interactions. When looking at model systems with longer carbon chains, for $\mathbf{H H}$ and $\mathbf{H F}$ the relative contributions of the different interactions do not change significantly (Supporting Information). However, for FF the relative contribution of the F1-F2 interactions decreases as the chain length is increased and correlates linearly with the relative molar fluorine content (Supporting Information).

An alternative approach to interrogate the origin of dispersion is provided by the dispersion interaction density (DID) developed recently by Wuttke and Mata. ${ }^{49}$ DIDs are determined on the basis of local orbital spaces using wavefunction methods and extract the origin of dispersion as a 3D scalar field in molecules. ${ }^{49}$ This scalar field can be visualized using voxel plots showing the extent of dispersion provided by a specific region in a molecule. The DID plots of 1-HH1, 1-HF1 and 1-FF1 are depicted in Figure 3 (DID plots of additional complexes are provided in the Supporting Information). They show again that dispersion in $\mathbf{1 - H}$ originates mainly from the electron density of the $\mathrm{C}-\mathrm{H}$ bonds and the carbon atom and in 1-F mainly from the contacting fluorine atoms. This is fully consistent with the results from LED (vide supra). 
a) 1-HH1.

b) 1-HF1.

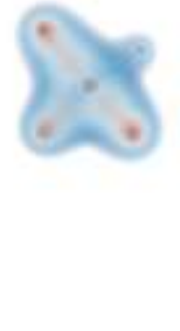

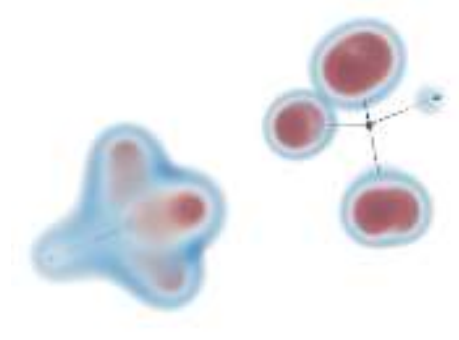

c) 1-FF2.

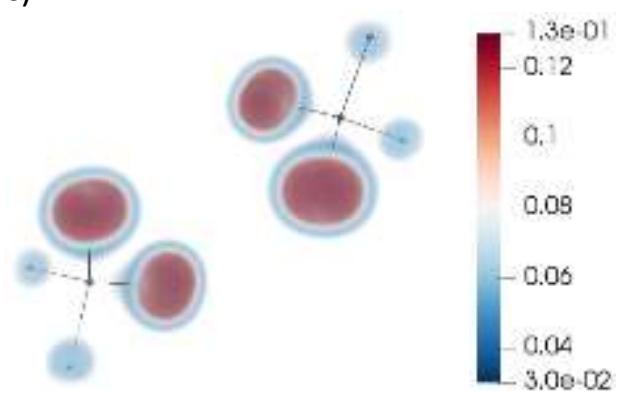

Figure 3. DID plots of complexes of 1-H and 1-F. a) 1-HH1. b) 1-HF1. c) 1-FF2. Red depicts strong contributions towards dispersion, blue depicts weak contributions.

Yet another approach to gauge the origin of dispersion is to estimate atomic polarizabilities using a quantum theory of atoms in molecules (QTAIM) approach. ${ }^{50}$ We employed the software PolaBer ${ }^{51}$ which uses the partitioning of molecular (hyper)polarizabilities proposed by Keith and implemented in AIMAII. ${ }^{22}$ The atomic polarizabilities were determined from MP2/def2-TZVPPD relaxed electron densities and visualized by tri-axial ellipsoids. The three axes of the ellipsoids represent the anisotropic polarizabilities with their volumes proportional to the corresponding isotropic values (Figure 4). The atomic polarizabilities in 1-H and 1-F are compared to the isolated ground-state values in Table 3.

a) $\mathbf{1 - H}$

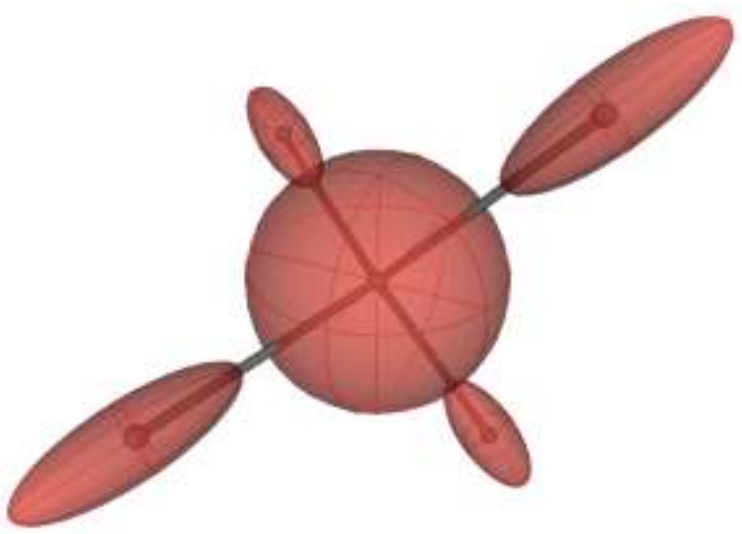

b) 1-F

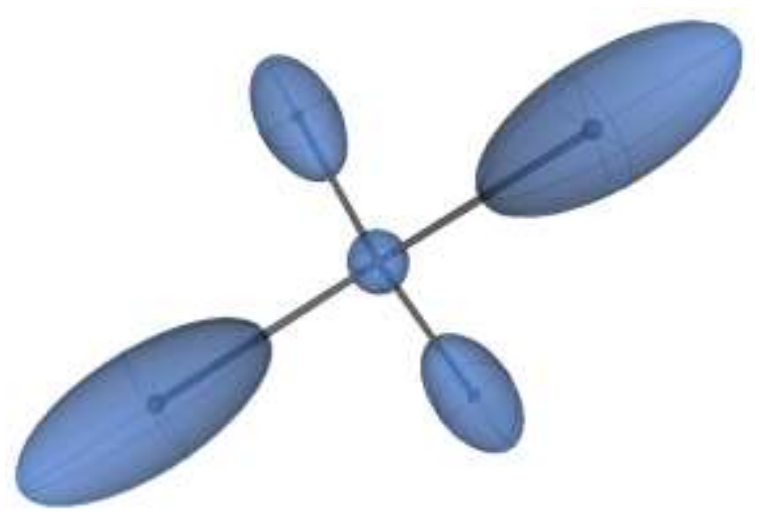

Figure 4. PolaBer plots visualizing the atomic polarizabilities of a) $\mathbf{1 - H}$ and b) $\mathbf{1 - F}$ using tri-axial ellipsoids representing the anisotropy with volumes depicting the isotropic values.

Table 3. Polarizabilities of carbon, hydrogen and fluorine as free atoms and in $\mathrm{CH}_{4}$ or $\mathrm{CF}_{4}$ molecules, respectively, as obtained from PolaBer.

\begin{tabular}{|c|c|c|c|}
\hline \multirow{2}{*}{ Atom Type } & \multicolumn{3}{|c|}{ Polarizabilities $\left[\AA^{3}\right]$} \\
\hline & Free Atom & $\mathrm{CH}_{4}$ & $\mathrm{CF}_{4}$ \\
\hline C & $1.76^{53}$ & 0.867 & 0.283 \\
\hline $\mathrm{H}$ & $0.667^{54}$ & 0.376 & - \\
\hline $\mathrm{F}$ & $0.557^{53}$ & - & 0.618 \\
\hline
\end{tabular}

The results show that in 1-H the atomic polarizability of the carbon atom is the biggest while in 1-F the atomic polarizabilities of the fluorine atoms are larger (cf. Figure 4). This is in contrast to the relative magnitude of the polarizabilities of atomic carbon and fluorine (atomic carbon is more polarizable than atomic fluorine, cf. Table 3). ${ }^{55}$ This suggests that London dispersion in FF systems originates mainly from fluorine atoms because not only are they most polarizable, they are also on the outside of the molecules. 
Simple Interaction Models. After EDA, we were interested in using simple interaction models to describe trends in dispersion. The idea was to use the London dispersion formula ${ }^{56}$ (Equation 6) to approximate the interaction between the interacting molecules, which would effectively be treated as interacting spheres having the properties of the whole molecules:

$$
E_{\text {disp }}=-\frac{3}{2} \frac{I_{1} I_{2}}{I_{1}+I_{2}} \frac{\alpha_{1} \alpha_{2}}{R^{6}}
$$

For this admittedly crude model, we used MP2/CBS(34) ionization potentials and MP2/def2-TZVPD molecular polarizabilities. When using the average distance of the two carbon chains the resulting energies correlated poorly to benchmark BIEs (Supporting Information). Using the $\mathrm{R}^{-6}$-weighted distance between the atoms mainly responsible for dispersion, i.e. using carbon atoms in $\mathbf{H}$ and fluorine atoms in $\mathbf{F}$, a linear correlation was obtained (cf. Figure 5a).

a) Molecular London Dispersion Model.

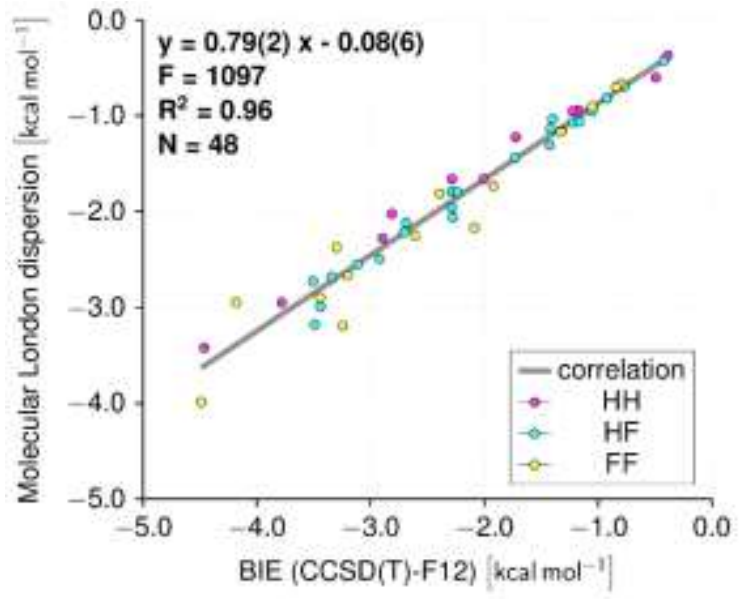

b) Atom-pairwise London Dispersion Model.

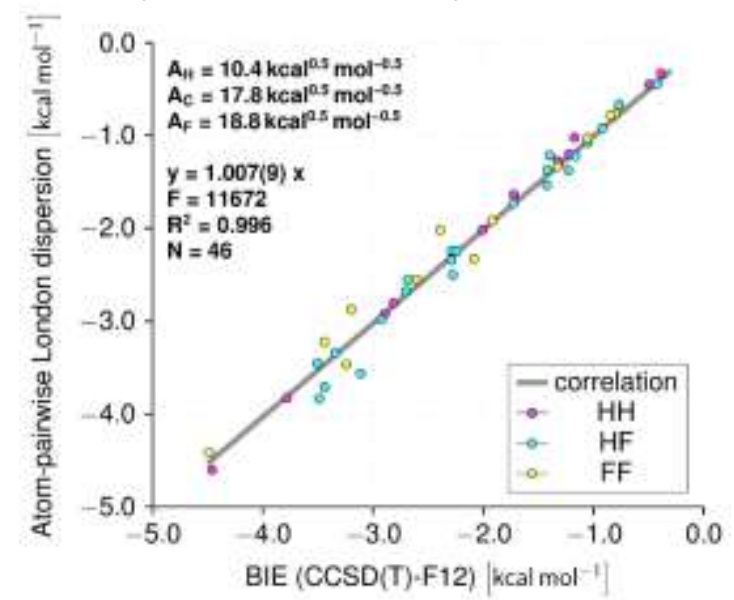

Figure 5. a) Intermolecular dispersion was estimated with the London dispersion formula (Equation 6) using molecular ionization potentials and molecular polarizabilities and compared to CCSD(T)-F12 BIEs. b) Intermolecular dispersion was estimated using atom-pairwise London dispersion by optimizing atom-type-dependent pre-factors A (cf. Equation 8) to reproduce the benchmark BIEs. The corresponding best set of $A$ parameters is provided in the top-left of the diagram.

As an alternative, we also applied an atomistic interaction model using the London dispersion formula (Equation 6), very much akin to the approach of atom-pairwise dispersion corrections. ${ }^{57} \mathrm{We}$ determined all atom-pairwise contributions to the overall BIEs using the atomic polarizabilities of the isolated non-interacting molecules as obtained from PolaBer. Then, we optimized the atom-type dependent parameters for the ionization potential contributions to best reproduce the benchmark energies. To do that, we used an approximation of the London dispersion formula explained in the following section simplifying the ionization potential pre-factor (which we term $A_{12}$ ) as product of atom-type dependent pre-factors $A_{1}$ and $A_{2}$ (cf. right-hand side of Equation 8). The motivation for using this approximation is explained in the following section. The corresponding results are depicted in Figure 5b. It shows that with the optimized set of A pre-factors the overall BIEs are reproduced well across all the complexes. Additionally, based on this model, we decomposed the overall dispersion into contributions of atom type pairs. The corresponding results provide even quantitatively comparable results to LED (Details in the Supporting Information). 
Interaction Descriptors. After having looked into interaction models, we were interested in simple interaction descriptors that would characterize the dispersive interaction capabilities of $\mathbf{H}$ and $\mathbf{F}$. Dunitz proposed the ratio $Q$ of polarizability $\alpha$ and volume $V$, a dimensionless quantity, as a simple measure to quantify dispersion of atoms and molecules: ${ }^{3}$

$$
Q=\frac{\alpha}{V}
$$

However, as he also acknowledged, ${ }^{3}$ he ignored the linear dimensions of the molecules and did not account for the actual polarizabilities of the atoms in the molecules and their spatial distribution. When comparing the definition of $Q$ (Equation 7) and the London dispersion formula (Equation 6) one can see that the dimensionless fraction of the product of polarizabilities $\alpha_{1}$ and $\alpha_{2}$ divided by the sixth power of the distance $R$ is effectively the product of two $Q$ values $\left(Q_{1}\right.$ and $\left.Q_{2}\right)$ :

$$
E_{\text {disp }}=-\frac{3}{2} \frac{I_{1} I_{2}}{I_{1}+I_{2}} \frac{\alpha_{1} \alpha_{2}}{R^{6}}=-\frac{3}{2} \frac{I_{1} I_{2}}{I_{1}+I_{2}} \frac{\alpha_{1} \alpha_{2}}{R^{3} R^{3}}=A_{12} Q_{1} Q_{2} \approx A_{1} A_{2} Q_{1} Q_{2}
$$

From this comparison, it is clear that the volume in Equation 7 should not be an atomic or a molecular volume but rather the "interaction volume" $R^{3}$, i.e. a volume derived from the interaction distance of the atoms. Using "interaction volumes" one explicitly accounts for the linear dimensions of molecules and the spatial distribution of polarizability therein. Therefore, we defined an alternative atomic $Q$ metric called $Q_{\text {atom }}$ as the fraction of the atomic polarizability of an atom in a molecule and the interaction volume $R^{3}$ of the atom with respect to a specific point in space. Hence, $Q_{\text {atom }}$ is a function of the reference point used for its computation. It quantifies the dispersive interaction capability of specific atoms in a molecule with respect to that point in space and explicitly accounts for the position of an atom in a molecule. Using this definition, we computed the maximum $Q_{\text {atom }}$ of hydrogen, carbon and fluorine in $\mathbf{H}$ and $\mathbf{F}$ of different carbon chain lengths with respect to points on their van der Waals surface (Details in the Supporting Information). The corresponding results are depicted in Figure 6a showing that the maximum $\mathbf{Q}_{\text {atom }}$ of fluorine in $\mathbf{F}$ is larger than the value of either hydrogen or carbon in $\mathbf{H}$. However, in $\mathbf{F}$ the value of carbon is reduced significantly compared to $\mathbf{H}$.
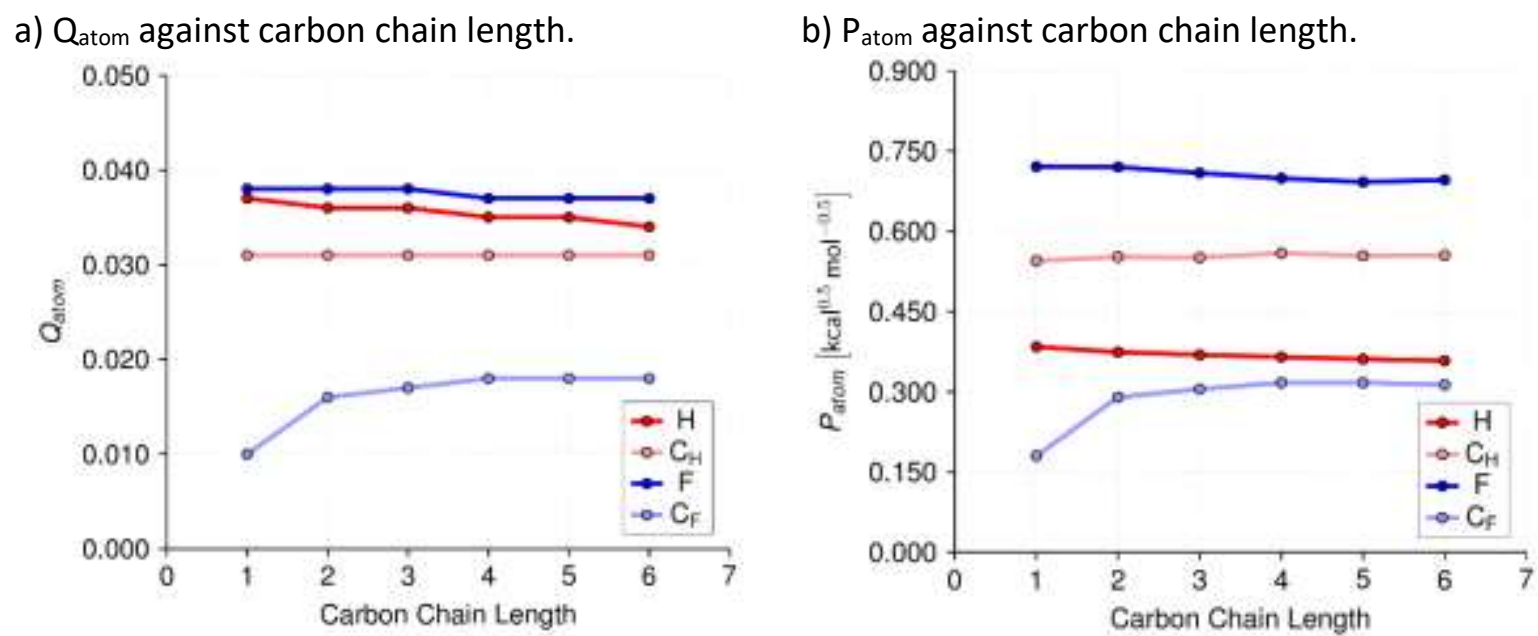

Figure 6. a) Maximum $Q_{\text {atom }}$ values on the van der Waals surface for all atom types in $\mathbf{H}$ and $\mathbf{F}$ (cf. Equation 7). b) Maximum $P_{\text {atom }}$ values on the van der Waals surface for all atom types in $\mathbf{H}$ and $\mathbf{F}$ (cf. Equation 9). $C_{H}$ refers to carbon atoms in $\mathrm{H}, \mathrm{C}_{\mathrm{F}}$ to carbon atoms in $\mathbf{F}$. 
To judge also the total dispersive interaction capability of a molecule we defined a second type of $Q$ metric termed $Q_{\text {tot }}$ as the sum of all $Q_{\text {atom }}$ of a molecule and hence it quantifies the dispersive interaction capability of a molecule with respect to a specific point in space. This metric explicitly accounts for the linear dimensions and the spatial distribution of polarizability of a molecule. Using this definition, we computed the average $Q_{\text {tot }}$ of $\mathbf{H}$ and $\mathbf{F}$ with respect to points on their van der Waals surface (Details in the Supporting Information). We find that $Q_{\text {tot }}$ of $\mathbf{F}$ having one to six carbon atoms is about $83 \%$ of $Q_{\text {tot }}$ of the corresponding $\mathbf{H}$. The main reasons for the lower $Q_{\text {tot }}$ in $\mathbf{F}$ are the significantly reduced $\mathbf{Q}_{\text {atom }}$ of carbon in $\mathbf{F}$ compared to $\mathbf{H}$ and the larger size of fluorine compared to hydrogen. Using Dunitz' Q metric (i.e. using molecular polarizability and molecular volume) one would estimate the corresponding ratio to be only 64\%. These results show that Dunitz' $Q$ metric significantly underestimates the interaction capabilities of $\mathbf{F}$ and, hence, stress the importance of accounting for linear dimensions and spatial polarizability distributions.

These results also show that, from $\mathbf{Q}_{\text {tot }}$, one would estimate the dispersive interaction capabilities of $\mathbf{F}$ to be generally weaker than $\mathbf{H}$. However, when looking again at the London dispersion formula (Equation 8, vide supra) we can see that in order to determine the dispersion energy we need the product of $Q_{1}$ and $Q_{2}$ as well as the pre-factor $A_{12}$. In the London dispersion formula, the pre-factor is estimated from the ionization potentials $I_{1}$ and $I_{2}$ of the interacting atoms. When comparing the dispersive interaction capability of different classes of compounds, which likely have distinct atomic ionization potentials, the pre-factor needs to be taken into account. We assumed that $A_{12}$ can be separated into the product of atom-type-dependent factors $A_{1}$ and $A_{2}$ (cf. Equation 8, right-hand side). We determined these factors for carbon, hydrogen and fluorine by reproducing the benchmark BIEs of all our model systems using the atom-pairwise London dispersion model (vide supra). With the optimized parameters, the benchmark energies could be reproduced with an overall MADrel of $5 \%$ and all subsystems were reproduced equally well. Using these atom-type-dependent factors, we defined a new measure to quantify dispersive interaction capabilities termed $P$. It is the product of the atomtype-dependent factor $A$ and $Q$ and has the dimension of the square root of energy. It is defined such that the dispersion energy could be estimated as the product of two $P$ measures, $P_{1}$ and $P_{2}$ (cf. righthand side of Equation 8):

$$
P=A Q=A \frac{\alpha}{V}
$$

Analogously to $Q_{\text {atom }}$ and $Q_{\text {tot }}$, we defined $P_{\text {atom }}$ and $P_{\text {tot }}$ and determined $P_{\text {atom }}$ values of hydrogen, carbon and fluorine atoms in $\mathbf{H}$ and $\mathbf{F}$. The results are depicted in Figure $6 \mathrm{~b}$. Compared to the relative magnitudes of the corresponding $Q_{\text {atom }}$ values, the $P_{\text {atom }}$ values of carbon and fluorine are elevated, the values of hydrogen reduced (the ratio of the pre-factors is $A_{H}: A_{C}: A_{F}=0.55: 0.94: 1.0$ ). In addition, we also determined the corresponding $P_{\text {tot }}$ values and found that the average value of $F$ is about $116 \%$ of the corresponding value of $\mathbf{H}$. Furthermore, the ratios of $\mathbf{P}_{\text {tot }}$ of $\mathbf{F}$ and $\mathbf{H}$ decrease linearly with the relative molar carbon content (Details in the Supporting Information). However, since the relative molar carbon content in $\mathbf{H}$ and $\mathbf{F}$ is always between a fifth and a third, based on this linear correlation, the lowest possible ratio of the $\mathbf{P}_{\text {tot }}$ of $\mathbf{F}$ and $\mathbf{H}$ is $108 \%$. This suggests that, at least in the respective extended conformer, no fully saturated $\mathbf{F}$ with a weaker intrinsic dispersion interaction capability than the analogous $\mathbf{H}$ can exist.

It should be noted that the difference in $\mathbf{P}_{\text {tot }}$ of $\mathbf{H}$ and $\mathbf{F}$ is only properly reflected in the interaction energies of 1-FF and 1-HF compared to 1-HH but not in the complexes with longer carbon chains. This suggests that the main reason for $\mathbf{F F}$ and $\mathbf{H H}$ having about equal interaction energies in longer systems 
(and $\mathbf{H F}$ having weaker interaction energies) is not the respective intrinsic capability for dispersive interactions but rather the interaction geometries. Therefore, we will investigate them in more detail in the following section.

Interaction Geometries. We already used DID plots to visualize the origin of disperion in the intermolecular complexes (vide supra). An alternative way to visualize non-covalent interactions ( $\mathrm{NCls)}$ is provided by the $\mathrm{NCl}$ plots. ${ }^{58}$ They are based on the analysis of electron densities and their derivatives and provide a visualization of interaction surfaces together with information about which portions of the surface are attractive or repulsive. ${ }^{58}$ Notably, they are based solely on electron density and cannot distinguish between different types of van der Waals interactions. ${ }^{58}$ They provide orthogonal information compared to DID plots as they allow assessing how well molecular complexes are aligned. Figure 7 shows 3D NCl plots of complexes of $\mathbf{5 - H}$ and $\mathbf{5 - F}$. The striking difference between the interaction surfaces in $\mathbf{5 - H H 1}, \mathbf{5}-\mathbf{H F} \mathbf{4}$ and $\mathbf{5 - F F \mathbf { 1 }}$ is the very regular shape in $\mathbf{5 - H H} \mathbf{H}$ compared to the increasingly irregular shapes observed in 5-HF4 and 5-FF1. Only certain portions of the interaction surfaces of 5-HF4 and 5-FF1 show strong interactions (attractive or repulsive) while in 5-HH1 strong interactions are distributed uniformly over the whole intermolecular interface. These observations suggest that the preferred intramolecular geometries of $\mathbf{F}$ are unsuitable for intermolecular interactions with either $\mathbf{H}$ or $\mathbf{F}$ which results in lower interaction energies for these complexes than would otherwise be attainable.

a) 5-HH1

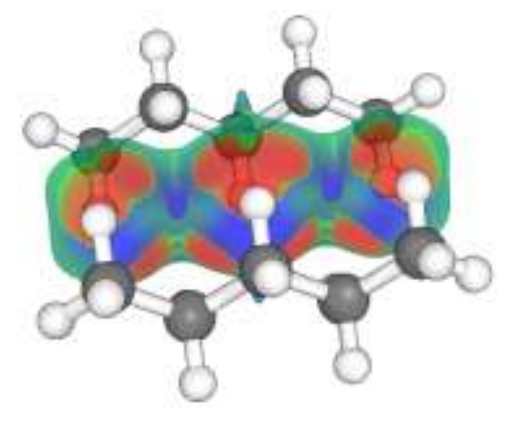

b) 5-HF4

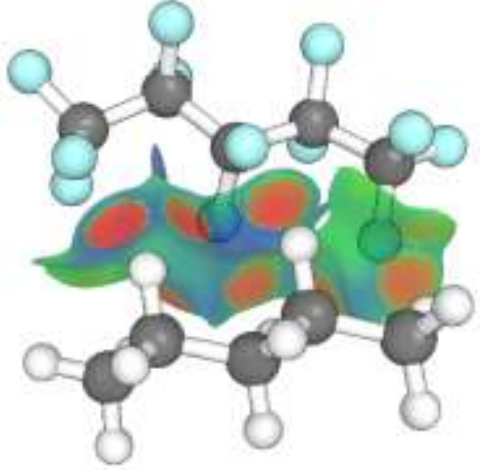

c) 5-FF1

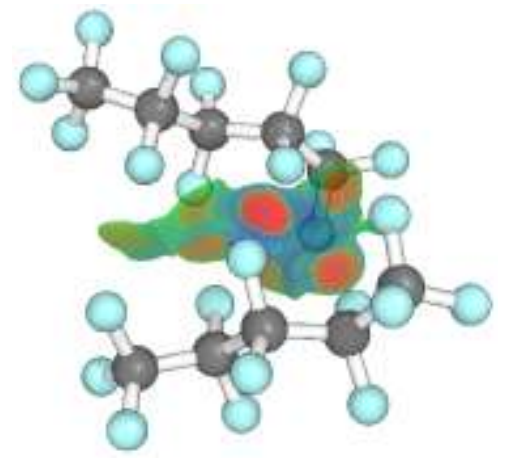

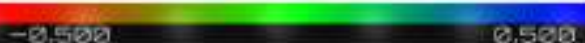

Figure 7. 3D NCl plots of complexes of pentanes: a) 5-HH1. b) 5-HF4. c) 5-FF1. Red depicts repulsive interactions, green depicts neither attractive nor repulsive interactions, and blue depicts attractive interactions. The selected conformers have the strongest BIEs of the corresponding type of complex.

Consequently, we sought to collect further evidence about the interaction geometries. Therefore, we looked into the scaling of the total surface area and the interaction surface area of the different complex classes. The total surfaces of $\mathbf{H}$ and $\mathbf{F}$ and the interaction surfaces of $\mathbf{H H}, \mathbf{H F}$ and $\mathbf{F F}$ increase linearly with the carbon chain length (Details in the Supporting Information). Using simple geometric models of cuboids to represent the interacting molecules we deduced that in FF only about $81 \%$ and in $\mathbf{H F}$ only about $85 \%$ of the additional interaction surface is used for interactions when the chains are elongated (Details in the Supporting Information). These results corroborate the visual impression of Figure $\mathbf{7}$ and confirm that in both $\mathbf{H F}$ and $\mathbf{F F}$ not all the potential surface is used for intermolecular interactions.

To obtain more direct evidence for the differences in interaction geometries of $\mathbf{H H}, \mathbf{H F}$ and $\mathbf{F F}$ we introduced a new geometric measure to characterize the alignment between two interacting fragments, which we term the number of van der Waals contacts $\left(n_{\text {contact }}\right)$. We defined one contact as 
two atoms at a distance equal to the sum of their van der Waals radii ( $\left.R_{v d w}\right)$. To have a continuous scale for atoms that are closer or farther apart than $R_{v d w}$ and to account for the distance dependence of van der Waals interactions we defined the number of contacts between two atoms as follows:

$$
n_{\text {contacts }}=\frac{R_{v d W}{ }^{6}}{R^{6}}
$$

The number of contacts between two interacting molecules then is the sum of all atom-pairwise contacts. We computed this new geometric measure for all complexes under study and investigated it as a function of carbon chain length (Figure 8a). There are several things to be noted. First, all the complexes with one carbon atom in the monomers have about the same number of contacts. Secondly, all classes of intermolecular complexes show a linear correlation of $n_{\text {contacts }}$ with the number of carbon atoms. Additionally, $\mathbf{H H}$ have a significantly larger increase of the number of contacts with the carbon chain length than either HF or FF. Furthermore, HF shows a marginally larger increase of the number of contacts with the carbon chain length than FF. These results provide additional evidence for the poor interaction geometries in $\mathbf{H F}$ and FF. Moreover, to test our newly introduced parameters, we correlated the BIEs of all the complexes against the product of $n_{\text {contacts }}$ and the $P_{\text {tot }}$ values of the respective interacting molecules and observed a reasonable correlation (cf. Figure $8 b$ ).

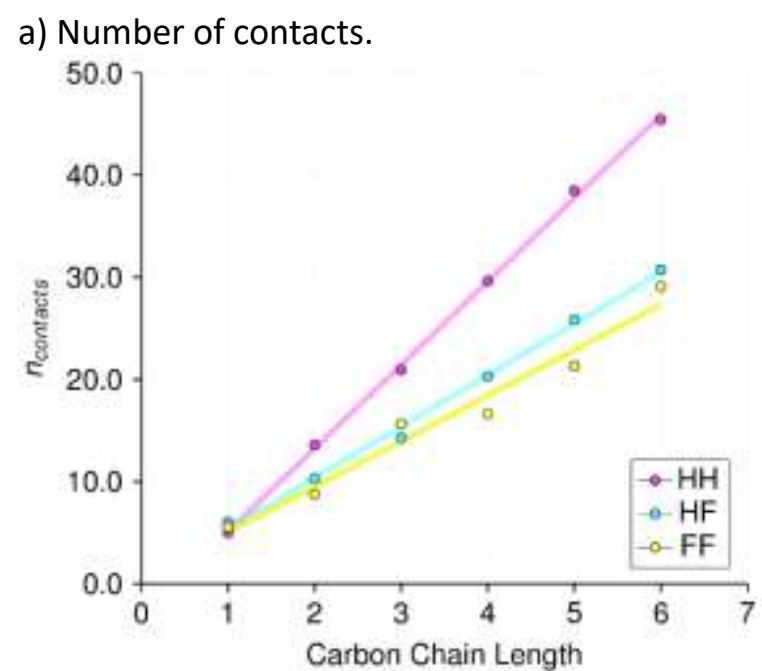

b) Correlation of BIE against $n_{\text {contacts }} P_{\text {tot }, 1} P_{\text {tot }, 2}$.

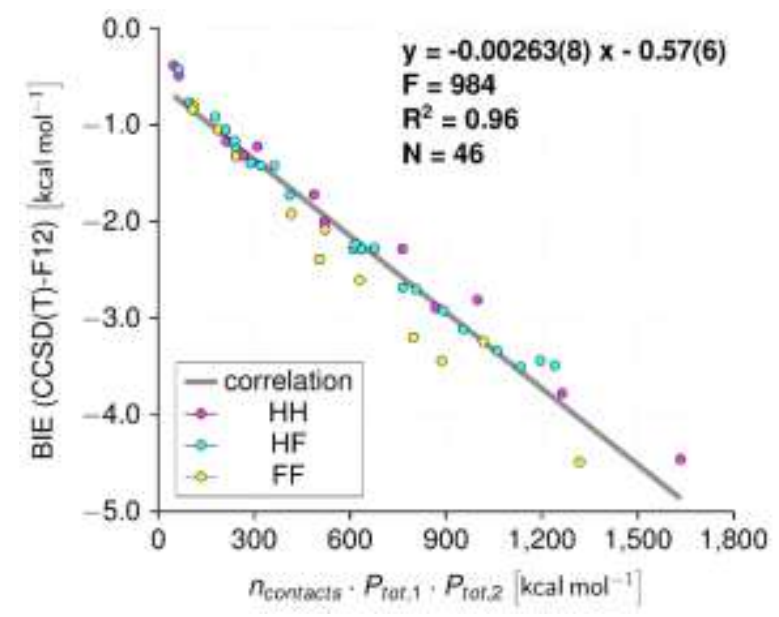

Figure 8. a) Number of van der Waals contacts ( $\left.n_{\text {contacts }}\right)$ in $\mathbf{H H}, \mathbf{H F}$ and $\mathbf{F F}$ as a function of carbon chain length. b) BIE of the intermolecular complexes correlated against the product of $n_{\text {contacts }}$ and the $P_{\text {tot }}$ values of the respective interacting molecules.

Computed Microscopic and Macroscopic Mixing Behavior. Having established reliable methods to compute the interaction energies of $\mathbf{H}$ and $\mathbf{F}$ we compared the relative BAEs of $\mathbf{H H}, \mathbf{H F}$ and $\mathbf{F F}$ as a function of carbon chain length (Figure 9a). As the carbon chain length is increased, the relative BAEs of both $\mathbf{H F}$ and $\mathbf{F F}$ get weaker compared to $\mathbf{H H}$. The effect is most pronounced in $\mathbf{H F}$ and somewhat less so in FF. Furthermore, assuming that $\mathbf{H H}, \mathbf{H F}$ and $\mathbf{F F}$ have comparable interaction geometries both in the molecular complexes and in the bulk phase (i.e. including packing effects), we estimated the bulk BAEs in pure $\mathbf{H}$, pure $\mathbf{F}$, as well as in mixed bulk phases of $\mathbf{H}$ and $\mathbf{F}$. To do that, we multiplied the association energies by the average coordination numbers derived from the molecular and the interaction surfaces, which is a treatment of the bulk phase at the level of Flory-Huggins solution theory (Details in the Supporting Information). ${ }^{59,60}$ The corresponding results are illustrated in Figure 9b. 
a) Relative Microscopic BAEs.

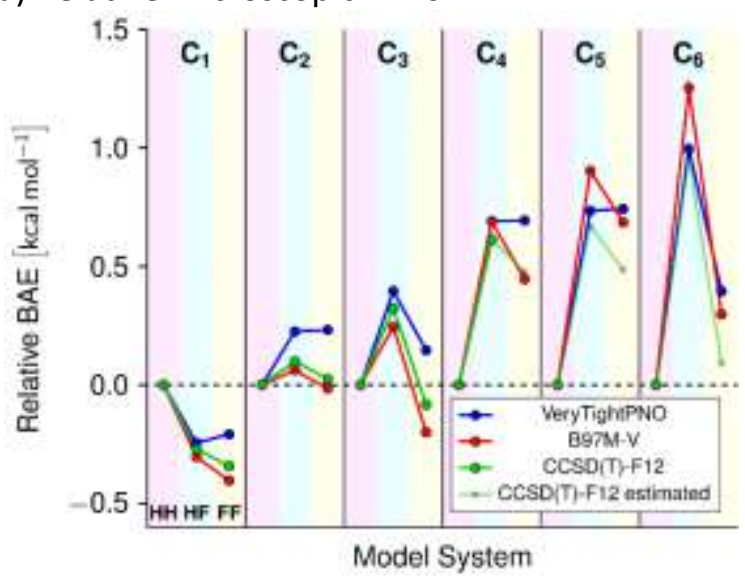

b) Relative Macroscopic BAEs.

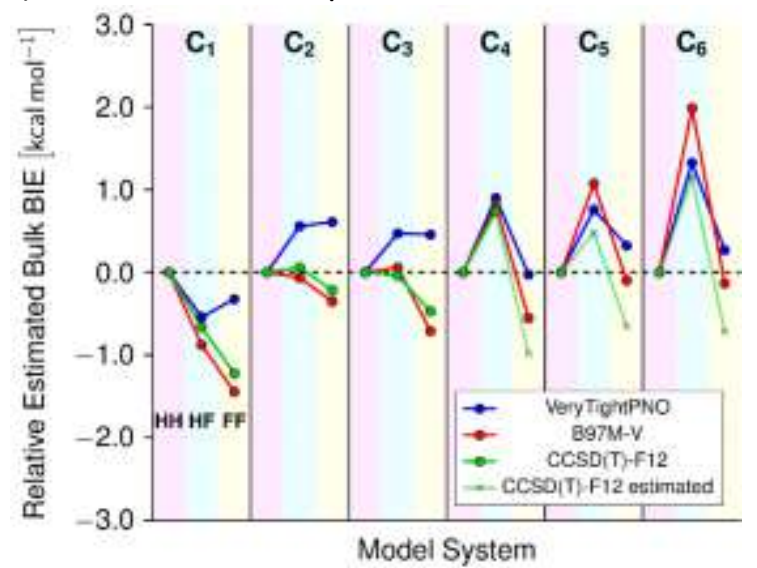

Figure 9. Relative BAEs of strongest binding isomers of $\mathbf{H F}$ and $\mathbf{F F}$ compared to the respective $\mathbf{H H}$ from one to six carbon atoms (labelled $C_{1}$ to $C_{6}$ ). a) Microscopic BAEs, b) bulk BAEs.

Additionally, we also looked into the relative energies of mixing of $\mathbf{H H}$ and $\mathbf{F F}$ to form two complexes of $\mathbf{H F}$ (Table 4, "CCSD(T)"). The relative energies of microscopic and macroscopic mixing on the basis of $\operatorname{CCSD}(T)-F 12$ change from favorable $\left(C_{1}\right)$ to increasingly unfavorable $\left(C_{2}\right.$ to $\left.C_{6}\right)$. As the carbon chain length is increased, two $\mathbf{H F}$ complexes are increasingly unfavorable compared to one $\mathbf{H H}$ and one $\mathbf{F F}$ complex. The mixing properties are almost equally determined when using the interaction energies of the crude molecular London dispersion model (Table 4, "London"). The trends of these energies are governed by the distances. When the ionization potential pre-factor and the polarizabilities are assumed to be one (with the appropriate unit), i.e. only the distances are taken into account, the computed mixing remains unaffected (Table 4, "Distance").

Table 4. Relative energies of mixing as a function of carbon chain length: $\mathrm{HH}+\mathrm{FF} \rightleftharpoons 2 \mathrm{HF}$.

\begin{tabular}{|c|c|c|c|c|c|c|c|}
\hline \multirow[b]{2}{*}{$\begin{array}{l}\text { Chain } \\
\text { Length }\end{array}$} & \multirow[b]{2}{*}{$\begin{array}{l}\text { Accounted } \\
\text { Isomers }\end{array}$} & \multicolumn{6}{|c|}{ Relative Energies of Mixing [ $\left.\mathrm{kcal} \mathrm{mol}^{-1}\right]$} \\
\hline & & $\begin{array}{c}\operatorname{CCSD}(T) \\
\text { Microscopic }\end{array}$ & $\begin{array}{c}\operatorname{CCSD}(T) \\
\text { Macroscopic }\end{array}$ & $\begin{array}{l}\text { London } \\
\text { Microscopic }\end{array}$ & $\begin{array}{c}\text { London } \\
\text { Macroscopic }\end{array}$ & $\begin{array}{c}\text { Distance } \\
\text { Microscopic }\end{array}$ & $\begin{array}{c}\text { Distance } \\
\text { Macroscopic }\end{array}$ \\
\hline \multirow{2}{*}{1} & Strongest & -0.20 & -0.19 & -0.06 & 1.29 & $-4.0 \cdot 10^{-5}$ & $33 \cdot 10^{-5}$ \\
\hline & Average & 0.07 & 0.32 & 0.04 & 0.14 & $1.9 \cdot 10^{-5}$ & $11 \cdot 10^{-5}$ \\
\hline \multirow{2}{*}{2} & Strongest & 0.18 & 0.67 & 0.17 & 0.24 & $4.9 \cdot 10^{-5}$ & $9.5 \cdot 10^{-5}$ \\
\hline & Average & 0.24 & 0.93 & 0.12 & 0.15 & $3.7 \cdot 10^{-5}$ & $3.8 \cdot 10^{-5}$ \\
\hline \multirow{2}{*}{3} & Strongest & 0.73 & 0.79 & 0.81 & 1.85 & $10 \cdot 10^{-5}$ & $16 \cdot 10^{-5}$ \\
\hline & Average & 0.62 & 0.95 & 0.68 & 1.53 & $8.2 \cdot 10^{-5}$ & $13 \cdot 10^{-5}$ \\
\hline \multirow{2}{*}{4} & Strongest & 0.75 & 6.31 & 0.37 & 0.21 & $3.2 \cdot 10^{-5}$ & $0.02 \cdot 10^{-5}$ \\
\hline & Average & 0.25 & 2.91 & -0.02 & 0.25 & $-0.2 \cdot 10^{-5}$ & $1.2 \cdot 10^{-5}$ \\
\hline \multirow{2}{*}{5} & Strongest & 0.85 & 3.23 & 0.67 & 1.98 & $3.7 \cdot 10^{-5}$ & $6.8 \cdot 10^{-5}$ \\
\hline & Average & 0.72 & 3.01 & 0.36 & 0.75 & $1.8 \cdot 10^{-5}$ & $2.0 \cdot 10^{-5}$ \\
\hline \multirow[b]{2}{*}{6} & Strongest & 1.84 & 6.01 & 0.93 & 0.93 & $3.5 \cdot 10^{-5}$ & $3.4 \cdot 10^{-5}$ \\
\hline & Average & 0.99 & 6.08 & 0.46 & 1.66 & $1.5 \cdot 10^{-5}$ & $4.4 \cdot 10^{-5}$ \\
\hline
\end{tabular}

"Strongest" denotes that only the strongest isomer was considered. "Average" denotes that the energies of all isomers were averaged. "CCSD(T)" values are based on CCSD(T)-F12/VDZ energies (Details in the Supporting Information). "London" values are based on the molecular London dispersion model (cf. Figure 5a). "Distance" values are based on the $\mathrm{R}^{-6}$-weighted average distances assuming the other factors to have numerical values of 1 with the appropriate units.

Trends in Experimental Properties. The last step was to look into trends of experimental properties of $\mathbf{H}$ and $\mathbf{F}$ and compare them to our computational results. The properties considered and the corresponding information they provide are summarized in Table 5.

Table 5. Experimental bulk properties of $\mathbf{H}$ and $\mathbf{F}$ selected and the information they provide.

\begin{tabular}{llll}
\hline Symbol & Bulk Property & Unit & Information
\end{tabular}




\begin{tabular}{|c|c|c|c|}
\hline$\Delta_{\text {vap }} H^{\circ}$ & Enthalpy of Vaporization & $\mathrm{kcal} \mathrm{mol}^{-1}$ & Strength of intermolecular interactions and packing in the liquid. \\
\hline$T_{\text {boil }}$ & Boiling Point & K & $\begin{array}{l}\text { Strength of intermolecular interactions, packing in the liquid and } \\
\text { conformational flexibility. }\end{array}$ \\
\hline$a / b$ & Critical Parameters Ratio & $\mathrm{kcal} \mathrm{mol}^{-1}$ & $\begin{array}{l}\text { Strength of intermolecular interactions and dependence on } \\
\text { intermolecular orientation in the fluid. }\end{array}$ \\
\hline$\gamma$ & Surface Tension & $\mathrm{mN} \mathrm{m} \mathrm{m}^{-1}$ & Strength of intermolecular interactions and packing in the liquid. \\
\hline$\pi_{T}$ & Internal Pressure & $\mathrm{MPa}$ & $\begin{array}{l}\text { Dependence of strength of intermolecular interactions on } \\
\text { incremental volume changes and packing in the liquid. }\end{array}$ \\
\hline$K$ & Bulk Modulus & $\mathrm{MPa}$ & $\begin{array}{l}\text { Dependence of strength of intermolecular interactions on } \\
\text { incremental volume changes and packing in the liquid. }\end{array}$ \\
\hline$B_{T}$ & Isothermal Compressibility & $\mathrm{GPa}^{-1}$ & $\begin{array}{l}\text { Dependence of strength of intermolecular interactions on } \\
\text { incremental volume changes and packing in the liquid. }\end{array}$ \\
\hline$\eta$ & Dynamic Viscosity & $\mathrm{mPa} \mathrm{s}$ & Strength of intermolecular interactions and shape of molecules. \\
\hline$c_{p}$ & Molar Heat Capacity & cal mol-1 $\mathrm{K}^{-1}$ & $\begin{array}{l}\text { Number of internal degrees of freedom and barrier height } \\
\text { between them. }\end{array}$ \\
\hline$B_{i j}$ & Second Virial Coefficients & $\mathrm{dm}^{3} \mathrm{~mol}^{-1}$ & $\begin{array}{l}\text { Strength of homomolecular and heteromolecular interactions } \\
\text { and orientational dependence of energies in the gas. }\end{array}$ \\
\hline
\end{tabular}

Most of the bulk properties investigated provide direct or indirect information about the strength of intermolecular interactions, about the packing in the liquid or a combination thereof. However, since they are based on distinct experimental measurements they provide mutually supporting evidence about the corresponding molecular origins. The comparison of the trends of all these experimental measures for $\mathbf{H}$ and $\mathbf{F}$ with respect to the carbon chain length is illustrated in Table 6 . Individual values with the corresponding references are provided in the Supporting Information. All properties considered except for the dynamic viscosity and the molar heat capacity show an inversion when $\mathbf{F}$ and $\mathbf{H}$ with increasing carbon chain length are compared. The enthalpies of vaporization show an inversion between seven and eight carbon atoms, the boiling points show an inversion between three and four carbon atoms and all the other properties show an inversion between one and two carbon atoms. Notably, the boiling point differences and the differences in the critical parameters ratio correlate reasonably with the relative molar carbon content in the molecules (Details in the Supporting Information). The ratio of surface tensions also correlates somewhat with the relative molar carbon content and the differences in the enthalpies of evaporation correlate with the number of carbon atoms.

Table 6. Comparison of trends of experimental bulk properties in $\mathbf{F}$ and $\mathbf{H}$. The second column specifies whether the difference between $\mathbf{F}$ and $\mathbf{H}$ or the ratio of $\mathbf{F}$ to $\mathbf{H}$ was used to compare the properties.

\begin{tabular}{|c|c|c|c|c|c|c|c|c|c|c|}
\hline \multicolumn{2}{|l|}{ Legend: } & \multicolumn{3}{|c|}{$>\mathrm{H}$} & \multicolumn{3}{|l|}{$F=H$} & \multicolumn{2}{|c|}{$\mathbf{F}<\mathbf{H}$} & \\
\hline \multirow{2}{*}{ Property } & \multirow{2}{*}{ Comparison } & \multicolumn{9}{|c|}{ Carbon Chain Length } \\
\hline & & 1 & 2 & 3 & 4 & 5 & 6 & 7 & 8 & 9 \\
\hline$\Delta_{v a p} H^{\circ}$ & Difference & 0.88 & 0.35 & 0.50 & 0.46 & 0.31 & 0.29 & 0.17 & -0.17 & -0.33 \\
\hline$T_{\text {boil }}$ & Difference & 34.1 & 10.4 & 2.9 & -2.0 & -6.6 & -11.6 & -16.5 & -19.7 & n.a. ${ }^{a}$ \\
\hline$a / b$ & Difference & 0.25 & -0.08 & -0.17 & -0.26 & -0.32 & -0.39 & -0.44 & -0.45 & n.a. ${ }^{a}$ \\
\hline$\gamma$ & Ratio - 1 & 0.28 & -0.22 & -0.23 & -0.30 & -0.37 & -0.38 & -0.34 & -0.34 & n.a. ${ }^{a}$ \\
\hline$\pi_{T}$ & Difference & 21 & -26 & -41 & -67 & -67 & -59 & -46 & -26 & n.a. ${ }^{a}$ \\
\hline K & Ratio - 1 & 0.48 & -0.33 & -0.54 & -0.57 & -0.52 & -0.42 & -0.40 & -0.38 & n.a. ${ }^{a}$ \\
\hline$B_{T}$ & Ratio - 1 & -0.32 & 0.21 & 0.94 & 0.76 & 1.35 & 0.92 & 0.58 & 0.39 & n.a. ${ }^{a}$ \\
\hline$\eta$ & Difference & 0.05 & 0.11 & 0.19 & 0.38 & 0.62 & 0.97 & 1.31 & 2.02 & n.a. ${ }^{a}$ \\
\hline$c_{p}$ & Difference & 5.8 & 13.6 & 19.9 & 30.0 & 35.4 & 37.7 & 45.1 & n.a. ${ }^{a}$ & n.a. ${ }^{a}$ \\
\hline
\end{tabular}

an.a. means data not available. 
In addition to the properties compared in Table 6, we used experimental second interaction virial coefficients ${ }^{61-63}$ to assess energies of mixing of $\mathbf{H}$ and $\mathbf{F}$ having equal carbon chain length (cf. Figure 10a). First, it should be noted that all the second interaction virial coefficients are negative indicating that they are measured below the respective Boyle temperature and, hence, in the attractive regime of intermolecular gas interactions. Secondly, the sum of the homomolecular second interaction virial coefficients is more attractive than the corresponding heteromolecular coefficients indicating that interactions between $\mathbf{H}$ and $\mathbf{F}$ are less favorable than interactions of $\mathbf{H}$ with $\mathbf{H}$ and $\mathbf{F}$ with $\mathbf{F}$. Additionally, Knobler et al. ${ }^{61-63}$ found the interactions in hydrocarbon-fluorocarbon mixtures to be about $10 \%$ weaker than estimated by the geometric mean. On average, we obtain comparable results when looking at $\operatorname{CCSD}(\mathrm{T})$-F12 energies, the London dispersion interaction model and the $\mathrm{R}^{-6}$-weighted distances $(11 \%, 9 \%$ and $9 \%$ weaker interactions on average, respectively, Details in the Supporting Information).

a) Second Interaction Virial Coefficients.

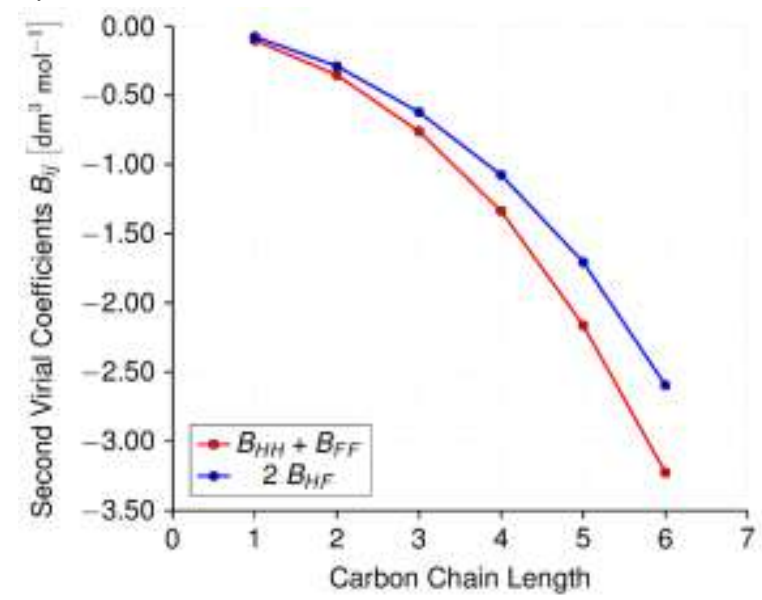

b) $\Delta_{v a p} H^{\circ}$ against bulk BAEs.

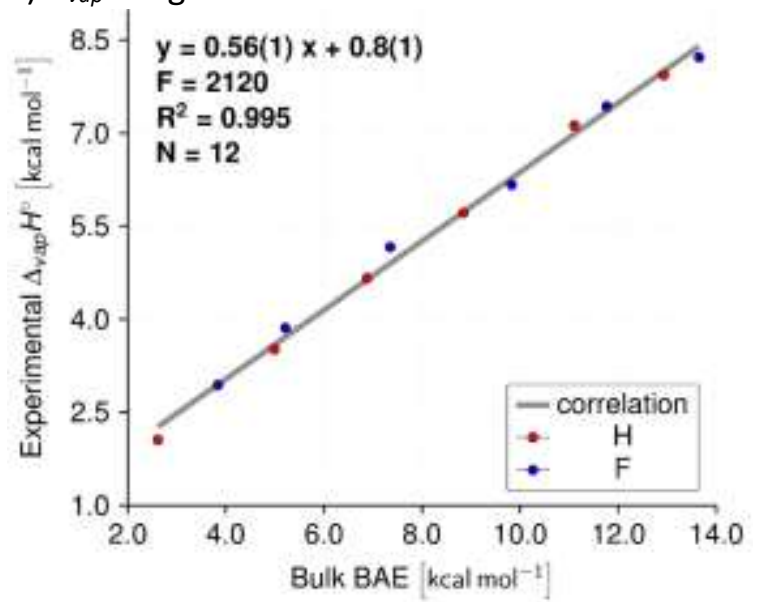

Figure 10. a) Comparison of experimental second virial coefficients of homomolecular (red) and heteromolecular (blue) interactions as a function of carbon chain length. Homomolecular interactions are more attractive. b) Experimental enthalpies of evaporation $\left(\Delta_{\mathrm{vap}} \mathrm{H}^{\circ}\right)$ of $\mathbf{H}$ and $\mathbf{F}$ of increasing carbon chain length correlated against computed bulk BAEs from CCSD(T)-F12/VDZ.

Lastly, we also compared the estimated bulk-phase interaction energies in $\mathbf{H}$ and $\mathbf{F}$ against experimental enthalpies of evaporation (Figure 10b). For these values to be as comparable as possible, we chose experimental values at rather low and comparable temperatures (Details in the Supporting Information). Figure $10 \mathrm{~b}$ shows a good correlation suggesting the computational BAEs to be a good estimate of the strength of intermolecular interactions. However, it needs to be emphasized that we are only considering enthalpies here and, hence, neglect entropy.

\section{Discussion}

Summarizing our results leads to four main conclusions:

i. Most well-established computational methods for describing dispersion have difficulties to properly describe the interactions in alkane-perfluoroalkane and perfluoroalkaneperfluoroalkane complexes.

ii. The dispersive interactions in perfluoroalkane dimers come mainly from interactions between fluorine atoms. 
iii. Perfluoroalkanes have a higher intrinsic ability for intermolecular dispersion than alkanes with equal carbon chain length but the unsuitable interaction geometries of perfluoroalkane dimers compared to alkane dimers result overall in roughly equal interaction energies.

iv. The differing trends of various experimental porperties of alkanes and perfluoroalkanes with increasing carbon chain length can be explained by the concomitant increasing relative molar carbon content and the increasingly unsuitable interaction geometries of perfluoroalkanes.

We will investigate these four claims in detail.

Benchmarking Interaction Energies. The accurate description of noncovalent intermolecular interactions, especially of London dispersion, has been one of the main themes of research in the field of computational chemistry in recent years. ${ }^{57,64-68}$ In DFT, several dispersion correction schemes have been developed ${ }^{57,64}$ in order to obtain accurate intermolecular interaction energies of (partially) dispersion-bound molecular complexes and many of them have been shown to be accurate in describing the interactions of "ordinary" organic molecules. ${ }^{67,69,78-80,70-77}$ Within this work, we made use of the D3 correction, ${ }^{81,82}$ the XDM correction, ${ }^{36-39,83}$ the $\mathrm{dDsC}$ correction, ${ }^{84-86}$ the VV10 correction, ${ }^{87}$ Minnesota functionals ${ }^{88,89}$ with implicit treatment of dispersion and the vdW-DF10 density functional ${ }^{90}$ to test their performance in the computation of intermolecular complexes of $\mathbf{H}$ and $\mathbf{F}$. In WFT, CCSD(T) energies at the CBS limit are considered the gold standard for computing interaction energies of dispersion-bound systems. ${ }^{16,66,75,91-95}$ In addition, recently, the low scaling DLPNO-CCSD(T) approach has been developed which promises near $\operatorname{CCSD}(T)$ accuracy at significantly lower computational cost, especially for medium-sized molecules with 20-200 atoms, or even larger systems. ${ }^{29-31,96-99}$ However, the systematic investigation of interaction energies of perfluoroalkanes has not received wide attention ${ }^{15-17,26,27}$ and, to the best of our knowledge, there are currently no good benchmark data available for the interaction between linear perfluoroalkanes of increasing chain length. Our CCSD(T)F12/VDZ interaction energies fill that gap. Surprisingly, we find that many computational methods designed to describe dispersion accurately fail in reproducing benchmark interaction energies of $\mathbf{H F}$ and, especially, FF. Even DLPNO-CCSD(T), the most systematic low-cost computational method we tested, requires tighter than usual cutoff parameters to obtain most accurate results and even then the errors still amount to $9 \%$ of the benchmark energies for FF.

We find that the most robust and efficient level of theory to determine the interaction energies of alkane and perfluoroalkane dimers is B97M-V. ${ }^{34}$ Most notably, it performs as well as DLPNO-CCSD(T) with extremely tight parameters (ExtremePNO, Details in the Supporting Information) across all the systems, and performs by far the best for FF with an $M A D_{\text {rel }}$ of only $3 \%$ (cf. Table 2). $\omega B 97 M-V$ performs only slightly worse but is also more expensive. Additionally, the XDM dispersion correction performs very well compared to most of the alternative corrections. B3LYP-XDM yields excellent results for both $\mathbf{H H}$ and $\mathbf{H F}$, and is acceptable for FF despite significant underestimation of the corresponding BIEs. Overall, many DFT and WFT approaches show different problems in calculating the BIEs of our model systems and, therefore, there is likely not one universal explanation for their failure (Details in the Supporting Information). While most approaches perform well for estimating the interaction energies of $\mathbf{H H}, \mathbf{H F}$ and $\mathbf{F F}$ seem to be problematic for most of the methods used. The ad-hoc explanation for that failure is the absence of good benchmark data for these types of interactions. Therefore, the benchmark data provided will contribute to the development of more accurate (cheap) electronic structure methods. Additionally, the benchmarking was the stepping-stone for all subsequent analyses to understand the quality and quantity of the intermolecular interactions in the investigated complexes. 
Energy Decomposition Analysis and Interaction Models. The decomposition of interaction energies of molecular complexes is one of the main computational approaches to gain physical and chemical insight into structure and reactivity. ${ }^{100}$ Symmetry-adapted perturbation theory (SAPT) ${ }^{43-46}$ belongs to the more widely used methods of EDA. It decomposes the overall interaction energy into electrostatics, exchange, induction and dispersion in a well-defined way. Local energy decomposition (LED), which is based on DLPNO methods, is a very recent addition to the multitude of EDA methods. ${ }^{47}$ However, only the dispersion energy components of LED and SAPT can be directly compared because the underlying theoretical framework for the other components is different. Therefore, we decided to use SAPT for interpretation of all the energy components provided therein and only looked into the dispersion energy components of LED. In addition to classical EDA methods, an increasing number of methods for the visualization of intermolecular interactions and their origin have been developed, of which DORI, ${ }^{101}$ $\mathrm{NCl}^{102} \mathrm{DID}^{49}$ and PolaBer ${ }^{103}$ are just a few more recent examples. Their appeal lies in the connection of computed numbers indicating interaction strengths and visual images illustrating the results, which makes them easier for the human brain to grasp.

As would be expected for complexes of nonpolar molecules, ${ }^{3}$ SAPT determines repulsive exchange and dispersion to be the major energy components in our test systems. Based on Figure 1, no significant difference in the contributions of the energy components between $\mathbf{H H}, \mathbf{H F}$ and $\mathbf{F F}$ can be seen, suggesting that the general type of interaction is of the same nature. However, when looking closer at the effective dispersion contributions in the three subclasses, the contribution of dispersion increases slightly when going from $\mathbf{H H}$ via $\mathbf{H F}$ to $\mathbf{F F}$ (Supporting Information). These results are in contrast to the results of previous EDA studies of $\mathbf{H}$ and $\mathbf{F}$ homo- and heterodimers which found effective dispersion to be weakest in $\mathbf{H F}$ and strongest in $\mathbf{H H} .{ }^{3}$ Notably, SAPT does not estimate the electrostatic component to be bigger in $\mathbf{H F}$ than in either $\mathbf{H H}$ or $\mathbf{F F}$, which has also been found by previous EDA studies. ${ }^{3}$ This might seem surprising as the hydrogen and fluorine atoms on the outside of the interacting molecules have opposite polarization. However, for every attractive electrostatic contribution stemming from interactions between hydrogen and fluorine atoms there are repulsive electrostatic contributions between hydrogen and carbon atoms and between carbon and fluorine atoms which compensate the attractive components. ${ }^{3}$

The major finding of LED, the DIDs and the PolaBer plots is the identification of fluorine atoms being the major dispersion energy donors in $\mathbf{F}$. The strong electronegativity difference between carbon and fluorine results in an Umpolarisierung of the isolated atomic polarizabilities, a phenomenon wellknown in the computational community but its implications are usually not clearly expressed. ${ }^{36,39,57,64,82,104,105}$ This Umpolarisierung can be readily understood using Sanderson's electronegativity equalization principle. ${ }^{106-110}$ Fluorine atoms are much more electronegative than carbon atoms. Hence, electronegativity equalization estimates transfer of electron density from carbon to fluorine until the corresponding local electronegativites are equal. The local electronegativity of fluorine bonded to carbon will be lower than its isolated atomic electronegativity. The corresponding local electronegativity of carbon will be higher. Polarizability was shown to be correlated reasonably well to electronegativity ${ }^{111}$ resulting in the estimation that the polarizability of fluorine bonded to carbon will be higher than its isolated atomic polarizability and that the corresponding polarizability of carbon will be lower with the caveat that the presence of the bond between the two atoms reduces both polarizabilities. Alternatively, the change in local polarizabilities can also be explained using hardness and softness from conceptual density functional theory, ${ }^{109,110,112,113}$ which are closely related to the concept of hard and soft acids and bases (HSAB). ${ }^{114,115}$ Electronegativity equalization of fluorine and carbon will lead to a local positive charge 
on carbon and a local negative charge on fluorine. The local positive charge on carbon will increase its local hardness and, hence, decrease its local softness. Softness has been shown to be correlated reasonably well to polarizability ${ }^{109,110}$ inferring a concomitant decrease in polarizability. The local negative charge on fluorine will decrease its local hardness and increase its local softness leading to a concomitant increase in polarizability. Therefore, Umpolarisierung, i.e. effective transfer of polarizability from one bonding partner to another, is expected to occur whenever polar covalent bonds are formed and can therefore be understood using well-established chemical principles.

Further support is obtained from the simple interaction models used to estimate the BIEs in the complexes (cf. Figure 5). Using the molecular interaction model, only when dispersion was assumed to originate from carbons in $\mathbf{H}$ and fluorines in $\mathbf{F}$ were we able to reproduce the general BIE trends (Figure 5a). This simple interaction model using only molecular polarizabilities, molecular ionization potentials and the $\mathrm{R}^{-6}$-weighted average interaction distance demonstrates that qualitative trends in dispersion can be estimated using very crude approaches. While many of the electronic structure methods show distinct deviations in the three subclasses of complexes (cf. Table 2), the molecular London dispersion model shows uniform systematic deviations from the benchmark BIEs indicating its robustness. Using the atom-pairwise dispersion interaction model, the benchmark BIE values were reproduced with an $M A D_{\text {rel }}$ of $5 \%$ which is not much worse than the best ab initio methods employed (cf. Table 2). Together with the fact that also the decomposition into atomic contributions compares surprisingly well with the LED results, it suggests that the underlying model is physically meaningful and its parameters can be used and interpreted quantitatively. However, notably, the atom-pairwise interaction model estimates effective dispersion, which is a sum of the attractive and repulsive contributions to the overall interaction. This complicates the direct interpretation of the pre-factors obtained as differences in the repulsive part of the interaction potential will also impact their values.

Interaction Descriptors and Interaction Geometries. The recent paradigm shift in the recognition of the importance of dispersion in small molecules ${ }^{116,117}$ has initiated a search for reliable dispersion energy donor descriptors that could quantitatively assess the ability of substituents in molecules for dispersion. Already several years ago, Dunitz proposed the ratio $Q$ (Equation 7) as dimensionless quantity to gauge the potential of molecules for dispersive interactions. ${ }^{117}$ He estimated atomic $Q$ values for hydrogen, carbon and fluorine using polarizabilities and volumes of the free atoms and found that $Q$ of fluorine is significantly smaller than $Q$ of either carbon or hydrogen. ${ }^{3} \mathrm{He}$ also found the corresponding molecular $Q$ values (using molecular polarizabilities and molecular volumes) of $\mathbf{F}$ to be significantly lower compared to $\mathbf{Q}$ values of $\mathbf{H}$. Consequently, he assigned these low $Q$ values as the origin of the distinct properties of $\mathbf{F}$ compared to $\mathbf{H}^{3}$

Based on that work we defined an alternative $Q$ measure using the "interaction volume" instead of the molecular volume. This is important because it explicitly accounts for the spatial distribution of polarizability within a molecule and the fact that close contacts result in higher dispersion. Using the molecular volume instead leads to a significant underestimation of the dispersive interaction abilities of $\mathbf{F}$ because the biggest portion of the polarizability is on the outside of these molecules (cf. Figure 4 and Table 3). To adequately quantify dispersive interaction capabilities across different classes of compounds, we introduced the $\mathrm{P}$ measure, which also accounts for the influence of the ionization potential of atoms on dispersion. The London dispersion formula estimates stronger dispersive interactions the larger the ionization potential of an atom. It is instructive in that regard to note that the trends of the ionization potentials in the periodic table are opposite to the trends of polarizabilities 
which suggests that only looking at polarizabilities when estimating the strength of dispersion is insufficient.

The average $P_{\text {tot }}$ on the van der Waals surface estimates $\mathbf{F}$ between one and six carbon atoms to have a stronger dispersive interaction ability compared to the corresponding $\mathbf{H}$. In that regard it gives the opposite answer about $\mathbf{F}$ to the $\mathbf{Q}$ values proposed by Dunitz ${ }^{3}$ and even the modified $Q$ values proposed in this work. Additionally, it is counter to the general notion in literature that $\mathbf{F}$ have a low propensity to interact with other molecules, ${ }^{3,8}$ despite the fact that both experimental $\left.\right|^{4,12}$ and computational $\left.\right|^{15-17}$ results show that, at least for 1-FF, the interactions are significantly stronger compared to 1-HH (vide supra). The average $P_{\text {tot }}$ on the van der Waals surface does not quantify how strong molecules actually interact by dispersion but rather how strong molecules in principle could interact by dispersion if interaction geometries were ideal. However, they are not ideal in $\mathbf{H F}$ and FF with more than one carbon atom (cf. Figure 7) which is a consequence of the ground-state geometries of $\mathbf{F}$ being unsuitable for interaction and of the rigidity of $\mathbf{F}$, a property well-established within the field of organofluorine chemistry. ${ }^{7}$ Deformation of $\mathbf{F}$ from the ground-state geometries requires a significant portion of additional energy and is overall unfavorable. The ground-state geometries of short chain $\mathbf{H}$ are already ideal for intermolecular interaction and therefore no significant deformation is required for $\mathbf{H H}$.

There is another notable aspect to the differences in the interaction geometries of $\mathbf{H H}$ and $\mathbf{F F}$. While two molecules of $\mathbf{H}$ try to bring mainly the carbon atoms close together, two molecules of $\mathbf{F}$ try to bring as many fluorine atoms as possible close together to gain intermolecular interaction energy. Therefore, even if $\mathbf{F}$ was not too rigid the ideal interaction geometries of $\mathbf{F F}$ would still differ from $\mathbf{H H}$ because carbon atoms are in the center of the structures while fluorine atoms are on the outside of $\mathbf{F}$. This is reflected in the interaction geometries of 2-FF and 3-FF. Figure 11 shows that while 3-HH prefers the conformer which is based on inversion followed by translation normal to the plane of the carbon atoms, 3-FF prefers the conformer that is based on inversion followed by translation in that plane (with some additional displacement normal to the plane). This observation supports our conclusions even further because this intrinsic difference in the interaction geometry preference also makes the groundstate geometries of dimers of increasingly long $\mathbf{F}$ less ideal for intermolecular interactions compared to $\mathbf{H}$. It is easier for $\mathbf{H}$ to align many carbon atoms than it is for $\mathbf{F}$ to align many fluorine atoms.

a) 3-HH1

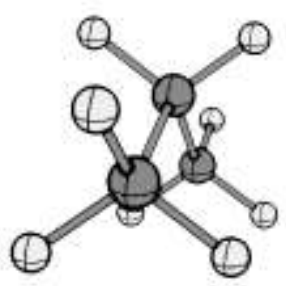

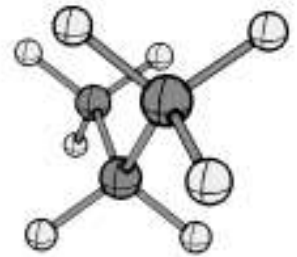

b) 3-FF2

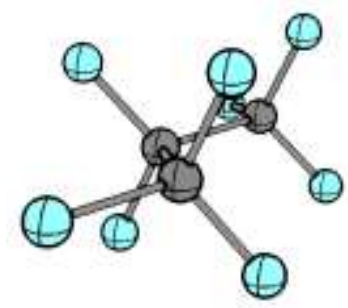

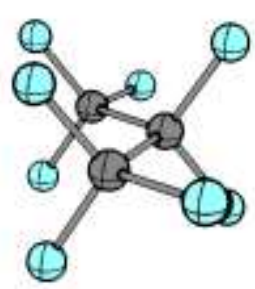

Figure 11. Comparison of most stable conformers of a) 3-HH and b) 3-FF.

The number of contacts ( $\mathrm{n}_{\text {contacts, }}$ cf. Equation 10) is a quantitative measure to assess the interaction geometries and determines the number of van der Waals contacts between two interacting molecules. The idea is to define one van der Waals contact as two atoms at a distance corresponding to the sum of their van der Waals radii. This basic concept is closely related to the normalized contact distance which is commonly used in the analysis of intermolecular interactions in crystal structures. ${ }^{118}$ The main difference is the use of the sixth power of the ratio of distances to account explicitly for the distance dependence of van der Waals interactions. The number of contacts does not suffer from significant systematic discrepancies for the different atom types as can be gleaned from the close values for 1- 
HH1, 1-HF1 and 1-FF2, which all have close to ideal interaction geometries. In addition, for each group of complexes, $\mathrm{n}_{\text {contacts }}$ shows a linear correlation with the number of carbon atoms demonstrating that $\mathrm{n}_{\text {contacts }}$ scales linearly with interaction surface (the interaction surface scales linearly with the number of carbon atoms in these complexes, cf. Supporting Information) which is a very attractive property of this purely geometric measure. The newly introduced parameters $P_{\text {tot }}$ and $n_{\text {contacts }}$ are a first step towards simple quantitative parameters to describe London dispersion between molecules or groups of atoms, which is demonstrated by the reasonable correlation observed between BIEs and the product of $\mathrm{n}_{\text {contacts }}$ and the $\mathrm{P}_{\text {tot }}$ values of the interacting molecules (cf. Figure $8 \mathrm{~b}$ ). While $\mathrm{P}_{\text {tot }}$ quantifies the average strength of a dispersive contact, $n_{\text {contacts }}$ quantifies the number of contacts. Hence, in order to judge the overall interaction energy, both parameters need to be considered.

Based on these results, we propose that the reason for the weak interactions of $\mathbf{F}$ compared to $\mathbf{H}$ is their ground-state geometries, which are not ideal for intermolecular interactions. This is fully consistent with the cavity-based solubility model used to explain the solvent properties of fluorous solvents. ${ }^{2,119}$ It is observed that molecular size plays an important role whether a solute is soluble in $\mathbf{F}$ or not. It is commonly stated in literature that, because of low intermolecular forces, $\mathbf{F}$ have large cavities in the bulk phase. ${ }^{2,119}$ These cavities can be occupied readily by small molecules of suitable size. We would argue that the main reasons for the formation of cavities are the ground-state geometries and the high rigidity of $\mathbf{F}^{7}$ Hence, we effectively argue for an inversion in the cause-effect relationship. Not the relatively weak intermolecular interactions are the reason for the presence of cavities but rather the presence of cavities are the reason for the relatively weak intermolecular interactions. This is the case for both intermolecular complexes, in which "cavities" refers to molecular surface not used for interaction, and bulk phases, in which there are real cavities.

Mixing Behavior and Trends in Experimental Properties. The widely known fluorophobic effect or fluorous effect refers to the low miscibility of $\mathbf{F}$ with $\mathbf{H}$ in the liquid state. ${ }^{8-11}$ Notably, the immiscibility of $\mathbf{F}$ with common organic solvents increases with carbon chain length of $\mathbf{F}$. Additionally, the fluorous properties of $\mathbf{F}$ only begin to appear at a certain chain length. Compounds with perfluoroalkyl groups of six carbon atoms are already well soluble in typical fluorous solvents. ${ }^{2}$ The critical solution temperature of mixtures of $\mathbf{5}-\mathbf{H}$ and $\mathbf{5}-\mathbf{F}$, i.e. the temperature above which mixtures of two liquids always form one homogeneous phase, is $-8^{\circ} \mathrm{C},{ }^{120}$ for mixtures of $\mathbf{6 - H}$ and $\mathbf{6 - F}$ it is $22.7^{\circ} \mathrm{C} .{ }^{121}$ Therefore, it is reasonable to say that fluorous properties of linear $\mathbf{F}$ start to become significant for $\mathbf{5}-\mathbf{F}$ and $\mathbf{6}-\mathbf{F}$, which are the two longest linear $\mathbf{F}$ investigated in this work. This also suggests that it is not the fluorine content making an organic molecule fluorous, but rather the number of carbon atoms bound to fluorine.

It is commonly argued that fluorous molecules, because of the low polarizability of fluorine, have a low propensity for interaction with any other molecule, even themselves, compared to nonfluorous molecules and therefore favor phase separation to minimize unfavorable interactions. ${ }^{8}$ However, our relative microscopic and macroscopic BAEs of $\mathbf{H H}, \mathbf{H F}$ and $\mathbf{F F}$ show that, except for $\mathbf{1 - H F}$, all the $\mathbf{H F}$ complexes have a smaller BAE than the corresponding $\mathbf{H H}$ and FF complexes. These observations argue against the notion that $\mathbf{F}$ are interacting weakly with any other molecule compared to $\mathbf{H}$, i.e. indifference for interaction with anything, but rather support the view that homo-aggregation of fluorinated molecules is preferential, at least for $\mathbf{F}$ with up to six carbon atoms.

From the microscopic and macroscopic energies of mixing (cf. Table 4), the overall mixing behavior can be decomposed into the contribution of the distances and the contribution of the intrinsic dispersive 
interaction ability. The distances quantify the impact of the interaction geometries on the mixing behavior. The system has a choice between one $\mathbf{H H}$, which has close to ideal interaction geometry, plus one FF, which has non-ideal geometry, and two HF, which both have non-ideal geometry. In the absence of other influences, one ideal plus one non-ideal geometry is preferred over two non-ideal geometries. In that regard, the interaction geometries of $\mathbf{F}$ show indifference for interaction with any other molecule. On the other hand, the intrinsic dispersive interaction ability shows preferred homoaggregation of both $\mathbf{H}$ and $\mathbf{F}$, respectively. To put it simply, $\mathbf{F}$ have a stronger intrinsic dispersion ability, $\mathbf{H}$ have a weaker intrinsic dispersion ability, which is estimated based on their average $P_{\text {tot }}$ measures (vide supra). In the absence of other influences, the system has a choice between one $\mathbf{H H}$, which is a contact between two weaker interactors, plus one $\mathbf{F F}$, which is a contact between two stronger interactors, and two HF, which is a contact between one weaker and one stronger interactor. Because the overall interaction energy is mathematically described as product of two P measures (cf. Equations 8 and 9), one strong-strong interaction plus one weak-weak interaction is generally favored over two strong-weak interactions. It is intriguing that these two very different mechanisms of unmixing favor the exact same outcome. Additionally, it is important to point out that this reasoning is based purely on enthalpic arguments. They compete with the entropy of mixing which favors mixing of $\mathbf{H}$ and $\mathbf{F}$, which is the reason for the temperature-dependent phase separation of $\mathbf{H}$ and $\mathbf{F}$.

We also carried out a comprehensive trend comparison of experimental bulk properties (cf. Table 5 and Table 6) of $\mathbf{F}$ and $\mathbf{H}$. Several of the properties investigated show an inversion, i.e. they change from being smaller in $\mathbf{H}$ to being larger, or vice versa, when the carbon chain is elongated. All the properties showing an inversion, i.e. enthalpy of evaporation, boiling point, critical parameter ratio, surface tension, internal pressure, bulk modulus and compressibility, provide information about intermolecular interactions and the corresponding interaction geometries. They can be roughly divided into properties quantifying the interaction energy (enthalpy of evaporation, boiling point, critical parameter ratio and surface tension) and properties quantifying the change of interaction energy with volume (internal pressure, bulk modulus and compressibility). Looking more closely into the properties considered, the high compressibilities of the longer $\mathbf{F}$ have been rationalized by the presence of cavities in the bulk liquid explaining their higher gas solubilities compared to $\mathbf{H}$. The inverted trend for 1-F suggests less cavities which likely relates to the good interaction geometries we found in 1-FF complexes (cf. Figure 8a). From the properties not showing an inversion, the molar heat capacity actually does not even quantify intermolecular interactions. However, we looked into it because an inversion has been suggested in the literature. ${ }^{122}$ However, using current experimental values we could not find evidence for it. In addition, the consistently higher viscosity of $\mathbf{F}$ compared to $\mathbf{H}$ can mainly be explained by the higher roughness of $\mathbf{F}$ and is therefore not a consequence of differences in intermolecular interaction energies. ${ }^{123}$

To the best of our knowledge, only the boiling point inversion between $\mathbf{H}$ and $\mathbf{F}$ has been pointed out in the literature before. It was first reported by Grosse and Cady in $1947 .{ }^{12}$ In that paper, which reported results obtained during the Manhattan Project at Columbia University during World War II, the physical properties of perfluorinated and partially fluorinated hydrocarbons were determined and documented comprehensively. To the best of our knowledge, no satisfactory explanation has been provided for the observed boiling point inversion. All the observed inversions in experimental bulk properties, including the boiling point inversion, can be explained by two synergistic mechanisms. First, we found that the dispersive interaction abilities of $\mathbf{F}$, quantified by $P_{\text {tot }}$, relative to $\mathbf{H}$, decreases with increasing carbon chain length because of a concomitant decrease in the relative molar fluorine content. Secondly, the interaction geometries of dimers of increasingly long $\mathbf{F}$ are less ideal compared 
to the corresponding dimers of $\mathbf{H}$ as quantified by the trends of $\mathrm{n}_{\text {contacts }}$ (Figure 8). $\mathbf{F}$ with a short chain length have a higher intrinsic interaction ability than the corresponding $\mathbf{H}$ and the corresponding interaction geometries are still good which results in overall stronger intermolecular interactions. Increasing the carbon chain length results in a decrease of the intrinsic interaction abilities of $\mathbf{F}$ compared to $\mathbf{H}$ and also in less ideal interaction geometries. Overall, this explains the inversions of all the bulk properties considered.

Understanding the mixing behavior of fluorous substances is critical for solving the current problems associated with perfluorooctanoic acid (PFOA) and its salts. PFOA is extremely persistent and bioaccumulative because of its chemical inertness under environmental conditions ${ }^{124}$ and its mixing properties. ${ }^{125}$ In humans, it accumulates in serum and has been detected in populations worldwide. ${ }^{124}$ Furthermore, it is, including other toxicities, toxic to the liver, to reproduction and the immune system and is carcinogenic. ${ }^{126}$ However, PFOA owes its main applications as emulsifier for the production of fluoroelastomers and fluoropolymers like polytetrafluoroethylene (PTFE) ${ }^{126}$ or as oil and dirt repellent in textile and paper production ${ }^{127}$ to its fluorous properties which govern its mixing properties. Because of its toxicity, PFOA was added to the restricted substances of REACH and by July 2020 will be forbidden to be produced or distributed in the European Union. ${ }^{127}$ Therefore, good substitutes for PFOA are required, which retain its attractive properties as much as possible. Understanding the origin of the fluorous properties of PFOA is one step towards suitable substitutes which underlines the importance of our findings. They suggest that potential substitutes could be molecules with sufficient rigidity and ground-state geometries unsuitable for interaction with both themselves and other molecules.

We would like to close this section by pointing out two relatively recent experimental studies, which found stronger than expected interaction energies of perfluorinated molecules but did not put the corresponding results into the right perspective. First, in a recent study of the binding of benzenesulfonamides substituted with either alkyl or perfluoroalkyl groups to human carbon anhydrase II it was found that both groups favor binding to a similar extent both enthalpically and entropically. ${ }^{128}$ The authors concluded that the favorable enthalpic contributions are a result of nonoptimal hydration of the hydrophobic wall of human carbon anhydrase II (HCA II). However, in order to reach that conclusion, they explicitly disregarded the possibility of equally strong dispersive interactions of the alkyl and perfluoroalkyl analogues because of considerably smaller bulk polarizabilities of perfluoroalkanes. ${ }^{128}$ However, based on our results, equally strong interactions of alkyl and perfluoroalkyl substituents with a carbon chain length of one to five with the hydrophobic pocket of HCA II cannot be excluded as explanation for the observed similarly strong enthalpic binding energy contributions. On the contrary, our results suggest they are even likely to be similar in strength. Secondly, in the recently conducted HYDROPHOBE challenge, ${ }^{129}$ a computational challenge for reproducing experimental host-guest binding free energies in water, it was found that using state-ofthe art quantum mechanical methods on the basis of PBEh- $3 c^{130}$ geometries and DLPNO-CCSD(T)/CBS single point energies with TightPNO cutoff parameters resulted in significantly underestimated binding of perfluorohexane and perfluorocyclohexane. Experimentally, it was found that the perfluorinated molecules have very similar Gibbs free energies of binding, within $1 \mathrm{kcal} \mathrm{mol}^{-1}$, compared to the unfluorinated analogues. However, the computed values showed deviations of up to $8 \mathrm{kcal} \mathrm{mol}^{-1}$. ${ }^{129}$ On the basis of our benchmark results (cf. Table 2), this is not surprising, as DFT-D3 methods without other means of accounting for dispersion significantly underestimate the interactions of $\mathbf{F}$, which hints towards possible errors in the computed geometries. Additionally, TightPNO cutoff parameters for DLPNO-CCSD(T) calculations were shown not to be sufficiently accurate for $\mathbf{F}$ as well (cf. Table 2 ) and this would likely lead to significant underestimation of the interaction energies in the corresponding 
host-guest complexes. These two recent instances of underestimated binding energies of perfluorinated molecules demonstrate the general implications of this study.

\section{Conclusions}

In this work, we report an extensive computational investigation of the interaction energies of alkane and perfluoroalkane homo- and heterodimers. We conclude that most well established computational approaches used to describe dispersion-bound systems are inappropriate to describe the interaction energies in perfluoroalkane homo- and heterodimers accurately. Therefore, we provide a systematic benchmark set of high-level computed interaction energies of alkanes and perfluoroalkanes that will likely contribute to the development of more robust (cheap) ab initio methods to describe dispersion in general, not only in fluorinated molecules, as well as to the development of accurate force fields for fluorous molecules. We also found that the B97M- $V$ functional performs best among all tested methods and is most efficient for the estimation of the corresponding interaction energies.

Additionally, spatial energy decomposition revealed that perfluoroalkanes provide most of their intermolecular dispersion via the fluorine atoms. Not only are the fluorine atoms on the outside, but the large electronegativity difference between carbon and fluorine also results in Umpolarisierung so that the fluorine atoms are at least about equally polarizable as the carbon atoms in perfluoroalkanes. As a result, when both the influence of polarizabilities and ionization potentials are accounted for, perfluoroalkanes show a higher intrinsic ability for intermolecular dispersion compared to analogous alkanes, as quantified by the newly proposed $P_{\text {tot }}$ measure. However, the higher ability for dispersion is impeded by the ground-state geometries of perfluoroalkanes, which are unsuitable for interaction with either perfluoroalkanes or alkanes, as well as their high rigidity, which make deformation of the ground-state geometries highly unfavorable. The result is that intermolecular homodimers of linear alkanes and the corresponding perfluoroalkanes with up to six carbon atoms have, except for methane and perfluoromethane, roughly equal interaction energies. Overall, we propose the unsuitable interaction geometries to be the main reason for the notion that perfluoroalkanes interact weakly with other molecules by dispersion compared to alkanes. Additionally, our study contributes to the understanding of dispersion in molecules in general. $P_{\text {tot }}$ can be used as a simple measure to gauge the potential for dispersion of any molecule and is therefore a first stepping-stone towards the development of simple quantitative dispersion energy donor descriptors. Further work investigating the use of $\mathrm{P}$ measures for the quantitative description of dispersion energy donors is underway.

Moreover, as the carbon chain is elongated, the relative molar fluorine content decreases and, hence, the number of attractive fluorine-fluorine contacts per carbon atom decreases. Additionally, the geometries of perfluoroalkanes become less ideal for intermolecular interactions. These synergistic trends directly influence their intrinsic ability for dispersion and has important consequences for their bulk properties. This is apparent from the inversion of various properties of perfluoroalkanes compared to analogous alkanes. Overall, our findings contribute towards the understanding of fluorous phases in general. They implicitly propose a mechanism for the transition of an increasingly fluorinated molecule from non-fluorous behavior towards fluorous behavior. As more fluorine atoms are incorporated into a molecule, its ground-state geometry is altered favoring phase separation because of indifference. Furthermore, the growing fluorous regions of the molecule also favor selfaggregation because of stronger dispersive contacts compared to contacts with non-fluorous regions. After surpassing a critical fluorine content, the synergism between these two mechanisms make phase separation of fluorous and non-fluorous matter favorable. 


\section{Associated Content}

Supporting Information

Computational details, supplementary results and discussion, mathematical derivations and raw computational data (PDF)

XYZ coordinates for all the model compounds (ZIP)

Output files of COSMO calculations (ZIP)

Atomic polarizabilities of all the atoms in the molecular complexes (ZIP)

\section{Author Information}

Corresponding Author

peter.chen@org.chem.ethz.ch

\section{Acknowledgments}

We thank Marek Bot, Eno Paenurk and Dr. Renana Poranne for helpful discussions and Prof. Ricardo Mata and Axel Wuttke for help with preparing the DID plots. The work was supported financially by the ETH Zürich, the Schweizerischer Nationalfonds, the Deutsche Forschungsgemeinschaft (SPP1807), and the ACS Petroleum Research Fund.

\section{References}

(1) O'Hagan, D. Understanding Organofluorine Chemistry. An Introduction to the C-F Bond. Chem. Soc. Rev. 2008, 37 (2), 308-319. https://doi.org/10.1039/B711844A.

(2) Horvath, I. T. Fluorous Chemistry; Springer, 2012; Vol. 308. https://doi.org/10.1016/03024598(80)87026-7.

(3) Dunitz, J. D. Organic Fluorine: Odd Man Out. ChemBioChem 2004, 5 (5), 614-621. https://doi.org/10.1002/cbic.200300801.

(4) Smart, B. E. Fluorine Substituent Effects (on Bioactivity). J. Fluor. Chem. 2001, 109 (1), 3-11. https://doi.org/10.1016/S0022-1139(01)00375-X.

(5) Lemal, D. M. Perspective on Fluorocarbon Chemistry. J. Org. Chem. 2004, 69 (1), 1-11. https://doi.org/10.1021/J00302556.

(6) Schlosser, M.; Michel, D. About the "Physiological Size" of Fluorine Substituents: Comparison of Sensorially Active Compounds with Fluorine and Methyl Substituted Analogues. Tetrahedron 1996, 52 (1), 99-108. https://doi.org/10.1016/0040-4020(95)00886-D.

(7) Eaton, D. F.; Smart, B. E. Are Fluorocarbon Chains "Stiffer" Than Hydrocarbon Chains? Dynamics of End-to-End Cyclization in a C8F16 Segment Monitored by Fluorescence. J. Am. Chem. Soc. 1990, 112 (7), 2821-2823. https://doi.org/10.1021/ja00163a065.

(8) Cametti, M.; Crousse, B.; Metrangolo, P.; Milani, R.; Resnati, G. The Fluorous Effect in Biomolecular Applications. Chem. Soc. Rev. 2012, 41 (1), 31-42.

https://doi.org/10.1039/C1CS15084G. 
(9) Johansson, G.; Percec, V.; Ungar, G.; Smith, K. Fluorophobic Effect Generates a Systematic Approach to the Synthesis of the Simplest Class of Rodlike Liquid Crystals Containing a Single Benzene Unit. Chem. Mater. 1997, 9 (1), 164-175. https://doi.org/10.1021/cm960267q.

(10) Johansson, G.; Percec, V.; Ungar, G.; Zhou, J. P. Fluorophobic Effect in the Self-Assembly of Polymers and Model Compounds Containing Tapered Groups into Supramolecular Columns. Macromolecules 1996, 29 (2), 646-660. https://doi.org/10.1021/ma9511558.

(11) Percec, V.; Schlueter, D.; Kwon, Y. K.; Blackwell, J.; Möller, M.; Slangen, P. J. Dramatic Stabilization of a Hexagonal Columnar Mesophase Generated from Supramolecular and Macromolecular Columns by the Semifluorination of the Alkyl Groups of Their Tapered Building Blocks. Macromolecules 1995, 28 (26), 8807-8818.

https://doi.org/10.1021/ma00130a013.

(12) Grosse, A. V.; Cady, G. H. Properties of Fluorocarbons. Ind. Eng. Chem. 1947, 39 (3), 367-374. https://doi.org/10.1021/ie50447a627.

(13) Krafft, M. P.; Riess, J. G. Chemistry, Physical Chemistry, and Uses of Molecular FluorocarbonHydrocarbon Diblocks, Triblocks, and Related Compounds-Unique "Apolar" Components for Self-Assembled Colloid and Interface Engineering. Chem. Rev. 2009, 109 (5), 1714-1792. https://doi.org/10.1021/cr800260k.

(14) London, F. The General Theory of Molecular Forces. Trans. Faraday Soc. 1937, 33 (8), 8-26. https://doi.org/10.1039/tf937330008b.

(15) Chattoraj, J.; Risthaus, T.; Rubner, O.; Heuer, A.; Grimme, S. A Multi-Scale Approach to Characterize Pure CH4, CF4, and CH4/CF4 Mixtures. J. Chem. Phys. 2015, 142 (16), 164508. https://doi.org/10.1063/1.4919079.

(16) Biller, M. J.; Mecozzi, S. A High Level Computational Study of the CH4 /CF4 Dimer: How Does It Compare with the CH4 /CH4 and CF4 /CF4 Dimers? Mol. Phys. 2012, 110 (7), 377-387. https://doi.org/10.1080/00268976.2011.648961.

(17) Tsuzuki, S.; Uchimaru, T.; Mikami, M.; Urata, S. Magnitude and Orientation Dependence of Intermolecular Interaction between Perfluoroalkanes: High Level Ab Initio Calculations of CF4 and C2F6 Dimers. J. Chem. Phys. 2002, 116 (8), 3309-3315. https://doi.org/10.1063/1.1436468.

(18) Neese, F. The ORCA Program System. Wiley Interdiscip. Rev. Comput. Mol. Sci. 2012, 2 (1), 7378. https://doi.org/10.1002/wcms.81.

(19) Neese, F. Software Update: The ORCA Program System, Version 4.0. Wiley Interdiscip. Rev. Comput. Mol. Sci. 2017, 1-6. https://doi.org/10.1002/wcms.1327.

(20) Turney, J. M.; Simmonett, A. C.; Parrish, R. M.; Hohenstein, E. G.; Evangelista, F. A.; Fermann, J. T.; Mintz, B. J.; Burns, L. A.; Wilke, J. J.; Abrams, M. L.; Russ, N. J.; Leininger, M. L.; Janssen, C. L.; Seidl, E. T.; Allen, W. D.; Schaefer, H. F.; King, R. A.; Valeev, E. F.; Sherrill, C. D.; Crawford, T. D. Psi4: An Open-Source Ab Initio Electronic Structure Program. Wiley Interdiscip. Rev. Comput. Mol. Sci. 2012, 2 (4), 556-565. https://doi.org/10.1002/wcms.93.

(21) Parrish, R. M.; Burns, L. A.; Smith, D. G. A.; Simmonett, A. C.; DePrince, A. E.; Hohenstein, E. G.; Bozkaya, U.; Sokolov, A. Y.; Di Remigio, R.; Richard, R. M.; Gonthier, J. F.; James, A. M.; McAlexander, H. R.; Kumar, A.; Saitow, M.; Wang, X.; Pritchard, B. P.; Verma, P.; Schaefer, H. F.; Patkowski, K.; King, R. A.; Valeev, E. F.; Evangelista, F. A.; Turney, J. M.; Crawford, T. D.; Sherrill, C. D. Psi4 1.1: An Open-Source Electronic Structure Program Emphasizing Automation, Advanced Libraries, and Interoperability. J. Chem. Theory Comput. 2017, 13 (7), 3185-3197. 
https://doi.org/10.1021/acs.jctc.7b00174.

(22) Werner, H. J.; Knowles, P. J.; Knizia, G.; Manby, F. R.; Schütz, M. Molpro: A General-Purpose Quantum Chemistry Program Package. Wiley Interdiscip. Rev. Comput. Mol. Sci. 2012, 2 (2), 242-253. https://doi.org/10.1002/wcms.82.

(23) Frisch, M. J.; Trucks, G. W.; Schlegel, H. B.; Scuseria, G. E.; Robb, M. A.; Cheeseman, J. R.; Scalmani, G.; Barone, V.; Mennucci, B.; Petersson, G. A.; Nakatsuji, H.; Caricato, M.; Li, X.; Hratchian, H. P.; Izmaylov, A. F.; Bloino, J.; Zheng, G.; Sonnenberg, J. L.; Hada, M.; Ehara, M.; Toyota, K.; Fukuda, R.; Hasegawa, J.; Ishida, M.; Nakajima, T.; Honda, Y.; Kitao, O.; Nakai, H.; Vreven, T.; Montgomery Jr., J. A.; Peralta, J. E.; Ogliaro, F.; Bearpark, M.; Heyd, J. J.; Brothers, E.; Kudin, K. N.; Staroverov, V. N.; Kobayashi, R.; Normand, J.; Raghavachari, K.; Rendell, A.; Burant, J. C.; Iyengar, S. S.; Tomasi, J.; Cossi, M.; Rega, N.; Millam, J. M.; Klene, M.; Knox, J. E.; Cross, J. B.; Bakken, V.; Adamo, C.; Jaramillo, J.; Gomperts, R.; Stratmann, R. E.; Yazyev, O.; Austin, A. J.; Cammi, R.; Pomelli, C.; Ochterski, J. W.; Martin, R. L.; Morokuma, K.; Zakrzewski, V. G.; Voth, G. A.; Salvador, P.; Dannenberg, J. J.; Dapprich, S.; Daniels, A. D.; Farkas, O.; Foresman, J. B.; Ortiz, J. V.; Cioslowski, J.; Fox, D. J. Gaussian09 Revision D.01. Gaussian 09. Gaussian Inc. Wallingford CT 2009.

(24) Baerends, E. J.; Ziegler, T.; Autschbach, J.; Bashford, D.; Berces, A.; Bickelhaupt, F. M.; Bo, C.; Boerrigter, P. M.; Cavallo, L.; Chong, D. P.; Deng, L.; Dickson, R. M.; Ellis, D. E.; van Faassen, M.; Fan, L.; Fischer, T. H.; Fonseca Guerra, C.; Franchini, M.; Ghysels, A.; Giammona, A. ADF2017. ADF2017. SCM, Theoretical Chemistry, Vrije Universiteit, Amsterdam, The Netherlands, http://www.scm.com.

(25) Shao, Y.; Gan, Z.; Epifanovsky, E.; Gilbert, A. T. B.; Wormit, M.; Kussmann, J.; Lange, A. W.; Behn, A.; Deng, J.; Feng, X.; Ghosh, D.; Goldey, M.; Horn, P. R.; Jacobson, L. D.; Kaliman, I.; Khaliullin, R. Z.; Kü̈, T.; Landau, A.; Liu, J.; Proynov, E. I.; Rhee, Y. M.; Richard, R. M.; Rohrdanz, M. A.; Steele, R. P.; Sundstrom, E. J.; Woodcock, H. L.; Zimmerman, P. M.; Zuev, D.; Albrecht, B.; Alguire, E.; Austin, B.; Beran, G. J. O.; Bernard, Y. A.; Berquist, E.; Brandhorst, K.; Bravaya, K. B.; Brown, S. T.; Casanova, D.; Chang, C. M.; Chen, Y.; Chien, S. H.; Closser, K. D.; Crittenden, D. L.; Diedenhofen, M.; Distasio, R. A.; Do, H.; Dutoi, A. D.; Edgar, R. G.; Fatehi, S.; FustiMolnar, L.; Ghysels, A.; Golubeva-Zadorozhnaya, A.; Gomes, J.; Hanson-Heine, M. W. D.; Harbach, P. H. P.; Hauser, A. W.; Hohenstein, E. G.; Holden, Z. C.; Jagau, T. C.; Ji, H.; Kaduk, B.; Khistyaev, K.; Kim, J.; Kim, J.; King, R. A.; Klunzinger, P.; Kosenkov, D.; Kowalczyk, T.; Krauter, C. M.; Lao, K. U.; Laurent, A. D.; Lawler, K. V.; Levchenko, S. V.; Lin, C. Y.; Liu, F.; Livshits, E.; Lochan, R. C.; Luenser, A.; Manohar, P.; Manzer, S. F.; Mao, S. P.; Mardirossian, N.; Marenich, A. V.; Maurer, S. A.; Mayhall, N. J.; Neuscamman, E.; Oana, C. M.; Olivares-Amaya, R.; Oneill, D. P.; Parkhill, J. A.; Perrine, T. M.; Peverati, R.; Prociuk, A.; Rehn, D. R.; Rosta, E.; Russ, N. J.; Sharada, S. M.; Sharma, S.; Small, D. W.; Sodt, A.; Stein, T.; Stück, D.; Su, Y. C.; Thom, A. J. W.; Tsuchimochi, T.; Vanovschi, V.; Vogt, L.; Vydrov, O.; Wang, T.; Watson, M. A.; Wenzel, J.; White, A.; Williams, C. F.; Yang, J.; Yeganeh, S.; Yost, S. R.; You, Z. Q.; Zhang, I. Y.; Zhang, X.; Zhao, Y.; Brooks, B. R.; Chan, G. K. L.; Chipman, D. M.; Cramer, C. J.; Goddard, W. A.; Gordon, M. S.; Hehre, W. J.; Klamt, A.; Schaefer, H. F.; Schmidt, M. W.; Sherrill, C. D.; Truhlar, D. G.; Warshel, A.; Xu, X.; Aspuru-Guzik, A.; Baer, R.; Bell, A. T.; Besley, N. A.; Chai, J. Da; Dreuw, A.; Dunietz, B. D.; Furlani, T. R.; Gwaltney, S. R.; Hsu, C. P.; Jung, Y.; Kong, J.; Lambrecht, D. S.; Liang, W.; Ochsenfeld, C.; Rassolov, V. A.; Slipchenko, L. V.; Subotnik, J. E.; Van Voorhis, T.; Herbert, J. M.; Krylov, A. I.; Gill, P. M. W.; Head-Gordon, M. Advances in Molecular Quantum Chemistry Contained in the Q-Chem 4 Program Package. Mol. Phys. 2015, 113 (2), 184-215. https://doi.org/10.1080/00268976.2014.952696.

(26) Mahlanen, R.; Jalkanen, J.-P.; Pakkanen, T. A. Potential Energy Surfaces of CF4, CCl4 and CBr4 Dimers. Chem. Phys. 2005, 313 (1), 271-277.

https://doi.org/10.1016/j.chemphys.2005.01.018. 
(27) Tsuzuki, S.; Uchimaru, T.; Mikami, M.; Urata, S. Magnitude and Orientation Dependence of Intermolecular Interaction of Perfluoropropane Dimer Studied by High-Level Ab Initio Calculations: Comparison with Propane Dimer. J. Chem. Phys. 2004, 121 (20), 9917-9924. https://doi.org/10.1063/1.1809603.

(28) Kozuch, S.; Martin, J. M. L. Spin-Component-Scaled Double Hybrids: An Extensive Search for the Best Fifth-Rung Functionals Blending DFT and Perturbation Theory. J. Comput. Chem. 2013, 34 (27), 2327-2344. https://doi.org/10.1002/jcc.23391.

(29) Riplinger, C.; Neese, F. An Efficient and near Linear Scaling Pair Natural Orbital Based Local Coupled Cluster Method. J. Chem. Phys. 2013, 138 (3), 034106. https://doi.org/10.1063/1.4773581.

(30) Riplinger, C.; Sandhoefer, B.; Hansen, A.; Neese, F. Natural Triple Excitations in Local Coupled Cluster Calculations with Pair Natural Orbitals. J. Chem. Phys. 2013, 139 (13), 134101. https://doi.org/10.1063/1.4821834.

(31) Liakos, D. G.; Neese, F. Is It Possible to Obtain Coupled Cluster Quality Energies at near Density Functional Theory Cost? Domain-Based Local Pair Natural Orbital Coupled Cluster vs Modern Density Functional Theory. J. Chem. Theory Comput. 2015, 11 (9), 4054-4063. https://doi.org/10.1021/acs.jctc.5b00359.

(32) Adler, T. B.; Knizia, G.; Werner, H. J. A Simple and Efficient CCSD(T)-F12 Approximation. J. Chem. Phys. 2007, 127 (22), 221106. https://doi.org/10.1063/1.2817618.

(33) Knizia, G.; Adler, T. B.; Werner, H. J. Simplified CCSD(T)-F12 Methods: Theory and Benchmarks. J. Chem. Phys. 2009, 130 (5), 054104. https://doi.org/10.1063/1.3054300.

(34) Mardirossian, N.; Head-Gordon, M. Mapping the Genome of Meta-Generalized Gradient Approximation Density Functionals: The Search for B97M-V. J. Chem. Phys. 2015, 142 (7), 074111. https://doi.org/10.1063/1.4907719.

(35) Mardirossian, N.; Head-Gordon, M. w B97M-V: A Combinatorially Optimized, RangeSeparated Hybrid, Meta-GGA Density Functional with VV10 Nonlocal Correlation. J. Chem. Phys. 2016, 144 (21), 214110. https://doi.org/10.1063/1.4952647.

(36) Johnson, E. R.; Becke, A. D. A Post-Hartree-Fock Model of Intermolecular Interactions. J. Chem. Phys. 2005, 123 (2), 024101. https://doi.org/10.1063/1.1949201.

(37) Johnson, E. R.; Becke, A. D. A Post-Hartree-Fock Model of Intermolecular Interactions: Inclusion of Higher-Order Corrections. J. Chem. Phys. 2006, 124 (17), 174104. https://doi.org/10.1063/1.2190220.

(38) Becke, A. D.; Johnson, E. R. Exchange-Hole Dipole Moment and the Dispersion Interaction. J. Chem. Phys. 2005, 122, 154104.

(39) Becke, A. D.; Johnson, E. R. Exchange-Hole Dipole Moment and the Dispersion Interaction Revisited. J. Chem. Phys. 2007, 127 (15), 154104. https://doi.org/10.1063/1.2795701.

(40) Moller, C.; Plesset, M. S. Note on an Approximation Treatment for Many-Electron Systems. Phys. Rev. 1934, 46 (7), 618-622.

(41) Feyereisen, M.; Fitzgerald, G.; Komornicki, A. Use of Approximate Integrals in Ab Initio Theory. An Application in MP2 Energy Calculations. Chem. Phys. Lett. 1993, 208 (5-6), 359-363. https://doi.org/10.1016/0009-2614(93)87156-W.

(42) Vahtras, O.; Almlöf, J.; Feyereisen, M. W. Integral Approximations for LCAO-SCF Calculations. 
Chem. Phys. Lett. 1993, 213 (5-6), 514-518. https://doi.org/10.1016/0009-2614(93)89151-7.

(43) Moszyński, R.; Jeziorski, B.; Szalewicz, K. Many-Body Theory of Exchange Effects in Intermolecular Interactions - 2Nd-Quantization Approach and Comparison With Full Configuration-Interaction Results. J. Chem. Phys. 1994, 100 (1994), 1312-1325.

(44) Rybak, S.; Jeziorski, B.; Szalewicz, K. Many-body Symmetry-adapted Perturbation Theory of Intermolecular Interactions. $\mathrm{H}_{2} \mathrm{O}$ and HF Dimers. J. Chem. Phys. 1991, 95 (9), 6576-6601. https://doi.org/10.1063/1.461528.

(45) Jeziorski, B.; Jeziorski, B.; Moszynski, R.; Moszynski, R.; Szalewicz, K.; Szalewicz, K. Perturbation Theory Approach to Intermolecular Potential Energy Surfaces of van Der Waals Complexes. Chem. Rev. 1994, 94 (7), 1887-1930. https://doi.org/10.1021/cr00031a008.

(46) Szalewicz, K. Symmetry-Adapted Perturbation Theory of Intermolecular Forces. Wiley Interdiscip. Rev. Comput. Mol. Sci. 2012, 2 (2), 254-272. https://doi.org/10.1002/wcms.86.

(47) Schneider, W. B.; Bistoni, G.; Sparta, M.; Saitow, M.; Riplinger, C.; Auer, A. A.; Neese, F. Decomposition of Intermolecular Interaction Energies within the Local Pair Natural Orbital Coupled Cluster Framework. J. Chem. Theory Comput. 2016, 12 (10), 4778-4792. https://doi.org/10.1021/acs.jctc.6b00523.

(48) Bistoni, G.; Auer, A. A.; Neese, F. Understanding the Role of Dispersion in Frustrated Lewis Pairs and Classical Lewis Adducts: A Domain-Based Local Pair Natural Orbital Coupled Cluster Study. Chem. - A Eur. J. 2017, 23 (4), 865-873. https://doi.org/10.1002/chem.201604127.

(49) Wuttke, A.; Mata, R. A. Visualizing Dispersion Interactions through the Use of Local Orbital Spaces. J. Comput. Chem. 2017, 38 (1), 15-23. https://doi.org/10.1002/jcc.24508.

(50) Hättig, C.; Jansen, G.; Hess, B. A.; Ángyán, J. G. Topologically Partitioned Dynamic Polarizabilities Using the Theory of Atoms in Molecules. Can. J. Chem. 1996, 74 (6), 976-987. https://doi.org/10.1139/v96-108.

(51) Krawczuk, A.; Pérez, D.; MacChi, P. PolaBer: A Program to Calculate and Visualize Distributed Atomic Polarizabilities Based on Electron Density Partitioning. J. Appl. Crystallogr. 2014, 47 (4), 1452-1458. https://doi.org/10.1107/S1600576714010838.

(52) Keith, T. A. AIMAll (Version 17.11.14). Todd A. Keith, TK Gristmill Software, Overland Park KS, USA, 2017 (aim.tkgristmill.com).

(53) Politzer, P.; Jin, P.; Murray, J. S. Atomic Polarizability, Volume and lonization Energy. J. Chem. Phys. 2002, 117 (18), 8197-8202. https://doi.org/10.1063/1.1511180.

(54) Schulman, J. M.; Musher, J. I. Hydrogen-Atom Polarizability as a Hartree-Fock Perturbation Expansion: A Geometric Approximation to Atomic Polarizabilities. J. Chem. Phys. 1968, 49 (11), 4845. https://doi.org/10.1063/1.1669968.

(55) CRC Handbook of Chemistry and Physics, 98th ed.; Rumble, J. R., Ed.; CRC Press/Taylor \& Francis: Boca Raton, FL, 2017.

(56) Eisenschitz, R.; London, F. Über Das Verhältnis Der van Der Waalsschen Kräfte Zu Den Homöopolaren Bindungskräften. Zeitschrift für Phys. 1930, 60 (7-8), 491-527. https://doi.org/10.1007/BF01341258.

(57) Grimme, S.; Hansen, A.; Brandenburg, J. G.; Bannwarth, C. Dispersion-Corrected Mean-Field Electronic Structure Methods. Chem. Rev. 2016, 116 (9), 5105-5154.

https://doi.org/10.1021/acs.chemrev.5b00533. 
(58) Contreras-Garcia, J.; Johnson, E. R.; Keinan, S.; Chaudret, R.; Piquemal, J. P.; Beratan, D. N.; Yang, W. NCIPLOT: A Program for Plotting Noncovalent Interaction Regions. J. Chem. Theory Comput. 2011, 7 (3), 625-632. https://doi.org/10.1021/ct100641a.

(59) Flory, P. J. Thermodynamics of High Polymer Solutions. J. Chem. Phys. 1942, 10 (51), 51-61. https://doi.org/10.1063/1.1723621.

(60) Huggins, M. L. Thermodynamic Properties of Solutions of Long-Chain Compounds. Ann. N.Y. Acad. Sci. 1942, 43 (828), 1-32.

(61) Dantzler Siebert, E. M.; Knobler, C. M. Interaction Virial Coefficients in HydrocarbonFluorocarbon Mixtures. J. Phys. Chem. 1971, 75 (25), 3863-3870. https://doi.org/10.1021/j100848a049.

(62) Dantzler, E. M.; Knobler, C. M.; Windsor, M. L. Interaction Virial Coefficients in Hydrocarbon Mixtures. J. Phys. Chem. 1968, 72 (2), 684. https://doi.org/10.1021/j100694a014.

(63) Dantzler, E. M.; Knobler, C. M. Interaction Virial Coefficients in Fluorocarbon Mixtures. J. Phys. Chem. 1969, 73 (5), 1335-1341. https://doi.org/10.1021/j100694a014.

(64) Hermann, J.; DiStasio, R. A.; Tkatchenko, A. First-Principles Models for van Der Waals Interactions in Molecules and Materials: Concepts, Theory, and Applications. Chem. Rev. 2017, 117 (6), 4714-4758. https://doi.org/10.1021/acs.chemrev.6b00446.

(65) Hohenstein, E. G.; Sherrill, C. D. Wavefunction Methods for Noncovalent Interactions. Wiley Interdiscip. Rev. Comput. Mol. Sci. 2012, 2 (2), 304-326. https://doi.org/10.1002/wcms.84.

(66) Burns, L. A.; Marshall, M. S.; Sherrill, C. D. Appointing Silver and Bronze Standards for Noncovalent Interactions: A Comparison of Spin-Component-Scaled (SCS), Explicitly Correlated (F12), and Specialized Wavefunction Approaches. J. Chem. Phys. 2014, 141 (23), 234111. https://doi.org/10.1063/1.4903765.

(67) Mardirossian, N.; Head-Gordon, M. Thirty Years of Density Functional Theory in Computational Chemistry: An Overview and Extensive Assessment of 200 Density Functionals. Mol. Phys. 2017, 8976 (July), 1-58. https://doi.org/10.1080/00268976.2017.1333644.

(68) Johnson, E. R.; Mackie, I. D.; DiLabio, G. A. Dispersion Interactions in Density-Functional Theory. J. Phys. Org. Chem. 2009, 22 (12), 1127-1135. https://doi.org/10.1002/poc.1606.

(69) Goerigk, L.; Kruse, H.; Grimme, S. Benchmarking Density Functional Methods against the S66 and S66x8 Datasets for Non-Covalent Interactions. ChemPhysChem 2011, 12 (17), 3421-3433. https://doi.org/10.1002/cphc.201100826.

(70) Mardirossian, N.; Head-Gordon, M. How Accurate Are the Minnesota Density Functionals for Noncovalent Interactions, Isomerization Energies, Thermochemistry, and Barrier Heights Involving Molecules Composed of Main-Group Elements? J. Chem. Theory Comput. 2016, 12 (9), 4303-4325. https://doi.org/10.1021/acs.jctc.6b00637.

(71) Otero-de-la-Roza, A.; Johnson, E. R. Non-Covalent Interactions and Thermochemistry Using XDM-Corrected Hybrid and Range-Separated Hybrid Density Functionals Non-Covalent Interactions and Thermochemistry Using XDM-Corrected Hybrid and Range-Separated Hybrid Density Functionals. J. Chem. Phys. 2013, 138, 204109. https://doi.org/10.1063/1.4807330.

(72) Kannemann, F. O.; Becke, A. D. Van Der Waals Interactions in Density-Functional Theory: Intermolecular Complexes. J. Chem. Theory Comput. 2010, 6, 1081-1088.

(73) Calbo, J.; Ortí, E.; Sancho-García, J. C.; Aragó, J. The Nonlocal Correlation Density Functional 
VV10: A Successful Attempt to Accurately Capture Noncovalent Interactions. Annu. Rep. Comput. Chem. 2015, 11, 37-102. https://doi.org/10.1016/bs.arcc.2015.09.002.

(74) Otero-De-La-Roza, A.; Johnson, E. R. Predicting Energetics of Supramolecular Systems Using the XDM Dispersion Model. J. Chem. Theory Comput. 2015, 11 (9), 4033-4040. https://doi.org/10.1021/acs.jctc.5b00044.

(75) Goerigk, L.; Grimme, S. A General Database for Main Group Thermochemistry, Kinetics, and Noncovalent Interactions - Assessment of Common and Reparameterized (Meta-)GGA Density Functionals. J. Chem. Theory Comput. 2010, 6 (1), 107-126. https://doi.org/10.1021/ct900489g.

(76) Risthaus, T.; Grimme, S. Benchmarking of London Dispersion-Accounting Density Functional Theory Methods on Very Large Molecular Complexes. J. Chem. Theory Comput. 2013, 9 (3), 1580-1591. https://doi.org/10.1021/ct301081n.

(77) Goerigk, L.; Grimme, S. Double-Hybrid Density Functionals. Wiley Interdiscip. Rev. Comput. Mol. Sci. 2014, 4 (6), 576-600. https://doi.org/10.1002/wcms.1193.

(78) Goerigk, L.; Grimme, S. A Thorough Benchmark of Density Functional Methods for General Main Group Thermochemistry, Kinetics, and Noncovalent Interactions. Phys. Chem. Chem. Phys. 2011, 13, 6670-6688. https://doi.org/10.1039/C0CP02984J.

(79) Grimme, S. Supramolecular Binding Thermodynamics by Dispersion-Corrected Density Functional Theory. Chem. Eur. J. 2012, 18 (32), 9955-9964. https://doi.org/10.1002/chem.201200497.

(80) Calbo, J.; Ortí, E.; Sancho-García, J. C.; Aragó, J. Accurate Treatment of Large Supramolecular Complexes by Double-Hybrid Density Functionals Coupled with Nonlocal van Der Waals Corrections. J. Chem. Theory Comput. 2015, 11 (3), 932-939. https://doi.org/10.1021/acs.jctc.5b00002.

(81) Grimme, S. Accurate Description of van Der Waals Complexes by Density Functional Theory Including Empirical Corrections. J. Comput. Chem. 2004, 25 (12), 1463-1473. https://doi.org/10.1002/jcc.20078.

(82) Grimme, S.; Antony, J.; Ehrlich, S.; Krieg, H. A Consistent and Accurate Ab Initio Parametrization of Density Functional Dispersion Correction (DFT-D) for the 94 Elements $\mathrm{H}$ Pu. J. Chem. Phys. 2010, 132 (15), 154104. https://doi.org/10.1063/1.3382344.

(83) Becke, A. D.; Johnson, E. R. A Density-Functional Model of the Dispersion Interaction. J. Chem. Phys. 2005, 123 (15), 154101. https://doi.org/10.1063/1.2065267.

(84) Steinmann, S. N.; Corminboeuf, C. A System-Dependent Density-Based Dispersion Correction. J. Chem. Theory Comput. 2010, 6 (7), 1990-2001. https://doi.org/10.1021/ct1001494.

(85) Steinmann, S. N.; Corminboeuf, C. Comprehensive Benchmarking of a Density-Dependent Dispersion Correction. J. Chem. Theory Comput. 2011, 7 (11), 3567-3577. https://doi.org/10.1021/ct200602x.

(86) Steinmann, S. N.; Corminboeuf, C. A Generalized-Gradient Approximation Exchange Hole Model for Dispersion Coefficients. J. Chem. Phys. 2011, 134 (4), 044117. https://doi.org/10.1063/1.3545985.

(87) Vydrov, O. A.; Van Voorhis, T. Nonlocal van Der Waals Density Functional: The Simpler the Better. J. Chem. Phys. 2010, 133 (24), 244103. https://doi.org/10.1063/1.3521275. 
(88) Zhao, Y.; Truhlar, D. G. The M06 Suite of Density Functionals for Main Group Thermochemistry, Thermochemical Kinetics, Noncovalent Interactions, Excited States, and Transition Elements: Two New Functionals and Systematic Testing of Four M06-Class Functionals and 12 Other Function. Theor. Chem. Acc. 2008, 120 (1-3), 215-241. https://doi.org/10.1007/s00214-007-0310-x.

(89) Zhao, Y.; Truhlar, D. G. A New Local Density Functional for Main-Group Thermochemistry, Transition Metal Bonding, Thermochemical Kinetics, and Noncovalent Interactions. J. Chem. Phys. 2006, 125 (19), 194101. https://doi.org/10.1063/1.2370993.

(90) Lee, K.; Murray, É. D.; Kong, L.; Lundqvist, B. I.; Langreth, D. C. Higher-Accuracy van Der Waals Density Functional. Phys. Rev. B - Condens. Matter Mater. Phys. 2010, 82 (8), 3-6. https://doi.org/10.1103/PhysRevB.82.081101.

(91) Řezáč, J.; Šimová, L.; Hobza, P. CCSD[T] Describes Noncovalent Interactions Better than the $\operatorname{CCSD}(\mathrm{T}), \operatorname{CCSD}(\mathrm{TQ})$, and CCSDT Methods. J. Chem. Theory Comput. 2013, 9 (1), 364-369. https://doi.org/10.1021/ct3008777.

(92) Burns, L. A.; Marshall, M. S.; Sherrill, C. D. Comparing Counterpoise-Corrected, Uncorrected, and Averaged Binding Energies for Benchmarking Noncovalent Interactions. J. Chem. Theory Comput. 2014, 10 (1), 49-57. https://doi.org/10.1021/ct400149j.

(93) Řezáč, J.; Riley, K. E.; Hobza, P. Benchmark Calculations of Noncovalent Interactions of Halogenated Molecules. J. Chem. Theory Comput. 2012, 8 (11), 4285-4292. https://doi.org/10.1021/ct300647k.

(94) Jurecka, P.; Sponer, J.; Cerný, J.; Hobza, P. Benchmark Database of Accurate (MP2 and CCSD(T) Complete Basis Set Limit) Interaction Energies of Small Model Complexes, DNA Base Pairs, and Amino Acid Pairs. Phys. Chem. Chem. Phys. 2006, 8 (17), 1985-1993. https://doi.org/10.1039/b600027d.

(95) Cheng, L.; Gauss, J.; Ruscic, B.; Armentrout, P. B.; Stanton, J. F. Bond Dissociation Energies for Diatomic Molecules Containing 3d Transition Metals: Benchmark Scalar-Relativistic CoupledCluster Calculations for 20 Molecules. J. Chem. Theory Comput. 2017, 13 (3), 1044-1056. https://doi.org/10.1021/acs.jctc.6b00970.

(96) Liakos, D. G.; Sparta, M.; Kesharwani, M. K.; Martin, J. M. L.; Neese, F. Exploring the Accuracy Limits of Local Pair Natural Orbital Coupled-Cluster Theory. J. Chem. Theory Comput. 2015, 11 (4), 1525-1539. https://doi.org/10.1021/ct501129s.

(97) Minenkov, Y.; Chermak, E.; Cavallo, L. Accuracy of DLPNO-CCSD ( T) Method for NonCovalent Bond Dissociation Enthalpies from Coinage Metal Cation Complexes. J. Chem. Theory Comput. 2015, 11 (10), 1-65.

(98) Liakos, D. G.; Neese, F. Domain Based Pair Natural Orbital Coupled Cluster Studies on Linear and Folded Alkane Chains. J. Chem. Theory Comput. 2015, 11 (5), 2137-2143. https://doi.org/10.1021/acs.jctc.5b00265.

(99) Paulechka, E.; Kazakov, A. Efficient DLPNO-CCSD(T)-Based Estimation of Formation Enthalpies for $\mathrm{C}-, \mathrm{H}-, \mathrm{O}-$, and $\mathrm{N}$-Containing Closed-Shell Compounds Validated Against Critically Evaluated Experimental Data. J. Phys. Chem. A 2017, 121 (22), 4379-4387. https://doi.org/10.1021/acs.jpca.7b03195.

(100) Phipps, M. J. S.; Fox, T.; Tautermann, C. S.; Skylaris, C.-K. Energy Decomposition Analysis Approaches and Their Evaluation on Prototypical Protein-drug Interaction Patterns. Chem. Soc. Rev. 2015, 44 (10), 3177-3211. https://doi.org/10.1039/C4CS00375F. 
(101) De Silva, P.; Corminboeuf, C. Simultaneous Visualization of Covalent and Noncovalent Interactions Using Regions of Density Overlap. J. Chem. Theory Comput. 2014, 10 (9), 37453756. https://doi.org/10.1021/ct500490b.

(102) Johnson, E. R.; Keinan, S.; Mori-Sánchez, P.; Contreras-García, J.; Cohen, A. J.; Yang, W. Revealing Noncovalent Interactions. J. Am. Chem. Soc. 2010, 132 (18), 6498-6506. https://doi.org/10.1021/ja100936w.

(103) Krawczuk-Pantula, A.; Perez, D.; Macchi, P. Distributed Atomic Polarizabilities from Electron Density. 1. Motivations and Theory. Trans. Amer. Cryst. Ass. 2012, 42, 1-25.

(104) Tkatchenko, A.; Scheffler, M. Accurate Molecular van Der Waals Interactions from GroundState Electron Density and Free-Atom Reference Data. Phys. Rev. Lett. 2009, 102 (7), 6-9. https://doi.org/10.1103/PhysRevLett.102.073005.

(105) Caldeweyher, E.; Bannwarth, C.; Grimme, S. Extension of the D3 Dispersion Coefficient Model. J. Chem. Phys. 2017, 147 (3), 034112. https://doi.org/10.1063/1.4993215.

(106) Sanderson, R. T. Principles of Electronegativity Part I. General Nature. J. Chem. Educ. 1988, 65 (2), 112. https://doi.org/10.1021/ed065p112.

(107) Sanderson, R. T. Principles of Electronegativity Part II. Applications. J. Chem. Educ. 1988, 65 (3), 227. https://doi.org/10.1021/ed065p227.

(108) Parr, R. G.; Donnelly, R. A.; Levy, M.; Palke, W. E. Electronegativity: The Density Functional Viewpoint. J. Chem. Phys. 1978, 68 (8), 3801-3807. https://doi.org/10.1063/1.436185.

(109) Geerlings, P.; De Proft, F.; Langenaeker, W. Conceptual Density Functional Theory. Chem. Rev. 2003, 103 (5), 1793-1874. https://doi.org/10.1021/cr990029p.

(110) Proft, F. De; Ayers, P. W.; Geerlings, P. The Conceptual Density Functional Theory Perspective of Bonding. In The Chemical Bond; Frenking, G., Shaik, S., Eds.; 2014. https://doi.org/10.1002/9783527664696.ch7.

(111) Nagle, J. K. Atomic Polarizability and Electronegativity. J. Am. Chem. Soc. 1990, 112 (12), 4741-4747. https://doi.org/10.1021/ja00168a019.

(112) Pearson, R. G. Chemical Hardness and Density Functional Theory. J. Chem. Sci. 2005, 117 (5), 369-377. https://doi.org/10.1007/BF02708340.

(113) Parr, R. G.; Pearson, R. G. Absolute Hardness: Companion Parameter to Absolute Electronegativity. J. Am. Chem. Soc. 1983, 105 (26), 7512-7516. https://doi.org/10.1021/ja00364a005.

(114) Pearson, R. G. Hard and Soft Acids and Bases, HSAB, Part 1: Fundamental Principles. J. Chem. Educ. 1968, 45 (9), 581. https://doi.org/10.1021/ed045p581.

(115) Pearson, R. G. Hard and Soft Acids and Bases, HSAB, Part II: Underlying Theories. J. Chem. Educ. 1968, 45 (10), 643. https://doi.org/10.1021/ed045p643.

(116) Grimme, S.; Huenerbein, R.; Ehrlich, S. On the Importance of the Dispersion Energy for the Thermodynamic Stability of Molecules. ChemPhysChem 2011, 12 (7), 1258-1261. https://doi.org/10.1002/cphc.201100127.

(117) Wagner, J. P.; Schreiner, P. R. London Dispersion in Molecular Chemistry - Reconsidering Steric Effects. Angew. Chem. Int. Ed. 2015, 54 (42), 12274-12296. https://doi.org/10.1002/anie.201503476. 
(118) McKinnon, J. J.; Jayatilaka, D.; Spackman, M. A. Towards Quantitative Analysis of Intermolecular Interactions with Hirshfeld Surfaces. Chem. Commun. 2007, No. 37, 38143816. https://doi.org/10.1039/b704980c.

(119) Gladysz, J. A.; Emnet, C. Fluorous Solvents and Related Media. In Handbook of Fluorous Chemistry; Gladysz, J. A., Curran, D. P., Horvath, I. T., Eds.; WILEY-VCH Verlag: Weinheim, Germany, 2005; pp 11-23. https://doi.org/10.1002/3527603905.ch3.

(120) Simons, J. H.; Dunlap, R. D. The Properties of N-Pentforane and Its Mixtures with n-Pentane. J. Chem. Phys. 1950, 18 (3), 335-346. https://doi.org/10.1063/1.1747628.

(121) Bedford, R. G.; Dunlap, R. D. Solubilities and Volume Changes Attending Mixing for the System: Perfluoro-n-Hexane-n-Hexane. J. Am. Chem. Soc. 1958, 80 (2), 282-285. https://doi.org/10.1021/ja01535a007.

(122) Campos-Vallette, M.; Campos-Vallette, M.; Diaz-Fleming, G. Some Physical and Thermodynamic Properties of N-Cnf2n+2 Compounds with $n=4-8$. Semina: Ciências Agrárias. 1983, pp 405-409. https://doi.org/10.5433/1679-0359.1983v4n14p405.

(123) Morgado, P.; Laginhas, C. M. C.; Lewis, J. Ben; McCabe, C.; Martins, L. F. G.; Filipe, E. J. M. Viscosity of Liquid Perfluoroalkanes and Perfluoroalkylalkane Surfactants. J. Phys. Chem. B 2011, 115 (29), 9130-9139. https://doi.org/10.1021/jp201364k.

(124) Post, G. B.; Cohn, P. D.; Cooper, K. R. Perfluorooctanoic Acid (PFOA), an Emerging Drinking Water Contaminant: A Critical Review of Recent Literature. Environ. Res. 2012, 116, 93-117. https://doi.org/10.1016/j.envres.2012.03.007.

(125) Prevedouros, K.; Cousins, I. T.; Buck, R. C.; Korzeniowski, S. H. Sources, Fate and Transport of Perfluorocarboxylates. Environ. Sci. Technol. 2006, 40 (1), 32-44. https://doi.org/10.1021/es0512475.

(126) Lau, C.; Anitole, K.; Hodes, C.; Lai, D.; Pfahles-Hutchens, A.; Seed, J. Perfluoroalkyl Acids: A Review of Monitoring and Toxicological Findings. Toxicol. Sci. 2007, 99 (2), 366-394. https://doi.org/10.1093/toxsci/kfm128.

(127) L 150. Off. J. Eur. Union 2017, 60 (L150), 1-19.

(128) Mecinović, J.; Snyder, P. W.; Mirica, K. A.; Bai, S.; MacK, E. T.; Kwant, R. L.; Moustakas, D. T.; Héroux, A.; Whitesides, G. M. Fluoroalkyl and Alkyl Chains Have Similar Hydrophobicities in Binding to the "Hydrophobic Wall" of Carbonic Anhydrase. J. Am. Chem. Soc. 2011, 133 (35), 14017-14026. https://doi.org/10.1021/ja2045293.

(129) Assaf, K. I.; Florea, M.; Antony, J.; Henriksen, N. M.; Yin, J.; Hansen, A.; Qu, Z.; Sure, R.; Klapstein, D.; Gilson, M. K.; Grimme, S.; Nau, W. M. The Hydrophobe Challenge: A Joint Experimental and Computational Study on the Binding of Hydrocarbons to Cucurbiturils. J. Phys. Chem. B 2017, 121 (49), 11144-11162. https://doi.org/10.1021/acs.jpcb.7b09175.

(130) Grimme, S.; Brandenburg, J. G.; Bannwarth, C.; Hansen, A. Consistent Structures and Interactions by Density Functional Theory with Small Atomic Orbital Basis Sets. J. Chem. Phys. 2015, 143 (5), 054107. https://doi.org/10.1063/1.4927476. 
For Table of Contents Only:

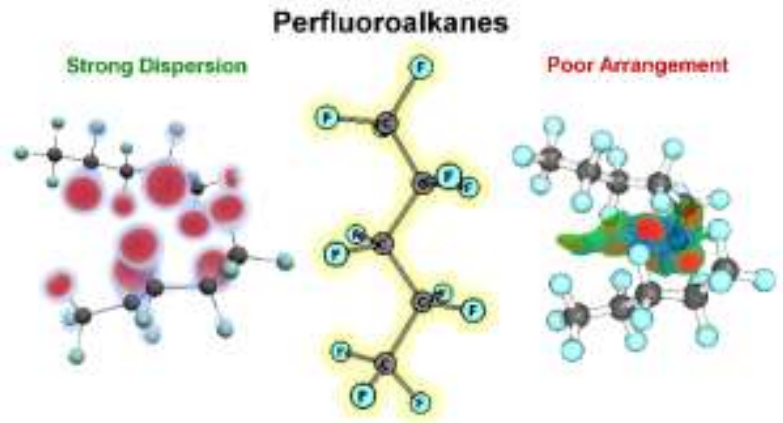


Electronic Supporting Information for

\title{
Origin of the Immiscibility of Alkanes and Perfluoroalkanes
}

\author{
Robert Pollice and Peter Chen* \\ Laboratorium für Organische Chemie, ETH Zürich, Vladimir-Prelog-Weg 2, 8093 Zürich, \\ Switzerland \\ E-mail: peter.chen@org.chem.ethz.ch
}




\section{Contents}

1 Computational Methods $\quad$ S5

1.1 Geometry Optimizations for Benchmarking . . . . . . . . . . . S5

1.2 Single Points . . . . . . . . . . . . . . . . . . S6

1.2.1 Semiempirical Methods . . . . . . . . . . . . . . . S7

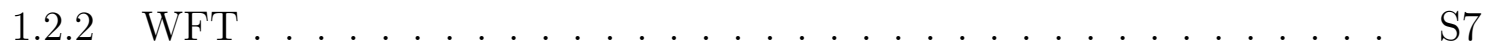

$1.2 .3 \mathrm{DFT} \ldots \ldots \ldots \ldots \ldots \ldots$

1.3 Energy Decomposition Schemes . . . . . . . . . . . . . . . S10

1.4 Visual Analyses . . . . . . . . . . . . . . . . . . . . S10

1.5 Additional Analyses . . . . . . . . . . . . . . . . . . . S11

2 Supplementary Results and Discussion $\quad$ S12

2.1 Benchmarking . . . . . . . . . . . . . . . . S12

2.1.1 Structures of the Complexes . . . . . . . . . . . . . S12

2.1 .2 Discussion . . . . . . . . . . . . . . . . . . S27

2.1.3 Benchmarking WFT Methods . . . . . . . . . . . . . . S31

2.1.4 Rigid Geometry Scans . . . . . . . . . . . . . . . . S35

2.1.5 Deformation Energies . . . . . . . . . . . . . . . S59

2.2 Energy Decomposition Analysis . . . . . . . . . . . . . . . . S59

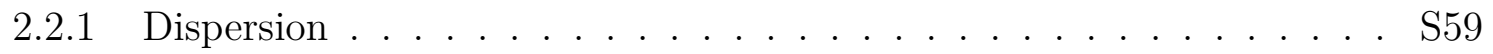

2.2.2 Electrostatics . . . . . . . . . . . . . . . . . . . S59

2.2.3 Comparison of SAPT2+ and LED . . . . . . . . . . . . . . . . S61

2.2.4 LED Decomposition . . . . . . . . . . . . . . . S62

2.2.5 Additional LED Results . . . . . . . . . . . . . . . . S65

2.2.6 Additional DID Plots . . . . . . . . . . . . . . S71

2.3 Simple Interaction Models . . . . . . . . . . . . . . . . . . . . . . S79

2.3.1 Molecular London Dispersion Model . . . . . . . . . . . . S79 
2.3.2 Atom-pairwise London Dispersion Models . . . . . . . . . . . . . S80

2.3.3 Atom-type Energy Decomposition . . . . . . . . . . . . . . . . . S82

2.3.4 Correlation of BIE with Interaction Surface . . . . . . . . . . . S90

2.4 Interaction Descriptors . . . . . . . . . . . . . . . . . . S S91

2.4 .1 Q Measure and P Measure . . . . . . . . . . . . . . . . . S91

2.5 Interaction Geometries . . . . . . . . . . . . . . . . . . . . S92

2.5 .1 Interaction Surface Areas . . . . . . . . . . . . . . . . . . . S92

2.6 Predicted Microscopic and Macroscopic Mixing Behavior . . . . . . . . . S95

2.6 .1 Average Coordination Number . . . . . . . . . . . . . . . . S95

2.7 Trends in Experimental Properties . . . . . . . . . . . . . . S95

2.7.1 Boiling Points of Alkanes and Perfluoroalkanes . . . . . . . . . . . . S95

2.7.2 Critical Parameters of Alkanes and Perfluoroalkanes . . . . . . . . . . S97

2.7.3 Enthalpies of Evaporation of Alkanes and Perfluoroalkanes . . . . . . S100

2.7.4 Bulk Moduli of Alkanes and Perfluoroalkanes . . . . . . . . . . . S101

2.7.5 Compressibilities of Alkanes and Perfluoroalkanes . . . . . . . . . . S102

2.7.6 Interal Pressures of Alkanes and Perfluoroalkanes . . . . . . . . . . S103

2.7.7 Surface Tensions of Alkanes and Perfluoroalkanes . . . . . . . . . . . S104

2.7.8 Molar Heat Capacities of Alkanes and Perfluoroalkanes . . . . . . . . S105

2.7.9 Dynamic Viscosities of Alkanes and Perfluoroalkanes . . . . . . . S106

2.7.10 Deviation of Computational Results from the Geometric Mean Rule . S107

3 Mathematical Derivations $\quad$ S110

3.1 Homomolecular Cuboid Model for Interaction Surfaces . . . . . . . . . S S110

3.2 Heteromolecular Cuboid Model for Interaction Surfaces . . . . . . . . . . . . S111

4 Computational Raw Data $\quad$ S114

4.1 Benchmarking . . . . . . . . . . . . . . . . . . . S114

4.2 Rigid Geometry Scans . . . . . . . . . . . . . . . . . . . . S166 
4.3 Energy Decomposition Analysis . . . . . . . . . . . . . . . . S173

4.4 Simple Interaction Models . . . . . . . . . . . . . . . . . . . . . . . S180

4.5 Interaction Descriptors . . . . . . . . . . . . . . . . . . S184

4.6 Interaction Geometries . . . . . . . . . . . . . . . . . . . S185

4.7 Predicted Microscopic and Macroscopic Mixing Behavior . . . . . . . . . . . S187

$\begin{array}{lr}\text { References } & \text { S189 }\end{array}$ 


\section{Computational Methods}

The following programs were employed for all the computations in this study:

- Orca (versions 3.0.3 and 4.0.1) ${ }^{1,2}$

- PSI4 (versions 1.1 and 1.2) ${ }^{3,4}$

- Molpro (version 2015.1.18) ${ }^{5}$

- Gaussian (version 09, revision D.01) ${ }^{6}$

- $\operatorname{ADF}\left(\right.$ version 2016) ${ }^{7}$

- Q-Chem (version 4.3$)^{8}$

- $\operatorname{postg}^{9,10}$

- BootD3 $3^{11,12}$

- xtb (version 5.8) ${ }^{13}$

- Paraview (version 5.5.0-RC3) 14,15

- PolaBer (version 0.02) ${ }^{16}$

- AIMAll (version 17.11.14) ${ }^{17}$

- multiwfn (version 3.4.1) 18

- NCIPLOT (version 3.0) 19,20

- PyMol (version 1.8.4) ${ }^{21}$

- Chemcraft (version 1.8, build 536b) ${ }^{22}$

- Python (version 3.6.0) ${ }^{23}$

\subsection{Geometry Optimizations for Benchmarking}

Geometry optimizations were performed in Orca (version 3.0.3) using the DSD-PBEP86 ${ }^{24} /$ def2TZVP-spd ${ }^{25,26}$ level of theory employing the RIJCOSX ${ }^{27,28}$ approximation in conjunction with the def2-TZVP $/ \mathrm{J}^{29}$ and def2-TZVP $/ \mathrm{C}^{30}$ fitting basis sets. We performed systematic exploration of the conformational space of the intermolecular complexes by starting DFT geometry optimizations from various initial geometries having distinct spatial arrangements 
of the interacting molecules. The exploration was performed starting from $\mathbf{1 - H H}, \mathbf{1 - H F}$ and $\mathbf{1 - F F}$ by increasing the carbon chains of the interacting molecules by one and finding the most stable intermolecular complexes for $\mathbf{2 - H H}, \mathbf{2 - H F}$ and $\mathbf{2 - F F}$ by systematically varying the position of the newly introduced groups and the spatial arrangement of the two interacting molecules. In addition, in a second cycle, we started from the most stable intermolecular complexes for $\mathbf{6 - H H}, \mathbf{6 - H F}$ and $\mathbf{6 - F F}$ and systematically reduced the carbon chain length by successively removing distinct end groups from the complexes. That way, the conformational space was explored step-by-step by systematically changing the carbon chain length from one to six and from six to one and performing conformational exploration at every step. The most stable conformers, judged on the basis of DSD-PBEP86/def2-QZVP single point energies, were selected for benchmarking. For $\mathbf{H H}$ and $\mathbf{F F}$, the two lowest energy conformers in each class of complexes was selected for further benchmarking. For $\mathbf{H F}$, except for $\mathbf{1 - H F}$ for which we selected only two conformers, the four lowest energy conformers in each class of complexes was selected for benchmarking.

\section{$1.2 \quad$ Single Points}

Wavefunction Theory (WFT) methods and Density Function Theory (DFT) methods were used for single point calculations. Single point calculations were performed using DSDPBEP86/def2-TZVP-spd geometries. Two-point complete basis set (CBS) extrapolations were performed using two single point energies calculated using the either the cc-pVTZ ${ }^{31}$ and cc-pVQZ ${ }^{31}$ or the aug-cc-pVTZ ${ }^{32}$ and aug-cc-pVQZ ${ }^{32}$ basis sets and are abbreviated herein as CBS(34) and CBS(aug34), respectively. For DLPNO-CCSD(T) calculations, we also used the following non-standard sets of thresholds together with the TightPNO ${ }^{33}$ keyword in Orca:

- VeryTightPNO: ${ }^{34}$ TCutPNO $=1 \cdot 10^{-8}$, TCutPairs $=1 \cdot 10^{-6}$, TCutMKN $=1 \cdot 10^{-4}$

- ExtremePNO: TCutPNO $=1 \cdot 10^{-9}$, TCutPairs $=1 \cdot 10^{-7}$, TCutMKN $=1 \cdot 10^{-5}$ 


\subsubsection{Semiempirical Methods}

GFN-xTB ${ }^{13}$ and GFN2-xTB ${ }^{35}$ calculations were performed using xtb (version 5.8).

\subsubsection{WFT}

\begin{tabular}{|c|c|c|c|}
\hline Method & Basis set & Software & Comments \\
\hline $\operatorname{CCSD}(\mathrm{T})-\mathrm{F} 12^{36,37}$ & cc-pVTZ-F12 $2^{38}$ & Molpro & - \\
\hline $\operatorname{CCSD}(\mathrm{T})-\mathrm{F} 12^{36,37}$ & cc-pVDZ-F12 $2^{38}$ & Molpro & - \\
\hline $\operatorname{CCSD}(\mathrm{T})^{39,40}$ & $\mathrm{CBS}(34)$ & Molpro & - \\
\hline $\operatorname{CCSD}(\mathrm{T})^{39,40}$ & aug-cc-pVDZ 32 & Molpro & $\begin{array}{l}\text { CBS(aug34) extrapolation was performed using Orca (version } \\
\text { 4.0.1) with RI-MP2. }{ }^{41,42}\end{array}$ \\
\hline $\operatorname{CCSD}(\mathrm{T})^{39,40}$ & aug-cc-pVDZ 32 & Molpro & $\begin{array}{l}\text { CBS(aug34) extrapolation was performed using Orca (version } \\
\text { 4.0.1) with LPNO-CEPA/1. }{ }^{43}\end{array}$ \\
\hline DLPNO-CCSD(T) ${ }^{44,45}$ & $\mathrm{CBS}(34)$ & Orca 4.0.1 & $\begin{array}{l}\text { The NormalPNO, }{ }^{33} \text { TightPNO, }{ }^{33} \text { VeryTightPNO and Ex- } \\
\text { tremePNO sets of threshold parameters were used, respectively. } \\
\text { The RI-JK }{ }^{46} \text { approximation for HF calculations was used. }\end{array}$ \\
\hline $\mathrm{MP} 2^{47}$ & $\mathrm{CBS}(34)$ & Orca 4.0.1 & - \\
\hline RI-MP2 $2^{41,42}$ & $\mathrm{CBS}(34)$ & Orca 4.0.1 & The RI-JK ${ }^{46}$ approximation for HF calculations was used. \\
\hline RI-SCS-MP2 $2^{41,42,48}$ & $\operatorname{CBS}(34)$ & Orca 4.0.1 & The RI-JK ${ }^{46}$ approximation for HF calculations was used. \\
\hline RI-MP3 $3^{41,42,49,50}$ & $\mathrm{CBS}(34)$ & Orca 4.0.1 & The RI-JK ${ }^{46}$ approximation for HF calculations was used. \\
\hline
\end{tabular}




\subsubsection{DFT}

\begin{tabular}{|c|c|c|c|}
\hline Method & Basis set & Software & Comments \\
\hline B97M-V 51 & def2-QZVP 25 & PSI4 1.2 & $\begin{array}{l}\text { The RI-J }{ }^{52-54} \text { approximation with the def } 2 / \mathrm{JK}^{55} \\
\text { fitting basis set was used. }\end{array}$ \\
\hline$\omega \mathrm{B} 97 \mathrm{M}-\mathrm{V}^{56}$ & def2-QZVP ${ }^{25}$ & PSI4 1.2 & $\begin{array}{l}\text { The RI-JK }{ }^{46} \text { approximation with the def } 2 / \mathrm{JK}^{55} \\
\text { fitting basis set was used. }\end{array}$ \\
\hline B3LYP ${ }^{57-59}-\mathrm{XDM}^{60-64}$ & aug-cc-pVTZ ${ }^{32}$ & Gaussian, postg & - \\
\hline $\mathrm{PBE}^{65}-\mathrm{XDM}^{60-64}$ & aug-cc-pVTZ ${ }^{32}$ & Gaussian, postg & - \\
\hline
\end{tabular}

\begin{tabular}{|c|c|c|c|}
\hline DSD-PBEP86 ${ }^{24}$ & def2-QZVP 25 & Orca 3.0.3 & $\begin{array}{l}\text { The RIJCOSX }{ }^{27,28} \text { approximation with the def2- } \\
\mathrm{TZVP} / \mathrm{J}^{29} \text { and def } 2 \text {-TZVP } / \mathrm{C}^{30} \text { fitting basis sets } \\
\text { was used. }\end{array}$ \\
\hline
\end{tabular}

\begin{tabular}{|c|c|c|c|}
\hline $\mathrm{BLYP}^{57,58}-\mathrm{XDM}^{60-64}$ & aug-cc-pVTZ ${ }^{32}$ & Gaussian, postg & - \\
\hline M06L 66 & def2-QZVP 25 & Orca 4.0.1 & $\begin{array}{l}\text { The RI-J }{ }^{52-54} \text { approximation with the } \operatorname{def} 2 / \mathrm{J}^{55} \\
\text { fitting basis set was used. }\end{array}$ \\
\hline B3LYP ${ }^{57-59}-\mathrm{NL}^{67,68}$ & def2-QZVP 25 & Orca 4.0.1 & $\begin{array}{l}\text { The RIJCOSX }{ }^{27,28} \text { approximation with the def2- } \\
\text { TZVP/J }{ }^{29} \text { fitting basis set was used. }\end{array}$ \\
\hline $\mathrm{PBE}^{65}-\mathrm{D} 3(\mathrm{BJ})^{11,69}$ & def2-QZVP 25 & Orca 4.0.1 & $\begin{array}{l}\text { The RI-J }{ }^{52-54} \text { approximation with the } \operatorname{def} 2 / \mathrm{J}^{55} \\
\text { fitting basis set was used. }\end{array}$ \\
\hline M06-2X ${ }^{70}$-D3(ZERO) ${ }^{11,69}$ & def2-QZVP 25 & Orca 4.0.1 & $\begin{array}{l}\text { The RIJCOSX }{ }^{27,28} \text { approximation with the def2- } \\
\text { TZVP/J }{ }^{29} \text { fitting basis set was used. }\end{array}$ \\
\hline $\mathrm{BLYP}^{57,58}-\mathrm{NL}^{67,68}$ & def2-QZVP 25 & Orca 4.0.1 & $\begin{array}{l}\text { The RI-J }{ }^{52-54} \text { approximation with the } \operatorname{def} 2 / \mathrm{J}^{55} \\
\text { fitting basis set was used. }\end{array}$ \\
\hline B3LYP ${ }^{57-59}-\mathrm{dDsC}^{71-73}$ & $\mathrm{QZ}_{4} \mathrm{P}^{74}$ & $\mathrm{ADF}$ & $\begin{array}{l}\text { Density fitting was performed using the RI approx- } \\
\text { imation }{ }^{74,75} \text { with the } \mathrm{QZ}_{4} \mathrm{P}^{74} \text { fitting basis set. }\end{array}$ \\
\hline
\end{tabular}




\begin{tabular}{|c|c|c|c|}
\hline Method & Basis set & Software & Comments \\
\hline M06L $^{66}$-D3(ZERO) ${ }^{11,69}$ & def2-QZVP 25 & Orca 4.0.1 & $\begin{array}{l}\text { The RI-J }{ }^{52-54} \text { approximation with the } \operatorname{def} 2 / \mathrm{J}^{55} \\
\text { fitting basis set was used. }\end{array}$ \\
\hline B2PLYP $76-D 3^{11,69}$ & def2-QZVP 25 & Orca 4.0.1 & $\begin{array}{l}\text { The RIJCOSX }{ }^{27,28} \text { approximation with the def2- } \\
\mathrm{TZVP} / \mathrm{J}^{29} \text { and } \operatorname{def} 2-\mathrm{TZVP} / \mathrm{C}^{30} \text { fitting basis sets } \\
\text { were used. }\end{array}$ \\
\hline TPSS $^{77}-\mathrm{D} 3(\mathrm{BJ})^{11,69}$ & def2-QZVP 25 & Orca 4.0.1 & $\begin{array}{l}\text { The RI-J }{ }^{52-54} \text { approximation with the } \operatorname{def} 2 / \mathrm{J}^{55} \\
\text { fitting basis set was used. }\end{array}$ \\
\hline B3LYP ${ }^{57-59-D 3(B J)}{ }^{11,69}$ & def2-QZVP 25 & Orca 4.0.1 & $\begin{array}{l}\text { The RIJCOSX }{ }^{27,28} \text { approximation with the def2- } \\
\mathrm{TZVP} / \mathrm{J}^{29} \text { fitting basis set was used. }\end{array}$ \\
\hline$\omega \mathrm{B} 97 \mathrm{X}-\mathrm{D} 3(\mathrm{BJ}){ }^{11,69,78}$ & def2-QZVP 25 & Orca 4.0.1 & $\begin{array}{l}\text { The RIJCOSX }{ }^{27,28} \text { approximation with the def2- } \\
\text { TZVP/J }{ }^{29} \text { fitting basis set was used. }\end{array}$ \\
\hline rPW86PW92-vdW-DF10 79 & pc- $3^{80-82}$ & Q-Chem & - \\
\hline $\mathrm{PBE}^{65}-\mathrm{dDsC}^{71-73}$ & $\mathrm{QZ4P}^{74}$ & $\mathrm{ADF}$ & $\begin{array}{l}\text { Density fitting was performed using the RI approx- } \\
\text { imation }{ }^{75} \text { with the QZ4P }{ }^{74} \text { fitting basis set. }\end{array}$ \\
\hline $\mathrm{BLYP}^{57,58}$-D3(BJ) ${ }^{11,69}$ & def2-QZVP 25 & Orca 4.0.1 & $\begin{array}{l}\text { The RI-J }{ }^{52-54} \text { approximation with the } \operatorname{def} 2 / \mathrm{J}^{55} \\
\text { fitting basis set was used. }\end{array}$ \\
\hline M06- $2 \mathrm{X}^{70}$ & def2-QZVP 25 & Orca 4.0.1 & $\begin{array}{l}\text { The RIJCOSX } 27,28 \text { approximation with the def2- } \\
\text { TZVP } / \mathrm{J}^{29} \text { fitting basis set was used. }\end{array}$ \\
\hline B97-D3(BJ) ${ }^{11,69,83}$ & def2-QZVP 25 & Orca 4.0.1 & $\begin{array}{l}\text { The RI-J }{ }^{52-54} \text { approximation with the } \operatorname{def} 2 / \mathrm{J}^{55} \\
\text { fitting basis set was used. }\end{array}$ \\
\hline
\end{tabular}




\subsection{Energy Decomposition Schemes}

\begin{tabular}{|c|c|c|c|}
\hline Method & Basis set & Software & Comments \\
\hline sSAPT0 $^{84}$ & jun-cc-pVDZ ${ }^{85,86}$ & PSI4 1.1 & $\begin{array}{l}\text { The RI-JK }{ }^{46} \text { approximation for HF calculations was used with } \\
\text { the jun-cc-pVDZ/JK }{ }^{87} \text { fitting basis set. }\end{array}$ \\
\hline $\mathrm{SAPT} 2+{ }^{84}$ & aug-cc-pVDZ ${ }^{32}$ & PSI4 1.1 & $\begin{array}{l}\text { The RI-JK }{ }^{46} \text { approximation for HF calculations was used with } \\
\text { the aug-cc-pVDZ/JK }{ }^{46} \text { fitting basis set. }\end{array}$ \\
\hline $\mathrm{SAPT} 2+(3) \delta \mathrm{MP} 2^{84}$ & aug-cc-pVTZ ${ }^{32}$ & PSI4 1.1 & $\begin{array}{l}\text { The RI-JK }{ }^{46} \text { approximation for HF calculations was used with } \\
\text { the aug-cc-pVTZ/JK }{ }^{46} \text { fitting basis set. }\end{array}$ \\
\hline $\mathrm{SAPT} 2+(\mathrm{CCD}) \delta \mathrm{MP} 2^{84}$ & aug-cc-pVTZ ${ }^{32}$ & PSI4 1.1 & $\begin{array}{l}\text { The RI-JK }{ }^{46} \text { approximation for HF calculations was used with } \\
\text { the aug-cc-pVTZ/JK }{ }^{46} \text { fitting basis set. }\end{array}$ \\
\hline LED $^{88}$-DLPNO-CCSD $(\mathrm{T})^{44,45}$ & cc-pVQZ ${ }^{31}$ & Orca 4.0.1 & $\begin{array}{l}\text { The RI-JK }{ }^{46} \text { approximation for HF calculations was used with } \\
\text { the cc-pVQZ/JK }{ }^{46} \text { fitting basis set. TightPNO }{ }^{33} \text { thresholds } \\
\text { were used. }\end{array}$ \\
\hline
\end{tabular}

\subsection{Visual Analyses}

\begin{tabular}{|c|c|c|c|}
\hline Method & Level of Theory & Software & Comments \\
\hline DID $^{89}$ & LMP2 ${ }^{90} /$ aug-cc-pVTZ & Molpro & $\begin{array}{l}\text { The cc-pVTZ basis set was used on } \mathrm{H} \text { atoms. The } \\
\text { RI-JK }{ }^{46} \text { approximation for HF calculations was } \\
\text { used with the automatically generated JKFIT basis } \\
\text { set. Visualization was performed using Paraview. }\end{array}$ \\
\hline PolaBer ${ }^{16}$ & $\mathrm{MP}^{91} /$ def2-TZVPPD ${ }^{25,92}$ & PolaBer, Orca 4.0.1 & $\begin{array}{l}\text { Calculations were performed once in the absence } \\
\text { and in the presence of electric fields of } 0.005 \text { a.u. in } \\
\text { the positive and negative directions of the cartesian } \\
\text { coordinate axes, respectively. }\end{array}$ \\
\hline
\end{tabular}




\begin{tabular}{|c|c|c|c|}
\hline Method & Level of Theory & Software & Comments \\
\hline NCIPLOT ${ }^{19,20}$ & $\mathrm{MP}^{91} /$ def2-TZVPPD ${ }^{25,92}$ & NCIPLOT, Orca 4.0.1 & $\begin{array}{l}\text { The intermolecular cut-off used was } 0.9 \text { to neglect } \\
\text { intramolecular interactions. The density cut-off } \\
\text { was } 0.1 \text {. The reduced density gradient cut-off was } \\
2.0 \text {. The density cut-off in the cube files was } 0.007 \text {, } \\
\text { the RDG cut-off in the cube files was } 0.5 \text {. Visual- } \\
\text { ization was performed using PyMol. }\end{array}$ \\
\hline
\end{tabular}

\subsection{Additional Analyses}

Van der Waals surfaces were calculated with an isovalue of 0.001 using MP2 ${ }^{91} /$ def2TZVPPD ${ }^{25,92}$ densities in multiwfn. COSMO $^{93}$ surfaces were calculated using ADF and the corresponding volumes and surfaces were obtained from the corresponding COSMO-RS module. ${ }^{94}$ Additional calculations including the simple interaction models, the Q values, the $P$ values and the $n_{\text {contacts }}$ were performed using python. 


\section{Supplementary Results and Discussion}

In this section, additional results, that did not fit into the main manuscript, are presented and discussed.

\subsection{Benchmarking}

\subsubsection{Structures of the Complexes}

Figures S1-S46 show the final structures of all the intermolecular complexes. The corresponding XYZ files are provided in the additional supporting material. For benchmarking of the DFT and WFT methods (cf. Table 2), the following types of complexes were used: 1-HH, 1-HF, 1-FF, 2-HH, 2-HF, 2-FF, 3-HH, 3-HF, 3-FF, 4-HH and 4-FF.
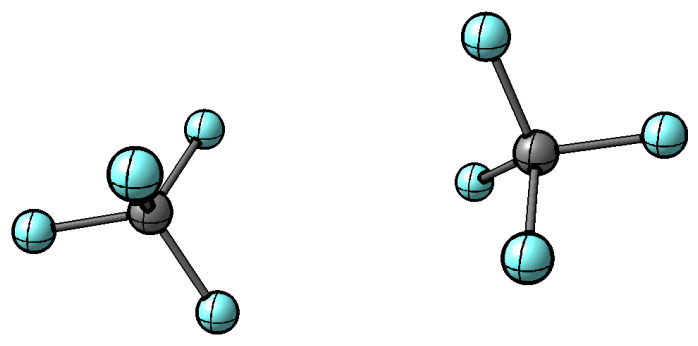

Figure S1: Final structure of complex 1-FF1.
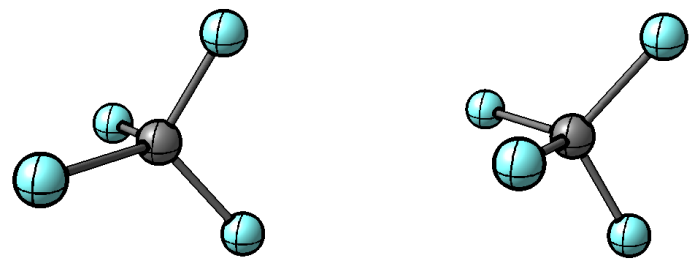

Figure S2: Final structure of complex 1-FF2. 

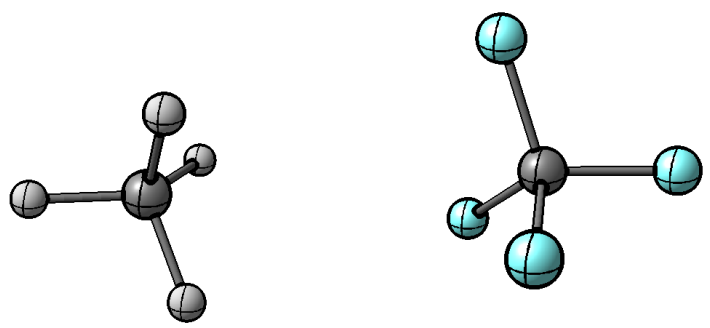

Figure S3: Final structure of complex 1-HF1.
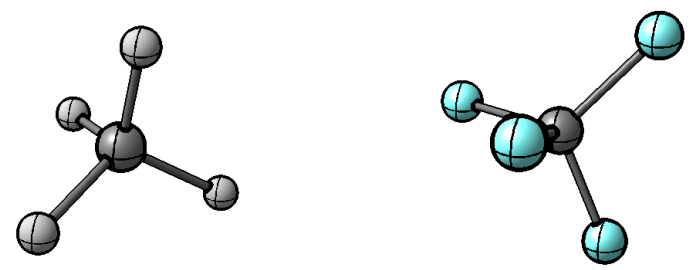

Figure S4: Final structure of complex 1-HF2.
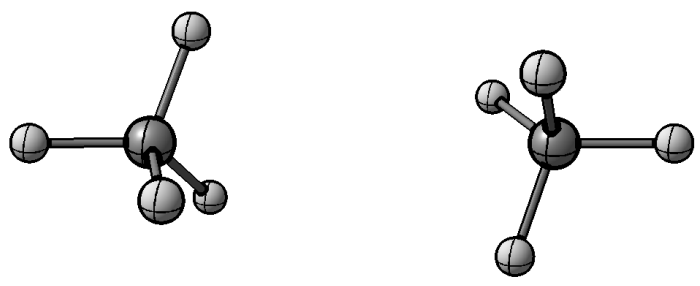

Figure S5: Final structure of complex 1-HH1.
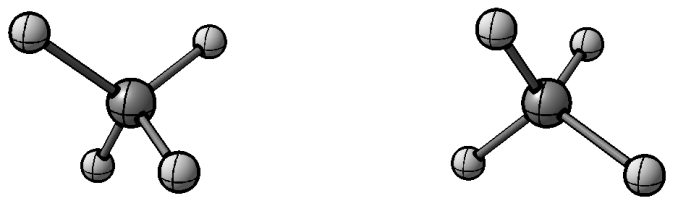

Figure S6: Final structure of complex 1-HH2.
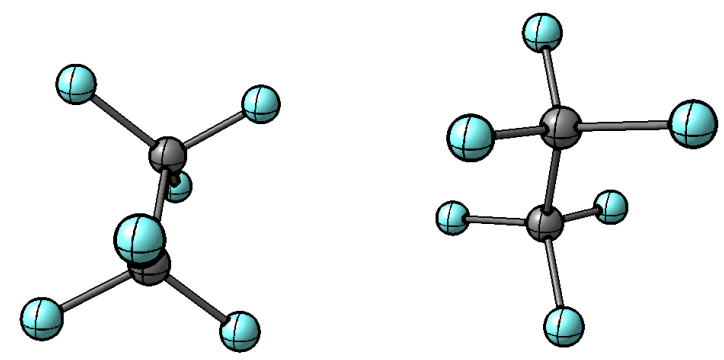

Figure S7: Final structure of complex 2-FF1. 

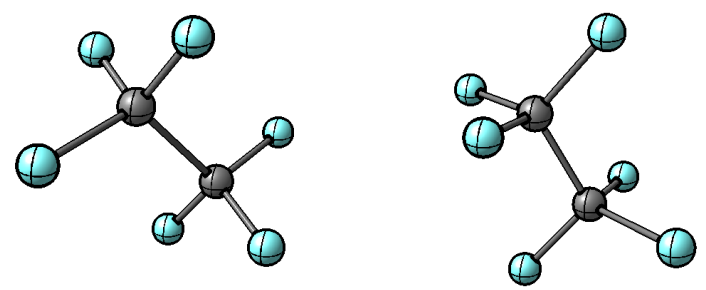

Figure S8: Final structure of complex 2-FF2.
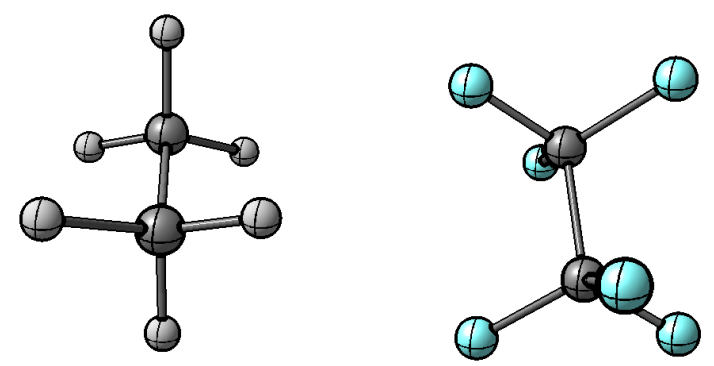

Figure S9: Final structure of complex 2-HF1.
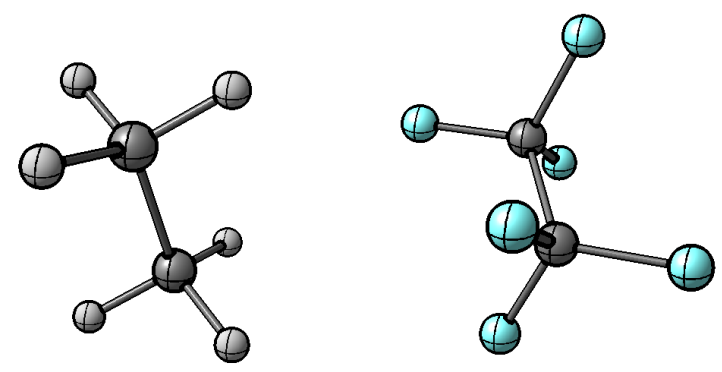

Figure S10: Final structure of complex 2-HF2.
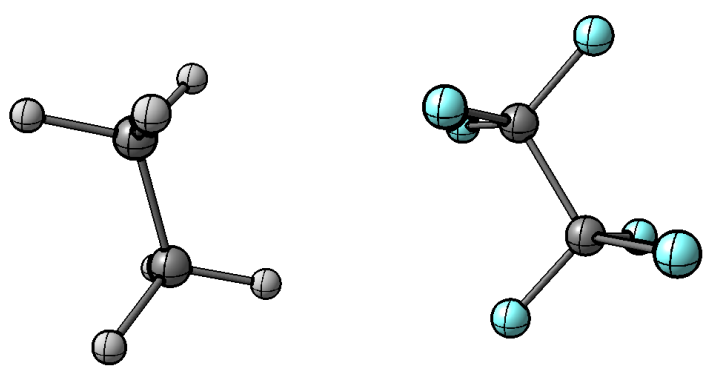

Figure S11: Final structure of complex 2-HF3. 

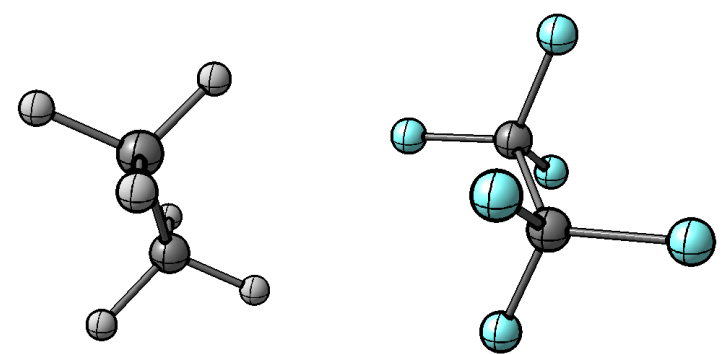

Figure S12: Final structure of complex 2-HF4.
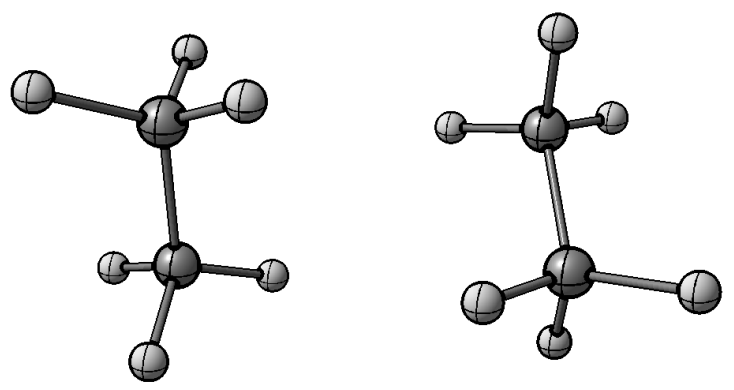

Figure S13: Final structure of complex 2-HH1.
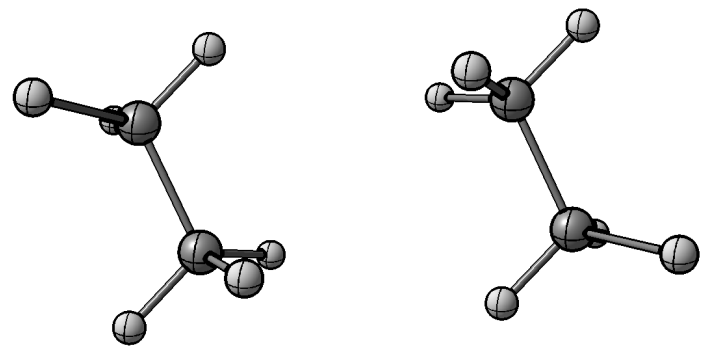

Figure S14: Final structure of complex 2-HH2.
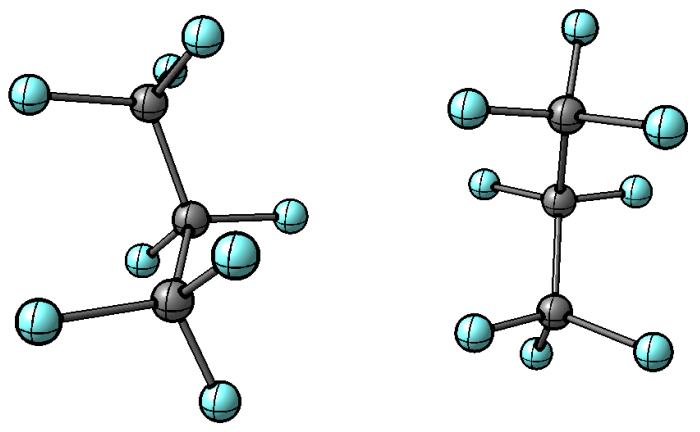

Figure S15: Final structure of complex 3-FF1. 

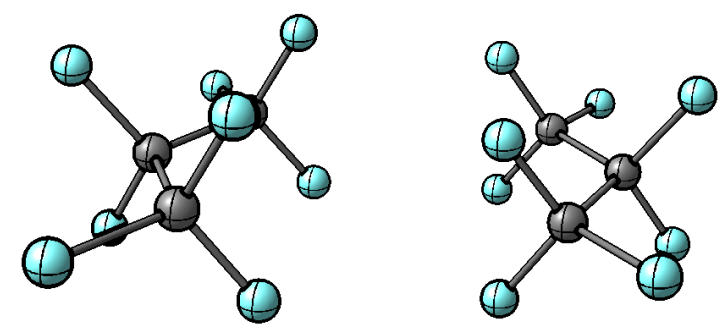

Figure S16: Final structure of complex 3-FF2.
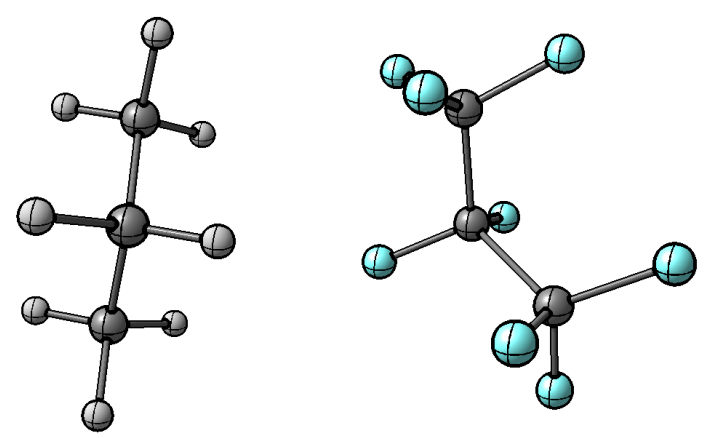

Figure S17: Final structure of complex 3-HF1.
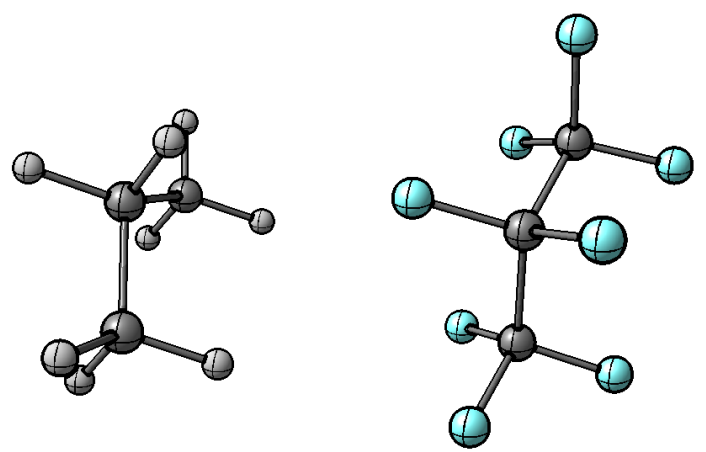

Figure S18: Final structure of complex 3-HF2.
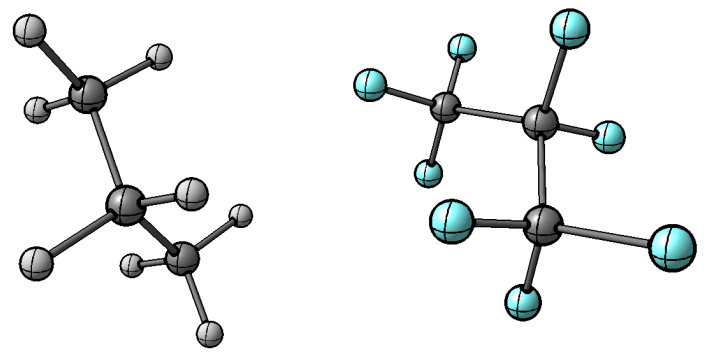

Figure S19: Final structure of complex 3-HF3. 

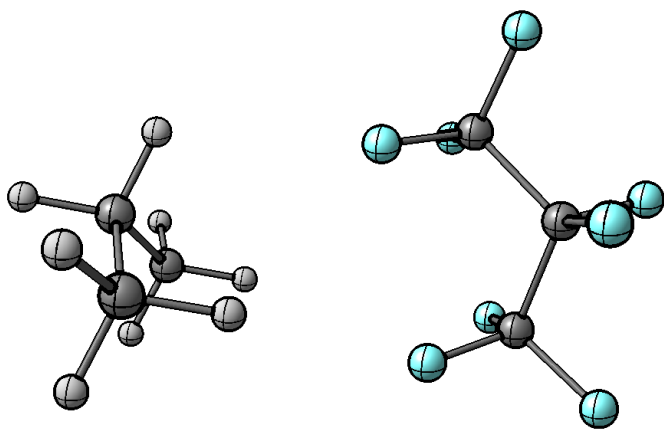

Figure S20: Final structure of complex 3-HF4.
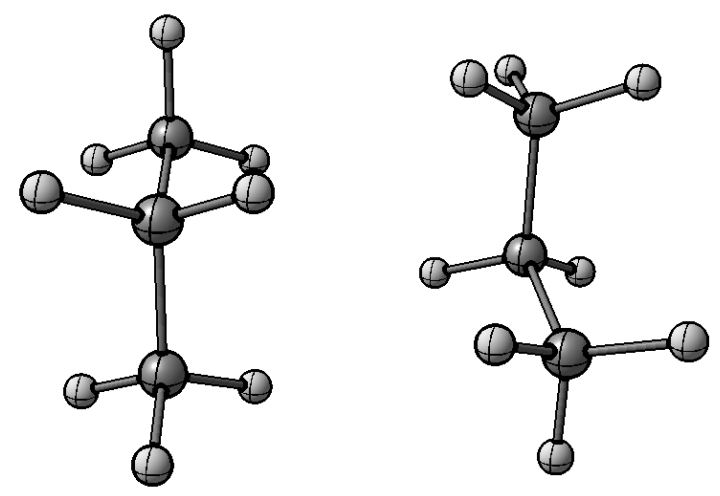

Figure S21: Final structure of complex 3-HH1.
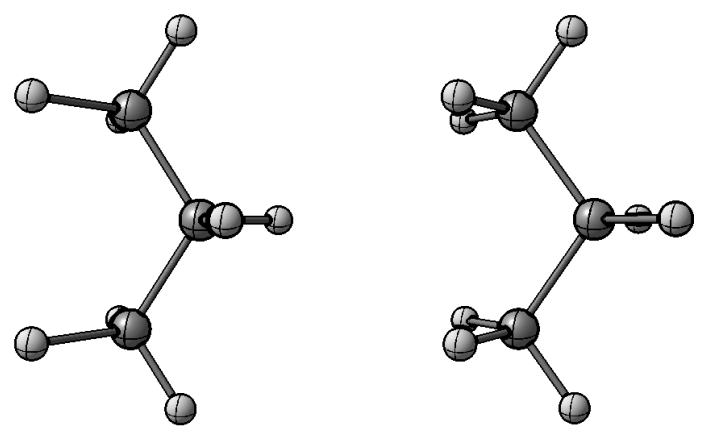

Figure S22: Final structure of complex $\mathbf{3 - H H 2 . ~}$ 

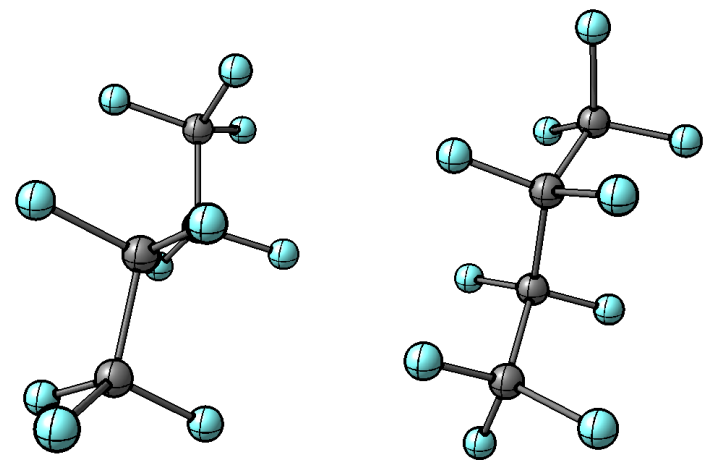

Figure S23: Final structure of complex 4-FF1.
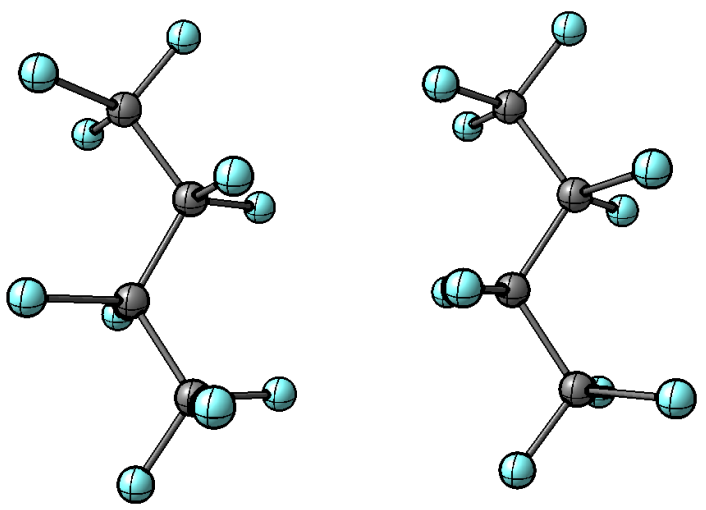

Figure S24: Final structure of complex 4-FF2.
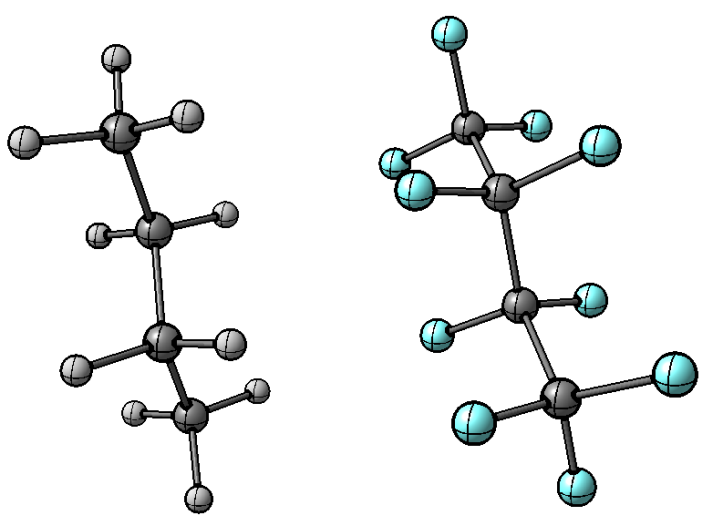

Figure S25: Final structure of complex 4-HF1. 

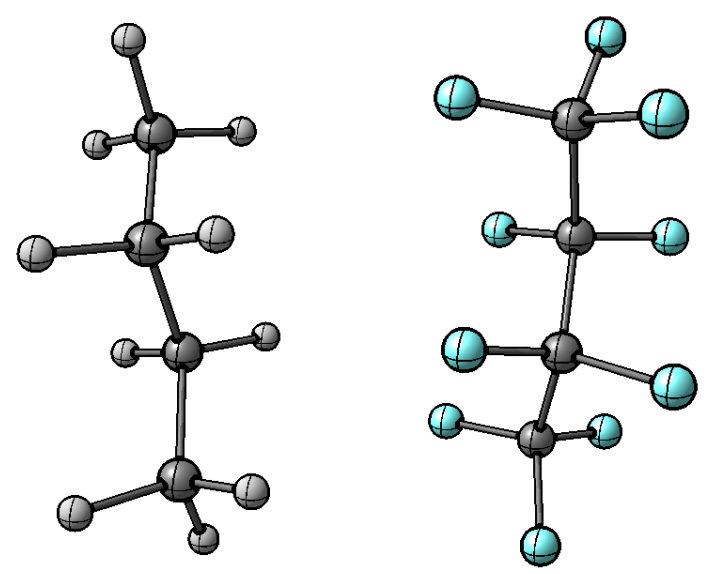

Figure S26: Final structure of complex 4-HF2.
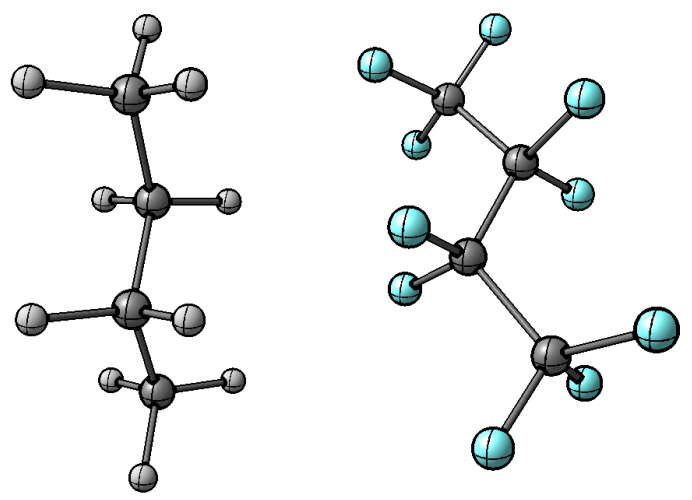

Figure S27: Final structure of complex 4-HF3.
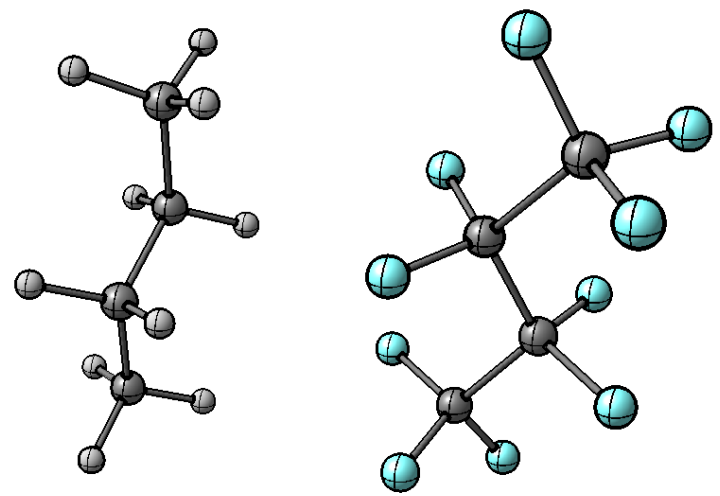

Figure S28: Final structure of complex 4-HF4. 

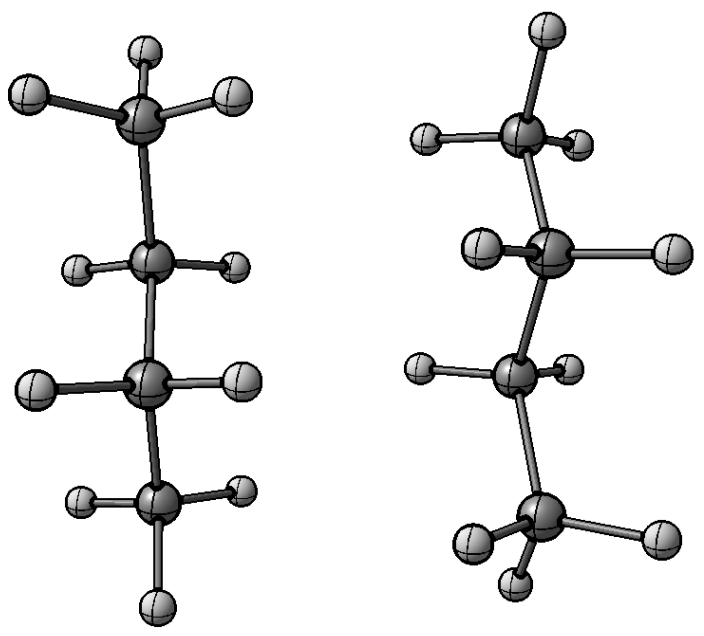

Figure S29: Final structure of complex 4-HH1.
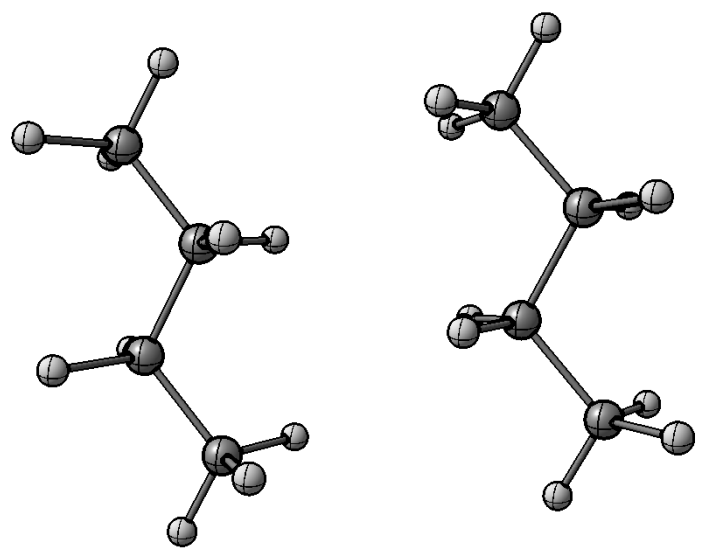

Figure S30: Final structure of complex 4-HH2.
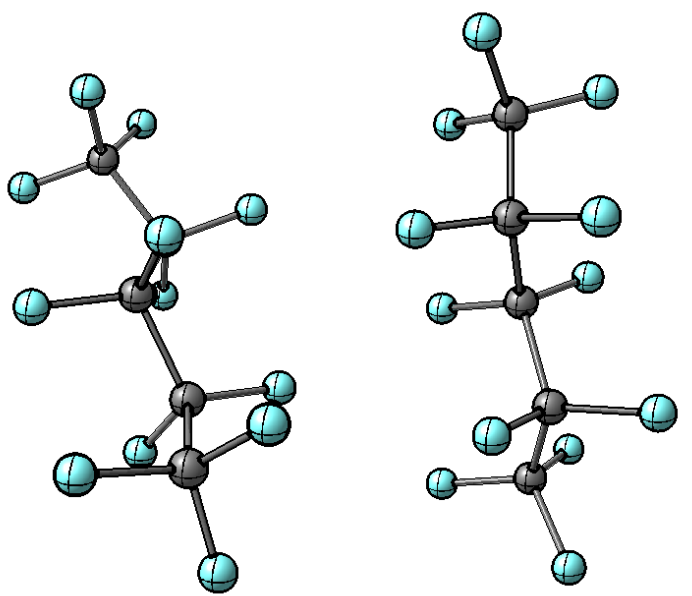

Figure S31: Final structure of complex 5-FF1. 

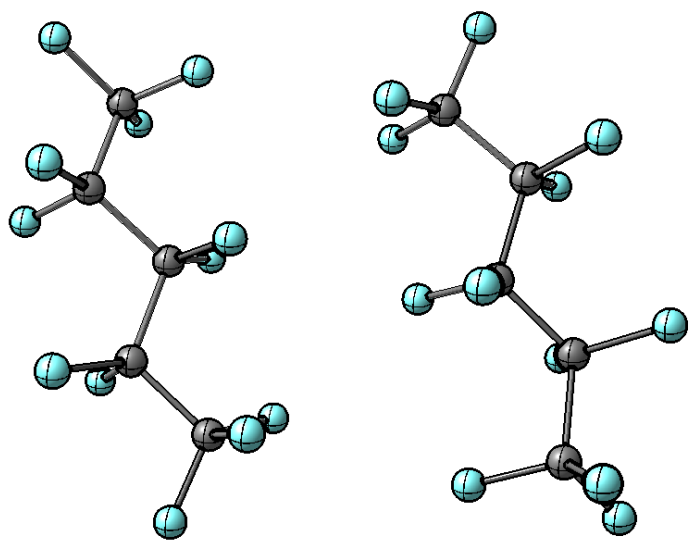

Figure S32: Final structure of complex 5-FF2.
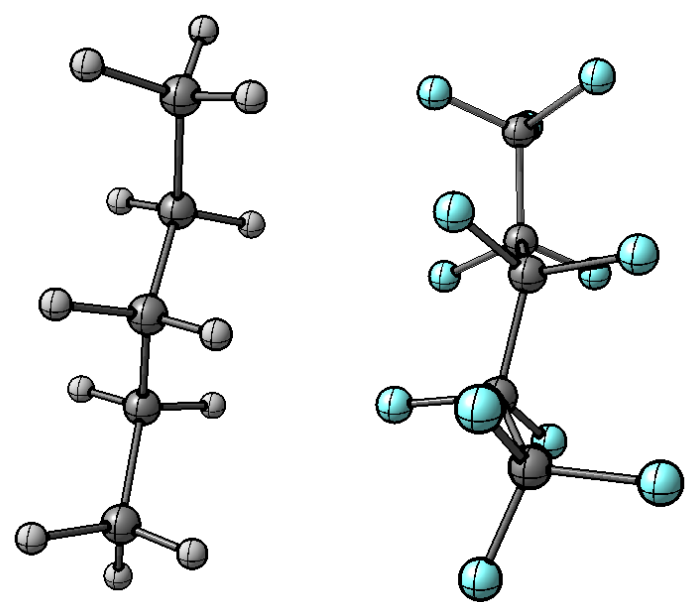

Figure S33: Final structure of complex 5-HF1.
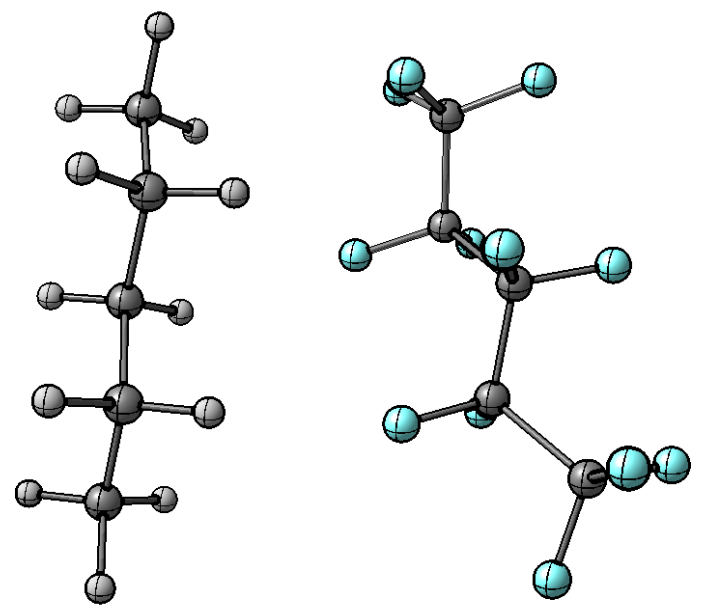

Figure S34: Final structure of complex 5-HF2. 

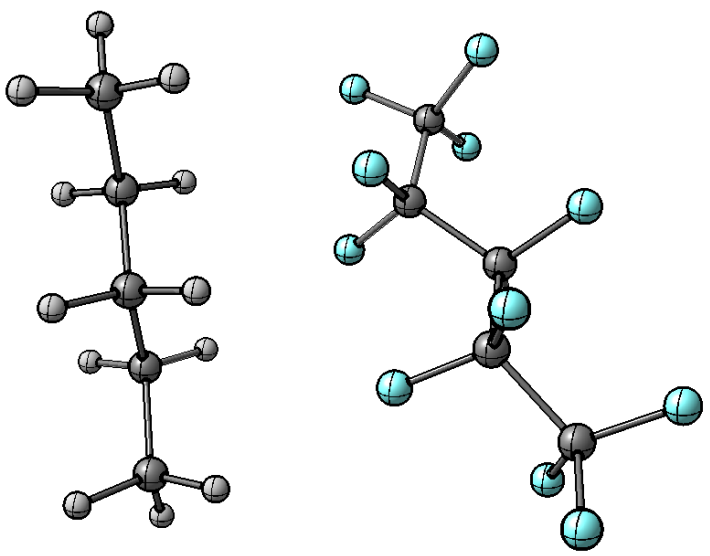

Figure S35: Final structure of complex 5-HF3.
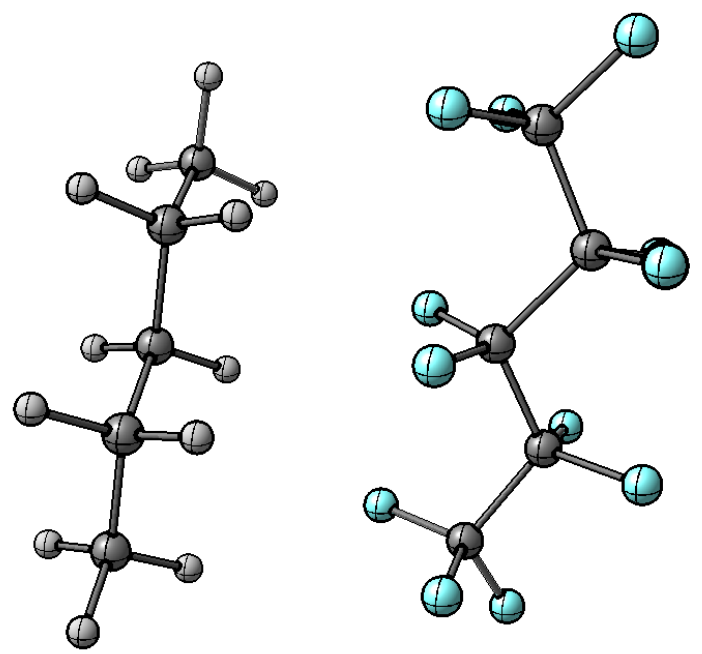

Figure S36: Final structure of complex 5-HF4. 

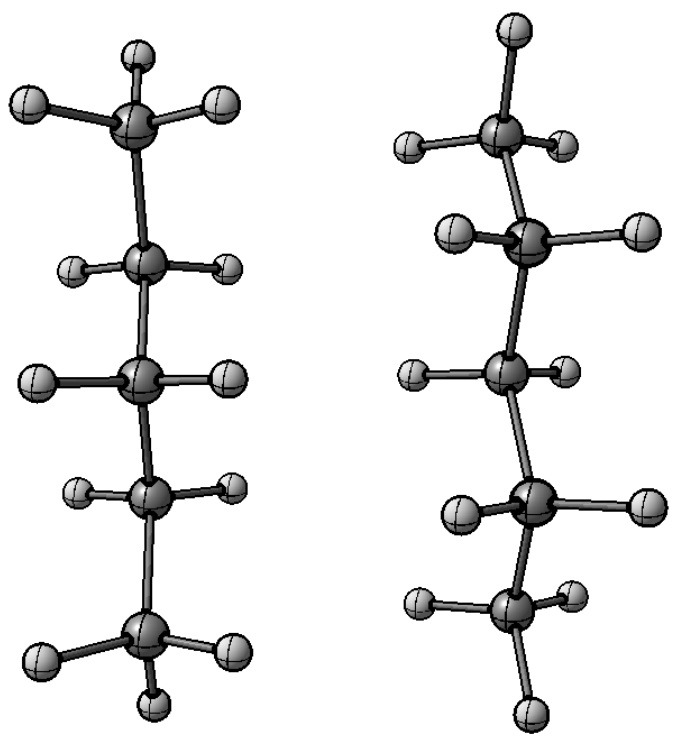

Figure S37: Final structure of complex 5-HH1.
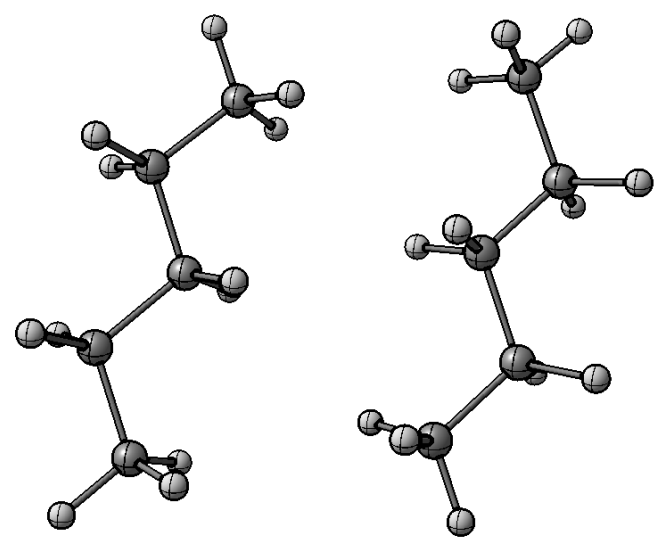

Figure S38: Final structure of complex 5-HH2. 

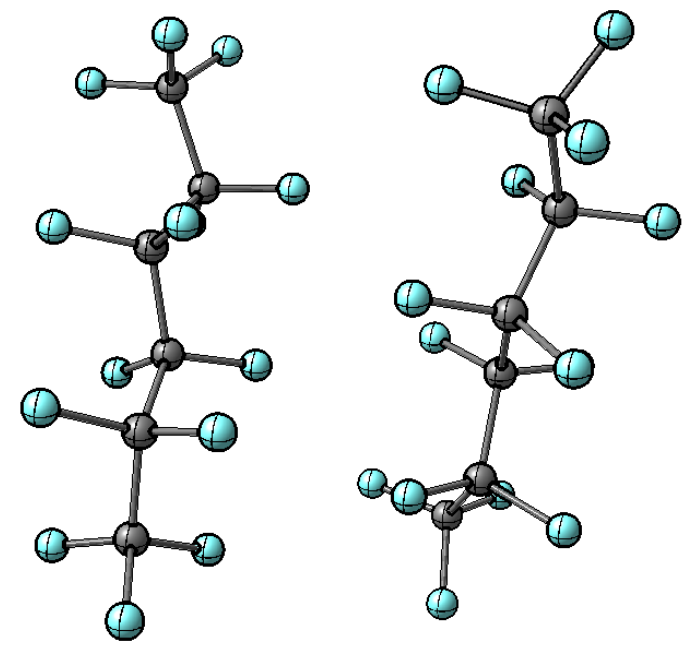

Figure S39: Final structure of complex 6-FF1.
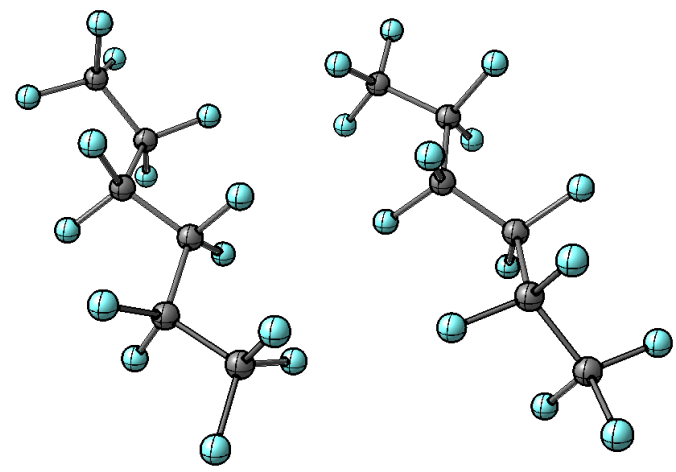

Figure S40: Final structure of complex 6-FF2.
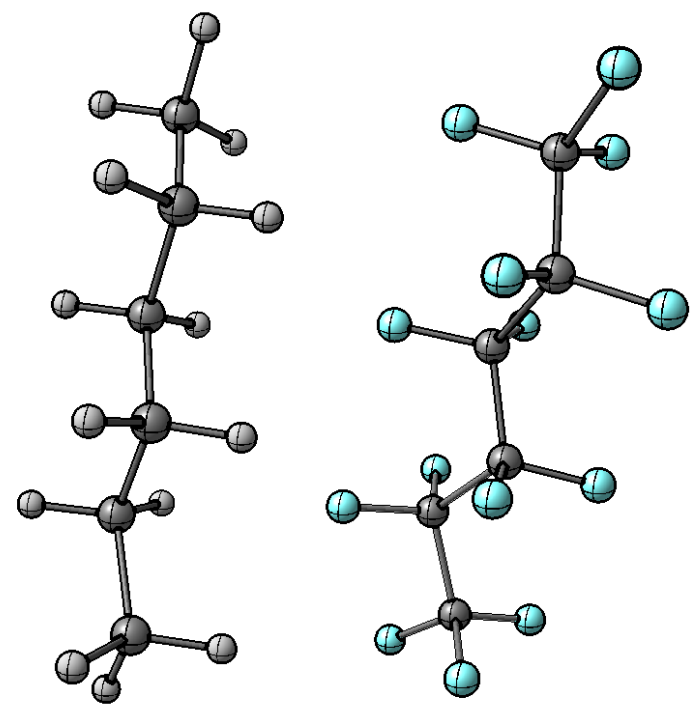

Figure S41: Final structure of complex 6-HF1. 

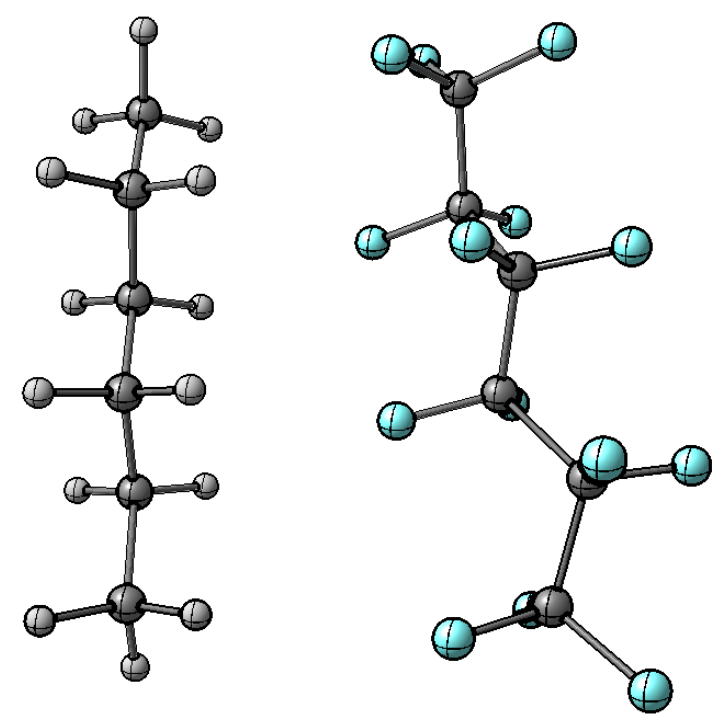

Figure S42: Final structure of complex 6-HF2.
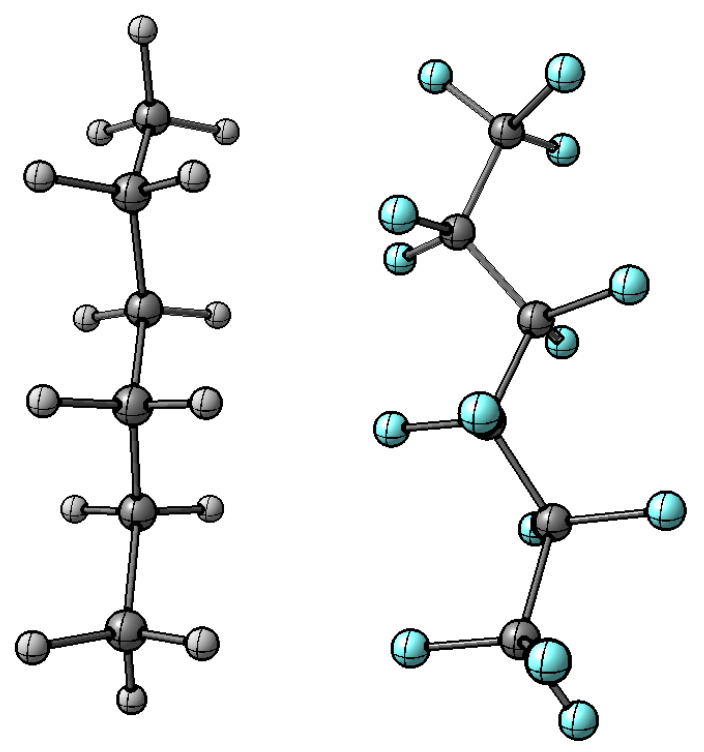

Figure S43: Final structure of complex 6-HF3. 


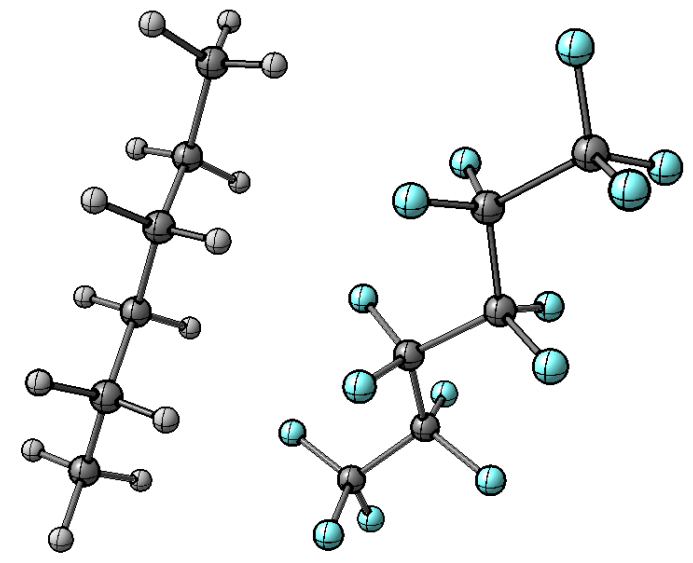

Figure S44: Final structure of complex 6-HF4.
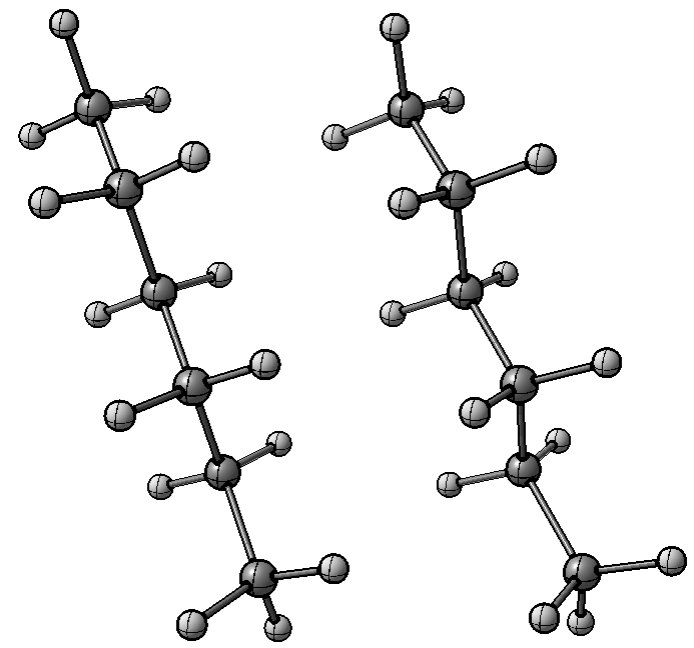

Figure S45: Final structure of complex 6-HH1. 

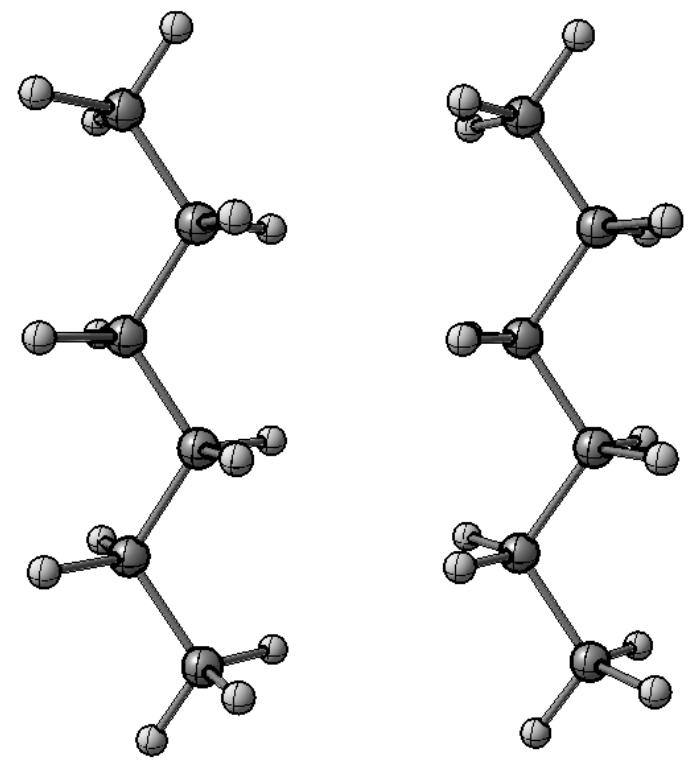

Figure S46: Final structure of complex 6-HH2.

\subsubsection{Discussion}

Systematic benchmark data for perfluoroalkane dimers of increasing carbon chain length is sparse. The only high-level benchmark data set that comes closest includes several halogenated small molecules, the most similar type of interaction energy computed being the molecular complex of trifluoromethane and methane. ${ }^{95}$ To the best of our knowledge, no $\operatorname{CCSD}(\mathrm{T})$ benchmark interaction energies estimated at the CBS limit of perfluoroalkane dimers larger than perfluoromethane have been reported before, probably because of the extremely high computational demands of these calculations.

The working horse for our benchmark data is the explicitly correlated CCSD(T)-F12 method. It provides BIEs close to the CBS limit already using the cc-pVDZ-F12 (VDZ-F12) basis set and therefore CCSD(T)-F12/VDZ-F12 is the most efficient benchmark method for the systems under investigation. We compared the VDZ-F12 numbers to the corresponding cc-pVTZ-F12 (VTZ-F12) numbers for methanes and ethanes and obtained almost the same results (vide supra). Explicitly correlated coupled cluster methods ${ }^{36,37,96-98}$ represent, at the moment, the best alternative to CBS extrapolation schemes and are in conjunction with 
CCSD $(\mathrm{T})$ computationally more affordable than CBS from triple- and quadruple-zeta basis set results.

Our benchmark results (cf. Table 2) also show that while for $\mathbf{H H}$ and $\mathbf{H F}$ systems TightPNO-DLPNO-CCSD(T) is almost converged to the canonical CCSD(T) results, using tighter cutoff parameters results only in slow convergence of the DLPNO-CCSD(T) results towards our CCSD(T)-F12/VDZ benchmark energies for FF complexes. The problems of DLPNO-CCSD $(\mathrm{T})$ in reproducing the benchmark interaction energies of perfluoroalkane complexes likely stem (at least to a significant extent) from the large contribution of the so-called weak pairs, which are treated using Local-MP2 and not further considered in the CCSD step. ${ }^{99}$ It should be noted that it has been shown before that for the accurate description of the interaction energies of some specific systems tighter cutoff thresholds than the standard TightPNO settings are necessary. ${ }^{34,100,101}$ The drawback is that these tighter cutoff thresholds make the corresponding DLPNO-CCSD $(\mathrm{T})$ computations much less affordable for increasingly large systems.

Additionally, it is also interesting to note that the commonly employed CBS extrapolation schemes used on top of CCSD(T) single points are not reproducing BIEs in $\mathbf{H F}$ and FF systems properly. Neither using MP2 nor using LPNO-CEPA/1 for extrapolation provides comparable numbers to either $\operatorname{CCSD}(\mathrm{T})-\mathrm{F} 12$ or $\operatorname{CCSD}(\mathrm{T}) / \mathrm{CBS}(34)$ (tested on methanes and ethanes). However, using MP2 for estimating the CBS correction to be added on top of $\operatorname{CCSD}(\mathrm{T})$ single points is commonly used for benchmark calculations. ${ }^{95,102}$ Extrapolation using LPNO-CEPA/1 has been proposed recently as alternative but has not yet been commonly applied. ${ }^{103}$

Looking at the performance of the various DFT methods, M06L performs acceptable across the whole series of molecules but still shows larger deviations for $\mathbf{H F}$ and $\mathbf{F F}$. The behavior of $\mathrm{dDsC}$ does not show a clear tendency as it significantly underestimates the BIEs in both $\mathbf{H F}$ and $\mathbf{F F}$ in conjunction with B3LYP but significantly overestimates the same BIEs in conjunction with PBE. While D3 performs well for $\mathbf{H H}$, it shows significant de- 
viations for $\mathbf{H F}$ already and even bigger deviations for $\mathbf{F F}$ complexes underestimating the dispersive contributions in these systems. It should be noted that it has been observed before that the standard D3 parametrization is inappropriate to describe the interaction of 1-FF. ${ }^{104}$ We also used the recently developed program BootD3 ${ }^{12}$ to test the uncertainties in the D3 dispersion contribution to the interaction energies. We obtained low uncertainties (i.e. generally below $10 \%$ ) with respect to the overall interaction energies. Therefore, uncertainties in the D3 correction cannot explain the large deviations from the benchmark values observed. The likely reason for the failure of D3 in perfluoroalkanes is the neglect of the change in polarizability due to the strongly polarized $\mathrm{C}-\mathrm{F}$ bonds. This effect is taken into account, at least to some extent, in the XDM correction and it is very strong in perfluoroalkanes as can be seen from the computed atomic polarizabilities in $\mathbf{1 - F}$ (cf. Table 3). It should be noted that this shortcoming of D3 is known and D4 has been developed to alleviate this problem. ${ }^{105}$ At the time this manuscript was prepared, we could only test the D4 correction as part of the semiempirical GFN2-xTB method. ${ }^{35}$ On the basis of our benchmarks, GFN2-xTB is a significant improvement over GFN-xTB. ${ }^{13}$ However, the performance of D4 in conjunction with DFT remains to be tested in the near future. VV10, abbreviated as either NL or $\mathrm{V}$ herein, on the other hand, in B97M-V and $\omega \mathrm{B} 97 \mathrm{M}-\mathrm{V}$, provides the most robust and efficient methods to predict BIEs of complexes of alkanes and perfluoroalkanes. On the other hand, in conjuction with BLYP and B3LYP it results in significant overestimation of the BIEs of $\mathbf{H F}$ and FF. This suggests that VV10 needs to be included in the overall functional parametrization rather than being used as correction on top of a given functional to obtain accurate results.

Having discussed the computational methods used to obtain accurate BIEs of our model complexes, we can now shift our attention to comparing the BIEs of $\mathbf{H H}, \mathbf{H F}$ and $\mathbf{F F}$ complexes having the same carbon chain length (cf. Figure 13). Looking at the actual CCSD(T)-F12 numbers and the estimated CCSD(T)-F12 numbers we observe that from carbon chains of one to three the FF complexes have a stronger BIE than the correspond- 
ing $\mathbf{H H}$ complexes. The estimated CCSD(T)-F12 numbers suggest that with carbon chain lengths of four and longer the relative BIEs invert so that the corresponding $\mathbf{H H}$ complexes have a stronger BIE. Interestingly, this change in the relative BIEs parallels the experimentally observed inversion of relative boiling points of alkanes and perfluoroalkanes at a carbon chain length of four (cf. Figure 14). The boiling point is a measure of the strength of intermolecular interactions so it is expected to somewhat reflect the BIEs of intermolecular complexes. The inversion of relative BIEs also reflects the trend observed in the experimental average attraction energies obtained from critical parameters (cf. Figure 15). The inversion in relative average attraction energies is observed already at a carbon chain length of two but the general trend is comparable.

From the benchmarked methods, only relative BIEs from B97M-V show the inversion at a carbon chain length of four, the third, fourth and fifth best methods according to our benchmarking, i.e. $\omega$ B97M-V/def2-QZVP, B3LYP-XDM/aug-cc-pVTZ and VeryTightDLPNO-CCSD(T)/CBS(34) would predict the inversion of BIEs already at a carbon chain length of two. It can be concluded that $\mathbf{H}$ and $\mathbf{F}$ homodimers with carbon chain lengths between one and six show similar BIEs. Overall, the benchmarking performed was necessary in order to be able to evaluate the relative BIEs of $\mathbf{H H}, \mathbf{H F}$ and $\mathbf{F F}$ properly. We find that the BIEs of the homodimers of alkanes and perfluoroalkanes having the same number of carbon atoms are similar (cf. Figure 13a) from one to six carbon atoms. Except for 1-HF, we find that the corresponding heterodimers have weaker BIEs than either of the homodimers.

Another important aspect are the geometries we chose for our benchmark data set. DSDPBEP86 is a double-hybrid functional and was selected on the basis of available benchmarks for "ordinary" organic molecules. ${ }^{24}$ While it is not the best functional to predict the BIEs of our model complexes (in our ranking of computational methods it is only the 11th rank), the rigid energy scans performed using Tight-LED-DLPNO-CCSD(T)/QZ show minima in the BIE at about the same intermolecular separations (vide supra) which suggests the interaction 
geometries to be adequate. Additionally, when looking at our ranking, the only better ranked methods to predict the BIEs, which could have been used for efficient geometry optimization as well, are MP2, PBE-XDM, B3LYP-XDM, B97M-V and $\omega$ B97M-V. For future computations, B97M-V geometries might be a cost-effective alternative to DSD-PBEP86 geometries as the additional cost of the HF and MP2 single points performed during the DSD-PBEP86 calculations are computationally more expensive than the additional cost of the VV10 correction in B97M-V.

\subsubsection{Benchmarking WFT Methods}

Using the optimized geometries we computed the interaction energies of the molecular complexes of the methane and ethane systems using high-level wavefunction methods. We used $\operatorname{CCSD}(\mathrm{T})-\mathrm{F} 12 / \mathrm{VTZ}$ as benchmark and compared several alternative methods against it. The corresponding results are summarized in Table S1.

One would expect the canonical coupled cluster methods CCSD(T)-F12 and CCSD(T)/CBS(34), which are extrapolated to the complete basis set limit, to be most accurate. On the basis of literature benchmarks ${ }^{36,37}$ we selected $\operatorname{CCSD}(\mathrm{T})$-F12/VTZ as reference method and were looking for a cheaper method that reproduces the corresponding interaction energies best. $\operatorname{CCSD}(\mathrm{T})-\mathrm{F} 12 / \mathrm{VDZ}$ shows only minor deviations from the reference method at much less computational cost (cf. Table S1). Using CCSD(T)-F12/VDZ we were able to perform benchmark calculations for most of our molecular complexes under study except for $\mathbf{F F}$ with a carbon chain length of four or higher, and $\mathbf{H F}$ with a carbon chain legnth of five or six.

We compared the corresponding $\operatorname{CCSD}(\mathrm{T})$-F12/VDZ interaction energies to DLPNO$\operatorname{CCSD}(\mathrm{T})$ results using various sets of cut-off thresholds. While many data points agree very well to the benchmark data, some selected data points show considerable deviations. We investigated these deviations further by analyzing all the data points of the corresponding subsystems HH, HF and FF separately (Figures S47-S49). 
Table S1: Benchmark of various wavefunction methods against CCSD(T)-F12/VTZ as reference for methane and ethane systems (MP3/CBS(34) numbers are only based on methane systems). CCSD(T)-F12/VDZ compares best and is still affordable for most of the complexes studied in this work.

\begin{tabular}{ccccc}
\hline Method & MAD [kcal mol $\left.{ }^{-1}\right]$ & MAD $_{\text {rel }}[\%]$ & MSD [kcal mol $\left.{ }^{-1}\right]$ & MSD $_{\text {rel }}[\%]$ \\
\hline CCSD(T)-F12/VDZ & $0.02(1)$ & $2(1)$ & $0.02(1)$ & $2(1)$ \\
CCSD(T)/CBS(34) & $0.03(3)$ & $3(3)$ & $0.02(3)$ & $2(4)$ \\
SAPT2(CCD)סMP2/aTZ & $0.03(3)$ & $3(3)$ & $0.02(4)$ & $2(4)$ \\
SAPT2+(3)סMP2/aTZ & $0.06(3)$ & $6(3)$ & $0.06(3)$ & $6(3)$ \\
CCSD(T)/CBS(LPNO-CEPA/1) & $0.07(4)$ & $8(4)$ & $-0.05(6)$ & $-4(8)$ \\
CCSD(T)/CBS(MP2) & $0.13(9)$ & $14(9)$ & $-0.13(9)$ & $-14(9)$ \\
MP2/CBS(34) & $0.13(8)$ & $14(7)$ & $0.12(10)$ & $13(9)$ \\
MP3/CBS(34) & $0.14(10)$ & $20(10)$ & $0.14(10)$ & $20(10)$ \\
\hline
\end{tabular}




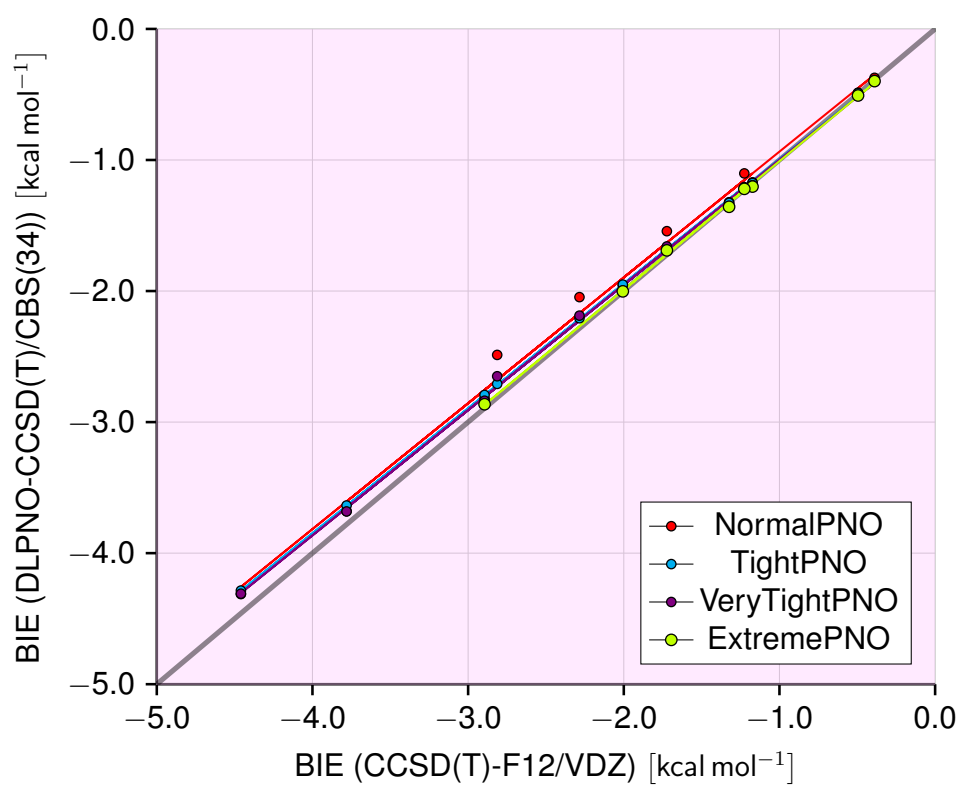

Figure S47: Interaction energies of molecular complexes from DLPNO-CCSD(T)/CBS using various cut-off thresholds against $\operatorname{CCSD}(\mathrm{T})-\mathrm{F} 12 / \mathrm{VDZ}$ as reference in $\mathbf{H H}$ complexes. Linear regressions illustrate the systematic deviations observed from the reference as a function of interaction energies.

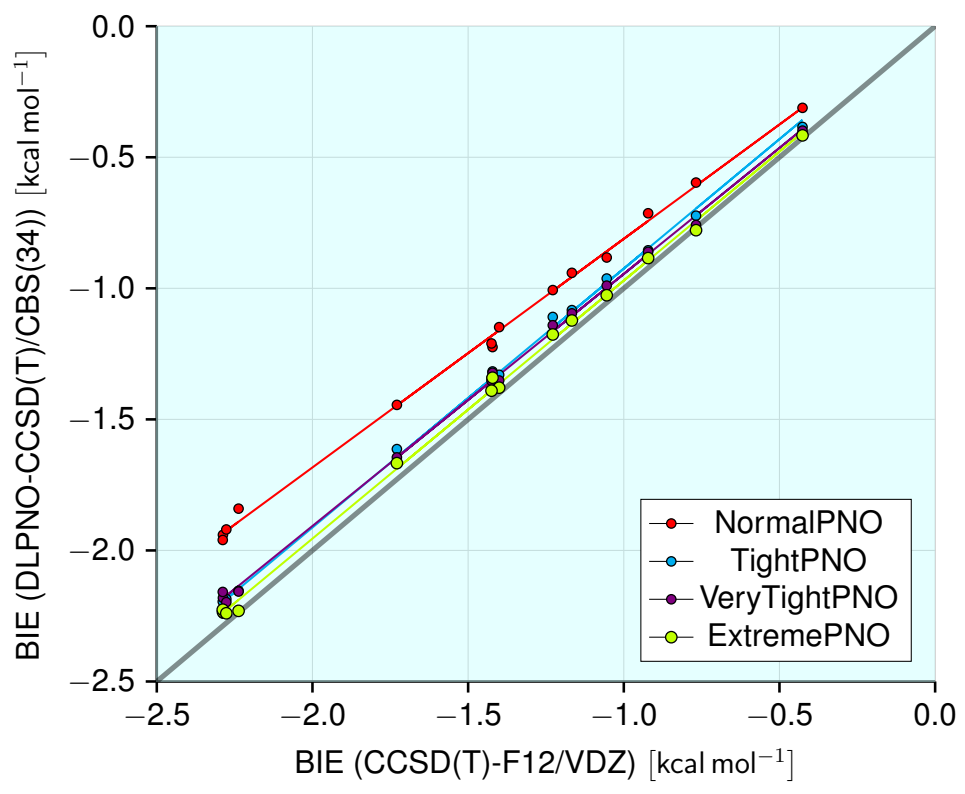

Figure S48: Interaction energies of molecular complexes from DLPNO-CCSD(T)/CBS using various cut-off thresholds against CCSD(T)-F12/VDZ as reference in $\mathbf{H F}$ complexes. Linear regressions illustrate the systematic deviations observed from the reference as a function of interaction energies. 


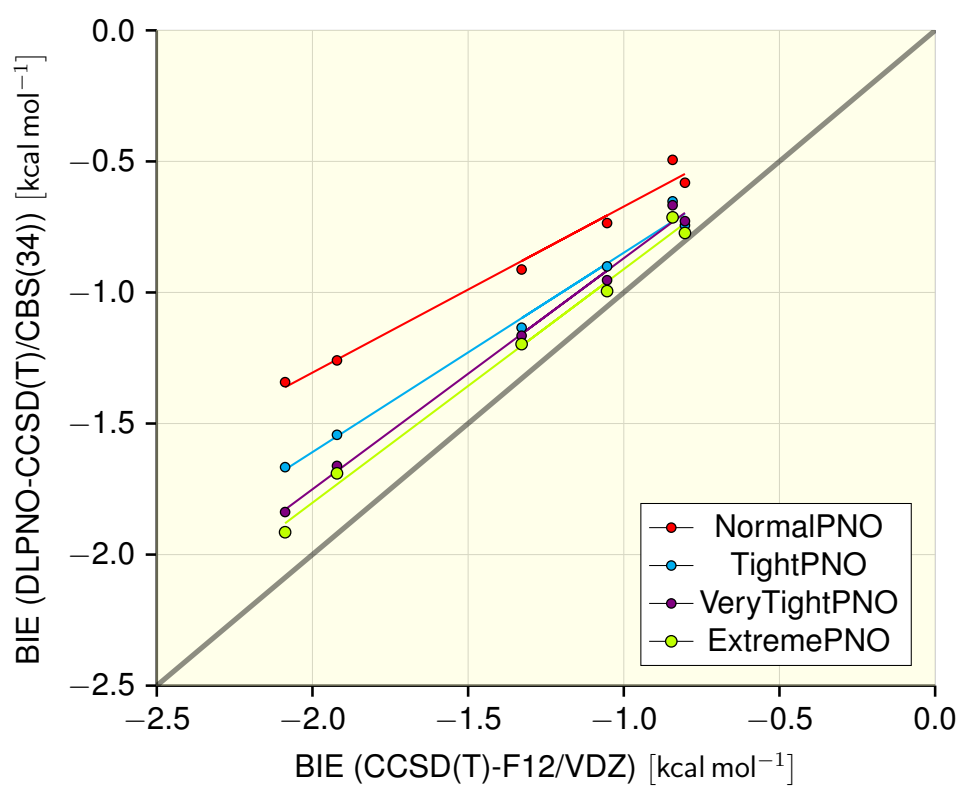

Figure S49: Interaction energies of molecular complexes from DLPNO-CCSD(T)/CBS using various cut-off thresholds against CCSD(T)-F12/VDZ as reference in FF complexes. Linear regressions illustrate the systematic deviations observed from the reference as a function of interaction energies.

Figures S47-S49 show that the data points with significant deviations are the FF subsystems. Additionally, the deviations are systematic as they approximately show linear scaling with interaction energy size. The corresponding linear regressions assessing the extent of the systematic deviations are shown in Table S2.

The parameters of the linear regressions from Table S2 can be interpreted as quantitative measure of the systematic deviations between DLPNO-CCSD(T)/CBS(34) and CCSD(T)F12/VDZ interaction energies. The slope $(\mathrm{k})$ is a measure of what percentage of the benchmark interaction energy is recovered by the DLPNO approach as the interaction energy increases (ideal would be a slope of one). The intercept (d) is a measure of the additional offset of all the interaction energies relative to the benchmark energies (ideal would be an intercept of zero). Table S2 demonstrates that all the intercepts are small but shows significant deviations of the slopes from one for the FF subsystems. This shows that DLPNOCCSD(T)/CBS(34) methods significantly underestimate the interaction of $\mathbf{F}$ with each other. 
Table S2: Systematic deviations of interaction energies of molecular complexes from DLPNOCCSD(T)/CBS using various cut-off thresholds against CCSD(T)-F12/VDZ as reference divided into the three subclasses $\mathbf{H H}, \mathbf{H F}$ and FF. Systematic deviations are negligible for $\mathbf{H H}$, small for $\mathbf{H F}$ but significant for $\mathbf{F F}$.

\begin{tabular}{|c|c|c|c|c|c|c|}
\hline \multirow[t]{2}{*}{ Truncation } & \multicolumn{2}{|r|}{ HH } & \multicolumn{2}{|r|}{$\mathbf{H F}$} & \multicolumn{2}{|r|}{ FF } \\
\hline & k & $\mathrm{d}\left[\mathrm{kcal} \mathrm{mol}{ }^{-1}\right]$ & k & $\mathrm{d}\left[\mathrm{kcal} \mathrm{mol}{ }^{-1}\right]$ & k & $\mathrm{d}\left[\mathrm{kcal} \mathrm{mol}{ }^{-1}\right]$ \\
\hline NormalPNO & $0.96(2)$ & $0.02(5)$ & $0.87(1)$ & $0.06(2)$ & $0.63(4)$ & $-0.04(6)$ \\
\hline TightPNO & $0.952(4)$ & $-0.039(9)$ & $0.99(1)$ & $0.06(1)$ & $0.76(4)$ & $-0.09(6)$ \\
\hline VeryTightPNO & $0.956(9)$ & $-0.04(2)$ & $0.962(9)$ & $0.02(1)$ & $0.88(3)$ & $0.01(5)$ \\
\hline ExtremePNO & $0.98(1)$ & $-0.03(2)$ & $0.98(1)$ & $0.01(2)$ & $0.89(4)$ & $-0.02(6)$ \\
\hline
\end{tabular}

It should be noted that while using increasingly tight cut-off thresholds remedies the problem, even ExtremePNO systematically underestimates the interaction by $11 \%$ (cf. Table S2). Additionally, we used the correlation of VeryTightPNO-DLPNO-CCSD(T)/CBS(34) against CCSD(T)-F12/VDZ to estimate benchmark interaction energies for HF complexes with five and six carbon atoms and FF complexes with four, five and six carbon atoms because the corresponding $\mathrm{CCSD}(\mathrm{T})-\mathrm{F} 12 / \mathrm{VDZ}$ calculations were too prohibitively expensive. These estimates agree reasonably well with the corresponding B97M-V BIEs.

\subsubsection{Rigid Geometry Scans}

To assess the performance of DSD-PBEP86/def2-TZVP(spd) in the geometry optimizations for our test systems we performed rigid geometry scans in which we systematically changed the distance between the interacting molecules but did not allow for geometry relaxation. We used both RI-MP2/CBS(34) and LED-DLPNO-CCSD(T)/QZ for the rigid distance scans. RI-MP2/CBS(34) was used for all the complexes studied, LED-DLPNO-CCSD(T)/QZ was used for complexes with one to three carbon atoms in the interacting molecules. The corresponding results are illustrated in Figures S50-S95. The interaction energy minima of both RI-MP2 and LED-DLPNO-CCSD(T)/QZ coincide well with the optimized intermolecular separation in the DSD-PBEP86 geometries. The good agreement between the interaction energy minima of LED-DLPNO-CCSD(T)/QZ, RI-MP2 and DSD-PBEP86/def2-TZVP(spd) 
suggests that these geometries are sufficiently accurate for all the molecular complexes under study.

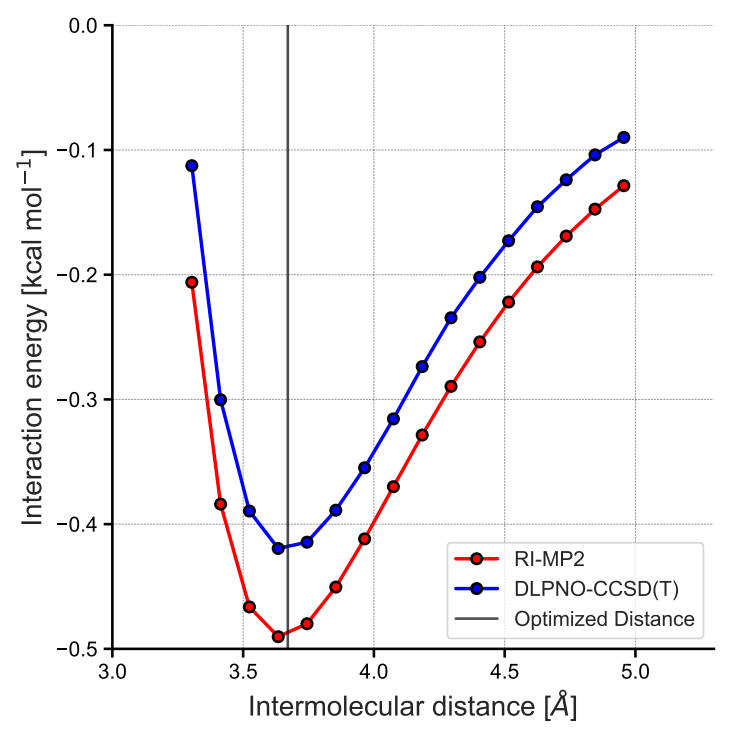

Figure S50: Rigid geometry scan of $\mathbf{1 - H H 1}$ and comparison to the optimized intermolecular separation.

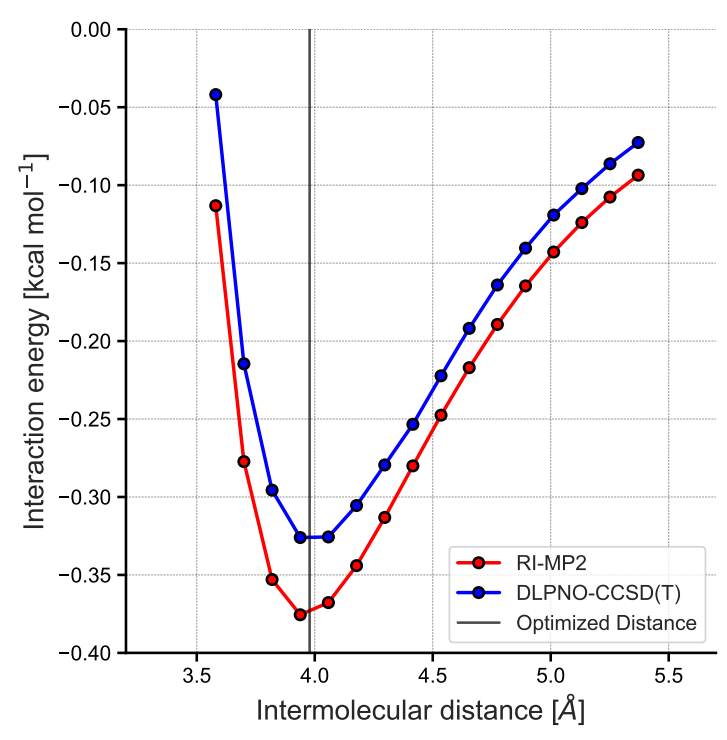

Figure S51: Rigid geometry scan of $\mathbf{1 - H H 2}$ and comparison to the optimized intermolecular separation. 


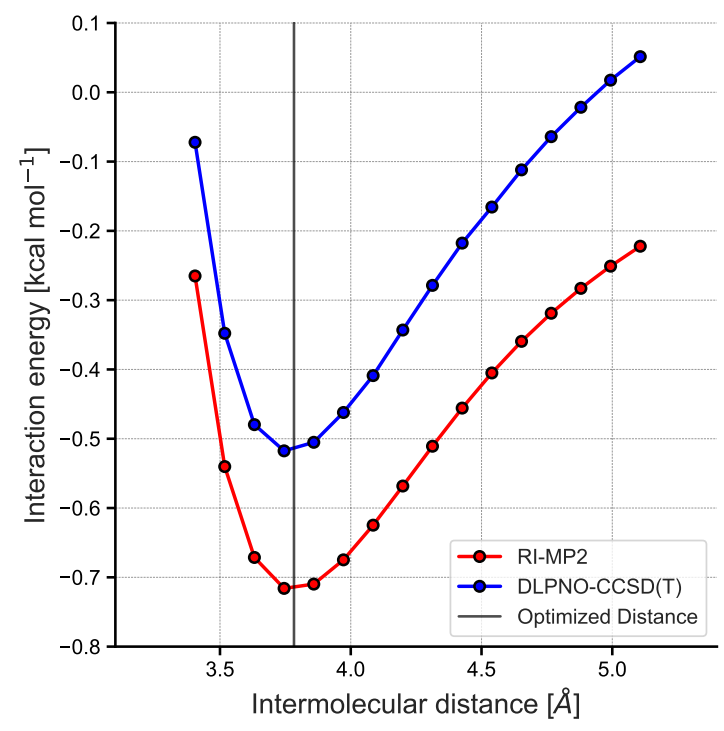

Figure S52: Rigid geometry scan of $\mathbf{1 - H F 1}$ and comparison to the optimized intermolecular separation.

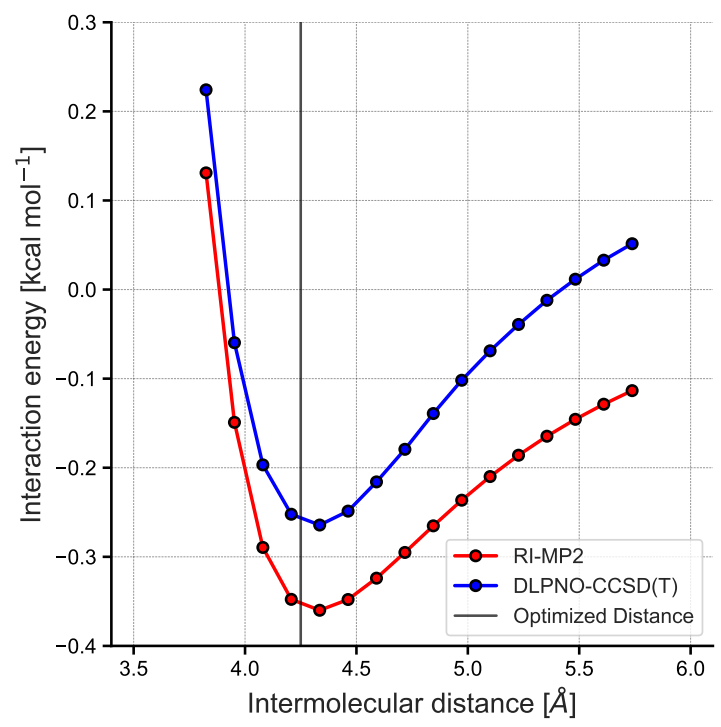

Figure S53: Rigid geometry scan of 1-HF2 and comparison to the optimized intermolecular separation. 


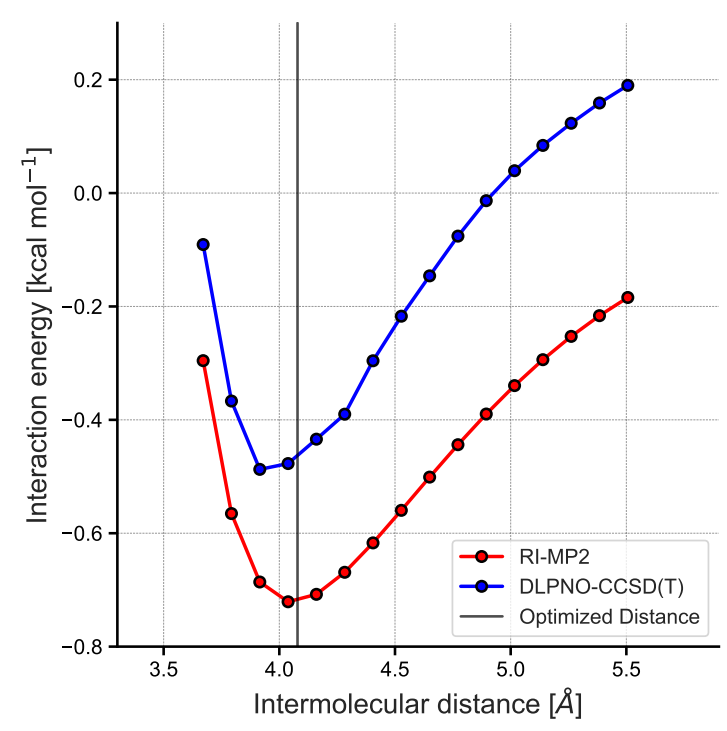

Figure S54: Rigid geometry scan of $\mathbf{1 - F F 1}$ and comparison to the optimized intermolecular separation.

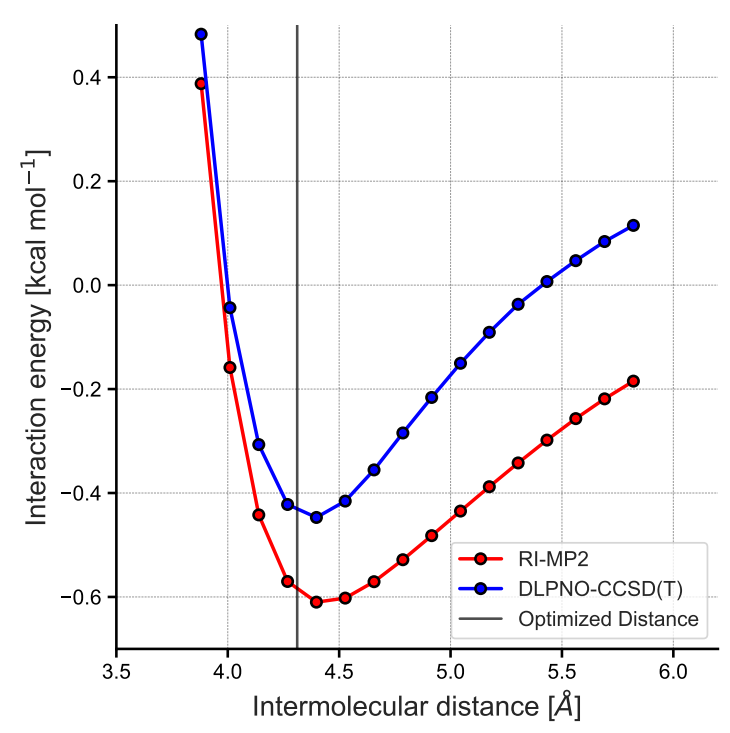

Figure S55: Rigid geometry scan of 1-FF2 and comparison to the optimized intermolecular separation. 


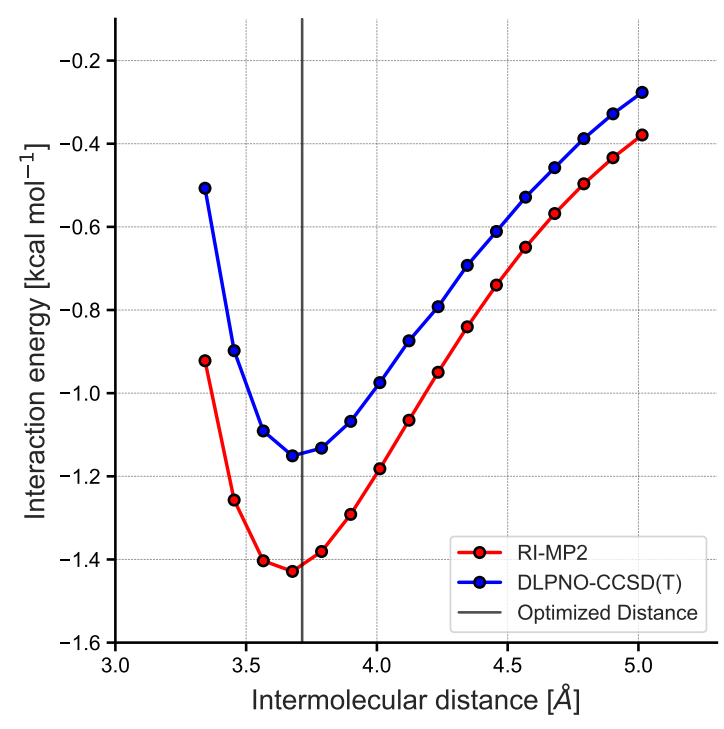

Figure S56: Rigid geometry scan of $\mathbf{2 - H H 1}$ and comparison to the optimized intermolecular separation.

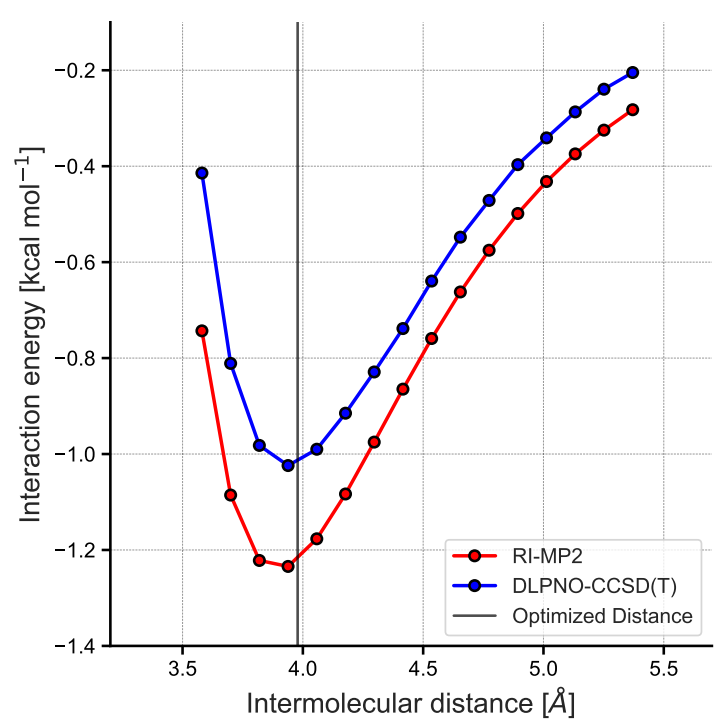

Figure S57: Rigid geometry scan of $\mathbf{2 - H H 2}$ and comparison to the optimized intermolecular separation. 


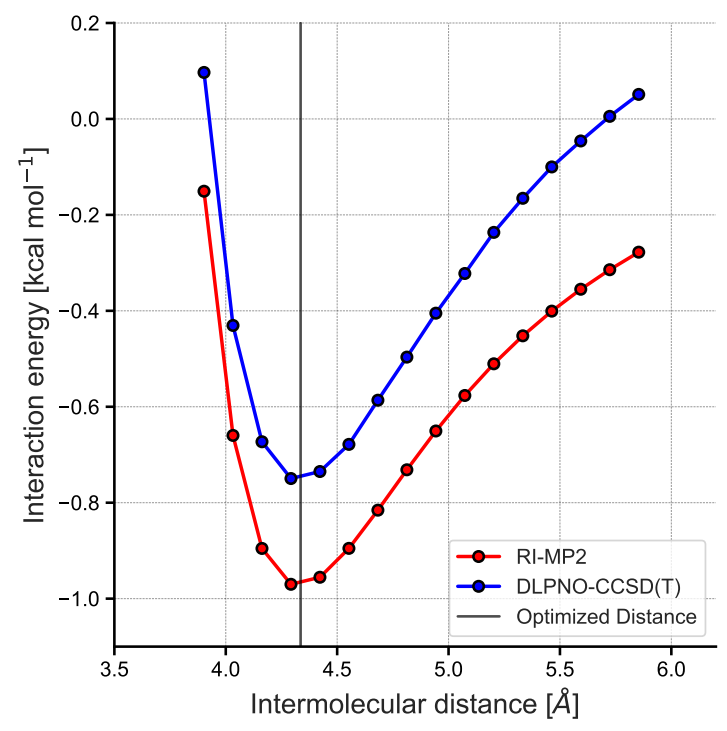

Figure S58: Rigid geometry scan of 2-HF1 and comparison to the optimized intermolecular separation.

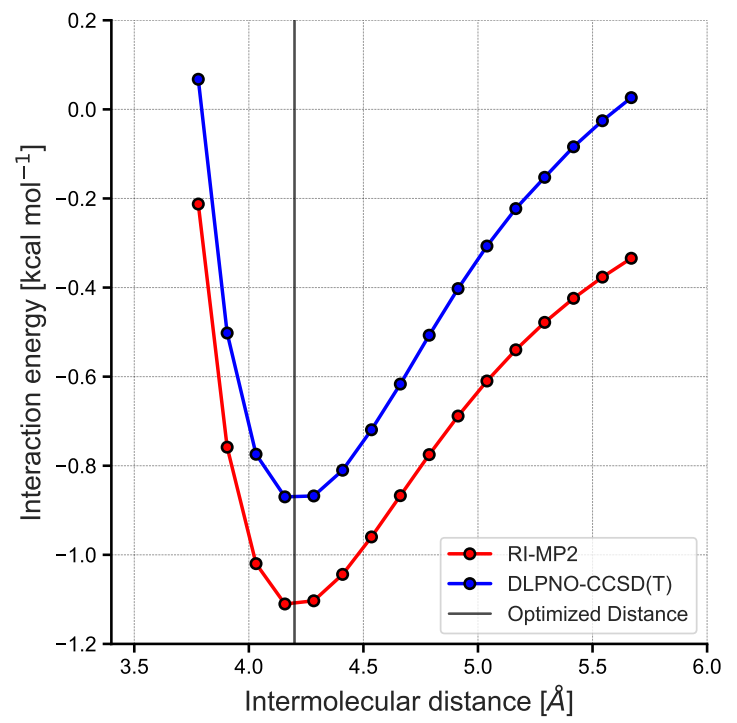

Figure S59: Rigid geometry scan of $\mathbf{2 - H F 2}$ and comparison to the optimized intermolecular separation. 


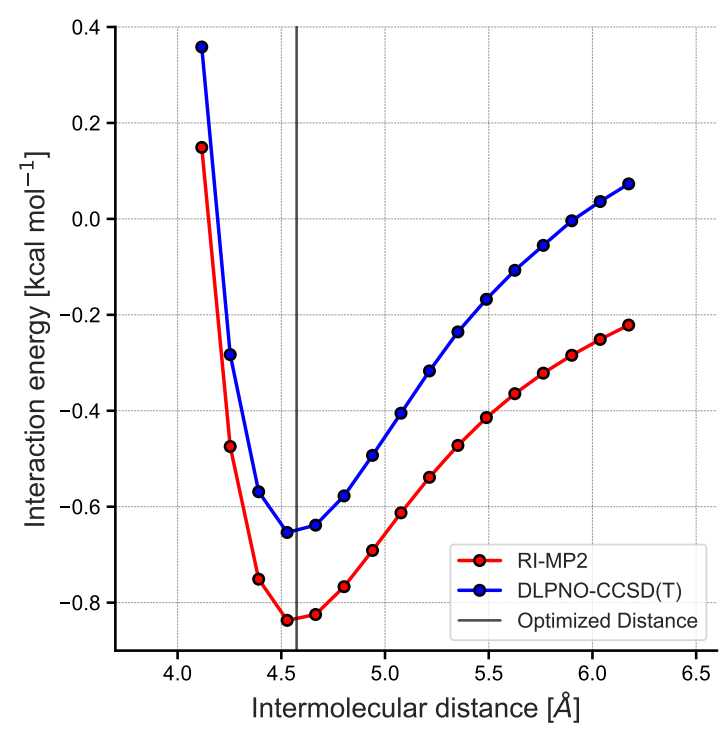

Figure S60: Rigid geometry scan of 2-HF3 and comparison to the optimized intermolecular separation.

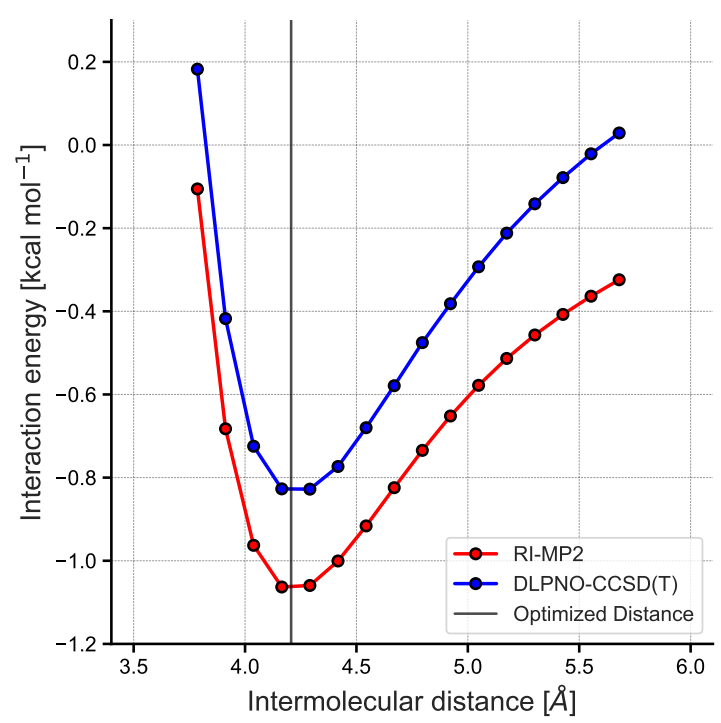

Figure S61: Rigid geometry scan of $\mathbf{2 - H F} 4$ and comparison to the optimized intermolecular separation. 


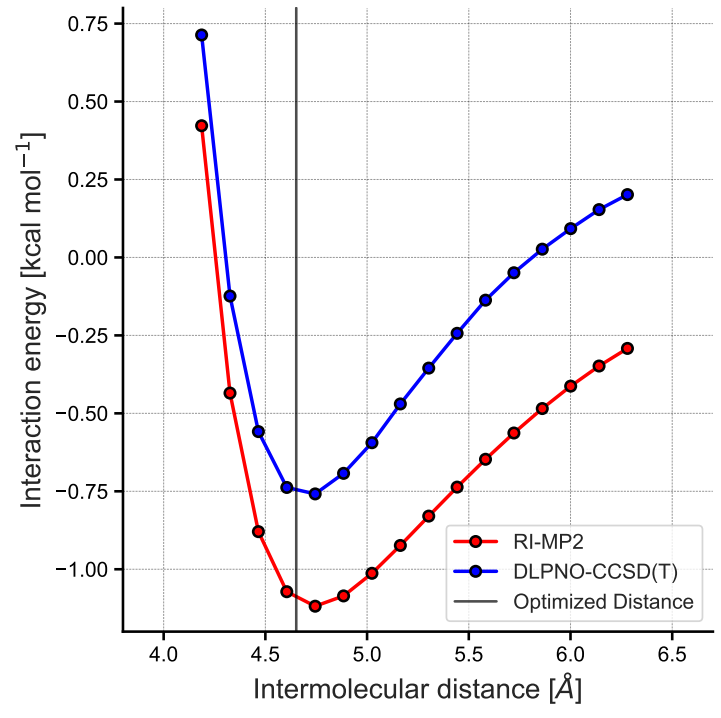

Figure S62: Rigid geometry scan of $\mathbf{2 - F F 1}$ and comparison to the optimized intermolecular separation.

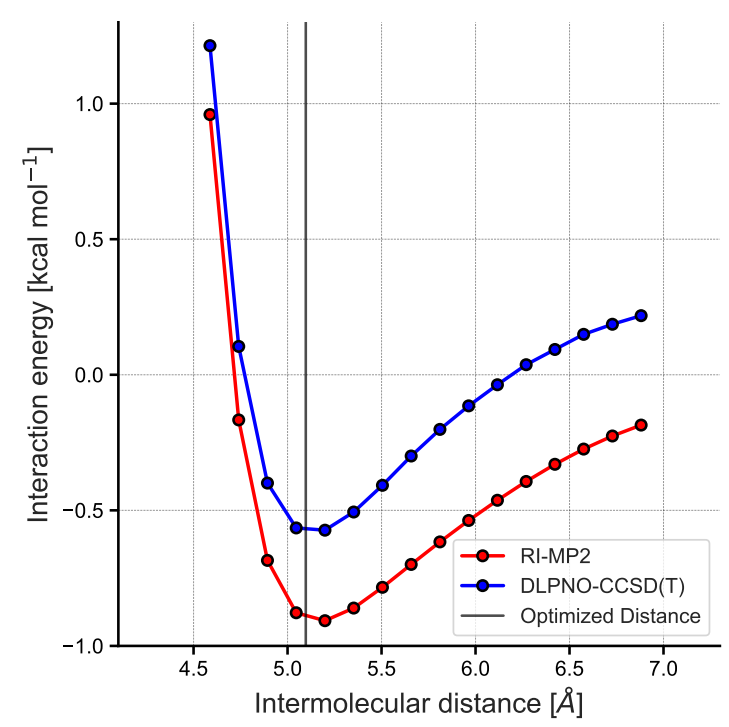

Figure S63: Rigid geometry scan of $\mathbf{2 - F F 2}$ and comparison to the optimized intermolecular separation. 


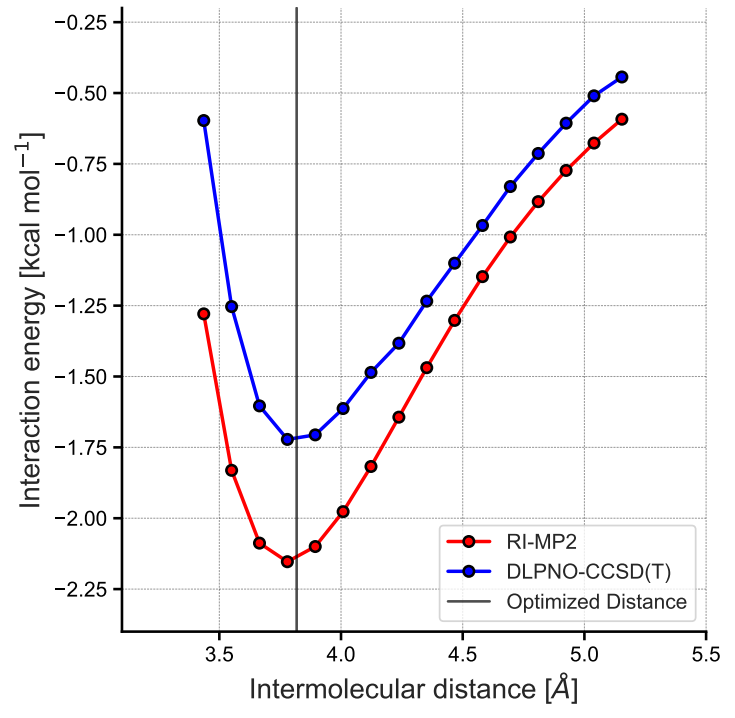

Figure S64: Rigid geometry scan of $\mathbf{3 - H H 1}$ and comparison to the optimized intermolecular separation.

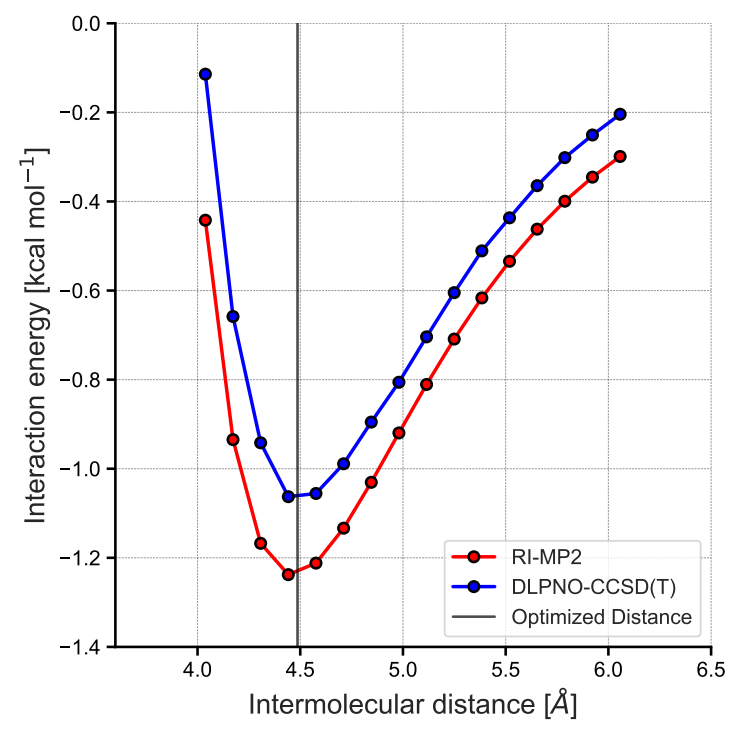

Figure S65: Rigid geometry scan of $\mathbf{3 - H H 2}$ and comparison to the optimized intermolecular separation. 


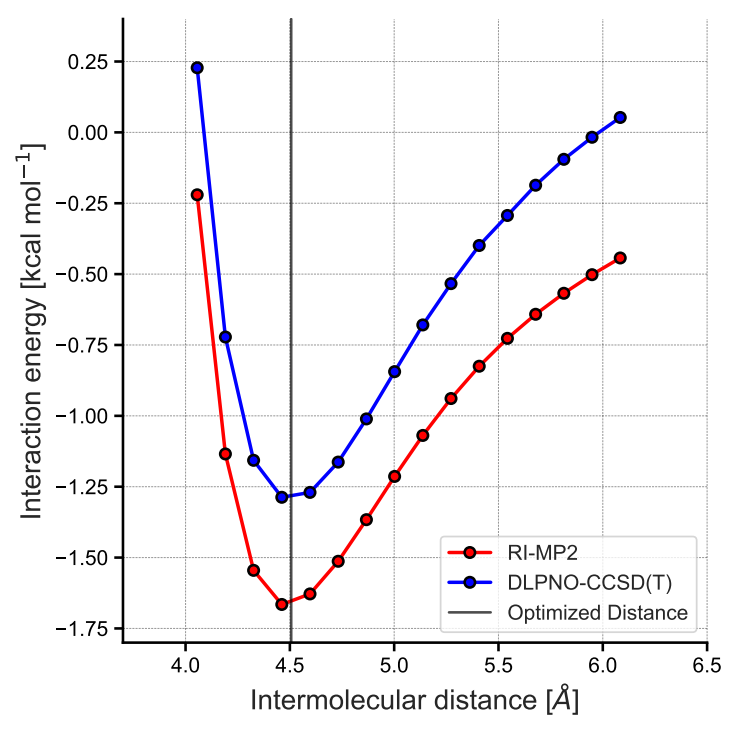

Figure S66: Rigid geometry scan of $\mathbf{3 - H F 1}$ and comparison to the optimized intermolecular separation.

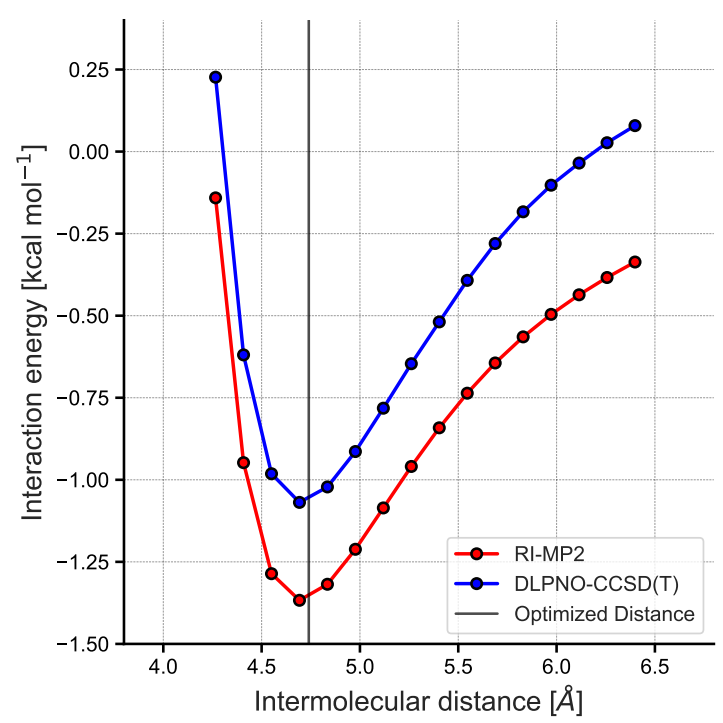

Figure S67: Rigid geometry scan of $\mathbf{3 - H F 2}$ and comparison to the optimized intermolecular separation. 


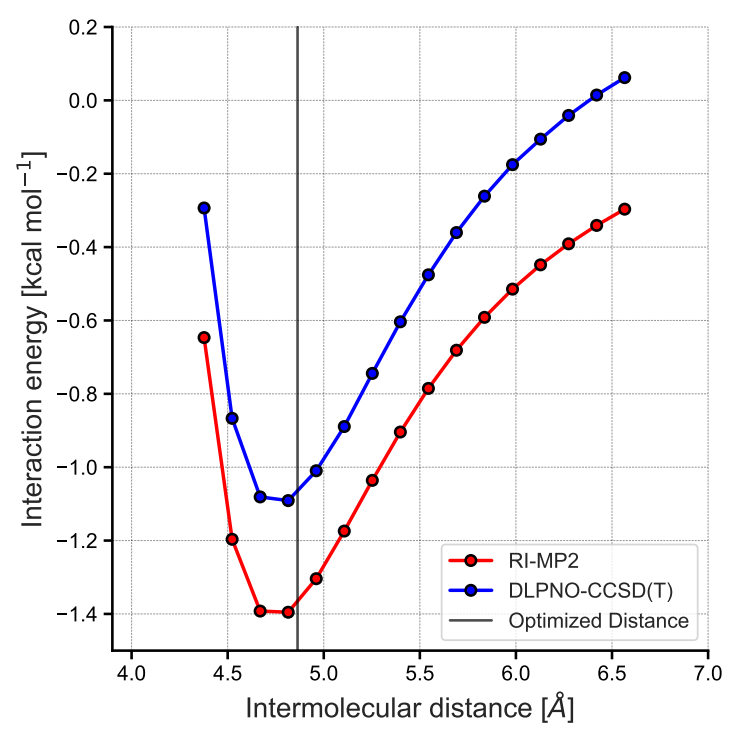

Figure S68: Rigid geometry scan of $\mathbf{3 - H F 3}$ and comparison to the optimized intermolecular separation.

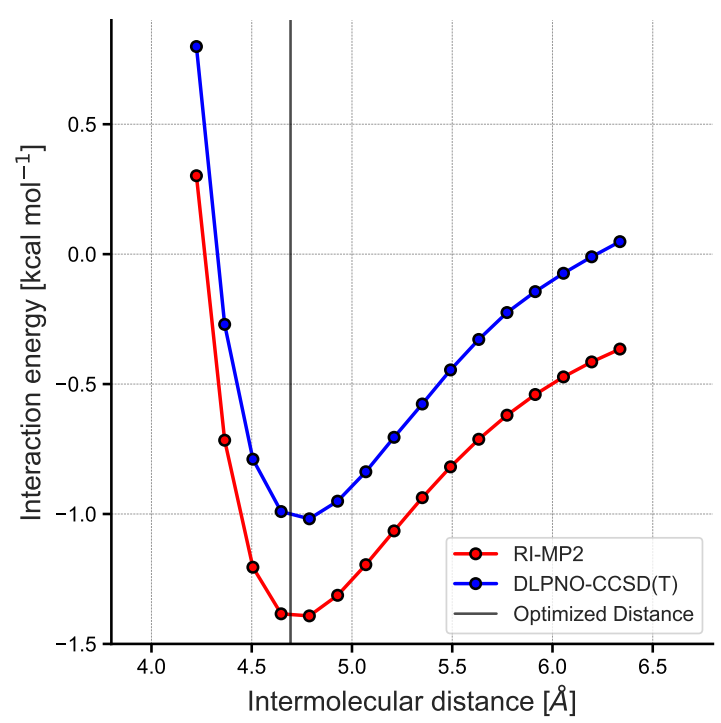

Figure S69: Rigid geometry scan of $\mathbf{3 - H F} 4$ and comparison to the optimized intermolecular separation. 


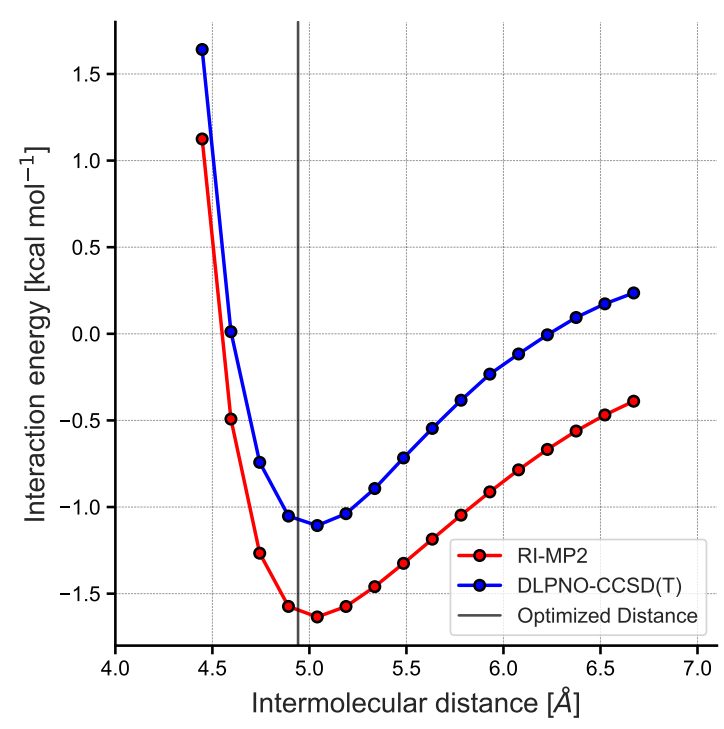

Figure S70: Rigid geometry scan of $\mathbf{3 - F F 1}$ and comparison to the optimized intermolecular separation.

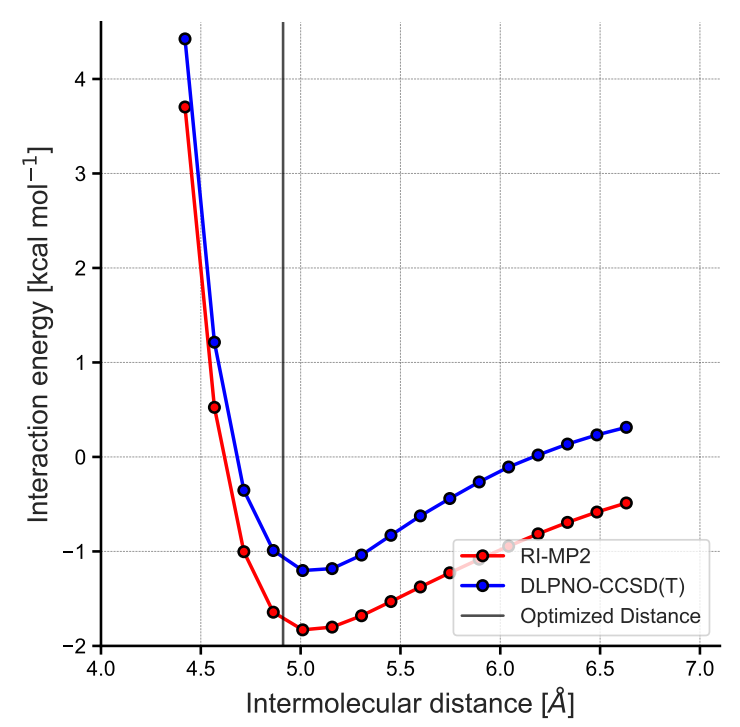

Figure S71: Rigid geometry scan of $\mathbf{3 - F F 2}$ and comparison to the optimized intermolecular separation. 


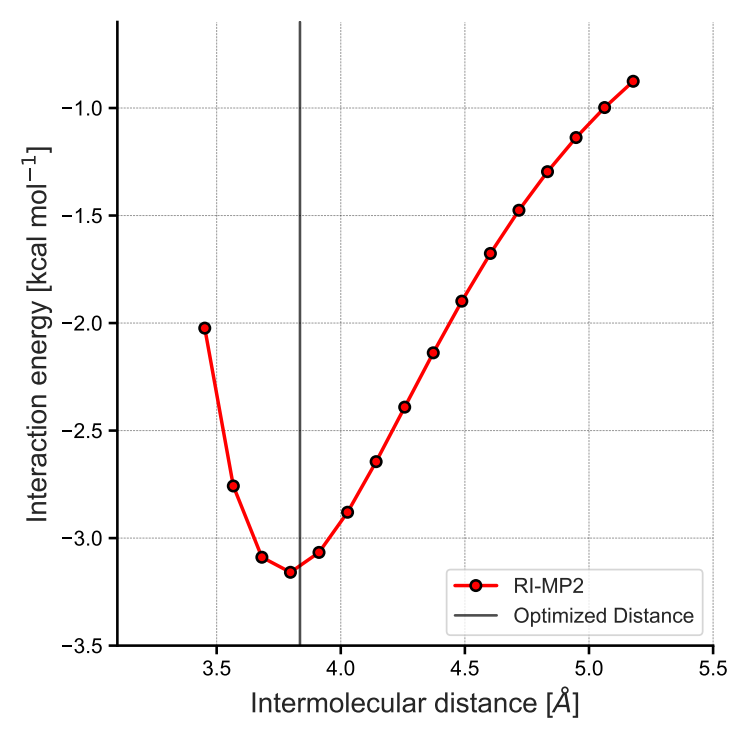

Figure S72: Rigid geometry scan of $\mathbf{4 - H H 1}$ and comparison to the optimized intermolecular separation.

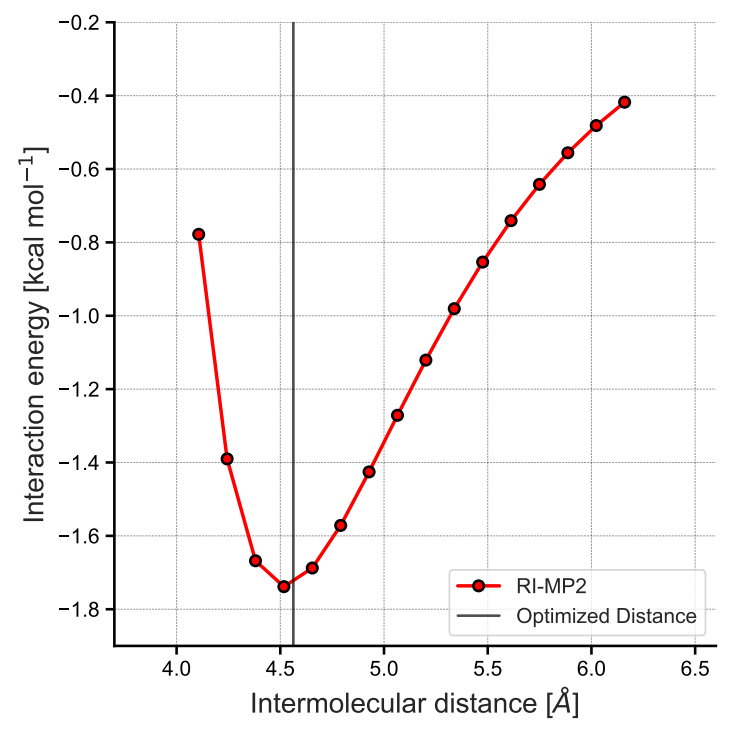

Figure S73: Rigid geometry scan of $\mathbf{4 - H H 2}$ and comparison to the optimized intermolecular separation. 


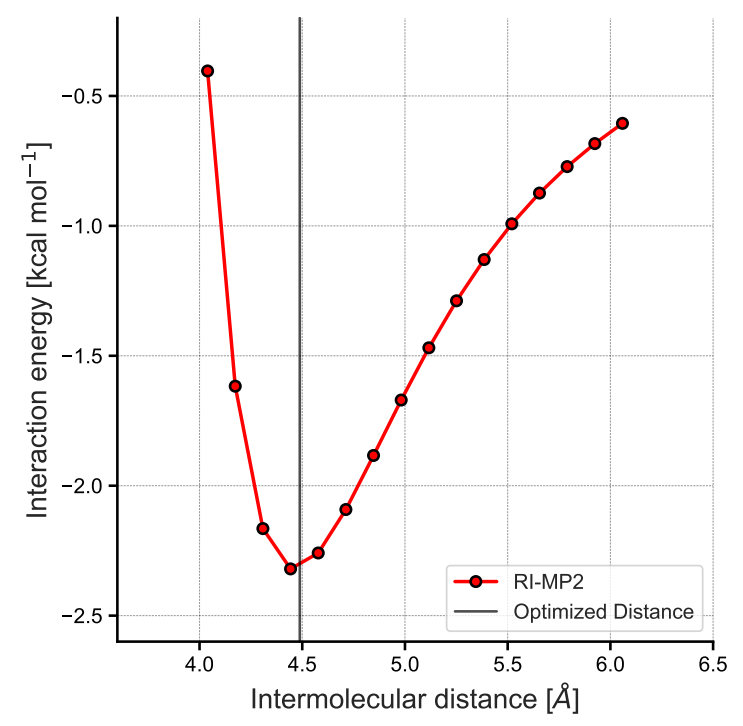

Figure S74: Rigid geometry scan of $\mathbf{4 - H F 1}$ and comparison to the optimized intermolecular separation.

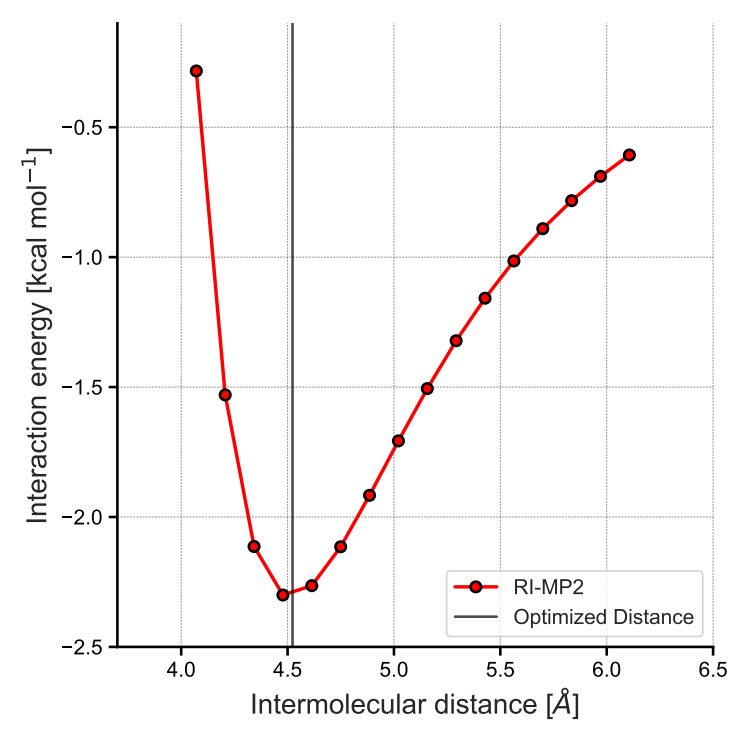

Figure S75: Rigid geometry scan of $\mathbf{4 - H F 2}$ and comparison to the optimized intermolecular separation. 


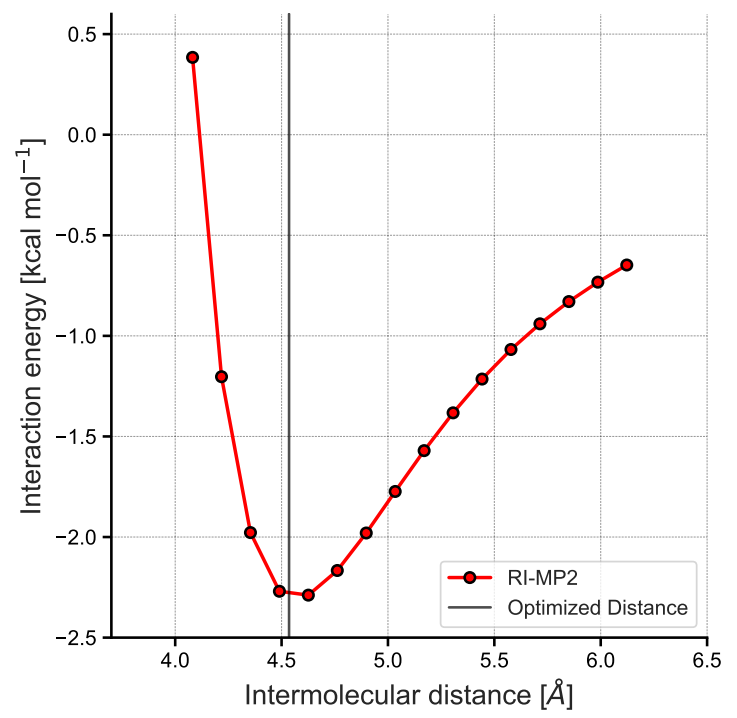

Figure S76: Rigid geometry scan of 4-HF3 and comparison to the optimized intermolecular separation.

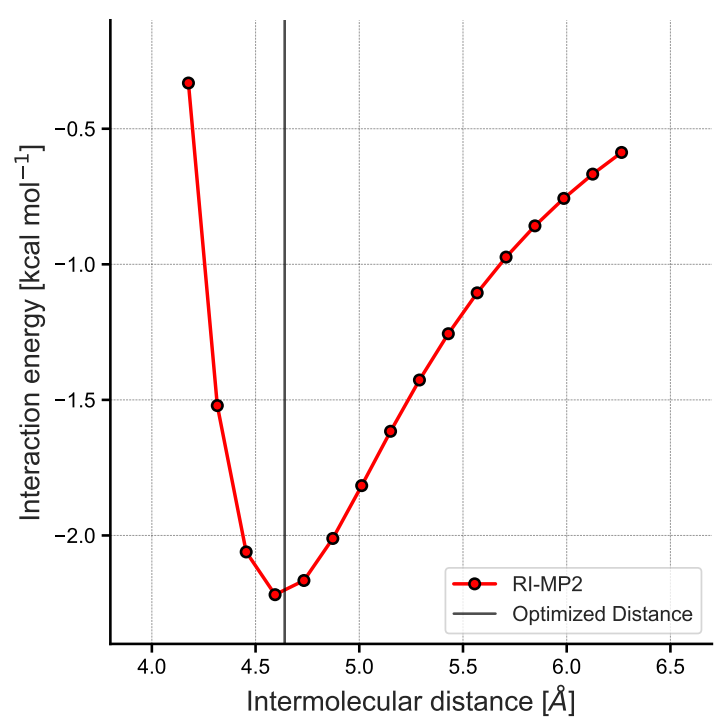

Figure S77: Rigid geometry scan of $\mathbf{4 - H F 4}$ and comparison to the optimized intermolecular separation. 


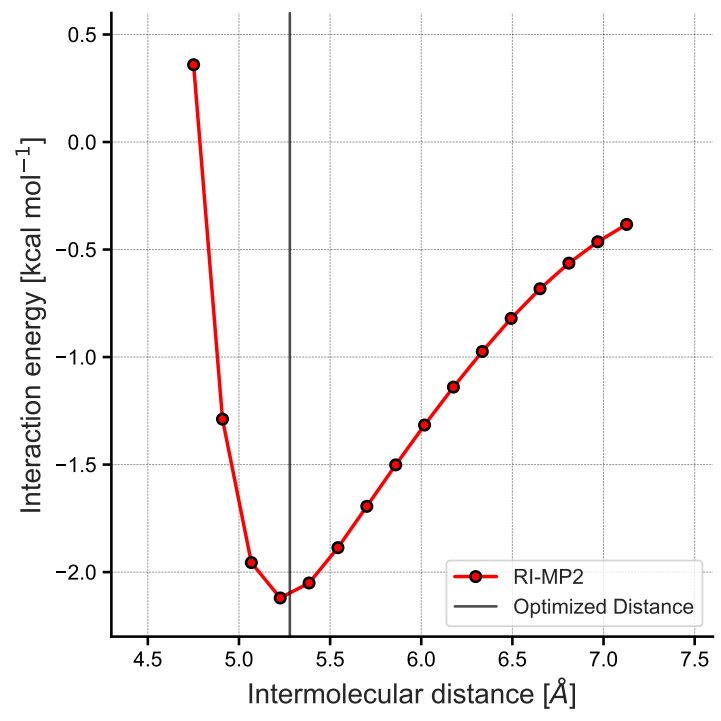

Figure S78: Rigid geometry scan of 4-FF1 and comparison to the optimized intermolecular separation.

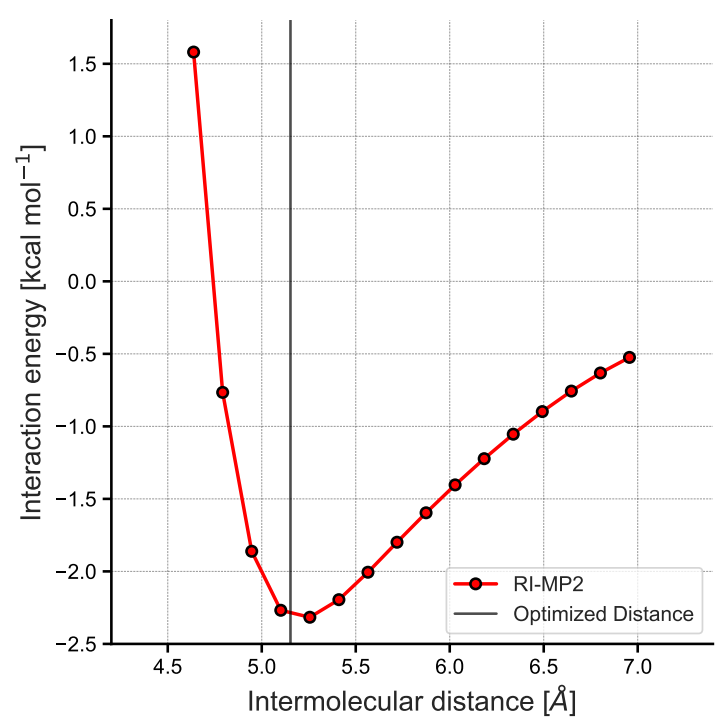

Figure S79: Rigid geometry scan of 4-FF2 and comparison to the optimized intermolecular separation. 


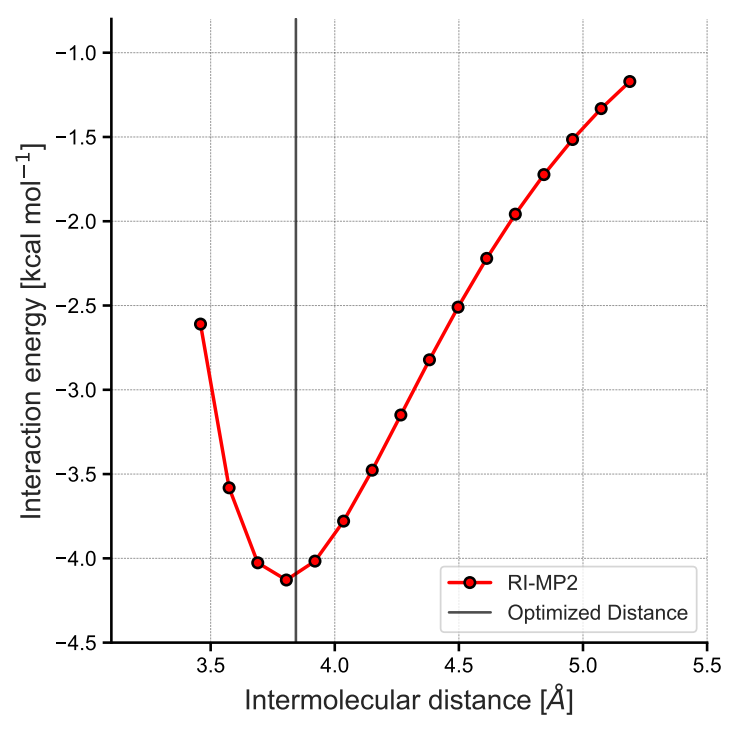

Figure S80: Rigid geometry scan of $\mathbf{5 - H H 1}$ and comparison to the optimized intermolecular separation.

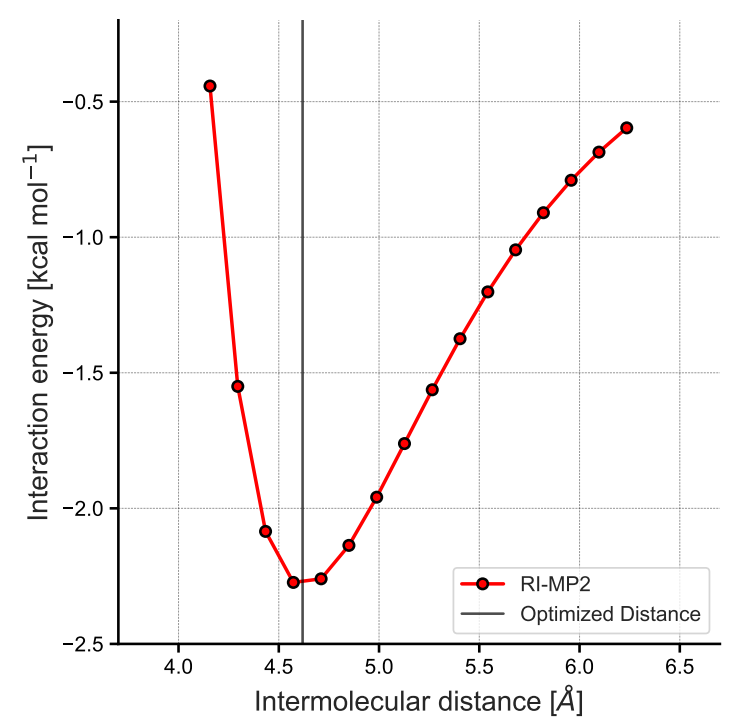

Figure S81: Rigid geometry scan of $\mathbf{5 - H H 2}$ and comparison to the optimized intermolecular separation. 


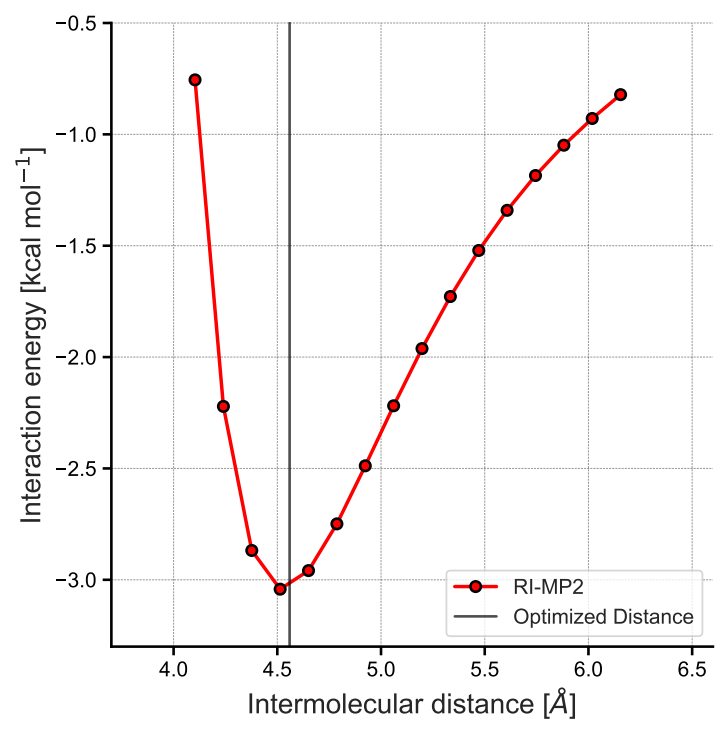

Figure S82: Rigid geometry scan of $\mathbf{5 - H F 1}$ and comparison to the optimized intermolecular separation.

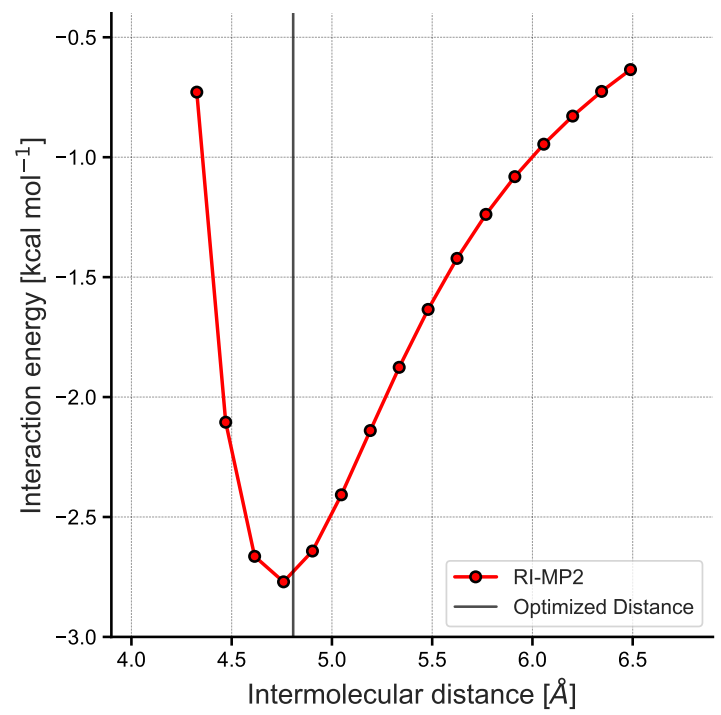

Figure S83: Rigid geometry scan of $\mathbf{5 - H F 2}$ and comparison to the optimized intermolecular separation. 


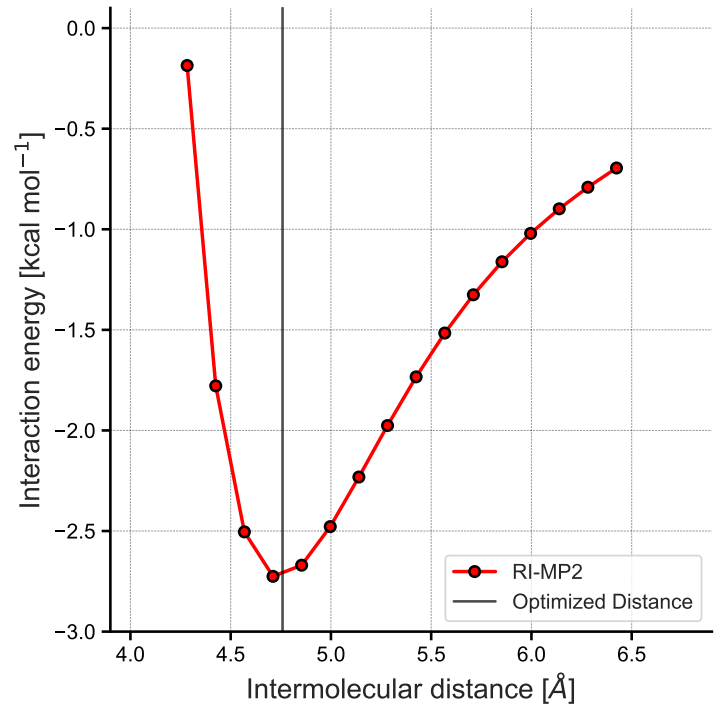

Figure S84: Rigid geometry scan of 5-HF3 and comparison to the optimized intermolecular separation.

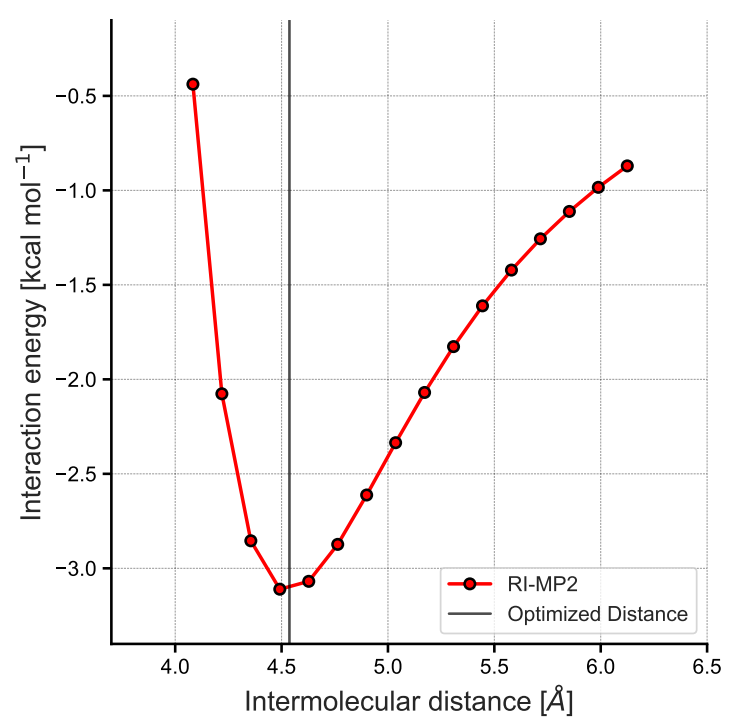

Figure S85: Rigid geometry scan of $\mathbf{5 - H F} 4$ and comparison to the optimized intermolecular separation. 


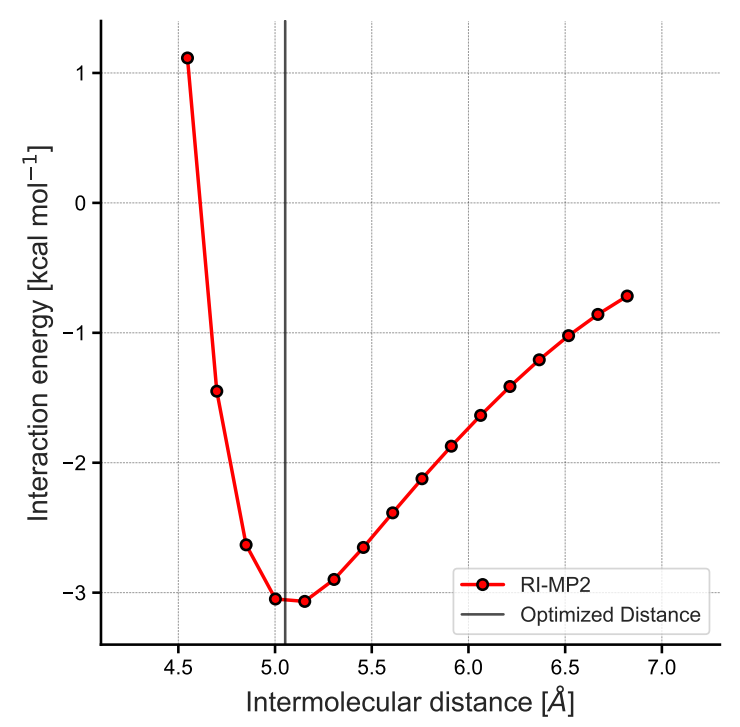

Figure S86: Rigid geometry scan of $\mathbf{5 - F F 1}$ and comparison to the optimized intermolecular separation.

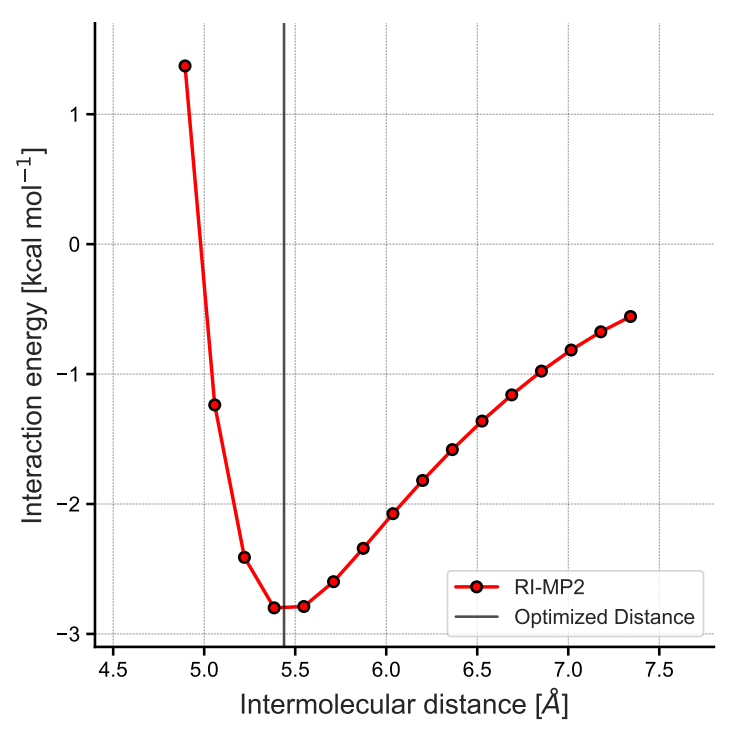

Figure S87: Rigid geometry scan of 5-FF2 and comparison to the optimized intermolecular separation. 


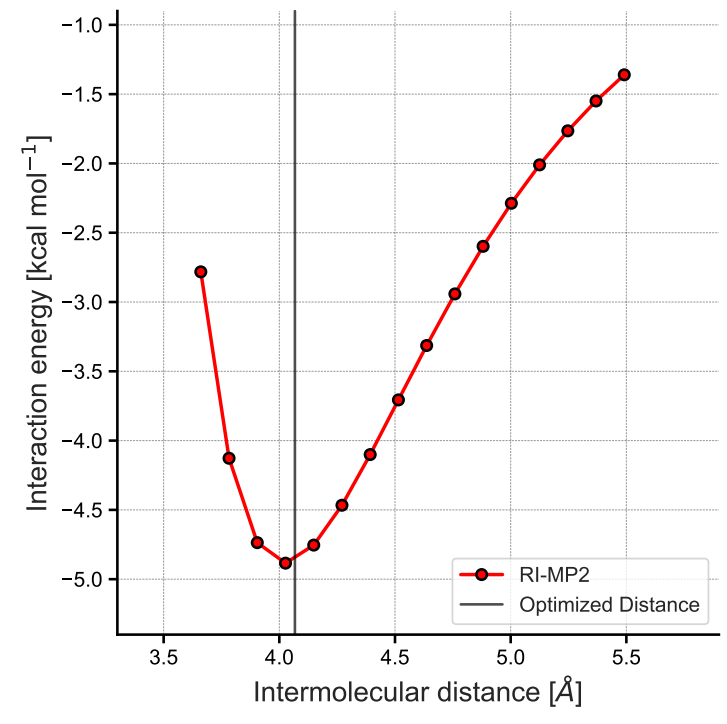

Figure S88: Rigid geometry scan of $\mathbf{6 - H H 1}$ and comparison to the optimized intermolecular separation.

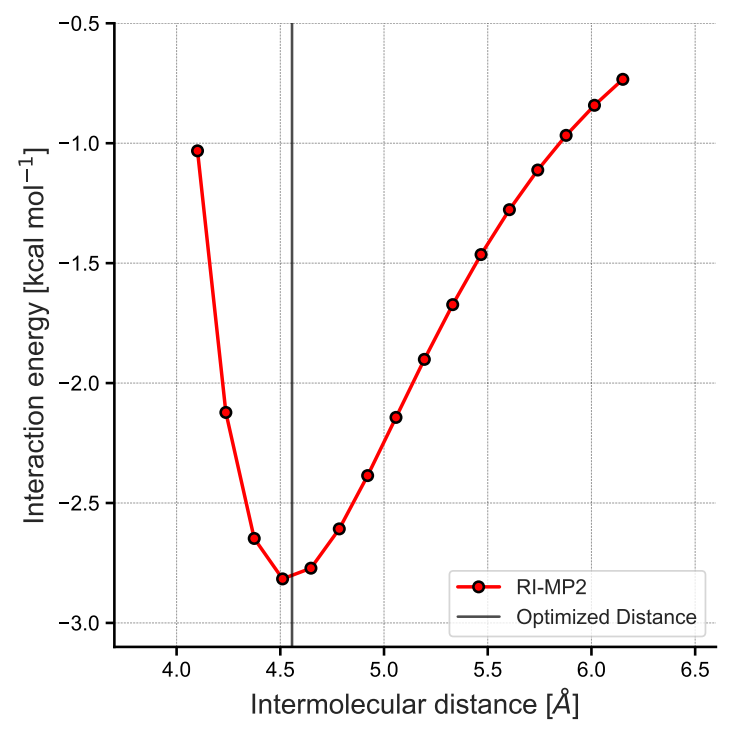

Figure S89: Rigid geometry scan of $\mathbf{6 - H H 2}$ and comparison to the optimized intermolecular separation. 


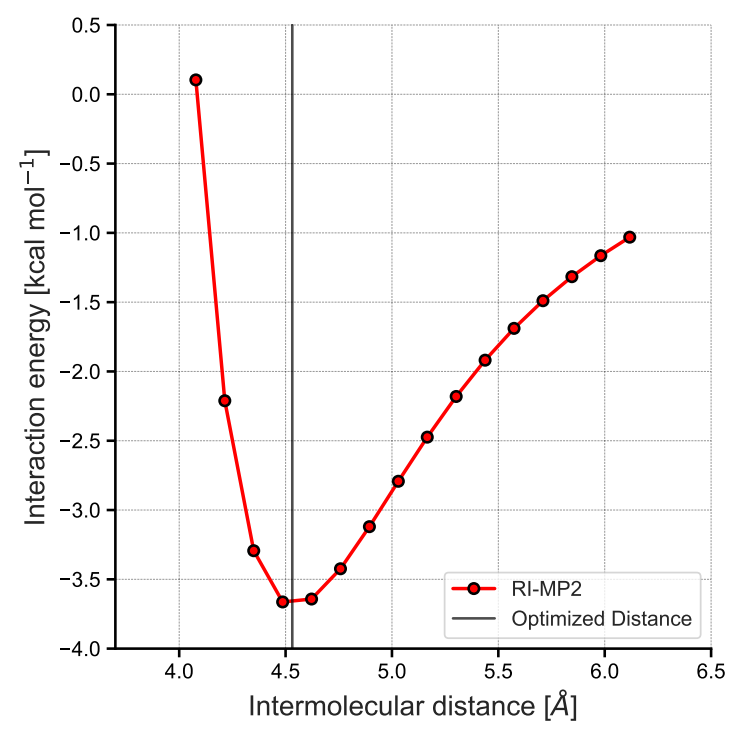

Figure S90: Rigid geometry scan of $\mathbf{6 - H F} \mathbf{1}$ and comparison to the optimized intermolecular separation.

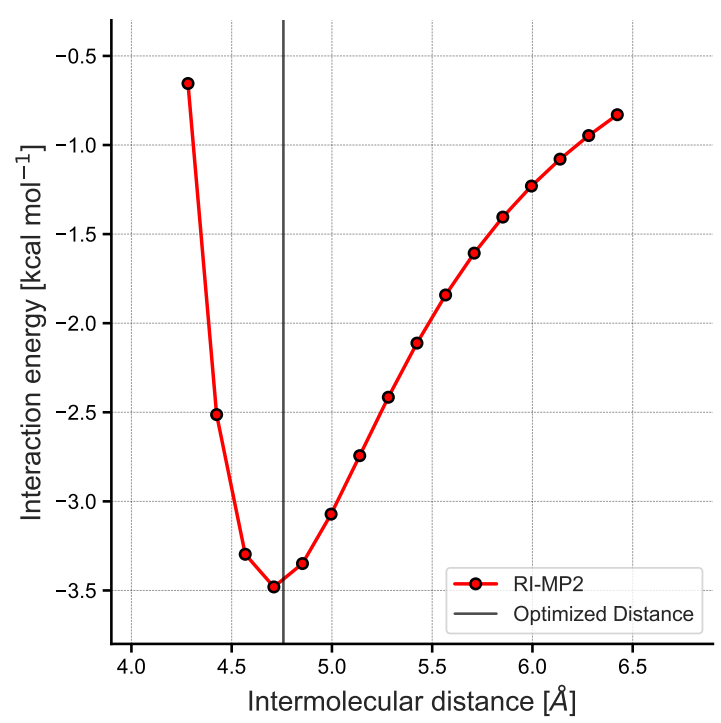

Figure S91: Rigid geometry scan of $\mathbf{6 - H F 2}$ and comparison to the optimized intermolecular separation. 


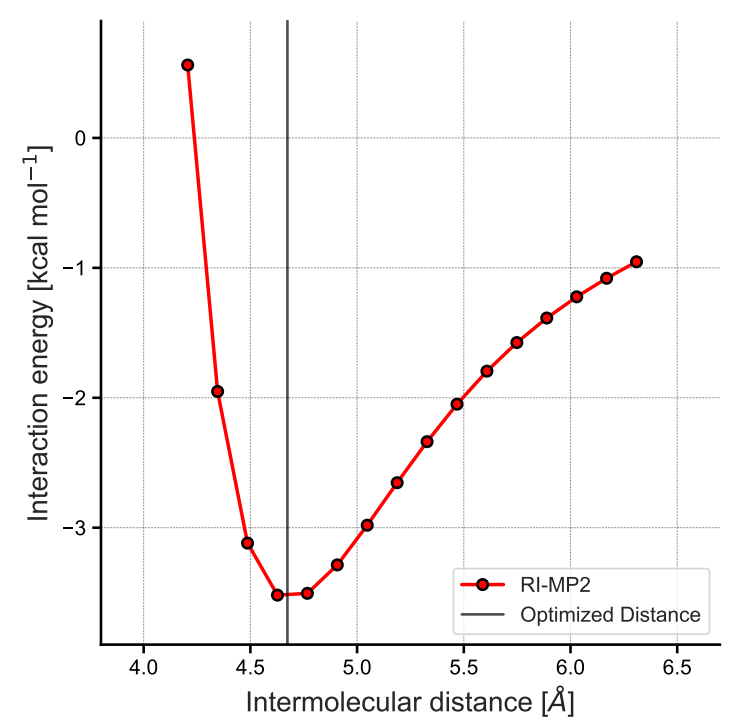

Figure S92: Rigid geometry scan of $\mathbf{6 - H F 3}$ and comparison to the optimized intermolecular separation.

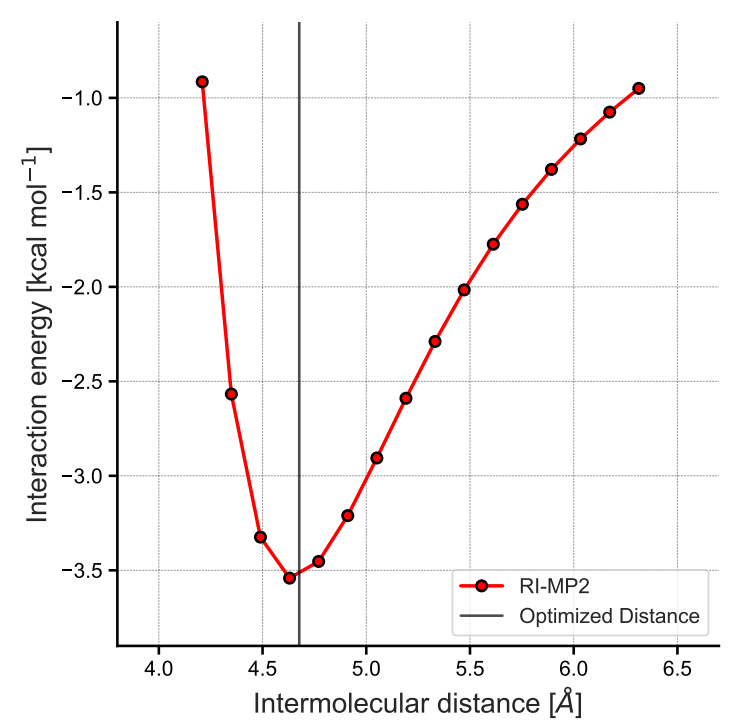

Figure S93: Rigid geometry scan of $\mathbf{6 - H F} 4$ and comparison to the optimized intermolecular separation. 


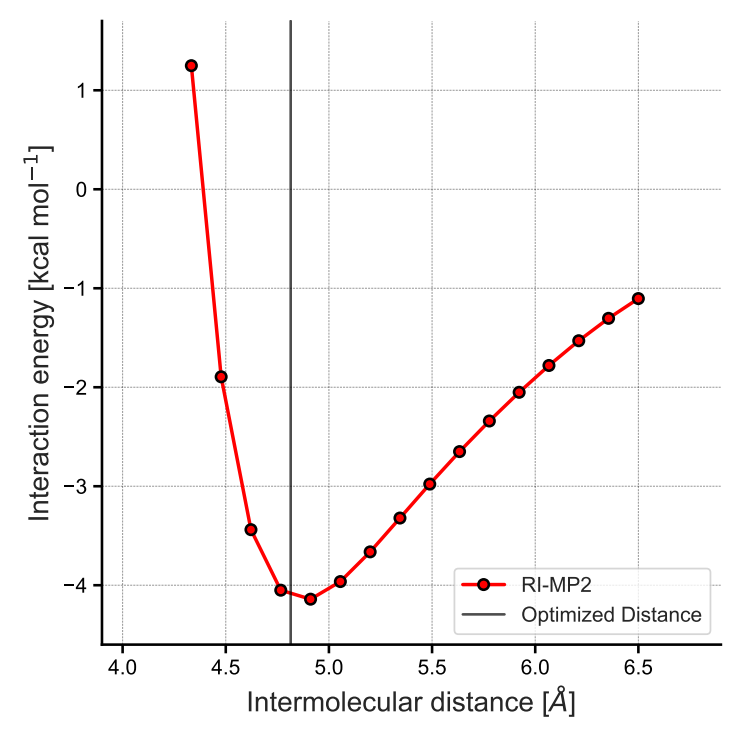

Figure S94: Rigid geometry scan of $\mathbf{6 - F F 1}$ and comparison to the optimized intermolecular separation.

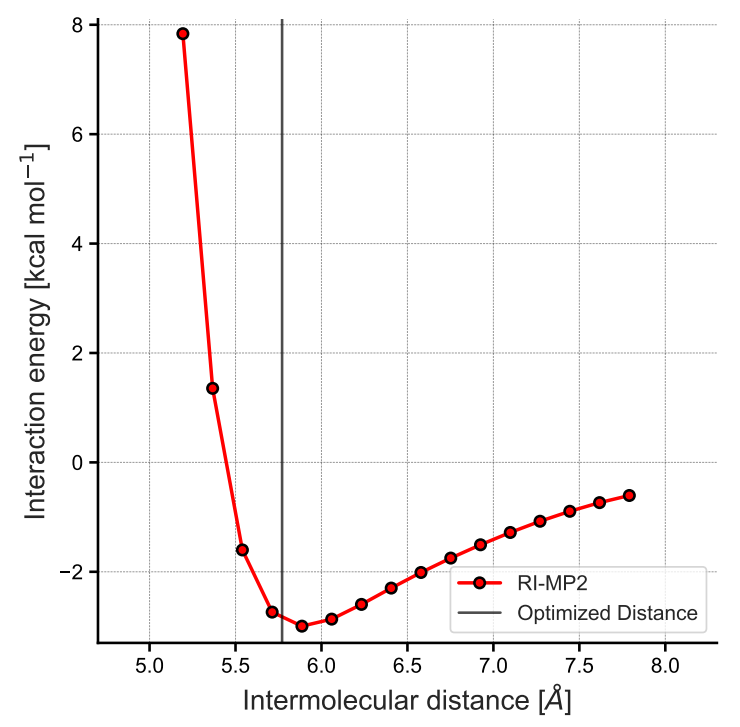

Figure S95: Rigid geometry scan of 6-FF2 and comparison to the optimized intermolecular separation. 


\subsubsection{Deformation Energies}

Deformation energies $\left(E_{d e f}\right)$ were estimated on the basis of the DSD-PBEP86/def2TZVP(spd) geometries using the benchmark method CCSD(T)-F12/VDZ and the bestperforming methods in the benchmarks for which results for all molecules under study were obtained (i.e. B97M-V/def2-QZVP, B3LYP-XMD/aug-cc-pVTZ and VeryTightPNODLPNO-CCSD(T)/CBS(3-4)). CCSD(T)-F12/VDZ values show that, on average, deformation energies amount only to about $(1 \pm 2) \%$ of the BIEs. Similarly, B97M-V predicts $(3 \pm 3) \%$, B3LYP-XDM predicts $(2 \pm 3) \%$ and VeryTightPNO-DLPNO-CCSD(T) predicts $(3 \pm 3) \%$. This shows that for these complexes, $E_{\text {def }}$ can be neglected without introducing too big errors because the error is on the same order of magnitude as the error in the computational methods or smaller than that, but not larger.

\subsection{Energy Decomposition Analysis}

\subsubsection{Dispersion}

We looked closer into the sum of the exchange and dispersion components and its importance in $\mathbf{H H}, \mathbf{H F}$ and $\mathbf{F F}$ complexes, respectively. Figure S96 shows this sum as a function of the overall BIE for the three different classes of complexes. It illustrates that the sum of exchange and dispersion is larger in FF systems than in $\mathbf{H F}$ and $\mathbf{H H}$. Figure S97 shows views across the interaction surface of exemplary complexes (labelled in Figure S96) of each class.

\subsubsection{Electrostatics}

We also looked closer into the electrostatics component and its importance in $\mathbf{H H}, \mathbf{H F}$ and FF complexes, respectively. Figure S98 shows the electrostatics contribution as a function of the overall BIE for the three different classes of complexes. It illustrates that while the sum

of exchange and dispersion was larger in $\mathbf{F F}$ systems than in $\mathbf{H F}$ and $\mathbf{H H}$ (vide supra), the 

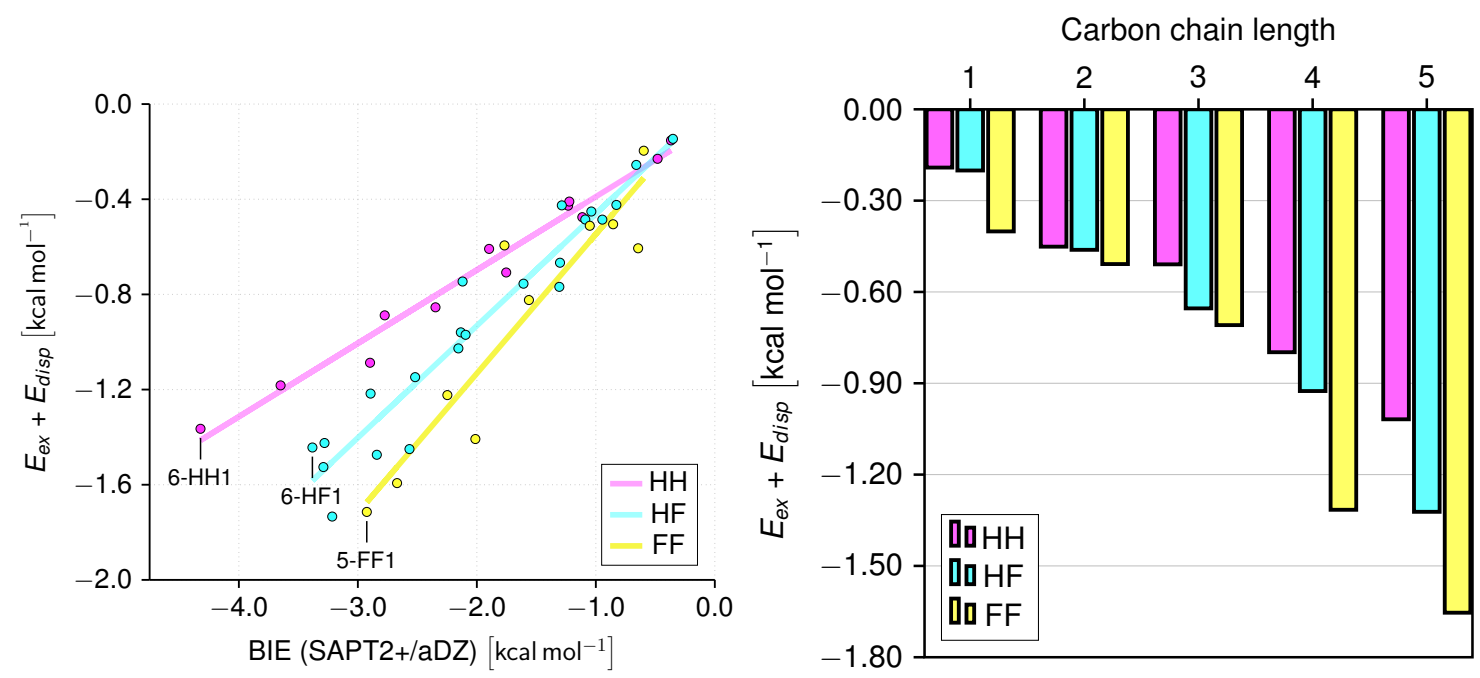

Figure S96: Sum of Pauli exchange and dispersion of the molecular complexes divided into the three subclasses HH, HF and FF. Left: Sum of Pauli exchange and dispersion plotted against overall interaction energies. Right: Sum of Pauli exchange and dispersion plotted against number of carbon atoms in the interacting molecules. Overall, the relative importance of dispersion increases when going from $\mathbf{H H}$ via $\mathbf{H F}$ to $\mathbf{F F}$.

\section{6-HH1}

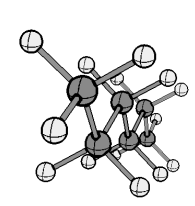

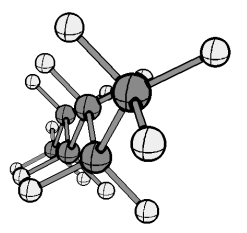

6-HF1

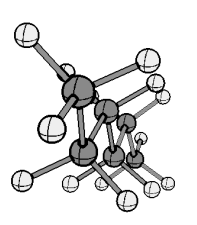

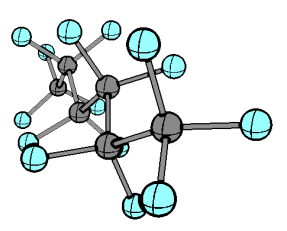

5-FF1

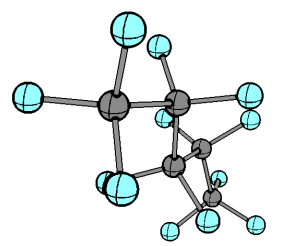

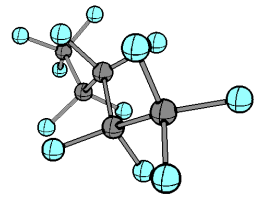

Figure S97: View along the interacting fragments of the labelled complexes 6-HH1, 6-HF1 and 5-FF1, respectively. 
corresponding ordering in the electrostatic contribution is reversed. It is striking that $\mathbf{H F}$ complexes do not show a larger contribution to the electrostatic component compared to the homomolecular complexes. It can be rationalized because the fluorine atoms of $\mathbf{F}$ prefer to maximize contacts with the carbon atoms of $\mathbf{H}$ to optimize attractive dispersion. However, these types of atoms are negatively polarized which results in a repulsive component to the overall electrostatics. Electrostatics could be maximized by increasing contacts of the fluorine atoms of $\mathbf{F}$ with the hydrogen atoms of $\mathbf{H}$ which, however, is not ideal for optimizing dispersion. This showcases that dispersion dominates the interaction and, hence, also the interaction geometries in all the intermolecular complexes studied.

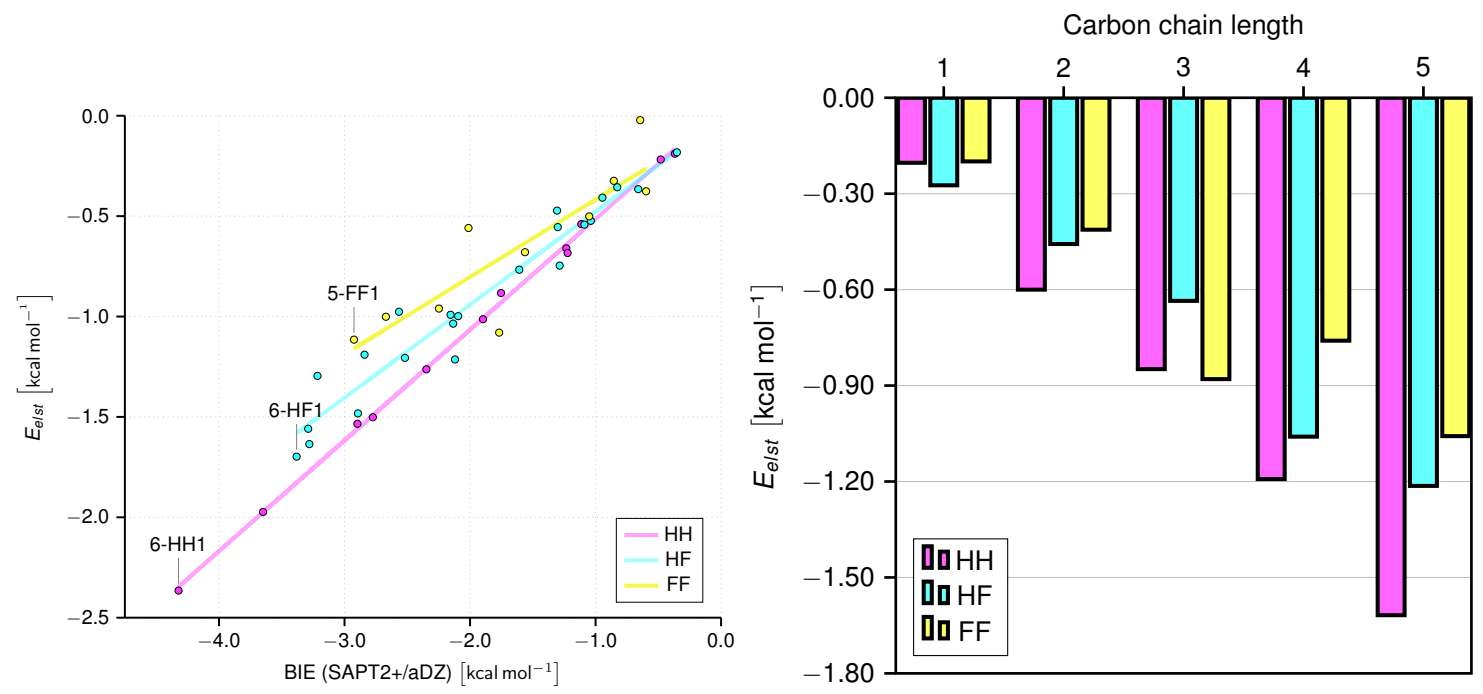

Figure S98: Electrostatics from SAPT of the molecular complexes divided into the three subclasses HH, HF and FF. Left: Electrostatics plotted against the BIE. Right: Electrostatics plotted against the number of carbon atoms.

\subsubsection{Comparison of SAPT2+ and LED}

We also looked into both the BIE and the dispersion energy obtained from LED-DLPNOCCSD(T)/QZ calculations and compared them to the results of the SAPT2+/aDZ calculations. The numbers showed equivalent trends compared to the energies from SAPT (Figure S99) which provides mutual support for the adequacy of these two method to discern the importance of dispersion in the overall interaction. 


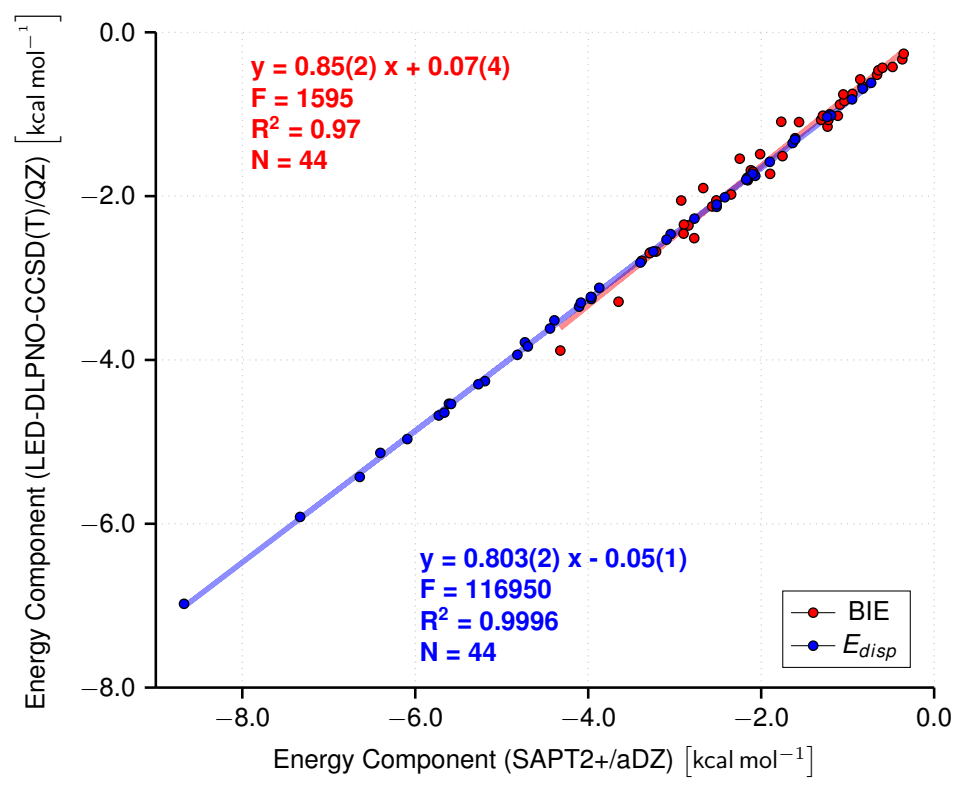

Figure S99: Comparison of interaction energies and dispersion energy components of molecular complexes obtained from SAPT2+/aDZ and LED-TightPNO-DLPNO-CCSD(T)/QZ, respectively. Systematic differences in the magnitudes are observed but trends are equivalent.

\subsubsection{LED Decomposition}

The decomposition of dispersion into contributions of atoms and bonds in complexes of $\mathbf{H}$ and $\mathbf{F}$ was performed using four distinct LED jobs (cf. Figure S100). First, LED was performed using two fragments corresponding to the two interacting molecules (job 1). Secondly, two LED jobs were performed with three fragments each (job 2 and job 3). In job 2 the hydrogen or fluorine atoms of the first interacting molecule were considered as a separate fragment from the corresponding carbon backbone. In job 3 the hydrogen or fluorine atoms of the second interacting molecule were considered as a separate fragment from the corresponding carbon backbone. In job 4 the hydrogen or fluorine atoms of both interacting molecules were considered as a separate fragment from the corresponding carbon backbone.

The results of these four LED jobs were evaluated to get nine different dispersion contributions. The corresponding formulas used for evaluation of the data are derived in this section. First, the parameters used are defined: 


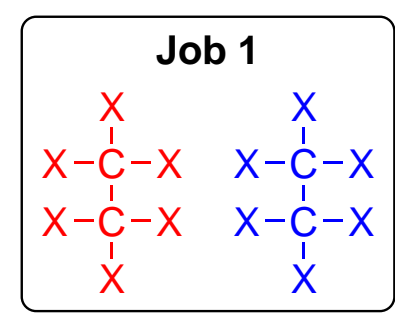

fragment 1 fragment 2

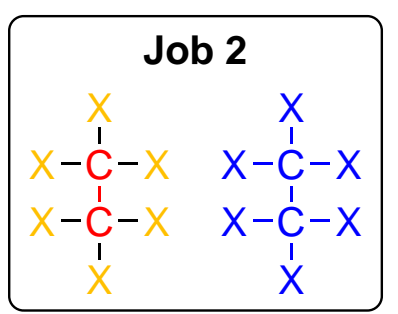

fragment 1 fragment 3

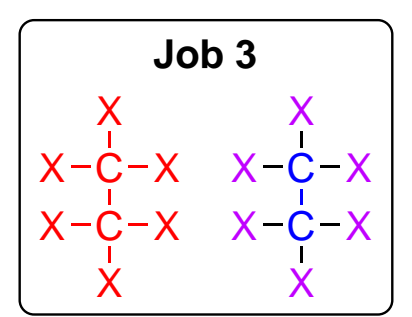

fragment 1 fragment 2

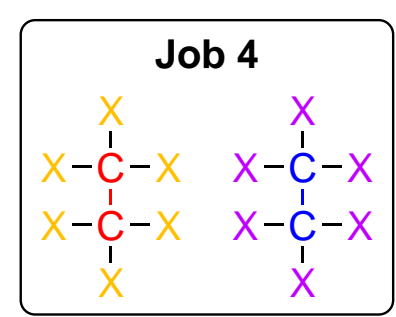

fragment 1 fragment 3

Figure S100: Required LED jobs to decompose the overall intermolecular dispersion into contributions from atoms and bonds.

$$
\begin{array}{lll}
D(n, A-B) & \ldots & \text { Intermolecular dispersion obtained from job } \mathrm{n} \text { between fragments } \mathrm{A} \text { and } \mathrm{B} \\
D_{t o t} & \ldots & \text { Total intermolecular dispersion } \\
D(C 1-C 2) & \ldots & \text { Dispersion between carbon chain } \mathrm{C} 1 \text { and carbon chain } \mathrm{C} 2 \\
D(C 1-X 2) & \ldots & \text { Dispersion between carbon chain } \mathrm{C} 1 \text { and atoms } \mathrm{X} 2(\mathrm{X}=\mathrm{H}, \mathrm{F}) \\
D(X 1-C 2) & \ldots & \text { Dispersion between atoms } \mathrm{X} 1(\mathrm{X}=\mathrm{H}, \mathrm{F}) \text { and carbon chain } \mathrm{C} 2 \\
D(X 1-X 2) & \ldots & \text { Dispersion between atoms } \mathrm{X} 1 \text { and atoms } \mathrm{X} 2(\mathrm{X}=\mathrm{H}, \mathrm{F}) \\
D(C 1-C X 2) & \ldots & \text { Dispersion between carbon chain } \mathrm{C} 1 \text { and } \mathrm{CX} 2 \text { bonds }(\mathrm{X}=\mathrm{H}, \mathrm{F}) \\
D(C X 1-C 2) & \ldots & \text { Dispersion between } \mathrm{CX} 1 \text { bonds }(\mathrm{X}=\mathrm{H}, \mathrm{F}) \text { and carbon chain } \mathrm{C} 2 \\
D(X 1-C X 2) & \ldots & \text { Dispersion between atoms X1 and CX2 bonds }(\mathrm{X}=\mathrm{H}, \mathrm{F}) \\
D(C X 1-X 2) & \ldots & \text { Dispersion between CX1 bonds and atoms } \mathrm{X} 2(\mathrm{X}=\mathrm{H}, \mathrm{F}) \\
D(C X 1-C X 2) & \ldots & \text { Dispersion between CX1 bonds and CX2 bonds }(\mathrm{X}=\mathrm{H}, \mathrm{F})
\end{array}
$$

The total dispersion energy is the sum of all its components (abbreviated as $D_{i j}$ for simplification):

$$
D_{t o t}=\sum_{i} \sum_{j} D_{i j}
$$

The following equations show which contributions are obtained from the 4 jobs:

$$
\begin{gathered}
D(1,1-2)=D_{\text {tot }} \\
D(2,1-3)=D(C 1-C 2)+D(C 1-X 2)+D(C 1-C X 2)
\end{gathered}
$$




$$
\begin{gathered}
D(2,2-3)=D(X 1-C 2)+D(X 1-X 2)+D(X 1-C X 2) \\
D(3,1-2)=D(C 1-C 2)+D(X 1-C 2)+D(C X 1-C 2) \\
D(3,1-3)=D(C 1-X 2)+D(X 1-X 2)+D(C X 1-X 2) \\
D(4,1-3)=D(C 1-C 2) \\
D(4,1-4)=D(C 1-X 2) \\
D(4,2-3)=D(X 1-C 2) \\
D(4,2-4)=D(X 1-X 2)
\end{gathered}
$$

Overall, we have ten independent linear equations and ten unknown parameters. Therefore, it should be possible to solve this system of equations for all unknown parameters. Five out of the ten unknown parameters are directly obtained from the jobs. Four additional parameters are obtained from equations S3-S6 by inserting the respective terms obtained from equations S7-S10. The last parameter is obtained from the energy balance in equation S1 by inserting all the other nine parameters. Using these equations, we obtained the overall intermolecular dispersion between the interacting molecules, as well as, nine distinct contributions to the overall intermolecular dispersion from interactions between different atoms and bonds with each other. The corresponding results of this decomposition are shown in the main text and in the following section. 


\subsubsection{Additional LED Results}

The relative contribution of the F1-F2 dispersive interaction correlates linearly with the relative molar fluorine content (cf. Figure S101). Plotting the ratio of the relative contributions of $\mathrm{C} 1$ and $\mathrm{C} 2$ of $\mathbf{F F}$ and $\mathbf{H H}$ against the relative molar carbon content also results in a linear regression (Figure S101). In other words, the smaller the relative number of fluorine atoms compared to the number of carbon atoms the smaller the number of favorable intermolecular fluorine-fluorine interactions. Since these dispersive interactions are most attractive, FF with longer carbon chains have lower BIEs normalized by the number of carbons compared to FF with shorter carbon chains. However, the relative carbon content levels at longer carbon chain lengths and, hence, this effect is most pronounced in the short and intermediate $\mathbf{H}$ and $\mathbf{F}$.
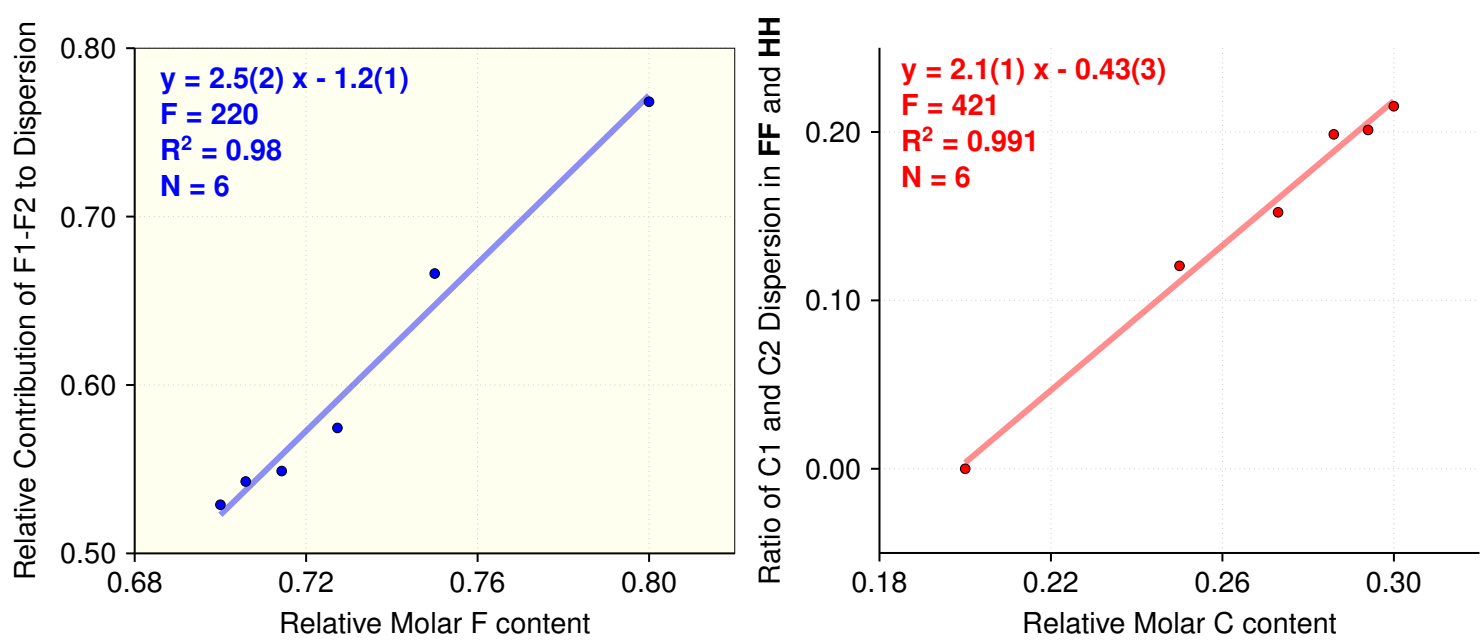

Figure S101: Left: Relative contribution of fluorine-fluorine interactions (F1-F2) in FF towards dispersion from LED-TightPNO-DLPNO-CCSD(T)/QZ as a function of the relative molar fluorine content. The relative importance of F1-F2 decreases with the fluorine content as the carbon chain is elongated. Right: Ratio of the contributions of $\mathrm{C} 1$ and $\mathrm{C} 2$ in FF and $\mathbf{H H}$, respectively, towards dispersion from LED-TightPNO-DLPNO-CCSD $(\mathrm{T}) / \mathrm{QZ}$ as a function of the relative molar carbon content.

Figures S102-S116 show the decomposition of LED-DLPNO-CCSD(T) dispersion energies into contributions of interactions between atoms and bonds for $\mathbf{H H}, \mathbf{H F}$ and $\mathbf{F F}$ with two to six carbon atoms in the respective interacting molecules. They show that that the results are 
relatively stable with respect to the number of carbon atoms. The most striking difference is observed in the relative contribution of F1-F2 in FF as discussed in the main text.

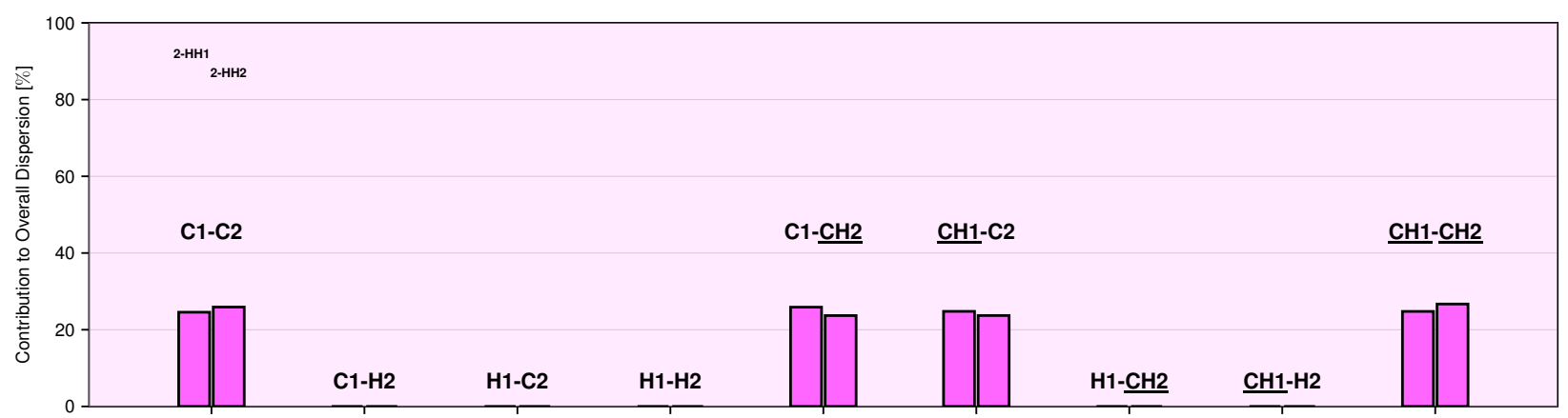

Figure S102: Decomposition of dispersion obtained from LED-TightPNO-DLPNO$\operatorname{CCSD}(\mathrm{T}) / \mathrm{QZ}$ into contributions of atoms and bonds for $\mathbf{2 -} \mathbf{H H}$ complexes.

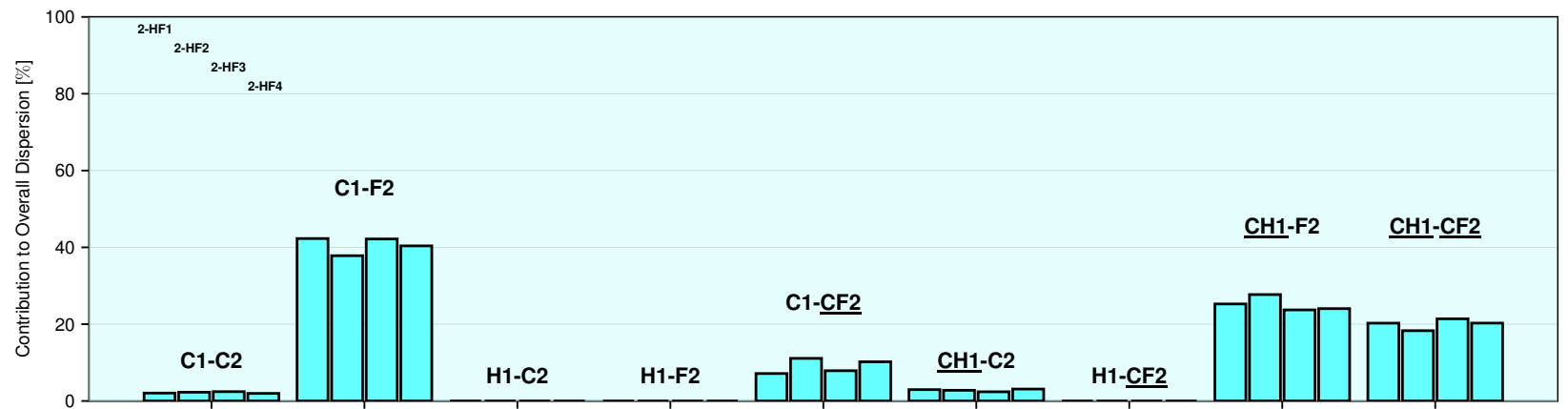

Figure S103: Decomposition of dispersion obtained from LED-TightPNO-DLPNOCCSD(T)/QZ into contributions of atoms and bonds for $\mathbf{2 - H F}$ complexes. 


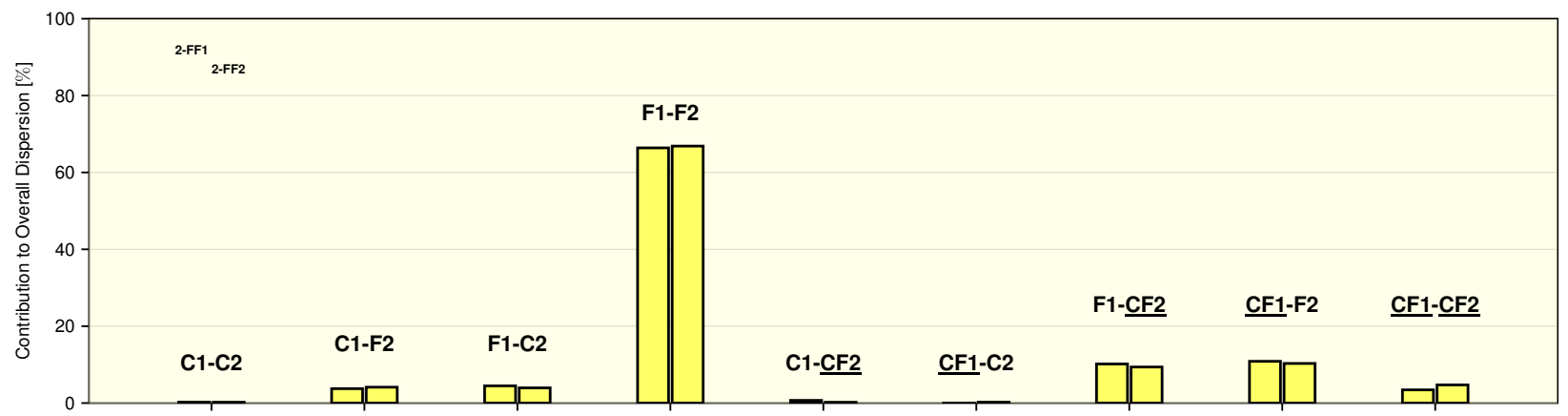

Figure S104: Decomposition of dispersion obtained from LED-TightPNO-DLPNOCCSD(T)/QZ into contributions of atoms and bonds for 2-FF complexes.

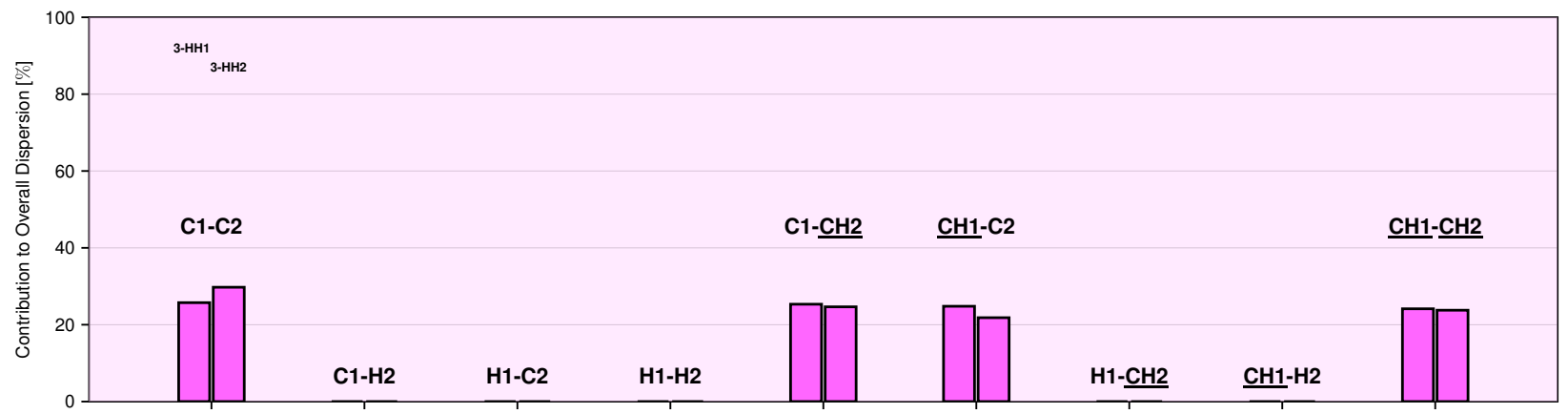

Figure S105: Decomposition of dispersion obtained from LED-TightPNO-DLPNOCCSD(T)/QZ into contributions of atoms and bonds for $\mathbf{3}-\mathbf{H H}$ complexes.

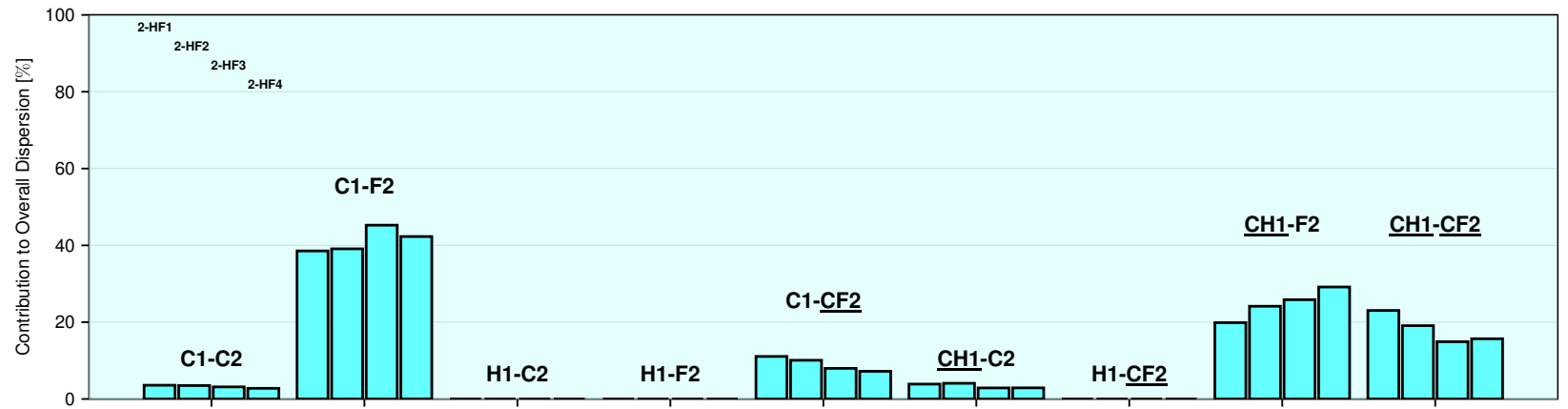

Figure S106: Decomposition of dispersion obtained from LED-TightPNO-DLPNOCCSD(T)/QZ into contributions of atoms and bonds for $\mathbf{3 - H F}$ complexes. 


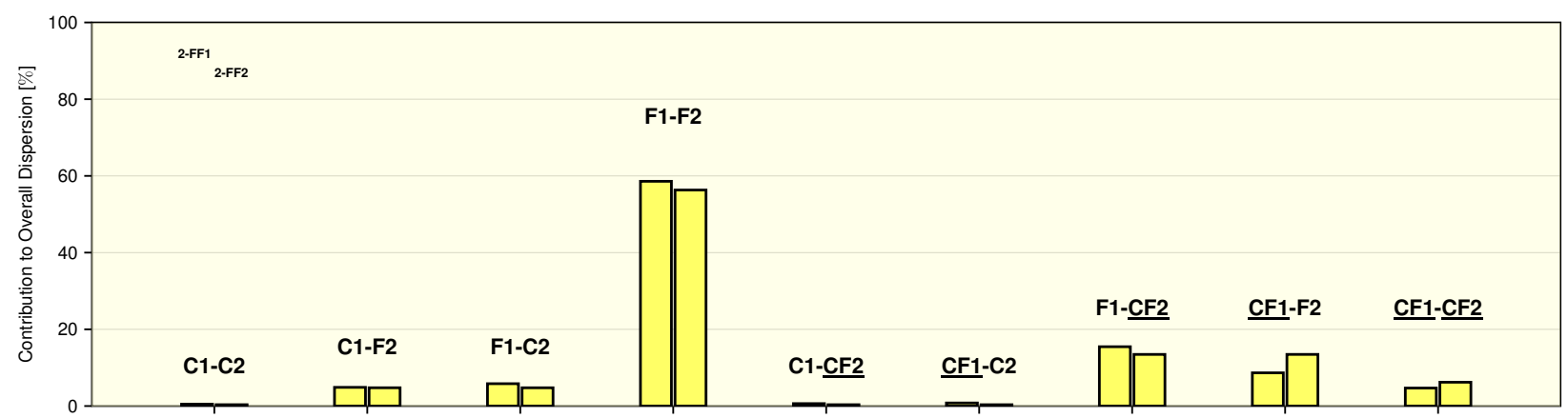

Figure S107: Decomposition of dispersion obtained from LED-TightPNO-DLPNOCCSD(T)/QZ into contributions of atoms and bonds for $\mathbf{3 - F F}$ complexes.

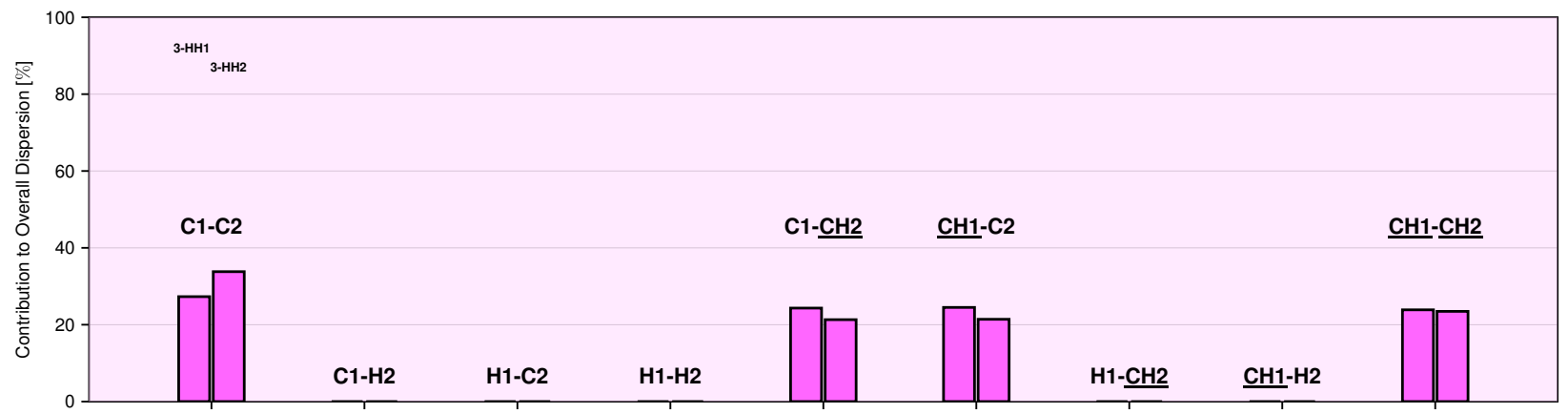

Figure S108: Decomposition of dispersion obtained from LED-TightPNO-DLPNOCCSD(T)/QZ into contributions of atoms and bonds for $\mathbf{4}-\mathbf{H H}$ complexes.

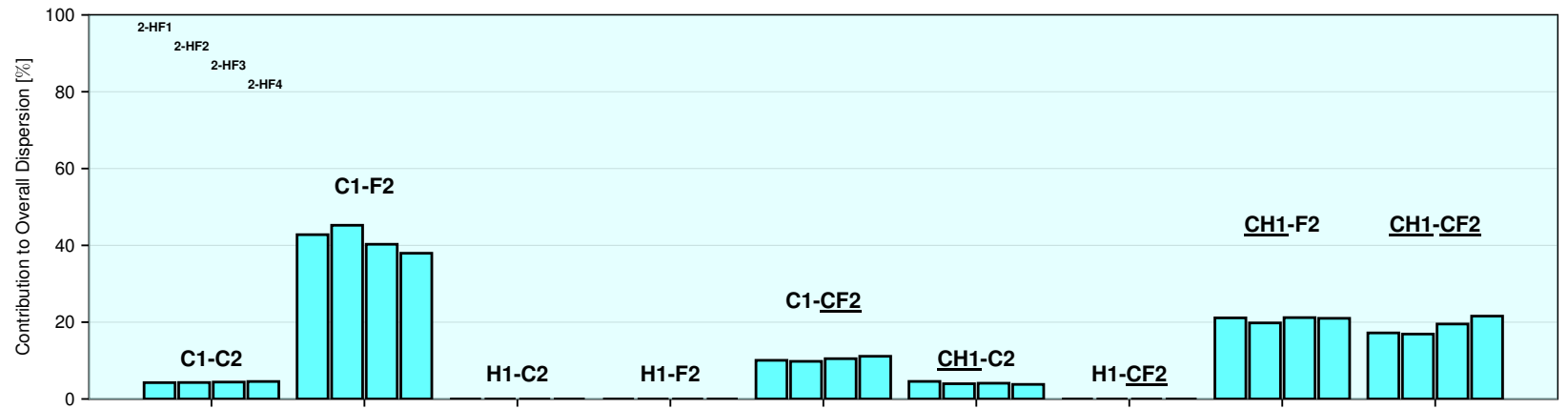

Figure S109: Decomposition of dispersion obtained from LED-TightPNO-DLPNOCCSD(T)/QZ into contributions of atoms and bonds for $\mathbf{4 - H F}$ complexes. 


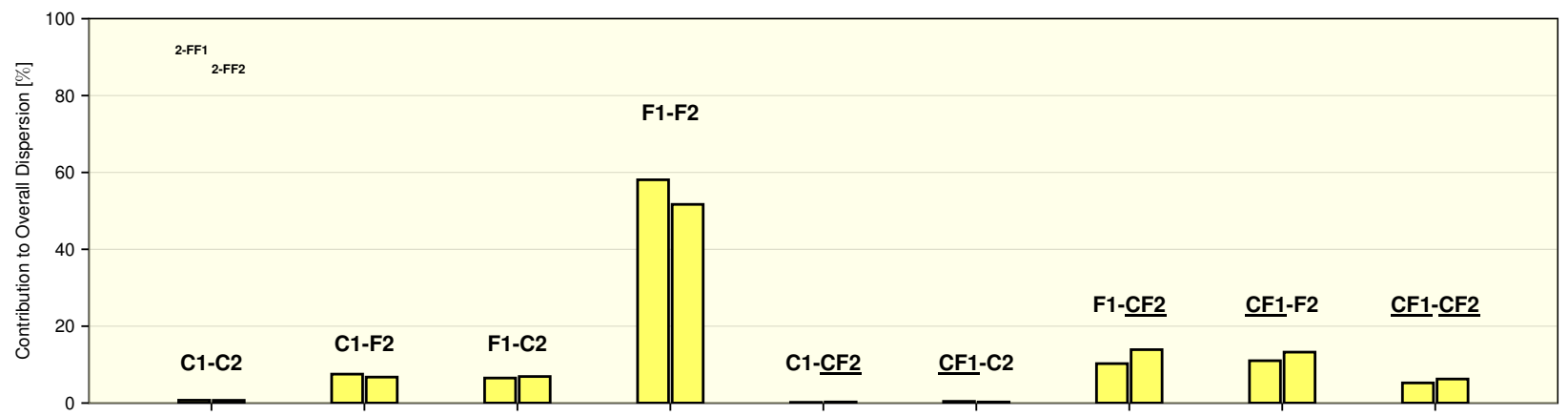

Figure S110: Decomposition of dispersion obtained from LED-TightPNO-DLPNOCCSD(T)/QZ into contributions of atoms and bonds for 4-FF complexes.

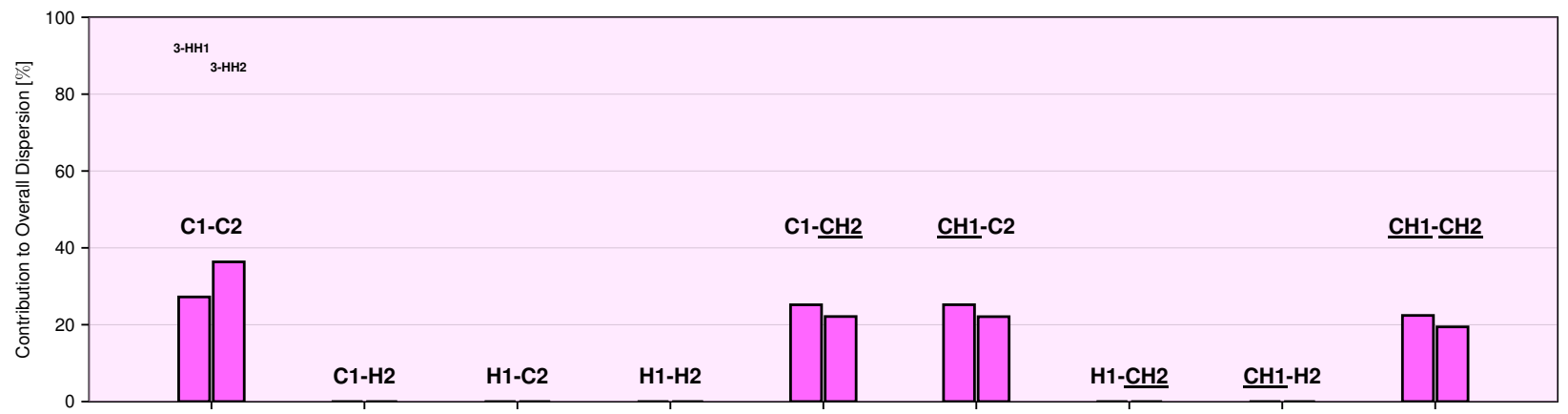

Figure S111: Decomposition of dispersion obtained from LED-TightPNO-DLPNOCCSD(T)/QZ into contributions of atoms and bonds for $\mathbf{5 - H H}$ complexes.

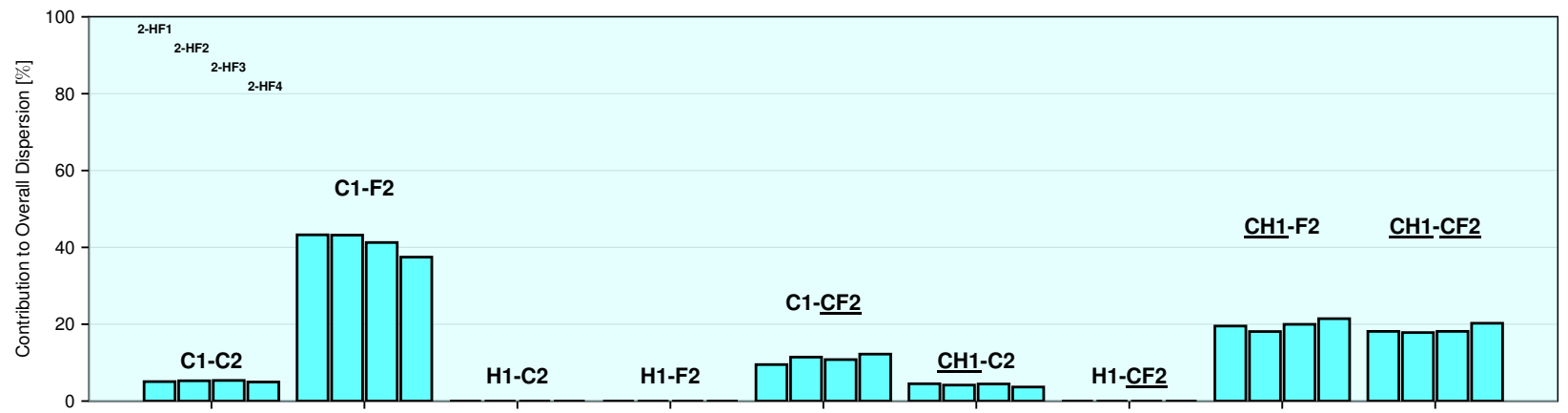

Figure S112: Decomposition of dispersion obtained from LED-TightPNO-DLPNOCCSD(T)/QZ into contributions of atoms and bonds for $\mathbf{5 - H F}$ complexes. 


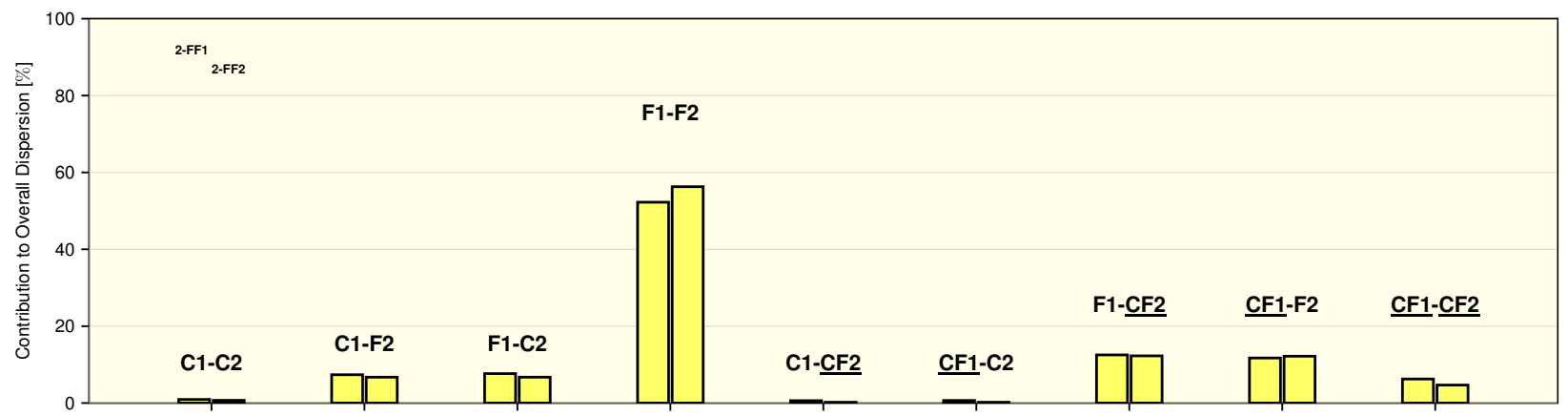

Figure S113: Decomposition of dispersion obtained from LED-TightPNO-DLPNOCCSD(T)/QZ into contributions of atoms and bonds for $\mathbf{5 - F F}$ complexes.

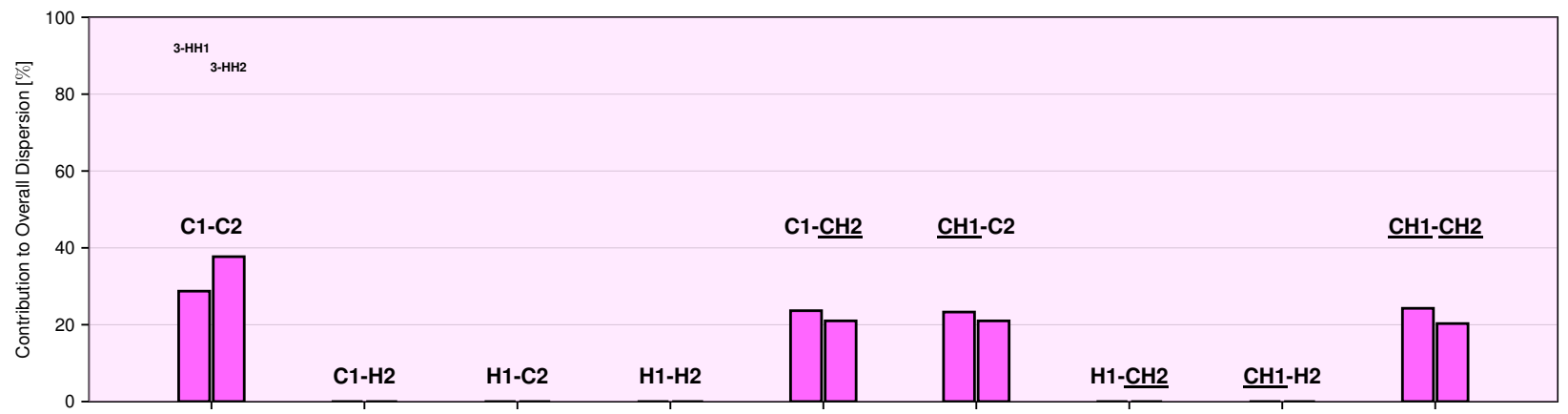

Figure S114: Decomposition of dispersion obtained from LED-TightPNO-DLPNOCCSD(T)/QZ into contributions of atoms and bonds for $\mathbf{6}-\mathbf{H H}$ complexes.

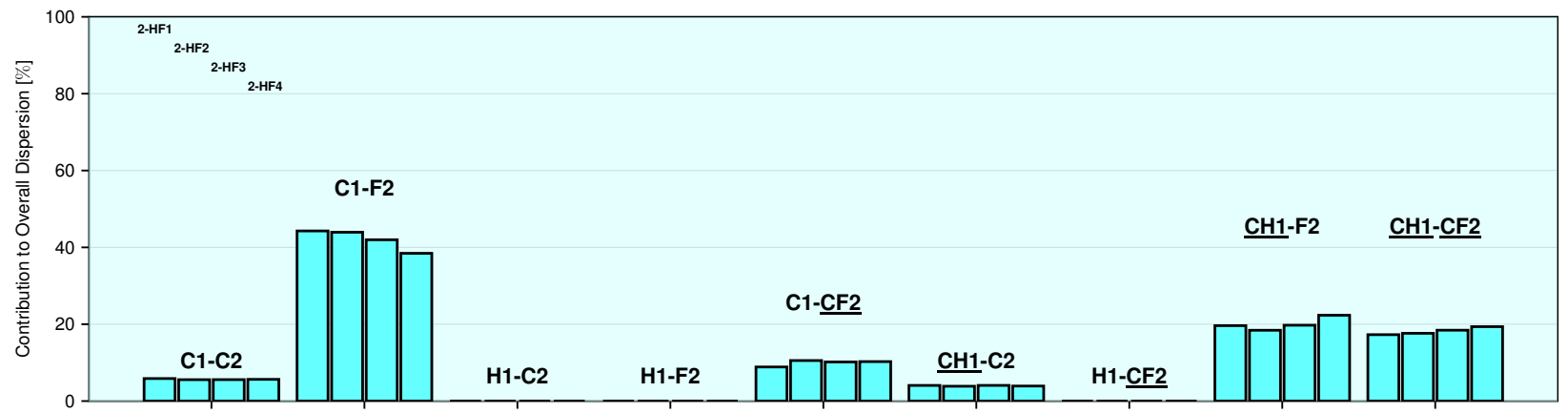

Figure S115: Decomposition of dispersion obtained from LED-TightPNO-DLPNO$\mathrm{CCSD}(\mathrm{T}) / \mathrm{QZ}$ into contributions of atoms and bonds for $\mathbf{6}-\mathbf{H F}$ complexes. 


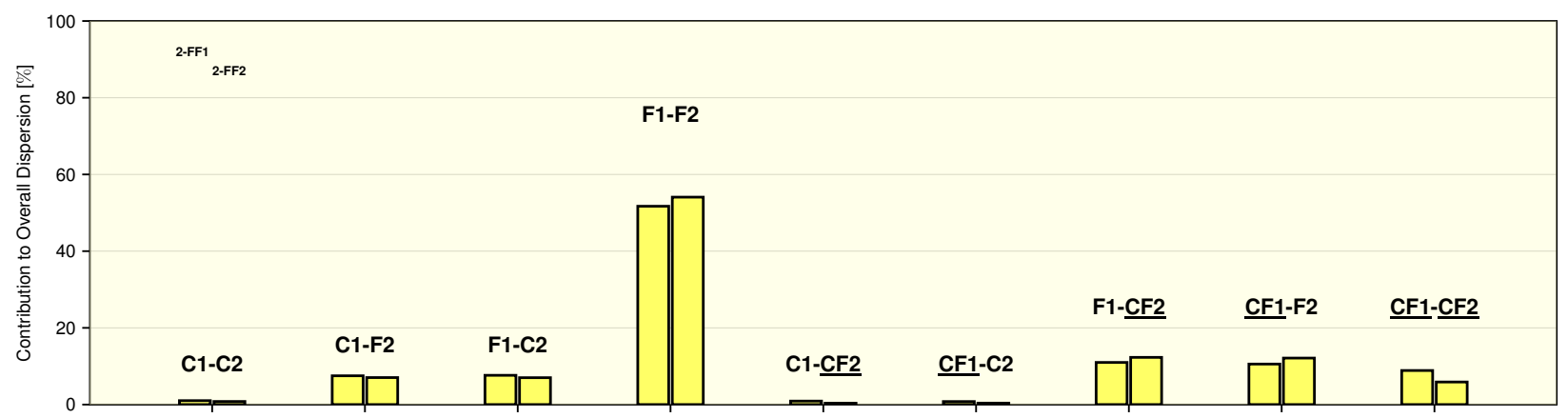

Figure S116: Decomposition of dispersion obtained from LED-TightPNO-DLPNOCCSD $(\mathrm{T}) / \mathrm{QZ}$ into contributions of atoms and bonds for $\mathbf{6 - F F}$ complexes.

\subsubsection{Additional DID Plots}

Figures S117-S131 show additional DID plots of the complexes investigated in this study. Qualitatively, they all illustrate that dispersion in $\mathbf{F}$ comes mainly from fluorine atoms and in $\mathbf{H}$ it is distributed between the carbon atoms and the carbon-hydrogen bonds. Additionally, they also show that the results are relatively stable with respect to the number of carbon atoms in the interacting complexes.

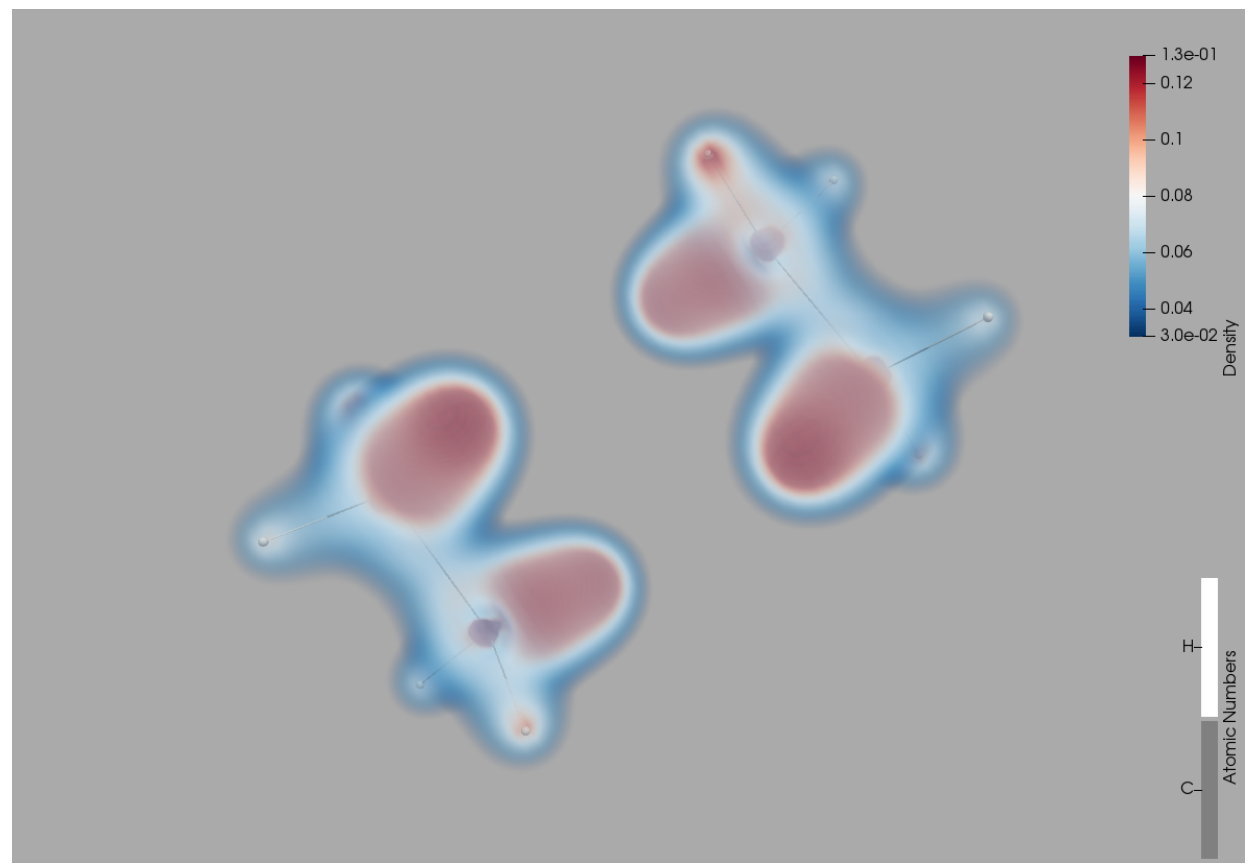

Figure S117: DID plot of 2-HH1. 


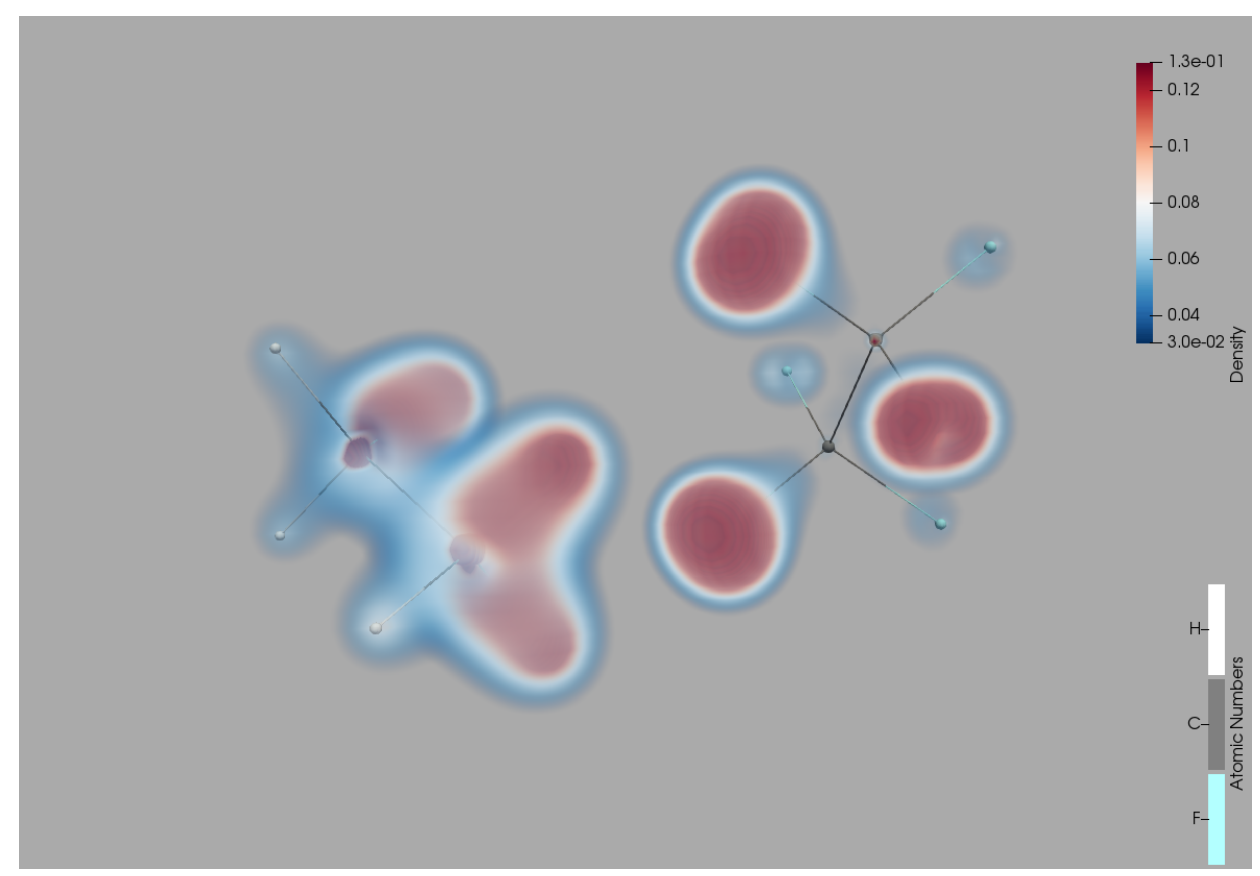

Figure S118: DID plot of $\mathbf{2 - H F 2 . ~}$

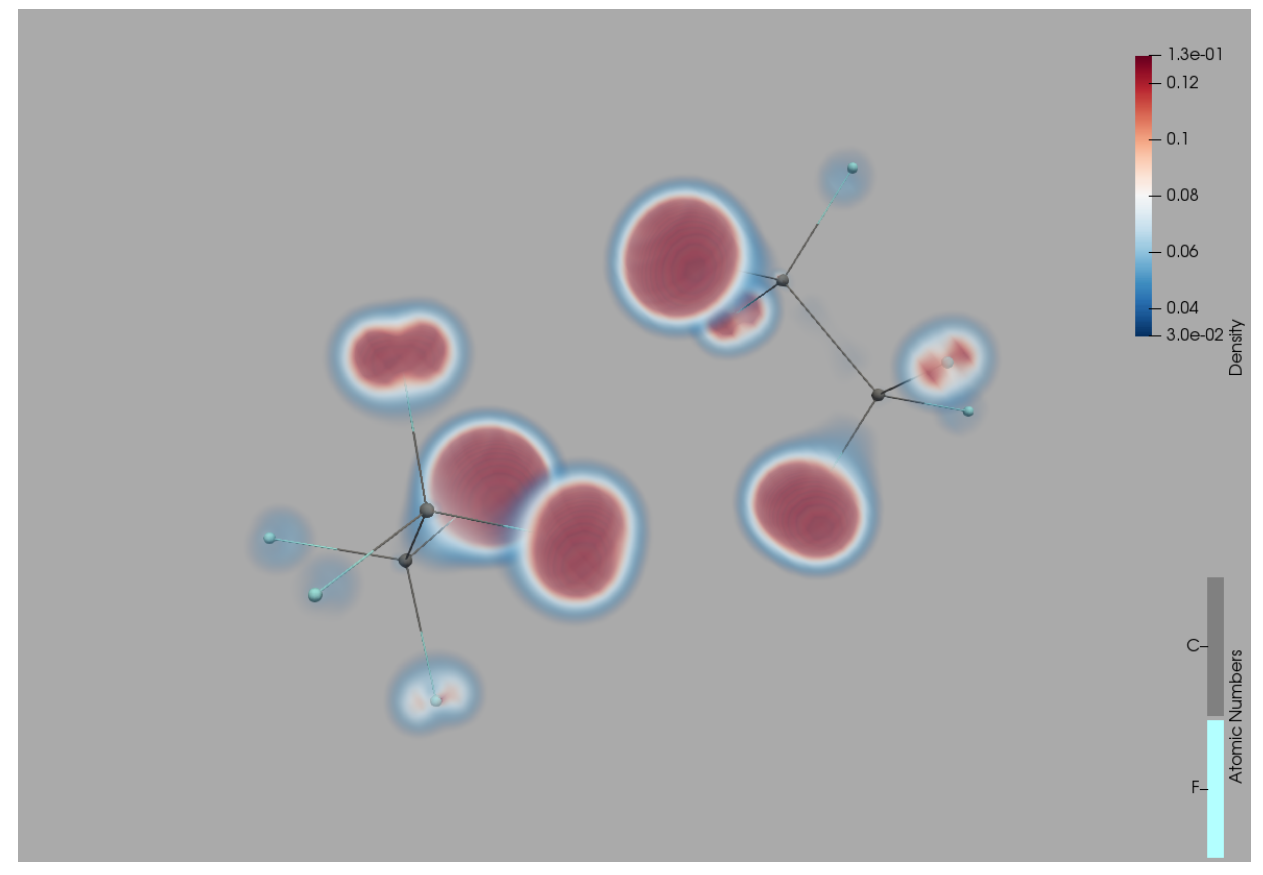

Figure S119: DID plot of 2-FF1. 


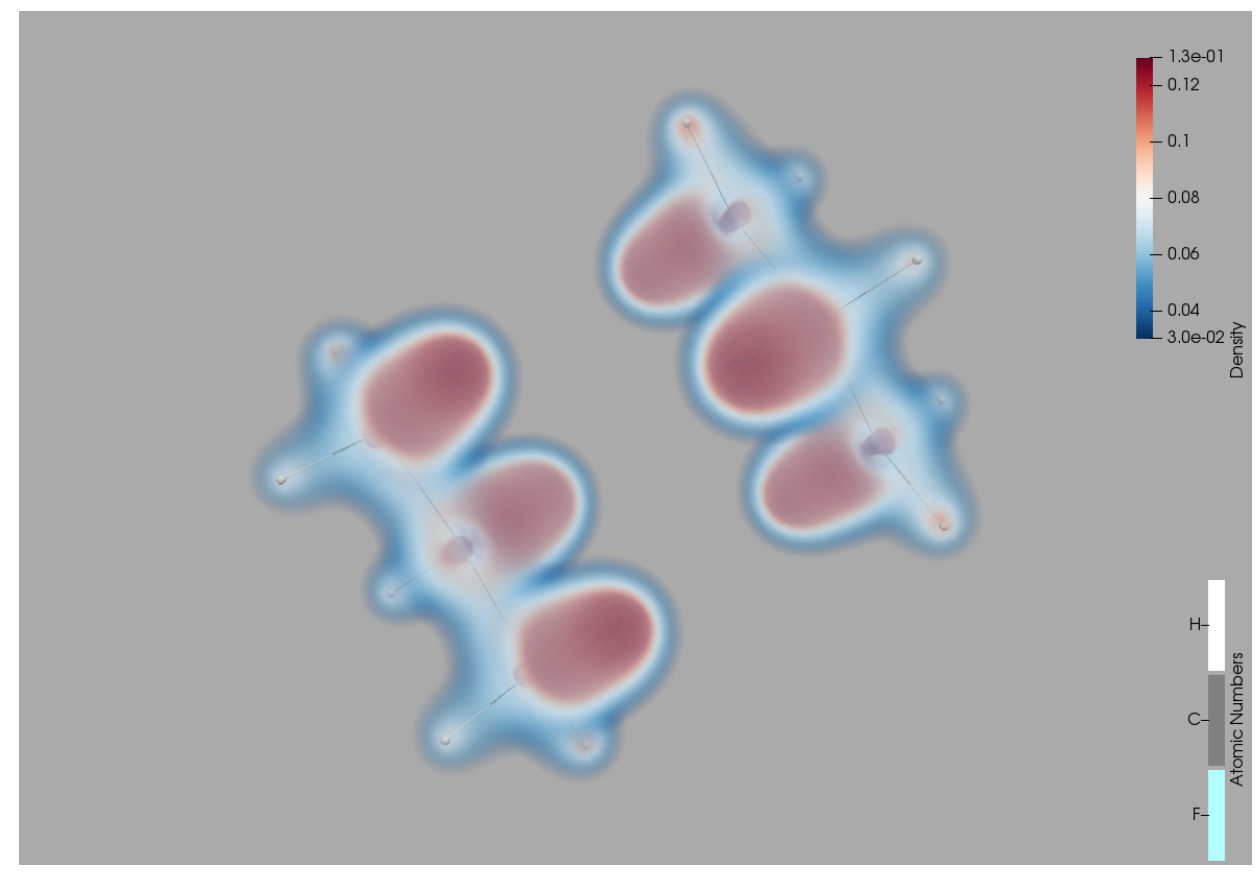

Figure S120: DID plot of $\mathbf{3 - H H 1 .}$

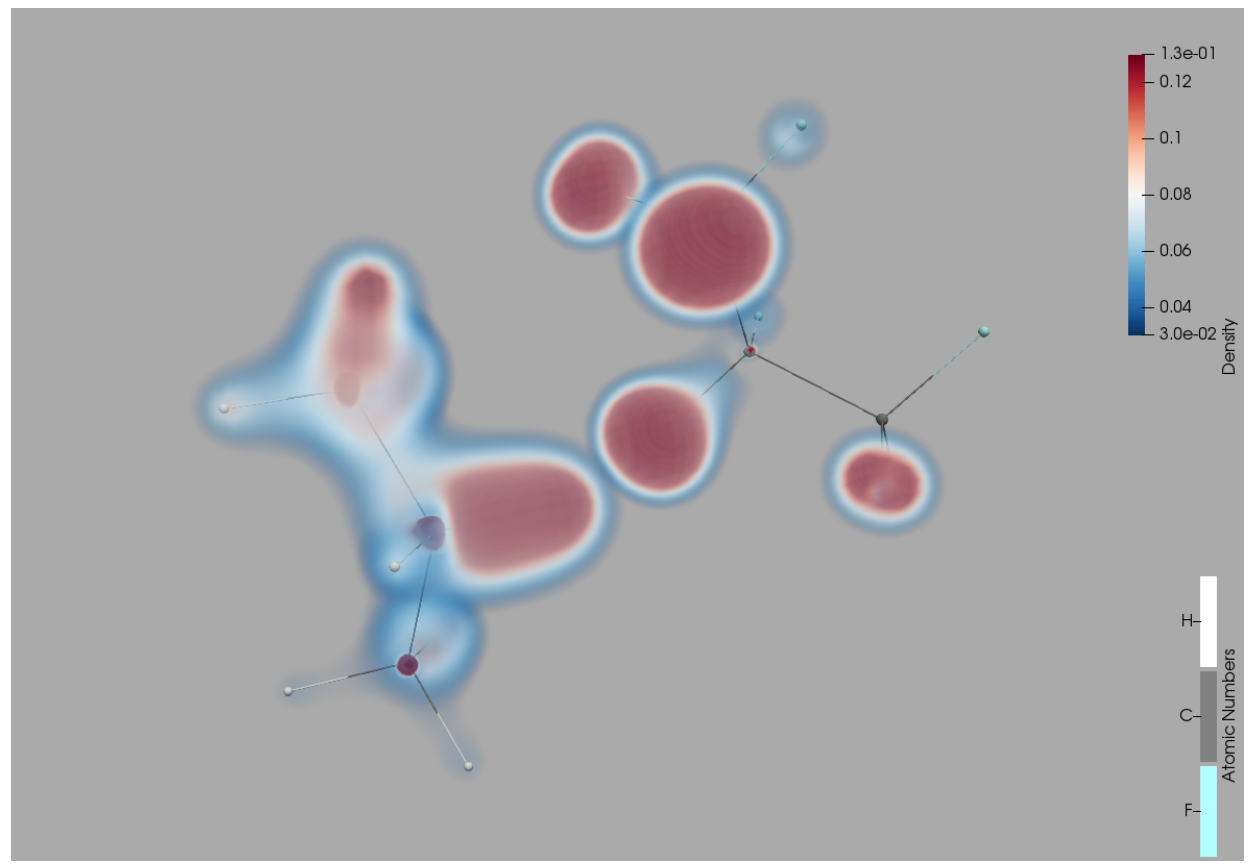

Figure S121: DID plot of $\mathbf{3}-\mathbf{H F} \mathbf{1}$. 


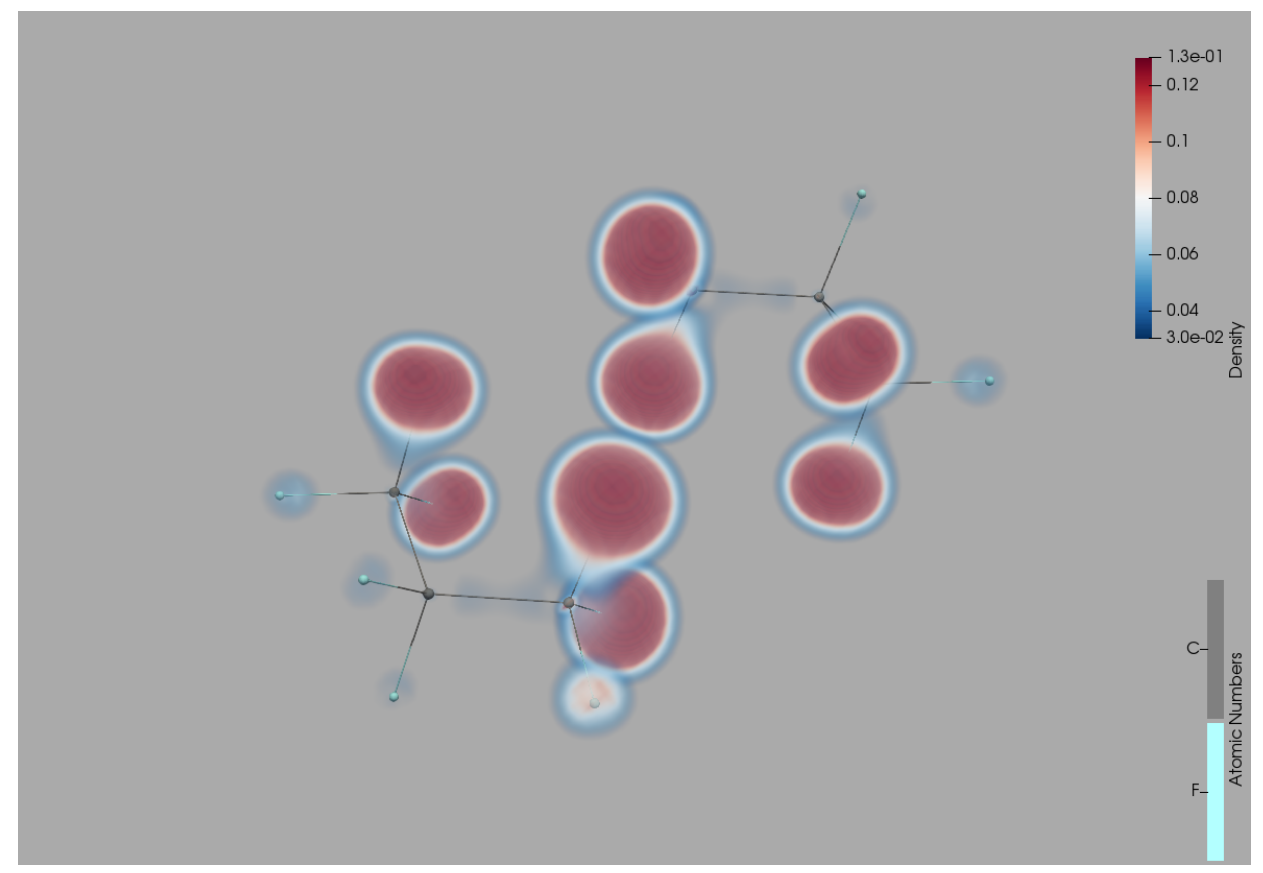

Figure S122: DID plot of 3-FF2.

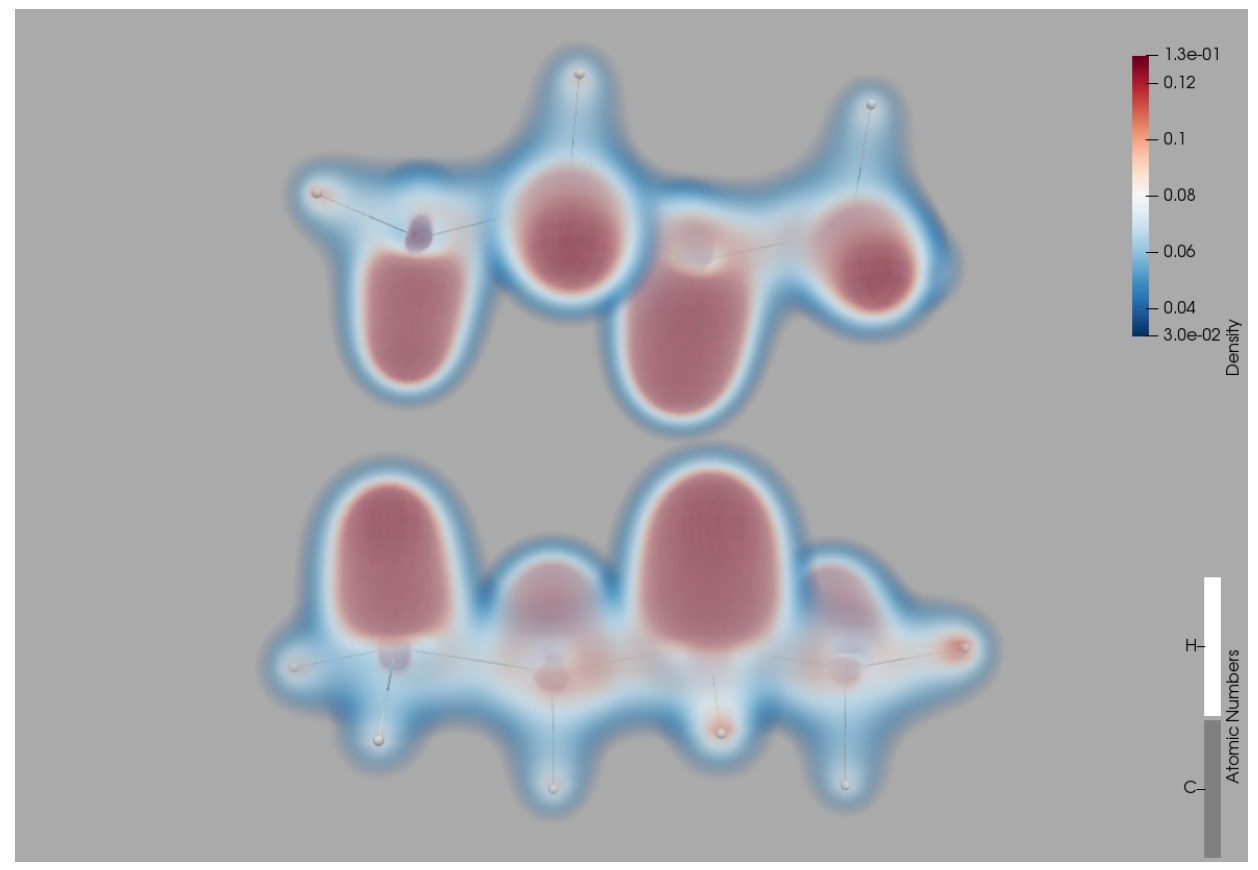

Figure S123: DID plot of $\mathbf{4}-\mathbf{H H} \mathbf{H}$. 


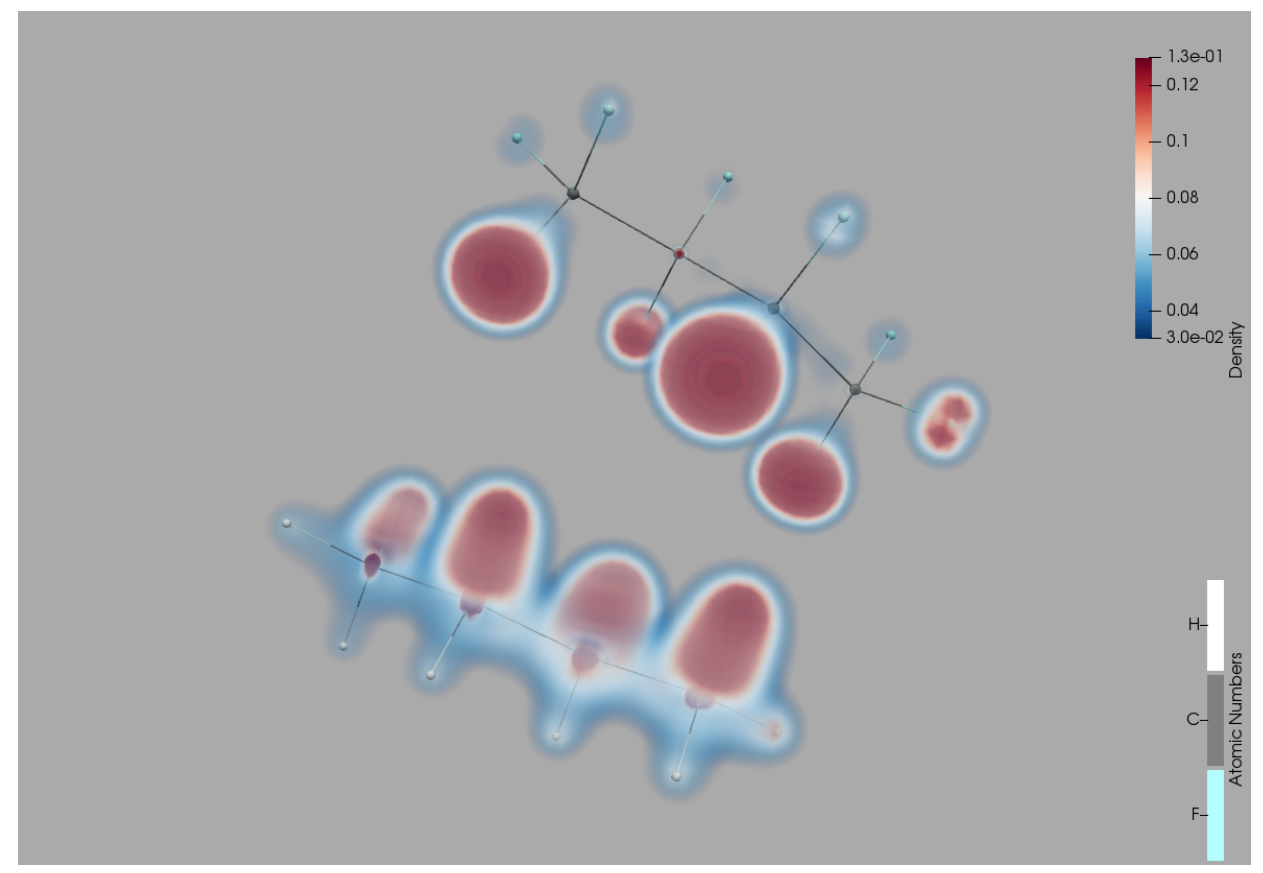

Figure S124: DID plot of $\mathbf{4 - H F 2 . ~}$

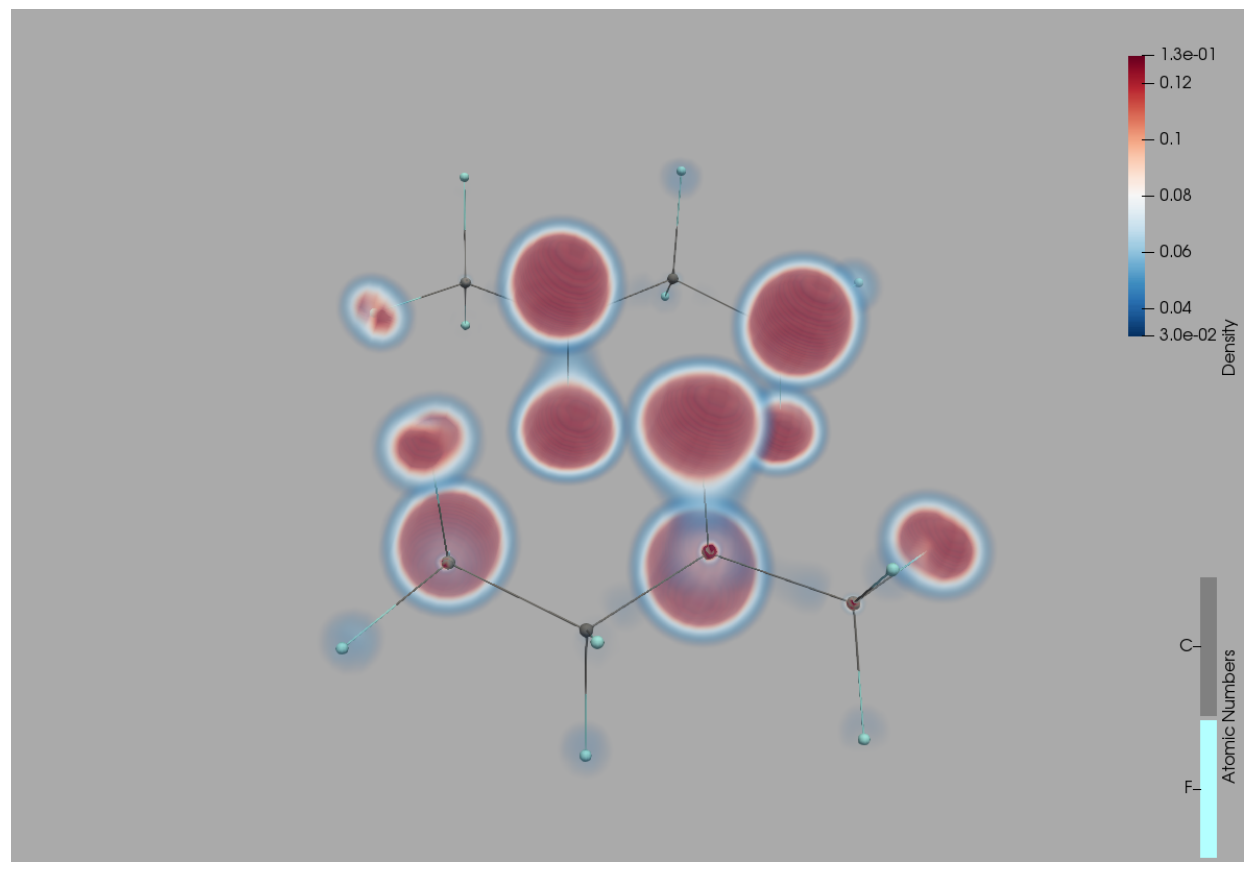

Figure S125: DID plot of 4-FF2. 


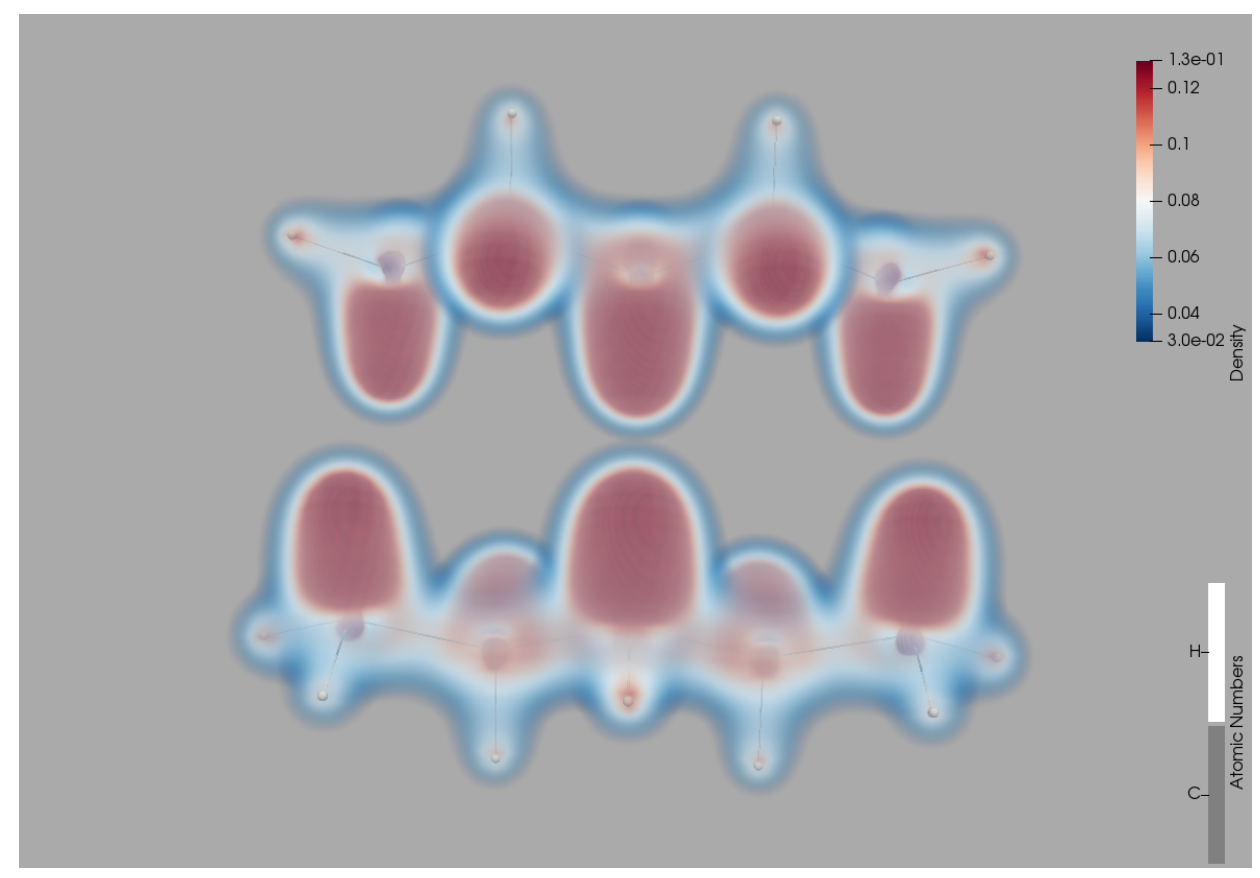

Figure S126: DID plot of $\mathbf{5}-\mathbf{H H} \mathbf{1}$

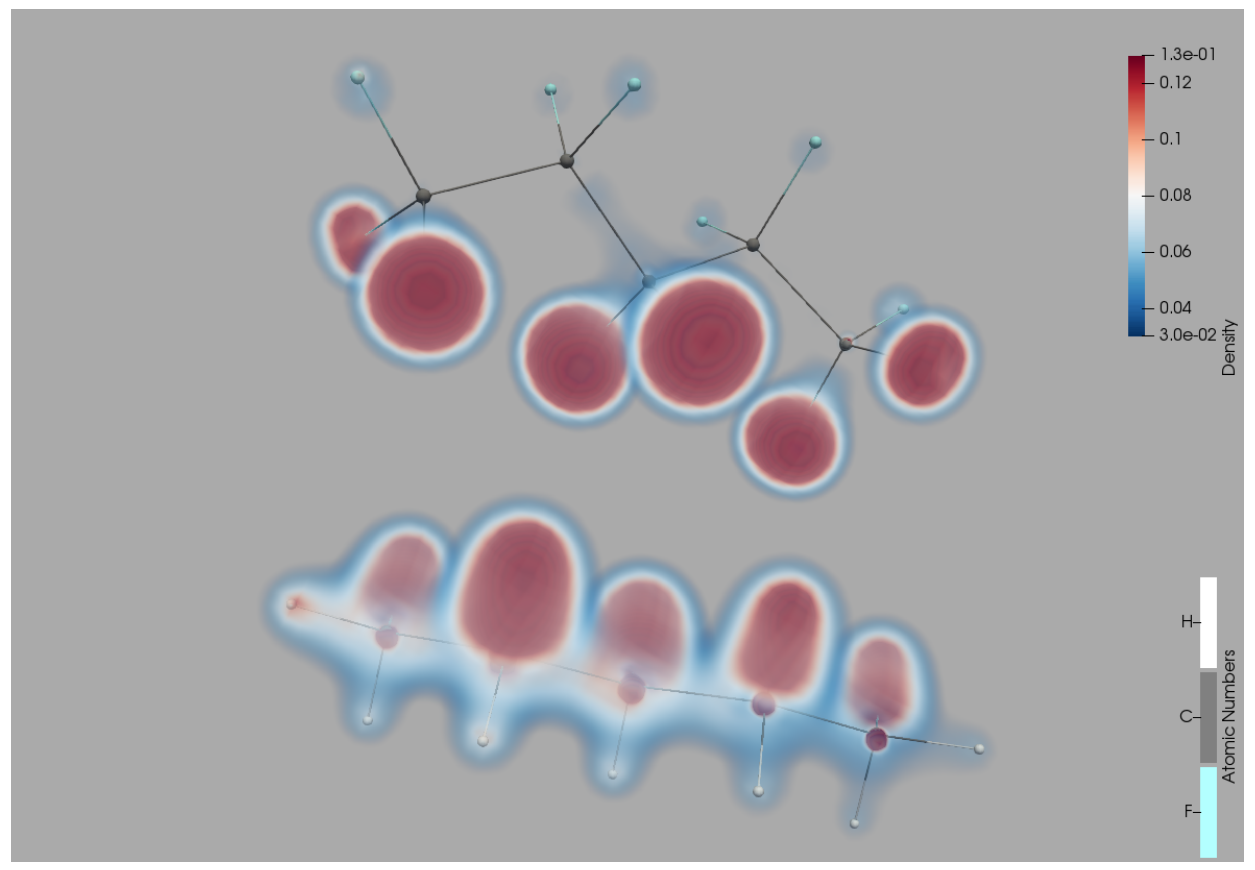

Figure S127: DID plot of 5 -HF4. 


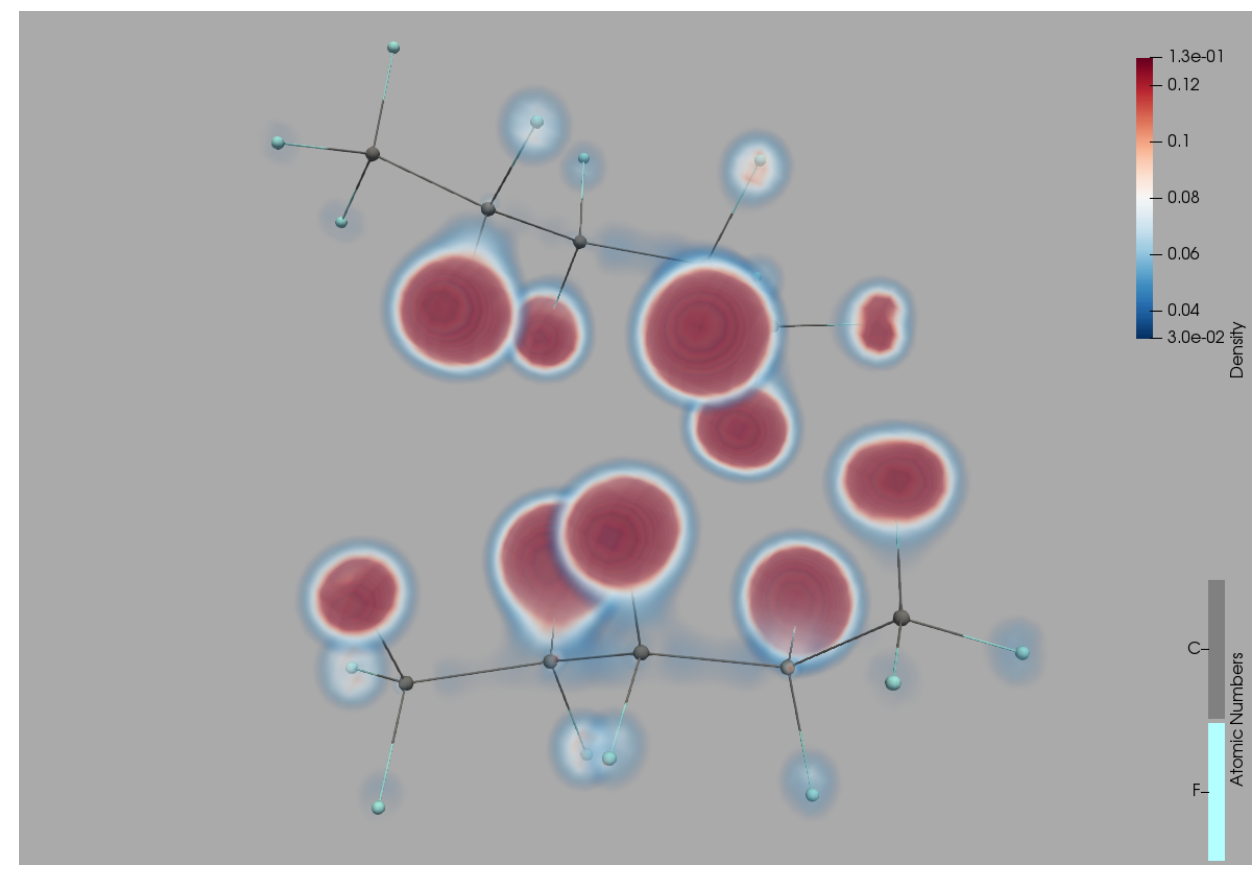

Figure S128: DID plot of 5-FF1.

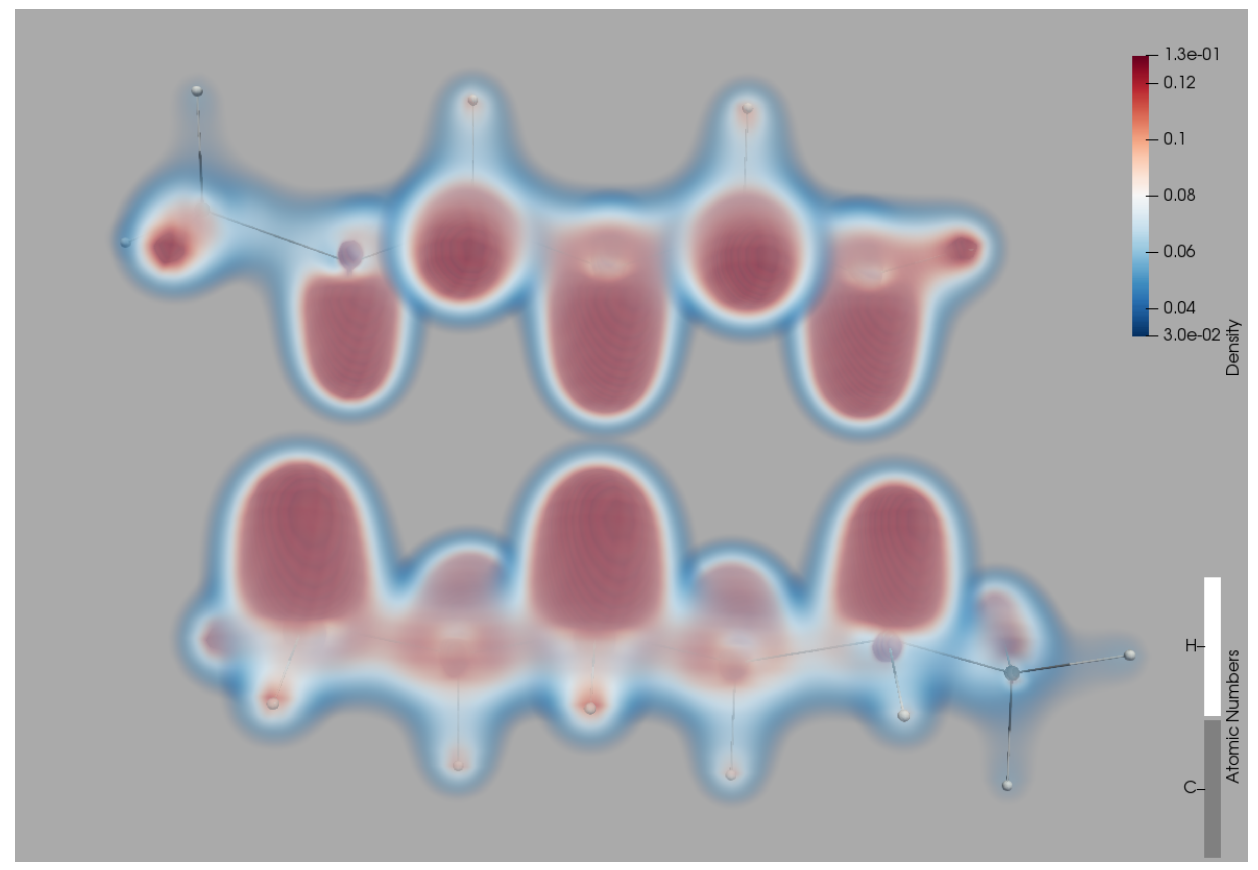

Figure S129: DID plot of $\mathbf{6 - H H 1 .}$ 


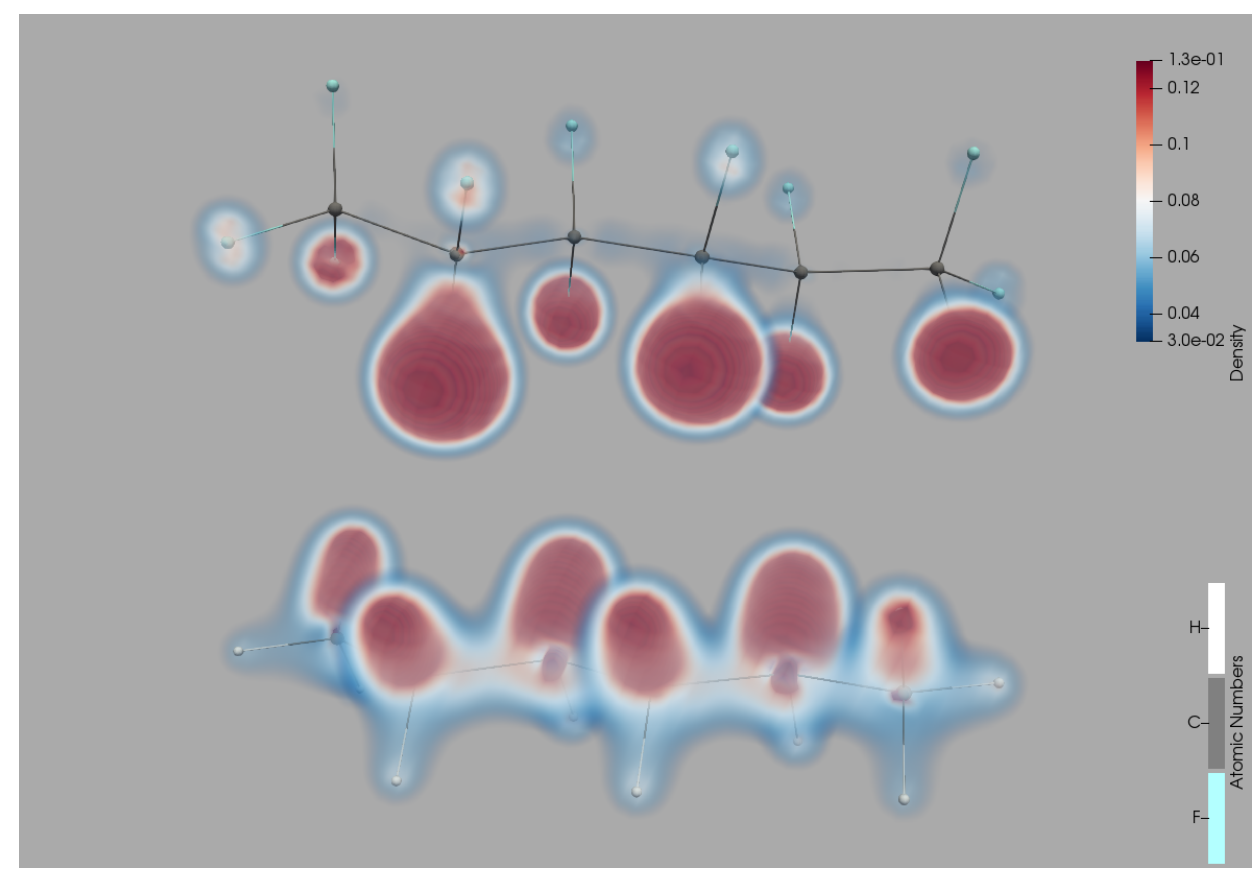

Figure S130: DID plot of 6-HF1.

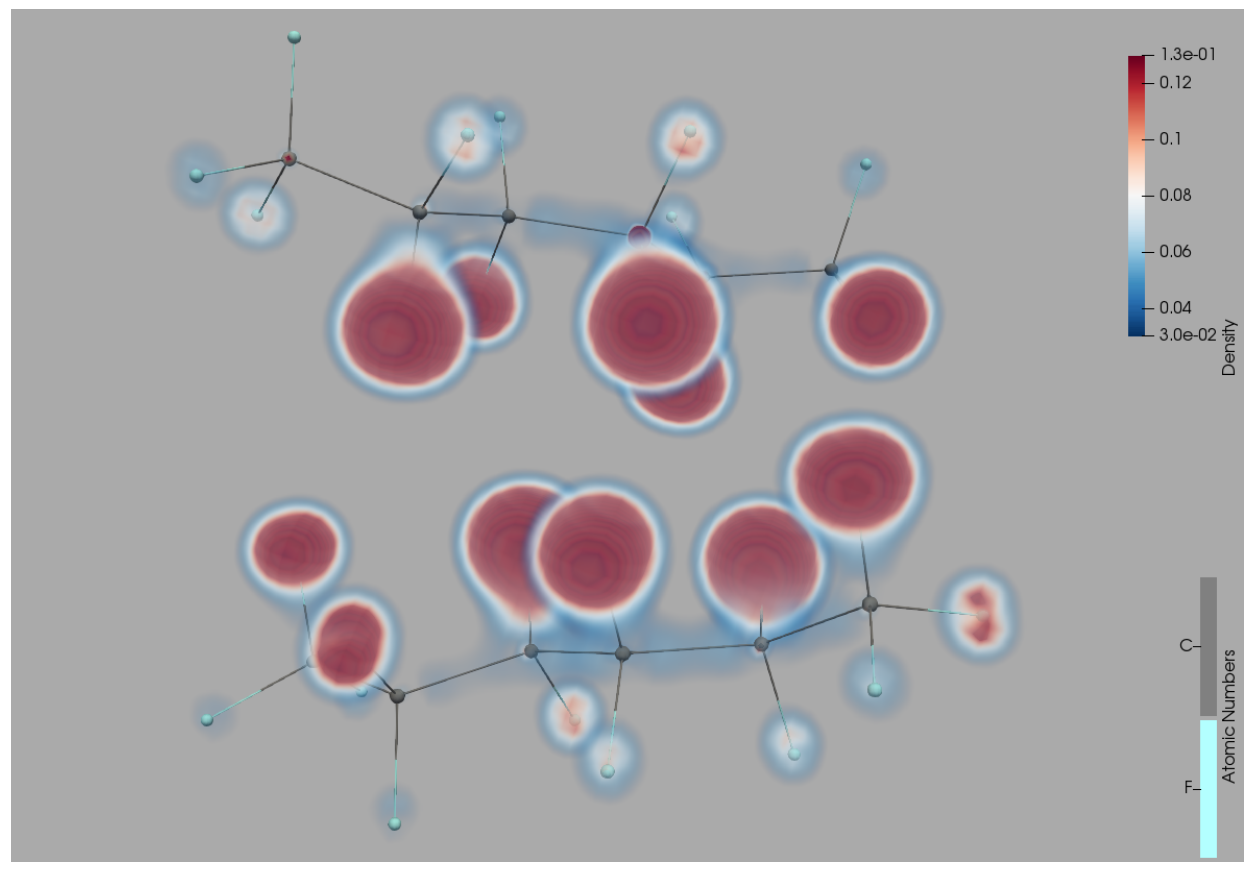

Figure S131: DID plot of $\mathbf{6 - F F 1 . ~}$ 


\subsection{Simple Interaction Models}

\subsubsection{Molecular London Dispersion Model}

For the molecular London dispersion model, ionization potentials $I_{1}$ and $I_{2}$ and polarizabilities $\alpha_{1}$ and $\alpha_{2}$ of the separate molecules with index 1 and 2 are required, as well as the intermolecular distance R. While it seems obvious to use the molecular ionization potentials and polarizabilities to describe intermolecular dispersion in this admittedly crude model, the choice of intermolecular distance to use between the molecules is not straightforward. Using MP2/CBS(34) ionization potentials and MP2/def2-TZVPD polarizabilities together with the average distance of the two carbon chains in each molecular complex resulted in the estimated London dispersion energies shown in Figure S132. It can be directly seen, when the estimated London dispersion energies are compared to $\operatorname{CCSD}(\mathrm{T})-\mathrm{F} 12$ BIEs, that $\mathbf{H H}$, HF and $\mathbf{F F}$ are not uniformly estimated and the three subclasses show three separate linear regression functions.

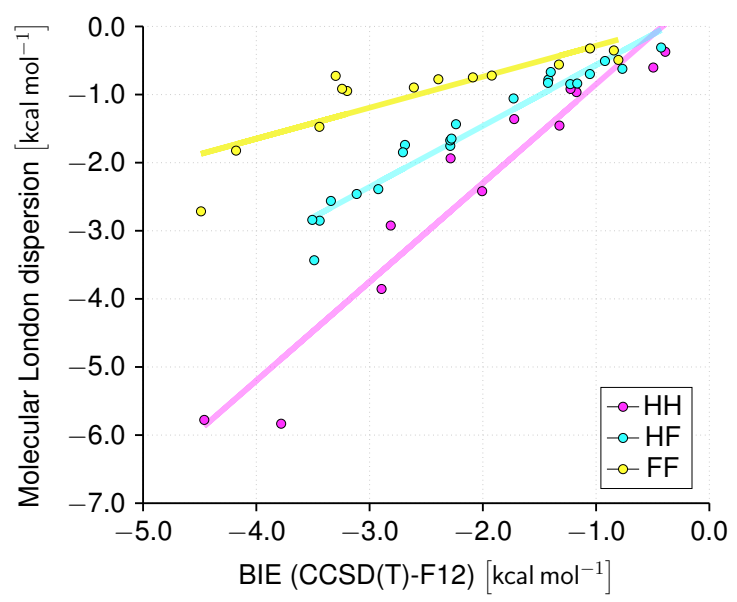

Figure S132: Intermolecular dispersion estimated with the London dispersion formula (cf. Equation 6 in the main text) using molecular ionization potentials and molecular polarizabilities compared to $\operatorname{CCSD}(\mathrm{T})-\mathrm{F} 12$ BIEs. The average distance between the carbon chains of the interacting molecules was used. 


\subsubsection{Atom-pairwise London Dispersion Models}

We used two atom-pairwise London dispersion models to fit interaction parameters of $\mathbf{H H}$, $\mathbf{H F}$ and $\mathbf{F F}$ and to estimate the corresponding BIEs. The idea was to find the best set of atom-type dependent parameters for the atomic ionization potentials to best reproduce the benchmark interaction energies (CCSD(T)-F12 level of theory). We performed this optimization using two different approaches. For the first, we used the London dispersion formula, as is, (cf. Equation 6 in the main text) and optimized atomic ionization potentials. For the second we used an approximation of the London dispersion formula explained in the main text simplifying the ionization potential pre-factor (which we term $\mathrm{A}_{12}$ ) as product of atom-type dependent pre-factors $\mathrm{A}_{1}$ and $\mathrm{A}_{2}$ (cf. right-hand side of Equation 8 in the main text) and therefore we optimized the atomic pre-factors A. The most basic assumption of all our simple interaction models is that the overall BIE of our model complexes can be approximated by accounting only for dispersion. This assumption is plausible on the basis of the results of the energy decomposition analyses employed. For the first model, we used the London dispersion formula, as is, ${ }^{106,107}$ and computed the overall BIE as sum of atom-pairwise terms:

$$
\mathrm{BIE} \approx \sum_{i} \sum_{j}-\frac{3}{2} \frac{I_{i} I_{j}}{I_{i}+I_{j}} \frac{\alpha_{i} \alpha_{j}}{R_{i j}^{6}}
$$

We used the optimized geometries at the DSD-PBEP86/def2-TZVP(spd) level of theory to determine the atom-pairwise interaction distances $R_{i j}$ and we determined the atomic polarizabilities $\alpha_{i}$ using PolaBer on the basis of MP2/def2-TZVPD densities. We assumed that the ionization potentials are atom-type dependent parameters and, hence, the model had three adjustable parameters (i.e. $I_{H}, I_{C}$ and $I_{F}$ ). These parameters were determined by minimizing the MAD between the estimated BIEs and the benchmark BIEs at the CCSD(T)F12/VDZ level of theory. After finding the best set of parameters, we compared the estimated BIEs to the benchmark BIEs. The corresponding results are illustrated in Figure S133. 
The MADrel of the fitted values is only $(5 \pm 4) \%$ and is therefore comparable to the best benchmarked DFT and WFT methods (cf. main text). It is also slightly better than the second atom-pairwise London dispersion model (vide infra).
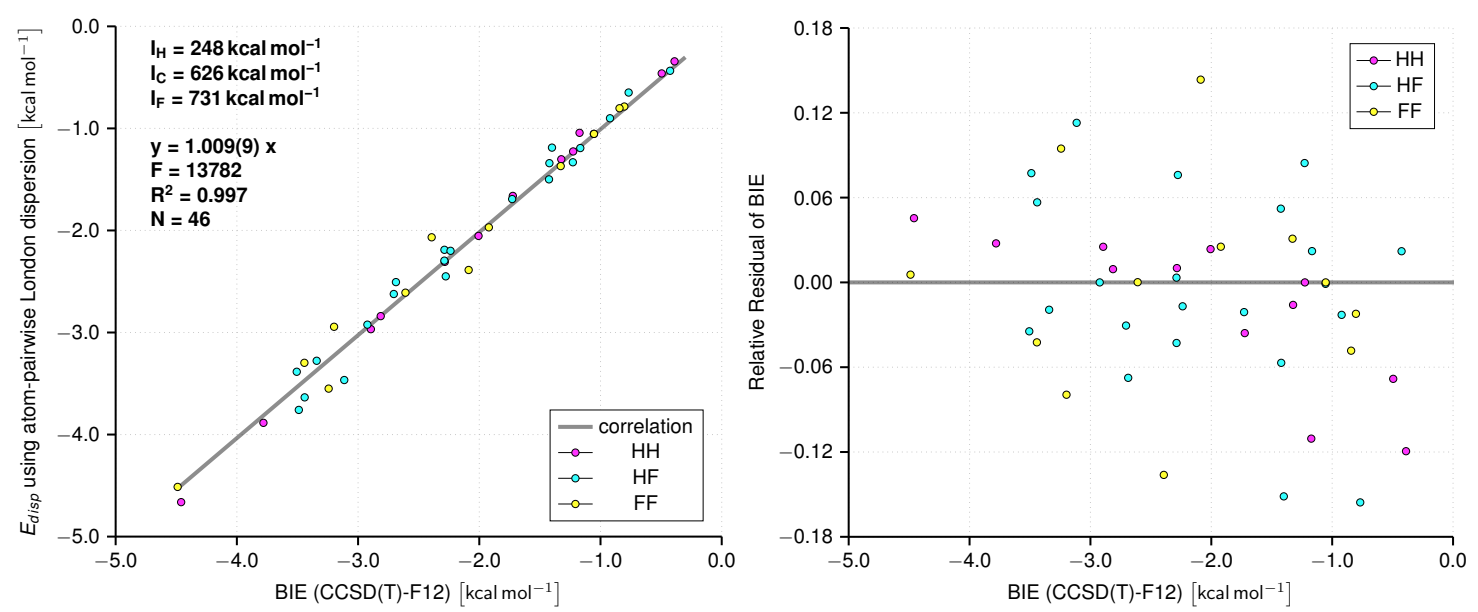

Figure S133: Intermolecular dispersion estimated using atom-pairwise London dispersion by optimizing atom-type dependent ionization potentials. Left: Results compared to reference data with the corresponding correlation. Right: Residual plot.

For the second model, we used an approximation of the London dispersion formula to describe the overall BIE of the complexes using instead of ionization potentials atom-type dependent pre-factors A:

$$
\mathrm{BIE} \approx \sum_{i} \sum_{j}-\frac{3}{2} \frac{A_{i} \alpha_{i} A_{j} \alpha_{j}}{R_{i j}^{6}}
$$

These atom-type dependent pre-factors formally have the dimension of the square-root of energy. The advantage of these pre-factors is that the dispersion can be formally decomposed into a product of two values which is only dependent on one of the interacting molecules each. The motivation for pursuing this approach is explained in detail in the main text. We used again optimized geometries at the DSD-PBEP86/def2-TZVP(spd) level of theory to determine the atom-pairwise interaction distances $R_{i j}$ and we also used the atomic polarizabilities $\alpha_{i}$ obtained from PolaBer on the basis of MP2/def2-TZVPD densities. In this model we optimized the atom-type dependent pre-factors as three adjustable parameters 
(i.e. $A_{H}, A_{C}$ and $A_{F}$ ). These parameters were again determined by minimizing the MAD between the estimated BIEs and the benchmark BIEs at the CCSD(T)-F12/VDZ level of theory. After finding the best set of parameters, we compared the estimated BIEs to the benchmark BIEs. The corresponding results are illustrated in Figure S134.
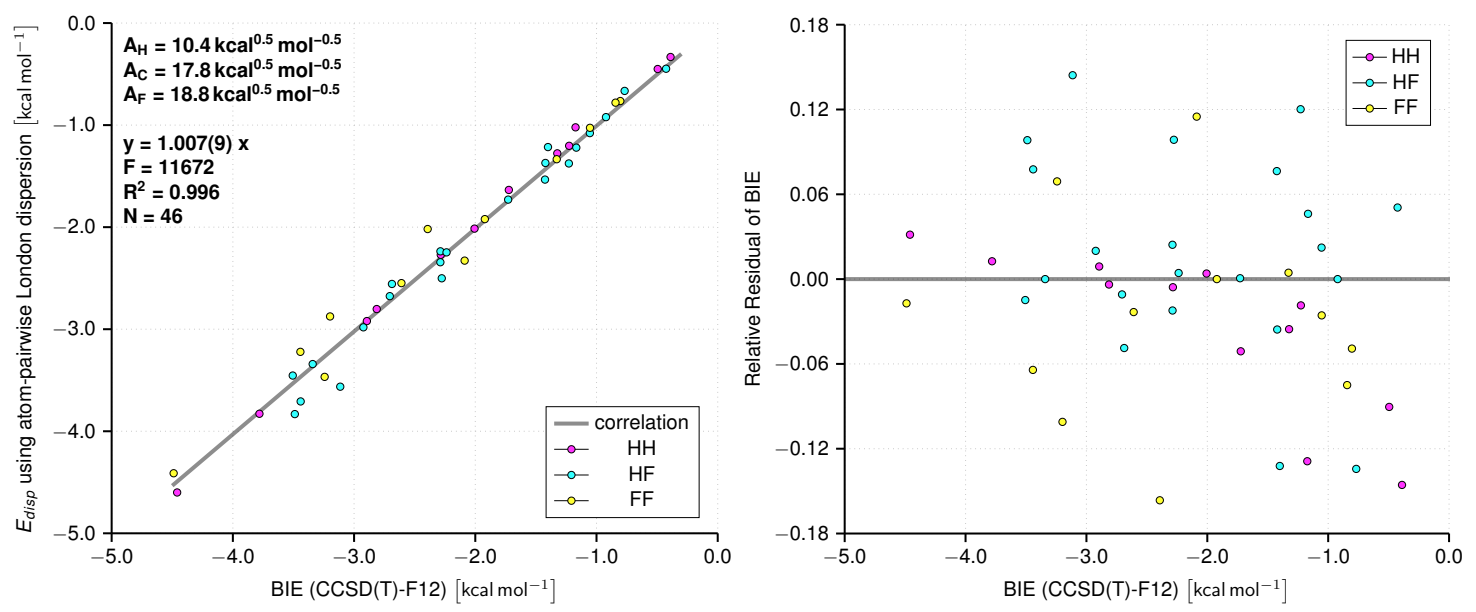

Figure S134: Intermolecular dispersion estimated using atom-pairwise London dispersion by optimizing atom-type dependent pre-factors A. Left: Results compared to reference data with the corresponding correlation. Right: Residual plot.

\subsubsection{Atom-type Energy Decomposition}

We also used the atom-pairwise London dispersion model to perform a decomposition of the overall dispersion into contributions of interactions between specific types of atoms. In that regard, it is similar to the decomposition into contributions of atoms and bonds we carried out using LED (vide supra). Hence, we obtain the following six energetic contributions:

$$
\begin{array}{lll}
D_{t o t} & \ldots & \text { Total intermolecular dispersion } \\
D(C-C) & \ldots & \text { Dispersion between two carbon atoms } \\
D(C-H) & \ldots & \text { Dispersion between a carbon atom and a hydrogen atom } \\
D(H-H) & \ldots & \text { Dispersion between two hydrogen atoms } \\
D(C-F) & \ldots & \text { Dispersion between one carbon and one fluorine atom } \\
D(H-F) & \ldots & \text { Dispersion between one hydrogen and one fluorine atom } \\
D(F-F) & \ldots & \text { Dispersion between two fluorine atoms }
\end{array}
$$

The corresponding results for all the molecular complexes studied are illustrated in Fig- 
ures S135-S152. The main difference between the LED decomposition into atoms and bonds and the decomposition performed using the atom-pairwise London dispersion model is that in the former the dispersion is also decomposed into contributions of bonds. In the atompairwise model the contributions of the bonds are distributed between the respective atomic contributions. This can be gleaned from the results of the complexes of $\mathbf{1 - H}$ and $\mathbf{1 - F}$. In 1-HH the contributions of the $\mathrm{C}-\mathrm{H}$ bonds are distributed among the $\mathrm{C}-\mathrm{H}$ and the $\mathrm{H}-\mathrm{H}$ contributions. In 1-HF the contributions of the $\mathrm{C}-\mathrm{H}$ and $\mathrm{C}-\mathrm{F}$ bonds are mainly distributed between the $\mathrm{C}-\mathrm{F}$ and $\mathrm{H}-\mathrm{F}$ contributions. In 1-FF the contributions of the $\mathrm{C}-\mathrm{F}$ bonds are mainly distributed between the C-F and F-F contributions.

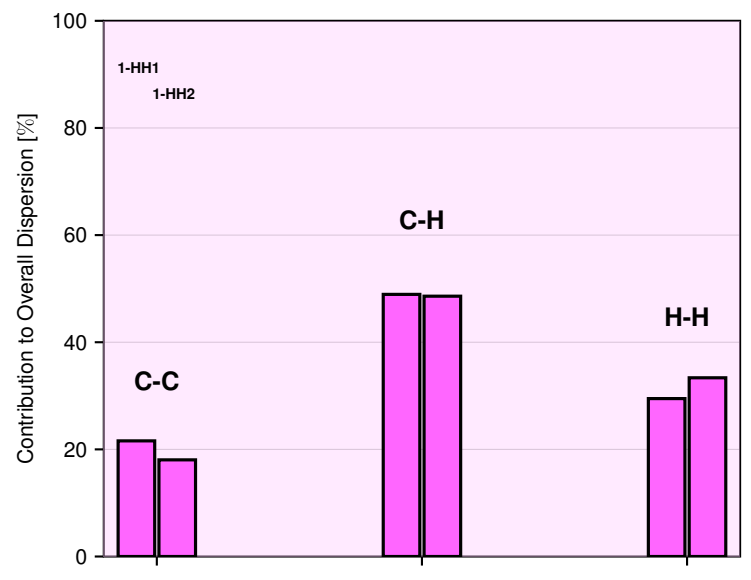

Figure S135: Decomposition of dispersion obtained from the atom-pairwise London dispersion model into contributions of interactions between types of atoms for $\mathbf{1 - H H}$ complexes.

Additionally, we investigated the relative contribution of F-F in FF as a function of the relative molar fluorine content, as well as, the relative contribution of $\mathrm{C}-\mathrm{C}$ in $\mathbf{H H}$ as a function of the relative molar carbon content (cf. Figure S153). In both cases we obtain reasonable linear correlations. These results indicate the direct impact of the relative molar carbon content on the dispersion interaction pattern. They also support the findings from LED. 


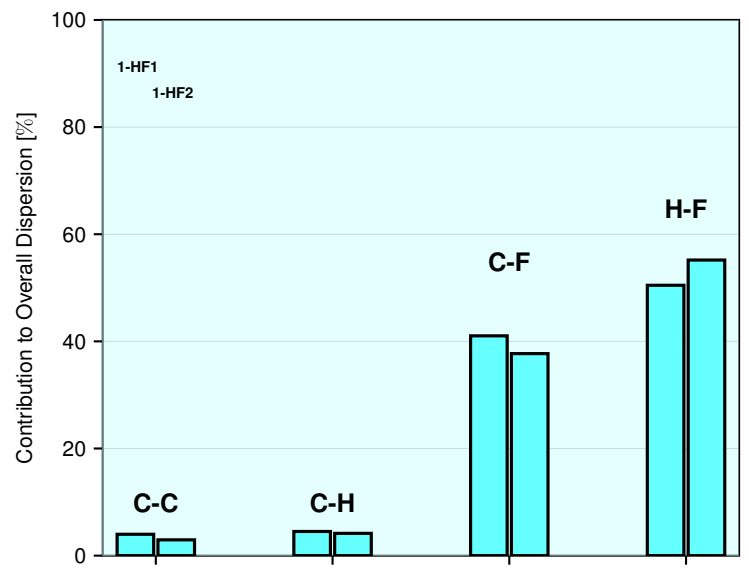

Figure S136: Decomposition of dispersion obtained from the atom-pairwise London dispersion model into contributions of interactions between types of atoms for $\mathbf{1 - H F}$ complexes.

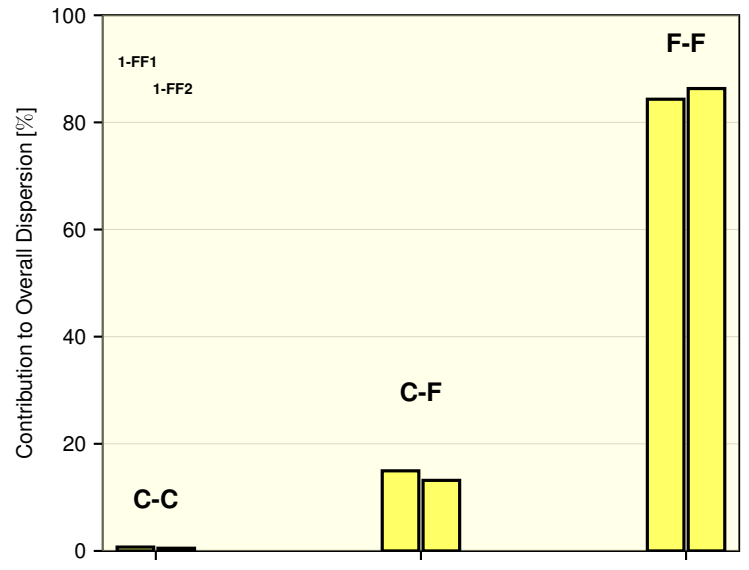

Figure S137: Decomposition of dispersion obtained from the atom-pairwise London dispersion model into contributions of interactions between types of atoms for $\mathbf{1 - F F}$ complexes. 


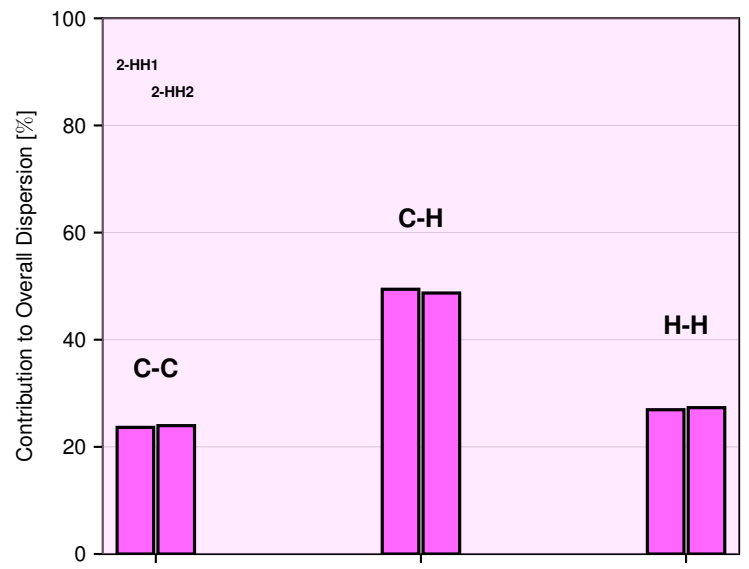

Figure S138: Decomposition of dispersion obtained from the atom-pairwise London dispersion model into contributions of interactions between types of atoms for $\mathbf{2}-\mathbf{H H}$ complexes.

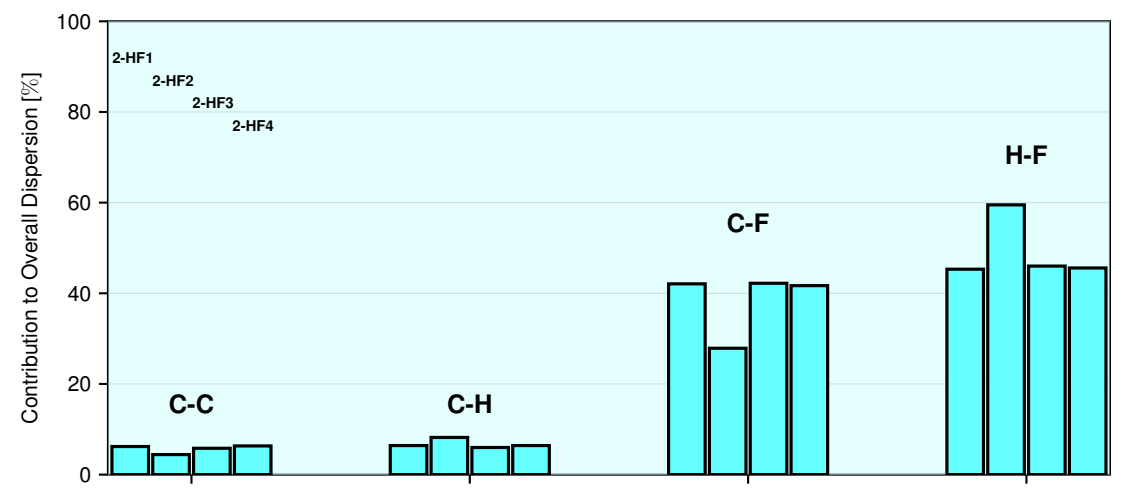

Figure S139: Decomposition of dispersion obtained from the atom-pairwise London dispersion model into contributions of interactions between types of atoms for $\mathbf{2}-\mathbf{H F}$ complexes.

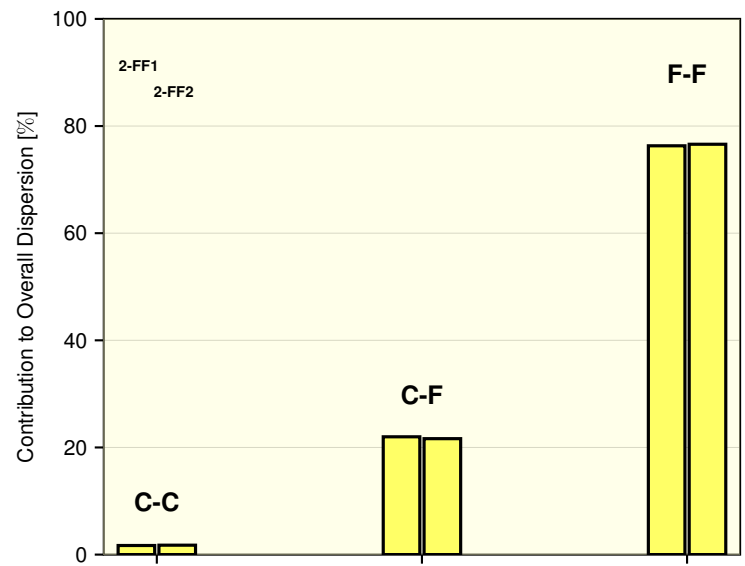

Figure S140: Decomposition of dispersion obtained from the atom-pairwise London dispersion model into contributions of interactions between types of atoms for $\mathbf{2}-\mathbf{F F}$ complexes. 


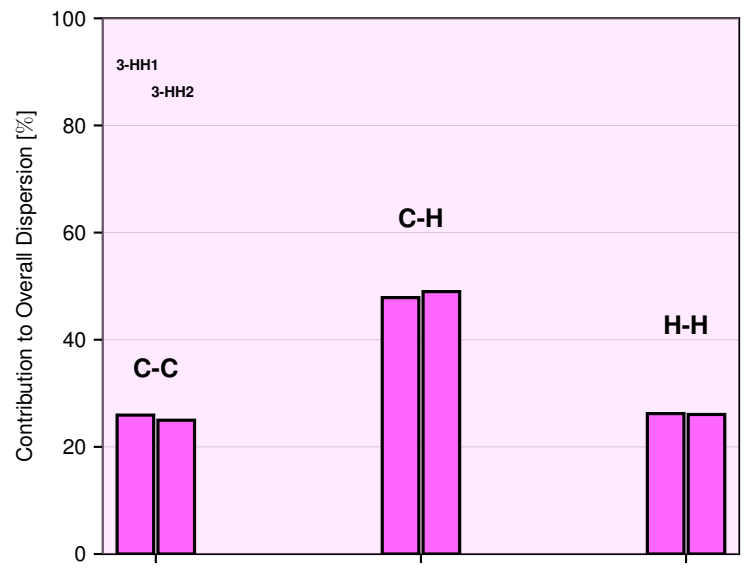

Figure S141: Decomposition of dispersion obtained from the atom-pairwise London dispersion model into contributions of interactions between types of atoms for $\mathbf{3}-\mathbf{H H}$ complexes.

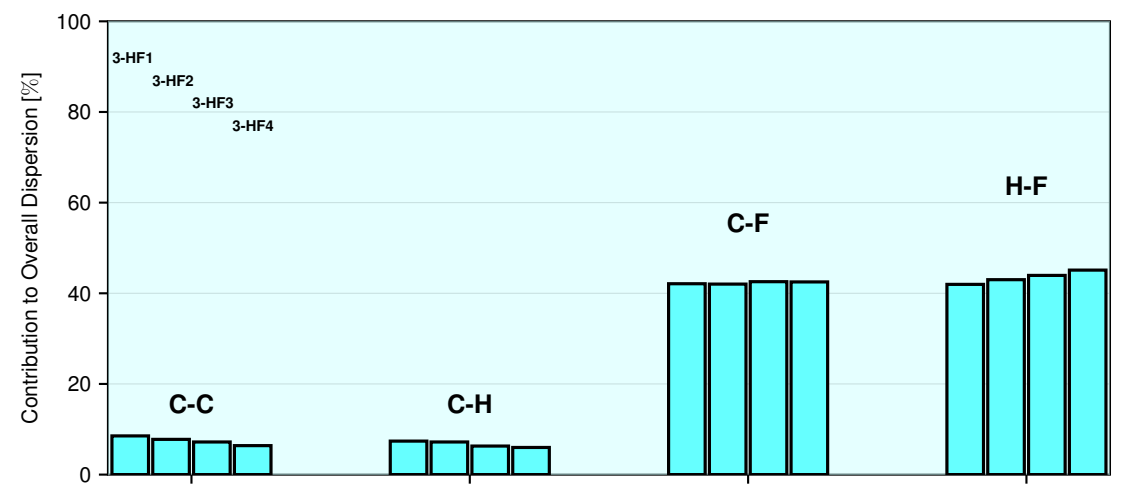

Figure S142: Decomposition of dispersion obtained from the atom-pairwise London dispersion model into contributions of interactions between types of atoms for $\mathbf{3}-\mathbf{H F}$ complexes.

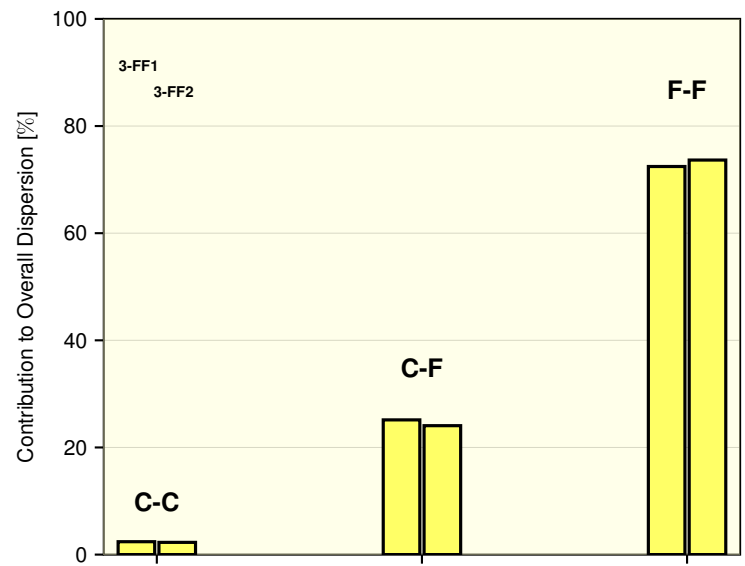

Figure S143: Decomposition of dispersion obtained from the atom-pairwise London dispersion model into contributions of interactions between types of atoms for $\mathbf{3}-\mathbf{F F}$ complexes. 


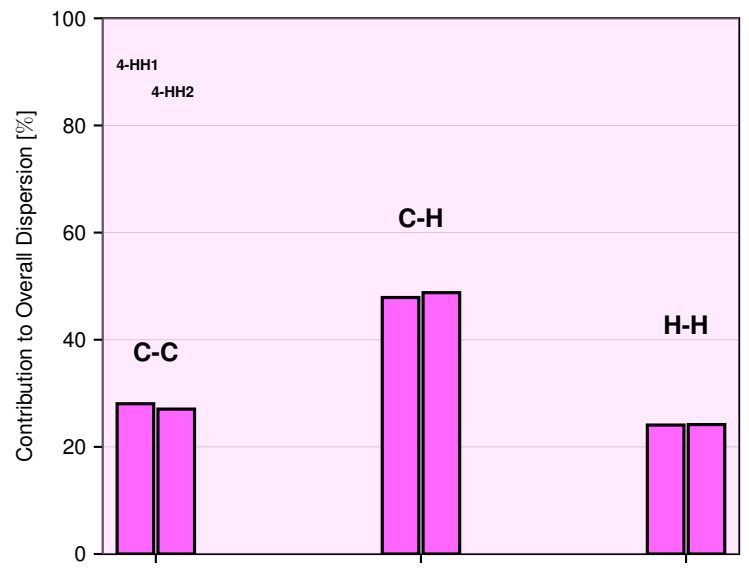

Figure S144: Decomposition of dispersion obtained from the atom-pairwise London dispersion model into contributions of interactions between types of atoms for $\mathbf{4} \mathbf{- H} \mathbf{H}$ complexes.

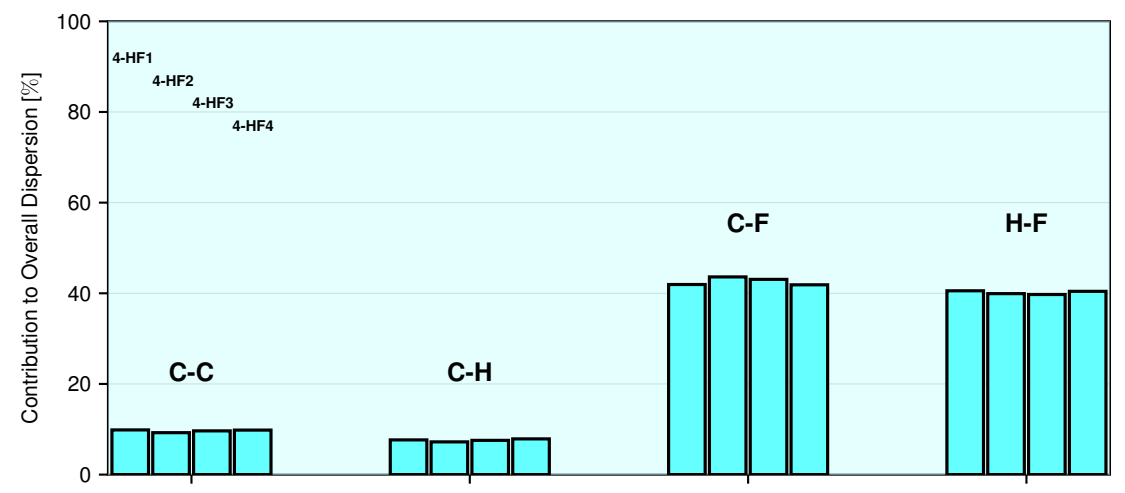

Figure S145: Decomposition of dispersion obtained from the atom-pairwise London dispersion model into contributions of interactions between types of atoms for $\mathbf{4 - H F}$ complexes.

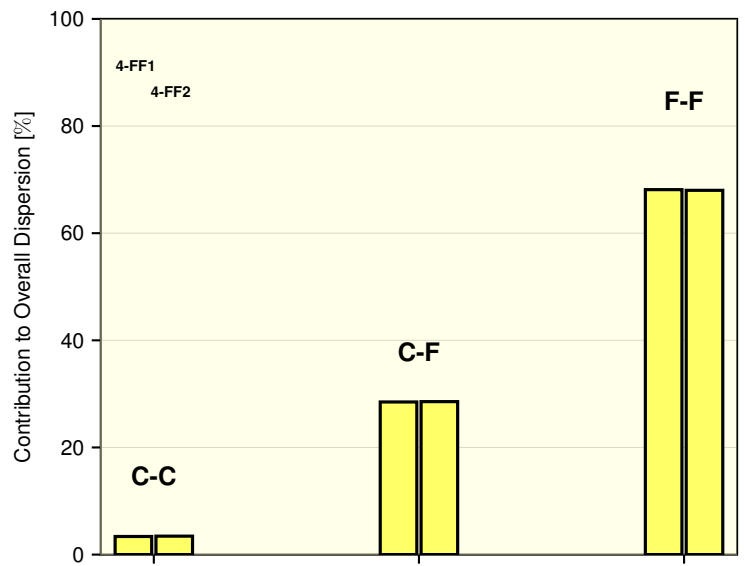

Figure S146: Decomposition of dispersion obtained from the atom-pairwise London dispersion model into contributions of interactions between types of atoms for $\mathbf{4}-\mathbf{F F}$ complexes. 


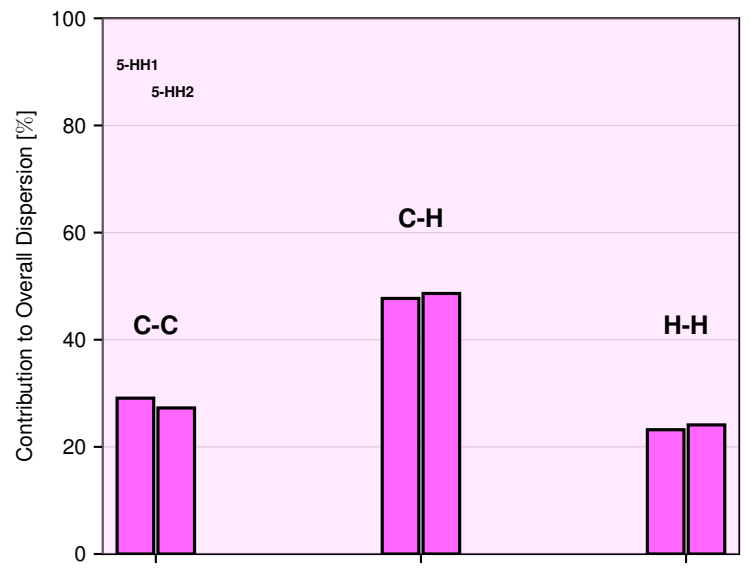

Figure S147: Decomposition of dispersion obtained from the atom-pairwise London dispersion model into contributions of interactions between types of atoms for $\mathbf{5}-\mathbf{H H}$ complexes.

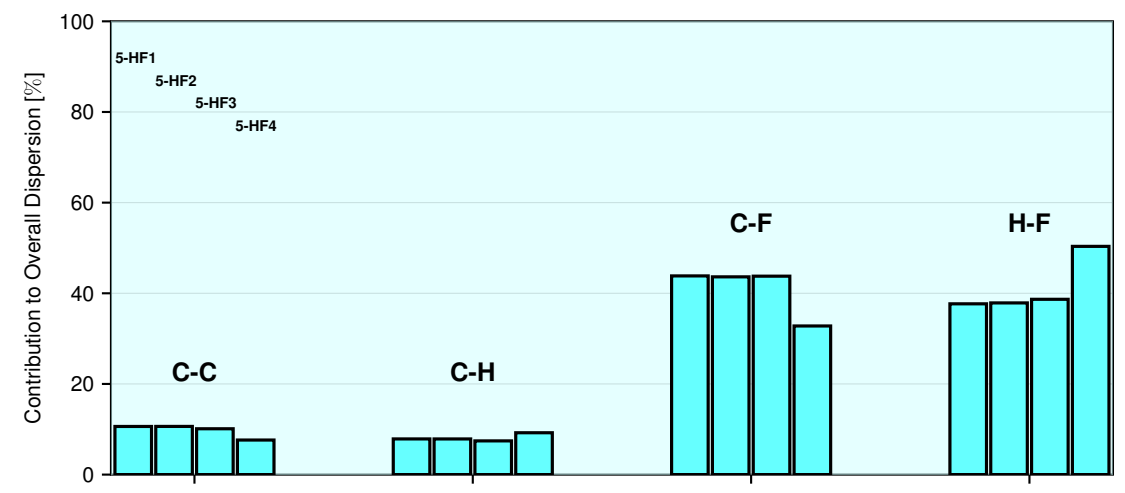

Figure S148: Decomposition of dispersion obtained from the atom-pairwise London dispersion model into contributions of interactions between types of atoms for $\mathbf{5 - H F}$ complexes.

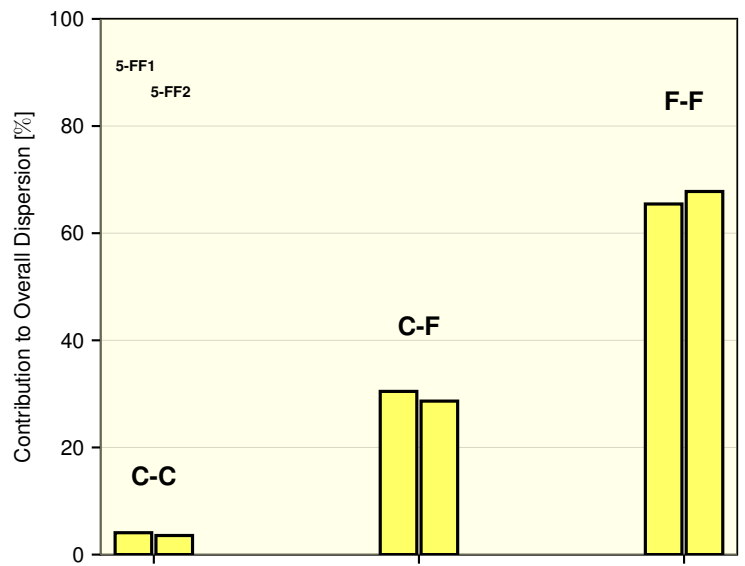

Figure S149: Decomposition of dispersion obtained from the atom-pairwise London dispersion model into contributions of interactions between types of atoms for $\mathbf{5 - F F}$ complexes. 


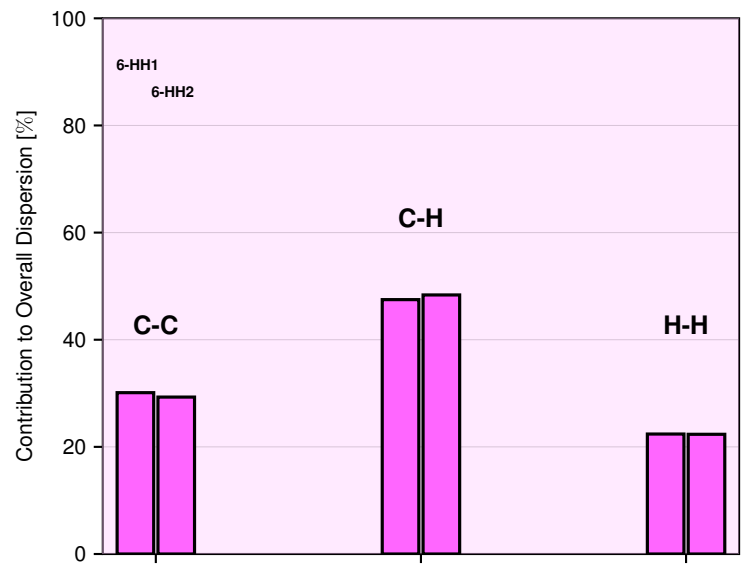

Figure S150: Decomposition of dispersion obtained from the atom-pairwise London dispersion model into contributions of interactions between types of atoms for $\mathbf{6}-\mathbf{H H}$ complexes.

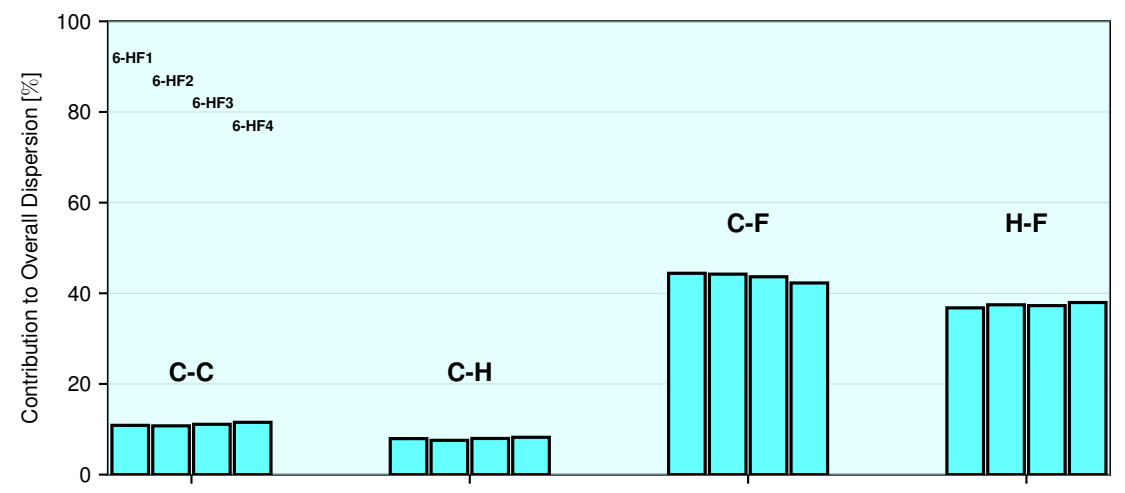

Figure S151: Decomposition of dispersion obtained from the atom-pairwise London dispersion model into contributions of interactions between types of atoms for $\mathbf{6}-\mathbf{H F}$ complexes.

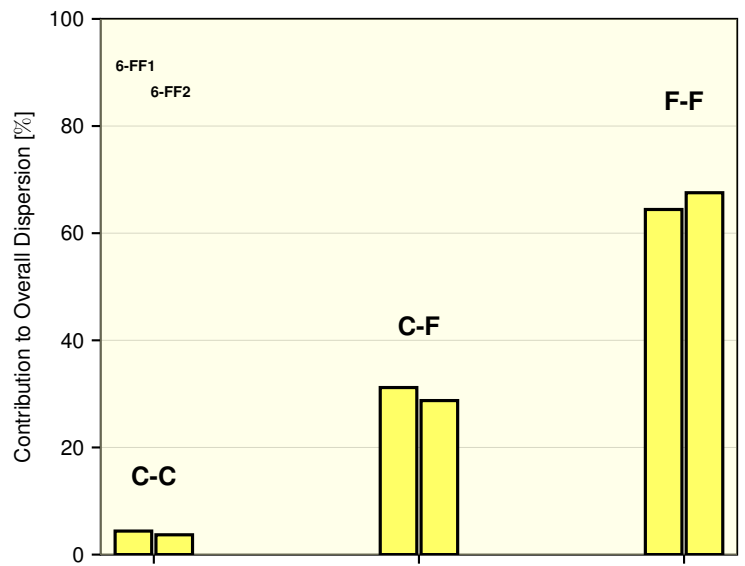

Figure S152: Decomposition of dispersion obtained from the atom-pairwise London dispersion model into contributions of interactions between types of atoms for $\mathbf{6}-\mathbf{F F}$ complexes. 

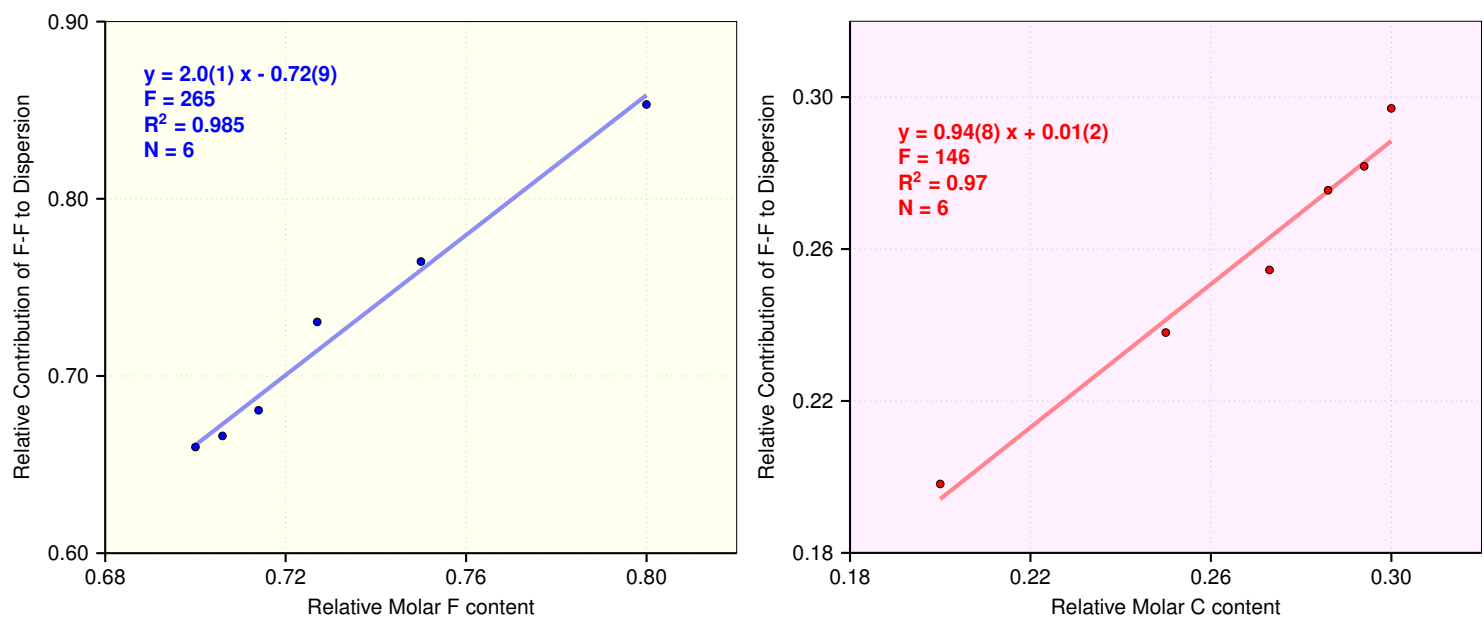

Figure S153: Left: Relative contribution of F-F in FF as a function of the relative molar fluorine content. Right: Relative contribution of $\mathrm{C}-\mathrm{C}$ in $\mathbf{H H}$ as a function of the relative molar carbon content from the atom-pairwise London dispersion interaction model.

\subsubsection{Correlation of BIE with Interaction Surface}

Furthermore, we were also interested whether alternative simple interaction models could be applied to predicting the interaction energies of $\mathbf{F}$. One common approximation is to regard it as interaction between exposed surfaces. Therefore, we computed the interaction surface area of all the molecular complexes using COSMO calculations and plotted the BIEs at the VeryTightPNO-DLPNO-CCSD(T)/CBS level of theory as well as the dispersion energy components at the LED-TightPNO-DLPNO-CCSD(T)/QZ level of theory against the corresponding interaction surface areas (cf. Figure S154). It can be seen that in both cases for each of the subsystems the BIE gets increasingly favorable as the interaction surface is increased and that reasonable linear correlations are observed. 

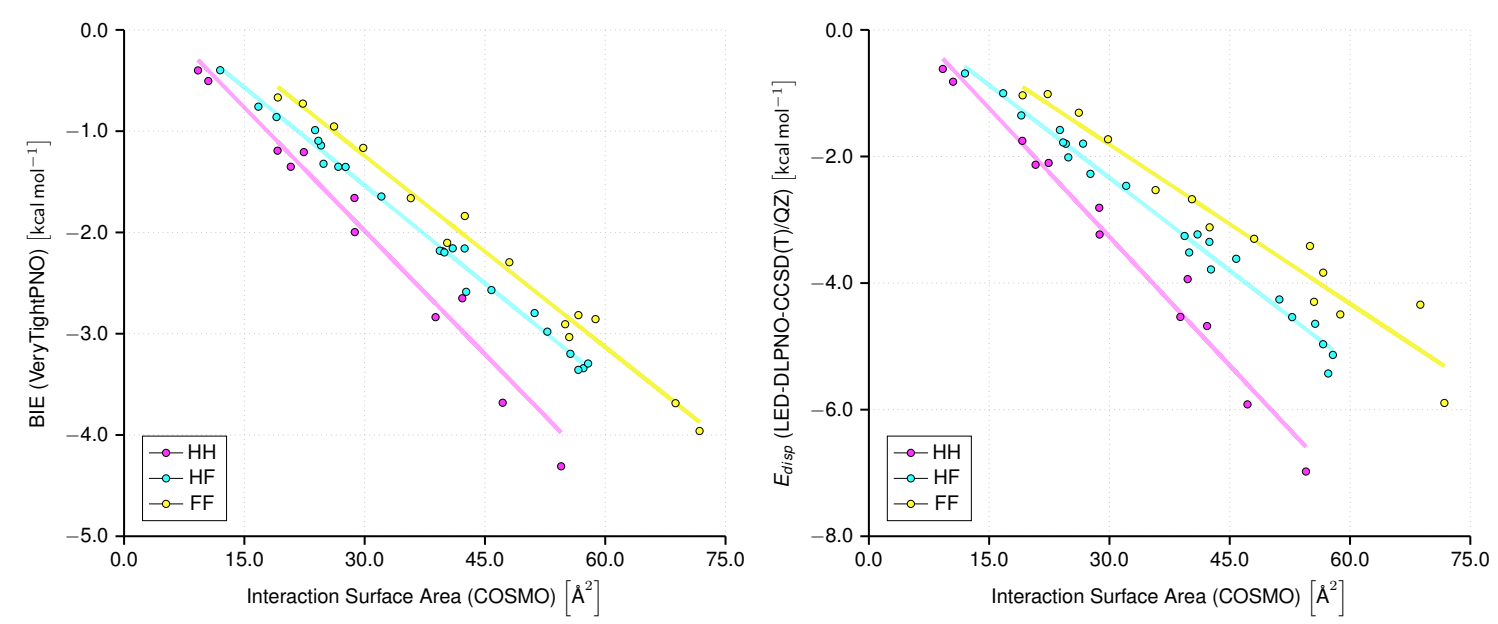

Figure S154: Left: Interaction energies obtained from VeryTightPNO-DLPNOCCSD(T)/CBS calculations plotted against the corresponding interaction surface areas. Right: Dispersion energy components of molecular complexes obtained from LEDTightPNO-DLPNO-CCSD(T)/QZ calculations plotted against the corresponding interaction surface areas. Within each of the three subclasses of molecular complexes, a linear correlation is observed.

\subsection{Interaction Descriptors}

\subsubsection{Q Measure and P Measure}

Qatom values were calculated as follows: For every point on the van der Waals surface (obtained from MP2/def2-TZVPD densities) the distance to one specific atom was calculated. The atomic polarizability (obtained using PolaBer) was divided by the third power of that distance to obtain the $\mathrm{Q}_{\text {atom }}$ value with respect to that specific point in space. The largest $Q_{\text {atom }}$ on the van der Waals surface was then the maximum $Q_{a t o m}$. To obtain $Q_{\text {tot }}$, for every point on the van der Waals surface the sum of all $Q_{\text {atom }}$ values of the molecule was calculated. $Q_{\text {tot }}$ was obtained as average of all these sums with respect to all points on the van der Waals surface. The corresponding $\mathrm{P}$ values were calculated analogously with the difference that multiplication by the respective atom-type dependent pre-factor A was carried out as well.

Figure $\mathrm{S} 155$ shows the correlation between the relative $\mathrm{P}_{\text {tot }}$ value of $\mathbf{F}$ with respect to the corresponding $\mathbf{H}$ and the ratio of the relative contributions of $\mathrm{C} 1$ and $\mathrm{C} 2$ of $\mathbf{F F}$ and $\mathbf{H H}$ from LED. This correlation suggests that the change in the relative importance of the interactions 
of the different atoms and bonds in the molecules is related to the change in the dispersive interaction capabilities of $\mathbf{H}$ with respect to $\mathbf{F}$. The relative $\mathrm{P}_{\text {tot }}$ value of $\mathbf{F}$ with respect to the corresponding $\mathbf{H}$ as a function of the relative molar carbon content is illustrated in Figure S155. The linear correlation observed illustrates that the dispersive interaction capability of $\mathbf{F}$ relative to $\mathbf{H}$ decreases with increasing carbon chain length because of a concomitant decreasing relative molar fluorine content.
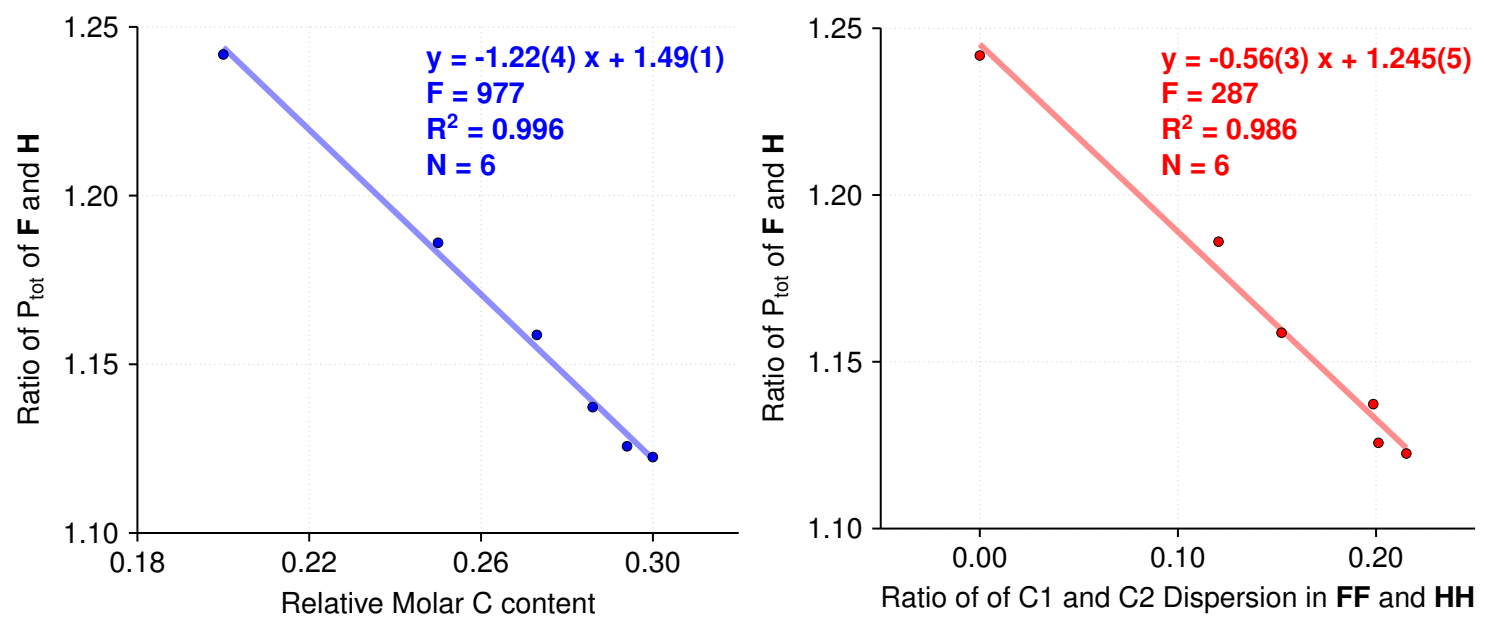

Figure S155: Correlation between the ratio of $\mathrm{P}_{\text {tot }}$ values of $\mathbf{F}$ and $\mathbf{H}$. Left: the relative molar carbon content; Right: the ratio of the relative contributions of $\mathrm{C} 1$ and $\mathrm{C} 2$ of $\mathbf{F F}$ and HH from LED.

\subsection{Interaction Geometries}

\subsubsection{Interaction Surface Areas}

We applied the geometric interaction model of cuboids (vide infra) to assess how well the interaction geometries of $\mathbf{H F}$ and $\mathbf{F F}$ compared to $\mathbf{H H}$ are. To do that, we determined the correlation of the molecular surfaces of $\mathbf{H}$ and $\mathbf{F}$, respectively, with the number of carbon atoms. For that, we used the COSMO surfaces $(\mathrm{S})$ of these molecules. The corresponding results are illustrated in Figure S156.

Consequently, we looked at the correlation of the COSMO interaction surfaces $\left(\mathrm{S}_{\text {int }}\right)$ of $\mathbf{H H}, \mathbf{H F}$ and $\mathbf{F F}$ against the number of carbon atoms. The COSMO interaction surfaces 


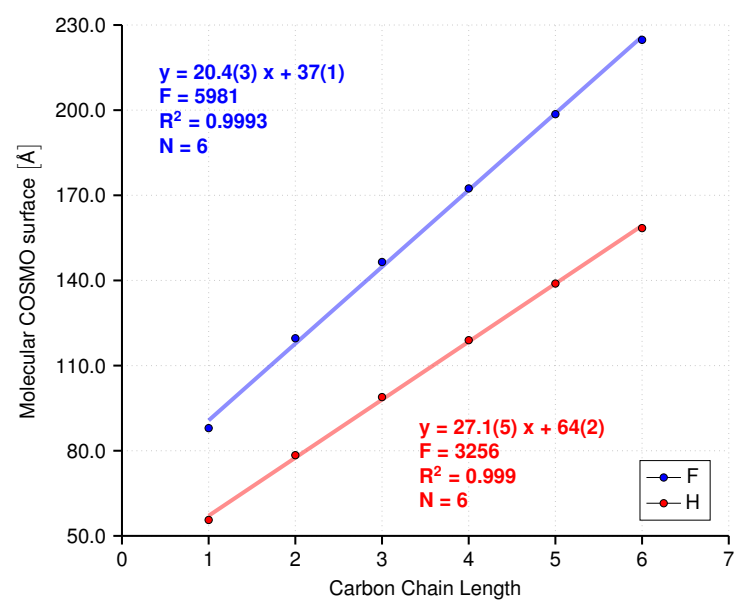

Figure S156: Molecular COSMO surface as a function of the number of carbon atoms for $\mathbf{H}$ and $\mathbf{F}$.

were determined as difference of the molecular COSMO surfaces of the complexes and the respective monomers. Figure S157 shows the correlation of the COSMO interaction surfaces against the number of carbon atoms for the three classes of complexes. For each number of carbon atoms we used the complex with the largest COSMO interaction surface for the correlation. The corresponding statistical parameters are summarized in Table S3.

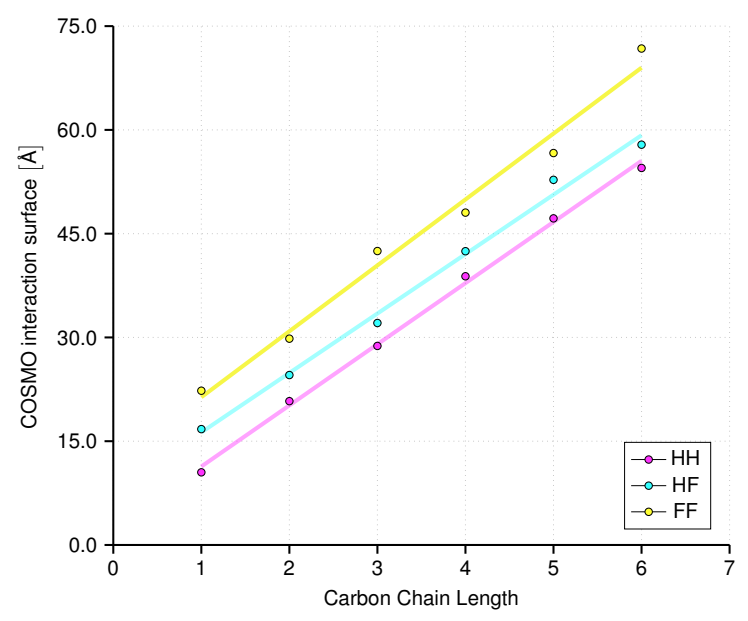

Figure S157: COSMO interaction surface as a function of the number of carbon atoms for HH, HF and FF.

From these correlations we can compare the interaction surfaces of $\mathbf{H H}, \mathbf{H F}$ and $\mathbf{F F}$. We assume that the interaction surface in $\mathbf{H H}$ is ideal. This assumption is based on the results of the NCI plots. On the basis of the interaction models of cuboids (vide infra) we 
Table S3: Statistical parameters of the correlation of the COSMO interaction surface with the number of carbon atoms for $\mathbf{H H}, \mathbf{H F}$ and $\mathbf{F F}$.

\begin{tabular}{cccc}
\multicolumn{4}{c}{$\mathrm{S}_{\text {int }}=\mathrm{k} \cdot \mathrm{n}(\mathrm{C})+\mathrm{d}$} \\
\hline Parameter & HH & HF & FF \\
\hline $\mathrm{k}[\AA]$ & $8.8(2)$ & $8.6(4)$ & $9.5(6)$ \\
$\mathrm{d}[\AA]$ & $2.5(9)$ & $8(1)$ & $12(2)$ \\
$\mathrm{F}$ & 1590 & 585 & 249 \\
$\mathrm{R}^{2}$ & 0.997 & 0.993 & 0.8 \\
$\mathrm{~N}$ & 6 & 6 & 6 \\
\hline
\end{tabular}

know that the ratio of the slope of the total surfaces of $\mathbf{H}$ and $\mathbf{F}$ as a function of the number of carbon atoms should correspond to the ratio of the slope of the interaction surfaces of $\mathbf{H H}$ and $\mathbf{F F}$ as a function of the number of carbon atoms if both are ideal. Hence, we need to compare the ratios of the molecular COSMO surfaces of $\mathbf{H}$ and $\mathbf{F}$ and the ratios of the COSMO interaction surfaces of $\mathbf{H H}$ and FF. The ratio of the slopes of the molecular COSMO surfaces of $\mathbf{F}$ and $\mathbf{H}$ is 1.32. The ratio of the slopes of the COSMO interaction surfaces of $\mathbf{F F}$ and $\mathbf{H H}$ is 1.1. Hence, the interaction surface in $\mathbf{F F}$ is $81 \%$ of what the ideal interaction surface estimated on the basis of the cuboid model should be. In addition, using the heteromolecular cuboid model (vide infra) we estimated that the ideal ratio of the slopes of the interaction surfaces of $\mathbf{H F}$ and $\mathbf{H H}$ should be 1.14. However, the ratio of the slopes of the COSMO interaction surfaces of $\mathbf{H F}$ and $\mathbf{H H}$ is 0.97 , i.e. it is $85 \%$ of what the ideal ratio is estimated to be. Overall, this suggests that the interaction surfaces in $\mathbf{H F}$ and $\mathbf{F F}$ are not ideal compared to the interaction surfaces in $\mathbf{H H}$.

For the computation of $\mathrm{n}_{\text {contacts }}$ we used the recalculated van der Waals radii of Alvarez. ${ }^{108}$ Hence, we used $1.20 \AA$ for hydrogen, $1.77 \AA$ for carbon and $1.46 \AA$ for fluorine. Table S4 gives the statistical parameters of $n_{\text {contacts }}$ as a function of the carbon chain length for $\mathbf{H H}, \mathbf{H F}$ and FF (cf. main text). 
Table S4: Statistical parameters of the correlation of $n_{\text {contacts }}$ with the number of carbon atoms for $\mathbf{H H}, \mathbf{H F}$ and $\mathbf{F F}$.

\begin{tabular}{cccc}
\multicolumn{4}{c}{$\mathrm{n}_{\text {contacts }}=\mathrm{k} \cdot \mathrm{n}(\mathrm{C})+\mathrm{d}$} \\
\hline Parameter & HH & HF & FF \\
\hline $\mathrm{k}$ & $8.2(1)$ & $5.0(3)$ & $4.5(4)$ \\
$\mathrm{d}$ & $-3.1(5)$ & $0.3(6)$ & $1(2)$ \\
$\mathrm{F}$ & 4159 & 908 & 112 \\
$\mathrm{R}^{2}$ & 0.999 & 0.996 & 0.97 \\
$\mathrm{~N}$ & 6 & 6 & 6 \\
\hline
\end{tabular}

\subsection{Predicted Microscopic and Macroscopic Mixing Behavior}

\subsubsection{Average Coordination Number}

In order to estimate macroscopic BIEs on the basis of Flory-Huggins solution theory, ${ }^{109,110}$ we needed to estimate the average coordination number of one molecule of the liquid in the bulk liquid. To do that, we assumed that the interaction geometries in the dimers we investigated in this work are akin to the interaction geometries in the liquid phase. Hence, we determined the average coordination number as ratio of the sum of the molecular COSMO surfaces of the interacting molecules in the molecular complex and the corresponding COSMO interaction surface in the molecular complex divided by two. The macroscopic interaction energies are then the product of the BIE of one intermolecular complex and the average coordination number.

\subsection{Trends in Experimental Properties}

\subsubsection{Boiling Points of Alkanes and Perfluoroalkanes}

When looking at the boiling points of $\mathbf{H}$ and $\mathbf{F}$ as a function of carbon chain length, linear $\mathbf{F}$ with a carbon chain length up to three have a higher boiling point compared to the corresponding H. ${ }^{111,112}$ Linear $\mathbf{F}$ with a longer carbon chain have a lower boiling point compared to the corresponding $\mathbf{H}$ (cf. Figure S158). Hence, there is a crossover in the boiling point curves between $\mathbf{H}$ and $\mathbf{F}$. As the boiling point is a measure of the strength of 
intermolecular interactions, the relative boiling point inversion indicates that the strength of the intermolecular interactions in $\mathbf{H}$ increase to a greater extent with carbon chain length than they do in $\mathbf{F}$. When the boiling point difference between $\mathbf{H}$ and the corresponding $\mathbf{F}$ is plotted against the relative molar carbon content, a linear correlation is observed (cf. Figure S158).
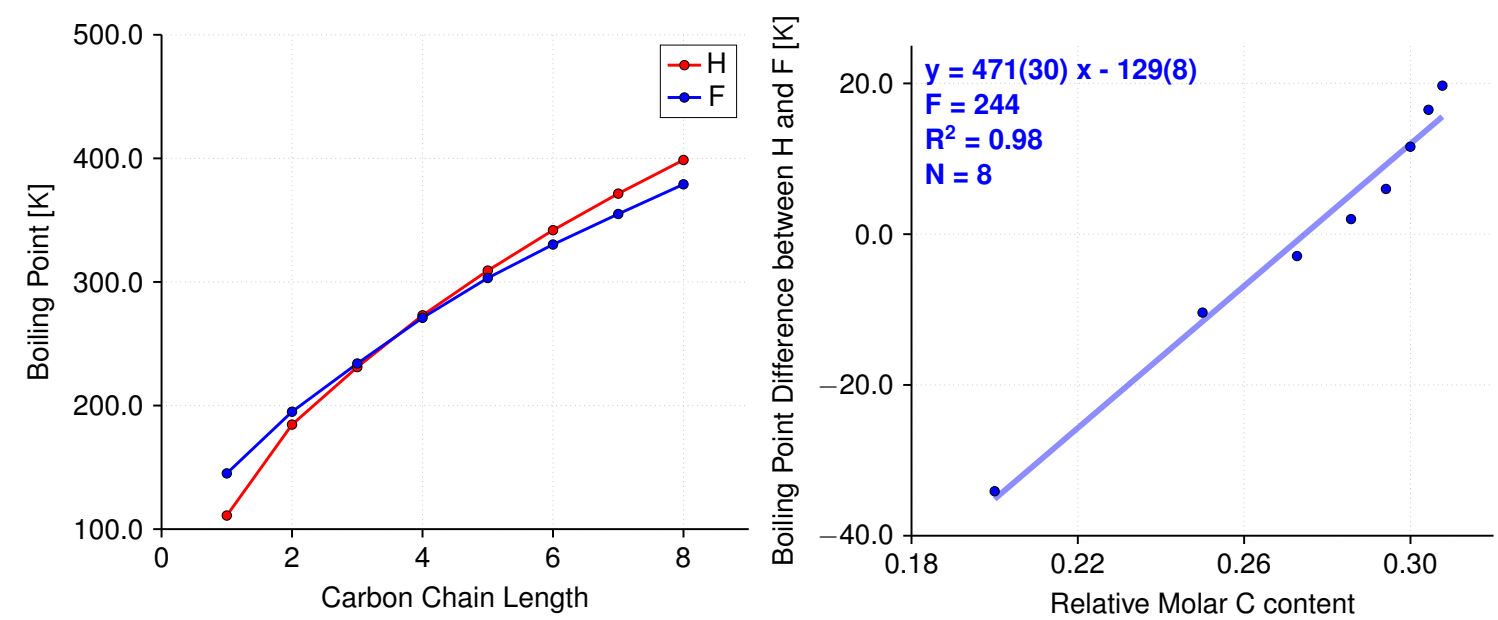

Figure S158: Left: Boiling points of $\mathbf{H}$ and $\mathbf{F}$ as a function of the respective carbon chain length. $\mathbf{H}$ have a higher boiling point at carbon chain lengths of four or higher. $\mathbf{F}$ have a higher boiling point with shorter carbon chains. Right: The boiling point difference between $\mathbf{H}$ and $\mathbf{F}$ of same carbon chain length as a function of the relative molar carbon content shows a linear correlation.

Table S5 contains the boiling points of $\mathbf{H}$ and $\mathbf{F}$ from one to eight carbon atoms and the corresponding references. 
Table S5: Boiling points $\left(\mathrm{T}_{\text {vap}}\right)$ of $\mathbf{H}$ and $\mathbf{F}$ from one to eight carbon atoms with the corresponding relative number of carbon atoms and the boiling point differences between $\mathbf{H}$ and $\mathbf{F}\left(\Delta \mathrm{T}_{\text {vap }}\right)$.

\begin{tabular}{ccccc}
\hline $\mathrm{n}(\mathrm{C})$ & $\mathrm{n}_{\text {rel }}(\mathrm{C})$ & $\mathrm{T}_{\text {vap }}(\mathbf{H})[\mathrm{K}]$ & $\mathrm{T}_{\text {vap }}(\mathbf{F})[\mathrm{K}]$ & $\Delta \mathrm{T}_{\text {vap }}(\mathbf{H}-\mathbf{F})[\mathrm{K}]$ \\
\hline 1 & 0.200 & $111^{113}$ & $145^{113}$ & -34 \\
2 & 0.250 & $185^{113}$ & $195^{113}$ & -10 \\
3 & 0.273 & $231^{113}$ & $234^{113}$ & -3 \\
4 & 0.286 & $273^{113}$ & $271^{114}$ & 2 \\
5 & 0.294 & $309^{113}$ & $303^{115}$ & 6 \\
6 & 0.300 & $342^{113}$ & $330^{115}$ & 12 \\
7 & 0.304 & $372^{113}$ & $355^{113}$ & 17 \\
8 & 0.308 & $399^{113}$ & $379^{116}$ & 20 \\
\hline
\end{tabular}

\subsubsection{Critical Parameters of Alkanes and Perfluoroalkanes}

Boiling points are only indirect measures of the strength of intermolecular interactions. Direct measures of intermolecular interactions in gases are provided by the van der Waals equation of state parameters $\mathrm{a}$ and $\mathrm{b}$ determined from critical point data. ${ }^{117}$ The ratio of a over b is an estimate of the minimum energy of the intermolecular potential energy surface which is apparent from the derivation of the van der Waals equation of state from statistical thermodynamics. ${ }^{118}$ Plotting the ratio a over b, determined from literature data (the references of all the individual data points are provided in Tables S6-S8), as a function of the carbon chain length for both $\mathbf{H}$ and $\mathbf{F}$, a similar trend compared to the boiling points is observed (cf. Figure S159). 1-F has stronger intermolecular interactions than 1-H, but already $\mathbf{2 - H}$ has stronger intermolecular interactions than $\mathbf{2 - F}$ and the difference between the two classes of compounds increases with carbon chain length. Plotting the difference in the ratio a over b between $\mathbf{H}$ and $\mathbf{F}$ of same carbon chain length against the respective relative molar carbon content, a linear correlation is observed (cf. Figure S159).

Tables S6-S8 contain the critical parameters of $\mathbf{H}$ and $\mathbf{F}$ from one to eight carbon atoms and the corresponding derived van der Waals parameters and average attraction energies between particles with the respective references. 

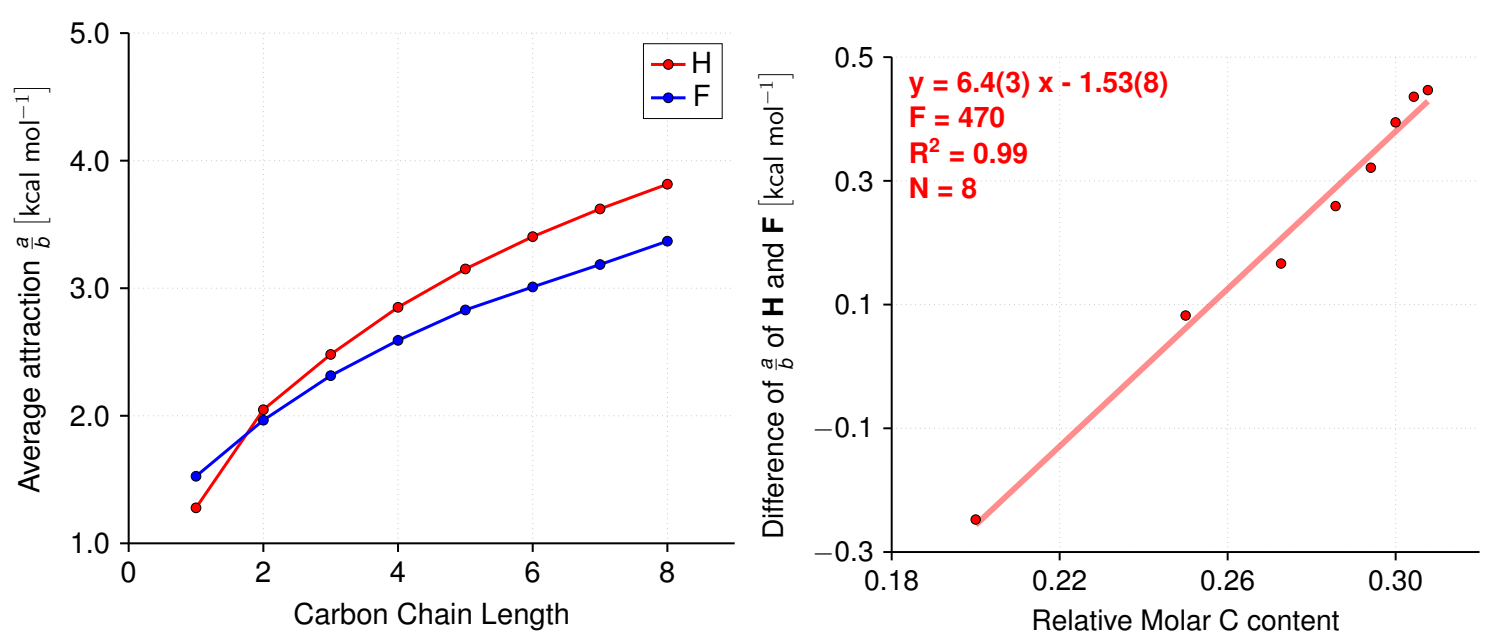

Figure S159: Left: Average attraction between molecules in the gas-phase as obtained from experimental van der Waals equation of state parameters at the critical point as a function of the carbon chain length. $\mathbf{1 - F}$ has stronger intermolecular attraction than $\mathbf{1 - H}$. At longer carbon chain lengths, $\mathbf{H}$ have stronger intermolecular attraction than the corresponding $\mathbf{F}$ having the same carbon chain length. Right: The difference in intermolecular attraction between $\mathbf{H}$ and $\mathbf{F}$ of same carbon chain length as a function of the relative molar carbon content shows a linear correlation.

Table S6: Critical parameters and van der Waals equation of state parameters a and b of $\mathbf{H}$ from one to eight carbon atoms. The ratio of a and b corresponds to the average attraction between particles.

\begin{tabular}{ccccccc}
\hline $\mathrm{n}(\mathrm{C})$ & $\mathrm{n}_{\mathrm{rel}}(\mathrm{C})$ & $\mathrm{T}_{\mathrm{c}}[\mathrm{K}]$ & $\mathrm{p}_{\mathrm{c}}[\mathrm{bar}]$ & $\mathrm{a}\left[\mathrm{J} \mathrm{m}^{3} \mathrm{~mol}^{-2}\right]$ & $\mathrm{b}\left[10^{-5} \mathrm{~m}^{3} \mathrm{~mol}^{-1}\right]$ & $\frac{\mathrm{a}}{\mathrm{b}}\left[\mathrm{kcal} \mathrm{mol}^{-1}\right]$ \\
\hline 1 & 0.200 & $190.6^{119}$ & $46.10^{119}$ & 0.2298 & 4.297 & 1.278 \\
2 & 0.250 & $305.3^{119}$ & $49.00^{119}$ & 0.5548 & 6.476 & 2.048 \\
3 & 0.273 & $369.9^{119}$ & $42.50^{119}$ & 0.9389 & 9.046 & 2.481 \\
4 & 0.286 & $425.0^{119}$ & $38.00^{119}$ & 1.386 & 11.62 & 2.850 \\
5 & 0.294 & $469.8^{119}$ & $33.60^{119}$ & 1.916 & 14.53 & 3.151 \\
6 & 0.300 & $507.6^{119}$ & $30.20^{119}$ & 2.488 & 17.47 & 3.404 \\
7 & 0.304 & $540.0^{119}$ & $27.40^{119}$ & 3.104 & 20.48 & 3.622 \\
8 & 0.308 & $568.9^{119}$ & $24.90^{119}$ & 3.791 & 23.75 & 3.816 \\
\hline
\end{tabular}


Table S7: Critical parameters and van der Waals equation of state parameters a and b of $\mathbf{F}$ from one to eight carbon atoms. The ratio of $\mathrm{a}$ and $\mathrm{b}$ corresponds to the average attraction between particles.

\begin{tabular}{ccccccc}
\hline $\mathrm{n}(\mathrm{C})$ & $\mathrm{n}_{\mathrm{rel}}(\mathrm{C})$ & $\mathrm{T}_{\mathrm{c}}[\mathrm{K}]$ & $\mathrm{p}_{\mathrm{c}}[\mathrm{bar}]$ & $\mathrm{a}\left[\mathrm{J} \mathrm{m}^{3} \mathrm{~mol}^{-2}\right]$ & $\mathrm{b}\left[10^{-5} \mathrm{~m}^{3} \mathrm{~mol}^{-1}\right]$ & $\frac{\mathrm{a}}{\mathrm{b}}\left[\mathrm{kcal} \mathrm{mol}^{-1}\right]$ \\
\hline 1 & 0.200 & $227.5^{119}$ & $37.45^{119}$ & 0.4031 & 6.314 & 1.526 \\
2 & 0.250 & $293.0^{120}$ & $30.42^{120}$ & 0.8232 & 10.01 & 1.965 \\
3 & 0.273 & $345.1^{121}$ & $26.75^{121}$ & 1.298 & 13.41 & 2.315 \\
4 & 0.286 & $386.4^{119}$ & $23.23^{119}$ & 1.874 & 17.28 & 2.591 \\
5 & 0.294 & $421.9^{119}$ & $20.37^{122}$ & 2.548 & 21.53 & 2.830 \\
6 & 0.300 & $448.8^{123}$ & $18.68^{123}$ & 3.144 & 24.97 & 3.010 \\
7 & 0.304 & $475.0^{124}$ & $16.50^{124}$ & 3.988 & 29.92 & 3.186 \\
8 & 0.308 & $502.3^{119}$ & $15.48^{122}$ & 4.753 & 33.72 & 3.369 \\
\hline
\end{tabular}

Table S8: Average attraction between particles of $\mathbf{H}$ and $\mathbf{F}$ with one to eight carbon atoms and the corresponding differences between $\mathbf{H}$ and $\mathbf{F}$.

\begin{tabular}{ccccc}
\hline $\mathrm{n}(\mathrm{C})$ & $\mathrm{n}_{\mathrm{rel}}(\mathrm{C})$ & $\frac{\mathrm{a}}{\mathrm{b}}(\mathbf{H})\left[\mathrm{kcal} \mathrm{mol}^{-1}\right]$ & $\frac{\mathrm{a}}{\mathrm{b}}(\mathbf{F})\left[\mathrm{kcal} \mathrm{mol}^{-1}\right]$ & $\Delta \frac{\mathrm{a}}{\mathrm{b}}(\mathbf{H}-\mathbf{F})\left[\mathrm{kcal} \mathrm{mol}^{-1}\right]$ \\
\hline 1 & 0.200 & 1.278 & 1.526 & -0.247 \\
2 & 0.250 & 2.048 & 1.965 & 0.082 \\
3 & 0.273 & 2.481 & 2.315 & 0.166 \\
4 & 0.286 & 2.850 & 2.591 & 0.259 \\
5 & 0.294 & 3.151 & 2.830 & 0.321 \\
6 & 0.300 & 3.404 & 3.010 & 0.395 \\
7 & 0.304 & 3.622 & 3.186 & 0.436 \\
8 & 0.308 & 3.816 & 3.369 & 0.447 \\
\hline
\end{tabular}




\subsubsection{Enthalpies of Evaporation of Alkanes and Perfluoroalkanes}

The enthalpies of evaporation of $\mathbf{H}$ and $\mathbf{F}$ correlate linearly with the carbon chain length (cf. Figure S160).

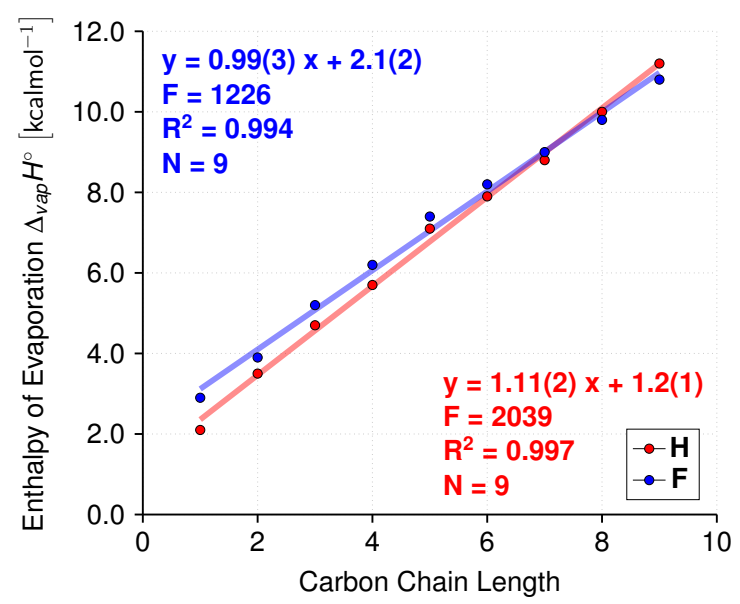

Figure S160: Enthalpies of evaporation of $\mathbf{H}$ and $\mathbf{F}$ of increasing carbon chain length.

Table S9 contains the enthalpies of evaporation of $\mathbf{H}$ and $\mathbf{F}$ with one to nine carbon atoms together with the corresponding references.

Table S9: Enthalpies of evaporation ( $\Delta \mathrm{H}_{\text {vap }}$ ) of $\mathbf{H}$ and $\mathbf{F}$ with one to nine carbon atoms with the corresponding temperature ranges for which the enthalpies are valid.

\begin{tabular}{ccccc}
\hline $\mathrm{n}(\mathrm{C})$ & $\Delta \mathrm{H}_{\text {vap }}(\mathbf{H})\left[\mathrm{kcal} \mathrm{mol}^{-1}\right]$ & $\mathrm{T}$-Range $[\mathrm{K}]$ & $\Delta \mathrm{H}_{\text {vap }}(\mathbf{F})\left[\mathrm{kcal} \mathrm{mol}^{-1}\right]$ & T-Range $[\mathrm{K}]$ \\
\hline 1 & $2.06^{125}$ & $90-120$ & $2.94^{125}$ & $89-163$ \\
2 & $3.51^{126}$ & 184 & $3.86^{116}$ & 195 \\
3 & $4.66^{125}$ & $165-248$ & $5.16^{125}$ & $193-237$ \\
4 & $5.71^{127}$ & $195-273$ & $6.17^{128}$ & $233-260$ \\
5 & $7.12^{125}$ & $223-352$ & $7.43^{125}$ & $221-303$ \\
6 & $7.93^{125}$ & $238-343$ & $8.22^{125}$ & $261-334$ \\
7 & $8.84^{129}$ & $288-348$ & $9.01^{125}$ & $271-379$ \\
8 & $10.01^{130}$ & $298-333$ & $9.85^{131}$ & $310-379$ \\
9 & $11.16^{132}$ & 299 & $10.83^{133}$ & $288-333$ \\
\hline
\end{tabular}




\subsubsection{Bulk Moduli of Alkanes and Perfluoroalkanes}

The bulk moduli of $\mathbf{H}$ and $\mathbf{F}$ show an inversion when the carbon chain length is increased (cf. Figure S161).

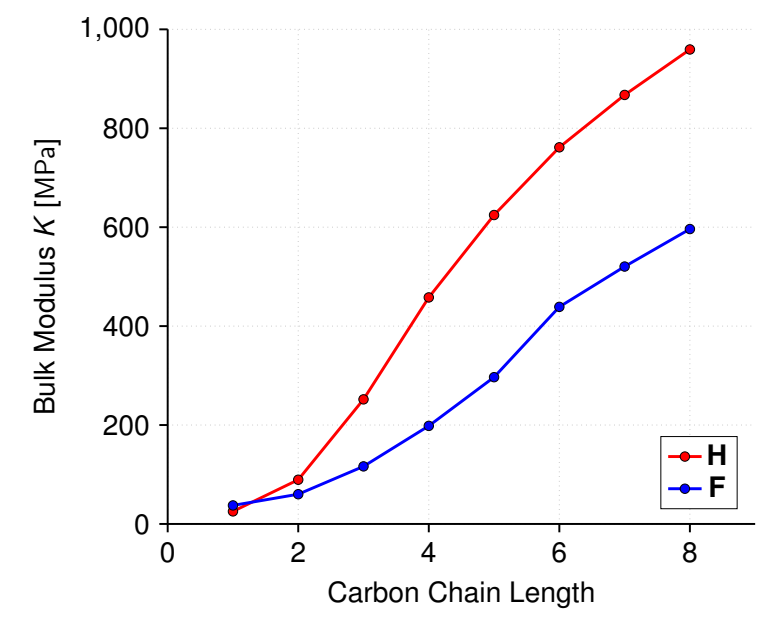

Figure S161: Bulk moduli of $\mathbf{H}$ and $\mathbf{F}$ of increasing carbon chain length.

Tables S10 and S11 contain the densities, speed of sound values and the derived bulk moduli of $\mathbf{H}$ and $\mathbf{F}$ with one to eight carbon atoms together with the corresponding references.

Table S10: Densities $(\rho)$, speed of sound values $(c)$ and derived bulk moduli $(K)$ of $\mathbf{H}$ at $298 \mathrm{~K}$ from one to eight carbon atoms with the corresponding pressures for which the values are valid. The bulk moduli are calculated as follows: $K=\rho c^{2}$.

\begin{tabular}{cccccc}
\hline $\mathrm{n}(\mathrm{C})$ & $\mathrm{n}_{\text {rel }}(\mathrm{C})$ & Pressure $[\mathrm{MPa}]$ & $\rho\left[\mathrm{kg} \mathrm{m}^{-3}\right]$ & $c\left[\mathrm{~m} \mathrm{~s}^{-1}\right]$ & $K[\mathrm{MPa}]$ \\
\hline 1 & 0.200 & 15.0 & $119.7^{134}$ & $460.6^{134}$ & 25.4 \\
2 & 0.250 & 7.5 & $362.0^{135}$ & $497.0^{135}$ & 89.4 \\
3 & 0.273 & 1.3 & $492.3^{136}$ & $715.3^{136}$ & 251.9 \\
4 & 0.286 & 0.6 & $573.3^{137}$ & $893.8^{138}$ & 458.0 \\
5 & 0.294 & 0.1 & $621.0^{139}$ & $1002.9^{139}$ & 624.6 \\
6 & 0.300 & 0.1 & $655.3^{140}$ & $1078.0^{140}$ & 761.5 \\
7 & 0.304 & 0.1 & $679.6^{141}$ & $1129.7^{141}$ & 867.4 \\
8 & 0.308 & 0.1 & $698.7^{141}$ & $1171.7^{141}$ & 959.3 \\
\hline
\end{tabular}


Table S11: Densities $(\rho)$, speed of sound values $(c)$ and derived bulk moduli $(K)$ of $\mathbf{F}$ at $298 \mathrm{~K}$ from one to eight carbon atoms with the corresponding pressures for which the values are valid. The bulk moduli are calculated as follows: $K=\rho c^{2}$.

\begin{tabular}{cccccc}
\hline $\mathrm{n}(\mathrm{C})$ & $\mathrm{n}_{\text {rel }}(\mathrm{C})$ & Pressure $[\mathrm{MPa}]$ & $\rho\left[\mathrm{kg} \mathrm{m}^{-3}\right]$ & $c\left[\mathrm{~m} \mathrm{~s}^{-1}\right]$ & $K[\mathrm{MPa}]$ \\
\hline 1 & 0.200 & 15.0 & $745.3^{142}$ & $224.3^{142}$ & 37.5 \\
2 & 0.250 & 7.5 & $1100.0^{142}$ & $233.8^{142}$ & 60.1 \\
3 & 0.273 & 1.3 & $1331.9^{142}$ & $295.6^{142}$ & 116.4 \\
4 & 0.286 & 0.6 & $1499.5^{143}$ & $363.6^{142}$ & 198.3 \\
5 & 0.294 & 0.1 & $1599.5^{144}$ & $430.7^{145}$ & 296.7 \\
6 & 0.300 & 0.1 & $1682.8^{146}$ & $510.7^{146}$ & 438.9 \\
7 & 0.304 & 0.1 & $1728.4^{146}$ & $548.7^{146}$ & 520.4 \\
8 & 0.308 & 0.1 & $1760.6^{146}$ & $581.9^{146}$ & 596.2 \\
\hline
\end{tabular}

\subsubsection{Compressibilities of Alkanes and Perfluoroalkanes}

The compressibilities of $\mathbf{H}$ and $\mathbf{F}$ show an inversion when the carbon chain length is increased (cf. Figure S162).

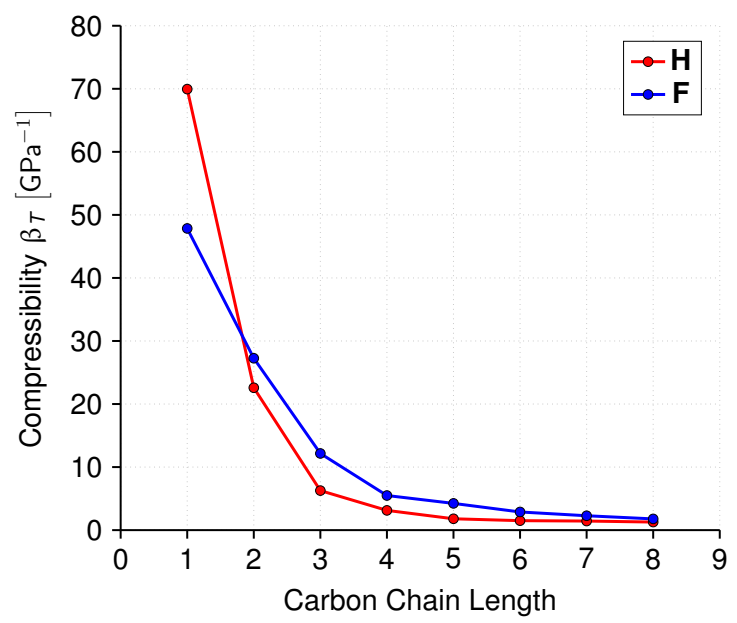

Figure S162: Isothermal compressibilities of $\mathbf{H}$ and $\mathbf{F}$ of increasing carbon chain length.

Table S12 contains the compressibilities of $\mathbf{H}$ and $\mathbf{F}$ with one to eight carbon atoms together with the corresponding references. 
Table S12: Isothermal compressibilities $\left(\beta_{\mathrm{T}}\right)$ of $\mathbf{H}$ and $\mathbf{F}$ at $298 \mathrm{~K}$ with one to eight carbon atoms with the corresponding pressures for which the values are valid.

\begin{tabular}{ccccc}
\hline $\mathrm{n}(\mathrm{C})$ & $\mathrm{n}_{\text {rel }}(\mathrm{C})$ & Pressure $[\mathrm{MPa}]$ & $\beta_{\mathrm{T}}(\mathbf{H})\left[\mathrm{GPa}^{-1}\right]$ & $\beta_{\mathrm{T}}(\mathbf{F})\left[\mathrm{GPa}^{-1}\right]$ \\
\hline 1 & 0.200 & 15.0 & $69.95^{142}$ & $47.85^{142}$ \\
2 & 0.250 & 7.5 & $22.58^{142}$ & $27.26^{142}$ \\
3 & 0.273 & 1.3 & $6.27^{147}$ & $12.16^{148}$ \\
4 & 0.286 & 0.6 & $3.13^{147}$ & $5.49^{149}$ \\
5 & 0.294 & 0.1 & $1.80^{149}$ & $4.24^{149}$ \\
6 & 0.300 & 0.1 & $1.50^{150}$ & $2.88^{151}$ \\
7 & 0.304 & 0.1 & $1.44^{151}$ & $2.28^{151}$ \\
8 & 0.308 & 0.1 & $1.28^{151}$ & $1.78^{151}$ \\
\hline
\end{tabular}

\subsubsection{Interal Pressures of Alkanes and Perfluoroalkanes}

The internal pressures of $\mathbf{H}$ and $\mathbf{F}$ show an inversion when the carbon chain length is increased (cf. Figure S163).

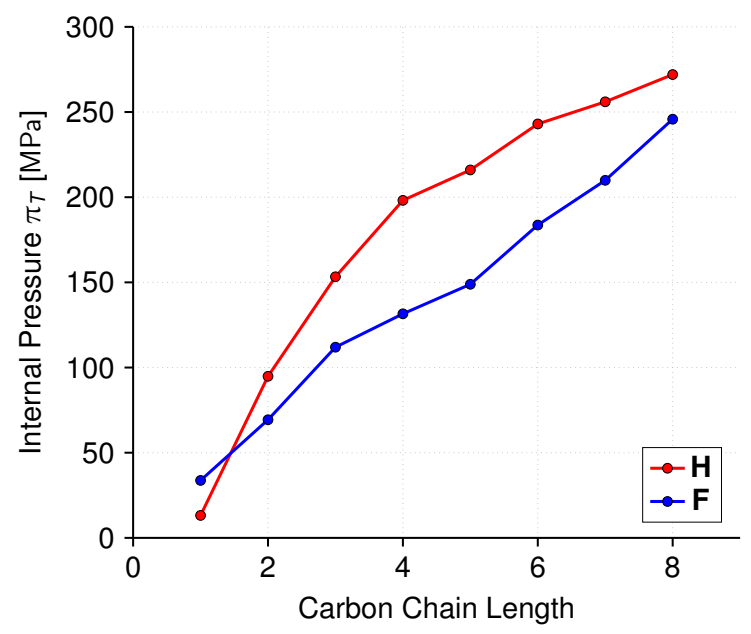

Figure S163: Internal pressures of $\mathbf{H}$ and $\mathbf{F}$ of increasing carbon chain length.

Table S13 contain the internal pressures of $\mathbf{H}$ and $\mathbf{F}$ with one to eight carbon atoms together with the corresponding references. 
Table S13: Internal pressures $\left(\pi_{\mathrm{T}}\right)$ of $\mathbf{H}$ and $\mathbf{F}$ at $298 \mathrm{~K}$ with one to eight carbon atoms with the corresponding pressures for which the values are valid.

\begin{tabular}{ccccc}
\hline $\mathrm{n}(\mathrm{C})$ & $\mathrm{n}_{\text {rel }}(\mathrm{C})$ & Pressure $[\mathrm{MPa}]$ & $\pi_{\mathrm{T}}(\mathbf{H})[\mathrm{MPa}]$ & $\pi_{\mathrm{T}}(\mathbf{F})[\mathrm{MPa}]$ \\
\hline 1 & 0.200 & 15.0 & $13^{142}$ & $34^{142}$ \\
2 & 0.250 & 7.5 & $95^{152}$ & $69^{142}$ \\
3 & 0.273 & 1.3 & $153^{147}$ & $112^{148}$ \\
4 & 0.286 & 0.6 & $198^{147}$ & $132^{149}$ \\
5 & 0.294 & 0.1 & $216^{150}$ & $149^{149}$ \\
6 & 0.300 & 0.1 & $243^{150}$ & $184^{151}$ \\
7 & 0.304 & 0.1 & $256^{151}$ & $210^{151}$ \\
8 & 0.308 & 0.1 & $272^{150}$ & $246^{146,151}$ \\
\hline
\end{tabular}

\subsubsection{Surface Tensions of Alkanes and Perfluoroalkanes}

The surface tensions of $\mathbf{H}$ and $\mathbf{F}$ show an inversion when the carbon chain length is increased (cf. Figure S164).

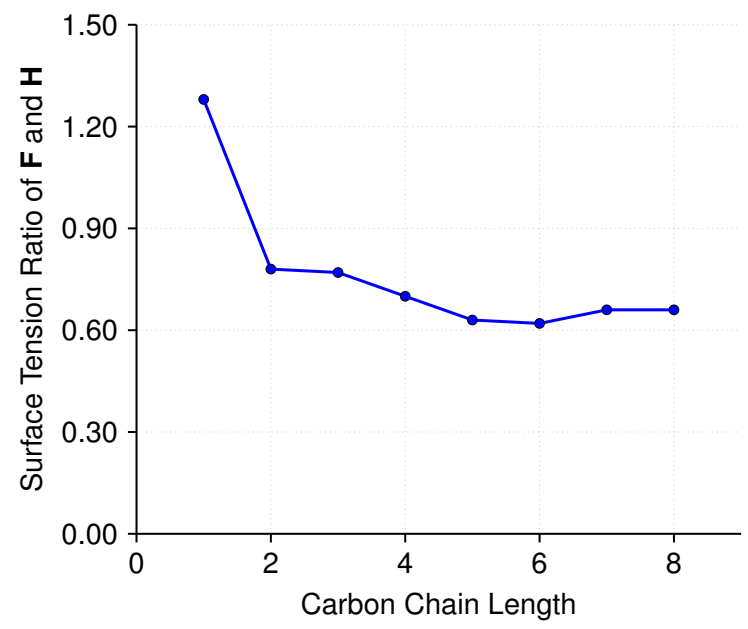

Figure S164: Ratio of surface tensions of $\mathbf{F}$ and $\mathbf{H}$ of increasing carbon chain length.

Table S14 contains the surface tensions of $\mathbf{H}$ and $\mathbf{F}$ with one to eight carbon atoms together with the corresponding references. 
Table S14: Surface tensions $(\gamma)$ of $\mathbf{H}$ and $\mathbf{F}$ with one to eight carbon atoms with the corresponding temperatures and pressures for which the values are valid.

\begin{tabular}{ccccccc}
\hline $\mathrm{n}(\mathrm{C})$ & Temperature $[\mathrm{K}]$ & Pressure $[\mathrm{MPa}]$ & $\gamma(\mathbf{H})\left[\mathrm{mN} \mathrm{m}^{-1}\right]$ & Temperature $[\mathrm{K}]$ & Pressure $[\mathrm{MPa}]$ & $\gamma(\mathbf{F})\left[\mathrm{mN} \mathrm{m}^{-1}\right]$ \\
\hline 1 & 90.7 & Triple point & $17.8^{153}$ & 89.6 & Triple point & $22.8^{154}$ \\
2 & 173.2 & 0.1 & $17.8^{155}$ & 173.2 & Triple point & $14.0^{154}$ \\
3 & 140.0 & 0.1 & $29.3^{156}$ & 140.0 & 0.1 & $22.5^{154}$ \\
4 & 193.2 & 0.1 & $25.3^{157}$ & 193.2 & 0.1 & $17.8^{154}$ \\
5 & 298.0 & 0.1 & $15.0^{158}$ & 298.0 & 0.1 & $9.4^{159}$ \\
6 & 298.0 & 0.1 & $17.8^{158}$ & 298.0 & 0.1 & $11.1^{160}$ \\
7 & 298.0 & 0.1 & $19.5^{158}$ & 298.0 & 0.1 & $12.9^{160}$ \\
8 & 298.0 & 0.1 & $21.3^{158}$ & 298.0 & 0.1 & $14.0^{160}$ \\
\hline
\end{tabular}

\subsubsection{Molar Heat Capacities of Alkanes and Perfluoroalkanes}

The molar heat capacities of $\mathbf{H}$ and $\mathbf{F}$ correlate linearly with the carbon chain length (cf. Figure S165).

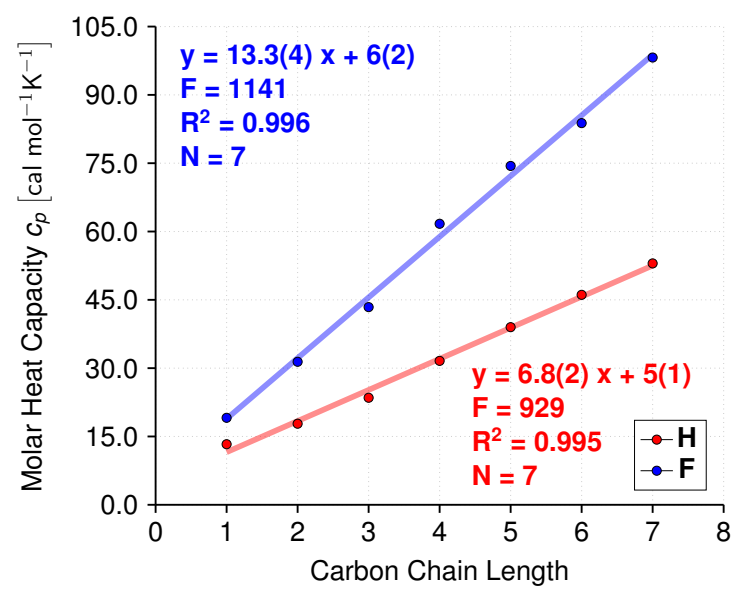

Figure S165: Molar heat capacities of $\mathbf{H}$ and $\mathbf{F}$ of increasing carbon chain length.

Table S15 contains the molar heat capacities of liquid $\mathbf{H}$ and $\mathbf{F}$ with one to seven carbon atoms together with the corresponding references. 
Table S15: Molar heat capacities $\left(c_{\mathrm{p}}\right)$ of $\mathbf{H}$ and $\mathbf{F}$ with one to seven carbon atoms with the corresponding temperatures for which the values are valid.

\begin{tabular}{ccccc}
\hline $\mathrm{n}(\mathrm{C})$ & Temperature $[\mathrm{K}]$ & $\mathrm{c}_{\mathrm{p}}(\mathbf{H})\left[\mathrm{cal} \mathrm{mol}^{-1} \mathrm{~K}^{-1}\right]$ & Temperature $[\mathrm{K}]$ & $\mathrm{c}_{\mathrm{p}}(\mathbf{F})\left[\mathrm{cal} \mathrm{mol}^{-1} \mathrm{~K}^{-1}\right]$ \\
\hline 1 & 111 & $13.3^{142}$ & 145 & $19.1^{161}$ \\
2 & 200 & $17.8^{162}$ & 195 & $31.4^{163}$ \\
3 & 230 & $23.5^{164}$ & 235 & $43.4^{165}$ \\
4 & 290 & $31.6^{166}$ & 293 & $61.7^{142}$ \\
5 & 290 & $39.0^{162}$ & 298 & $74.4^{145}$ \\
6 & 290 & $46.1^{149}$ & 293 & $83.8^{145}$ \\
7 & 290 & $53.0^{149}$ & 293 & $98.2^{167}$ \\
\hline
\end{tabular}

\subsubsection{Dynamic Viscosities of Alkanes and Perfluoroalkanes}

The dynamic viscosities of $\mathbf{H}$ and $\mathbf{F}$ do not show an inversion when the carbon chain length is increased (cf. Figure S166).

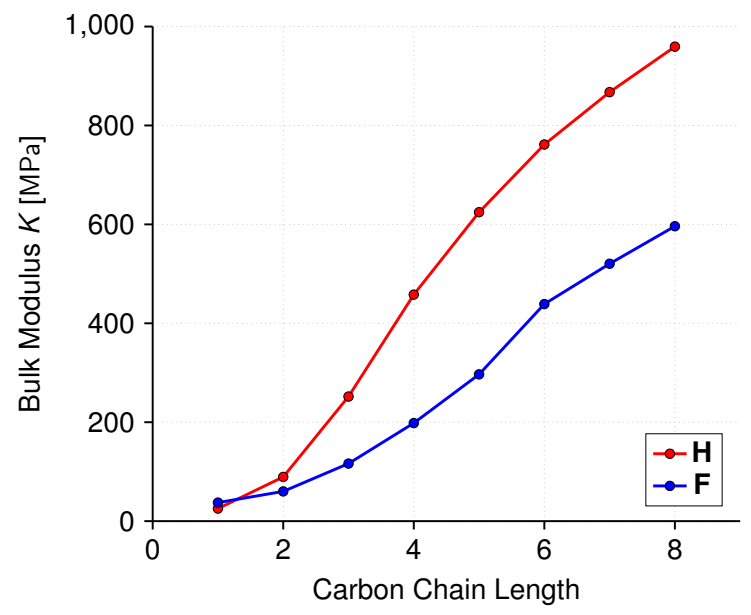

Figure S166: Dynamic viscosities of $\mathbf{H}$ and $\mathbf{F}$ of increasing carbon chain length.

Table S16 contains the dynamic viscosities of $\mathbf{H}$ and $\mathbf{F}$ with one to eight carbon atoms together with the corresponding references. 
Table S16: Dynamic viscosities $(\mu)$ of $\mathbf{H}$ and $\mathbf{F}$ at $253 \mathrm{~K}$ with one to eight carbon atoms with the corresponding pressures for which the values are valid.

\begin{tabular}{ccccc}
\hline $\mathrm{n}(\mathrm{C})$ & $\mathrm{n}_{\text {rel }}(\mathrm{C})$ & Pressure $[\mathrm{MPa}]$ & $\mu(\mathbf{H})[\mathrm{mPa} \mathrm{s}]$ & $\mu(\mathbf{F})[\mathrm{mPa} \mathrm{s}]$ \\
\hline 1 & 0.200 & 15 & $0.019^{142}$ & $0.071^{142}$ \\
2 & 0.250 & 7.5 & $0.084^{142}$ & $0.199^{142}$ \\
3 & 0.273 & 1.3 & $0.157^{142}$ & $0.343^{142}$ \\
4 & 0.286 & 0.1 & $0.250^{149}$ & $0.630^{168}$ \\
5 & 0.294 & 0.1 & $0.340^{149}$ & $0.957^{169}$ \\
6 & 0.300 & 0.1 & $0.450^{149}$ & $1.423^{169}$ \\
7 & 0.304 & 0.1 & $0.690^{149}$ & $2.000^{149}$ \\
8 & 0.308 & 0.1 & $0.980^{149}$ & $3.000^{149}$ \\
\hline
\end{tabular}

\subsubsection{Deviation of Computational Results from the Geometric Mean Rule}

We estimated the BIEs of $\mathbf{H F}$ from the corresponding BIEs of $\mathbf{H H}$ and $\mathbf{F F}$ using the respective average BIEs of all conformers considered and taking the geometric mean of the absolute of the two BIEs. The deviation of the actual BIE and the estimated BIE was determined using equation S13. We also performed the same calculations using BAEs instead of BIEs. The corresponding results are provided in Tables S17-S19.

$$
\Delta \mathrm{BIE}_{r e l}=\frac{\mathrm{BIE}_{g e o}-\mathrm{BIE}_{c o m p}}{\mathrm{BIE}_{c o m p}}
$$

Table S17: Estimation of the deviation of the BIEs of HF complexes from the geometric means of the corresponding $\mathbf{H H}$ and FF complexes using CCSD(T)-F12/VDZ, VeryTightPNO-DLPNO-CCSD(T)/CBS(34) and B97M-V/def2-QZVP interaction energies.

\begin{tabular}{|c|c|c|c|c|c|c|c|c|c|}
\hline \multirow{3}{*}{ System } & \multicolumn{3}{|c|}{$\operatorname{CCSD}(\mathrm{T})-\mathrm{F} 12$} & \multicolumn{3}{|c|}{ VeryTight-DLPNO-CCSD(T) } & \multicolumn{3}{|c|}{ B97M-V } \\
\hline & \multicolumn{2}{|c|}{$\mathrm{BIE}\left[\mathrm{kcal} \mathrm{mol}^{-1}\right]$} & \multirow[t]{2}{*}{$\Delta \mathrm{BIE}_{\mathrm{rel}}$} & \multicolumn{2}{|c|}{$\mathrm{BIE}\left[\mathrm{kcal} \mathrm{mol}{ }^{-1}\right]$} & \multirow[t]{2}{*}{$\Delta \mathrm{BIE}_{\mathrm{rel}}$} & \multicolumn{2}{|c|}{$\mathrm{BIE}\left[\mathrm{kcal} \mathrm{mol}^{-1}\right]$} & \multirow[t]{2}{*}{$\Delta \mathrm{BIE}_{\mathrm{re}}$} \\
\hline & $\mathrm{BIE}_{\mathrm{comp}}$ & $\mathrm{BIE}_{\text {geo }}$ & & $\mathrm{BIE}_{\text {comp }}$ & $\mathrm{BIE}_{\text {geo }}$ & & $\mathrm{BIE}_{\text {comp }}$ & $\mathrm{BIE}_{\text {geo }}$ & \\
\hline 1-HF & -0.60 & -0.60 & $1.1 \%$ & -0.58 & -0.56 & $-2.8 \%$ & -0.58 & -0.56 & $-3.4 \%$ \\
\hline 2-HF & -1.09 & -1.22 & $11.6 \%$ & -1.02 & -1.16 & $13.6 \%$ & -1.04 & -1.16 & $12.2 \%$ \\
\hline 3-HF & -1.49 & -1.80 & $20.5 \%$ & -1.42 & -1.67 & $18.1 \%$ & -1.50 & -1.83 & $22.2 \%$ \\
\hline 4-HF & -2.27 & -2.40 & $5.7 \%$ & -2.17 & -2.22 & $2.3 \%$ & -2.21 & -2.34 & $5.6 \%$ \\
\hline 5-HF & -2.86 & -3.17 & $11.1 \%$ & -2.73 & -2.93 & $7.2 \%$ & -2.92 & -3.26 & $11.5 \%$ \\
\hline $6-\mathrm{HF}$ & -3.44 & -3.75 & $8.9 \%$ & -3.30 & -3.44 & $4.4 \%$ & -3.52 & -3.84 & $9.0 \%$ \\
\hline Average & & & $10 \%$ & & & $7 \%$ & & & $10 \%$ \\
\hline
\end{tabular}


Table S18: Estimation of the deviation of the BIEs of HF complexes from the geometric means of the corresponding $\mathbf{H H}$ and $\mathbf{F F}$ complexes using the intermolecular London dispersion estimates and only the interaction distance components of the intermolecular London dispersion model (multiplied by the appropriate units to formally obtain energies).

\begin{tabular}{|c|c|c|c|c|c|c|}
\hline \multirow{3}{*}{ System } & \multicolumn{3}{|c|}{ Intermolecular London Dispersion } & \multicolumn{3}{|c|}{ Interaction Distances } \\
\hline & \multicolumn{2}{|c|}{$\mathrm{BIE}\left[\mathrm{kcal} \mathrm{mol}^{-1}\right]$} & \multirow{2}{*}{$\Delta \mathrm{BIE}_{\mathrm{rel}}$} & \multicolumn{2}{|c|}{$\mathrm{BIE}\left[\mathrm{kcal} \mathrm{mol}{ }^{-1}\right]$} & \multirow{2}{*}{$\Delta \mathrm{BIE}_{\mathrm{rel}}$} \\
\hline & $\mathrm{BIE}_{\mathrm{comp}}$ & $\mathrm{BIE}_{\text {geo }}$ & & $\mathrm{BIE}_{\text {comp }}\left[10^{-4}\right]$ & $\mathrm{BIE}_{\text {geo }}\left[10^{-4}\right]$ & \\
\hline 1-HF & -0.56 & -0.58 & $3.2 \%$ & -3.07 & -3.16 & $3.0 \%$ \\
\hline 2-HF & -0.98 & -1.04 & $6.9 \%$ & -2.10 & -2.24 & $6.7 \%$ \\
\hline 3-HF & -1.23 & -1.60 & $29.8 \%$ & 1.39 & -1.80 & $29.6 \%$ \\
\hline 4-HF & -1.90 & -1.89 & $-0.8 \%$ & -1.33 & -1.32 & $-1.0 \%$ \\
\hline 5-HF & -2.34 & -2.53 & $8.1 \%$ & -1.09 & -1.18 & $8.0 \%$ \\
\hline $6-\mathrm{HF}$ & -2.90 & -3.13 & $8.0 \%$ & -0.97 & -1.05 & $7.9 \%$ \\
\hline Average & & & $9 \%$ & & & $9 \%$ \\
\hline
\end{tabular}

Table S19: Estimation of the deviation of the BAEs of $\mathbf{H F}$ complexes from the geometric means of the corresponding $\mathbf{H H}$ and FF complexes using CCSD(T)-F12/VDZ, VeryTightPNO-DLPNO-CCSD(T)/CBS(34) and B97M-V/def2-QZVP interaction energies.

\begin{tabular}{|c|c|c|c|c|c|c|c|c|c|}
\hline \multirow{3}{*}{ System } & \multicolumn{3}{|c|}{$\mathrm{CCSD}(\mathrm{T})-\mathrm{F} 12$} & \multicolumn{3}{|c|}{ VeryTight-DLPNO-CCSD(T) } & \multicolumn{3}{|c|}{ B97M-V } \\
\hline & \multicolumn{2}{|c|}{$\mathrm{BAE}[\mathrm{kcal} \mathrm{mol}-1]$} & \multirow[t]{2}{*}{$\Delta \mathrm{BAE}_{\mathrm{rel}}$} & \multicolumn{2}{|c|}{$\mathrm{BAE}\left[\mathrm{kcal} \mathrm{mol}^{-1}\right]$} & \multirow[t]{2}{*}{$\Delta \mathrm{BAE}_{\mathrm{rel}}$} & \multicolumn{2}{|c|}{ BAE $\left[\mathrm{kcal} \mathrm{mol}^{-1}\right]$} & \multirow[t]{2}{*}{$\Delta \mathrm{BAE}_{\mathrm{rel}}$} \\
\hline & $\mathrm{BAE}_{\mathrm{comp}}$ & $\mathrm{BAE}_{\text {geo }}$ & & $\mathrm{BAE}_{\mathrm{comp}}$ & $\mathrm{BAE}_{\mathrm{geo}}$ & & $\mathrm{BAE}_{\mathrm{comp}}$ & $\mathrm{BAE}_{\mathrm{geo}}$ & \\
\hline 1-HF & -0.59 & -0.60 & $1.1 \%$ & -0.57 & -0.56 & $-2.5 \%$ & -0.57 & -0.55 & $-2.8 \%$ \\
\hline 2-HF & -1.09 & -1.21 & $11.0 \%$ & -1.01 & -1.14 & $13.0 \%$ & -1.02 & -1.14 & $11.6 \%$ \\
\hline $3-\mathrm{HF}$ & -1.46 & -1.77 & $20.8 \%$ & -1.38 & -1.65 & $19.4 \%$ & -1.45 & -1.81 & $24.2 \%$ \\
\hline $4-\mathrm{HF}$ & -2.20 & -2.33 & $5.8 \%$ & -2.08 & -2.16 & $4.1 \%$ & -2.13 & -2.29 & $7.3 \%$ \\
\hline 5-HF & -2.75 & -3.11 & $13.0 \%$ & -2.60 & -2.89 & $10.9 \%$ & -2.79 & -3.22 & $15.3 \%$ \\
\hline 6-HF & -3.19 & -3.68 & $15.5 \%$ & -3.02 & -3.41 & $12.7 \%$ & -3.22 & -3.82 & $18.6 \%$ \\
\hline Average & & & $11 \%$ & & & $10 \%$ & & & $12 \%$ \\
\hline
\end{tabular}


Tables S17-S19 show that, on average, the BIEs of HF complexes are roughly $10 \%$ weaker than estimated on the basis of the geometric mean rule. This has been observed experimentally on the basis of second interaction virial coefficients as well. ${ }^{170-172}$ The results in Table S18 suggest that the interaction distances seem to be somewhat longer in $\mathbf{H F}$ than would be expected on the basis of the interaction distances in $\mathbf{H H}$ and $\mathbf{F F}$. While the geometric mean rule is a commonly employed rule to estimate the strength of heteromolecular interactions on the basis of homomolecular interactions, there is no rigorous physical basis for it. The interaction of molecules interacting predominantly by dispersion can be estimated using the London dispersion formula. ${ }^{106,107}$ In the London dispersion formula, there is a combination rule for the ionization potentials of the interacting atoms and molecules, but it is not a geometric mean rule. Additionally, the geometric mean rule is commonly used to estimate heteroatomic $\mathrm{C}_{6}$ coefficients of interaction from homoatomic $\mathrm{C}_{6}$ coefficients of interaction because it empirically provides good estimates but not because of the underlying physical laws. Therefore, a priori, there is no reason that the geometric mean rule should describe the interactions in $\mathbf{H F}$ well. 


\section{Mathematical Derivations}

In this section mathematical derivations are presented and explained in detail. The corresponding results are applied to analyze and interpret the computational results.

\subsection{Homomolecular Cuboid Model for Interaction Surfaces}

The basic assumption is that the interacting molecules (linear alkanes and perfluoroalkanes) can be approximated as interacting cuboids (cf. Figure S167).

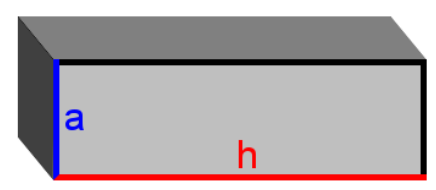

Figure S167: Cuboid model.

The total surface $(\mathrm{S})$ of the cuboid can be calculated as follows:

$$
S=2 a^{2}+4 a h
$$

The interaction surface corresponds to one of the long faces of the cuboid with the area:

$$
S_{\text {int }}=a h
$$

The length of the long face $(h)$ in this model is approximately a linear function of the length of the carbon chain and hence of the number of carbon atoms $(n)$ with $u$ and $v$ being the corresponding slope and intercept, respectively:

$$
h \approx u \cdot n+v
$$

It follows from the combination of equations S14 and S16 that the total surface is predicted to be a linear function (with intercept $q$ ) of the number of carbon atoms for each class 
of compounds (i.e. either $\mathbf{H}$ or $\mathbf{F}$ ):

$$
S \approx 2 a^{2}+4 a v+4 a n=4 a u \cdot n+q
$$

Combining equations S15 and S16 it can be seen that also the interaction surface is a linear function (with intercept $r$ ) of the number of carbon atoms with the slope being a quarter of the corresponding slope of the total surface.

$$
S_{\text {int }} \approx a u \cdot n+a v=a u \cdot n+r
$$

Therefore, on the basis of this simple model it can be shown that comparing the slope of the total surface of the isolated molecules to the slope of the interaction surface is a means to estimate whether the interaction surface is ideal or not. Additionally, when comparing the ratio of the slopes of the total surface of $\mathbf{H}$ and $\mathbf{F}$ against the ratio of the slopes of the interaction surface area of $\mathbf{H}$ and $\mathbf{F}$ provides a means to investigate whether $\mathbf{H}$ or $\mathbf{F}$ have a closer to ideal interaction surface. The application of this model has been shown in the Supplementary Results and Discussion section.

\subsection{Heteromolecular Cuboid Model for Interaction Surfaces}

The model discussed in the previous section is only valid when the two interacting molecules are identical, i.e. the interacting cuboids have the same size. When they do not, the interaction surface is not equal to the long face of either of the two molecules. This is illustrated in Figure S168.

The edge of the interaction surface $a_{i}$ can determined if we assume the following relationship:

$$
\frac{a_{1}}{a_{2}}=\frac{d_{1}}{d_{2}}
$$

Using the intercept theorem of elementary geometry we can derive the following relation- 


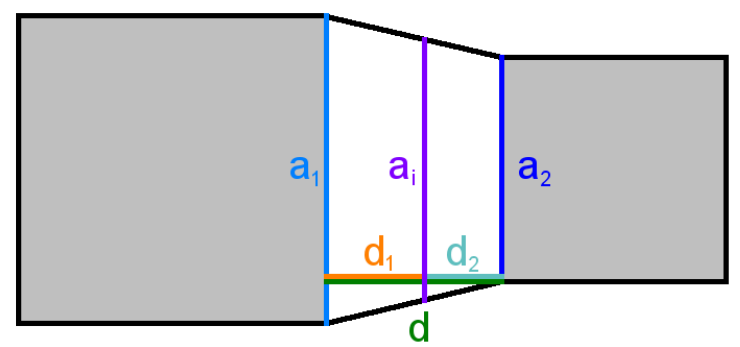

Figure S168: Cuboid model for heteromolecular interaction. View from the side on the volume of intercuboidal interaction.

ship:

$$
\frac{d_{1}}{a_{1}-a_{i}}=\frac{d_{2}}{a_{i}-a_{2}}
$$

Minor rearrangement leads to the following equation:

$$
\frac{d_{1}}{d_{2}}=\frac{a_{1}-a_{i}}{a_{i}-a_{2}}
$$

Combining equations S19 and S21 leads to:

$$
\frac{a_{1}}{a_{2}}=\frac{a_{1}-a_{i}}{a_{i}-a_{2}}
$$

Solving equation S22 for $a_{i}$ yields:

$$
\begin{gathered}
a_{1} a_{i}-a_{1} a_{2}=a_{1} a_{2}-a_{2} a_{i} \\
a_{i}\left(a_{1}+a_{2}\right)=2 a_{1} a_{2} \\
a_{i}=\frac{2 a_{1} a_{2}}{a_{1}+a_{2}}
\end{gathered}
$$

Using equation S25, $a_{i}$ can be estimated from both $a_{1}$ and $a_{2}$. Assuming that $a_{i}$ corre- 
sponds approximately to the slope of the interaction surface in heteromolecular complexes of $\mathbf{H}$ and $\mathbf{F}$ with respect $n$, it can be estimated how close to ideal the interaction geometries in the heteromolecular complexes are. The application of this model has been shown in the Supplementary Results and Discussion section. 


\section{Computational Raw Data}

In this section, all the computational results are provided in tables and the corresponding contents are explained briefly. In addition to the abbrevations used in the main text, we used abbrevations to refer to monomers at the dimer geometry. $\mathbf{H x} \mathbf{1}$ refers to the $\mathbf{H}$ molecule in either $\mathbf{H H 1}$ or $\mathbf{H F 1}$ at the dimer geometry, $\mathbf{x F} 2$ refers to the $\mathbf{F}$ molecule in either $\mathbf{H F} \mathbf{2}$ or FF2 at the dimer geometry. Analogous rules apply for all the additional abbreviations used. In the following tables, the corresponding dimer is always preceded by the respective monomers at the dimer geometries.

\subsection{Benchmarking}

The following tables provide the computational raw data for the benchmarking performed. E refers to the electronic energy, BAE refers to bond association energy, BIE refers to bond interaction energy and E-def refers to the deformation energy. 


\begin{tabular}{|c|c|c|c|c|c|c|c|c|}
\hline & \multicolumn{4}{|c|}{ DSD-PBEP86/def2-QZVP } & \multicolumn{4}{|c|}{ B97D3/def2-QZVP } \\
\hline & $\mathrm{E}[\mathrm{Ha}]$ & $\mathrm{BAE}\left[\mathrm{kcal} \mathrm{mol}^{-1}\right]$ & $\mathrm{BIE}\left[\mathrm{kcal} \mathrm{mol}^{-1}\right]$ & E-def $\left[\mathrm{kcal} \mathrm{mol}^{-1}\right]$ & $\mathrm{E}[\mathrm{Ha}]$ & $\mathrm{BAE}\left[\mathrm{kcal} \mathrm{mol}^{-1}\right]$ & BIE $\left[\mathrm{kcal} \mathrm{mol}^{-1}\right]$ & E-def $\left[\mathrm{kcal} \mathrm{mol}^{-1}\right]$ \\
\hline \multicolumn{9}{|c|}{ Methanes } \\
\hline $\mathrm{H}$ & -40.45340156171 & & & & -40.45340156171 & & & \\
\hline $\mathrm{F}$ & -437.174440690947 & & & & -437.174440690947 & & & \\
\hline Hx1 & -40.45330228048 & & & & -40.45330228048 & & & \\
\hline xH1 & -40.453285931633 & & & & -40.453285931633 & & & \\
\hline HH1 & -80.907464827431 & -0.415 & -0.55 & 0.135 & -80.907464827431 & -0.415 & -0.55 & 0.135 \\
\hline $\mathrm{Hx} 2$ & -40.453236937567 & & & & -40.453236937567 & & & \\
\hline $\mathrm{xH} 2$ & -40.45321606935 & & & & -40.45321606935 & & & \\
\hline $\mathrm{HH} 2$ & -80.907158820144 & -0.223 & -0.443 & 0.22 & -80.907158820144 & -0.223 & -0.443 & 0.22 \\
\hline $\mathrm{Hx} 1$ & -40.453289294462 & & & & -40.453289294462 & & & \\
\hline $\mathrm{xF} 1$ & -437.174380258028 & & & & -437.174380258028 & & & \\
\hline HF1 & -477.62881556709 & -0.611 & -0.719 & 0.108 & -477.62881556709 & -0.611 & -0.719 & 0.108 \\
\hline $\mathrm{Hx} 2$ & -40.45340085228 & & & & -40.45340085228 & & & \\
\hline $\mathrm{xF} 2$ & -437.174418523656 & & & & -437.174418523656 & & & \\
\hline $\mathrm{HF} 2$ & -477.628405703825 & -0.354 & -0.368 & 0.014 & -477.628405703825 & -0.354 & -0.368 & 0.014 \\
\hline Fx1 & -437.174376111767 & & & & -437.174376111767 & & & \\
\hline $\mathrm{xF} 1$ & -437.17436253555 & & & & -437.17436253555 & & & \\
\hline FF1 & -874.349672274866 & -0.496 & -0.586 & 0.09 & -874.349672274866 & -0.496 & -0.586 & 0.09 \\
\hline $\mathrm{Fx} 2$ & -437.174366684698 & & & & -437.174366684698 & & & \\
\hline $\mathrm{xF} 2$ & -437.174299461049 & & & & -437.174299461049 & & & \\
\hline $\mathrm{FF} 2$ & -874.349608232686 & -0.456 & -0.591 & 0.135 & -874.349608232686 & -0.456 & -0.591 & 0.135 \\
\hline \multicolumn{9}{|c|}{ Ethanes } \\
\hline $\mathrm{H}$ & -79.707934123378 & & & & -79.707934123378 & & & \\
\hline $\mathrm{F}$ & -674.775073674995 & & & & -674.775073674995 & & & \\
\hline $\mathrm{Hx} 1$ & -79.70770580573 & & & & -79.70770580573 & & & \\
\hline $\mathrm{xH} 1$ & -79.70758322253 & & & & -79.70758322253 & & & \\
\hline НH1 & -159.417497446015 & -1.022 & -1.386 & 0.363 & -159.417497446015 & -1.022 & -1.386 & 0.363 \\
\hline $\mathrm{Hx} 2$ & -79.707788275836 & & & & -79.707788275836 & & & \\
\hline $\mathrm{xH} 2$ & -79.70780953927 & & & & -79.70780953927 & & & \\
\hline $\mathrm{HH} 2$ & -159.417461471667 & -1 & -1.169 & 0.17 & -159.417461471667 & -1 & -1.169 & 0.17 \\
\hline $\mathrm{Hx} 1$ & -79.707701164699 & & & & -79.707701164699 & & & \\
\hline $\mathrm{xF} 1$ & -674.774994438047 & & & & -674.774994438047 & & & \\
\hline HF1 & -754.484186500296 & -0.74 & -0.936 & 0.196 & -754.484186500296 & -0.74 & -0.936 & 0.196 \\
\hline $\mathrm{Hx} 2$ & -79.707783090038 & & & & -79.707783090038 & & & \\
\hline $\mathrm{xF} 2$ & -674.774709820781 & & & & -674.774709820781 & & & \\
\hline $\mathrm{HF} 2$ & -754.484272866279 & -0.794 & -1.117 & 0.323 & -754.484272866279 & -0.794 & -1.117 & 0.323 \\
\hline $\mathrm{Hx} 3$ & -79.707711996956 & & & & -79.707711996956 & & & \\
\hline $\mathrm{xF} 3$ & -674.775069266901 & & & & -674.775069266901 & & & \\
\hline HF3 & -754.484063454228 & -0.662 & -0.805 & 0.142 & -754.484063454228 & -0.662 & -0.805 & 0.142 \\
\hline $\mathrm{Hx} 4$ & -79.707931884504 & & & & -79.707931884504 & & & \\
\hline $\mathrm{xF} 4$ & -674.77499362067 & & & & -674.77499362067 & & & \\
\hline $\mathrm{HF} 4$ & -754.484545931517 & -0.965 & -1.017 & 0.052 & -754.484545931517 & -0.965 & -1.017 & 0.052 \\
\hline $\mathrm{Fx} 1$ & -674.774912767867 & & & & -674.774912767867 & & & \\
\hline $\mathrm{xF} 1$ & -674.775027363192 & & & & -674.775027363192 & & & \\
\hline $\mathrm{FF} 1$ & -1349.551441293 & -0.812 & -0.942 & 0.13 & -1349.551441293 & -0.812 & -0.942 & 0.13 \\
\hline $\mathrm{Fx} 2$ & -674.774988794396 & & & & -674.774988794396 & & & \\
\hline $\mathrm{xF} 2$ & -674.775009188958 & & & & -674.775009188958 & & & \\
\hline $\mathrm{FF} 2$ & -1349.55119211352 & -0.656 & -0.749 & 0.094 & -1349.55119211352 & -0.656 & -0.749 & 0.094 \\
\hline
\end{tabular}




\begin{tabular}{|c|c|c|c|c|c|c|c|c|}
\hline & \multicolumn{4}{|c|}{ DSD-PBEP86/def2-QZVP } & \multicolumn{4}{|c|}{ B97D3/def2-QZVP } \\
\hline & $\mathrm{E}[\mathrm{Ha}]$ & $\mathrm{BAE}\left[\mathrm{kcal} \mathrm{mol}{ }^{-1}\right]$ & $\mathrm{BIE}\left[\mathrm{kcal} \mathrm{mol}^{-1}\right]$ & E-def $\left[\mathrm{kcal} \mathrm{mol}^{-1}\right]$ & $\mathrm{E}[\mathrm{Ha}]$ & $\mathrm{BAE}\left[\mathrm{kcal} \mathrm{mol}^{-1}\right]$ & $\mathrm{BIE}\left[\mathrm{kcal} \mathrm{mol}^{-1}\right]$ & E-def $\left[\mathrm{kcal} \mathrm{mol}^{-1}\right]$ \\
\hline \multicolumn{9}{|c|}{ Propanes } \\
\hline $\mathrm{H}$ & -118.9654774391 & & & & -119.122414400952 & & & \\
\hline $\mathrm{F}$ & -912.375873583156 & & & & -913.046964940022 & & & \\
\hline Hx1 & -118.965379676288 & & & & -119.122421422984 & & & \\
\hline $\mathrm{xH} 1$ & -118.965377856626 & & & & -119.122421602204 & & & \\
\hline HH1 & -237.934072723275 & -1.956 & -2.080 & 0.124 & -238.248439969699 & -2.266 & -2.257 & -0.009 \\
\hline $\mathrm{H} \times 2$ & -118.965384882435 & & & & -119.122432551183 & & & \\
\hline $\mathrm{xH} 2$ & -118.965388558935 & & & & -119.122421030765 & & & \\
\hline HH2 & -237.932811029255 & -1.165 & -1.279 & 0.114 & -238.247148535681 & -1.456 & -1.440 & -0.016 \\
\hline Hx1 & -118.965463991994 & & & & -119.122402248926 & & & \\
\hline $\mathrm{xF} 1$ & -912.375583166442 & & & & -913.04688980176 & & & \\
\hline HF1 & -1031.34352492383 & -1.364 & -1.555 & 0.191 & -1032.17109756073 & -1.078 & -1.133 & 0.055 \\
\hline $\mathrm{Hx} 2$ & -118.965640771484 & & & & -119.122412395297 & & & \\
\hline $\mathrm{xF} 2$ & -912.375407423381 & & & & -913.04699042111 & & & \\
\hline HF2 & -1031.3430212738 & -1.048 & -1.238 & 0.190 & -1032.17088793157 & -0.947 & -0.932 & -0.015 \\
\hline $\mathrm{Hx} 3$ & -118.965503780129 & & & & -119.122432528751 & & & \\
\hline $\mathrm{xF} 3$ & -912.375580479297 & & & & -913.046978971916 & & & \\
\hline HF3 & -1031.34301200984 & -1.042 & -1.210 & 0.167 & -1032.17111678507 & -1.090 & -1.070 & -0.020 \\
\hline $\mathrm{Hx} 4$ & -118.965583327392 & & & & -119.122405207464 & & & \\
\hline $\mathrm{xF} 4$ & -912.375365777955 & & & & -913.046941763001 & & & \\
\hline $\mathrm{HF} 4$ & -1031.34288682525 & -0.964 & -1.216 & 0.252 & -1032.17046407035 & -0.681 & -0.701 & 0.020 \\
\hline Fx1 & -912.375534725034 & & & & -913.046697884011 & & & \\
\hline $\mathrm{xF} 1$ & -912.375592306062 & & & & -913.046845007231 & & & \\
\hline FF1 & -1824.75336996114 & -1.018 & -1.407 & 0.389 & -1826.09418060133 & -0.157 & -0.400 & 0.243 \\
\hline $\mathrm{Fx} 2$ & -912.375833319941 & & & & -913.04695868885 & & & \\
\hline $\mathrm{xF} 2$ & -912.375818181067 & & & & -913.04695662918 & & & \\
\hline FF2 & -1824.75409611851 & -1.474 & -1.534 & 0.060 & -1826.09402560567 & -0.060 & -0.069 & 0.009 \\
\hline \multicolumn{9}{|c|}{ Butanes } \\
\hline $\mathrm{H}$ & -158.223459069769 & & & & -158.427561314396 & & & \\
\hline $\mathrm{F}$ & -1149.97602431515 & & & & -1150.8224663123 & & & \\
\hline $\mathrm{Hx} 1$ & -158.223116062729 & & & & -158.42755830191 & & & \\
\hline $\mathrm{xH} 1$ & -158.223173998622 & & & & -158.427569097518 & & & \\
\hline HH1 & -316.451027070645 & -2.578 & -2.973 & 0.394 & -316.860269979008 & -3.230 & -3.227 & -0.003 \\
\hline $\mathrm{H} \times 2$ & -158.223101087948 & & & & -158.427551787638 & & & \\
\hline $\mathrm{xH} 2$ & -158.223116907561 & & & & -158.427574181297 & & & \\
\hline HH2 & -316.449006884695 & -1.311 & -1.750 & 0.439 & -316.858282598001 & -1.983 & -1.981 & -0.002 \\
\hline $\mathrm{Hx} 1$ & -158.223390696939 & & & & -158.427561201376 & & & \\
\hline $\mathrm{xF} 1$ & -1149.97568757908 & & & & -1150.82248685748 & & & \\
\hline HF1 & -1308.20227841443 & -1.754 & -2.008 & 0.254 & -1309.25247799235 & -1.538 & -1.525 & -0.013 \\
\hline $\mathrm{Hx} 2$ & -158.223435367015 & & & & -158.42754573836 & & & \\
\hline $\mathrm{xF} 2$ & -1149.97598112465 & & & & -1150.82252645239 & & & \\
\hline HF2 & -1308.20255606827 & -1.928 & -1.970 & 0.042 & -1309.25232596798 & -1.442 & -1.414 & -0.028 \\
\hline $\mathrm{Hx} 3$ & -158.223328472696 & & & & -158.427517788679 & & & \\
\hline $\mathrm{xF} 3$ & -1149.97591924557 & & & & -1150.82258830489 & & & \\
\hline HF3 & -1308.20242964852 & -1.849 & -1.997 & 0.148 & -1309.25210539396 & -1.304 & -1.255 & -0.049 \\
\hline $\mathrm{Hx} 4$ & -158.22332087725 & & & & -158.427513693935 & & & \\
\hline $\mathrm{xF} 4$ & -1149.97605430886 & & & & -1150.82252023264 & & & \\
\hline $\mathrm{HF} 4$ & -1308.20254264709 & -1.920 & -1.988 & 0.068 & -1309.2524059484 & -1.492 & -1.488 & -0.004 \\
\hline $\mathrm{Fx} 1$ & -1149.97602564114 & & & & -1150.82251030736 & & & \\
\hline $\mathrm{xF} 1$ & -1149.97596481922 & & & & -1150.82242178566 & & & \\
\hline $\mathrm{FF} 1$ & -2299.9547418453 & -1.690 & -1.727 & 0.037 & -2301.64654471567 & -1.012 & -1.012 & 0.000 \\
\hline $\mathrm{Fx} 2$ & -1149.97593439025 & & & & -1150.82260514959 & & & \\
\hline $\mathrm{xF} 2$ & -1149.97592921202 & & & & -1150.8225410968 & & & \\
\hline FF2 & -2299.95485520245 & -1.761 & -1.877 & 0.116 & -2301.64627839915 & -0.844 & -0.710 & -0.134 \\
\hline
\end{tabular}




\begin{tabular}{|c|c|c|c|c|c|c|c|c|}
\hline & \multicolumn{4}{|c|}{ DSD-PBEP86/def2-QZVP } & \multicolumn{4}{|c|}{ B97D3/def2-QZVP } \\
\hline & $\mathrm{E}[\mathrm{Ha}]$ & $\mathrm{BAE}\left[\mathrm{kcal} \mathrm{mol}^{-1}\right]$ & $\mathrm{BIE}\left[\mathrm{kcal} \mathrm{mol}^{-1}\right]$ & E-def $\left[\mathrm{kcal} \mathrm{mol}^{-1}\right]$ & $\mathrm{E}[\mathrm{Ha}]$ & $\mathrm{BAE}\left[\mathrm{kcal} \mathrm{mol}^{-1}\right]$ & $\mathrm{BIE}\left[\mathrm{kcal} \mathrm{mol}^{-1}\right]$ & E-def $\left[\mathrm{kcal} \mathrm{mol}^{-1}\right]$ \\
\hline \multicolumn{9}{|c|}{ Pentanes } \\
\hline H & -197.481196827152 & & & & -197.732670441897 & & & \\
\hline $\mathrm{F}$ & -1387.57653321274 & & & & -1388.59812906709 & & & \\
\hline $\mathrm{Hx} 1$ & -197.48084504907 & & & & -197.732653420579 & & & \\
\hline $\mathrm{xH} 1$ & -197.480842571138 & & & & -197.732653309355 & & & \\
\hline нH1 & -394.967838590586 & -3.417 & -3.860 & 0.443 & -395.471991778568 & -4.173 & -4.195 & 0.021 \\
\hline $\mathrm{Hx} 2$ & -197.480766159988 & & & & -197.732681313157 & & & \\
\hline $\mathrm{xH} 2$ & -197.480773210228 & & & & -197.732681237827 & & & \\
\hline нH2 & -394.96525551959 & -1.796 & -2.332 & 0.536 & -395.469408219742 & -2.552 & -2.539 & -0.014 \\
\hline Hx1 & -197.48102673258 & & & & -197.732566918995 & & & \\
\hline $\mathrm{xF} 1$ & -1387.57639097769 & & & & -1388.59823044576 & & & \\
\hline HF1 & -1585.06157430192 & -2.412 & -2.608 & 0.196 & -1586.33376971721 & -1.864 & -1.865 & 0.001 \\
\hline $\mathrm{Hx} 2$ & -197.481153673457 & & & & -197.732635773032 & & & \\
\hline $\mathrm{xF} 2$ & -1387.57613843331 & & & & -1388.59797138019 & & & \\
\hline $\mathrm{HF} 2$ & -1585.06106681064 & -2.094 & -2.369 & 0.275 & -1586.33356768505 & -1.737 & -1.858 & 0.121 \\
\hline $\mathrm{Hx} 3$ & -197.481031793051 & & & & -197.732648518033 & & & \\
\hline $\mathrm{xF} 3$ & -1387.57641977966 & & & & -1388.59831798437 & & & \\
\hline HF3 & -1585.06118575447 & -2.168 & -2.343 & 0.175 & -1586.33360756567 & -1.762 & -1.657 & -0.105 \\
\hline $\mathrm{Hx} 4$ & -197.48109400322 & & & & -197.732632167301 & & & \\
\hline $\mathrm{xF} 4$ & -1387.57654488909 & & & & -1388.59823416254 & & & \\
\hline $\mathrm{HF} 4$ & -1585.06194506742 & -2.645 & -2.702 & 0.057 & -1586.3339052928 & -1.949 & -1.907 & -0.042 \\
\hline $\mathrm{Fx} 1$ & -1387.57643673039 & & & & -1388.59813354821 & & & \\
\hline $\mathrm{xF} 1$ & -1387.57640690226 & & & & -1388.59820175132 & & & \\
\hline $\mathrm{FF} 1$ & -2775.15676607437 & -2.322 & -2.461 & 0.140 & -2777.19769201507 & -0.900 & -0.851 & -0.048 \\
\hline $\mathrm{Fx} 2$ & -1387.5764108988 & & & & -1388.59810632768 & & & \\
\hline $\mathrm{xF} 2$ & -1387.57641498716 & & & & -1388.59811001759 & & & \\
\hline $\mathrm{FF} 2$ & -2775.15644650798 & -2.121 & -2.272 & 0.151 & -2777.19778724243 & -0.960 & -0.986 & 0.026 \\
\hline \multicolumn{9}{|c|}{ Hexanes } \\
\hline $\mathrm{H}$ & -236.738918710099 & & & & -237.037787409713 & & & \\
\hline $\mathrm{F}$ & -1625.17699658677 & & & & -1626.37391599793 & & & \\
\hline $\mathrm{Hx} 1$ & -236.738537546223 & & & & -237.037763704464 & & & \\
\hline $\mathrm{xH} 1$ & -236.73854118593 & & & & -237.037763465984 & & & \\
\hline HH1 & -473.484100704068 & -3.930 & -4.406 & 0.476 & -474.083371389092 & -4.892 & -4.922 & 0.030 \\
\hline $\mathrm{Hx} 2$ & -236.738444547433 & & & & -237.037801583698 & & & \\
\hline $\mathrm{xH} 2$ & -236.73842660549 & & & & -237.037801435229 & & & \\
\hline нH2 & -473.481152583191 & -2.080 & -2.687 & 0.606 & -474.080606193581 & -3.157 & -3.140 & -0.018 \\
\hline $\mathrm{Hx} 1$ & -236.738619938236 & & & & -237.037598539951 & & & \\
\hline $\mathrm{xF} 1$ & -1625.17631682686 & & & & -1626.373687184 & & & \\
\hline HF1 & -1861.91974242161 & -2.402 & -3.016 & 0.614 & -1863.41438394275 & -1.682 & -1.944 & 0.262 \\
\hline $\mathrm{Hx} 2$ & -236.738827977027 & & & & -237.037692122121 & & & \\
\hline $\mathrm{xF} 2$ & -1625.17650739874 & & & & -1626.37373561144 & & & \\
\hline $\mathrm{HF} 2$ & -1861.92002226058 & -2.577 & -2.941 & 0.364 & -1863.4150261913 & -2.085 & -2.258 & 0.173 \\
\hline $\mathrm{Hx} 3$ & -236.738635432864 & & & & -237.037659748394 & & & \\
\hline $\mathrm{xF} 3$ & -1625.17659352015 & & & & -1626.37365800472 & & & \\
\hline HF3 & -1861.91988138564 & -2.489 & -2.919 & 0.431 & -1863.41456568502 & -1.796 & -2.038 & 0.242 \\
\hline $\mathrm{Hx} 4$ & -236.73889737329 & & & & -237.037778039017 & & & \\
\hline $\mathrm{xF} 4$ & -1625.17695873229 & & & & -1626.37392881256 & & & \\
\hline $\mathrm{HF} 4$ & -1861.92063080005 & -2.959 & -2.996 & 0.037 & -1863.41531372376 & -2.266 & -2.263 & -0.002 \\
\hline $\mathrm{Fx} 1$ & -1625.17690990042 & & & & -1626.37384278893 & & & \\
\hline $\mathrm{xF} 1$ & -1625.17682451672 & & & & -1626.37384287734 & & & \\
\hline FF1 & -3250.35887153017 & -3.061 & -3.224 & 0.162 & -3252.74909255155 & -0.791 & -0.883 & 0.092 \\
\hline $\mathrm{Fx} 2$ & -1625.17684427736 & & & & -1626.37360981283 & & & \\
\hline $\mathrm{xF} 2$ & -1625.17684445309 & & & & -1626.37361437007 & & & \\
\hline $\mathrm{FF} 2$ & -3250.35718730193 & -2.004 & -2.195 & 0.191 & -3252.74774948449 & 0.052 & -0.330 & 0.381 \\
\hline
\end{tabular}




\begin{tabular}{|c|c|c|c|c|c|c|c|c|}
\hline & \multicolumn{4}{|c|}{ BLYP-D3/def2-QZVP } & \multicolumn{4}{|c|}{ BLYP-XDM/aug-cc-pVTZ } \\
\hline & $\mathrm{E}[\mathrm{Ha}]$ & $\mathrm{BAE}\left[\mathrm{kcal} \mathrm{mol}^{-1}\right]$ & $\mathrm{BIE}\left[\mathrm{kcal} \mathrm{mol}^{-1}\right]$ & E-def $\left[\mathrm{kcal} \mathrm{mol}^{-1}\right]$ & $\mathrm{E}[\mathrm{Ha}]$ & $\mathrm{BAE}\left[\mathrm{kcal} \mathrm{mol}^{-1}\right]$ & $\mathrm{BIE}\left[\mathrm{kcal} \mathrm{mol}^{-1}\right]$ & E-def $\left[\mathrm{kcal} \mathrm{mol}^{-1}\right]$ \\
\hline \multicolumn{9}{|c|}{ Methanes } \\
\hline $\mathrm{H}$ & -40.507666231781 & & & & -40.50408117046 & & & \\
\hline $\mathrm{F}$ & -437.680273179664 & & & & -437.6410851716 & & & \\
\hline $\mathrm{Hx} 1$ & -40.507663793825 & & & & -40.50407877668 & & & \\
\hline $\mathrm{xH} 1$ & -40.507663886296 & & & & -40.50407868563 & & & \\
\hline HH1 & -81.015929112992 & -0.374 & -0.377 & 0.003 & -81.00886638346 & -0.442 & -0.445 & 0.003 \\
\hline $\mathrm{Hx} 2$ & -40.50766597435 & & & & -40.50408190086 & & & \\
\hline $\mathrm{xH} 2$ & -40.507665958799 & & & & -40.50408235675 & & & \\
\hline $\mathrm{HH} 2$ & -81.015789170723 & -0.287 & -0.287 & 0.000 & -81.00864152956 & -0.301 & -0.299 & -0.001 \\
\hline $\mathrm{Hx} 1$ & -40.507665604689 & & & & -40.5040816505 & & & \\
\hline $\mathrm{xF} 1$ & -437.680354043638 & & & & -437.6411710577 & & & \\
\hline HF1 & -478.188747279681 & -0.507 & -0.457 & -0.050 & -478.1463467596 & -0.741 & -0.687 & -0.054 \\
\hline $\mathrm{Hx} 2$ & -40.507664068776 & & & & -40.50407954161 & & & \\
\hline $\mathrm{xF} 2$ & -437.680330454236 & & & & -437.6411447594 & & & \\
\hline HF2 & -478.188337704603 & -0.250 & -0.215 & -0.035 & -478.1457650304 & -0.376 & -0.339 & -0.036 \\
\hline Fx1 & -437.680320096013 & & & & -437.641134048 & & & \\
\hline $\mathrm{xF} 1$ & -437.680326661296 & & & & -437.641141319 & & & \\
\hline FF1 & -875.361278746623 & -0.460 & -0.397 & -0.063 & -875.2832595794 & -0.684 & -0.618 & -0.066 \\
\hline $\mathrm{Fx} 2$ & -437.680335021164 & & & & -437.6411522329 & & & \\
\hline $\mathrm{xF} 2$ & -437.680326358207 & & & & -437.6411422 & & & \\
\hline $\mathrm{FF} 2$ & -875.361199598805 & -0.410 & -0.338 & -0.072 & -875.2832100158 & -0.652 & -0.575 & -0.078 \\
\hline \multicolumn{9}{|c|}{ Ethanes } \\
\hline $\mathrm{H}$ & -79.815156616873 & & & & -79.80889741932 & & & \\
\hline $\mathrm{F}$ & -675.564112602858 & & & & -675.5041374099 & & & \\
\hline $\mathrm{Hx} 1$ & -79.815162341258 & & & & -79.80889965725 & & & \\
\hline $\mathrm{xH} 1$ & -79.815163018095 & & & & -79.80890186263 & & & \\
\hline HH1 & -159.632203093962 & -1.186 & -1.178 & -0.008 & -159.6199030076 & -1.323 & -1.319 & -0.004 \\
\hline $\mathrm{Hx} 2$ & -79.815158838352 & & & & -79.80889916564 & & & \\
\hline $\mathrm{xH} 2$ & -79.815159006277 & & & & -79.80889969434 & & & \\
\hline $\mathrm{HH} 2$ & -159.631993953908 & -1.055 & -1.052 & -0.003 & -159.6195886958 & -1.126 & -1.123 & -0.003 \\
\hline $\mathrm{Hx} 1$ & -79.815152287801 & & & & -79.80888922865 & & & \\
\hline $\mathrm{xF} 1$ & -675.564262678841 & & & & -675.5042854962 & & & \\
\hline $\mathrm{HF} 1$ & -755.380463708674 & -0.750 & -0.658 & -0.091 & -755.3146480048 & -1.012 & -0.924 & -0.088 \\
\hline $\mathrm{Hx} 2$ & -79.815154087394 & & & & -79.80889036107 & & & \\
\hline $\mathrm{xF} 2$ & -675.564294035234 & & & & -675.5043481975 & & & \\
\hline $\mathrm{HF} 2$ & -755.380730899065 & -0.917 & -0.805 & -0.112 & -755.3149848408 & -1.224 & -1.096 & -0.128 \\
\hline $\mathrm{Hx} 3$ & -79.815154450476 & & & & -79.80889115451 & & & \\
\hline $\mathrm{xF} 3$ & -675.564252803315 & & & & -675.5042672149 & & & \\
\hline HF3 & -755.380298349712 & -0.646 & -0.559 & -0.087 & -755.3144115428 & -0.864 & -0.786 & -0.078 \\
\hline $\mathrm{Hx} 4$ & -79.815153948316 & & & & -79.80888878577 & & & \\
\hline $\mathrm{xF} 4$ & -675.564202388235 & & & & -675.5042288107 & & & \\
\hline $\mathrm{HF} 4$ & -755.380531315403 & -0.792 & -0.737 & -0.055 & -755.3147866407 & -1.099 & -1.047 & -0.052 \\
\hline Fx1 & -675.564189783414 & & & & -675.5042101185 & & & \\
\hline $\mathrm{xF} 1$ & -675.564154778769 & & & & -675.5041725866 & & & \\
\hline $\mathrm{FF} 1$ & -1351.12925010851 & -0.643 & -0.568 & -0.075 & -1351.009942449 & -1.046 & -0.979 & -0.068 \\
\hline $\mathrm{Fx} 2$ & -675.564291496695 & & & & -675.504318521 & & & \\
\hline $\mathrm{xF} 2$ & -675.564181338857 & & & & -675.5041993049 & & & \\
\hline FF2 & -1351.1292612544 & -0.650 & -0.495 & -0.155 & -1351.009797189 & -0.955 & -0.803 & -0.152 \\
\hline
\end{tabular}




\begin{tabular}{|c|c|c|c|c|c|c|c|c|}
\hline & \multicolumn{4}{|c|}{ BLYP-D3/def2-QZVP } & \multicolumn{4}{|c|}{ BLYP-XDM/aug-cc-pVTZ } \\
\hline & $\mathrm{E}[\mathrm{Ha}]$ & $\mathrm{BAE}\left[\mathrm{kcal} \mathrm{mol}^{-1}\right]$ & $\mathrm{BIE}\left[\mathrm{kcal} \mathrm{mol}^{-1}\right]$ & E-def $\left[\mathrm{kcal} \mathrm{mol}^{-1}\right]$ & $\mathrm{E}[\mathrm{Ha}]$ & $\mathrm{BAE}\left[\mathrm{kcal} \mathrm{mol}^{-1}\right]$ & $\mathrm{BIE}\left[\mathrm{kcal} \mathrm{mol}^{-1}\right]$ & E-def $\left[\mathrm{kcal} \mathrm{mol}^{-1}\right]$ \\
\hline \multicolumn{9}{|c|}{ Propanes } \\
\hline $\mathrm{H}$ & -119.125618426848 & & & & -119.1166834021 & & & \\
\hline $\mathrm{F}$ & -913.446564564769 & & & & -913.365986134 & & & \\
\hline $\mathrm{Hx} 1$ & -119.125627103176 & & & & -119.1166912458 & & & \\
\hline $\mathrm{xH} 1$ & -119.125627538997 & & & & -119.1166921312 & & & \\
\hline HH1 & -238.254424509273 & -2.000 & -1.989 & -0.011 & -238.2368344256 & -2.176 & -2.166 & -0.010 \\
\hline $\mathrm{Hx} 2$ & -119.125645006767 & & & & -119.116707429 & & & \\
\hline $\mathrm{xH} 2$ & -119.125622640156 & & & & -119.1166834927 & & & \\
\hline $\mathrm{HH} 2$ & -238.253003046856 & -1.108 & -1.089 & -0.019 & -238.2351615894 & -1.126 & -1.111 & -0.015 \\
\hline $\mathrm{Hx} 1$ & -119.125602589088 & & & & -119.1166668358 & & & \\
\hline $\mathrm{xF} 1$ & -913.446550638558 & & & & -913.3659887147 & & & \\
\hline HF1 & -1032.5740183928 & -1.152 & -1.170 & 0.019 & -1032.485205206 & -1.591 & -1.600 & 0.009 \\
\hline $\mathrm{Hx} 2$ & -119.125616379723 & & & & -119.1166820355 & & & \\
\hline $\mathrm{xF} 2$ & -913.446663601517 & & & & -913.3661207528 & & & \\
\hline $\mathrm{HF} 2$ & -1032.57375782922 & -0.988 & -0.927 & -0.061 & -1032.484858136 & -1.373 & -1.290 & -0.084 \\
\hline $\mathrm{Hx} 3$ & -119.125646375114 & & & & -119.1167186599 & & & \\
\hline $\mathrm{xF} 3$ & -913.446657677995 & & & & -913.3661003844 & & & \\
\hline HF3 & -1032.57382843001 & -1.033 & -0.957 & -0.076 & -1032.484740837 & -1.300 & -1.206 & -0.094 \\
\hline $\mathrm{Hx} 4$ & -119.125607920419 & & & & -119.1166719854 & & & \\
\hline $\mathrm{xF} 4$ & -913.446620964252 & & & & -913.3660937807 & & & \\
\hline $\mathrm{HF} 4$ & -1032.57354083488 & -0.852 & -0.823 & -0.029 & -1032.484726896 & -1.291 & -1.231 & -0.060 \\
\hline $\mathrm{Fx} 1$ & -913.446292609158 & & & & -913.3657165162 & & & \\
\hline $\mathrm{xF} 1$ & -913.446456657543 & & & & -913.3659003131 & & & \\
\hline FF1 & -1826.89399714866 & -0.545 & -0.783 & 0.238 & -1826.733914083 & -1.219 & -1.442 & 0.223 \\
\hline $\mathrm{Fx} 2$ & -913.446557634289 & & & & -913.3659802527 & & & \\
\hline $\mathrm{xF} 2$ & -913.446553569967 & & & & -913.3659759849 & & & \\
\hline $\mathrm{FF} 2$ & -1826.89424203296 & -0.698 & -0.710 & 0.011 & -1826.734432243 & -1.544 & -1.554 & 0.010 \\
\hline \multicolumn{9}{|c|}{ Butanes } \\
\hline $\mathrm{H}$ & -158.436115915613 & & & & -158.4243687149 & & & \\
\hline $\mathrm{F}$ & -1151.32811369802 & & & & -1151.227134462 & & & \\
\hline $\mathrm{Hx} 1$ & -158.436111588814 & & & & -158.4243689258 & & & \\
\hline $\mathrm{xH} 1$ & -158.43612380173 & & & & -158.4243712635 & & & \\
\hline HH1 & -316.87685861193 & -2.903 & -2.901 & -0.002 & -316.8537749143 & -3.161 & -3.159 & -0.002 \\
\hline $\mathrm{Hx} 2$ & -158.436103500595 & & & & -158.4243560167 & & & \\
\hline $\mathrm{xH} 2$ & -158.436132747508 & & & & -158.4243833382 & & & \\
\hline $\mathrm{HH} 2$ & -316.874707176155 & -1.553 & -1.551 & -0.003 & -316.8512993621 & -1.608 & -1.606 & -0.001 \\
\hline $\mathrm{Hx} 1$ & -158.43611873873 & & & & -158.4243708947 & & & \\
\hline $\mathrm{xF} 1$ & -1151.32822958988 & & & & -1151.227208538 & & & \\
\hline HF1 & -1309.767022718 & -1.753 & -1.678 & -0.074 & -1309.655076815 & -2.242 & -2.195 & -0.048 \\
\hline $\mathrm{Hx} 2$ & -158.436103097384 & & & & -158.4243552504 & & & \\
\hline $\mathrm{xF} 2$ & -1151.32818601543 & & & & -1151.227193342 & & & \\
\hline HF2 & -1309.76678996666 & -1.607 & -1.569 & -0.037 & -1309.654930862 & -2.151 & -2.122 & -0.028 \\
\hline $\mathrm{Hx} 3$ & -158.4360622433 & & & & -158.4243063835 & & & \\
\hline $\mathrm{xF} 3$ & -1151.32824295549 & & & & -1151.227238587 & & & \\
\hline HF3 & -1309.76670931223 & -1.556 & -1.509 & -0.047 & -1309.654970275 & -2.176 & -2.149 & -0.026 \\
\hline $\mathrm{Hx} 4$ & -158.436064924797 & & & & -158.4243135422 & & & \\
\hline $\mathrm{xF} 4$ & -1151.32816625779 & & & & -1151.227171408 & & & \\
\hline $\mathrm{HF} 4$ & -1309.7667372783 & -1.574 & -1.573 & -0.001 & -1309.654885856 & -2.123 & -2.134 & 0.011 \\
\hline Fx1 & -1151.32812852056 & & & & -1151.227125241 & & & \\
\hline $\mathrm{xF} 1$ & -1151.32812130777 & & & & -1151.227165941 & & & \\
\hline FF1 & -2302.65820751612 & -1.243 & -1.228 & -0.014 & -2302.457352433 & -1.935 & -1.921 & -0.014 \\
\hline $\mathrm{Fx} 2$ & -1151.32828113473 & & & & -1151.227264197 & & & \\
\hline $\mathrm{xF} 2$ & -1151.32822586146 & & & & -1151.227214535 & & & \\
\hline FF2 & -2302.65831427216 & -1.310 & -1.134 & -0.175 & -2302.457714031 & -2.162 & -2.030 & -0.132 \\
\hline
\end{tabular}




\begin{tabular}{|c|c|c|c|c|c|c|c|c|}
\hline & \multicolumn{4}{|c|}{ BLYP-D3/def2-QZVP } & \multicolumn{4}{|c|}{ BLYP-XDM/aug-cc-pVTZ } \\
\hline & $\mathrm{E}[\mathrm{Ha}]$ & $\mathrm{BAE}\left[\mathrm{kcal} \mathrm{mol}^{-1}\right]$ & $\mathrm{BIE}\left[\mathrm{kcal} \mathrm{mol}^{-1}\right]$ & E-def $\left[\mathrm{kcal} \mathrm{mol}^{-1}\right]$ & $\mathrm{E}[\mathrm{Ha}]$ & $\mathrm{BAE}\left[\mathrm{kcal} \mathrm{mol}^{-1}\right]$ & $\mathrm{BIE}\left[\mathrm{kcal} \mathrm{mol}^{-1}\right]$ & E-def $\left[\mathrm{kcal} \mathrm{mol}^{-1}\right]$ \\
\hline \multicolumn{9}{|c|}{ Pentanes } \\
\hline H & -197.445522623464 & & & & -197.57021659243 & & & \\
\hline $\mathrm{F}$ & -1388.22912666694 & & & & -1388.00768686124 & & & \\
\hline $\mathrm{Hx} 1$ & -197.445500380936 & & & & -197.570199738717 & & & \\
\hline $\mathrm{xH} 1$ & -197.445500224481 & & & & -197.570199641368 & & & \\
\hline нH1 & -394.896884801564 & -3.664 & -3.692 & 0.028 & -395.146708964698 & -3.938 & -3.959 & 0.021 \\
\hline $\mathrm{Hx} 2$ & -197.445536493822 & & & & -197.570225693725 & & & \\
\hline $\mathrm{xH} 2$ & -197.44553642235 & & & & -197.570225603214 & & & \\
\hline нH2 & -394.894059851211 & -1.892 & -1.874 & -0.017 & -395.144494463869 & -2.548 & -2.537 & -0.011 \\
\hline Hx1 & -197.44542411314 & & & & -197.57010880511 & & & \\
\hline $\mathrm{xF} 1$ & -1388.22915542896 & & & & -1388.00771916376 & & & \\
\hline HF1 & -1585.68016328151 & -3.460 & -3.504 & 0.044 & -1585.58191489409 & -2.517 & -2.565 & 0.047 \\
\hline $\mathrm{Hx} 2$ & -197.445491204696 & & & & -197.570178336334 & & & \\
\hline $\mathrm{xF} 2$ & -1388.22894038678 & & & & -1388.00749476257 & & & \\
\hline $\mathrm{HF} 2$ & -1585.67945047163 & -3.013 & -3.149 & 0.137 & -1585.58155331671 & -2.290 & -2.435 & 0.145 \\
\hline $\mathrm{Hx} 3$ & -197.445487208859 & & & & -197.570185351125 & & & \\
\hline $\mathrm{xF} 3$ & -1388.2292550995 & & & & -1388.00782045027 & & & \\
\hline HF3 & -1585.6797459737 & -3.198 & -3.140 & -0.058 & -1585.58168345636 & -2.372 & -2.308 & -0.064 \\
\hline $\mathrm{Hx} 4$ & -197.445476716655 & & & & -197.57016950943 & & & \\
\hline $\mathrm{xF} 4$ & -1388.22919705012 & & & & -1388.00775596823 & & & \\
\hline $\mathrm{HF} 4$ & -1585.68047047778 & -3.653 & -3.637 & -0.015 & -1585.58219783442 & -2.695 & -2.681 & -0.014 \\
\hline $\mathrm{Fx} 1$ & -1388.22912719251 & & & & -1388.00767315185 & & & \\
\hline $\mathrm{xF} 1$ & -1388.2291652788 & & & & -1388.00771565856 & & & \\
\hline $\mathrm{FF} 1$ & -2776.46517364352 & -4.343 & -4.318 & -0.025 & -2776.01869143357 & -2.082 & -2.072 & -0.009 \\
\hline $\mathrm{Fx} 2$ & -1388.22905185176 & & & & -1388.00764289579 & & & \\
\hline $\mathrm{xF} 2$ & -1388.22905941403 & & & & -1388.00764784066 & & & \\
\hline $\mathrm{FF} 2$ & -2776.46428185928 & -3.783 & -3.872 & 0.089 & -2776.01853050655 & -1.981 & -2.033 & 0.052 \\
\hline \multicolumn{9}{|c|}{ Hexanes } \\
\hline $\mathrm{H}$ & -236.699295041123 & & & & -236.846336404762 & & & \\
\hline $\mathrm{F}$ & -1625.94218900133 & & & & -1625.68361908786 & & & \\
\hline $\mathrm{Hx} 1$ & -236.699265179933 & & & & -236.846316725267 & & & \\
\hline $\mathrm{xH} 1$ & -236.699264784254 & & & & -236.846316384078 & & & \\
\hline HH1 & -473.405643134184 & -4.426 & -4.464 & 0.038 & -473.699986719555 & -4.590 & -4.614 & 0.025 \\
\hline $\mathrm{Hx} 2$ & -236.699308990341 & & & & -236.846346638323 & & & \\
\hline $\mathrm{xH} 2$ & -236.699308885483 & & & & -236.846346514905 & & & \\
\hline нH2 & -473.402553715556 & -2.487 & -2.470 & -0.017 & -473.697531634804 & -3.049 & -3.036 & -0.013 \\
\hline $\mathrm{Hx} 1$ & -236.699089660894 & & & & -236.846138988141 & & & \\
\hline $\mathrm{xF} 1$ & -1625.94185271133 & & & & -1625.68330656475 & & & \\
\hline HF1 & -1862.64774318473 & -3.928 & -4.268 & 0.340 & -1862.53404066957 & -2.563 & -2.883 & 0.320 \\
\hline $\mathrm{Hx} 2$ & -236.699196616116 & & & & -236.84624436968 & & & \\
\hline $\mathrm{xF} 2$ & -1625.94194449193 & & & & -1625.68338448685 & & & \\
\hline $\mathrm{HF} 2$ & -1862.64751687209 & -3.786 & -4.001 & 0.215 & -1862.53440114756 & -2.790 & -2.995 & 0.205 \\
\hline $\mathrm{Hx} 3$ & -236.699177057735 & & & & -236.846202507192 & & & \\
\hline $\mathrm{xF} 3$ & -1625.94191343566 & & & & -1625.68335515976 & & & \\
\hline HF3 & -1862.64771262641 & -3.908 & -4.155 & 0.247 & -1862.53421875789 & -2.675 & -2.925 & 0.250 \\
\hline $\mathrm{Hx} 4$ & -236.699286156833 & & & & -236.846318193451 & & & \\
\hline $\mathrm{xF} 4$ & -1625.94218207656 & & & & -1625.68360831792 & & & \\
\hline $\mathrm{HF} 4$ & -1862.64808895805 & -4.145 & -4.155 & 0.010 & -1862.53480090783 & -3.041 & -3.059 & 0.018 \\
\hline $\mathrm{Fx} 1$ & -1625.94210998791 & & & & -1625.68353590655 & & & \\
\hline $\mathrm{xF} 1$ & -1625.94211897862 & & & & -1625.68355150013 & & & \\
\hline FF1 & -3251.89344391726 & -5.689 & -5.782 & 0.094 & -3251.37108429254 & -2.413 & -2.508 & 0.095 \\
\hline $\mathrm{Fx} 2$ & -1625.94197145041 & & & & -1625.68339492751 & & & \\
\hline $\mathrm{xF} 2$ & -1625.94197372558 & & & & -1625.68339760666 & & & \\
\hline $\mathrm{FF} 2$ & -3251.89053102413 & -3.861 & -4.133 & 0.272 & -3251.36963231621 & -1.502 & -1.782 & 0.280 \\
\hline
\end{tabular}




\begin{tabular}{|c|c|c|c|c|c|c|c|c|}
\hline & \multicolumn{4}{|c|}{ BLYP-NL/def2-QZVP } & \multicolumn{4}{|c|}{ PBE-D3/def2-QZVP } \\
\hline & $\mathrm{E}[\mathrm{Ha}]$ & $\mathrm{BAE}\left[\mathrm{kcal} \mathrm{mol}^{-1}\right]$ & $\mathrm{BIE}\left[\mathrm{kcal} \mathrm{mol}^{-1}\right]$ & E-def $\left[\mathrm{kcal} \mathrm{mol}^{-1}\right]$ & $\mathrm{E}[\mathrm{Ha}]$ & $\mathrm{BAE}\left[\mathrm{kcal} \mathrm{mol}^{-1}\right]$ & $\mathrm{BIE}\left[\mathrm{kcal} \mathrm{mol}^{-1}\right]$ & E-def $\left[\mathrm{kcal} \mathrm{mol}^{-1}\right]$ \\
\hline \multicolumn{9}{|c|}{ Methanes } \\
\hline $\mathrm{H}$ & -40.433704361338 & & & & -40.468623686438 & & & \\
\hline $\mathrm{F}$ & -437.375877997406 & & & & -437.301896329176 & & & \\
\hline $\mathrm{Hx} 1$ & -40.43370239369 & & & & -40.468620808704 & & & \\
\hline $\mathrm{xH} 1$ & -40.433702316336 & & & & -40.468620904186 & & & \\
\hline HH1 & -80.867774570902 & -0.230 & -0.232 & 0.003 & -80.938353180285 & -0.694 & -0.697 & 0.004 \\
\hline $\mathrm{Hx} 2$ & -40.433703893428 & & & & -40.468623342435 & & & \\
\hline $\mathrm{xH} 2$ & -40.433703649388 & & & & -40.468623349292 & & & \\
\hline $\mathrm{HH} 2$ & -80.8676809263 & -0.171 & -0.172 & 0.001 & -80.938198496086 & -0.597 & -0.597 & 0.000 \\
\hline Hx1 & -40.433704613238 & & & & -40.468622899117 & & & \\
\hline $\mathrm{xF} 1$ & -437.375949360451 & & & & -437.301950949965 & & & \\
\hline HF1 & -477.810725967763 & -0.718 & -0.673 & -0.045 & -477.771926223092 & -0.882 & -0.849 & -0.034 \\
\hline $\mathrm{Hx} 2$ & -40.433702074606 & & & & -40.468621198987 & & & \\
\hline $\mathrm{xF} 2$ & -437.375928651571 & & & & -437.301935300114 & & & \\
\hline $\mathrm{HF} 2$ & -477.810209906563 & -0.394 & -0.363 & -0.030 & -477.771434722621 & -0.574 & -0.551 & -0.023 \\
\hline Fx1 & -437.375918634265 & & & & -437.301924589287 & & & \\
\hline $\mathrm{xF} 1$ & -437.375926270295 & & & & -437.301934202071 & & & \\
\hline $\mathrm{FF} 1$ & -874.7533666248 & -1.011 & -0.955 & -0.056 & -874.605151852988 & -0.853 & -0.811 & -0.041 \\
\hline $\mathrm{Fx} 2$ & -437.375933951719 & & & & -437.301940042731 & & & \\
\hline $\mathrm{xF} 2$ & -437.375927525666 & & & & -437.301933667468 & & & \\
\hline $\mathrm{FF} 2$ & -874.753349886422 & -1.000 & -0.934 & -0.066 & -874.604968104437 & -0.738 & -0.687 & -0.051 \\
\hline \multicolumn{9}{|c|}{ Ethanes } \\
\hline $\mathrm{H}$ & -79.684223508866 & & & & -79.7417710803 & & & \\
\hline $\mathrm{F}$ & -675.090003134662 & & & & -674.979472457831 & & & \\
\hline Hx1 & -79.684231747825 & & & & -79.741779422275 & & & \\
\hline $\mathrm{xH} 1$ & -79.684227308279 & & & & -79.741779217914 & & & \\
\hline HH1 & -159.370019547555 & -0.987 & -0.979 & -0.008 & -159.485940254543 & -1.505 & -1.494 & -0.010 \\
\hline $\mathrm{Hx} 2$ & -79.684225243405 & & & & -79.741777772561 & & & \\
\hline $\mathrm{xH} 2$ & -79.684225849981 & & & & -79.741777983379 & & & \\
\hline $\mathrm{HH} 2$ & -159.369803890498 & -0.851 & -0.849 & -0.003 & -159.485766956578 & -1.396 & -1.388 & -0.009 \\
\hline Hx1 & -79.68421724922 & & & & -79.741767618115 & & & \\
\hline $\mathrm{xF} 1$ & -675.090129486775 & & & & -674.979579223335 & & & \\
\hline HF1 & -754.776058014443 & -1.149 & -1.074 & -0.075 & -754.723054213834 & -1.136 & -1.071 & -0.065 \\
\hline $\mathrm{Hx} 2$ & -79.684221309085 & & & & -79.741769849808 & & & \\
\hline $\mathrm{xF} 2$ & -675.090177897775 & & & & -674.979609886189 & & & \\
\hline $\mathrm{HF} 2$ & -754.776442739331 & -1.391 & -1.282 & -0.108 & -754.723344923298 & -1.319 & -1.233 & -0.085 \\
\hline Hx3 & -79.684219224531 & & & & -79.741770127112 & & & \\
\hline $\mathrm{xF} 3$ & -675.090114248897 & & & & -674.979570389162 & & & \\
\hline HF3 & -754.775792444717 & -0.983 & -0.916 & -0.067 & -754.722857752047 & -1.013 & -0.952 & -0.061 \\
\hline $\mathrm{Hx} 4$ & -79.684222548284 & & & & -79.741767240933 & & & \\
\hline $\mathrm{xF} 4$ & -675.090077904307 & & & & -674.979542830408 & & & \\
\hline $\mathrm{HF} 4$ & -754.776219880308 & -1.251 & -1.204 & -0.046 & -754.723153773823 & -1.199 & -1.157 & -0.042 \\
\hline $\mathrm{Fx} 1$ & -675.09008029082 & & & & -674.979524491432 & & & \\
\hline $\mathrm{xF} 1$ & -675.090045036977 & & & & -674.979494959116 & & & \\
\hline $\mathrm{FF} 1$ & -1350.18266065928 & -1.666 & -1.591 & -0.075 & -1349.96060986308 & -1.045 & -0.998 & -0.047 \\
\hline $\mathrm{Fx} 2$ & -675.090151396854 & & & & -674.979598011236 & & & \\
\hline $\mathrm{xF} 2$ & -675.090059544163 & & & & -674.979525454922 & & & \\
\hline $\mathrm{FF} 2$ & -1350.18221849205 & -1.388 & -1.260 & -0.128 & -1349.9605408618 & -1.001 & -0.889 & -0.112 \\
\hline
\end{tabular}




\begin{tabular}{|c|c|c|c|c|c|c|c|c|}
\hline & \multicolumn{4}{|c|}{ BLYP-NL/def2-QZVP } & \multicolumn{4}{|c|}{ PBE-D3/def2-QZVP } \\
\hline & $\mathrm{E}[\mathrm{Ha}]$ & $\mathrm{BAE}\left[\mathrm{kcal} \mathrm{mol}{ }^{-1}\right]$ & $\mathrm{BIE}\left[\mathrm{kcal} \mathrm{mol}^{-1}\right]$ & E-def $\left[\mathrm{kcal} \mathrm{mol}^{-1}\right]$ & $\mathrm{E}[\mathrm{Ha}]$ & $\mathrm{BAE}\left[\mathrm{kcal} \mathrm{mol}^{-1}\right]$ & $\mathrm{BIE}\left[\mathrm{kcal} \mathrm{mol}^{-1}\right]$ & E-def $\left[\mathrm{kcal} \mathrm{mol}^{-1}\right]$ \\
\hline \multicolumn{9}{|c|}{ Propanes } \\
\hline $\mathrm{H}$ & -118.937964398449 & & & & -119.0179306436 & & & \\
\hline $\mathrm{F}$ & -912.803367592912 & & & & -912.656089243053 & & & \\
\hline Hx1 & -118.937973621097 & & & & -119.017937008877 & & & \\
\hline $\mathrm{xH} 1$ & -118.937973526147 & & & & -119.017936890239 & & & \\
\hline HH1 & -237.878782195759 & -1.791 & -1.779 & -0.012 & -238.039446723487 & -2.250 & -2.242 & -0.008 \\
\hline $\mathrm{Hx} 2$ & -118.93798960294 & & & & -119.017945348766 & & & \\
\hline $\mathrm{xH} 2$ & -118.937971327401 & & & & -119.017936616299 & & & \\
\hline HH2 & -237.877471248851 & -0.968 & -0.948 & -0.020 & -238.03818755883 & -1.460 & -1.447 & -0.013 \\
\hline $\mathrm{Hx} 1$ & -118.937949662138 & & & & -119.017917311258 & & & \\
\hline $\mathrm{xF} 1$ & -912.803374073348 & & & & -912.656065869378 & & & \\
\hline HF1 & -1031.74434606743 & -1.891 & -1.897 & 0.005 & -1031.67655924824 & -1.593 & -1.617 & 0.023 \\
\hline $\mathrm{Hx} 2$ & -118.937965036793 & & & & -119.017929374897 & & & \\
\hline $\mathrm{xF} 2$ & -912.803492943316 & & & & -912.656157661562 & & & \\
\hline HF2 & -1031.74388838031 & -1.604 & -1.525 & -0.079 & -1031.67623821865 & -1.392 & -1.350 & -0.042 \\
\hline $\mathrm{Hx} 3$ & -118.938000364735 & & & & -119.017960554628 & & & \\
\hline $\mathrm{xF} 3$ & -912.8034622063 & & & & -912.656149631115 & & & \\
\hline HF3 & -1031.74381371527 & -1.557 & -1.475 & -0.082 & -1031.67634746339 & -1.461 & -1.404 & -0.057 \\
\hline $\mathrm{Hx} 4$ & -118.937953983659 & & & & -119.017915606786 & & & \\
\hline $\mathrm{xF} 4$ & -912.803464449168 & & & & -912.656130860455 & & & \\
\hline $\mathrm{HF} 4$ & -1031.74386142261 & -1.587 & -1.533 & -0.054 & -1031.67606736184 & -1.285 & -1.268 & -0.017 \\
\hline $\mathrm{Fx} 1$ & -912.803125555602 & & & & -912.655866762547 & & & \\
\hline $\mathrm{xF} 1$ & -912.803281832585 & & & & -912.656007062583 & & & \\
\hline FF1 & -1825.61014285935 & -2.138 & -2.344 & 0.206 & -1825.313888666106 & -1.073 & -1.264 & 0.191 \\
\hline $\mathrm{Fx} 2$ & -912.80336131173 & & & & -912.656083826031 & & & \\
\hline $\mathrm{xF} 2$ & -912.803356795842 & & & & -912.656081341146 & & & \\
\hline FF2 & -1825.61098804215 & -2.669 & -2.679 & 0.011 & -1825.31412272449 & -1.220 & -1.228 & 0.008 \\
\hline \multicolumn{9}{|c|}{ Butanes } \\
\hline $\mathrm{H}$ & -158.191753745149 & & & & -158.294103788252 & & & \\
\hline $\mathrm{F}$ & -1150.5161489168 & & & & -1150.33182471274 & & & \\
\hline $\mathrm{Hx} 1$ & -158.191749045443 & & & & -158.294102252034 & & & \\
\hline $\mathrm{xH} 1$ & -158.191767521511 & & & & -158.294108591526 & & & \\
\hline HH1 & -316.387843615443 & -2.721 & -2.715 & -0.006 & -316.593129784883 & -3.089 & -3.087 & -0.002 \\
\hline $\mathrm{H} \times 2$ & -158.191748874118 & & & & -158.294094121973 & & & \\
\hline $\mathrm{xH} 2$ & -158.191772853829 & & & & -158.294113582637 & & & \\
\hline HH2 & -316.385782429455 & -1.428 & -1.419 & -0.009 & -316.591327043135 & -1.957 & -1.957 & 0.000 \\
\hline $\mathrm{Hx} 1$ & -158.19176013785 & & & & -158.294099492185 & & & \\
\hline $\mathrm{xF} 1$ & -1150.51608945163 & & & & -1150.33180376386 & & & \\
\hline HF1 & -1308.71212141691 & -2.647 & -2.681 & 0.033 & -1308.62929373477 & -2.112 & -2.128 & 0.016 \\
\hline $\mathrm{H} \times 2$ & -158.19173724032 & & & & -158.294082741885 & & & \\
\hline $\mathrm{xF} 2$ & -1150.51617953716 & & & & -1150.33186209905 & & & \\
\hline HF2 & -1308.71215188482 & -2.666 & -2.658 & -0.009 & -1308.6291995376 & -2.053 & -2.042 & -0.010 \\
\hline Hx3 & -158.191700094947 & & & & -158.29405097961 & & & \\
\hline $\mathrm{xF} 3$ & -1150.5161873807 & & & & -1150.33188837845 & & & \\
\hline HF3 & -1308.71210370547 & -2.636 & -2.646 & 0.010 & -1308.62901678959 & -1.938 & -1.931 & -0.007 \\
\hline $\mathrm{Hx} 4$ & -158.191707327736 & & & & -158.294055257055 & & & \\
\hline $\mathrm{xF} 4$ & -1150.51618299674 & & & & -1150.3318529682 & & & \\
\hline $\mathrm{HF} 4$ & -1308.71198142107 & -2.559 & -2.567 & 0.008 & -1308.62917291173 & -2.036 & -2.049 & 0.013 \\
\hline $\mathrm{Fx} 1$ & -1150.51609225676 & & & & -1150.33182013943 & & & \\
\hline $\mathrm{xF} 1$ & -1150.51615388232 & & & & -1150.33181697288 & & & \\
\hline FF1 & -2301.03681570544 & -2.835 & -2.867 & 0.032 & -2300.66634028935 & -1.689 & -1.696 & 0.008 \\
\hline $\mathrm{Fx} 2$ & -1150.51620020772 & & & & -1150.33189325135 & & & \\
\hline $\mathrm{xF} 2$ & -1150.51618824372 & & & & -1150.33185347854 & & & \\
\hline FF2 & -2301.03752343686 & -3.279 & -3.222 & -0.057 & -2300.66637367249 & -1.709 & -1.648 & -0.061 \\
\hline
\end{tabular}




\begin{tabular}{|c|c|c|c|c|c|c|c|c|}
\hline & \multicolumn{4}{|c|}{ BLYP-NL/def2-QZVP } & \multicolumn{4}{|c|}{ PBE-D3/def2-QZVP } \\
\hline & $\mathrm{E}[\mathrm{Ha}]$ & $\mathrm{BAE}\left[\mathrm{kcal} \mathrm{mol}^{-1}\right]$ & BIE $\left[\mathrm{kcal} \mathrm{mol}^{-1}\right]$ & E-def $\left[\mathrm{kcal} \mathrm{mol}^{-1}\right]$ & $\mathrm{E}[\mathrm{Ha}]$ & $\mathrm{BAE}\left[\mathrm{kcal} \mathrm{mol}^{-1}\right]$ & $\mathrm{BIE}\left[\mathrm{kcal} \mathrm{mol}^{-1}\right]$ & E-def $\left[\mathrm{kcal} \mathrm{mol}^{-1}\right]$ \\
\hline \multicolumn{9}{|c|}{ Pentanes } \\
\hline H & -197.445522623464 & & & & -197.57021659243 & & & \\
\hline $\mathrm{F}$ & -1388.22912666694 & & & & -1388.00768686124 & & & \\
\hline $\mathrm{Hx} 1$ & -197.445500380936 & & & & -197.570199738717 & & & \\
\hline $\mathrm{xH} 1$ & -197.445500224481 & & & & -197.570199641368 & & & \\
\hline нH1 & -394.896884801564 & -3.664 & -3.692 & 0.028 & -395.146708964698 & -3.938 & -3.959 & 0.021 \\
\hline $\mathrm{Hx} 2$ & -197.445536493822 & & & & -197.570225693725 & & & \\
\hline $\mathrm{xH} 2$ & -197.44553642235 & & & & -197.570225603214 & & & \\
\hline нH2 & -394.894059851211 & -1.892 & -1.874 & -0.017 & -395.144494463869 & -2.548 & -2.537 & -0.011 \\
\hline Hx1 & -197.44542411314 & & & & -197.57010880511 & & & \\
\hline $\mathrm{xF} 1$ & -1388.22915542896 & & & & -1388.00771916376 & & & \\
\hline HF1 & -1585.68016328151 & -3.460 & -3.504 & 0.044 & -1585.58191489409 & -2.517 & -2.565 & 0.047 \\
\hline $\mathrm{Hx} 2$ & -197.445491204696 & & & & -197.570178336334 & & & \\
\hline $\mathrm{xF} 2$ & -1388.22894038678 & & & & -1388.00749476257 & & & \\
\hline $\mathrm{HF} 2$ & -1585.67945047163 & -3.013 & -3.149 & 0.137 & -1585.58155331671 & -2.290 & -2.435 & 0.145 \\
\hline $\mathrm{Hx} 3$ & -197.445487208859 & & & & -197.570185351125 & & & \\
\hline $\mathrm{xF} 3$ & -1388.2292550995 & & & & -1388.00782045027 & & & \\
\hline HF3 & -1585.6797459737 & -3.198 & -3.140 & -0.058 & -1585.58168345636 & -2.372 & -2.308 & -0.064 \\
\hline $\mathrm{Hx} 4$ & -197.445476716655 & & & & -197.57016950943 & & & \\
\hline $\mathrm{xF} 4$ & -1388.22919705012 & & & & -1388.00775596823 & & & \\
\hline $\mathrm{HF} 4$ & -1585.68047047778 & -3.653 & -3.637 & -0.015 & -1585.58219783442 & -2.695 & -2.681 & -0.014 \\
\hline $\mathrm{Fx} 1$ & -1388.22912719251 & & & & -1388.00767315185 & & & \\
\hline $\mathrm{xF} 1$ & -1388.2291652788 & & & & -1388.00771565856 & & & \\
\hline $\mathrm{FF} 1$ & -2776.46517364352 & -4.343 & -4.318 & -0.025 & -2776.01869143357 & -2.082 & -2.072 & -0.009 \\
\hline $\mathrm{Fx} 2$ & -1388.22905185176 & & & & -1388.00764289579 & & & \\
\hline $\mathrm{xF} 2$ & -1388.22905941403 & & & & -1388.00764784066 & & & \\
\hline $\mathrm{FF} 2$ & -2776.46428185928 & -3.783 & -3.872 & 0.089 & -2776.01853050655 & -1.981 & -2.033 & 0.052 \\
\hline \multicolumn{9}{|c|}{ Hexanes } \\
\hline $\mathrm{H}$ & -236.699295041123 & & & & -236.846336404762 & & & \\
\hline $\mathrm{F}$ & -1625.94218900133 & & & & -1625.68361908786 & & & \\
\hline $\mathrm{Hx} 1$ & -236.699265179933 & & & & -236.846316725267 & & & \\
\hline $\mathrm{xH} 1$ & -236.699264784254 & & & & -236.846316384078 & & & \\
\hline HH1 & -473.405643134184 & -4.426 & -4.464 & 0.038 & -473.699986719555 & -4.590 & -4.614 & 0.025 \\
\hline $\mathrm{Hx} 2$ & -236.699308990341 & & & & -236.846346638323 & & & \\
\hline $\mathrm{xH} 2$ & -236.699308885483 & & & & -236.846346514905 & & & \\
\hline нH2 & -473.402553715556 & -2.487 & -2.470 & -0.017 & -473.697531634804 & -3.049 & -3.036 & -0.013 \\
\hline $\mathrm{Hx} 1$ & -236.699089660894 & & & & -236.846138988141 & & & \\
\hline $\mathrm{xF} 1$ & -1625.94185271133 & & & & -1625.68330656475 & & & \\
\hline HF1 & -1862.64774318473 & -3.928 & -4.268 & 0.340 & -1862.53404066957 & -2.563 & -2.883 & 0.320 \\
\hline $\mathrm{Hx} 2$ & -236.699196616116 & & & & -236.84624436968 & & & \\
\hline $\mathrm{xF} 2$ & -1625.94194449193 & & & & -1625.68338448685 & & & \\
\hline $\mathrm{HF} 2$ & -1862.64751687209 & -3.786 & -4.001 & 0.215 & -1862.53440114756 & -2.790 & -2.995 & 0.205 \\
\hline $\mathrm{Hx} 3$ & -236.699177057735 & & & & -236.846202507192 & & & \\
\hline $\mathrm{xF} 3$ & -1625.94191343566 & & & & -1625.68335515976 & & & \\
\hline HF3 & -1862.64771262641 & -3.908 & -4.155 & 0.247 & -1862.53421875789 & -2.675 & -2.925 & 0.250 \\
\hline $\mathrm{Hx} 4$ & -236.699286156833 & & & & -236.846318193451 & & & \\
\hline $\mathrm{xF} 4$ & -1625.94218207656 & & & & -1625.68360831792 & & & \\
\hline $\mathrm{HF} 4$ & -1862.64808895805 & -4.145 & -4.155 & 0.010 & -1862.53480090783 & -3.041 & -3.059 & 0.018 \\
\hline $\mathrm{Fx} 1$ & -1625.94210998791 & & & & -1625.68353590655 & & & \\
\hline $\mathrm{xF} 1$ & -1625.94211897862 & & & & -1625.68355150013 & & & \\
\hline FF1 & -3251.89344391726 & -5.689 & -5.782 & 0.094 & -3251.37108429254 & -2.413 & -2.508 & 0.095 \\
\hline $\mathrm{Fx} 2$ & -1625.94197145041 & & & & -1625.68339492751 & & & \\
\hline $\mathrm{xF} 2$ & -1625.94197372558 & & & & -1625.68339760666 & & & \\
\hline $\mathrm{FF} 2$ & -3251.89053102413 & -3.861 & -4.133 & 0.272 & -3251.36963231621 & -1.502 & -1.782 & 0.280 \\
\hline
\end{tabular}




\begin{tabular}{|c|c|c|c|c|c|c|c|c|}
\hline & \multicolumn{4}{|c|}{ PBE-dDsC/QZ4P } & \multicolumn{4}{|c|}{ PBE-XDM/aug-cc-pVTZ } \\
\hline & $\mathrm{E}[\mathrm{Ha}]$ & $\mathrm{BAE}\left[\mathrm{kcal} \mathrm{mol}^{-1}\right]$ & $\mathrm{BIE}\left[\mathrm{kcal} \mathrm{mol}^{-1}\right]$ & E-def $\left[\mathrm{kcal} \mathrm{mol}^{-1}\right]$ & $\mathrm{E}[\mathrm{Ha}]$ & $\mathrm{BAE}\left[\mathrm{kcal} \mathrm{mol}^{-1}\right]$ & $\mathrm{BIE}\left[\mathrm{kcal} \mathrm{mol}^{-1}\right]$ & E-def $\left[\mathrm{kcal} \mathrm{mol}^{-1}\right]$ \\
\hline \multicolumn{9}{|c|}{ Methanes } \\
\hline $\mathrm{H}$ & -0.883839257049025 & & & & -40.46393269993 & & & \\
\hline $\mathrm{F}$ & -0.956055451608807 & & & & -437.2587215495 & & & \\
\hline $\mathrm{Hx} 1$ & -0.883827775253054 & & & & -40.46392923761 & & & \\
\hline xH1 & -0.883830659545519 & & & & -40.46392943039 & & & \\
\hline HH1 & -1.76871316340648 & -0.649 & -0.662 & 0.013 & -80.92882932944 & -0.605 & -0.609 & 0.004 \\
\hline $\mathrm{Hx} 2$ & -0.883832356826416 & & & & -40.46393246336 & & & \\
\hline $\mathrm{xH} 2$ & -0.88382980334505 & & & & -40.46393271425 & & & \\
\hline $\mathrm{HH} 2$ & -1.76857791682207 & -0.564 & -0.575 & 0.010 & -80.92871381574 & -0.532 & -0.533 & 0.000 \\
\hline $\mathrm{Hx} 1$ & -0.883836412011288 & & & & -40.46393176796 & & & \\
\hline $\mathrm{xF} 1$ & -0.956129725276525 & & & & -437.258783099 & & & \\
\hline HF1 & -1.84151490521401 & -1.017 & -0.972 & -0.045 & -477.7240729407 & -0.890 & -0.852 & -0.038 \\
\hline $\mathrm{Hx} 2$ & -0.883836662738119 & & & & -40.46393013783 & & & \\
\hline $\mathrm{xF} 2$ & -0.956133370790201 & & & & -437.2587644449 & & & \\
\hline $\mathrm{HF} 2$ & -1.84101927097868 & -0.706 & -0.658 & -0.047 & -477.7235847436 & -0.584 & -0.559 & -0.025 \\
\hline Fx1 & -0.956092092491527 & & & & -437.2587550137 & & & \\
\hline $\mathrm{xF} 1$ & -0.956094119926821 & & & & -437.2587619069 & & & \\
\hline FF1 & -1.91385777454594 & -1.096 & -1.049 & -0.047 & -874.5189381449 & -0.938 & -0.892 & -0.046 \\
\hline $\mathrm{Fx} 2$ & -0.95609624362216 & & & & -437.258769224 & & & \\
\hline $\mathrm{xF} 2$ & -0.95602509416178 & & & & -437.2587615512 & & & \\
\hline FF2 & -1.91376273109257 & -1.037 & -1.030 & -0.007 & -874.5186795373 & -0.776 & -0.721 & -0.055 \\
\hline \multicolumn{9}{|l|}{ Ethanes } \\
\hline $\mathrm{H}$ & -1.49005255386608 & & & & -79.73259089238 & & & \\
\hline $\mathrm{F}$ & -1.58681376474656 & & & & -674.9131339434 & & & \\
\hline $\mathrm{Hx} 1$ & -1.49008654602944 & & & & -79.73259887991 & & & \\
\hline $\mathrm{xH} 1$ & -1.49009823624231 & & & & -79.73259832731 & & & \\
\hline HH1 & -2.98292756409948 & -1.771 & -1.721 & -0.050 & -159.467230186 & -1.285 & -1.276 & -0.010 \\
\hline $\mathrm{Hx} 2$ & -1.49007356230251 & & & & -79.73259725759 & & & \\
\hline $\mathrm{xH} 2$ & -1.49007736045316 & & & & -79.73259770695 & & & \\
\hline $\mathrm{HH} 2$ & -2.9826034868582 & -1.568 & -1.539 & -0.029 & -159.4671164218 & -1.214 & -1.206 & -0.008 \\
\hline $\mathrm{Hx} 1$ & -1.49007795529372 & & & & -79.73258682409 & & & \\
\hline $\mathrm{xF} 1$ & -1.58687673275345 & & & & -674.9132483746 & & & \\
\hline HF1 & -3.079205506044 & -1.468 & -1.412 & -0.055 & -754.6476587458 & -1.214 & -1.144 & -0.069 \\
\hline $\mathrm{Hx} 2$ & -1.49004580754786 & & & & -79.73258841946 & & & \\
\hline $\mathrm{xF} 2$ & -1.58690666669895 & & & & -674.9132887973 & & & \\
\hline HF2 & -3.07954016480729 & -1.678 & -1.624 & -0.054 & -754.6479035879 & -1.367 & -1.272 & -0.096 \\
\hline $\mathrm{Hx} 3$ & -1.49006065006389 & & & & -79.73258886887 & & & \\
\hline $\mathrm{xF} 3$ & -1.58685863991975 & & & & -674.9132384075 & & & \\
\hline HF3 & -3.07892690451456 & -1.293 & -1.260 & -0.033 & -754.6474456455 & -1.080 & -1.016 & -0.064 \\
\hline $\mathrm{Hx} 4$ & -1.4900495194976 & & & & -79.73258457144 & & & \\
\hline $\mathrm{xF} 4$ & -1.58683697604237 & & & & -674.9132071271 & & & \\
\hline $\mathrm{HF} 4$ & -3.0793480387753 & -1.557 & -1.545 & -0.013 & -754.6477383198 & -1.263 & -1.222 & -0.042 \\
\hline Fx1 & -1.58680801477139 & & & & -674.9131870047 & & & \\
\hline $\mathrm{xF} 1$ & -1.58677107218408 & & & & -674.9131543035 & & & \\
\hline FF1 & -3.17610281557898 & -1.553 & -1.584 & 0.030 & -1349.828160055 & -1.187 & -1.141 & -0.046 \\
\hline $\mathrm{Fx} 2$ & -1.58692960879544 & & & & -674.9132697437 & & & \\
\hline $\mathrm{xF} 2$ & -1.58674990194849 & & & & -674.9131862992 & & & \\
\hline FF2 & -3.17584009588139 & -1.388 & -1.356 & -0.033 & -1349.828059295 & -1.124 & -1.006 & -0.118 \\
\hline
\end{tabular}




\begin{tabular}{|c|c|c|c|c|c|c|c|c|}
\hline & \multicolumn{4}{|c|}{ PBE-dDsC/QZ4P } & \multicolumn{4}{|c|}{ PBE-XDM/aug-cc-pVTZ } \\
\hline & $\mathrm{E}[\mathrm{Ha}]$ & $\mathrm{BAE}\left[\mathrm{kcal} \mathrm{mol}^{-1}\right]$ & $\mathrm{BIE}\left[\mathrm{kcal} \mathrm{mol}^{-1}\right]$ & E-def $\left[\mathrm{kcal} \mathrm{mol}^{-1}\right]$ & $\mathrm{E}[\mathrm{Ha}]$ & $\mathrm{BAE}\left[\mathrm{kcal} \mathrm{mol}^{-1}\right]$ & $\mathrm{BIE}\left[\mathrm{kcal} \mathrm{mol}^{-1}\right]$ & E-def $\left[\mathrm{kcal} \mathrm{mol}^{-1}\right]$ \\
\hline \multicolumn{9}{|c|}{ Propanes } \\
\hline $\mathrm{H}$ & -2.09975507610939 & & & & -119.00413359 & & & \\
\hline $\mathrm{F}$ & -2.21718363507822 & & & & -912.5664076754 & & & \\
\hline $\mathrm{Hx} 1$ & -2.09970939794028 & & & & -119.0041425869 & & & \\
\hline $\mathrm{xH} 1$ & -2.09971467118466 & & & & -119.0041429721 & & & \\
\hline HH1 & -4.20369749236946 & -2.628 & -2.682 & 0.054 & -238.0114446451 & -1.994 & -1.982 & -0.012 \\
\hline $\mathrm{Hx} 2$ & -2.09972476723893 & & & & -119.0041498969 & & & \\
\hline $\mathrm{xH} 2$ & -2.09971601644477 & & & & -119.0041393643 & & & \\
\hline $\mathrm{HH} 2$ & -4.20219829983119 & -1.687 & -1.730 & 0.044 & -238.010345195 & -1.304 & -1.290 & -0.014 \\
\hline $\mathrm{Hx} 1$ & -2.09972870659801 & & & & -119.0041188039 & & & \\
\hline $\mathrm{xF} 1$ & -2.21713179304622 & & & & -912.5663868836 & & & \\
\hline HF1 & -4.32049165871626 & -2.230 & -2.279 & 0.049 & -1031.573263688 & -1.708 & -1.731 & 0.022 \\
\hline $\mathrm{Hx} 2$ & -2.09972919709301 & & & & -119.0041325896 & & & \\
\hline $\mathrm{xF} 2$ & -2.21719940278449 & & & & -912.5664868161 & & & \\
\hline $\mathrm{HF} 2$ & -4.31993208459369 & -1.878 & -1.885 & 0.006 & -1031.572985263 & -1.534 & -1.485 & -0.049 \\
\hline $\mathrm{Hx} 3$ & -2.09978812294241 & & & & -119.0041689222 & & & \\
\hline $\mathrm{xF} 3$ & -2.21724102296353 & & & & -912.5664751765 & & & \\
\hline HF3 & -4.31996925484445 & -1.902 & -1.845 & -0.057 & -1031.57313218 & -1.626 & -1.561 & -0.065 \\
\hline $\mathrm{Hx} 4$ & -2.09966068122946 & & & & -119.0041194317 & & & \\
\hline $\mathrm{xF} 4$ & -2.21722004243295 & & & & -912.5664582747 & & & \\
\hline $\mathrm{HF} 4$ & -4.31988888630637 & -1.851 & -1.888 & 0.036 & -1031.572828909 & -1.436 & -1.413 & -0.023 \\
\hline $\mathrm{Fx} 1$ & -2.21695164738816 & & & & -912.566183157 & & & \\
\hline $\mathrm{xF} 1$ & -2.21713917826022 & & & & -912.5663243669 & & & \\
\hline FF1 & -4.43765371921319 & -2.062 & -2.236 & 0.173 & -1825.134883308 & -1.298 & -1.491 & 0.193 \\
\hline $\mathrm{Fx} 2$ & -2.2171596281897 & & & & -912.5664029928 & & & \\
\hline $\mathrm{xF} 2$ & -2.21718049196758 & & & & -912.5664005967 & & & \\
\hline $\mathrm{FF} 2$ & -4.43826148281025 & -2.444 & -2.461 & 0.017 & -1825.135184858 & -1.487 & -1.494 & 0.007 \\
\hline \multicolumn{9}{|c|}{ Butanes } \\
\hline $\mathrm{H}$ & -2.70961929396179 & & & & -158.275702988 & & & \\
\hline $\mathrm{F}$ & -2.84708193038427 & & & & -1150.218885049 & & & \\
\hline $\mathrm{Hx} 1$ & -2.70963410406778 & & & & -158.2757069788 & & & \\
\hline $\mathrm{xH} 1$ & -2.70959075838609 & & & & -158.27570423 & & & \\
\hline HH1 & -5.42545776374939 & -3.903 & -3.911 & 0.009 & -316.5558400812 & -2.782 & -2.779 & -0.003 \\
\hline $\mathrm{Hx} 2$ & -2.70955261344846 & & & & -158.2756898051 & & & \\
\hline $\mathrm{xH} 2$ & -2.70957196991216 & & & & -158.2757117823 & & & \\
\hline $\mathrm{HH} 2$ & -5.42298600967028 & -2.352 & -2.423 & 0.072 & -316.5542335187 & -1.774 & -1.777 & 0.003 \\
\hline $\mathrm{Hx} 1$ & -2.7095540019397 & & & & -158.2756978399 & & & \\
\hline $\mathrm{xF} 1$ & -2.84698126399749 & & & & -1150.218826054 & & & \\
\hline HF1 & -5.5613175934172 & -2.897 & -3.001 & 0.104 & -1308.498298315 & -2.328 & -2.368 & 0.040 \\
\hline $\mathrm{Hx} 2$ & -2.70958307998382 & & & & -158.2756819432 & & & \\
\hline $\mathrm{xF} 2$ & -2.84710998549403 & & & & -1150.21890975 & & & \\
\hline HF2 & -5.56140455437863 & -2.951 & -2.957 & 0.005 & -1308.498186541 & -2.258 & -2.256 & -0.002 \\
\hline $\mathrm{Hx} 3$ & -2.70954178726408 & & & & -158.2756414865 & & & \\
\hline $\mathrm{xF} 3$ & -2.8471206574374 & & & & -1150.218925325 & & & \\
\hline HF3 & -5.56152197536837 & -3.025 & -3.049 & 0.024 & -1308.497954154 & -2.112 & -2.126 & 0.013 \\
\hline $\mathrm{Hx} 4$ & -2.70952651159695 & & & & -158.2756502936 & & & \\
\hline $\mathrm{xF} 4$ & -2.84707225082311 & & & & -1150.218904814 & & & \\
\hline $\mathrm{HF} 4$ & -5.5614434094447 & -2.976 & -3.040 & 0.064 & -1308.498165539 & -2.245 & -2.266 & 0.021 \\
\hline Fx1 & -2.84702286044624 & & & & -1150.218857941 & & & \\
\hline $\mathrm{xF} 1$ & -2.84705376190627 & & & & -1150.218875907 & & & \\
\hline FF1 & -5.698487416525 & -2.713 & -2.768 & 0.055 & -2300.440924977 & -1.980 & -2.002 & 0.023 \\
\hline $\mathrm{Fx} 2$ & -2.84709677386872 & & & & -1150.21892819 & & & \\
\hline $\mathrm{xF} 2$ & -2.84706551099736 & & & & -1150.21889262 & & & \\
\hline FF2 & -5.69912143711902 & -3.111 & -3.112 & 0.001 & -2300.441021462 & -2.040 & -2.008 & -0.032 \\
\hline
\end{tabular}




\begin{tabular}{|c|c|c|c|c|c|c|c|c|}
\hline & \multicolumn{4}{|c|}{ PBE-dDsC/QZ4P } & \multicolumn{4}{|c|}{ PBE-XDM/aug-cc-pVTZ } \\
\hline & $\mathrm{E}[\mathrm{Ha}]$ & $\mathrm{BAE}\left[\mathrm{kcal} \mathrm{mol}^{-1}\right]$ & $\mathrm{BIE}\left[\mathrm{kcal} \mathrm{mol}^{-1}\right]$ & E-def $\left[\mathrm{kcal} \mathrm{mol}^{-1}\right]$ & $\mathrm{E}[\mathrm{Ha}]$ & $\mathrm{BAE}\left[\mathrm{kcal} \mathrm{mol}^{-1}\right]$ & $\mathrm{BIE}\left[\mathrm{kcal} \mathrm{mol}^{-1}\right]$ & E-def $\left[\mathrm{kcal} \mathrm{mol}^{-1}\right]$ \\
\hline \multicolumn{9}{|c|}{ Pentanes } \\
\hline $\mathrm{H}$ & -3.31940480555648 & & & & -197.5472260191 & & & \\
\hline $\mathrm{F}$ & -3.47714194207951 & & & & -1387.871512522 & & & \\
\hline $\mathrm{Hx} 1$ & -3.31939806439905 & & & & -197.5472150548 & & & \\
\hline $\mathrm{xH} 1$ & -3.31939680965446 & & & & -197.5472149286 & & & \\
\hline HH1 & -6.64697608220883 & -5.125 & -5.134 & 0.009 & -395.1001452695 & -3.573 & -3.586 & 0.014 \\
\hline $\mathrm{Hx} 2$ & -3.31936708365482 & & & & -197.5472345331 & & & \\
\hline $\mathrm{xH} 2$ & -3.31936971469356 & & & & -197.5472344538 & & & \\
\hline HH2 & -6.64393142317421 & -3.214 & -3.260 & 0.046 & -395.0980889143 & -2.282 & -2.272 & -0.011 \\
\hline $\mathrm{Hx} 1$ & -3.31923178027 & & & & -197.5471273811 & & & \\
\hline $\mathrm{xF} 1$ & -3.47718625953986 & & & & -1387.871521025 & & & \\
\hline HF1 & -6.80247613150678 & -3.721 & -3.802 & 0.081 & -1585.423217183 & -2.810 & -2.867 & 0.057 \\
\hline $\mathrm{Hx} 2$ & -3.31936018092275 & & & & -197.5471873085 & & & \\
\hline $\mathrm{xF} 2$ & -3.47694171588405 & & & & -1387.871267836 & & & \\
\hline HF2 & -6.80181484911669 & -3.306 & -3.459 & 0.154 & -1585.422773914 & -2.532 & -2.710 & 0.178 \\
\hline $\mathrm{Hx} 3$ & -3.31937575387692 & & & & -197.5471946523 & & & \\
\hline $\mathrm{xF} 3$ & -3.47723193927879 & & & & -1387.871634834 & & & \\
\hline HF3 & -6.80228465793758 & -3.601 & -3.562 & -0.038 & -1585.42287852 & -2.598 & -2.541 & -0.057 \\
\hline $\mathrm{Hx} 4$ & -3.31936222636314 & & & & -197.5471763614 & & & \\
\hline $\mathrm{xF} 4$ & -3.47715366582953 & & & & -1387.871571467 & & & \\
\hline $\mathrm{HF} 4$ & -6.80293938538261 & -4.011 & -4.031 & 0.019 & -1585.423529689 & -3.006 & -3.001 & -0.006 \\
\hline Fx1 & -3.47711779787034 & & & & -1387.871498195 & & & \\
\hline $\mathrm{xF} 1$ & -3.47715613780696 & & & & -1387.871530325 & & & \\
\hline FF1 & -6.96036034423541 & -3.813 & -3.819 & 0.006 & -2775.747050454 & -2.526 & -2.524 & -0.002 \\
\hline $\mathrm{Fx} 2$ & -3.4771672942031 & & & & -1387.87145405 & & & \\
\hline $\mathrm{xF} 2$ & -3.47717461137172 & & & & -1387.871459409 & & & \\
\hline FF2 & -6.96013933851824 & -3.674 & -3.638 & -0.036 & -2775.74687086 & -2.413 & -2.483 & 0.070 \\
\hline \multicolumn{9}{|c|}{ Hexanes } \\
\hline $\mathrm{H}$ & -3.9292195839309 & & & & -236.8187678111 & & & \\
\hline $\mathrm{F}$ & -4.10746799143434 & & & & -1625.524209883 & & & \\
\hline $\mathrm{Hx} 1$ & -3.92925110925325 & & & & -236.8187493839 & & & \\
\hline $\mathrm{xH} 1$ & -3.92925398967885 & & & & -236.818749242 & & & \\
\hline HH1 & -7.86818250932209 & -6.114 & -6.073 & -0.041 & -473.6442197439 & -4.194 & -4.218 & 0.023 \\
\hline $\mathrm{Hx} 2$ & -3.92917456949484 & & & & -236.8187663229 & & & \\
\hline $\mathrm{xH} 2$ & -3.92916923776214 & & & & -236.818766197 & & & \\
\hline $\mathrm{HH} 2$ & -7.86466338246352 & -3.906 & -3.966 & 0.060 & -473.6419155928 & -2.748 & -2.750 & 0.002 \\
\hline $\mathrm{Hx} 1$ & -3.92903047433647 & & & & -236.8185697565 & & & \\
\hline $\mathrm{xF} 1$ & -4.1069535621265 & & & & -1625.523843552 & & & \\
\hline $\mathrm{HF} 1$ & -8.0431659763061 & -4.065 & -4.507 & 0.441 & -1862.347600792 & -2.901 & -3.255 & 0.354 \\
\hline $\mathrm{Hx} 2$ & -3.92915437006568 & & & & -236.8186722824 & & & \\
\hline $\mathrm{xF} 2$ & -4.10716999495156 & & & & -1625.523932796 & & & \\
\hline HF2 & -8.04317603696079 & -4.072 & -4.299 & 0.228 & -1862.34796281 & -3.128 & -3.362 & 0.234 \\
\hline $\mathrm{Hx} 3$ & -3.92903106095917 & & & & -236.8186386995 & & & \\
\hline $\mathrm{xF} 3$ & -4.10717084700408 & & & & -1625.523916445 & & & \\
\hline HF3 & -8.04347448264836 & -4.259 & -4.564 & 0.305 & -1862.347786671 & -3.018 & -3.283 & 0.265 \\
\hline $\mathrm{Hx} 4$ & -3.92918353874802 & & & & -236.8187333724 & & & \\
\hline $\mathrm{xF} 4$ & -4.10737798606225 & & & & -1625.524187215 & & & \\
\hline $\mathrm{HF} 4$ & -8.0439177495198 & -4.537 & -4.616 & 0.079 & -1862.348382683 & -3.392 & -3.428 & 0.036 \\
\hline $\mathrm{Fx} 1$ & -4.10726645637324 & & & & -1625.524132314 & & & \\
\hline $\mathrm{xF} 1$ & -4.10721697794202 & & & & -1625.52413722 & & & \\
\hline FF1 & -8.22237083026386 & -4.665 & -4.949 & 0.284 & -3251.053228483 & -3.018 & -3.112 & 0.094 \\
\hline Fx2 & -4.10720896650448 & & & & -1625.524003844 & & & \\
\hline $\mathrm{xF} 2$ & -4.10722032565206 & & & & -1625.524005858 & & & \\
\hline FF2 & -8.22031744483011 & -3.377 & -3.695 & 0.318 & -3251.051648578 & -2.026 & -2.283 & 0.257 \\
\hline
\end{tabular}




\begin{tabular}{|c|c|c|c|c|c|c|c|c|}
\hline & \multicolumn{4}{|c|}{ TPSS-D3/def2-QZVP } & \multicolumn{4}{|c|}{ M06L/def2-QZVP } \\
\hline & $\mathrm{E}[\mathrm{Ha}]$ & $\mathrm{BAE}\left[\mathrm{kcal} \mathrm{mol}^{-1}\right]$ & $\mathrm{BIE}\left[\mathrm{kcal} \mathrm{mol}^{-1}\right]$ & E-def $\left[\mathrm{kcal} \mathrm{mol}^{-1}\right]$ & $\mathrm{E}[\mathrm{Ha}]$ & $\mathrm{BAE}\left[\mathrm{kcal} \mathrm{mol}^{-1}\right]$ & $\mathrm{BIE}\left[\mathrm{kcal} \mathrm{mol}^{-1}\right]$ & E-def $\left[\mathrm{kcal} \mathrm{mol}^{-1}\right]$ \\
\hline \multicolumn{9}{|c|}{ Methanes } \\
\hline $\mathrm{H}$ & -40.545876889156 & & & & -40.531313296368 & & & \\
\hline $\mathrm{F}$ & -437.741373399662 & & & & -437.638884588424 & & & \\
\hline $\mathrm{Hx} 1$ & -40.545875208204 & & & & -40.531310430462 & & & \\
\hline $\mathrm{xH} 1$ & -40.545875210792 & & & & -40.53131106774 & & & \\
\hline HH1 & -81.092700187038 & -0.594 & -0.596 & 0.002 & -81.063416060228 & -0.495 & -0.499 & 0.003 \\
\hline $\mathrm{Hx} 2$ & -40.545876566182 & & & & -40.531311957368 & & & \\
\hline $\mathrm{xH} 2$ & -40.545876519163 & & & & -40.531312292159 & & & \\
\hline $\mathrm{HH} 2$ & -81.09253878652 & -0.493 & -0.493 & 0.000 & -81.06301260005 & -0.242 & -0.244 & 0.001 \\
\hline Hx1 & -40.545876513073 & & & & -40.531311709867 & & & \\
\hline $\mathrm{xF} 1$ & -437.741419399716 & & & & -437.638871944456 & & & \\
\hline $\mathrm{HF} 1$ & -478.288406784797 & -0.726 & -0.697 & -0.029 & -478.171407636663 & -0.759 & -0.768 & 0.009 \\
\hline $\mathrm{Hx} 2$ & -40.545875171279 & & & & -40.531312910727 & & & \\
\hline $\mathrm{xF} 2$ & -437.741406663173 & & & & -437.638874470425 & & & \\
\hline HF2 & -478.287995815984 & -0.468 & -0.448 & -0.020 & -478.17056066646 & -0.228 & -0.234 & 0.007 \\
\hline Fx1 & -437.741394039167 & & & & -437.63886022921 & & & \\
\hline $\mathrm{xF} 1$ & -437.741408167293 & & & & -437.638879102913 & & & \\
\hline $\mathrm{FF} 1$ & -875.483951384018 & -0.756 & -0.721 & -0.035 & -875.279010518116 & -0.779 & -0.798 & 0.019 \\
\hline $\mathrm{Fx} 2$ & -437.741412927962 & & & & -437.638877748562 & & & \\
\hline $\mathrm{xF} 2$ & -437.741406689847 & & & & -437.638862726542 & & & \\
\hline $\mathrm{FF} 2$ & -875.483641211926 & -0.561 & -0.516 & -0.046 & -875.278623687304 & -0.536 & -0.554 & 0.018 \\
\hline \multicolumn{9}{|c|}{ Ethanes } \\
\hline $\mathrm{H}$ & -79.883012891806 & & & & -79.854027492189 & & & \\
\hline $\mathrm{F}$ & -675.664573839115 & & & & -675.503338370446 & & & \\
\hline Hx1 & -79.883015573347 & & & & -79.854032286033 & & & \\
\hline $\mathrm{xH} 1$ & -79.883016462659 & & & & -79.854030519234 & & & \\
\hline HH1 & -159.767932292748 & -1.196 & -1.192 & -0.004 & -159.70997177197 & -1.203 & -1.198 & -0.005 \\
\hline $\mathrm{Hx} 2$ & -79.883015127177 & & & & -79.854027399091 & & & \\
\hline $\mathrm{xH} 2$ & -79.883015124224 & & & & -79.854026017108 & & & \\
\hline $\mathrm{HH} 2$ & -159.767884199177 & -1.166 & -1.163 & -0.003 & -159.709714805679 & -1.042 & -1.043 & 0.001 \\
\hline Hx1 & -79.88300848243 & & & & -79.85402710114 & & & \\
\hline $\mathrm{xF} 1$ & -675.664677033957 & & & & -675.503336062214 & & & \\
\hline HF1 & -755.549074354432 & -0.933 & -0.872 & -0.062 & -755.359026159167 & -1.042 & -1.044 & 0.002 \\
\hline $\mathrm{Hx} 2$ & -79.883011290343 & & & & -79.854027138122 & & & \\
\hline $\mathrm{xF} 2$ & -675.664723690195 & & & & -675.503484037444 & & & \\
\hline $\mathrm{HF} 2$ & -755.549319535961 & -1.087 & -0.994 & -0.093 & -755.359385947453 & -1.268 & -1.176 & -0.091 \\
\hline Hx3 & -79.883011523823 & & & & -79.854029980694 & & & \\
\hline $\mathrm{xF} 3$ & -675.664657924814 & & & & -675.503355005165 & & & \\
\hline HF3 & -755.548898059841 & -0.823 & -0.771 & -0.052 & -755.358531030086 & -0.731 & -0.719 & -0.012 \\
\hline $\mathrm{Hx} 4$ & -79.883010287708 & & & & -79.854027234807 & & & \\
\hline $\mathrm{xF} 4$ & -675.664645991311 & & & & -675.503401231715 & & & \\
\hline $\mathrm{HF} 4$ & -755.549102791869 & -0.951 & -0.908 & -0.044 & -755.359249579626 & -1.182 & -1.143 & -0.039 \\
\hline $\mathrm{Fx} 1$ & -675.664617425776 & & & & -675.503322051978 & & & \\
\hline $\mathrm{xF} 1$ & -675.664587200765 & & & & -675.503300080168 & & & \\
\hline $\mathrm{FF} 1$ & -1351.33046337945 & -0.826 & -0.790 & -0.036 & -1351.0084710138 & -1.126 & -1.160 & 0.034 \\
\hline $\mathrm{Fx} 2$ & -675.664690876929 & & & & -675.503342738584 & & & \\
\hline $\mathrm{xF} 2$ & -675.6646218685888 & & & & -675.503351695798 & & & \\
\hline $\mathrm{FF} 2$ & -1351.33047437217 & -0.833 & -0.729 & -0.104 & -1351.00770039685 & -0.642 & -0.631 & -0.011 \\
\hline
\end{tabular}




\begin{tabular}{|c|c|c|c|c|c|c|c|c|}
\hline & \multicolumn{4}{|c|}{ TPSS-D3/def2-QZVP } & \multicolumn{4}{|c|}{ M06L/def2-QZVP } \\
\hline & $\mathrm{E}[\mathrm{Ha}]$ & $\mathrm{BAE}\left[\mathrm{kcal} \mathrm{mol}{ }^{-1}\right]$ & $\operatorname{BIE}\left[\mathrm{kcal} \mathrm{mol}^{-1}\right]$ & E-def $\left[\mathrm{kcal} \mathrm{mol}^{-1}\right]$ & $\mathrm{E}[\mathrm{Ha}]$ & $\mathrm{BAE}\left[\mathrm{kcal} \mathrm{mol}{ }^{-1}\right]$ & $\mathrm{BIE}\left[\mathrm{kcal} \mathrm{mol}^{-1}\right]$ & E-def $\left[\mathrm{kcal} \mathrm{mol}^{-1}\right]$ \\
\hline \multicolumn{9}{|c|}{ Propanes } \\
\hline H & -119.222649222907 & & & & -119.179099827686 & & & \\
\hline $\mathrm{F}$ & -913.586518906826 & & & & -913.367681855226 & & & \\
\hline $\mathrm{Hx} 1$ & -119.222652996912 & & & & -119.179120817425 & & & \\
\hline $\mathrm{xH} 1$ & -119.222653631568 & & & & -119.179120891353 & & & \\
\hline нH1 & -238.448285505759 & -1.874 & -1.869 & -0.005 & -238.361394812013 & -2.005 & -1.979 & -0.026 \\
\hline $\mathrm{Hx} 2$ & -119.222663212444 & & & & -119.179106907271 & & & \\
\hline $\mathrm{xH} 2$ & -119.222650776032 & & & & -119.179114463103 & & & \\
\hline HH2 & -238.447294250109 & -1.252 & -1.243 & -0.010 & -238.360028800746 & -1.148 & -1.134 & -0.014 \\
\hline $\mathrm{Hx} 1$ & -119.222637779103 & & & & -119.179096092706 & & & \\
\hline $\mathrm{xF} 1$ & -913.586506961758 & & & & -913.367662713436 & & & \\
\hline HF1 & -1032.81130609176 & -1.342 & -1.356 & 0.015 & -1032.54942797309 & -1.661 & -1.675 & 0.014 \\
\hline $\mathrm{Hx} 2$ & -119.222648383933 & & & & -119.179101577484 & & & \\
\hline $\mathrm{xF} 2$ & -913.586594959763 & & & & -913.367787273952 & & & \\
\hline HF2 & -1032.81102824425 & -1.167 & -1.120 & -0.047 & -1032.54871122369 & -1.211 & -1.144 & -0.067 \\
\hline $\mathrm{Hx} 3$ & -119.222665994781 & & & & -119.179084150446 & & & \\
\hline $\mathrm{xF} 3$ & -913.586594429269 & & & & -913.367672486197 & & & \\
\hline HF3 & -1032.81121911606 & -1.287 & -1.229 & -0.058 & -1032.54867005664 & -1.185 & -1.201 & 0.016 \\
\hline $\mathrm{Hx} 4$ & -119.222640517255 & & & & -119.179092651281 & & & \\
\hline $\mathrm{xF} 4$ & -913.586586246972 & & & & -913.367819067733 & & & \\
\hline $\mathrm{HF} 4$ & -1032.81077039232 & -1.005 & -0.969 & -0.037 & -1032.5486422136 & -1.168 & -1.086 & -0.082 \\
\hline $\mathrm{Fx} 1$ & -913.586331786741 & & & & -913.367601110676 & & & \\
\hline $\mathrm{xF} 1$ & -913.586437691329 & & & & -913.367697999224 & & & \\
\hline FF1 & -1827.17431975313 & -0.804 & -0.973 & 0.168 & -1826.7380290992 & -1.673 & -1.713 & 0.041 \\
\hline $\mathrm{Fx} 2$ & -913.586517451097 & & & & -913.367681747519 & & & \\
\hline $\mathrm{xF} 2$ & -913.586514967614 & & & & -913.367676438581 & & & \\
\hline FF2 & -1827.17428008465 & -0.780 & -0.783 & 0.003 & -1826.73817057167 & -1.761 & -1.765 & 0.003 \\
\hline \multicolumn{9}{|c|}{ Butanes } \\
\hline $\mathrm{H}$ & -158.56234695919 & & & & -158.504212619942 & & & \\
\hline F & -1151.50774503466 & & & & -1151.23169986605 & & & \\
\hline $\mathrm{Hx} 1$ & -158.562342713572 & & & & -158.504211882524 & & & \\
\hline $\mathrm{xH} 1$ & -158.562346989899 & & & & -158.504227177329 & & & \\
\hline HH1 & -317.12888303279 & -2.629 & -2.631 & 0.003 & -317.013345940843 & -3.088 & -3.079 & -0.009 \\
\hline $\mathrm{Hx} 2$ & -158.562336484009 & & & & -158.504210227948 & & & \\
\hline $\mathrm{xH} 2$ & -158.562354961524 & & & & -158.504216918158 & & & \\
\hline HH2 & -317.127468254398 & -1.741 & -1.742 & 0.002 & -317.011147942597 & -1.709 & -1.707 & -0.001 \\
\hline $\mathrm{Hx} 1$ & -158.562346534096 & & & & -158.504192021048 & & & \\
\hline $\mathrm{xF} 1$ & -1151.50763748558 & & & & -1151.23116793546 & & & \\
\hline HF1 & -1310.07287900113 & -1.749 & -1.817 & 0.068 & -1309.73820569109 & -1.439 & -1.786 & 0.347 \\
\hline $\mathrm{Hx} 2$ & -158.562333645964 & & & & -158.504195645872 & & & \\
\hline $\mathrm{xF} 2$ & -1151.50774102669 & & & & -1151.23166288416 & & & \\
\hline HF2 & -1310.07278794136 & -1.692 & -1.703 & 0.011 & -1309.73931301338 & -2.134 & -2.168 & 0.034 \\
\hline $\mathrm{Hx} 3$ & -158.562304669652 & & & & -158.504175269299 & & & \\
\hline $\mathrm{xF} 3$ & -1151.50775628473 & & & & -1151.23160089333 & & & \\
\hline HF3 & -1310.07251134893 & -1.518 & -1.538 & 0.019 & -1309.73906834421 & -1.980 & -2.066 & 0.086 \\
\hline $\mathrm{Hx} 4$ & -158.562299787449 & & & & -158.504185404812 & & & \\
\hline $\mathrm{xF} 4$ & -1151.50774544679 & & & & -1151.23166362074 & & & \\
\hline $\mathrm{HF} 4$ & -1310.07280540381 & -1.703 & -1.732 & 0.029 & -1309.73915565291 & -2.035 & -2.075 & 0.040 \\
\hline $\mathrm{Fx} 1$ & -1151.50770754885 & & & & -1151.23162894804 & & & \\
\hline $\mathrm{xF} 1$ & -1151.50771870599 & & & & -1151.23167791501 & & & \\
\hline FF1 & -2303.01778161085 & -1.438 & -1.478 & 0.040 & -2302.46635841842 & -1.857 & -1.915 & 0.058 \\
\hline $\mathrm{Fx} 2$ & -1151.50774122351 & & & & -1151.23147183144 & & & \\
\hline $\mathrm{xF} 2$ & -1151.50768784112 & & & & -1151.23150039952 & & & \\
\hline FF2 & -2303.01749786521 & -1.260 & -1.298 & 0.038 & -2302.46671580194 & -2.081 & -2.349 & 0.268 \\
\hline
\end{tabular}




\begin{tabular}{|c|c|c|c|c|c|c|c|c|}
\hline & \multicolumn{4}{|c|}{ TPSS-D3/def2-QZVP } & \multicolumn{4}{|c|}{ M06L/def2-QZVP } \\
\hline & $\mathrm{E}[\mathrm{Ha}]$ & $\mathrm{BAE}\left[\mathrm{kcal} \mathrm{mol}^{-1}\right]$ & BIE $\left[\mathrm{kcal} \mathrm{mol}^{-1}\right]$ & E-def $\left[\mathrm{kcal} \mathrm{mol}^{-1}\right]$ & $\mathrm{E}[\mathrm{Ha}]$ & $\mathrm{BAE}\left[\mathrm{kcal} \mathrm{mol}^{-1}\right]$ & $\mathrm{BIE}\left[\mathrm{kcal} \mathrm{mol}^{-1}\right]$ & E-def $\left[\mathrm{kcal} \mathrm{mol}^{-1}\right]$ \\
\hline \multicolumn{9}{|c|}{ Pentanes } \\
\hline H & -197.902019791865 & & & & -197.829207208924 & & & \\
\hline $\mathrm{F}$ & -1389.42908497232 & & & & -1389.09577099359 & & & \\
\hline $\mathrm{Hx} 1$ & -197.902004204388 & & & & -197.829246212566 & & & \\
\hline $\mathrm{xH} 1$ & -197.902004050381 & & & & -197.829246291122 & & & \\
\hline нH1 & -395.809390727747 & -3.358 & -3.378 & 0.02 & -395.664740278264 & -3.97 & -3.921 & -0.049 \\
\hline $\mathrm{Hx} 2$ & -197.902017496276 & & & & -197.82923276239 & & & \\
\hline $\mathrm{xH} 2$ & -197.902017377496 & & & & -197.829232623606 & & & \\
\hline нH2 & -395.807596376246 & -2.232 & -2.235 & 0.003 & -395.662425874782 & -2.517 & -2.485 & -0.032 \\
\hline Hx1 & -197.901916612921 & & & & -197.829090470193 & & & \\
\hline $\mathrm{xF} 1$ & -1389.42902030315 & & & & -1389.09559494498 & & & \\
\hline HF1 & -1587.33446999986 & -2.112 & -2.217 & 0.105 & -1586.9295349048 & -2.859 & -3.043 & 0.184 \\
\hline $\mathrm{Hx} 2$ & -197.901990335994 & & & & -197.829163666231 & & & \\
\hline $\mathrm{xF} 2$ & -1389.4287809975 & & & & -1389.09532405391 & & & \\
\hline $\mathrm{HF} 2$ & -1587.3341659679 & -1.921 & -2.13 & 0.209 & -1586.92844471739 & -2.175 & -2.483 & 0.308 \\
\hline $\mathrm{Hx} 3$ & -197.901993556334 & & & & -197.829209911164 & & & \\
\hline $\mathrm{xF} 3$ & -1389.42917547656 & & & & -1389.09573258885 & & & \\
\hline HF3 & -1587.33425127319 & -1.974 & -1.934 & -0.04 & -1586.9286525406 & -2.306 & -2.328 & 0.022 \\
\hline $\mathrm{Hx} 4$ & -197.901979411593 & & & & -197.829188437833 & & & \\
\hline $\mathrm{xF} 4$ & -1389.42913183573 & & & & -1389.0957270257 & & & \\
\hline $\mathrm{HF} 4$ & -1587.3346836789 & -2.246 & -2.242 & -0.004 & -1586.92964572275 & -2.929 & -2.968 & 0.039 \\
\hline $\mathrm{Fx} 1$ & -1389.42903955758 & & & & -1389.09572045492 & & & \\
\hline $\mathrm{xF} 1$ & -1389.42905257742 & & & & -1389.09565299646 & & & \\
\hline $\mathrm{FF} 1$ & -2778.86074818065 & -1.618 & -1.667 & 0.049 & -2778.19610154589 & -2.861 & -2.967 & 0.106 \\
\hline $\mathrm{Fx} 2$ & -1389.42901969083 & & & & -1389.09569757717 & & & \\
\hline $\mathrm{xF} 2$ & -1389.42902355845 & & & & -1389.09569756873 & & & \\
\hline $\mathrm{FF} 2$ & -2778.86072872366 & -1.606 & -1.685 & 0.08 & -2778.19566561915 & -2.588 & -2.68 & 0.092 \\
\hline \multicolumn{9}{|c|}{ Hexanes } \\
\hline $\mathrm{H}$ & -237.241693278491 & & & & -237.154200718982 & & & \\
\hline $\mathrm{F}$ & -1627.35045818311 & & & & -1626.95982446733 & & & \\
\hline $\mathrm{Hx} 1$ & -237.241676867185 & & & & -237.154258624144 & & & \\
\hline $\mathrm{xH} 1$ & -237.241676696576 & & & & -237.154258152089 & & & \\
\hline HH1 & -474.489698483428 & -3.961 & -3.981 & 0.021 & -474.315850648367 & -4.674 & -4.602 & -0.072 \\
\hline $\mathrm{Hx} 2$ & -237.241698189312 & & & & -237.15424527861 & & & \\
\hline $\mathrm{xH} 2$ & -237.241698222044 & & & & -237.154245192201 & & & \\
\hline нH2 & -474.4877403738 & -2.732 & -2.726 & -0.006 & -474.313284969139 & -3.064 & -3.009 & -0.056 \\
\hline $\mathrm{Hx} 1$ & -237.241509247322 & & & & -237.15402395451 & & & \\
\hline $\mathrm{xF} 1$ & -1627.35000388271 & & & & -1626.95917484718 & & & \\
\hline HF1 & -1864.59537016007 & -2.02 & -2.42 & 0.401 & -1864.11852106709 & -2.821 & -3.34 & 0.519 \\
\hline $\mathrm{Hx} 2$ & -237.241609105753 & & & & -237.154120938445 & & & \\
\hline $\mathrm{xF} 2$ & -1627.35012070514 & & & & -1626.95922897748 & & & \\
\hline $\mathrm{HF} 2$ & -1864.59588996368 & -2.346 & -2.611 & 0.265 & -1864.11807012286 & -2.538 & -2.962 & 0.424 \\
\hline $\mathrm{Hx} 3$ & -237.241564505658 & & & & -237.15407286172 & & & \\
\hline $\mathrm{xF} 3$ & -1627.35014529302 & & & & -1626.95938614533 & & & \\
\hline HF3 & -1864.59560733316 & -2.169 & -2.446 & 0.277 & -1864.11844143075 & -2.771 & -3.127 & 0.355 \\
\hline $\mathrm{Hx} 4$ & -237.24168388154 & & & & -237.154219531913 & & & \\
\hline $\mathrm{xF} 4$ & -1627.35041899558 & & & & -1626.95973174262 & & & \\
\hline $\mathrm{HF} 4$ & -1864.59629351458 & -2.599 & -2.63 & 0.03 & -1864.11885655251 & -3.032 & -3.078 & 0.046 \\
\hline $\mathrm{Fx} 1$ & -1627.35033876335 & & & & -1626.95968274096 & & & \\
\hline $\mathrm{xF} 1$ & -1627.35036472861 & & & & -1626.95970909978 & & & \\
\hline FF1 & -3254.70384325965 & -1.837 & -1.97 & 0.134 & -3253.92605123883 & -4.018 & -4.179 & 0.161 \\
\hline $\mathrm{Fx} 2$ & -1627.3502755816 & & & & -1626.95968417419 & & & \\
\hline $\mathrm{xF} 2$ & -1627.35027637325 & & & & -1626.9596817135 & & & \\
\hline $\mathrm{FF} 2$ & -3254.70260179171 & -1.058 & -1.286 & 0.229 & -3253.92265301019 & -1.885 & -2.063 & 0.178 \\
\hline
\end{tabular}




\begin{tabular}{|c|c|c|c|c|c|c|c|c|}
\hline & \multicolumn{4}{|c|}{ M06L-D3/def2-QZVP } & \multicolumn{4}{|c|}{ rPW86PW92-vdW-DF10/pc-3 } \\
\hline & $\mathrm{E}[\mathrm{Ha}]$ & $\mathrm{BAE}\left[\mathrm{kcal} \mathrm{mol}^{-1}\right]$ & $\mathrm{BIE}\left[\mathrm{kcal} \mathrm{mol}^{-1}\right]$ & E-def $\left[\mathrm{kcal} \mathrm{mol}^{-1}\right]$ & $\mathrm{E}[\mathrm{Ha}]$ & $\mathrm{BAE}\left[\mathrm{kcal} \mathrm{mol}^{-1}\right]$ & $\mathrm{BIE}\left[\mathrm{kcal} \mathrm{mol}^{-1}\right]$ & E-def $\left[\mathrm{kcal} \mathrm{mol}^{-1}\right]$ \\
\hline \multicolumn{9}{|c|}{ Methanes } \\
\hline $\mathrm{H}$ & -40.531313494154 & & & & -40.8510656396 & & & \\
\hline $\mathrm{F}$ & -437.63888562999 & & & & -439.6412093037 & & & \\
\hline $\mathrm{Hx} 1$ & -40.531310628153 & & & & -40.8510693484 & & & \\
\hline $\mathrm{xH} 1$ & -40.531311265437 & & & & -40.8510740856 & & & \\
\hline HH1 & -81.063540656048 & -0.573 & -0.577 & 0.003 & -81.7031539731 & -0.642 & -0.634 & -0.008 \\
\hline $\mathrm{H} \times 2$ & -40.531312155147 & & & & -40.8510589574 & & & \\
\hline $\mathrm{xH} 2$ & -40.531312489944 & & & & -40.8510547251 & & & \\
\hline HH2 & -81.06316146489 & -0.335 & -0.337 & 0.001 & -81.7027364161 & -0.380 & -0.391 & 0.011 \\
\hline $\mathrm{Hx} 1$ & -40.531311907622 & & & & -40.851056957 & & & \\
\hline $\mathrm{xF} 1$ & -437.638872988599 & & & & -439.6413398624 & & & \\
\hline HF1 & -478.171625635736 & -0.895 & -0.904 & 0.009 & -480.4939410165 & -1.045 & -0.969 & -0.076 \\
\hline $\mathrm{Hx} 2$ & -40.531313108451 & & & & -40.8510949362 & & & \\
\hline $\mathrm{xF} 2$ & -437.638875513836 & & & & -439.6412358168 & & & \\
\hline HF2 & -478.170774711101 & -0.361 & -0.368 & 0.007 & -480.4933560313 & -0.678 & -0.643 & -0.035 \\
\hline $\mathrm{Fx} 1$ & -437.63886127239 & & & & -439.6412659791 & & & \\
\hline $\mathrm{xF} 1$ & -437.63888014626 & & & & -439.6412143835 & & & \\
\hline FF1 & -875.279222636535 & -0.911 & -0.929 & 0.019 & -879.2841866738 & -1.109 & -1.071 & -0.039 \\
\hline $\mathrm{Fx} 2$ & -437.638878792144 & & & & -439.641181742 & & & \\
\hline $\mathrm{xF} 2$ & -437.63886376985 & & & & -439.6412362103 & & & \\
\hline FF2 & -875.278836451879 & -0.668 & -0.686 & 0.018 & -879.2840123925 & -1.000 & -1.001 & 0.000 \\
\hline \multicolumn{9}{|c|}{ Ethanes } \\
\hline $\mathrm{H}$ & -79.854039272244 & & & & -80.4514493009 & & & \\
\hline $\mathrm{F}$ & -675.503370187583 & & & & -678.6297265259 & & & \\
\hline $\mathrm{Hx} 1$ & -79.8540440827 & & & & -80.451449053 & & & \\
\hline $\mathrm{xH} 1$ & -79.854042316937 & & & & -80.4514339121 & & & \\
\hline HH1 & -159.710360601872 & -1.432 & -1.427 & -0.005 & -160.9052225053 & -1.458 & -1.468 & 0.010 \\
\hline $\mathrm{H} \times 2$ & -79.854039175391 & & & & -80.4514491704 & & & \\
\hline $\mathrm{xH} 2$ & -79.854037794075 & & & & -80.4514452927 & & & \\
\hline $\mathrm{HH} 2$ & -159.710087936175 & -1.261 & -1.262 & 0.001 & -160.9050497817 & -1.350 & -1.352 & 0.003 \\
\hline $\mathrm{Hx} 1$ & -79.85403888336 & & & & -80.4513612733 & & & \\
\hline $\mathrm{xF} 1$ & -675.503368020086 & & & & -678.6299899071 & & & \\
\hline HF1 & -755.359543885376 & -1.339 & -1.341 & 0.002 & -759.0838300134 & -1.666 & -1.555 & -0.110 \\
\hline $\mathrm{Hx} 2$ & -79.854038911322 & & & & -80.4514242626 & & & \\
\hline $\mathrm{xF} 2$ & -675.503515992153 & & & & -678.6300688824 & & & \\
\hline HF2 & -755.35991293815 & -1.571 & -1.480 & -0.091 & -759.0840111592 & -1.779 & -1.580 & -0.199 \\
\hline $\mathrm{Hx} 3$ & -79.854041757239 & & & & -80.4514557207 & & & \\
\hline $\mathrm{xF} 3$ & -675.503387014904 & & & & -678.6299769076 & & & \\
\hline HF3 & -755.35902395819 & -1.013 & -1.001 & -0.012 & -759.0835257052 & -1.475 & -1.313 & -0.161 \\
\hline $\mathrm{Hx} 4$ & -79.854039016178 & & & & -80.4513758026 & & & \\
\hline $\mathrm{xF} 4$ & -675.503433180248 & & & & -678.6297990257 & & & \\
\hline HF4 & -755.359775026334 & -1.484 & -1.445 & -0.039 & -759.0838229011 & -1.661 & -1.662 & 0.001 \\
\hline Fx1 & -675.50335390688 & & & & -678.6297825313 & & & \\
\hline $\mathrm{xF} 1$ & -675.503331919154 & & & & -678.6298903181 & & & \\
\hline FF1 & -1351.00902692263 & -1.435 & -1.469 & 0.034 & -1357.262660815 & -2.013 & -1.875 & -0.138 \\
\hline Fx2 & -675.503374723874 & & & & -678.6299160046 & & & \\
\hline $\mathrm{xF} 2$ & -675.50338363883 & & & & -678.6299071005 & & & \\
\hline FF2 & -1351.00820143222 & -0.917 & -0.906 & -0.011 & -1357.2623402932 & -1.812 & -1.580 & -0.232 \\
\hline
\end{tabular}




\begin{tabular}{|c|c|c|c|c|c|c|c|c|}
\hline & \multicolumn{4}{|c|}{ M06L-D3/def2-QZVP } & \multicolumn{4}{|c|}{ rPW86PW92-vdW-DF10/pc-3 } \\
\hline & $\mathrm{E}[\mathrm{Ha}]$ & $\mathrm{BAE}\left[\mathrm{kcal} \mathrm{mol}^{-1}\right]$ & $\mathrm{BIE}\left[\mathrm{kcal} \mathrm{mol}^{-1}\right]$ & E-def $\left[\mathrm{kcal} \mathrm{mol}^{-1}\right]$ & $\mathrm{E}[\mathrm{Ha}]$ & $\mathrm{BAE}\left[\mathrm{kcal} \mathrm{mol}^{-1}\right]$ & $\mathrm{BIE}\left[\mathrm{kcal} \mathrm{mol}^{-1}\right]$ & E-def $\left[\mathrm{kcal} \mathrm{mol}^{-1}\right]$ \\
\hline \multicolumn{9}{|c|}{ Propanes } \\
\hline $\mathrm{H}$ & -119.179174685064 & & & & -120.0545345692 & & & \\
\hline $\mathrm{F}$ & -913.367837461475 & & & & -917.6166781956 & & & \\
\hline $\mathrm{Hx} 1$ & -119.179195935982 & & & & -120.0545433359 & & & \\
\hline $\mathrm{xH} 1$ & -119.179196019286 & & & & -120.054544287 & & & \\
\hline HH1 & -238.362220065339 & -2.429 & -2.402 & -0.027 & -240.1128279819 & -2.359 & -2.347 & -0.012 \\
\hline $\mathrm{Hx} 2$ & -119.179182095252 & & & & -120.0545573652 & & & \\
\hline $\mathrm{xH} 2$ & -119.179189505402 & & & & -120.0545333223 & & & \\
\hline $\mathrm{HH} 2$ & -238.360764436726 & -1.515 & -1.502 & -0.014 & -240.1115329459 & -1.546 & -1.533 & -0.014 \\
\hline $\mathrm{Hx} 1$ & -119.179170847752 & & & & -120.0545253421 & & & \\
\hline $\mathrm{xF} 1$ & -913.367818203814 & & & & -917.6167229623 & & & \\
\hline HF1 & -1032.55043458467 & -2.148 & -2.162 & 0.014 & -1037.6749357906 & -2.336 & -2.314 & -0.022 \\
\hline $\mathrm{Hx} 2$ & -119.179176463796 & & & & -120.0545346031 & & & \\
\hline $\mathrm{xF} 2$ & -913.36794252332 & & & & -917.6168435381 & & & \\
\hline HF2 & -1032.54971371423 & -1.695 & -1.628 & -0.067 & -1037.6744857594 & -2.054 & -1.950 & -0.104 \\
\hline $\mathrm{Hx} 3$ & -119.179159110814 & & & & -120.0545501035 & & & \\
\hline $\mathrm{xF} 3$ & -913.367828045902 & & & & -917.6168318342 & & & \\
\hline HF3 & -1032.54965869595 & -1.661 & -1.676 & 0.016 & -1037.674481074 & -2.051 & -1.945 & -0.106 \\
\hline $\mathrm{Hx} 4$ & -119.179167561595 & & & & -120.0545319813 & & & \\
\hline $\mathrm{xF} 4$ & -913.36797403723 & & & & -917.616806891 & & & \\
\hline HF 4 & -1032.54966079082 & -1.662 & -1.581 & -0.081 & -1037.6744097202 & -2.006 & -1.927 & -0.079 \\
\hline Fx1 & -913.367757029184 & & & & -917.6163891716 & & & \\
\hline $\mathrm{xF} 1$ & -913.367853075201 & & & & -917.6165921804 & & & \\
\hline $\mathrm{FF} 1$ & -1826.73915142545 & -2.182 & -2.222 & 0.041 & -1835.2367320651 & -2.118 & -2.354 & 0.235 \\
\hline $\mathrm{Fx} 2$ & -913.367837394936 & & & & -917.6166736657 & & & \\
\hline $\mathrm{xF} 2$ & -913.367832093387 & & & & -917.6166665186 & & & \\
\hline $\mathrm{FF} 2$ & -1826.73929114994 & -2.269 & -2.273 & 0.003 & -1835.2375886881 & -2.656 & -2.666 & 0.010 \\
\hline \multicolumn{9}{|c|}{ Butanes } \\
\hline $\mathrm{H}$ & -158.504405429392 & & & & -159.6577013044 & & & \\
\hline $\mathrm{F}$ & -1151.23205085392 & & & & -1156.6027837611 & & & \\
\hline Hx1 & -158.504404762333 & & & & -159.6577088502 & & & \\
\hline $\mathrm{xH} 1$ & -158.504420022722 & & & & -159.657712478 & & & \\
\hline HH1 & -317.014768736239 & -3.739 & -3.730 & -0.009 & -319.3203436152 & -3.101 & -3.089 & -0.012 \\
\hline $\mathrm{Hx} 2$ & -158.504402982743 & & & & -159.6576874725 & & & \\
\hline $\mathrm{xH} 2$ & -158.504409827564 & & & & -159.6577182613 & & & \\
\hline $\mathrm{HH} 2$ & -317.01242018095 & -2.265 & -2.264 & -0.001 & -319.3184440044 & -1.909 & -1.907 & -0.002 \\
\hline Hx1 & -158.5043848569 & & & & -159.6577115253 & & & \\
\hline $\mathrm{xF} 1$ & -1151.23151472739 & & & & -1156.602908989 & & & \\
\hline HF1 & -1309.74000445396 & -2.227 & -2.576 & 0.349 & -1316.2655957806 & -3.207 & -3.122 & -0.085 \\
\hline $\mathrm{Hx} 2$ & -158.504388489769 & & & & -159.6576948043 & & & \\
\hline $\mathrm{xF} 2$ & -1151.23201292053 & & & & -1156.6029340376 & & & \\
\hline HF2 & -1309.74109149874 & -2.909 & -2.943 & 0.034 & -1316.2653016023 & -3.022 & -2.932 & -0.090 \\
\hline $\mathrm{Hx} 3$ & -158.504367975808 & & & & -159.6576578688 & & & \\
\hline $\mathrm{xF} 3$ & -1151.2319501625 & & & & -1156.602864446 & & & \\
\hline HF3 & -1309.74078932977 & -2.719 & -2.806 & 0.087 & -1316.2652381355 & -2.983 & -2.959 & -0.023 \\
\hline $\mathrm{Hx} 4$ & -158.504378234664 & & & & -159.6576927182 & & & \\
\hline $\mathrm{xF} 4$ & -1151.2320140071 & & & & -1156.6028528897 & & & \\
\hline HF 4 & -1309.74086119089 & -2.764 & -2.804 & 0.040 & -1316.2652212975 & -2.972 & -2.934 & -0.038 \\
\hline $\mathrm{Fx} 1$ & -1151.23197855604 & & & & -1156.6027323388 & & & \\
\hline $\mathrm{xF} 1$ & -1151.23202832642 & & & & -1156.6028807005 & & & \\
\hline FF1 & -2302.4681530638 & -2.542 & -2.602 & 0.060 & -2313.2101861807 & -2.898 & -2.870 & -0.029 \\
\hline $\mathrm{Fx} 2$ & -1151.23182029732 & & & & -1156.6030145974 & & & \\
\hline $\mathrm{xF} 2$ & -1151.23184890978 & & & & -1156.602804627 & & & \\
\hline FF2 & -2302.46852549155 & -2.776 & -3.047 & 0.271 & -2313.2109816812 & -3.397 & -3.239 & -0.158 \\
\hline
\end{tabular}




\begin{tabular}{|c|c|c|c|c|c|c|c|c|}
\hline & \multicolumn{4}{|c|}{ M06L-D3/def2-QZVP } & \multicolumn{4}{|c|}{ rPW86PW92-vdW-DF10/pc-3 } \\
\hline & $\mathrm{E}[\mathrm{Ha}]$ & $\mathrm{BAE}\left[\mathrm{kcal} \mathrm{mol}{ }^{-1}\right]$ & BIE $\left[\mathrm{kcal} \mathrm{mol}^{-1}\right]$ & E-def $\left[\mathrm{kcal} \mathrm{mol}^{-1}\right]$ & E [Ha] & BAE $\left[\mathrm{kcal} \mathrm{mol}^{-1}\right]$ & $\mathrm{BIE}\left[\mathrm{kcal} \mathrm{mol}^{-1}\right]$ & E-def $\left[\mathrm{kcal} \mathrm{mol}^{-1}\right]$ \\
\hline \multicolumn{9}{|c|}{ Pentanes } \\
\hline $\mathrm{H}$ & -197.829546912061 & & & & -199.2606853165 & & & \\
\hline $\mathrm{F}$ & -1389.09635173789 & & & & -1395.588991017 & & & \\
\hline Hx1 & -197.829585908694 & & & & -199.2606771086 & & & \\
\hline xH1 & -197.829585987278 & & & & -199.2606788554 & & & \\
\hline HH1 & -395.666853816336 & -4.869 & -4.820 & -0.049 & -398.5283621744 & -4.387 & -4.396 & 0.009 \\
\hline $\mathrm{Hx} 2$ & -197.829572457672 & & & & -199.2607009512 & & & \\
\hline $\mathrm{xH} 2$ & -197.829572319054 & & & & -199.2607019433 & & & \\
\hline нH2 & -395.664329804598 & -3.286 & -3.254 & -0.032 & -398.52585015 & -2.811 & -2.791 & -0.020 \\
\hline Hx1 & -197.829430387443 & & & & -199.2608299172 & & & \\
\hline $\mathrm{xF} 1$ & -1389.09617298117 & & & & -1395.5891164208 & & & \\
\hline HF1 & -1586.93208436091 & -3.882 & -4.067 & 0.185 & -1594.8560653088 & -4.009 & -3.840 & -0.169 \\
\hline $\mathrm{Hx} 2$ & -197.829503425025 & & & & -199.2607321568 & & & \\
\hline $\mathrm{xF} 2$ & -1389.09590115683 & & & & -1395.5890465671 & & & \\
\hline $\mathrm{HF} 2$ & -1586.93083565244 & -3.098 & -3.408 & 0.310 & -1594.8554131765 & -3.600 & -3.536 & -0.064 \\
\hline Hx3 & -197.829549637961 & & & & -199.2607080866 & & & \\
\hline $\mathrm{xF} 3$ & -1389.09631197743 & & & & -1395.5893589664 & & & \\
\hline HF3 & -1586.93105857581 & -3.238 & -3.261 & 0.023 & -1594.8553784205 & -3.578 & -3.333 & -0.245 \\
\hline $\mathrm{Hx} 4$ & -197.829528116067 & & & & -199.2606733081 & & & \\
\hline $\mathrm{xF} 4$ & -1389.09630670999 & & & & -1395.5891777798 & & & \\
\hline $\mathrm{HF} 4$ & -1586.93219755266 & -3.953 & -3.993 & 0.040 & -1594.8563005292 & -4.157 & -4.047 & -0.110 \\
\hline Fx1 & -1389.09630074287 & & & & -1395.5890811807 & & & \\
\hline $\mathrm{xF} 1$ & -1389.09623211052 & & & & -1395.5891323758 & & & \\
\hline $\mathrm{FF} 1$ & -2778.19885254001 & -3.859 & -3.966 & 0.107 & -2791.1845148135 & -4.099 & -3.954 & -0.145 \\
\hline $\mathrm{Fx} 2$ & -1389.09627708287 & & & & -1395.5889126617 & & & \\
\hline $\mathrm{xF} 2$ & -1389.09627708811 & & & & -1395.5889853576 & & & \\
\hline $\mathrm{FF} 2$ & -2778.19826636464 & -3.491 & -3.584 & 0.094 & -2791.1840640257 & -3.817 & -3.869 & 0.053 \\
\hline \multicolumn{9}{|c|}{ Hexanes } \\
\hline $\mathrm{H}$ & -237.154697232514 & & & & -238.8638768436 & & & \\
\hline F & -1626.96064808145 & & & & -1634.5753070568 & & & \\
\hline $\mathrm{Hx1}$ & -237.154755174877 & & & & -238.8638584254 & & & \\
\hline xH1 & -237.154754702224 & & & & -238.8638607577 & & & \\
\hline HH1 & -474.318634788667 & -5.798 & -5.726 & -0.072 & -477.735112896 & -4.618 & -4.640 & 0.022 \\
\hline $\mathrm{Hx} 2$ & -237.154741846345 & & & & -238.863865576 & & & \\
\hline $\mathrm{xH} 2$ & -237.15474176022 & & & & -238.863878442 & & & \\
\hline HH2 & -474.315825402905 & -4.035 & -3.980 & -0.056 & -477.7327391994 & -3.128 & -3.135 & 0.006 \\
\hline Hx1 & -237.154520998888 & & & & -238.8638527077 & & & \\
\hline $\mathrm{xF} 1$ & -1626.95999282742 & & & & -1634.5753072981 & & & \\
\hline HF1 & -1864.12189613397 & -4.111 & -4.632 & 0.522 & -1873.4461234753 & -4.355 & -4.370 & 0.015 \\
\hline $\mathrm{Hx} 2$ & -237.154617823874 & & & & -238.8637670022 & & & \\
\hline $\mathrm{xF} 2$ & -1626.96004847542 & & & & -1634.5751558421 & & & \\
\hline HF2 & -1864.12132916802 & -3.755 & -4.181 & 0.426 & -1873.445887794 & -4.207 & -4.371 & 0.164 \\
\hline Hx3 & -237.154569569288 & & & & -238.8639312758 & & & \\
\hline $\mathrm{xF} 3$ & -1626.96020765417 & & & & -1634.5750455547 & & & \\
\hline HF3 & -1864.12174521149 & -4.016 & -4.372 & 0.356 & -1873.4460397874 & -4.302 & -4.432 & 0.130 \\
\hline $\mathrm{Hx} 4$ & -237.154716136238 & & & & -238.8639121156 & & & \\
\hline $\mathrm{xF} 4$ & -1626.96055416028 & & & & -1634.5755362197 & & & \\
\hline $\mathrm{HF} 4$ & -1864.12214472893 & -4.267 & -4.314 & 0.047 & -1873.4464576914 & -4.564 & -4.398 & -0.166 \\
\hline Fx1 & -1626.96050589548 & & & & -1634.5750833833 & & & \\
\hline $\mathrm{xF} 1$ & -1626.96053246581 & & & & -1634.5753596311 & & & \\
\hline $\mathrm{FF} 1$ & -3253.92982130881 & -5.350 & -5.511 & 0.162 & -3269.1590413137 & -5.288 & -5.396 & 0.107 \\
\hline $\mathrm{Fx} 2$ & -1626.96051003683 & & & & -1634.5749524227 & & & \\
\hline $\mathrm{xF} 2$ & -1626.96050748751 & & & & -1634.5749457219 & & & \\
\hline $\mathrm{FF} 2$ & -3253.92603885371 & -2.976 & -3.151 & 0.175 & -3269.1567315811 & -3.839 & -4.288 & 0.449 \\
\hline
\end{tabular}




\begin{tabular}{|c|c|c|c|c|c|c|c|c|}
\hline & \multicolumn{4}{|c|}{ M06-2X/def2-QZVP } & \multicolumn{4}{|c|}{ M06-2X-D3/def2-QZVP } \\
\hline & $\mathrm{E}[\mathrm{Ha}]$ & $\mathrm{BAE}\left[\mathrm{kcal} \mathrm{mol}^{-1}\right]$ & $\mathrm{BIE}\left[\mathrm{kcal} \mathrm{mol}^{-1}\right]$ & E-def $\left[\mathrm{kcal} \mathrm{mol}^{-1}\right]$ & $\mathrm{E}[\mathrm{Ha}]$ & $\mathrm{BAE}\left[\mathrm{kcal} \mathrm{mol}^{-1}\right]$ & BIE $\left[\mathrm{kcal} \mathrm{mol}^{-1}\right]$ & E-def $\left[\mathrm{kcal} \mathrm{mol}^{-1}\right]$ \\
\hline \multicolumn{9}{|c|}{ Methanes } \\
\hline $\mathrm{H}$ & -40.506624277959 & & & & -40.506624419795 & & & \\
\hline $\mathrm{F}$ & -437.573388251481 & & & & -437.573388998429 & & & \\
\hline Hx1 & -40.506533736772 & & & & -40.50653387854 & & & \\
\hline $\mathrm{xH} 1$ & -40.506525030411 & & & & -40.506525172184 & & & \\
\hline HH1 & -81.013751947257 & -0.316 & -0.435 & 0.119 & -81.013857181298 & -0.382 & -0.501 & 0.119 \\
\hline $\mathrm{Hx} 2$ & -40.506494112138 & & & & -40.50649425397 & & & \\
\hline $\mathrm{xH} 2$ & -40.506481858911 & & & & -40.506482000747 & & & \\
\hline $\mathrm{HH} 2$ & -81.013440737748 & -0.121 & -0.292 & 0.171 & -81.013570468737 & -0.202 & -0.373 & 0.171 \\
\hline Hx1 & -40.506531614822 & & & & -40.506531756636 & & & \\
\hline $\mathrm{xF} 1$ & -437.573295171184 & & & & -437.57329591998 & & & \\
\hline HF1 & -478.080871528725 & -0.539 & -0.656 & 0.117 & -478.08105536543 & -0.654 & -0.770 & 0.117 \\
\hline $\mathrm{Hx} 2$ & -40.506623946011 & & & & -40.506624087803 & & & \\
\hline $\mathrm{xF} 2$ & -437.573352489113 & & & & -437.573353237384 & & & \\
\hline $\mathrm{HF} 2$ & -478.08034858822 & -0.211 & -0.234 & 0.023 & -478.08053883961 & -0.330 & -0.352 & 0.023 \\
\hline Fx1 & -437.573285703867 & & & & -437.573286451973 & & & \\
\hline $\mathrm{xF} 1$ & -437.573309479055 & & & & -437.57331022728 & & & \\
\hline $\mathrm{FF} 1$ & -875.147447246175 & -0.421 & -0.535 & 0.114 & -875.147624324572 & -0.531 & -0.645 & 0.114 \\
\hline $\mathrm{Fx} 2$ & -437.573309538083 & & & & -437.573310286477 & & & \\
\hline $\mathrm{xF} 2$ & -437.573257142947 & & & & -437.573257891144 & & & \\
\hline $\mathrm{FF} 2$ & -875.147392588487 & -0.387 & -0.518 & 0.132 & -875.147580909556 & -0.504 & -0.635 & 0.132 \\
\hline \multicolumn{9}{|c|}{ Ethanes } \\
\hline $\mathrm{H}$ & -79.814933219858 & & & & -79.814941721041 & & & \\
\hline $\mathrm{F}$ & -675.401082451369 & & & & -675.40110567909 & & & \\
\hline Hx1 & -79.814932721472 & & & & -79.814941234748 & & & \\
\hline $\mathrm{xH} 1$ & -79.814824946182 & & & & -79.814833460212 & & & \\
\hline HH1 & -159.631744332222 & -1.178 & -1.247 & 0.068 & -159.632077942442 & -1.377 & -1.445 & 0.068 \\
\hline $\mathrm{Hx} 2$ & -79.814990133969 & & & & -79.814998632432 & & & \\
\hline $\mathrm{xH} 2$ & -79.815004418129 & & & & -79.815012917078 & & & \\
\hline HH2 & -159.631483163056 & -1.015 & -0.934 & -0.080 & -159.631807916467 & -1.208 & -1.127 & -0.080 \\
\hline Hx1 & -79.814920621854 & & & & -79.814929124594 & & & \\
\hline $\mathrm{xF} 1$ & -675.401064094147 & & & & -675.401087427405 & & & \\
\hline HF1 & -755.217045976799 & -0.647 & -0.666 & 0.019 & -755.217500515714 & -0.912 & -0.931 & 0.019 \\
\hline $\mathrm{Hx} 2$ & -79.814986592888 & & & & -79.814995089083 & & & \\
\hline $\mathrm{xF} 2$ & -675.400870979205 & & & & -675.400894310354 & & & \\
\hline $\mathrm{HF} 2$ & -755.217122137153 & -0.694 & -0.794 & 0.099 & -755.217583936528 & -0.964 & -1.063 & 0.099 \\
\hline $\mathrm{Hx} 3$ & -79.814927859366 & & & & -79.814936357994 & & & \\
\hline $\mathrm{xF} 3$ & -675.401166900331 & & & & -675.401190272276 & & & \\
\hline HF3 & -755.216917432915 & -0.566 & -0.516 & -0.050 & -755.217354947991 & -0.820 & -0.771 & -0.050 \\
\hline $\mathrm{Hx} 4$ & -79.81510495871 & & & & -79.815113460836 & & & \\
\hline $\mathrm{xF} 4$ & -675.40114701712 & & & & -675.401170343237 & & & \\
\hline $\mathrm{HF} 4$ & -755.21738550104 & -0.860 & -0.711 & -0.148 & -755.21784826173 & -1.130 & -0.982 & -0.148 \\
\hline $\mathrm{Fx} 1$ & -675.401057099872 & & & & -675.401080355952 & & & \\
\hline $\mathrm{xF} 1$ & -675.401160696272 & & & & -675.401183939684 & & & \\
\hline $\mathrm{FF} 1$ & -1350.8033431572 & -0.739 & -0.706 & -0.033 & -1350.80382902467 & -1.015 & -0.982 & -0.033 \\
\hline $\mathrm{Fx} 2$ & -675.401063976818 & & & & -675.401087330707 & & & \\
\hline $\mathrm{xF} 2$ & -675.401200970927 & & & & -675.401224292829 & & & \\
\hline FF2 & -1350.80297288156 & -0.507 & -0.444 & -0.063 & -1350.80340945068 & -0.752 & -0.689 & -0.063 \\
\hline
\end{tabular}




\begin{tabular}{|c|c|c|c|c|c|c|c|c|}
\hline & \multicolumn{4}{|c|}{ M06-2X/def2-QZVP } & \multicolumn{4}{|c|}{ M06-2X-D3/def2-QZVP } \\
\hline & $\mathrm{E}[\mathrm{Ha}]$ & $\mathrm{BAE}\left[\mathrm{kcal} \mathrm{mol}{ }^{-1}\right]$ & $\mathrm{BIE}\left[\mathrm{kcal} \mathrm{mol}^{-1}\right]$ & E-def $\left[\mathrm{kcal} \mathrm{mol}^{-1}\right]$ & $\mathrm{E}[\mathrm{Ha}]$ & $\mathrm{BAE}\left[\mathrm{kcal} \mathrm{mol}^{-1}\right]$ & $\mathrm{BIE}\left[\mathrm{kcal} \mathrm{mol}^{-1}\right]$ & E-def $\left[\mathrm{kcal} \mathrm{mol}^{-1}\right]$ \\
\hline \multicolumn{9}{|c|}{ Propanes } \\
\hline H & -119.126434379236 & & & & -119.126492988755 & & & \\
\hline $\mathrm{F}$ & -913.229549836054 & & & & -913.229673716665 & & & \\
\hline $\mathrm{Hx} 1$ & -119.12636663932 & & & & -119.126425475808 & & & \\
\hline $\mathrm{xH} 1$ & -119.126364669245 & & & & -119.126423514884 & & & \\
\hline HН1 & -238.255476484992 & -1.636 & -1.723 & 0.086 & -238.25618709045 & -2.009 & -2.095 & 0.086 \\
\hline $\mathrm{Hx} 2$ & -119.126372708028 & & & & -119.126431604503 & & & \\
\hline $\mathrm{xH} 2$ & -119.12637788495 & & & & -119.126436656174 & & & \\
\hline HH2 & -238.254320845063 & -0.911 & -0.985 & 0.074 & -238.254963569123 & -1.241 & -1.315 & 0.074 \\
\hline $\mathrm{Hx} 1$ & -119.126429880875 & & & & -119.126488401389 & & & \\
\hline $\mathrm{xF} 1$ & -913.229337999544 & & & & -913.229461728969 & & & \\
\hline HF1 & -1032.35756346057 & -0.991 & -1.127 & 0.136 & -1032.35844735369 & -1.431 & -1.567 & 0.136 \\
\hline $\mathrm{Hx} 2$ & -119.126570466029 & & & & -119.126629099655 & & & \\
\hline $\mathrm{xF} 2$ & -913.229178809165 & & & & -913.229302351329 & & & \\
\hline $\mathrm{HF} 2$ & -1032.35693072129 & -0.594 & -0.741 & 0.147 & -1032.35781749967 & -1.036 & -1.184 & 0.148 \\
\hline $\mathrm{Hx} 3$ & -119.126460035265 & & & & -119.126518732339 & & & \\
\hline $\mathrm{xF} 3$ & -913.229269792262 & & & & -913.229393614863 & & & \\
\hline HF3 & -1032.35687144408 & -0.557 & -0.716 & 0.160 & -1032.35774283106 & -0.989 & -1.149 & 0.160 \\
\hline $\mathrm{Hx} 4$ & -119.126509969017 & & & & -119.126568625486 & & & \\
\hline $\mathrm{xF} 4$ & -913.229140940132 & & & & -913.22926423021 & & & \\
\hline $\mathrm{HF} 4$ & -1032.35671925713 & -0.461 & -0.670 & 0.209 & -1032.35761952757 & -0.912 & -1.121 & 0.209 \\
\hline Fx1 & -913.229340746907 & & & & -913.229464861789 & & & \\
\hline $\mathrm{xF} 1$ & -913.229376958566 & & & & -913.229500361599 & & & \\
\hline FF1 & -1826.46028011037 & -0.741 & -0.980 & 0.240 & -1826.46126954961 & -1.206 & -1.446 & 0.240 \\
\hline $\mathrm{Fx} 2$ & -913.22954028812 & & & & -913.229664212406 & & & \\
\hline $\mathrm{xF} 2$ & -913.22953011019 & & & & -913.22965404266 & & & \\
\hline FF2 & -1826.4606776204 & -0.990 & -1.009 & 0.018 & -1826.46165598907 & -1.449 & -1.467 & 0.018 \\
\hline \multicolumn{9}{|c|}{ Butanes } \\
\hline $\mathrm{H}$ & -158.438067270029 & & & & -158.438227672519 & & & \\
\hline $\mathrm{F}$ & -1151.05752770185 & & & & -1151.05782043843 & & & \\
\hline Hx1 & -158.437795653278 & & & & -158.437956121148 & & & \\
\hline $\mathrm{xH} 1$ & -158.437862534786 & & & & -158.438022974135 & & & \\
\hline Hн1 & -316.879829205757 & -2.318 & -2.617 & 0.299 & -316.881068992786 & -2.895 & -3.194 & 0.299 \\
\hline $\mathrm{Hx} 2$ & -158.437799967936 & & & & -158.437960322424 & & & \\
\hline $\mathrm{xH} 2$ & -158.437811227434 & & & & -158.43797172662 & & & \\
\hline HH2 & -316.877879759955 & -1.095 & -1.424 & 0.328 & -316.879000723385 & -1.597 & -1.926 & 0.328 \\
\hline Hx1 & -158.438009561757 & & & & -158.438169989744 & & & \\
\hline $\mathrm{xF} 1$ & -1151.05688945467 & & & & -1151.05717936607 & & & \\
\hline $\mathrm{HF} 1$ & -1309.49657842429 & -0.617 & -1.054 & 0.437 & -1309.49816708333 & -1.330 & -1.768 & 0.438 \\
\hline $\mathrm{Hx} 2$ & -158.438051732325 & & & & -158.438212171805 & & & \\
\hline $\mathrm{xF} 2$ & -1151.05747194133 & & & & -1151.0577640165 & & & \\
\hline $\mathrm{HF} 2$ & -1309.49745796476 & -1.169 & -1.214 & 0.045 & -1309.4990232083 & -1.867 & -1.912 & 0.045 \\
\hline $\mathrm{Hx} 3$ & -158.43795787314 & & & & -158.438118181865 & & & \\
\hline $\mathrm{xF} 3$ & -1151.0573595921 & & & & -1151.05765123724 & & & \\
\hline HF3 & -1309.49737123463 & -1.115 & -1.289 & 0.174 & -1309.4988937269 & -1.786 & -1.961 & 0.175 \\
\hline $\mathrm{Hx} 4$ & -158.437946224707 & & & & -158.438106640633 & & & \\
\hline $\mathrm{xF} 4$ & -1151.05752046813 & & & & -1151.0578128187 & & & \\
\hline $\mathrm{HF} 4$ & -1309.49740087116 & -1.133 & -1.214 & 0.080 & -1309.49890259192 & -1.791 & -1.872 & 0.081 \\
\hline Fx1 & -1151.05746013013 & & & & -1151.05775193612 & & & \\
\hline $\mathrm{xF} 1$ & -1151.05747866447 & & & & -1151.05777094118 & & & \\
\hline FF1 & -2302.11664533737 & -0.998 & -1.071 & 0.073 & -2302.11823777794 & -1.630 & -1.704 & 0.074 \\
\hline $\mathrm{Fx} 2$ & -1151.05727793152 & & & & -1151.0575689947 & & & \\
\hline $\mathrm{xF} 2$ & -1151.05729509385 & & & & -1151.05758610052 & & & \\
\hline FF2 & -2302.11657161285 & -0.951 & -1.254 & 0.303 & -2302.11816703545 & -1.585 & -1.890 & 0.305 \\
\hline
\end{tabular}




\begin{tabular}{|c|c|c|c|c|c|c|c|c|}
\hline & \multicolumn{4}{|c|}{ M06-2X/def2-QZVP } & \multicolumn{4}{|c|}{ M06-2X-D3/def2-QZVP } \\
\hline & $\mathrm{E}[\mathrm{Ha}]$ & $\mathrm{BAE}\left[\mathrm{kcal} \mathrm{mol}{ }^{-1}\right]$ & BIE $\left[\mathrm{kcal} \mathrm{mol}^{-1}\right]$ & E-def $\left[\mathrm{kcal} \mathrm{mol}^{-1}\right]$ & $\mathrm{E}[\mathrm{Ha}]$ & BAE $\left[\mathrm{kcal} \mathrm{mol}^{-1}\right]$ & BIE $\left[\mathrm{kcal} \mathrm{mol}^{-1}\right]$ & E-def $\left[\mathrm{kcal} \mathrm{mol}^{-1}\right]$ \\
\hline \multicolumn{9}{|c|}{ Pentanes } \\
\hline н & -197.749441168558 & & & & -197.749731142782 & & & \\
\hline $\mathrm{F}$ & -1388.88577417831 & & & & -1388.88626869996 & & & \\
\hline $\mathrm{Hx} 1$ & -197.749177593733 & & & & -197.749467565986 & & & \\
\hline $\mathrm{xH} 1$ & -197.749175663617 & & & & -197.749465635858 & & & \\
\hline нH1 & -395.503705449188 & -3.027 & -3.359 & 0.332 & -395.505564225936 & -3.829 & -4.161 & 0.332 \\
\hline Hx2 & -197.749122886594 & & & & -197.749412875496 & & & \\
\hline $\mathrm{xH} 2$ & -197.749128166813 & & & & -197.749418155953 & & & \\
\hline нH2 & -395.50137342704 & -1.563 & -1.959 & 0.396 & -395.503060881127 & -2.258 & -2.654 & 0.396 \\
\hline Hx1 & -197.749278094786 & & & & -197.749568201345 & & & \\
\hline $\mathrm{xF} 1$ & -1388.88552577365 & & & & -1388.88601837898 & & & \\
\hline HF1 & -1586.63762765877 & -1.514 & -1.772 & 0.258 & -1586.63989234664 & -2.443 & -2.702 & 0.259 \\
\hline $\mathrm{Hx} 2$ & -197.74940852951 & & & & -197.749698556699 & & & \\
\hline $\mathrm{xF} 2$ & -1388.88523418015 & & & & -1388.88572603339 & & & \\
\hline $\mathrm{HF} 2$ & -1586.63692310455 & -1.072 & -1.431 & 0.359 & -1586.63905065418 & -1.914 & -2.275 & 0.361 \\
\hline Hx3 & -197.74929966772 & & & & -197.749589673233 & & & \\
\hline $\mathrm{xF} 3$ & -1388.88561624516 & & & & -1388.88610973775 & & & \\
\hline HF3 & -1586.63711300479 & -1.191 & -1.379 & 0.188 & -1586.63926189702 & -2.047 & -2.235 & 0.189 \\
\hline $\mathrm{Hx} 4$ & -197.749349470575 & & & & -197.749639399292 & & & \\
\hline $\mathrm{xF} 4$ & -1388.88573977882 & & & & -1388.8862335089 & & & \\
\hline $\mathrm{HF} 4$ & -1586.63771947515 & -1.571 & -1.650 & 0.079 & -1586.63998034057 & -2.498 & -2.577 & 0.080 \\
\hline $\mathrm{Fx} 1$ & -1388.88569965021 & & & & -1388.88619393033 & & & \\
\hline $\mathrm{xF} 1$ & -1388.88554901496 & & & & -1388.88604236892 & & & \\
\hline FF1 & -2777.77373030164 & -1.369 & -1.557 & 0.188 & -2777.77618334649 & -2.288 & -2.477 & 0.189 \\
\hline $\mathrm{Fx} 2$ & -1388.88564790934 & & & & -1388.88614150698 & & & \\
\hline $\mathrm{xF} 2$ & -1388.88565173059 & & & & -1388.88614533993 & & & \\
\hline $\mathrm{FF} 2$ & -2777.77351411219 & -1.234 & -1.390 & 0.156 & -2777.77582504292 & -2.063 & -2.220 & 0.157 \\
\hline \multicolumn{9}{|c|}{ Hexanes } \\
\hline $\mathrm{H}$ & -237.060759966413 & & & & -237.061189291471 & & & \\
\hline $\mathrm{F}$ & -1626.71387372348 & & & & -1626.71458324202 & & & \\
\hline $\mathrm{Hx} 1$ & -237.060504392305 & & & & -237.060933750037 & & & \\
\hline $\mathrm{xH} 1$ & -237.060506485745 & & & & -237.060935842857 & & & \\
\hline HH1 & -474.127217935721 & -3.576 & -3.895 & 0.319 & -474.129679192762 & -4.581 & -4.901 & 0.319 \\
\hline $\mathrm{Hx} 2$ & -237.060452099559 & & & & -237.060881467488 & & & \\
\hline $\mathrm{xH} 2$ & -237.060441021288 & & & & -237.060870389517 & & & \\
\hline нH2 & -474.124676814695 & -1.981 & -2.374 & 0.393 & -474.126942416905 & -2.864 & -3.257 & 0.393 \\
\hline $\mathrm{Hx} 1$ & -237.060525268677 & & & & -237.060954958168 & & & \\
\hline $\mathrm{xF} 1$ & -1626.71300192605 & & & & -1626.71370739825 & & & \\
\hline HF1 & -1863.77666272148 & -1.273 & -1.968 & 0.694 & -1863.77967947148 & -2.452 & -3.148 & 0.697 \\
\hline $\mathrm{Hx} 2$ & -237.060679164864 & & & & -237.061108787726 & & & \\
\hline $\mathrm{xF} 2$ & -1626.71323419026 & & & & -1626.71394069975 & & & \\
\hline $\mathrm{HF} 2$ & -1863.77658346246 & -1.224 & -1.676 & 0.452 & -1863.77949570621 & -2.336 & -2.790 & 0.454 \\
\hline Hx3 & -237.060536990814 & & & & -237.06096642472 & & & \\
\hline $\mathrm{xF} 3$ & -1626.71343238351 & & & & -1626.71414039438 & & & \\
\hline HF3 & -1863.77676347337 & -1.336 & -1.753 & 0.417 & -1863.77971166819 & -2.472 & -2.890 & 0.418 \\
\hline $\mathrm{Hx} 4$ & -237.060774225589 & & & & -237.061203615144 & & & \\
\hline $\mathrm{xF} 4$ & -1626.71378669138 & & & & -1626.71449534598 & & & \\
\hline $\mathrm{HF} 4$ & -1863.77717786123 & -1.596 & -1.642 & 0.046 & -1863.78010989712 & -2.722 & -2.768 & 0.046 \\
\hline $\mathrm{Fx} 1$ & -1626.71382053582 & & & & -1626.71452976613 & & & \\
\hline $\mathrm{xF} 1$ & -1626.71371185139 & & & & -1626.71442127604 & & & \\
\hline FF1 & -3253.43092916116 & -1.997 & -2.132 & 0.135 & -3253.43430036363 & -3.222 & -3.357 & 0.135 \\
\hline $\mathrm{Fx} 2$ & -1626.71383095927 & & & & -1626.7145420632 & & & \\
\hline $\mathrm{xF} 2$ & -1626.71382757215 & & & & -1626.71453860626 & & & \\
\hline $\mathrm{FF} 2$ & -3253.42921985684 & -0.924 & -0.980 & 0.056 & -3253.43223904757 & -1.928 & -1.982 & 0.054 \\
\hline
\end{tabular}




\begin{tabular}{|c|c|c|c|c|c|c|c|c|}
\hline & \multicolumn{4}{|c|}{ B3LYP-D3/def2-QZVP } & \multicolumn{4}{|c|}{ B3LYP-dDsC/QZ4P } \\
\hline & $\mathrm{E}[\mathrm{Ha}]$ & $\mathrm{BAE}\left[\mathrm{kcal} \mathrm{mol}^{-1}\right]$ & $\mathrm{BIE}\left[\mathrm{kcal} \mathrm{mol}^{-1}\right]$ & E-def $\left[\mathrm{kcal} \mathrm{mol}^{-1}\right]$ & $\mathrm{E}[\mathrm{Ha}]$ & $\mathrm{BAE}\left[\mathrm{kcal} \mathrm{mol}^{-1}\right]$ & BIE $\left[\mathrm{kcal} \mathrm{mol}^{-1}\right]$ & E-def $\left[\mathrm{kcal} \mathrm{mol}^{-1}\right]$ \\
\hline \multicolumn{9}{|c|}{ Methanes } \\
\hline $\mathrm{H}$ & -40.508353810813 & & & & -0.997054523256134 & & & \\
\hline $\mathrm{F}$ & -437.554162389672 & & & & -1.20895404065298 & & & \\
\hline $\mathrm{Hx} 1$ & -40.508323911401 & & & & -0.997046912167397 & & & \\
\hline $\mathrm{xH} 1$ & -40.508319036247 & & & & -0.997046064187006 & & & \\
\hline HH1 & -81.017407549216 & -0.439 & -0.480 & 0.041 & -1.9948341514964 & -0.455 & -0.465 & 0.010 \\
\hline $\mathrm{Hx} 2$ & -40.50830460883 & & & & -0.997022863079061 & & & \\
\hline $\mathrm{xH} 2$ & -40.50829832882 & & & & -0.997051984170406 & & & \\
\hline HH2 & -81.017206276302 & -0.313 & -0.379 & 0.066 & -1.99467653524753 & -0.356 & -0.378 & 0.021 \\
\hline $\mathrm{Hx} 1$ & -40.508320525556 & & & & -0.997043172266109 & & & \\
\hline $\mathrm{xF} 1$ & -437.554164428607 & & & & -1.20899379040633 & & & \\
\hline HF1 & -478.063455294679 & -0.589 & -0.609 & 0.020 & -2.20704863374822 & -0.653 & -0.635 & -0.018 \\
\hline $\mathrm{Hx} 2$ & -40.508352870386 & & & & -0.997054041876732 & & & \\
\hline $\mathrm{xF} 2$ & -437.554173188624 & & & & -1.20899682317528 & & & \\
\hline HF2 & -478.063018697603 & -0.315 & -0.309 & -0.006 & -2.20666040117139 & -0.409 & -0.382 & -0.027 \\
\hline $\mathrm{Fx} 1$ & -437.554152374313 & & & & -1.20896730375955 & & & \\
\hline $\mathrm{xF} 1$ & -437.554149622471 & & & & -1.20895840960563 & & & \\
\hline $\mathrm{FF} 1$ & -875.109123155343 & -0.501 & -0.515 & 0.014 & -2.41884083309372 & -0.585 & -0.574 & -0.011 \\
\hline $\mathrm{Fx} 2$ & -437.554153563222 & & & & -1.20896316362528 & & & \\
\hline $\mathrm{xF} 2$ & -437.554127927127 & & & & -1.20888550538817 & & & \\
\hline FF2 & -875.109097581252 & -0.485 & -0.512 & 0.027 & -2.41893497945686 & -0.644 & -0.682 & 0.037 \\
\hline \multicolumn{9}{|c|}{ Ethanes } \\
\hline $\mathrm{H}$ & -79.812303022174 & & & & -1.68017047467535 & & & \\
\hline $\mathrm{F}$ & -675.36868477534 & & & & -1.98569503969456 & & & \\
\hline Hx1 & -79.812303025414 & & & & -1.68027599243754 & & & \\
\hline $\mathrm{xH} 1$ & -79.812262434402 & & & & -1.68028143449678 & & & \\
\hline HH1 & -159.626642396514 & -1.278 & -1.303 & 0.025 & -3.36320553821355 & -1.798 & -1.662 & -0.136 \\
\hline $\mathrm{Hx} 2$ & -79.812325584565 & & & & -1.6802344960833 & & & \\
\hline $\mathrm{xH} 2$ & -79.812331912901 & & & & -1.68023059195844 & & & \\
\hline HH2 & -159.626482791287 & -1.178 & -1.145 & -0.032 & -3.36268782620929 & -1.473 & -1.395 & -0.078 \\
\hline Hx1 & -79.812292350121 & & & & -1.68020751873251 & & & \\
\hline $\mathrm{xF} 1$ & -675.368741016648 & & & & -1.98567652827987 & & & \\
\hline HF1 & -755.18232476599 & -0.839 & -0.810 & -0.029 & -3.66729405065374 & -0.896 & -0.885 & -0.012 \\
\hline $\mathrm{Hx} 2$ & -79.812326604564 & & & & -1.68021959152825 & & & \\
\hline $\mathrm{xF} 2$ & -675.368656220824 & & & & -1.98560407575361 & & & \\
\hline HF2 & -755.18254319798 & -0.976 & -0.979 & 0.003 & -3.66763532723768 & -1.111 & -1.137 & 0.026 \\
\hline Hx3 & -79.812300940751 & & & & -1.68018270017829 & & & \\
\hline xF3 & -675.368766824769 & & & & -1.98564618668733 & & & \\
\hline HF3 & -755.18216954899 & -0.742 & -0.691 & -0.050 & -3.66703273800714 & -0.732 & -0.755 & 0.023 \\
\hline $\mathrm{Hx} 4$ & -79.812371197768 & & & & -1.68018221215156 & & & \\
\hline $\mathrm{xF} 4$ & -675.368721459916 & & & & -1.98564763735756 & & & \\
\hline HF 4 & -755.182522821893 & -0.963 & -0.897 & -0.066 & -3.66748792047934 & -1.018 & -1.040 & 0.022 \\
\hline Fx1 & -675.368688456292 & & & & -1.98564455503553 & & & \\
\hline $\mathrm{xF} 1$ & -675.368703352579 & & & & -1.9856074618755 & & & \\
\hline FF1 & -1350.73865895424 & -0.809 & -0.795 & -0.014 & -3.9725782550825 & -0.746 & -0.832 & 0.087 \\
\hline $\mathrm{Fx} 2$ & -675.368746871734 & & & & -1.98570551135143 & & & \\
\hline $\mathrm{xF} 2$ & -675.36872158297 & & & & -1.9855777713251 & & & \\
\hline FF2 & -1350.7385106709 & -0.716 & -0.654 & -0.062 & -3.97226126327924 & -0.547 & -0.614 & 0.067 \\
\hline
\end{tabular}




\begin{tabular}{|c|c|c|c|c|c|c|c|c|}
\hline & \multicolumn{4}{|c|}{ B3LYP-D3/def2-QZVP } & \multicolumn{4}{|c|}{ B3LYP-dDsC/QZ4P } \\
\hline & $\mathrm{E}[\mathrm{Ha}]$ & $\mathrm{BAE}\left[\mathrm{kcal} \mathrm{mol}{ }^{-1}\right]$ & $\mathrm{BIE}\left[\mathrm{kcal} \mathrm{mol}^{-1}\right]$ & E-def $\left[\mathrm{kcal} \mathrm{mol}^{-1}\right]$ & $\mathrm{E}[\mathrm{Ha}]$ & $\mathrm{BAE}\left[\mathrm{kcal} \mathrm{mol}{ }^{-1}\right]$ & BIE $\left[\mathrm{kcal} \mathrm{mol}^{-1}\right]$ & E-def $\left[\mathrm{kcal} \mathrm{mol}^{-1}\right]$ \\
\hline \multicolumn{9}{|c|}{ Propanes } \\
\hline H & -119.119219214312 & & & & -2.36714715632625 & & & \\
\hline $\mathrm{F}$ & -913.182649236586 & & & & -2.76210974389371 & & & \\
\hline $\mathrm{Hx} 1$ & -119.11920191317 & & & & -2.36706632980892 & & & \\
\hline $\mathrm{xH} 1$ & -119.119201781452 & & & & -2.3670493889605 & & & \\
\hline HН1 & -238.241715108446 & -2.056 & -2.078 & 0.022 & -4.73857531500969 & -2.686 & -2.798 & 0.112 \\
\hline $\mathrm{Hx} 2$ & -119.119209050169 & & & & -2.36710943950061 & & & \\
\hline $\mathrm{xH} 2$ & -119.11920316734 & & & & -2.36709130400842 & & & \\
\hline $\mathrm{HH} 2$ & -238.240343040799 & -1.195 & -1.212 & 0.016 & -4.736507343554 & -1.389 & -1.447 & 0.059 \\
\hline Hx1 & -119.119209555224 & & & & -2.36714181956329 & & & \\
\hline $\mathrm{xF} 1$ & -913.182508169149 & & & & -2.76203626172459 & & & \\
\hline HF1 & -1032.30390540466 & -1.278 & -1.373 & 0.095 & -5.13195340563505 & -1.692 & -1.742 & 0.049 \\
\hline $\mathrm{Hx} 2$ & -119.119271328837 & & & & -2.36715869854355 & & & \\
\hline $\mathrm{xF} 2$ & -913.18251612529 & & & & -2.76204321888593 & & & \\
\hline HF2 & -1032.30350984179 & -1.030 & -1.081 & 0.051 & -5.13127784362148 & -1.268 & -1.303 & 0.035 \\
\hline Hx3 & -119.119232677646 & & & & -2.36718211964487 & & & \\
\hline $\mathrm{xF} 3$ & -913.182556697026 & & & & -2.76212653548463 & & & \\
\hline HF3 & -1032.30353365825 & -1.045 & -1.095 & 0.050 & -5.1312450424375 & -1.248 & -1.215 & -0.032 \\
\hline $\mathrm{Hx} 4$ & -119.119253166967 & & & & -2.36706559788857 & & & \\
\hline $\mathrm{xF} 4$ & -913.182479316403 & & & & -2.76209065428599 & & & \\
\hline $\mathrm{HF} 4$ & -1032.30334789651 & -0.928 & -1.014 & 0.085 & -5.13140551676806 & -1.348 & -1.411 & 0.063 \\
\hline Fx1 & -913.182391540217 & & & & -2.76192719192125 & & & \\
\hline $\mathrm{xF} 1$ & -913.182505601146 & & & & -2.76212374052011 & & & \\
\hline FF1 & -1826.3666473172 & -0.846 & -1.098 & 0.252 & -5.52633962922619 & -1.330 & -1.436 & 0.106 \\
\hline $\mathrm{Fx} 2$ & -913.182641137748 & & & & -2.76209999590492 & & & \\
\hline $\mathrm{xF} 2$ & -913.182636100043 & & & & -2.76213965001451 & & & \\
\hline FF2 & -1826.36709157574 & -1.125 & -1.139 & 0.013 & -5.52673757602075 & -1.580 & -1.567 & -0.013 \\
\hline \multicolumn{9}{|c|}{ Butanes } \\
\hline $\mathrm{H}$ & -158.426236778255 & & & & -3.05413458301858 & & & \\
\hline $\mathrm{F}$ & -1150.99577796131 & & & & -3.53814802563191 & & & \\
\hline $\mathrm{Hx} 1$ & -158.426138319369 & & & & -3.05412734697419 & & & \\
\hline $\mathrm{xH} 1$ & -158.426158936112 & & & & -3.0541148613696 & & & \\
\hline HH1 & -316.85707412121 & -2.887 & -2.998 & 0.111 & -6.11497067281755 & -4.205 & -4.222 & 0.017 \\
\hline $\mathrm{Hx} 2$ & -158.426125888057 & & & & -3.0539861726772 & & & \\
\hline $\mathrm{xH} 2$ & -158.42614701502 & & & & -3.05405243526411 & & & \\
\hline Hн2 & -316.854975298649 & -1.570 & -1.696 & 0.126 & -6.1113762243495 & -1.950 & -2.094 & 0.145 \\
\hline $\mathrm{Hx} 1$ & -158.42621214639 & & & & -3.05406171503111 & & & \\
\hline $\mathrm{xF} 1$ & -1150.99566511654 & & & & -3.53790483499211 & & & \\
\hline $\mathrm{HF} 1$ & -1309.42486579861 & -1.789 & -1.875 & 0.086 & -6.59566950916554 & -2.125 & -2.324 & 0.198 \\
\hline $\mathrm{Hx} 2$ & -158.426223340603 & & & & -3.05412213466942 & & & \\
\hline $\mathrm{xF} 2$ & -1150.99580685855 & & & & -3.53815157758183 & & & \\
\hline $\mathrm{HF} 2$ & -1309.42487163233 & -1.793 & -1.783 & -0.010 & -6.59590559199684 & -2.273 & -2.279 & 0.006 \\
\hline $\mathrm{Hx} 3$ & -158.426174472668 & & & & -3.05407739409964 & & & \\
\hline $\mathrm{xF} 3$ & -1150.99580787547 & & & & -3.53821539174974 & & & \\
\hline $\mathrm{HF} 3$ & -1309.42476943426 & -1.729 & -1.749 & 0.020 & -6.59609499233364 & -2.392 & -2.386 & -0.006 \\
\hline $\mathrm{Hx} 4$ & -158.426163155924 & & & & -3.05405086550945 & & & \\
\hline $\mathrm{xF} 4$ & -1150.99581880253 & & & & -3.53815185219887 & & & \\
\hline $\mathrm{HF} 4$ & -1309.42483058123 & -1.767 & -1.788 & 0.021 & -6.59581485136437 & -2.217 & -2.267 & 0.050 \\
\hline Fx1 & -1150.99580288325 & & & & -3.5381420883391 & & & \\
\hline $\mathrm{xF} 1$ & -1150.9957332409 & & & & -3.53810973870566 & & & \\
\hline FF1 & -2301.99390942518 & -1.477 & -1.489 & 0.012 & -7.0787081224622 & -1.514 & -1.541 & 0.028 \\
\hline $\mathrm{Fx} 2$ & -1150.9958170141 & & & & -3.53814654050713 & & & \\
\hline $\mathrm{xF} 2$ & -1150.99578610147 & & & & -3.53796166792821 & & & \\
\hline FF2 & -2301.99402091801 & -1.547 & -1.517 & -0.030 & -7.07899798029486 & -1.695 & -1.813 & 0.118 \\
\hline
\end{tabular}




\begin{tabular}{|c|c|c|c|c|c|c|c|c|}
\hline & \multicolumn{4}{|c|}{ B3LYP-D3/def2-QZVP } & \multicolumn{4}{|c|}{ B3LYP-dDsC/QZ4P } \\
\hline & $\mathrm{E}[\mathrm{Ha}]$ & $\mathrm{BAE}\left[\mathrm{kcal} \mathrm{mol}^{-1}\right]$ & $\mathrm{BIE}\left[\mathrm{kcal} \mathrm{mol}^{-1}\right]$ & E-def $\left[\mathrm{kcal} \mathrm{mol}^{-1}\right]$ & $\mathrm{E}[\mathrm{Ha}]$ & $\mathrm{BAE}\left[\mathrm{kcal} \mathrm{mol}^{-1}\right]$ & $\mathrm{BIE}\left[\mathrm{kcal} \mathrm{mol}^{-1}\right]$ & E-def $\left[\mathrm{kcal} \mathrm{mol}^{-1}\right]$ \\
\hline \multicolumn{9}{|c|}{ Pentanes } \\
\hline H & -197.733150514393 & & & & -3.74100920430532 & & & \\
\hline $\mathrm{F}$ & -1388.809137313 & & & & -4.31416645591214 & & & \\
\hline $\mathrm{Hx} 1$ & -197.733039082328 & & & & -3.74106507503068 & & & \\
\hline $\mathrm{xH} 1$ & -197.733038898408 & & & & -3.74106066460062 & & & \\
\hline HH1 & -395.472349513822 & -3.795 & -3.935 & 0.140 & -7.49125042280308 & -5.793 & -5.726 & -0.067 \\
\hline $\mathrm{Hx} 2$ & -197.733032827674 & & & & -3.74091576568679 & & & \\
\hline $\mathrm{xH} 2$ & -197.733033165663 & & & & -3.74091237868381 & & & \\
\hline нH2 & -395.469579099767 & -2.057 & -2.205 & 0.147 & -7.4865145102586 & -2.821 & -2.941 & 0.119 \\
\hline Hx1 & -197.733026822065 & & & & -3.74087673241662 & & & \\
\hline $\mathrm{xF} 1$ & -1388.80913396051 & & & & -4.31418222324668 & & & \\
\hline HF1 & -1586.54584320238 & -2.231 & -2.311 & 0.080 & -8.05980476585605 & -2.905 & -2.978 & 0.073 \\
\hline $\mathrm{Hx} 2$ & -197.733114611753 & & & & -3.74097135483946 & & & \\
\hline $\mathrm{xF} 2$ & -1388.80892381137 & & & & -4.3138706767912 & & & \\
\hline HF2 & -1586.54550693547 & -2.020 & -2.177 & 0.157 & -8.05915003671939 & -2.494 & -2.703 & 0.209 \\
\hline $\mathrm{Hx} 3$ & -197.733084956732 & & & & -3.74100361177355 & & & \\
\hline $\mathrm{xF} 3$ & -1388.80919451388 & & & & -4.31427426559792 & & & \\
\hline HF3 & -1586.54563955873 & -2.103 & -2.108 & 0.005 & -8.05974693715521 & -2.869 & -2.804 & -0.064 \\
\hline $\mathrm{Hx} 4$ & -197.733098652071 & & & & -3.7409619200154 & & & \\
\hline $\mathrm{xF} 4$ & -1388.80919109966 & & & & -4.3141525904642 & & & \\
\hline $\mathrm{HF} 4$ & -1586.5461817346 & -2.443 & -2.442 & -0.001 & -8.06030841849651 & -3.221 & -3.259 & 0.038 \\
\hline $\mathrm{Fx} 1$ & -1388.80911531471 & & & & -4.31419119145626 & & & \\
\hline $\mathrm{xF} 1$ & -1388.80912736768 & & & & -4.31417416426328 & & & \\
\hline FF1 & -2777.62145927768 & -1.998 & -2.018 & 0.020 & -8.63236726693781 & -2.532 & -2.511 & -0.020 \\
\hline $\mathrm{Fx} 2$ & -1388.80908651043 & & & & -4.31429870946289 & & & \\
\hline $\mathrm{xF} 2$ & -1388.80909045596 & & & & -4.31428571277507 & & & \\
\hline $\mathrm{FF} 2$ & -2777.62113807622 & -1.797 & -1.858 & 0.061 & -8.63187375800452 & -2.222 & -2.064 & -0.158 \\
\hline \multicolumn{9}{|c|}{ Hexanes } \\
\hline $\mathrm{H}$ & -237.040062124583 & & & & -4.42798094604148 & & & \\
\hline $\mathrm{F}$ & -1626.62254094344 & & & & -5.0904208285254 & & & \\
\hline $\mathrm{Hx} 1$ & -237.039946402381 & & & & -4.42800501641623 & & & \\
\hline $\mathrm{xH} 1$ & -237.039946748938 & & & & -4.42801128104791 & & & \\
\hline HH1 & -474.087256613648 & -4.476 & -4.621 & 0.145 & -8.86688887116302 & -6.857 & -6.823 & -0.034 \\
\hline $\mathrm{Hx} 2$ & -237.0399440548 & & & & -4.42784034456552 & & & \\
\hline $\mathrm{xH} 2$ & -237.039942212862 & & & & -4.42783163507668 & & & \\
\hline нH2 & -474.084273826733 & -2.604 & -2.753 & 0.149 & -8.86141644369284 & -3.423 & -3.605 & 0.182 \\
\hline $\mathrm{Hx} 1$ & -237.039858557189 & & & & -4.42778226592321 & & & \\
\hline $\mathrm{xF} 1$ & -1626.62217796736 & & & & -5.08986153677259 & & & \\
\hline HF1 & -1863.66631481909 & -2.329 & -2.685 & 0.356 & -9.52370079737084 & -3.325 & -3.801 & 0.476 \\
\hline $\mathrm{Hx} 2$ & -237.039972842364 & & & & -4.42792188817633 & & & \\
\hline $\mathrm{xF} 2$ & -1626.6222702608 & & & & -5.09002550880112 & & & \\
\hline $\mathrm{HF} 2$ & -1863.66655857684 & -2.482 & -2.708 & 0.226 & -9.5234030053296 & -3.138 & -3.423 & 0.285 \\
\hline $\mathrm{Hx} 3$ & -237.039891059779 & & & & -4.42775945462131 & & & \\
\hline $\mathrm{xF} 3$ & -1626.62227446402 & & & & -5.09011661231521 & & & \\
\hline HF3 & -1863.66643076806 & -2.402 & -2.676 & 0.275 & -9.52380077722309 & -3.388 & -3.718 & 0.330 \\
\hline $\mathrm{Hx} 4$ & -237.040049327837 & & & & -4.42786230693905 & & & \\
\hline $\mathrm{xF} 4$ & -1626.62253357553 & & & & -5.09028091465909 & & & \\
\hline $\mathrm{HF} 4$ & -1863.66702299448 & -2.774 & -2.786 & 0.013 & -9.52420160022139 & -3.639 & -3.802 & 0.162 \\
\hline $\mathrm{Fx} 1$ & -1626.62248565959 & & & & -5.09022452776762 & & & \\
\hline $\mathrm{xF} 1$ & -1626.62244553499 & & & & -5.09013088581869 & & & \\
\hline FF1 & -3253.24903898184 & -2.483 & -2.578 & 0.095 & -10.1859513340609 & -3.206 & -3.511 & 0.305 \\
\hline $\mathrm{Fx} 2$ & -1626.62233964131 & & & & -5.09022673896258 & & & \\
\hline $\mathrm{xF} 2$ & -1626.62234210278 & & & & -5.09023467932742 & & & \\
\hline $\mathrm{FF} 2$ & -3253.24744307792 & -1.482 & -1.733 & 0.251 & -10.1844317405797 & -2.253 & -2.491 & 0.239 \\
\hline
\end{tabular}




\begin{tabular}{|c|c|c|c|c|c|c|c|c|}
\hline & \multicolumn{4}{|c|}{ B3LYP-XDM/aug-cc-pVTZ } & \multicolumn{4}{|c|}{ B3LYP-NL/def2-QZVP } \\
\hline & $\mathrm{E}[\mathrm{Ha}]$ & $\mathrm{BAE}\left[\mathrm{kcal} \mathrm{mol}^{-1}\right]$ & $\mathrm{BIE}\left[\mathrm{kcal} \mathrm{mol}^{-1}\right]$ & E-def $\left[\mathrm{kcal} \mathrm{mol}^{-1}\right]$ & $\mathrm{E}[\mathrm{Ha}]$ & $\mathrm{BAE}\left[\mathrm{kcal} \mathrm{mol}^{-1}\right]$ & $\mathrm{BIE}\left[\mathrm{kcal} \mathrm{mol}^{-1}\right]$ & E-def $\left[\mathrm{kcal} \mathrm{mol}^{-1}\right]$ \\
\hline \multicolumn{9}{|c|}{ Methanes } \\
\hline $\mathrm{H}$ & -40.54004623483 & & & & -40.450478955403 & & & \\
\hline $\mathrm{F}$ & -437.6729650577 & & & & -437.316494422571 & & & \\
\hline $\mathrm{Hx} 1$ & -40.54004539424 & & & & -40.450449310548 & & & \\
\hline $\mathrm{xH} 1$ & -40.54004531584 & & & & -40.450444325613 & & & \\
\hline HH1 & -81.08090732403 & -0.511 & -0.512 & 0.001 & -80.901395160982 & -0.274 & -0.315 & 0.040 \\
\hline $\mathrm{Hx} 2$ & -40.54004647956 & & & & -40.450429536079 & & & \\
\hline $\mathrm{xH} 2$ & -40.54004668809 & & & & -40.45042308801 & & & \\
\hline $\mathrm{HH} 2$ & -81.08069053054 & -0.375 & -0.375 & 0.000 & -80.9012505642 & -0.184 & -0.250 & 0.066 \\
\hline $\mathrm{Hx} 1$ & -40.54004655243 & & & & -40.45044619739 & & & \\
\hline $\mathrm{xF} 1$ & -437.6729909055 & & & & -437.316489488932 & & & \\
\hline HF1 & -478.2142820835 & -0.797 & -0.781 & -0.016 & -477.768112140567 & -0.715 & -0.738 & 0.024 \\
\hline $\mathrm{Hx} 2$ & -40.54004547394 & & & & -40.450477893705 & & & \\
\hline $\mathrm{xF} 2$ & -437.6729821742 & & & & -437.316500472315 & & & \\
\hline $\mathrm{HF} 2$ & -478.2136827772 & -0.421 & -0.411 & -0.010 & -477.767615192459 & -0.403 & -0.400 & -0.003 \\
\hline Fx1 & -437.6729765161 & & & & -437.316479767398 & & & \\
\hline $\mathrm{xF} 1$ & -437.6729803442 & & & & -437.316477693023 & & & \\
\hline $\mathrm{FF} 1$ & -875.3471212641 & -0.747 & -0.731 & -0.017 & -874.634451116533 & -0.918 & -0.937 & 0.020 \\
\hline $\mathrm{Fx} 2$ & -437.6729853342 & & & & -437.316481129966 & & & \\
\hline $\mathrm{xF} 2$ & -437.6729814414 & & & & -437.316456918576 & & & \\
\hline $\mathrm{FF} 2$ & -875.3470913289 & -0.729 & -0.706 & -0.023 & -874.634482533851 & -0.937 & -0.969 & 0.032 \\
\hline \multicolumn{9}{|c|}{ Ethanes } \\
\hline $\mathrm{H}$ & -79.86938267815 & & & & -79.709616974232 & & & \\
\hline $\mathrm{F}$ & -675.5569747212 & & & & -674.998274255037 & & & \\
\hline Hx1 & -79.8693829113 & & & & -79.709618653591 & & & \\
\hline $\mathrm{xH} 1$ & -79.86938425842 & & & & -79.709574807682 & & & \\
\hline HH1 & -159.7408395862 & -1.302 & -1.300 & -0.001 & -159.420849465373 & -1.014 & -1.039 & 0.025 \\
\hline $\mathrm{Hx} 2$ & -79.86938333018 & & & & -79.709639034483 & & & \\
\hline $\mathrm{xH} 2$ & -79.86938357455 & & & & -79.709645710091 & & & \\
\hline $\mathrm{HH} 2$ & -159.7406054811 & -1.155 & -1.154 & -0.001 & -159.420711172813 & -0.927 & -0.895 & -0.032 \\
\hline $\mathrm{Hx} 1$ & -79.86937718941 & & & & -79.709604808579 & & & \\
\hline $\mathrm{xF} 1$ & -675.5570247102 & & & & -674.998312855404 & & & \\
\hline HF1 & -755.4280621249 & -1.070 & -1.042 & -0.028 & -754.709649826696 & -1.104 & -1.087 & -0.017 \\
\hline $\mathrm{Hx} 2$ & -79.86937931324 & & & & -79.709640965284 & & & \\
\hline $\mathrm{xF} 2$ & -675.5570424826 & & & & -674.998240837602 & & & \\
\hline $\mathrm{HF} 2$ & -755.4283541596 & -1.253 & -1.213 & -0.040 & -754.709946837673 & -1.290 & -1.296 & 0.006 \\
\hline $\mathrm{Hx} 3$ & -79.86937972961 & & & & -79.709613134606 & & & \\
\hline $\mathrm{xF} 3$ & -675.5570249865 & & & & -674.998335271991 & & & \\
\hline HF3 & -755.4278119583 & -0.913 & -0.883 & -0.030 & -754.709431502109 & -0.967 & -0.931 & -0.036 \\
\hline $\mathrm{Hx} 4$ & -79.8693781419 & & & & -79.709686859244 & & & \\
\hline $\mathrm{xF} 4$ & -675.5570123418 & & & & -674.998299516033 & & & \\
\hline $\mathrm{HF} 4$ & -755.4282352374 & -1.178 & -1.158 & -0.021 & -754.70991224001 & -1.268 & -1.208 & -0.060 \\
\hline $\mathrm{Fx} 1$ & -675.5569918754 & & & & -674.998277841144 & & & \\
\hline $\mathrm{xF} 1$ & -675.5569592576 & & & & -674.998293334715 & & & \\
\hline FF1 & -1351.115759828 & -1.136 & -1.135 & -0.001 & -1349.99910062199 & -1.601 & -1.587 & -0.014 \\
\hline $\mathrm{Fx} 2$ & -675.55703413 & & & & -674.998313721572 & & & \\
\hline $\mathrm{xF} 2$ & -675.5569987096 & & & & -674.998301628671 & & & \\
\hline FF2 & -1351.115487244 & -0.965 & -0.913 & -0.052 & -1349.99860191231 & -1.289 & -1.247 & -0.042 \\
\hline
\end{tabular}




\begin{tabular}{|c|c|c|c|c|c|c|c|c|}
\hline & \multicolumn{4}{|c|}{ B3LYP-XDM/aug-cc-pVTZ } & \multicolumn{4}{|c|}{ B3LYP-NL/def2-QZVP } \\
\hline & $\mathrm{E}[\mathrm{Ha}]$ & $\mathrm{BAE}\left[\mathrm{kcal} \mathrm{mol}^{-1}\right]$ & $\mathrm{BIE}\left[\mathrm{kcal} \mathrm{mol}^{-1}\right]$ & E-def $\left[\mathrm{kcal} \mathrm{mol}^{-1}\right]$ & $\mathrm{E}[\mathrm{Ha}]$ & BAE $\left[\mathrm{kcal} \mathrm{mol}^{-1}\right]$ & $\mathrm{BIE}\left[\mathrm{kcal} \mathrm{mol}^{-1}\right]$ & E-def $\left[\mathrm{kcal} \mathrm{mol}^{-1}\right]$ \\
\hline \multicolumn{9}{|c|}{ Propanes } \\
\hline $\mathrm{H}$ & -119.201598301 & & & & -118.971860350801 & & & \\
\hline $\mathrm{F}$ & -913.4402027813 & & & & -912.679965030856 & & & \\
\hline $\mathrm{Hx} 1$ & -119.2016081296 & & & & -118.971843577662 & & & \\
\hline $\mathrm{xH} 1$ & -119.2016090068 & & & & -118.97184304787 & & & \\
\hline HH1 & -238.4065410993 & -2.099 & -2.086 & -0.013 & -237.9464806476 & -1.732 & -1.753 & 0.021 \\
\hline $\mathrm{Hx} 2$ & -119.2016122714 & & & & -118.97184957987 & & & \\
\hline $\mathrm{xH} 2$ & -119.2016043885 & & & & -118.971846332987 & & & \\
\hline $\mathrm{HH} 2$ & -238.4050731668 & -1.178 & -1.165 & -0.013 & -237.945284491948 & -0.981 & -0.997 & 0.016 \\
\hline $\mathrm{Hx} 1$ & -119.2015884645 & & & & -118.971851307233 & & & \\
\hline $\mathrm{xF} 1$ & -913.4401371672 & & & & -912.679839542536 & & & \\
\hline HF1 & -1032.644468677 & -1.674 & -1.721 & 0.047 & -1031.65467464866 & -1.788 & -1.872 & 0.084 \\
\hline $\mathrm{Hx} 2$ & -119.2015966378 & & & & -118.971915556045 & & & \\
\hline $\mathrm{xF} 2$ & -913.4402177558 & & & & -912.679851308916 & & & \\
\hline $\mathrm{HF} 2$ & -1032.64399395 & -1.376 & -1.368 & -0.008 & -1031.6541517172 & -1.460 & -1.497 & 0.037 \\
\hline $\mathrm{Hx} 3$ & -119.2016043111 & & & & -118.971879792334 & & & \\
\hline $\mathrm{xF} 3$ & -913.4402000295 & & & & -912.679873969651 & & & \\
\hline HF3 & -1032.643960281 & -1.355 & -1.353 & -0.002 & -1031.65407237792 & -1.410 & -1.455 & 0.045 \\
\hline $\mathrm{Hx} 4$ & -119.2015958418 & & & & -118.971894438584 & & & \\
\hline $\mathrm{xF} 4$ & -913.4401984519 & & & & -912.679825485673 & & & \\
\hline $\mathrm{HF} 4$ & -1032.643912768 & -1.325 & -1.329 & 0.004 & -1031.65412941019 & -1.446 & -1.512 & 0.066 \\
\hline $\mathrm{Fx} 1$ & -913.4399953516 & & & & -912.679728197594 & & & \\
\hline $\mathrm{xF} 1$ & -913.4401331848 & & & & -912.679838440776 & & & \\
\hline FF1 & -1826.882704387 & -1.443 & -1.616 & 0.174 & -1825.36326651251 & -2.094 & -2.322 & 0.228 \\
\hline $\mathrm{Fx} 2$ & -913.4401981268 & & & & -912.679957388913 & & & \\
\hline $\mathrm{xF} 2$ & -913.440197866 & & & & -912.679952054382 & & & \\
\hline $\mathrm{FF} 2$ & -1826.883262696 & -1.793 & -1.799 & 0.006 & -1825.36416043466 & -2.655 & -2.668 & 0.013 \\
\hline \multicolumn{9}{|c|}{ Butanes } \\
\hline $\mathrm{H}$ & -158.5337904438 & & & & -158.234217622374 & & & \\
\hline $\mathrm{F}$ & -1151.322804154 & & & & -1150.36106990829 & & & \\
\hline $\mathrm{Hx} 1$ & -158.5337950507 & & & & -158.234118115911 & & & \\
\hline $\mathrm{xH} 1$ & -158.5337928382 & & & & -158.234143573152 & & & \\
\hline HH1 & -317.0723802489 & -3.012 & -3.007 & -0.004 & -316.472461878985 & -2.527 & -2.636 & 0.109 \\
\hline $\mathrm{Hx} 2$ & -158.5337775663 & & & & -158.234111196688 & & & \\
\hline $\mathrm{xH} 2$ & -158.5337983939 & & & & -158.234129018892 & & & \\
\hline $\mathrm{HH} 2$ & -317.0702106113 & -1.650 & -1.653 & 0.003 & -316.470561581613 & -1.334 & -1.457 & 0.122 \\
\hline $\mathrm{Hx} 1$ & -158.5337893695 & & & & -158.234195051681 & & & \\
\hline $\mathrm{xF} 1$ & -1151.322659884 & & & & -1150.36082658845 & & & \\
\hline HF1 & -1309.860124587 & -2.215 & -2.306 & 0.091 & -1308.59913723586 & -2.416 & -2.583 & 0.167 \\
\hline $\mathrm{Hx} 2$ & -158.5337769999 & & & & -158.234201543534 & & & \\
\hline $\mathrm{xF} 2$ & -1151.322831049 & & & & -1150.36106814525 & & & \\
\hline HF2 & -1309.860178792 & -2.249 & -2.241 & -0.008 & -1308.59933611743 & -2.541 & -2.552 & 0.011 \\
\hline $\mathrm{Hx} 3$ & -158.5337512364 & & & & -158.234154643924 & & & \\
\hline $\mathrm{xF} 3$ & -1151.322828531 & & & & -1150.3610319958 & & & \\
\hline HF3 & -1309.860120772 & -2.213 & -2.222 & 0.009 & -1308.59925292141 & -2.488 & -2.552 & 0.063 \\
\hline $\mathrm{Hx} 4$ & -158.5337451139 & & & & -158.234146167724 & & & \\
\hline $\mathrm{xF} 4$ & -1151.322828296 & & & & -1150.36109677636 & & & \\
\hline $\mathrm{HF} 4$ & -1309.86014538 & -2.228 & -2.241 & 0.013 & -1308.59919878773 & -2.454 & -2.482 & 0.028 \\
\hline Fx1 & -1151.322800231 & & & & -1150.36104091546 & & & \\
\hline $\mathrm{xF} 1$ & -1151.32276843 & & & & -1150.36102432983 & & & \\
\hline FF1 & -2302.648844431 & -2.031 & -2.056 & 0.025 & -2300.72650548168 & -2.739 & -2.786 & 0.047 \\
\hline $\mathrm{Fx} 2$ & -1151.322812817 & & & & -1150.36102132587 & & & \\
\hline $\mathrm{xF} 2$ & -1151.322790862 & & & & -1150.36102394809 & & & \\
\hline FF2 & -2302.649147154 & -2.221 & -2.224 & 0.003 & -2300.72706800781 & -3.092 & -3.152 & 0.059 \\
\hline
\end{tabular}




\begin{tabular}{|c|c|c|c|c|c|c|c|c|}
\hline & \multicolumn{4}{|c|}{ B3LYP-XDM/aug-cc-pVTZ } & \multicolumn{4}{|c|}{ B3LYP-NL/def2-QZVP } \\
\hline & $\mathrm{E}[\mathrm{Ha}]$ & $\mathrm{BAE}\left[\mathrm{kcal} \mathrm{mol}^{-1}\right]$ & $\mathrm{BIE}\left[\mathrm{kcal} \mathrm{mol}^{-1}\right]$ & E-def $\left[\mathrm{kcal} \mathrm{mol}^{-1}\right]$ & $\mathrm{E}[\mathrm{Ha}]$ & $\mathrm{BAE}\left[\mathrm{kcal} \mathrm{mol}{ }^{-1}\right]$ & $\mathrm{BIE}\left[\mathrm{kcal} \mathrm{mol}{ }^{-1}\right]$ & E-def $\left[\mathrm{kcal} \mathrm{mol}^{-1}\right]$ \\
\hline \multicolumn{9}{|c|}{ Pentanes } \\
\hline $\mathrm{H}$ & -197.8659489804 & & & & -197.496482909712 & & & \\
\hline $\mathrm{F}$ & -1389.205622122 & & & & -1388.04244626475 & & & \\
\hline $\mathrm{Hx} 1$ & -197.8659433981 & & & & -197.496370920763 & & & \\
\hline $\mathrm{xH} 1$ & -197.8659433077 & & & & -197.496370742356 & & & \\
\hline HH1 & -395.7381838128 & -3.944 & -3.951 & 0.007 & -394.998388860074 & -3.403 & -3.544 & 0.141 \\
\hline $\mathrm{H} \times 2$ & -197.8659519449 & & & & -197.496364500639 & & & \\
\hline $\mathrm{xH} 2$ & -197.8659518889 & & & & -197.496364815149 & & & \\
\hline $\mathrm{HH} 2$ & -395.7354185071 & -2.209 & -2.205 & -0.004 & -394.99579381963 & -1.775 & -1.923 & 0.148 \\
\hline $\mathrm{Hx} 1$ & -197.865852492 & & & & -197.496360722111 & & & \\
\hline $\mathrm{xF} 1$ & -1389.205596126 & & & & -1388.04235714598 & & & \\
\hline HF1 & -1587.076100221 & -2.842 & -2.919 & 0.077 & -1585.54404865606 & -3.213 & -3.345 & 0.133 \\
\hline $\mathrm{Hx} 2$ & -197.8659135457 & & & & -197.496446410431 & & & \\
\hline $\mathrm{xF} 2$ & -1389.205393922 & & & & -1388.04216195353 & & & \\
\hline $\mathrm{HF} 2$ & -1587.075597068 & -2.526 & -2.692 & 0.165 & -1585.54341962774 & -2.818 & -3.019 & 0.201 \\
\hline $\mathrm{Hx} 3$ & -197.86593217 & & & & -197.496409475625 & & & \\
\hline $\mathrm{xF} 3$ & -1389.205692479 & & & & -1388.0424375512 & & & \\
\hline HF3 & -1587.0757676 & -2.633 & -2.600 & -0.034 & -1585.54363859131 & -2.955 & -3.007 & 0.052 \\
\hline $\mathrm{Hx} 4$ & -197.8659151811 & & & & -197.496427024841 & & & \\
\hline $\mathrm{xF} 4$ & -1389.205658447 & & & & -1388.04246456623 & & & \\
\hline $\mathrm{HF} 4$ & -1587.076465052 & -3.071 & -3.069 & -0.002 & -1585.544449251 & -3.464 & -3.487 & 0.024 \\
\hline Fx1 & -1389.205612339 & & & & -1388.04241412298 & & & \\
\hline $\mathrm{xF} 1$ & -1389.205615196 & & & & -1388.04238298251 & & & \\
\hline FF1 & -2778.415868008 & -2.901 & -2.912 & 0.010 & -2776.09146253957 & -4.123 & -4.183 & 0.060 \\
\hline $\mathrm{Fx} 2$ & -1389.205593735 & & & & -1388.04236809276 & & & \\
\hline $\mathrm{xF} 2$ & -1389.205596532 & & & & -1388.04237257564 & & & \\
\hline $\mathrm{FF} 2$ & -2778.415479694 & -2.658 & -2.692 & 0.034 & -2776.09075739337 & -3.680 & -3.776 & 0.095 \\
\hline \multicolumn{9}{|c|}{ Hexanes } \\
\hline $\mathrm{H}$ & -237.1981241153 & & & & -236.758745814544 & & & \\
\hline F & -1627.088508879 & & & & -1625.72382769832 & & & \\
\hline Hx1 & -237.1981125141 & & & & -236.758631934014 & & & \\
\hline $\mathrm{xH} 1$ & -237.1981125193 & & & & -236.758632128411 & & & \\
\hline HH1 & -474.403658019 & -4.650 & -4.664 & 0.015 & -473.524009146021 & -4.090 & -4.233 & 0.143 \\
\hline $\mathrm{Hx} 2$ & -237.1981249154 & & & & -236.758629631867 & & & \\
\hline $\mathrm{xH} 2$ & -237.1981247898 & & & & -236.758627791465 & & & \\
\hline HH2 & -474.4005742217 & -2.715 & -2.714 & -0.001 & -473.521196078234 & -2.325 & -2.472 & 0.147 \\
\hline $\mathrm{Hx} 1$ & -237.1979436564 & & & & -236.758535587396 & & & \\
\hline $\mathrm{xF} 1$ & -1627.088138214 & & & & -1625.72334756807 & & & \\
\hline HF1 & -1864.291567151 & -3.096 & -3.442 & 0.346 & -1862.48833751328 & -3.617 & -4.050 & 0.433 \\
\hline $\mathrm{Hx} 2$ & -237.198031358 & & & & -236.758656104133 & & & \\
\hline $\mathrm{xF} 2$ & -1627.088215248 & & & & -1625.7234691238 & & & \\
\hline $\mathrm{HF} 2$ & -1864.291600104 & -3.117 & -3.359 & 0.242 & -1862.48821653987 & -3.541 & -3.822 & 0.281 \\
\hline $\mathrm{Hx} 3$ & -237.1980042966 & & & & -236.758580029361 & & & \\
\hline $\mathrm{xF} 3$ & -1627.088247962 & & & & -1625.72353392107 & & & \\
\hline HF3 & -1864.291688815 & -3.173 & -3.411 & 0.239 & -1862.48839934114 & -3.656 & -3.944 & 0.288 \\
\hline $\mathrm{Hx} 4$ & -237.198104247 & & & & -236.758733091263 & & & \\
\hline $\mathrm{xF} 4$ & -1627.088486132 & & & & -1625.72379892127 & & & \\
\hline $\mathrm{HF} 4$ & -1864.292106221 & -3.435 & -3.461 & 0.027 & -1862.48883097724 & -3.927 & -3.953 & 0.026 \\
\hline Fx1 & -1627.088442847 & & & & -1625.72377353118 & & & \\
\hline $\mathrm{xF} 1$ & -1627.088441655 & & & & -1625.7237226291 & & & \\
\hline FF1 & -3254.182882935 & -3.680 & -3.764 & 0.084 & -3251.4564103505 & -5.494 & -5.594 & 0.100 \\
\hline $\mathrm{Fx} 2$ & -1627.088332941 & & & & -1625.72369557978 & & & \\
\hline $\mathrm{xF} 2$ & -1627.088334003 & & & & -1625.72369592969 & & & \\
\hline FF2 & -3254.180988466 & -2.492 & -2.712 & 0.220 & -3251.45379994909 & -3.856 & -4.021 & 0.166 \\
\hline
\end{tabular}




\begin{tabular}{|c|c|c|c|c|c|c|c|c|}
\hline & \multicolumn{4}{|c|}{$\omega B 97 X-D 3 /$ def2-QZVP } & \multicolumn{4}{|c|}{ B2PLYP-D3/def2-QZVP } \\
\hline & $\mathrm{E}[\mathrm{Ha}]$ & $\mathrm{BAE}\left[\mathrm{kcal} \mathrm{mol}^{-1}\right]$ & $\mathrm{BIE}\left[\mathrm{kcal} \mathrm{mol}^{-1}\right]$ & E-def $\left[\mathrm{kcal} \mathrm{mol}^{-1}\right]$ & $\mathrm{E}[\mathrm{Ha}]$ & $\mathrm{BAE}\left[\mathrm{kcal} \mathrm{mol}^{-1}\right]$ & $\mathrm{BIE}\left[\mathrm{kcal} \mathrm{mol}^{-1}\right]$ & E-def $\left[\mathrm{kcal} \mathrm{mol}^{-1}\right]$ \\
\hline \multicolumn{9}{|c|}{ Methanes } \\
\hline $\mathrm{H}$ & -40.523989637157 & & & & -40.501525442575 & & & \\
\hline $\mathrm{F}$ & -437.604661912271 & & & & -437.541182245921 & & & \\
\hline Hx1 & -40.523958818407 & & & & -40.501446942421 & & & \\
\hline $\mathrm{xH} 1$ & -40.523952957948 & & & & -40.501434196638 & & & \\
\hline HH1 & -81.048851651381 & -0.547 & -0.590 & 0.042 & -81.003553381963 & -0.315 & -0.422 & 0.107 \\
\hline $\mathrm{Hx} 2$ & -40.523938643624 & & & & -40.501394733956 & & & \\
\hline $\mathrm{xH} 2$ & -40.523934636742 & & & & -40.501378294744 & & & \\
\hline $\mathrm{HH} 2$ & -81.048619995006 & -0.402 & -0.469 & 0.067 & -81.003311524393 & -0.164 & -0.338 & 0.174 \\
\hline Hx1 & -40.523954720257 & & & & -40.501436128894 & & & \\
\hline $\mathrm{xF} 1$ & -437.604640614853 & & & & -437.541140557009 & & & \\
\hline HF1 & -478.129715242635 & -0.667 & -0.703 & 0.035 & -478.043568259918 & -0.540 & -0.622 & 0.082 \\
\hline $\mathrm{Hx} 2$ & -40.52398868047 & & & & -40.501525205944 & & & \\
\hline $\mathrm{xF} 2$ & -437.604655117936 & & & & -437.541172358575 & & & \\
\hline $\mathrm{HF} 2$ & -478.129197680029 & -0.343 & -0.348 & 0.005 & -478.043195963977 & -0.306 & -0.313 & 0.006 \\
\hline Fx1 & -437.604636895287 & & & & -437.541133784091 & & & \\
\hline $\mathrm{xF} 1$ & -437.604633921286 & & & & -437.541122660487 & & & \\
\hline FF1 & -875.210027471615 & -0.442 & -0.475 & 0.033 & -875.083127853354 & -0.479 & -0.547 & 0.068 \\
\hline $\mathrm{Fx} 2$ & -437.604636547421 & & & & -437.541126333705 & & & \\
\hline $\mathrm{xF} 2$ & -437.60461399148 & & & & -437.541069463641 & & & \\
\hline $\mathrm{FF} 2$ & -875.209681141116 & -0.224 & -0.270 & 0.046 & -875.083115092591 & -0.471 & -0.577 & 0.106 \\
\hline \multicolumn{9}{|c|}{ Ethanes } \\
\hline $\mathrm{H}$ & -79.841952885754 & & & & -79.80171482493 & & & \\
\hline $\mathrm{F}$ & -675.448326150121 & & & & -675.348142403687 & & & \\
\hline $\mathrm{Hx} 1$ & -79.841965343636 & & & & -79.801710238646 & & & \\
\hline $\mathrm{xH} 1$ & -79.841926996393 & & & & -79.801607002674 & & & \\
\hline HH1 & -159.686114201806 & -1.386 & -1.394 & 0.008 & -159.605183749063 & -1.101 & -1.171 & 0.071 \\
\hline $\mathrm{Hx} 2$ & -79.841983721594 & & & & -79.801774040706 & & & \\
\hline $\mathrm{xH} 2$ & -79.841987498528 & & & & -79.80179036455 & & & \\
\hline $\mathrm{HH} 2$ & -159.685931987125 & -1.271 & -1.230 & -0.041 & -159.605162209406 & -1.087 & -1.003 & -0.085 \\
\hline $\mathrm{Hx} 1$ & -79.84195629888 & & & & -79.801699202244 & & & \\
\hline $\mathrm{xF} 1$ & -675.448348741906 & & & & -675.34817193922 & & & \\
\hline HF1 & -755.291684980701 & -0.882 & -0.866 & -0.016 & -755.15124127724 & -0.869 & -0.860 & -0.009 \\
\hline $\mathrm{Hx} 2$ & -79.841987844431 & & & & -79.801772945268 & & & \\
\hline $\mathrm{xF} 2$ & -675.448272357927 & & & & -675.347943484417 & & & \\
\hline $\mathrm{HF} 2$ & -755.291863624704 & -0.994 & -1.006 & 0.012 & -755.15135631432 & -0.941 & -1.029 & 0.088 \\
\hline $\mathrm{Hx} 3$ & -79.841960791932 & & & & -79.801713402284 & & & \\
\hline $\mathrm{xF} 3$ & -675.448381829863 & & & & -675.348231835444 & & & \\
\hline HF3 & -755.29145741176 & -0.739 & -0.700 & -0.040 & -755.151128114893 & -0.797 & -0.742 & -0.055 \\
\hline $\mathrm{Hx} 4$ & -79.842024474054 & & & & -79.801889026458 & & & \\
\hline $\mathrm{xF} 4$ & -675.448346068047 & & & & -675.348160777516 & & & \\
\hline $\mathrm{HF} 4$ & -755.291839284107 & -0.979 & -0.922 & -0.057 & -755.151543381146 & -1.058 & -0.937 & -0.121 \\
\hline $\mathrm{Fx} 1$ & -675.448303824525 & & & & -675.348099109757 & & & \\
\hline $\mathrm{xF} 1$ & -675.448320250528 & & & & -675.348185542113 & & & \\
\hline $\mathrm{FF} 1$ & -1350.89738287022 & -0.458 & -0.476 & 0.018 & -1350.69779134423 & -0.945 & -0.945 & 0.000 \\
\hline Fx2 & -675.448352028375 & & & & -675.348170135301 & & & \\
\hline $\mathrm{xF} 2$ & -675.448355279647 & & & & -675.34817248712 & & & \\
\hline FF2 & -1350.89732933296 & -0.425 & -0.390 & -0.035 & -1350.6975505268 & -0.794 & -0.758 & -0.036 \\
\hline
\end{tabular}




\begin{tabular}{|c|c|c|c|c|c|c|c|c|}
\hline & \multicolumn{4}{|c|}{$\omega B 97 X-D 3 /$ def2-QZVP } & \multicolumn{4}{|c|}{ B2PLYP-D3/def2-QZVP } \\
\hline & $\mathrm{E}[\mathrm{Ha}]$ & $\mathrm{BAE}\left[\mathrm{kcal} \mathrm{mol}{ }^{-1}\right]$ & $\mathrm{BIE}\left[\mathrm{kcal} \mathrm{mol}^{-1}\right]$ & E-def $\left[\mathrm{kcal} \mathrm{mol}^{-1}\right]$ & $\mathrm{E}[\mathrm{Ha}]$ & $\mathrm{BAE}\left[\mathrm{kcal} \mathrm{mol}{ }^{-1}\right]$ & BIE $\left[\mathrm{kcal} \mathrm{mol}^{-1}\right]$ & E-def $\left[\mathrm{kcal} \mathrm{mol}^{-1}\right]$ \\
\hline \multicolumn{9}{|c|}{ Propanes } \\
\hline H & -119.162726065561 & & & & -119.105210215662 & & & \\
\hline $\mathrm{F}$ & -913.291719045601 & & & & -913.155050293842 & & & \\
\hline $\mathrm{Hx} 1$ & -119.162695070299 & & & & -119.10514416426 & & & \\
\hline $\mathrm{xH} 1$ & -119.162692889302 & & & & -119.105143096442 & & & \\
\hline HН1 & -238.328850095919 & -2.132 & -2.173 & 0.040 & -238.213251959607 & -1.777 & -1.860 & 0.084 \\
\hline $\mathrm{Hx} 2$ & -119.162706441179 & & & & -119.105147026118 & & & \\
\hline $\mathrm{xH} 2$ & -119.162703605184 & & & & -119.105149844914 & & & \\
\hline $\mathrm{HH} 2$ & -238.327705821858 & -1.414 & -1.441 & 0.026 & -238.212028075626 & -1.009 & -1.086 & 0.078 \\
\hline Hx1 & -119.162716984175 & & & & -119.105202967554 & & & \\
\hline $\mathrm{xF} 1$ & -913.29155321797 & & & & -913.15480750992 & & & \\
\hline HF1 & -1032.45660270664 & -1.354 & -1.464 & 0.110 & -1032.26234259515 & -1.307 & -1.463 & 0.157 \\
\hline $\mathrm{Hx} 2$ & -119.162772681517 & & & & -119.105343906942 & & & \\
\hline $\mathrm{xF} 2$ & -913.291550162414 & & & & -913.154685909976 & & & \\
\hline HF2 & -1032.45604535644 & -1.004 & -1.081 & 0.077 & -1032.26190882555 & -1.034 & -1.179 & 0.145 \\
\hline Hx3 & -119.162738260944 & & & & -119.10522666714 & & & \\
\hline $\mathrm{xF} 3$ & -913.291567156462 & & & & -913.154825449426 & & & \\
\hline HF3 & -1032.45609706258 & -1.037 & -1.124 & 0.088 & -1032.26190458293 & -1.032 & -1.162 & 0.131 \\
\hline $\mathrm{Hx} 4$ & -119.162768485575 & & & & -119.10530053403 & & & \\
\hline $\mathrm{xF} 4$ & -913.291522226192 & & & & -913.15464452575 & & & \\
\hline $\mathrm{HF} 4$ & -1032.45581721102 & -0.861 & -0.958 & 0.097 & -1032.26176526591 & -0.944 & -1.142 & 0.198 \\
\hline Fx1 & -913.291493984464 & & & & -913.154750679567 & & & \\
\hline $\mathrm{xF} 1$ & -913.291582517194 & & & & -913.154832708672 & & & \\
\hline FF1 & -1826.58395235351 & -0.323 & -0.550 & 0.227 & -1826.31180125185 & -1.067 & -1.392 & 0.325 \\
\hline $\mathrm{Fx} 2$ & -913.291712160794 & & & & -913.155041347573 & & & \\
\hline $\mathrm{xF} 2$ & -913.291705256765 & & & & -913.155029570048 & & & \\
\hline FF2 & -1826.58408810623 & -0.408 & -0.421 & 0.013 & -1826.31242949808 & -1.461 & -1.480 & 0.019 \\
\hline \multicolumn{9}{|c|}{ Butanes } \\
\hline $\mathrm{H}$ & -158.483603881961 & & & & -158.408887655819 & & & \\
\hline $\mathrm{F}$ & -1151.13425298519 & & & & -1150.96116059696 & & & \\
\hline $\mathrm{Hx} 1$ & -158.48349004351 & & & & -158.408626897532 & & & \\
\hline $\mathrm{xH} 1$ & -158.483511459698 & & & & -158.40866740057 & & & \\
\hline HH1 & -316.972065459881 & -3.048 & -3.178 & 0.129 & -316.821645845627 & -2.429 & -2.731 & 0.302 \\
\hline $\mathrm{Hx} 2$ & -158.483466320688 & & & & -158.408610158148 & & & \\
\hline $\mathrm{xH} 2$ & -158.483486572891 & & & & -158.408627419287 & & & \\
\hline Hн2 & -316.970241636398 & -1.904 & -2.064 & 0.160 & -316.819664647858 & -1.186 & -1.523 & 0.337 \\
\hline $\mathrm{Hx} 1$ & -158.48354732995 & & & & -158.408830755428 & & & \\
\hline $\mathrm{xF} 1$ & -1151.1339725387 & & & & -1150.96092036433 & & & \\
\hline $\mathrm{HF} 1$ & -1309.62039470818 & -1.593 & -1.804 & 0.211 & -1309.37294829445 & -1.820 & -2.006 & 0.186 \\
\hline $\mathrm{Hx} 2$ & -158.483588010416 & & & & -158.408873263134 & & & \\
\hline $\mathrm{xF} 2$ & -1151.13424019064 & & & & -1150.96114954222 & & & \\
\hline $\mathrm{HF} 2$ & -1309.62065279537 & -1.754 & -1.772 & 0.018 & -1309.37308930181 & -1.908 & -1.924 & 0.016 \\
\hline $\mathrm{Hx} 3$ & -158.483515962317 & & & & -158.4087814023 & & & \\
\hline $\mathrm{xF} 3$ & -1151.13422976682 & & & & -1150.96111582064 & & & \\
\hline $\mathrm{HF} 3$ & -1309.62054294867 & -1.686 & -1.755 & 0.070 & -1309.37297091449 & -1.834 & -1.929 & 0.095 \\
\hline $\mathrm{Hx} 4$ & -158.483518180739 & & & & -158.408773871218 & & & \\
\hline $\mathrm{xF} 4$ & -1151.13427030414 & & & & -1150.96120481247 & & & \\
\hline $\mathrm{HF} 4$ & -1309.62077132318 & -1.829 & -1.872 & 0.043 & -1309.3730294492 & -1.871 & -1.914 & 0.044 \\
\hline Fx1 & -1151.13425764979 & & & & -1150.96118010287 & & & \\
\hline $\mathrm{xF} 1$ & -1151.13416854247 & & & & -1150.96111256877 & & & \\
\hline FF1 & -2302.27002381851 & -0.952 & -1.003 & 0.050 & -2301.92513364923 & -1.765 & -1.783 & 0.018 \\
\hline $\mathrm{Fx} 2$ & -1151.13420064653 & & & & -1150.96113065191 & & & \\
\hline $\mathrm{xF} 2$ & -1151.13416285207 & & & & -1150.9611182198 & & & \\
\hline FF2 & -2302.26990137633 & -0.876 & -0.965 & 0.089 & -2301.92525259683 & -1.839 & -1.885 & 0.045 \\
\hline
\end{tabular}




\begin{tabular}{|c|c|c|c|c|c|c|c|c|}
\hline & \multicolumn{4}{|c|}{$\omega B 97 X-D 3 /$ def2-QZVP } & \multicolumn{4}{|c|}{ B2PLYP-D3/def2-QZVP } \\
\hline & $\mathrm{E}[\mathrm{Ha}]$ & $\mathrm{BAE}\left[\mathrm{kcal} \mathrm{mol}^{-1}\right]$ & BIE $\left[\mathrm{kcal} \mathrm{mol}^{-1}\right]$ & E-def $\left[\mathrm{kcal} \mathrm{mol}^{-1}\right]$ & $\mathrm{E}[\mathrm{Ha}]$ & $\mathrm{BAE}\left[\mathrm{kcal} \mathrm{mol}^{-1}\right]$ & $\mathrm{BIE}\left[\mathrm{kcal} \mathrm{mol}^{-1}\right]$ & E-def $\left[\mathrm{kcal} \mathrm{mol}^{-1}\right]$ \\
\hline \multicolumn{9}{|c|}{ Pentanes } \\
\hline H & -197.804347904371 & & & & -197.712361038809 & & & \\
\hline $\mathrm{F}$ & -1388.97697980544 & & & & -1388.76760173007 & & & \\
\hline $\mathrm{Hx} 1$ & -197.804223766805 & & & & -197.712087919601 & & & \\
\hline $\mathrm{xH} 1$ & -197.804222482112 & & & & -197.712086405016 & & & \\
\hline нH1 & -395.615046486167 & -3.985 & -4.142 & 0.157 & -395.429871069013 & -3.231 & -3.575 & 0.344 \\
\hline $\mathrm{Hx} 2$ & -197.804224068999 & & & & -197.712028914123 & & & \\
\hline $\mathrm{xH} 2$ & -197.804225500517 & & & & -197.71203319141 & & & \\
\hline нH2 & -395.612918324993 & -2.650 & -2.804 & 0.155 & -395.427117971479 & -1.503 & -1.918 & 0.414 \\
\hline Hx1 & -197.804224254005 & & & & -197.712204066394 & & & \\
\hline $\mathrm{xF1}$ & -1388.97691771509 & & & & -1388.76752380962 & & & \\
\hline HF1 & -1586.78499878521 & -2.304 & -2.420 & 0.117 & -1586.48375666583 & -2.381 & -2.528 & 0.147 \\
\hline $\mathrm{Hx} 2$ & -197.804311161946 & & & & -197.712324581324 & & & \\
\hline $\mathrm{xF} 2$ & -1388.97670903974 & & & & -1388.76728895971 & & & \\
\hline HF2 & -1586.78448732797 & -1.983 & -2.176 & 0.193 & -1586.48334090172 & -2.120 & -2.339 & 0.219 \\
\hline $\mathrm{Hx} 3$ & -197.804293796985 & & & & -197.71222655078 & & & \\
\hline $\mathrm{xF} 3$ & -1388.97700660947 & & & & -1388.76755484894 & & & \\
\hline HF3 & -1586.78463209596 & -2.074 & -2.091 & 0.017 & -1586.48345527394 & -2.192 & -2.305 & 0.114 \\
\hline $\mathrm{Hx} 4$ & -197.804301042282 & & & & -197.712283326489 & & & \\
\hline $\mathrm{xF} 4$ & -1388.97700293732 & & & & -1388.76763965797 & & & \\
\hline $\mathrm{HF} 4$ & -1586.78532871107 & -2.511 & -2.526 & 0.015 & -1586.48412805957 & -2.614 & -2.639 & 0.025 \\
\hline $\mathrm{Fx} 1$ & -1388.97695969876 & & & & -1388.7675389959 & & & \\
\hline $\mathrm{xF} 1$ & -1388.97691785676 & & & & -1388.76753067382 & & & \\
\hline $\mathrm{FF} 1$ & -2777.95575603242 & -1.127 & -1.179 & 0.051 & -2777.53912677457 & -2.462 & -2.546 & 0.084 \\
\hline $\mathrm{Fx} 2$ & -1388.9769331607 & & & & -1388.76751015058 & & & \\
\hline $\mathrm{xF} 2$ & -1388.97693387251 & & & & -1388.76751466087 & & & \\
\hline $\mathrm{FF} 2$ & -2777.95577326012 & -1.138 & -1.196 & 0.058 & -2777.53869663949 & -2.192 & -2.304 & 0.112 \\
\hline \multicolumn{9}{|c|}{ Hexanes } \\
\hline $\mathrm{H}$ & -237.125118325372 & & & & -237.015822517878 & & & \\
\hline $\mathrm{F}$ & -1626.81975475692 & & & & -1626.57403644275 & & & \\
\hline $\mathrm{Hx} 1$ & -237.124964081574 & & & & -237.015536615949 & & & \\
\hline $\mathrm{xH} 1$ & -237.12496706719 & & & & -237.015538978194 & & & \\
\hline HH1 & -474.257659667059 & -4.658 & -4.850 & 0.192 & -474.037695992657 & -3.797 & -4.154 & 0.357 \\
\hline $\mathrm{Hx} 2$ & -237.12496511198 & & & & -237.015471000698 & & & \\
\hline $\mathrm{xH} 2$ & -237.124959201718 & & & & -237.015460028627 & & & \\
\hline Hн2 & -474.255328318038 & -3.195 & -3.391 & 0.196 & -474.034799057409 & -1.979 & -2.427 & 0.448 \\
\hline $\mathrm{Hx} 1$ & -237.124901996346 & & & & -237.015565777105 & & & \\
\hline $\mathrm{xF1}$ & -1626.81925712925 & & & & -1626.57347489366 & & & \\
\hline HF1 & -1863.94854834778 & -2.306 & -2.754 & 0.448 & -1863.59376760533 & -2.453 & -2.966 & 0.513 \\
\hline $\mathrm{Hx} 2$ & -237.125029189562 & & & & -237.015735172815 & & & \\
\hline $\mathrm{xF} 2$ & -1626.8193767355 & & & & -1626.57363065423 & & & \\
\hline HF2 & -1863.94866330396 & -2.378 & -2.672 & 0.293 & -1863.59402880792 & -2.617 & -2.926 & 0.309 \\
\hline $\mathrm{Hx} 3$ & -237.124922336206 & & & & -237.015576673154 & & & \\
\hline $\mathrm{xF} 3$ & -1626.8194470484 & & & & -1626.57369241852 & & & \\
\hline HF3 & -1863.9487005088 & -2.402 & -2.718 & 0.316 & -1863.59387350454 & -2.519 & -2.889 & 0.370 \\
\hline $\mathrm{Hx} 4$ & -237.125094592074 & & & & -237.015803753604 & & & \\
\hline $\mathrm{xF} 4$ & -1626.819717558 & & & & -1626.57401459891 & & & \\
\hline HF4 & -1863.94934295751 & -2.805 & -2.843 & 0.038 & -1863.59457044326 & -2.956 & -2.982 & 0.025 \\
\hline Fx1 & -1626.81969422358 & & & & -1626.57396307011 & & & \\
\hline $\mathrm{xF} 1$ & -1626.8196370696 & & & & -1626.57389438932 & & & \\
\hline FF1 & -3253.64174854085 & -1.405 & -1.517 & 0.112 & -3253.15315918619 & -3.192 & -3.327 & 0.135 \\
\hline $\mathrm{Fx} 2$ & -1626.81958770749 & & & & -1626.57386470851 & & & \\
\hline $\mathrm{xF} 2$ & -1626.81958872181 & & & & -1626.57386612718 & & & \\
\hline $\mathrm{FF} 2$ & -3253.6402260916 & -0.450 & -0.659 & 0.209 & -3253.15137948001 & -2.075 & -2.290 & 0.215 \\
\hline
\end{tabular}




\begin{tabular}{|c|c|c|c|c|c|c|c|c|}
\hline & \multicolumn{4}{|c|}{ B97M-V/def2-QZVP } & \multicolumn{4}{|c|}{$\omega B 97 \mathrm{M}-\mathrm{V} / \mathrm{def2}-\mathrm{QZVP}$} \\
\hline & $\mathrm{E}[\mathrm{Ha}]$ & $\mathrm{BAE}\left[\mathrm{kcal} \mathrm{mol}^{-1}\right]$ & $\mathrm{BIE}\left[\mathrm{kcal} \mathrm{mol}^{-1}\right]$ & E-def $\left[\mathrm{kcal} \mathrm{mol}^{-1}\right]$ & $\mathrm{E}[\mathrm{Ha}]$ & $\mathrm{BAE}\left[\mathrm{kcal} \mathrm{mol}^{-1}\right]$ & $\mathrm{BIE}\left[\mathrm{kcal} \mathrm{mol}^{-1}\right]$ & E-def $\left[\mathrm{kcal} \mathrm{mol}^{-1}\right]$ \\
\hline \multicolumn{9}{|c|}{ Methanes } \\
\hline $\mathrm{H}$ & -40.5346275635259 & & & & -40.4957485046463 & & & \\
\hline $\mathrm{F}$ & -437.567748985576 & & & & -437.613280285495 & & & \\
\hline Hx1 & -40.5346279941849 & & & & -40.4957476962338 & & & \\
\hline $\mathrm{xH1}$ & -40.5346247297872 & & & & -40.4957471970221 & & & \\
\hline HH1 & -81.0699247132812 & -0.420 & -0.422 & 0.002 & -80.9922062646482 & -0.445 & -0.446 & 0.001 \\
\hline $\mathrm{Hx} 2$ & -40.5346145302907 & & & & -40.4957483738133 & & & \\
\hline $\mathrm{xH} 2$ & -40.5346145512595 & & & & -40.4957478718937 & & & \\
\hline $\mathrm{HH} 2$ & -81.069772953125 & -0.325 & -0.341 & 0.016 & -80.9920540519836 & -0.350 & -0.350 & 0.000 \\
\hline Hx1 & -40.5346178172627 & & & & -40.4957471698063 & & & \\
\hline $\mathrm{xF} 1$ & -437.567736175916 & & & & -437.613286002039 & & & \\
\hline HF1 & -478.103530368362 & -0.724 & -0.738 & 0.014 & -478.110294093698 & -0.794 & -0.791 & -0.003 \\
\hline $\mathrm{Hx} 2$ & -40.5346206892954 & & & & -40.4957467708123 & & & \\
\hline $\mathrm{xF} 2$ & -437.567733592544 & & & & -437.613278250184 & & & \\
\hline $\mathrm{HF} 2$ & -478.10302721959 & -0.408 & -0.422 & 0.014 & -478.109724388104 & -0.436 & -0.439 & 0.002 \\
\hline Fx1 & -437.567739928678 & & & & -437.613283661763 & & & \\
\hline $\mathrm{xF} 1$ & -437.56774125294 & & & & -437.613285923743 & & & \\
\hline FF1 & -875.136811462286 & -0.824 & -0.835 & 0.011 & -875.228020669945 & -0.916 & -0.911 & -0.006 \\
\hline $\mathrm{Fx} 2$ & -437.567737482119 & & & & -437.613282413706 & & & \\
\hline $\mathrm{xF} 2$ & -437.567741311718 & & & & -437.613284222453 & & & \\
\hline $\mathrm{FF} 2$ & -875.13677297774 & -0.800 & -0.812 & 0.012 & -875.227932974225 & -0.861 & -0.857 & -0.004 \\
\hline \multicolumn{9}{|c|}{ Ethanes } \\
\hline $\mathrm{H}$ & -79.8634676185315 & & & & -79.799771959001 & & & \\
\hline $\mathrm{F}$ & -675.401483616651 & & & & -675.463447286677 & & & \\
\hline $\mathrm{Hx} 1$ & -79.8634844120543 & & & & -79.7997728170933 & & & \\
\hline $\mathrm{xH} 1$ & -79.863460913367 & & & & -79.79977221734 & & & \\
\hline HH1 & -159.728890124617 & -1.227 & -1.220 & -0.006 & -159.601538230249 & -1.251 & -1.251 & -0.001 \\
\hline $\mathrm{Hx} 2$ & -79.8634675051755 & & & & -79.7997721413314 & & & \\
\hline $\mathrm{xH} 2$ & -79.8634685947661 & & & & -79.7997722602839 & & & \\
\hline HH2 & -159.728711983365 & -1.115 & -1.114 & -0.001 & -159.601272359127 & -1.085 & -1.084 & 0.000 \\
\hline $\mathrm{Hx} 1$ & -79.8634630201355 & & & & -79.7997690954954 & & & \\
\hline $\mathrm{xF} 1$ & -675.401451126738 & & & & -675.463452268948 & & & \\
\hline HF1 & -755.266529348869 & -0.990 & -1.014 & 0.023 & -755.264975628052 & -1.102 & -1.101 & -0.001 \\
\hline $\mathrm{Hx} 2$ & -79.8634810660224 & & & & -79.7997721460025 & & & \\
\hline $\mathrm{xF} 2$ & -675.401447878314 & & & & -675.463462280309 & & & \\
\hline $\mathrm{HF} 2$ & -755.266806810393 & -1.164 & -1.178 & 0.014 & -755.265257510737 & -1.279 & -1.270 & -0.010 \\
\hline $\mathrm{Hx} 3$ & -79.8634860682731 & & & & -79.7997723075836 & & & \\
\hline $\mathrm{xF} 3$ & -675.40145709312 & & & & -675.463453606389 & & & \\
\hline HF3 & -755.266293482318 & -0.842 & -0.847 & 0.005 & -755.264707655317 & -0.934 & -0.930 & -0.004 \\
\hline $\mathrm{Hx} 4$ & -79.8634696687753 & & & & -79.799770814186 & & & \\
\hline $\mathrm{xF} 4$ & -675.40146509215 & & & & -675.463456299702 & & & \\
\hline $\mathrm{HF} 4$ & -755.266701156412 & -1.098 & -1.108 & 0.010 & -755.265131492954 & -1.200 & -1.195 & -0.005 \\
\hline $\mathrm{Fx} 1$ & -675.401462834626 & & & & -675.463444391719 & & & \\
\hline $\mathrm{xF} 1$ & -675.401425395704 & & & & -675.4634096174 & & & \\
\hline $\mathrm{FF} 1$ & -1350.80494647974 & -1.242 & -1.292 & 0.050 & -1350.92912982858 & -1.403 & -1.428 & 0.025 \\
\hline Fx2 & -675.401443320877 & & & & -675.46345649593 & & & \\
\hline $\mathrm{xF} 2$ & -675.401457663878 & & & & -675.463443424911 & & & \\
\hline FF2 & -1350.80453933562 & -0.987 & -1.028 & 0.042 & -1350.92869936838 & -1.133 & -1.129 & -0.003 \\
\hline
\end{tabular}




\begin{tabular}{|c|c|c|c|c|c|c|c|c|}
\hline & \multicolumn{4}{|c|}{ B97M-V/def2-QZVP } & \multicolumn{4}{|c|}{$\omega B 97 \mathrm{M}-\mathrm{V} / \mathrm{def} 2-\mathrm{QZVP}$} \\
\hline & $\mathrm{E}[\mathrm{Ha}]$ & $\mathrm{BAE}\left[\mathrm{kcal} \mathrm{mol}{ }^{-1}\right]$ & $\mathrm{BIE}\left[\mathrm{kcal} \mathrm{mol}^{-1}\right]$ & E-def $\left[\mathrm{kcal} \mathrm{mol}^{-1}\right]$ & $\mathrm{E}[\mathrm{Ha}]$ & $\mathrm{BAE}\left[\mathrm{kcal} \mathrm{mol}{ }^{-1}\right]$ & BIE $\left[\mathrm{kcal} \mathrm{mol}^{-1}\right]$ & E-def $\left[\mathrm{kcal} \mathrm{mol}^{-1}\right]$ \\
\hline \multicolumn{9}{|c|}{ Propanes } \\
\hline $\mathrm{H}$ & -119.195746349543 & & & & -119.107038420108 & & & \\
\hline $\mathrm{F}$ & -913.235611080036 & & & & -913.313990815918 & & & \\
\hline $\mathrm{Hx} 1$ & -119.195751040919 & & & & -119.107040019672 & & & \\
\hline $\mathrm{xH} 1$ & -119.195751235565 & & & & -119.107040513007 & & & \\
\hline HН1 & -238.39464926539 & -1.981 & -1.975 & -0.006 & -238.217140494273 & -1.922 & -1.920 & -0.002 \\
\hline $\mathrm{Hx} 2$ & -119.195739574173 & & & & -119.107038139443 & & & \\
\hline $\mathrm{xH} 2$ & -119.195751301128 & & & & -119.10703954224 & & & \\
\hline HH2 & -238.393564206149 & -1.300 & -1.301 & 0.001 & -238.215968431749 & -1.187 & -1.186 & -0.001 \\
\hline Hx1 & -119.195747158019 & & & & -119.107034110003 & & & \\
\hline $\mathrm{xF} 1$ & -913.235520036749 & & & & -913.313926445028 & & & \\
\hline HF1 & -1032.43412446875 & -1.736 & -1.793 & 0.057 & -1032.42389439752 & -1.798 & -1.841 & 0.043 \\
\hline $\mathrm{Hx} 2$ & -119.195743229829 & & & & -119.107036115086 & & & \\
\hline $\mathrm{xF} 2$ & -913.235560448272 & & & & -913.313977042428 & & & \\
\hline HF2 & -1032.43353788276 & -1.368 & -1.402 & 0.034 & -1032.42330712558 & -1.429 & -1.439 & 0.010 \\
\hline Hx3 & -119.195723331443 & & & & -119.107032036408 & & & \\
\hline $\mathrm{xF} 3$ & -913.235563818858 & & & & -913.313967916387 & & & \\
\hline HF3 & -1032.43346160282 & -1.320 & -1.364 & 0.044 & -1032.42327916938 & -1.412 & -1.430 & 0.018 \\
\hline $\mathrm{Hx} 4$ & -119.195749362503 & & & & -119.107037004719 & & & \\
\hline $\mathrm{xF} 4$ & -913.23555428915 & & & & -913.313966963938 & & & \\
\hline $\mathrm{HF} 4$ & -1032.43357510583 & -1.392 & -1.425 & 0.034 & -1032.42329048709 & -1.419 & -1.435 & 0.016 \\
\hline Fx1 & -913.235469341764 & & & & -913.31383468207 & & & \\
\hline $\mathrm{xF} 1$ & -913.235578542832 & & & & -913.313949338467 & & & \\
\hline FF1 & -1826.47408473935 & -1.796 & -1.906 & 0.109 & -1826.63103234849 & -1.914 & -2.038 & 0.124 \\
\hline Fx2 & -913.235612323517 & & & & -913.313987791468 & & & \\
\hline $\mathrm{xF} 2$ & -913.235615386666 & & & & -913.313989209126 & & & \\
\hline FF2 & -1826.47469791244 & -2.181 & -2.178 & -0.003 & -1826.63157041598 & -2.252 & -2.255 & 0.003 \\
\hline \multicolumn{9}{|c|}{ Butanes } \\
\hline $\mathrm{H}$ & -158.528256438714 & & & & -158.414400341613 & & & \\
\hline $\mathrm{F}$ & -1151.06934645219 & & & & -1151.1640864102 & & & \\
\hline $\mathrm{Hx} 1$ & -158.528262190079 & & & & -158.414400900464 & & & \\
\hline $\mathrm{xH} 1$ & -158.52825623994 & & & & -158.414398581128 & & & \\
\hline HH1 & -317.061134263997 & -2.900 & -2.896 & -0.003 & -316.833382584199 & -2.875 & -2.876 & 0.001 \\
\hline $\mathrm{Hx} 2$ & -158.528249584874 & & & & -158.414392074961 & & & \\
\hline $\mathrm{xH} 2$ & -158.528251642459 & & & & -158.414399841277 & & & \\
\hline HH2 & -317.059063280091 & -1.600 & -1.608 & 0.007 & -316.831466359715 & -1.673 & -1.678 & 0.006 \\
\hline $\mathrm{Hx} 1$ & -158.52825034829 & & & & -158.414397671149 & & & \\
\hline $\mathrm{xF} 1$ & -1151.06898226145 & & & & -1151.16366761481 & & & \\
\hline $\mathrm{HF} 1$ & -1309.60073427327 & -1.965 & -2.197 & 0.232 & -1309.58192322833 & -2.156 & -2.421 & 0.264 \\
\hline $\mathrm{Hx} 2$ & -158.52823774443 & & & & -158.414385410504 & & & \\
\hline $\mathrm{xF} 2$ & -1151.06933204032 & & & & -1151.16407399966 & & & \\
\hline $\mathrm{HF} 2$ & -1309.60105583654 & -2.167 & -2.188 & 0.021 & -1309.5823188575 & -2.405 & -2.422 & 0.017 \\
\hline $\mathrm{Hx} 3$ & -158.528239204002 & & & & -158.414375365031 & & & \\
\hline $\mathrm{xF} 3$ & -1151.06927688162 & & & & -1151.16401449552 & & & \\
\hline $\mathrm{HF} 3$ & -1309.60112646316 & -2.211 & -2.266 & 0.054 & -1309.58221193721 & -2.338 & -2.398 & 0.061 \\
\hline $\mathrm{Hx} 4$ & -158.52821183069 & & & & -158.414357925721 & & & \\
\hline $\mathrm{xF} 4$ & -1151.06935916862 & & & & -1151.16409150155 & & & \\
\hline $\mathrm{HF} 4$ & -1309.60108941313 & -2.188 & -2.208 & 0.020 & -1309.58217743759 & -2.316 & -2.339 & 0.023 \\
\hline Fx1 & -1151.06930291287 & & & & -1151.16402939764 & & & \\
\hline $\mathrm{xF} 1$ & -1151.06928533926 & & & & -1151.1640367469 & & & \\
\hline FF1 & -2302.14220640668 & -2.205 & -2.270 & 0.066 & -2302.33203272416 & -2.422 & -2.489 & 0.067 \\
\hline $\mathrm{Fx} 2$ & -1151.06921196654 & & & & -1151.16394851121 & & & \\
\hline $\mathrm{xF} 2$ & -1151.06926765903 & & & & -1151.16398611436 & & & \\
\hline FF2 & -2302.14260320689 & -2.454 & -2.588 & 0.134 & -2302.33234525607 & -2.618 & -2.768 & 0.149 \\
\hline
\end{tabular}




\begin{tabular}{|c|c|c|c|c|c|c|c|c|}
\hline & \multicolumn{4}{|c|}{ B97M-V/def2-QZVP } & \multicolumn{4}{|c|}{$\omega B 97 \mathrm{M}-\mathrm{V} /$ def2-QZVP } \\
\hline & $\mathrm{E}[\mathrm{Ha}]$ & $\mathrm{BAE}\left[\mathrm{kcal} \mathrm{mol}^{-1}\right]$ & BIE $\left[\mathrm{kcal} \mathrm{mol}^{-1}\right]$ & E-def $\left[\mathrm{kcal} \mathrm{mol}^{-1}\right]$ & $\mathrm{E}[\mathrm{Ha}]$ & $\mathrm{BAE}\left[\mathrm{kcal} \mathrm{mol}^{-1}\right]$ & BIE $\left[\mathrm{kcal} \mathrm{mol}^{-1}\right]$ & E-def $\left[\mathrm{kcal} \mathrm{mol}^{-1}\right]$ \\
\hline \multicolumn{9}{|c|}{ Pentanes } \\
\hline н & -197.860420190153 & & & & -197.721684828828 & & & \\
\hline $\mathrm{F}$ & -1388.90335480808 & & & & -1389.01437615101 & & & \\
\hline $\mathrm{Hx} 1$ & -197.860427954992 & & & & -197.72168355061 & & & \\
\hline $\mathrm{xH} 1$ & -197.860428009057 & & & & -197.721683538158 & & & \\
\hline нH1 & -395.72725042996 & -4.022 & -4.013 & -0.010 & -395.449466982842 & -3.826 & -3.828 & 0.002 \\
\hline Hx2 & -197.860419374304 & & & & -197.72168020287 & & & \\
\hline $\mathrm{xH} 2$ & -197.860419308318 & & & & -197.721680192709 & & & \\
\hline нH2 & -395.724782048332 & -2.473 & -2.474 & 0.001 & -395.447055883623 & -2.313 & -2.319 & 0.006 \\
\hline Hx1 & -197.860362802645 & & & & -197.721592909566 & & & \\
\hline $\mathrm{xF} 1$ & -1388.90313394693 & & & & -1389.01419305294 & & & \\
\hline HF1 & -1586.76833452294 & -2.861 & -3.036 & 0.175 & -1586.74095269262 & -3.070 & -3.242 & 0.173 \\
\hline $\mathrm{Hx} 2$ & -197.860386963496 & & & & -197.721651403275 & & & \\
\hline $\mathrm{xF} 2$ & -1388.90297341392 & & & & -1389.01398801318 & & & \\
\hline $\mathrm{HF} 2$ & -1586.76769650998 & -2.461 & -2.721 & 0.260 & -1586.74026116524 & -2.636 & -2.900 & 0.265 \\
\hline Hx3 & -197.860419965385 & & & & -197.721673539593 & & & \\
\hline $\mathrm{xF} 3$ & -1388.90327547579 & & & & -1389.01433273858 & & & \\
\hline HF3 & -1586.76811046652 & -2.721 & -2.770 & 0.050 & -1586.7405471167 & -2.815 & -2.849 & 0.034 \\
\hline $\mathrm{Hx} 4$ & -197.860403595305 & & & & -197.721654258961 & & & \\
\hline $\mathrm{xF} 4$ & -1388.90330580609 & & & & -1389.01434436298 & & & \\
\hline $\mathrm{HF} 4$ & -1586.76874488396 & -3.119 & -3.160 & 0.041 & -1586.74126733087 & -3.267 & -3.306 & 0.039 \\
\hline $\mathrm{Fx} 1$ & -1388.90332861873 & & & & -1389.01434732485 & & & \\
\hline $\mathrm{xF} 1$ & -1388.90322727535 & & & & -1389.01426868359 & & & \\
\hline $\mathrm{FF} 1$ & -2777.81202574803 & -3.336 & -3.432 & 0.096 & -2778.03455550893 & -3.642 & -3.727 & 0.086 \\
\hline $\mathrm{Fx} 2$ & -1388.90329675328 & & & & -1389.01430694186 & & & \\
\hline $\mathrm{xF} 2$ & -1388.90329883243 & & & & -1389.01430989324 & & & \\
\hline $\mathrm{FF} 2$ & -2777.81154904871 & -3.037 & -3.108 & 0.072 & -2778.03400258757 & -3.295 & -3.380 & 0.085 \\
\hline \multicolumn{9}{|c|}{ Hexanes } \\
\hline $\mathrm{H}$ & -237.192833856703 & & & & -237.029020009266 & & & \\
\hline $\mathrm{F}$ & -1626.73729652087 & & & & -1626.86466363313 & & & \\
\hline $\mathrm{Hx} 1$ & -237.19284438643 & & & & -237.029016679569 & & & \\
\hline $\mathrm{xH} 1$ & -237.192844278365 & & & & -237.029016563773 & & & \\
\hline HH1 & -474.393219481582 & -4.739 & -4.726 & -0.013 & -474.065308054862 & -4.561 & -4.565 & 0.004 \\
\hline $\mathrm{Hx} 2$ & -237.19283615573 & & & & -237.029017404975 & & & \\
\hline $\mathrm{xH} 2$ & -237.19283597222 & & & & -237.029017250157 & & & \\
\hline нH2 & -474.390341587435 & -2.933 & -2.930 & -0.003 & -474.062647937146 & -2.892 & -2.895 & 0.003 \\
\hline $\mathrm{Hx} 1$ & -237.192672123572 & & & & -237.028850242377 & & & \\
\hline $\mathrm{xF} 1$ & -1626.73663012081 & & & & -1626.86406776417 & & & \\
\hline HF1 & -1863.93516865747 & -3.162 & -3.681 & 0.520 & -1863.8991389047 & -3.423 & -3.904 & 0.480 \\
\hline $\mathrm{Hx} 2$ & -237.19277205658 & & & & -237.028936080516 & & & \\
\hline $\mathrm{xF} 2$ & -1626.73677829964 & & & & -1626.86419522586 & & & \\
\hline $\mathrm{HF} 2$ & -1863.93487160703 & -2.975 & -3.339 & 0.364 & -1863.89888879657 & -3.266 & -3.613 & 0.347 \\
\hline Hx3 & -237.192733450481 & & & & -237.028904043426 & & & \\
\hline $\mathrm{xF} 3$ & -1626.73699148147 & & & & -1626.86434932274 & & & \\
\hline HF3 & -1863.93532506853 & -3.260 & -3.514 & 0.254 & -1863.89920268402 & -3.463 & -3.733 & 0.270 \\
\hline $\mathrm{Hx} 4$ & -237.192820554453 & & & & -237.028999680929 & & & \\
\hline $\mathrm{xF} 4$ & -1626.73721092159 & & & & -1626.86459071103 & & & \\
\hline $\mathrm{HF} 4$ & -1863.93568561775 & -3.486 & -3.548 & 0.062 & -1863.89952682884 & -3.667 & -3.725 & 0.059 \\
\hline $\mathrm{Fx} 1$ & -1626.73724201235 & & & & -1626.86461397394 & & & \\
\hline $\mathrm{xF} 1$ & -1626.73721598677 & & & & -1626.86458414399 & & & \\
\hline FF1 & -3253.48167011514 & -4.441 & -4.526 & 0.085 & -3253.73707536975 & -4.862 & -4.943 & 0.081 \\
\hline $\mathrm{Fx} 2$ & -1626.73729341561 & & & & -1626.86462285499 & & & \\
\hline $\mathrm{xF} 2$ & -1626.7372905027 & & & & -1626.8646201186 & & & \\
\hline $\mathrm{FF} 2$ & -3253.4796346857 & -3.164 & -3.169 & 0.006 & -3253.73473241604 & -3.392 & -3.445 & 0.053 \\
\hline
\end{tabular}




\begin{tabular}{|c|c|c|c|c|c|c|c|c|}
\hline & \multicolumn{4}{|c|}{ GFN1-xTB } & \multicolumn{4}{|c|}{ GFN2-xTB } \\
\hline & $\mathrm{E}[\mathrm{Ha}]$ & $\mathrm{BAE}\left[\mathrm{kcal} \mathrm{mol}^{-1}\right]$ & $\mathrm{BIE}\left[\mathrm{kcal} \mathrm{mol}^{-1}\right]$ & E-def $\left[\mathrm{kcal} \mathrm{mol}^{-1}\right]$ & $\mathrm{E}[\mathrm{Ha}]$ & $\mathrm{BAE}\left[\mathrm{kcal} \mathrm{mol}^{-1}\right]$ & BIE $\left[\mathrm{kcal} \mathrm{mol}^{-1}\right]$ & E-def $\left[\mathrm{kcal} \mathrm{mol}^{-1}\right]$ \\
\hline \multicolumn{9}{|c|}{ Methanes } \\
\hline $\mathrm{H}$ & -4.2742696 & & & & -4.1751772 & & & \\
\hline $\mathrm{F}$ & -22.6940918 & & & & -21.0784782 & & & \\
\hline $\mathrm{Hx} 1$ & -4.2742692 & & & & -4.1751781 & & & \\
\hline $\mathrm{xH} 1$ & -4.2742691 & & & & -4.1751779 & & & \\
\hline HH1 & -8.5491539 & -0.386 & -0.386 & 0.001 & -8.3509684 & -0.385 & -0.384 & -0.001 \\
\hline $\mathrm{Hx} 2$ & -4.2742694 & & & & -4.175177 & & & \\
\hline $\mathrm{xH} 2$ & -4.2742694 & & & & -4.175177 & & & \\
\hline $\mathrm{HH} 2$ & -8.5490736 & -0.335 & -0.336 & 0.000 & -8.3509953 & -0.402 & -0.402 & 0.000 \\
\hline Hx1 & -4.2742695 & & & & -4.1751775 & & & \\
\hline $\mathrm{xF} 1$ & -22.6940844 & & & & -21.078461 & & & \\
\hline $\mathrm{HF} 1$ & -26.9692182 & -0.538 & -0.542 & 0.005 & -25.2552051 & -0.972 & -0.983 & 0.011 \\
\hline $\mathrm{Hx} 2$ & -4.274269 & & & & -4.1751774 & & & \\
\hline $\mathrm{xF} 2$ & -22.6940862 & & & & -21.0784656 & & & \\
\hline $\mathrm{HF} 2$ & -26.9689881 & -0.393 & -0.397 & 0.004 & -25.2543451 & -0.433 & -0.441 & 0.008 \\
\hline Fx1 & -22.6940837 & & & & -21.0784644 & & & \\
\hline $\mathrm{xF} 1$ & -22.6940859 & & & & -21.0784657 & & & \\
\hline FF1 & -45.3888706 & -0.431 & -0.440 & 0.009 & -42.157921 & -0.605 & -0.622 & 0.017 \\
\hline $\mathrm{Fx} 2$ & -22.6940858 & & & & -21.0784645 & & & \\
\hline $\mathrm{xF} 2$ & -22.6940851 & & & & -21.0784651 & & & \\
\hline FF2 & -45.389012 & -0.520 & -0.528 & 0.008 & -42.1587683 & -1.137 & -1.154 & 0.017 \\
\hline \multicolumn{9}{|c|}{ Ethanes } \\
\hline $\mathrm{H}$ & -7.4698011 & & & & -7.3362538 & & & \\
\hline F & -35.0833459 & & & & -32.6629925 & & & \\
\hline $\mathrm{Hx} 1$ & -7.4697937 & & & & -7.3362454 & & & \\
\hline $\mathrm{xH} 1$ & -7.4697931 & & & & -7.3362451 & & & \\
\hline HH1 & -14.9405102 & -0.570 & -0.579 & 0.010 & -14.6737975 & -0.809 & -0.820 & 0.011 \\
\hline $\mathrm{Hx} 2$ & -7.469805 & & & & -7.3362563 & & & \\
\hline $\mathrm{xH} 2$ & -7.4698048 & & & & -7.336256 & & & \\
\hline $\mathrm{HH} 2$ & -14.9407083 & -0.694 & -0.689 & -0.005 & -14.6738098 & -0.817 & -0.814 & -0.003 \\
\hline $\mathrm{Hx} 1$ & -7.4697961 & & & & -7.3362487 & & & \\
\hline $\mathrm{xF} 1$ & -35.0833841 & & & & -32.6630363 & & & \\
\hline HF1 & -42.5546094 & -0.918 & -0.897 & -0.021 & -40.000975 & -1.085 & -1.060 & -0.024 \\
\hline $\mathrm{Hx} 2$ & -7.4698035 & & & & -7.3362565 & & & \\
\hline $\mathrm{xF} 2$ & -35.0833714 & & & & -32.6630238 & & & \\
\hline $\mathrm{HF} 2$ & -42.5547999 & -1.037 & -1.020 & -0.018 & -40.0014073 & -1.356 & -1.335 & -0.021 \\
\hline Hx3 & -7.4698018 & & & & -7.3362549 & & & \\
\hline $\mathrm{xF} 3$ & -35.0834075 & & & & -32.6630687 & & & \\
\hline HF3 & -42.5544514 & -0.819 & -0.779 & -0.039 & -40.0007921 & -0.970 & -0.921 & -0.049 \\
\hline $\mathrm{Hx} 4$ & -7.4697963 & & & & -7.3362503 & & & \\
\hline $\mathrm{xF} 4$ & -35.0833905 & & & & -32.663046 & & & \\
\hline $\mathrm{HF} 4$ & -42.5547811 & -1.025 & -1.000 & -0.025 & -40.00123 & -1.245 & -1.213 & -0.031 \\
\hline $\mathrm{Fx} 1$ & -35.0833412 & & & & -32.6629955 & & & \\
\hline $\mathrm{xF} 1$ & -35.0833382 & & & & -32.6629733 & & & \\
\hline FF1 & -70.1680971 & -0.882 & -0.890 & 0.008 & -65.3283223 & -1.467 & -1.477 & 0.010 \\
\hline $\mathrm{Fx} 2$ & -35.0833867 & & & & -32.6630406 & & & \\
\hline $\mathrm{xF} 2$ & -35.0833903 & & & & -32.6630414 & & & \\
\hline FF2 & -70.1678576 & -0.732 & -0.678 & -0.053 & -65.327514 & -0.959 & -0.899 & -0.061 \\
\hline
\end{tabular}




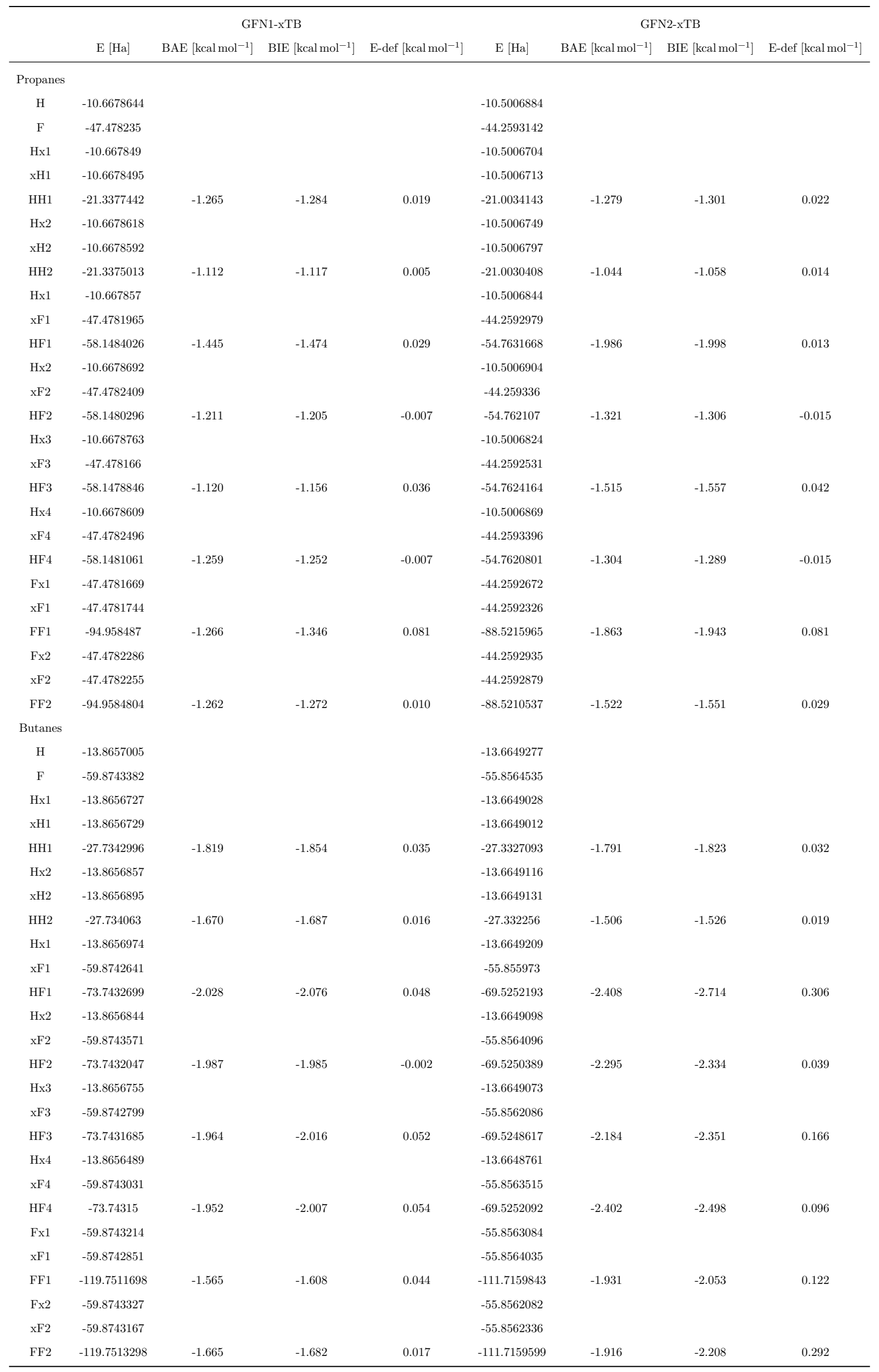




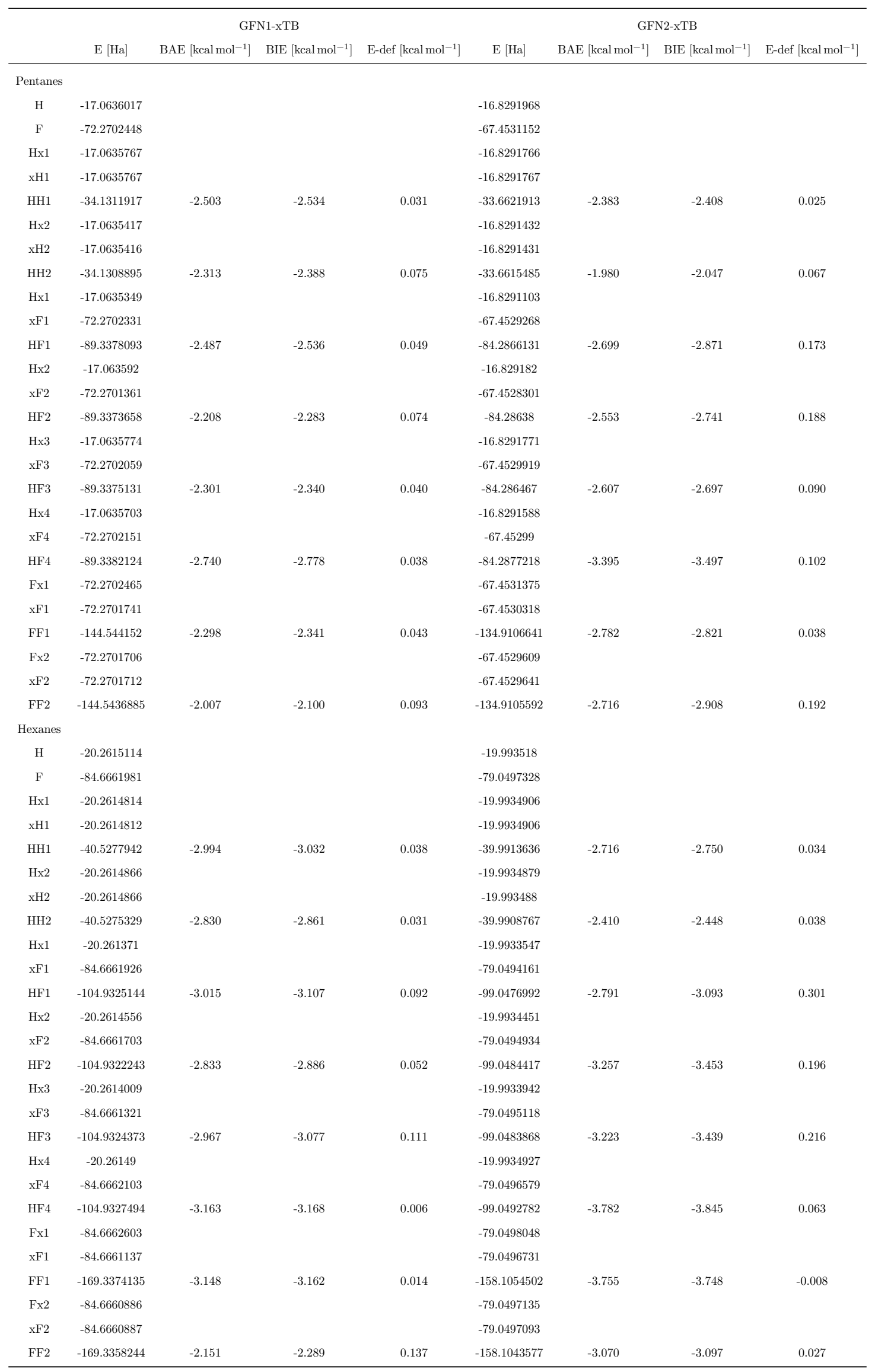




\begin{tabular}{|c|c|c|c|c|c|c|c|c|}
\hline & \multicolumn{4}{|c|}{$\operatorname{CCSD}(\mathrm{T}) / \operatorname{CBS}(34)$} & \multicolumn{4}{|c|}{ CCSD(T)-F12/cc-pVTZ-F12 } \\
\hline & $\mathrm{E}[\mathrm{Ha}]$ & $\mathrm{BAE}\left[\mathrm{kcal} \mathrm{mol}^{-1}\right]$ & $\mathrm{BIE}\left[\mathrm{kcal} \mathrm{mol}^{-1}\right]$ & E-def $\left[\mathrm{kcal} \mathrm{mol}^{-1}\right]$ & $\mathrm{E}[\mathrm{Ha}]$ & $\mathrm{BAE}\left[\mathrm{kcal} \mathrm{mol}^{-1}\right]$ & $\mathrm{BIE}\left[\mathrm{kcal} \mathrm{mol}^{-1}\right]$ & E-def $\left[\mathrm{kcal} \mathrm{mol}^{-1}\right]$ \\
\hline \multicolumn{9}{|c|}{ Methanes } \\
\hline $\mathrm{H}$ & -40.458835527101 & & & & -40.45435366861 & & & \\
\hline $\mathrm{F}$ & -437.223954430823 & & & & -437.196105711662 & & & \\
\hline $\mathrm{Hx} 1$ & -40.458834706746 & & & & -40.454352711424 & & & \\
\hline $\mathrm{xH} 1$ & -40.458834717192 & & & & -40.454352729683 & & & \\
\hline HH1 & -80.918492058307 & -0.515 & -0.516 & 0.001 & -80.909526118263 & -0.514 & -0.515 & 0.001 \\
\hline $\mathrm{Hx} 2$ & -40.458835276684 & & & & -40.454353408584 & & & \\
\hline $\mathrm{xH} 2$ & -40.458835303851 & & & & -40.454353444011 & & & \\
\hline $\mathrm{HH} 2$ & -80.918319964781 & -0.407 & -0.407 & 0.000 & -80.909349843905 & -0.403 & -0.403 & 0.000 \\
\hline Hx1 & -40.458835268385 & & & & -40.454353365614 & & & \\
\hline $\mathrm{xF} 1$ & -437.223941360422 & & & & -437.196096332932 & & & \\
\hline $\mathrm{HF} 1$ & -477.684050987955 & -0.791 & -0.800 & 0.008 & -477.651705876698 & -0.782 & -0.788 & 0.006 \\
\hline $\mathrm{Hx} 2$ & -40.458834628794 & & & & -40.454352680522 & & & \\
\hline $\mathrm{xF} 2$ & -437.223944619657 & & & & -437.196098546992 & & & \\
\hline HF2 & -477.683463240106 & -0.422 & -0.429 & 0.007 & -477.65115094201 & -0.434 & -0.439 & 0.005 \\
\hline Fx1 & -437.223941775041 & & & & -437.196095349424 & & & \\
\hline $\mathrm{xF} 1$ & -437.223944397226 & & & & -437.196098227114 & & & \\
\hline $\mathrm{FF} 1$ & -874.449194822515 & -0.807 & -0.821 & 0.014 & -874.39350561 & -0.812 & -0.823 & 0.011 \\
\hline $\mathrm{Fx} 2$ & -437.22394399808 & & & & -437.196098160978 & & & \\
\hline $\mathrm{xF} 2$ & -437.22394355781 & & & & -437.196097317972 & & & \\
\hline $\mathrm{FF} 2$ & -874.449076217401 & -0.733 & -0.746 & 0.013 & -874.39354404 & -0.836 & -0.846 & 0.010 \\
\hline \multicolumn{9}{|c|}{ Ethanes } \\
\hline $\mathrm{H}$ & -79.7138450153 & & & & -79.70583503191 & & & \\
\hline $\mathrm{F}$ & -674.847888197 & & & & -674.80435257993 & & & \\
\hline Hx1 & -79.7138452637 & & & & -79.705835449029 & & & \\
\hline $\mathrm{xH} 1$ & -79.7138454003 & & & & -79.705835579999 & & & \\
\hline HH1 & -159.429906212 & -1.391 & -1.390 & 0.000 & -159.413841250544 & -1.362 & -1.362 & -0.001 \\
\hline $\mathrm{Hx} 2$ & -79.7138461745 & & & & -79.705836312437 & & & \\
\hline $\mathrm{xH} 2$ & -79.7138461967 & & & & -79.705836348597 & & & \\
\hline HH2 & -159.429649819 & -1.230 & -1.228 & -0.001 & -159.413578137522 & -1.197 & -1.196 & -0.002 \\
\hline Hx1 & -79.7138414857 & & & & -79.705831263297 & & & \\
\hline $\mathrm{xF} 1$ & -674.847870399 & & & & -674.804342241885 & & & \\
\hline $\mathrm{HF} 1$ & -754.563396384 & -1.044 & -1.057 & 0.013 & -754.511897859996 & -1.073 & -1.082 & 0.009 \\
\hline $\mathrm{Hx} 2$ & -79.7138447957 & & & & -79.705834711312 & & & \\
\hline $\mathrm{xF} 2$ & -674.847863242 & & & & -674.804337089012 & & & \\
\hline $\mathrm{HF} 2$ & -754.56365468 & -1.206 & -1.222 & 0.016 & -754.51217783368 & -1.249 & -1.259 & 0.010 \\
\hline Hx3 & -79.7138447729 & & & & -79.705834703832 & & & \\
\hline $\mathrm{xF} 3$ & -674.847874756 & & & & -674.804346513413 & & & \\
\hline HF3 & -754.563187335 & -0.912 & -0.921 & 0.009 & -754.511681101996 & -0.937 & -0.941 & 0.004 \\
\hline $\mathrm{Hx} 4$ & -79.7138426297 & & & & -79.705832418504 & & & \\
\hline $\mathrm{xF} 4$ & -674.847881523 & & & & -674.804350855375 & & & \\
\hline $\mathrm{HF} 4$ & -754.5635855 & -1.162 & -1.168 & 0.006 & -754.512089033869 & -1.193 & -1.196 & 0.003 \\
\hline Fx1 & -674.847870779 & & & & -674.804338967135 & & & \\
\hline $\mathrm{xF} 1$ & -674.847842902 & & & & -674.804311243574 & & & \\
\hline $\mathrm{FF} 1$ & -1349.69774921 & -1.238 & -1.277 & 0.039 & -1349.61078209367 & -1.303 & -1.338 & 0.034 \\
\hline $\mathrm{Fx} 2$ & -674.847862818 & & & & -674.804336120495 & & & \\
\hline $\mathrm{xF} 2$ & -674.847878159 & & & & -674.804346634521 & & & \\
\hline FF2 & -1349.69740997 & -1.025 & -1.047 & 0.022 & -1349.6103776125 & -1.049 & -1.064 & 0.014 \\
\hline
\end{tabular}




\begin{tabular}{|c|c|c|c|c|c|c|c|c|}
\hline & \multicolumn{4}{|c|}{$\operatorname{CCSD}(\mathrm{T}) / \mathrm{CBS}(\mathrm{LPNO}-\mathrm{CEPA} / 1)$} & \multicolumn{4}{|c|}{$\operatorname{CCSD}(\mathrm{T}) / \mathrm{CBS}(\mathrm{MP} 2)$} \\
\hline & $\mathrm{E}[\mathrm{Ha}]$ & $\mathrm{BAE}\left[\mathrm{kcal} \mathrm{mol}^{-1}\right]$ & $\mathrm{BIE}\left[\mathrm{kcal} \mathrm{mol}^{-1}\right]$ & E-def $\left[\mathrm{kcal} \mathrm{mol}^{-1}\right]$ & $\mathrm{E}[\mathrm{Ha}]$ & $\mathrm{BAE}\left[\mathrm{kcal} \mathrm{mol}^{-1}\right]$ & $\mathrm{BIE}\left[\mathrm{kcal} \mathrm{mol}^{-1}\right]$ & E-def $\left[\mathrm{kcal} \mathrm{mol}^{-1}\right]$ \\
\hline \multicolumn{9}{|c|}{ Methanes } \\
\hline $\mathrm{H}$ & -40.4564853618 & & & & -40.4632579788 & & & \\
\hline $\mathrm{F}$ & -437.207904623 & & & & -437.225925685 & & & \\
\hline $\mathrm{Hx} 1$ & -40.4564845778 & & & & -40.4632569638 & & & \\
\hline $\mathrm{xH} 1$ & -40.4564845839 & & & & -40.4632569849 & & & \\
\hline HH1 & -80.913723895 & -0.473 & -0.474 & 0.001 & -80.927347218 & -0.522 & -0.523 & 0.001 \\
\hline $\mathrm{Hx} 2$ & -40.4564851119 & & & & -40.4632577139 & & & \\
\hline $\mathrm{xH} 2$ & -40.4564851404 & & & & -40.4632577534 & & & \\
\hline HH2 & -80.9135240798 & -0.347 & -0.348 & 0.000 & -80.9271742318 & -0.413 & -0.413 & 0.000 \\
\hline $\mathrm{Hx} 1$ & -40.4564851171 & & & & -40.4632576571 & & & \\
\hline $\mathrm{xF} 1$ & -437.207886651 & & & & -437.225917531 & & & \\
\hline HF1 & -477.665767595 & -0.864 & -0.876 & 0.011 & -477.690700627 & -0.952 & -0.957 & 0.005 \\
\hline $\mathrm{Hx} 2$ & -40.4564844873 & & & & -40.4632569533 & & & \\
\hline $\mathrm{xF} 2$ & -437.207892259 & & & & -437.225919405 & & & \\
\hline HF2 & -477.665121095 & -0.459 & -0.467 & 0.008 & -477.690079237 & -0.562 & -0.567 & 0.005 \\
\hline Fx1 & -437.207888553 & & & & -437.2259161 & & & \\
\hline $\mathrm{xF} 1$ & -437.207891506 & & & & -437.225919049 & & & \\
\hline $\mathrm{FF} 1$ & -874.41713011 & -0.829 & -0.847 & 0.018 & -874.453433305 & -0.993 & -1.003 & 0.010 \\
\hline $\mathrm{Fx} 2$ & -437.207890753 & & & & -437.225919088 & & & \\
\hline $\mathrm{xF} 2$ & -437.207888541 & & & & -437.225918125 & & & \\
\hline $\mathrm{FF} 2$ & -874.417154865 & -0.844 & -0.863 & 0.019 & -874.453417584 & -0.983 & -0.992 & 0.009 \\
\hline \multicolumn{9}{|c|}{ Ethanes } \\
\hline $\mathrm{H}$ & -79.7091919342 & & & & -79.7212675812 & & & \\
\hline $\mathrm{F}$ & -674.822345166 & & & & -674.852088075 & & & \\
\hline $\mathrm{Hx} 1$ & -79.7091881043 & & & & -79.7212678453 & & & \\
\hline $\mathrm{xH} 1$ & -79.7091902863 & & & & -79.7212680513 & & & \\
\hline HH1 & -159.420507656 & -1.333 & -1.336 & 0.003 & -159.444715599 & -1.368 & -1.368 & 0.000 \\
\hline $\mathrm{Hx} 2$ & -79.7091843925 & & & & -79.7212685565 & & & \\
\hline $\mathrm{xH} 2$ & -79.7091840582 & & & & -79.7212685932 & & & \\
\hline HH2 & -159.420317214 & -1.213 & -1.223 & 0.010 & -159.444450167 & -1.202 & -1.200 & -0.001 \\
\hline $\mathrm{Hx} 1$ & -79.7091861193 & & & & -79.7212637383 & & & \\
\hline $\mathrm{xF} 1$ & -674.82231885 & & & & -674.852079549 & & & \\
\hline HF1 & -754.533408886 & -1.175 & -1.195 & 0.020 & -754.575383674 & -1.273 & -1.280 & 0.008 \\
\hline $\mathrm{Hx} 2$ & -79.7091893595 & & & & -79.7212670805 & & & \\
\hline $\mathrm{xF} 2$ & -674.822316344 & & & & -674.85207526 & & & \\
\hline HF2 & -754.533737159 & -1.381 & -1.400 & 0.020 & -754.575703261 & -1.473 & -1.481 & 0.008 \\
\hline $\mathrm{Hx} 3$ & -79.7091916675 & & & & -79.7212670955 & & & \\
\hline xF3 & -674.822327984 & & & & -674.852082795 & & & \\
\hline HF3 & -754.533186584 & -1.035 & -1.046 & 0.011 & -754.575109569 & -1.101 & -1.104 & 0.004 \\
\hline $\mathrm{Hx} 4$ & -79.7091914325 & & & & -79.7212649525 & & & \\
\hline $\mathrm{xF} 4$ & -674.822329425 & & & & -674.852086932 & & & \\
\hline $\mathrm{HF} 4$ & -754.533616941 & -1.305 & -1.315 & 0.010 & -754.575592487 & -1.404 & -1.406 & 0.002 \\
\hline $\mathrm{Fx} 1$ & -674.822316889 & & & & -674.852075278 & & & \\
\hline $\mathrm{xF} 1$ & -674.822509246 & & & & -674.852049182 & & & \\
\hline $\mathrm{FF} 1$ & -1349.64711979 & -1.525 & -1.439 & -0.085 & -1349.70662753 & -1.538 & -1.571 & 0.032 \\
\hline $\mathrm{Fx} 2$ & -674.822308924 & & & & -674.852073779 & & & \\
\hline $\mathrm{xF} 2$ & -674.822327979 & & & & -674.852082752 & & & \\
\hline FF2 & -1349.64646961 & -1.117 & -1.150 & 0.034 & -1349.70611771 & -1.218 & -1.231 & 0.012 \\
\hline
\end{tabular}




\begin{tabular}{|c|c|c|c|c|c|c|c|c|}
\hline & \multicolumn{4}{|c|}{$\operatorname{CCSD}(\mathrm{T}) / \mathrm{CBS}(\mathrm{LPNO}-\mathrm{CEPA} / 1)$} & \multicolumn{4}{|c|}{$\operatorname{CCSD}(\mathrm{T}) / \mathrm{CBS}(\mathrm{MP} 2)$} \\
\hline & $\mathrm{E}[\mathrm{Ha}]$ & BAE $\left[\mathrm{kcal} \mathrm{mol}^{-1}\right]$ & BIE $\left[\mathrm{kcal} \mathrm{mol}^{-1}\right]$ & E-def $\left[\mathrm{kcal} \mathrm{mol}^{-1}\right]$ & $\mathrm{E}[\mathrm{Ha}]$ & $\mathrm{BAE}\left[\mathrm{kcal} \mathrm{mol}^{-1}\right]$ & BIE $\left[\mathrm{kcal} \mathrm{mol}^{-1}\right]$ & E-def $\left[\mathrm{kcal} \mathrm{mol}^{-1}\right]$ \\
\hline \multicolumn{9}{|c|}{ Propanes } \\
\hline $\mathrm{H}$ & -118.965353755 & & & & -118.982669996 & & & \\
\hline $\mathrm{F}$ & -912.437655968 & & & & -912.478703185 & & & \\
\hline $\mathrm{Hx} 1$ & -118.965348083 & & & & -118.982665214 & & & \\
\hline $\mathrm{xH} 1$ & -118.965349908 & & & & -118.982665615 & & & \\
\hline нH1 & -237.933847853 & -1.971 & -1.977 & 0.006 & -237.968529133 & -2.001 & -2.007 & 0.006 \\
\hline $\mathrm{Hx} 2$ & -118.965348909 & & & & -118.982666863 & & & \\
\hline $\mathrm{xH} 2$ & -118.965349885 & & & & -118.982665607 & & & \\
\hline HH2 & -237.93270847 & -1.256 & -1.261 & 0.005 & -237.967300652 & -1.230 & -1.235 & 0.005 \\
\hline $\mathrm{Hx1}$ & -118.965348859 & & & & -118.982664774 & & & \\
\hline $\mathrm{xF} 1$ & -912.437461087 & & & & -912.478628787 & & & \\
\hline $\mathrm{HF} 1$ & -1031.40590608 & -1.817 & -1.943 & 0.125 & -1031.46454325 & -1.989 & -2.039 & 0.050 \\
\hline $\mathrm{Hx} 2$ & -118.9653506 & & & & -118.982667866 & & & \\
\hline $\mathrm{xF} 2$ & -912.437577231 & & & & -912.478667995 & & & \\
\hline HF2 & -1031.40538374 & -1.490 & -1.541 & 0.051 & -1031.46396683 & -1.628 & -1.651 & 0.023 \\
\hline Hx3 & -118.965351507 & & & & -118.982672817 & & & \\
\hline $\mathrm{xF} 3$ & -912.437516045 & & & & -912.478658971 & & & \\
\hline HF3 & -1031.40527507 & -1.422 & -1.511 & 0.089 & -1031.46392093 & -1.599 & -1.625 & 0.026 \\
\hline $\mathrm{Hx} 4$ & -118.965347544 & & & & -118.982664418 & & & \\
\hline $\mathrm{xF} 4$ & -912.437622762 & & & & -912.478657386 & & & \\
\hline $\mathrm{HF} 4$ & -1031.40545173 & -1.532 & -1.557 & 0.025 & -1031.46400574 & -1.652 & -1.684 & 0.032 \\
\hline Fx1 & -912.43739287 & & & & -912.478550971 & & & \\
\hline $\mathrm{xF} 1$ & -912.437577494 & & & & -912.478663612 & & & \\
\hline $\mathrm{FF} 1$ & -1824.87823528 & -1.834 & -2.049 & 0.214 & -1824.96079523 & -2.127 & -2.247 & 0.120 \\
\hline $\mathrm{Fx} 2$ & -912.437637249 & & & & -912.478699771 & & & \\
\hline $\mathrm{xF} 2$ & -912.43763246 & & & & -912.478702192 & & & \\
\hline $\mathrm{FF} 2$ & -1824.8789201 & -2.264 & -2.291 & 0.026 & -1824.96135146 & -2.476 & -2.478 & 0.003 \\
\hline \multicolumn{9}{|l|}{ Butanes } \\
\hline H & -158.221640465 & & & & -158.24418516 & & & \\
\hline F & -1150.05230427 & & & & -1150.1049852 & & & \\
\hline $\mathrm{Hx} 1$ & -158.221639528 & & & & -158.244180372 & & & \\
\hline $\mathrm{xH} 1$ & -158.22163919 & & & & -158.244179874 & & & \\
\hline нH1 & -316.447849443 & -2.867 & -2.868 & 0.001 & -316.492931507 & -2.862 & -2.869 & 0.006 \\
\hline $\mathrm{H} \times 2$ & -158.22163578 & & & & -158.244177995 & & & \\
\hline $\mathrm{xH} 2$ & -158.221639435 & & & & -158.244184027 & & & \\
\hline HH2 & -316.446048691 & -1.737 & -1.740 & 0.004 & -316.491078586 & -1.699 & -1.705 & 0.005 \\
\hline Hx1 & -158.221639274 & & & & -158.244182832 & & & \\
\hline $\mathrm{xF} 1$ & -1150.05190277 & & & & -1150.10461504 & & & \\
\hline $\mathrm{HF} 1$ & -1308.27754688 & -2.260 & -2.513 & 0.253 & -1308.35302406 & -2.418 & -2.652 & 0.234 \\
\hline $\mathrm{Hx2}$ & -158.221627277 & & & & -158.244169385 & & & \\
\hline $\mathrm{xF} 2$ & -1150.05225178 & & & & -1150.10496833 & & & \\
\hline HF2 & -1308.27786347 & -2.459 & -2.500 & 0.041 & -1308.35336097 & -2.630 & -2.650 & 0.020 \\
\hline $\mathrm{Hx3}$ & -158.22161186 & & & & -158.244154117 & & & \\
\hline $\mathrm{xF} 3$ & -1150.05235518 & & & & -1150.10490613 & & & \\
\hline HF3 & -1308.27792249 & -2.496 & -2.482 & -0.014 & -1308.35327611 & -2.576 & -2.645 & 0.069 \\
\hline $\mathrm{Hx} 4$ & -158.221594367 & & & & -158.24413582 & & & \\
\hline $\mathrm{xF} 4$ & -1150.05227058 & & & & -1150.10498577 & & & \\
\hline $\mathrm{HF} 4$ & -1308.27772791 & -2.374 & -2.424 & 0.050 & -1308.35323683 & -2.552 & -2.582 & 0.031 \\
\hline \multicolumn{9}{|l|}{ Fx1 } \\
\hline \multicolumn{9}{|l|}{$\mathrm{xF} 1$} \\
\hline \multicolumn{9}{|l|}{$\mathrm{FF} 1$} \\
\hline \multicolumn{9}{|l|}{$\mathrm{Fx} 2$} \\
\hline \multicolumn{9}{|l|}{$\mathrm{xF} 2$} \\
\hline $\mathrm{FF} 2$ & & & & & & & & \\
\hline
\end{tabular}




\begin{tabular}{|c|c|c|c|c|c|c|c|c|}
\hline & \multicolumn{4}{|c|}{ CCSD(T)-F12/cc-pVDZ-F12 } & \multicolumn{4}{|c|}{ ExtremePNO-DLPNO-CCSD(T)/CBS(34) } \\
\hline & $\mathrm{E}[\mathrm{Ha}]$ & $\mathrm{BAE}\left[\mathrm{kcal} \mathrm{mol}^{-1}\right]$ & $\mathrm{BIE}\left[\mathrm{kcal} \mathrm{mol}{ }^{-1}\right]$ & E-def $\left[\mathrm{kcal} \mathrm{mol}^{-1}\right]$ & $\mathrm{E}[\mathrm{Ha}]$ & $\mathrm{BAE}\left[\mathrm{kcal} \mathrm{mol}^{-1}\right]$ & $\mathrm{BIE}\left[\mathrm{kcal} \mathrm{mol}^{-1}\right]$ & E-def $\left[\mathrm{kcal} \mathrm{mol}^{-1}\right]$ \\
\hline \multicolumn{9}{|c|}{ Methanes } \\
\hline $\mathrm{H}$ & -40.447999976925 & & & & -40.45883236544 & & & \\
\hline $\mathrm{F}$ & -437.122744137689 & & & & -437.222431231796 & & & \\
\hline $\mathrm{Hx} 1$ & -40.447999035723 & & & & -40.458831557834 & & & \\
\hline $\mathrm{xH} 1$ & -40.447999052373 & & & & -40.458831565443 & & & \\
\hline HH1 & -80.896787083326 & -0.494 & -0.495 & 0.001 & -80.918472574501 & -0.507 & -0.508 & 0.001 \\
\hline $\mathrm{Hx} 2$ & -40.447999717363 & & & & -40.458832115762 & & & \\
\hline $\mathrm{xH} 2$ & -40.447999751484 & & & & -40.458832142124 & & & \\
\hline $\mathrm{HH} 2$ & -80.8966197003 & -0.389 & -0.389 & 0.000 & -80.91829878399 & -0.398 & -0.398 & 0.000 \\
\hline $\mathrm{Hx} 1$ & -40.447999679402 & & & & -40.458832110835 & & & \\
\hline $\mathrm{xF} 1$ & -437.122738504592 & & & & -437.22241605469 & & & \\
\hline HF1 & -477.571962046076 & -0.764 & -0.768 & 0.004 & -477.682488234706 & -0.768 & -0.778 & 0.010 \\
\hline $\mathrm{Hx} 2$ & -40.447998998258 & & & & -40.458831475222 & & & \\
\hline $\mathrm{xF} 2$ & -437.122739653581 & & & & -437.222420508373 & & & \\
\hline HF2 & -477.571417478363 & -0.423 & -0.426 & 0.003 & -477.681915605826 & -0.409 & -0.416 & 0.007 \\
\hline Fx1 & -437.122736072511 & & & & -437.222420849004 & & & \\
\hline $\mathrm{xF} 1$ & -437.12273923139 & & & & -437.222420265875 & & & \\
\hline $\mathrm{FF} 1$ & -874.246755931905 & -0.795 & -0.804 & 0.008 & -874.446072896779 & -0.760 & -0.773 & 0.013 \\
\hline $\mathrm{Fx} 2$ & -437.122739514482 & & & & -437.222420272937 & & & \\
\hline $\mathrm{xF} 2$ & -437.122738258817 & & & & -437.222419671603 & & & \\
\hline $\mathrm{FF} 2$ & -874.246821616374 & -0.837 & -0.843 & 0.007 & -874.445976788717 & -0.699 & -0.713 & 0.014 \\
\hline \multicolumn{9}{|c|}{ Ethanes } \\
\hline $\mathrm{H}$ & -79.693734466298 & & & & -79.713724795789 & & & \\
\hline $\mathrm{F}$ & -674.691619616535 & & & & -674.845120739521 & & & \\
\hline $\mathrm{Hx} 1$ & -79.693734710715 & & & & -79.713725039495 & & & \\
\hline $\mathrm{xH} 1$ & -79.693734890698 & & & & -79.713725166933 & & & \\
\hline HH1 & -159.389578828691 & -1.324 & -1.324 & 0.000 & -159.429612933537 & -1.358 & -1.357 & 0.000 \\
\hline $\mathrm{Hx} 2$ & -79.693735549941 & & & & -79.713725933073 & & & \\
\hline $\mathrm{xH} 2$ & -79.693735582632 & & & & -79.71372595889 & & & \\
\hline HH2 & -159.389340370727 & -1.174 & -1.173 & -0.001 & -159.429369053514 & -1.204 & -1.203 & -0.001 \\
\hline $\mathrm{Hx} 1$ & -79.693730594262 & & & & -79.713721272974 & & & \\
\hline $\mathrm{xF} 1$ & -674.691616880024 & & & & -674.845096137316 & & & \\
\hline HF1 & -754.387028471939 & -1.051 & -1.055 & 0.004 & -754.560453063559 & -1.009 & -1.026 & 0.018 \\
\hline $\mathrm{Hx} 2$ & -79.693734076935 & & & & -79.713724566739 & & & \\
\hline $\mathrm{xF} 2$ & -674.691613869448 & & & & -674.845091893913 & & & \\
\hline $\mathrm{HF} 2$ & -754.387305120539 & -1.224 & -1.228 & 0.004 & -754.560690601218 & -1.158 & -1.176 & 0.018 \\
\hline $\mathrm{Hx} 3$ & -79.693734078752 & & & & -79.713724557059 & & & \\
\hline $\mathrm{xF} 3$ & -674.691620113649 & & & & -674.845102202878 & & & \\
\hline HF3 & -754.38682317727 & -0.922 & -0.922 & 0.000 & -754.56023653073 & -0.873 & -0.885 & 0.012 \\
\hline $\mathrm{Hx} 4$ & -79.693731851176 & & & & -79.713722423374 & & & \\
\hline $\mathrm{xF} 4$ & -674.691622374721 & & & & -674.845111211258 & & & \\
\hline $\mathrm{HF} 4$ & -754.387213663146 & -1.167 & -1.167 & 0.000 & -754.560622627775 & -1.115 & -1.123 & 0.007 \\
\hline $\mathrm{Fx} 1$ & -674.691609506162 & & & & -674.84509839362 & & & \\
\hline $\mathrm{xF} 1$ & -674.691584467383 & & & & -674.845070795438 & & & \\
\hline $\mathrm{FF} 1$ & -1349.38531064049 & -1.300 & -1.328 & 0.028 & -1349.69207685299 & -1.152 & -1.197 & 0.045 \\
\hline $\mathrm{Fx} 2$ & -674.691612139525 & & & & -674.845090367952 & & & \\
\hline $\mathrm{xF} 2$ & -674.691617643655 & & & & -674.845107306051 & & & \\
\hline $\mathrm{FF} 2$ & -1349.38490896877 & -1.048 & -1.054 & 0.006 & -1349.69178287617 & -0.967 & -0.995 & 0.027 \\
\hline
\end{tabular}




\begin{tabular}{|c|c|c|c|c|c|c|c|c|}
\hline & \multicolumn{4}{|c|}{$\operatorname{CCSD}(\mathrm{T})$-F12/cc-pVDZ-F12 } & \multicolumn{4}{|c|}{ ExtremePNO-DLPNO-CCSD(T)/CBS(34) } \\
\hline & $\mathrm{E}[\mathrm{Ha}]$ & $\mathrm{BAE}\left[\mathrm{kcal} \mathrm{mol}^{-1}\right]$ & $\mathrm{BIE}\left[\mathrm{kcal} \mathrm{mol}^{-1}\right]$ & E-def $\left[\mathrm{kcal} \mathrm{mol}^{-1}\right]$ & $\mathrm{E}[\mathrm{Ha}]$ & $\mathrm{BAE}\left[\mathrm{kcal} \mathrm{mol}^{-1}\right]$ & BIE $\left[\mathrm{kcal} \mathrm{mol}^{-1}\right]$ & E-def $\left[\mathrm{kcal} \mathrm{mol}^{-1}\right]$ \\
\hline \multicolumn{9}{|c|}{ Propanes } \\
\hline H & -118.942844355675 & & & & -118.972067786382 & & & \\
\hline $\mathrm{F}$ & -912.260942870335 & & & & -912.468011042762 & & & \\
\hline Hx1 & -118.942839980005 & & & & -118.972064160139 & & & \\
\hline $\mathrm{xH} 1$ & -118.942840442653 & & & & -118.972064477887 & & & \\
\hline HН1 & -237.888877693659 & -2.001 & -2.006 & 0.005 & -237.947319736954 & -1.998 & -2.002 & 0.004 \\
\hline $\mathrm{Hx} 2$ & -118.942841158761 & & & & -118.972063930754 & & & \\
\hline $\mathrm{xH} 2$ & -118.942840842427 & & & & -118.972065101736 & & & \\
\hline HH2 & -237.887635306472 & -1.222 & -1.226 & 0.004 & -237.946073662057 & -1.216 & -1.220 & 0.004 \\
\hline $\mathrm{Hx} 1$ & -118.942839183879 & & & & -118.972063607711 & & & \\
\hline $\mathrm{xF} 1$ & -912.260867578635 & & & & -912.467944538586 & & & \\
\hline HF1 & -1031.20646186905 & -1.678 & -1.729 & 0.050 & -1031.44266454604 & -1.623 & -1.667 & 0.044 \\
\hline $\mathrm{Hx} 2$ & -118.94284228285 & & & & -118.972065686724 & & & \\
\hline $\mathrm{xF} 2$ & -912.260913751534 & & & & -912.467957771246 & & & \\
\hline $\mathrm{HF} 2$ & -1031.20602157557 & -1.402 & -1.422 & 0.020 & -1031.44216043208 & -1.306 & -1.341 & 0.035 \\
\hline $\mathrm{Hx} 3$ & -118.942846184931 & & & & -118.972067499376 & & & \\
\hline $\mathrm{xF} 3$ & -912.260901002422 & & & & -912.467959335761 & & & \\
\hline HF3 & -1031.20597874094 & -1.375 & -1.400 & 0.025 & -1031.44222455346 & -1.346 & -1.379 & 0.033 \\
\hline $\mathrm{Hx} 4$ & -118.942839145392 & & & & -118.972063186267 & & & \\
\hline $\mathrm{xF} 4$ & -912.260903629426 & & & & -912.467944476894 & & & \\
\hline $\mathrm{HF} 4$ & -1031.2060135717 & -1.397 & -1.425 & 0.028 & -1031.44222367377 & -1.346 & -1.391 & 0.045 \\
\hline Fx1 & -912.260782171931 & & & & -912.467890738825 & & & \\
\hline $\mathrm{xF} 1$ & -912.260901047332 & & & & -912.46797888394 & & & \\
\hline FF1 & -1824.52474494826 & -1.794 & -1.921 & 0.127 & -1824.93856280435 & -1.594 & -1.690 & 0.096 \\
\hline $\mathrm{Fx} 2$ & -912.260939151683 & & & & -912.468006315844 & & & \\
\hline $\mathrm{xF} 2$ & -912.260941184676 & & & & -912.468010463395 & & & \\
\hline FF2 & -1824.5252075978 & -2.084 & -2.088 & 0.003 & -1824.93906806307 & -1.911 & -1.915 & 0.003 \\
\hline \multicolumn{9}{|c|}{ Butanes } \\
\hline $\mathrm{H}$ & -158.192066667656 & & & & -158.230530166731 & & & \\
\hline $\mathrm{F}$ & -1149.82992018262 & & & & -1150.09061289021 & & & \\
\hline Hx1 & -158.192061823679 & & & & -158.2305277631 & & & \\
\hline $\mathrm{xH} 1$ & -158.192061035021 & & & & -158.230525723237 & & & \\
\hline Hн1 & -316.388735463913 & -2.888 & -2.894 & 0.007 & -316.465617221183 & -2.859 & -2.864 & 0.004 \\
\hline $\mathrm{Hx} 2$ & -158.192059116789 & & & & -158.230524542646 & & & \\
\hline $\mathrm{xH} 2$ & -158.192064851329 & & & & -158.230527376907 & & & \\
\hline HH2 & -316.386870245669 & -1.717 & -1.723 & 0.006 & -316.463744573946 & -1.684 & -1.690 & 0.005 \\
\hline Hx1 & -158.192063771568 & & & & -158.230526351675 & & & \\
\hline $\mathrm{xF} 1$ & -1149.82961839877 & & & & -1150.09016731751 & & & \\
\hline $\mathrm{HF} 1$ & -1308.0253277113 & -2.096 & -2.288 & 0.191 & -1308.32425492421 & -1.953 & -2.235 & 0.282 \\
\hline $\mathrm{Hx} 2$ & -158.192050371468 & & & & -158.230515759292 & & & \\
\hline $\mathrm{xF} 2$ & -1149.82991506622 & & & & -1150.090587977 & & & \\
\hline $\mathrm{HF} 2$ & -1308.02561181709 & -2.275 & -2.288 & 0.013 & -1308.32465110068 & -2.201 & -2.226 & 0.025 \\
\hline $\mathrm{Hx} 3$ & -158.192036332102 & & & & -158.230503871159 & & & \\
\hline $\mathrm{xF} 3$ & -1149.82986329354 & & & & -1150.09052306273 & & & \\
\hline HF3 & -1308.02552777129 & -2.222 & -2.277 & 0.055 & -1308.3245961659 & -2.167 & -2.240 & 0.073 \\
\hline $\mathrm{Hx} 4$ & -158.192017187497 & & & & -158.230485613852 & & & \\
\hline $\mathrm{xF} 4$ & -1149.82992664017 & & & & -1150.09061091914 & & & \\
\hline $\mathrm{HF} 4$ & -1308.02550927225 & -2.210 & -2.237 & 0.027 & -1308.32465110068 & -2.201 & -2.231 & 0.029 \\
\hline Fx1 & & & & & -1150.09055716374 & & & \\
\hline $\mathrm{xF} 1$ & & & & & -1150.0905491159 & & & \\
\hline FF1 & & & & & -2300.1845317021 & -2.074 & -2.149 & 0.075 \\
\hline $\mathrm{Fx} 2$ & & & & & -1150.09045281713 & & & \\
\hline $\mathrm{xF} 2$ & & & & & -1150.09047895973 & & & \\
\hline FF2 & & & & & -2300.18475070843 & -2.212 & -2.396 & 0.184 \\
\hline
\end{tabular}




\begin{tabular}{|c|c|c|c|c|c|c|c|c|}
\hline & \multicolumn{4}{|c|}{ NormalPNO-DLPNO-CCSD(T)/CBS(34) } & \multicolumn{4}{|c|}{ TightPNO-DLPNO-CCSD(T)/CBS (34) } \\
\hline & $\mathrm{E}[\mathrm{Ha}]$ & $\mathrm{BAE}\left[\mathrm{kcal} \mathrm{mol}^{-1}\right]$ & $\mathrm{BIE}\left[\mathrm{kcal} \mathrm{mol}^{-1}\right]$ & E-def $\left[\mathrm{kcal} \mathrm{mol}^{-1}\right]$ & $\mathrm{E}[\mathrm{Ha}]$ & $\mathrm{BAE}\left[\mathrm{kcal} \mathrm{mol}^{-1}\right]$ & BIE $\left[\mathrm{kcal} \mathrm{mol}^{-1}\right]$ & E-def $\left[\mathrm{kcal} \mathrm{mol}^{-1}\right]$ \\
\hline \multicolumn{9}{|c|}{ Methanes } \\
\hline $\mathrm{H}$ & -40.458979789634 & & & & -40.458900385254 & & & \\
\hline $\mathrm{F}$ & -437.220645058674 & & & & -437.221777818776 & & & \\
\hline Hx1 & -40.458979070777 & & & & -40.458899578178 & & & \\
\hline $\mathrm{xH} 1$ & -40.458979077608 & & & & -40.458899585582 & & & \\
\hline HH1 & -80.91873445578 & -0.486 & -0.487 & 0.001 & -80.918578889665 & -0.488 & -0.489 & 0.001 \\
\hline $\mathrm{Hx} 2$ & -40.458979540573 & & & & -40.458900135613 & & & \\
\hline $\mathrm{xH} 2$ & -40.458979566395 & & & & -40.458900161934 & & & \\
\hline $\mathrm{HH} 2$ & -80.918553742962 & -0.373 & -0.373 & 0.000 & -80.91842154816 & -0.390 & -0.390 & 0.000 \\
\hline Hx1 & -40.45897956448 & & & & -40.458900130834 & & & \\
\hline $\mathrm{xF} 1$ & -437.2206309157 & & & & -437.221765937366 & & & \\
\hline HF1 & -477.680560993753 & -0.587 & -0.596 & 0.009 & -477.681817954508 & -0.715 & -0.723 & 0.008 \\
\hline $\mathrm{Hx} 2$ & -40.45897895856 & & & & -40.458899495379 & & & \\
\hline $\mathrm{xF} 2$ & -437.220634165649 & & & & -437.221769183984 & & & \\
\hline $\mathrm{HF} 2$ & -477.680109428997 & -0.304 & -0.311 & 0.007 & -477.681280973822 & -0.378 & -0.384 & 0.006 \\
\hline Fx1 & -437.220632521522 & & & & -437.221765711528 & & & \\
\hline $\mathrm{xF} 1$ & -437.220632066669 & & & & -437.22176977829 & & & \\
\hline FF1 & -874.44219105764 & -0.565 & -0.581 & 0.016 & -874.444720881471 & -0.731 & -0.744 & 0.013 \\
\hline $\mathrm{Fx} 2$ & -437.220630217326 & & & & -437.22176920529 & & & \\
\hline $\mathrm{xF} 2$ & -437.22063446222 & & & & -437.221768841067 & & & \\
\hline $\mathrm{FF} 2$ & -874.442052003138 & -0.478 & -0.494 & 0.016 & -874.44457747893 & -0.641 & -0.652 & 0.011 \\
\hline \multicolumn{9}{|c|}{ Ethanes } \\
\hline $\mathrm{H}$ & -79.713874124651 & & & & -79.713808937866 & & & \\
\hline $\mathrm{F}$ & -674.84225036989 & & & & -674.843921099744 & & & \\
\hline Hx1 & -79.713872984657 & & & & -79.713809212295 & & & \\
\hline $\mathrm{xH} 1$ & -79.713874471439 & & & & -79.713809324241 & & & \\
\hline HH1 & -159.429854718811 & -1.322 & -1.322 & 0.000 & -159.429733420158 & -1.328 & -1.327 & 0.000 \\
\hline $\mathrm{Hx} 2$ & -79.713875126026 & & & & -79.713810055344 & & & \\
\hline $\mathrm{xH} 2$ & -79.713875151483 & & & & -79.713810081251 & & & \\
\hline HH2 & -159.429616023382 & -1.172 & -1.171 & -0.001 & -159.429493298095 & -1.177 & -1.175 & -0.001 \\
\hline Hx1 & -79.713872503343 & & & & -79.713807074755 & & & \\
\hline $\mathrm{xF} 1$ & -674.842225642529 & & & & -674.84390260749 & & & \\
\hline HF1 & -754.557504425239 & -0.866 & -0.882 & 0.017 & -754.559243623915 & -0.950 & -0.963 & 0.013 \\
\hline $\mathrm{Hx} 2$ & -79.713874433134 & & & & -79.713808702769 & & & \\
\hline $\mathrm{xF} 2$ & -674.842226803085 & & & & -674.843906279609 & & & \\
\hline $\mathrm{HF} 2$ & -754.557705400251 & -0.992 & -1.007 & 0.015 & -754.559482525291 & -1.100 & -1.109 & 0.009 \\
\hline $\mathrm{Hx} 3$ & -79.71387385788 & & & & -79.713808693182 & & & \\
\hline $\mathrm{xF} 3$ & -674.842228598466 & & & & -674.843907832661 & & & \\
\hline HF3 & -754.557239608612 & -0.700 & -0.714 & 0.014 & -754.559079021046 & -0.846 & -0.855 & 0.008 \\
\hline $\mathrm{Hx} 4$ & -79.713871135672 & & & & -79.713806646741 & & & \\
\hline $\mathrm{xF} 4$ & -674.842239667514 & & & & -674.843917052641 & & & \\
\hline $\mathrm{HF} 4$ & -754.557610235492 & -0.932 & -0.941 & 0.009 & -754.559449746863 & -1.079 & -1.083 & 0.004 \\
\hline $\mathrm{Fx} 1$ & -674.842230574223 & & & & -674.843904021205 & & & \\
\hline $\mathrm{xF} 1$ & -674.842247809953 & & & & -674.843869802666 & & & \\
\hline $\mathrm{FF} 1$ & -1349.68593238178 & -0.898 & -0.912 & 0.014 & -1349.6895815669 & -1.091 & -1.134 & 0.043 \\
\hline $\mathrm{Fx} 2$ & -674.842227722824 & & & & -674.843886664823 & & & \\
\hline $\mathrm{xF} 2$ & -674.842244913549 & & & & -674.843910702744 & & & \\
\hline FF2 & -1349.68564419369 & -0.718 & -0.735 & 0.018 & -1349.68923248412 & -0.872 & -0.901 & 0.028 \\
\hline
\end{tabular}




\begin{tabular}{|c|c|c|c|c|c|c|c|c|}
\hline & \multicolumn{4}{|c|}{ NormalPNO-DLPNO-CCSD(T)/CBS(34) } & \multicolumn{4}{|c|}{ TightPNO-DLPNO-CCSD(T)/CBS(34) } \\
\hline & $\mathrm{E}[\mathrm{Ha}]$ & $\mathrm{BAE}\left[\mathrm{kcal} \mathrm{mol}{ }^{-1}\right]$ & $\mathrm{BIE}\left[\mathrm{kcal} \mathrm{mol}^{-1}\right]$ & E-def $\left[\mathrm{kcal} \mathrm{mol}^{-1}\right]$ & $\mathrm{E}[\mathrm{Ha}]$ & $\mathrm{BAE}\left[\mathrm{kcal} \mathrm{mol}^{-1}\right]$ & $\mathrm{BIE}\left[\mathrm{kcal} \mathrm{mol}^{-1}\right]$ & E-def $\left[\mathrm{kcal} \mathrm{mol}^{-1}\right]$ \\
\hline \multicolumn{9}{|c|}{ Propanes } \\
\hline H & -118.972132968789 & & & & -118.972167148961 & & & \\
\hline $\mathrm{F}$ & -912.463755063015 & & & & -912.466215512477 & & & \\
\hline $\mathrm{Hx} 1$ & -118.972126403027 & & & & -118.972158957498 & & & \\
\hline $\mathrm{xH} 1$ & -118.972126506231 & & & & -118.972159476421 & & & \\
\hline Hн1 & -237.947370069894 & -1.948 & -1.956 & 0.008 & -237.947426946602 & -1.941 & -1.951 & 0.010 \\
\hline $\mathrm{Hx} 2$ & -118.972126637966 & & & & -118.972158904248 & & & \\
\hline $\mathrm{xH} 2$ & -118.972127230858 & & & & -118.972160491154 & & & \\
\hline HH2 & -237.946010605398 & -1.095 & -1.102 & 0.008 & -237.94626001514 & -1.208 & -1.218 & 0.009 \\
\hline Hx1 & -118.972124584908 & & & & -118.972160730635 & & & \\
\hline $\mathrm{xF} 1$ & -912.463837010732 & & & & -912.466129682442 & & & \\
\hline HF1 & -1031.4382638712 & -1.491 & -1.445 & -0.046 & -1031.44086140384 & -1.555 & -1.613 & 0.058 \\
\hline $\mathrm{Hx} 2$ & -118.972126160584 & & & & -118.972162530038 & & & \\
\hline $\mathrm{xF} 2$ & -912.463729571619 & & & & -912.46615666121 & & & \\
\hline $\mathrm{HF} 2$ & -1031.4378065979 & -1.204 & -1.224 & 0.020 & -1031.44041814971 & -1.277 & -1.317 & 0.040 \\
\hline $\mathrm{Hx} 3$ & -118.972128306644 & & & & -118.972164158349 & & & \\
\hline $\mathrm{xF} 3$ & -912.463800210181 & & & & -912.466163376212 & & & \\
\hline HF3 & -1031.43775808144 & -1.173 & -1.148 & -0.025 & -1031.44044683014 & -1.295 & -1.330 & 0.035 \\
\hline $\mathrm{Hx} 4$ & -118.972126524598 & & & & -118.972160357895 & & & \\
\hline $\mathrm{xF} 4$ & -912.463707942626 & & & & -912.46613786162 & & & \\
\hline $\mathrm{HF} 4$ & -1031.43776318789 & -1.177 & -1.210 & 0.034 & -1031.4404378602 & -1.290 & -1.343 & 0.053 \\
\hline Fx1 & -912.463828656995 & & & & -912.46608508582 & & & \\
\hline $\mathrm{xF} 1$ & -912.463758260495 & & & & -912.466172958188 & & & \\
\hline FF1 & -1824.92959361174 & -1.307 & -1.259 & -0.048 & -1824.93471712658 & -1.435 & -1.543 & 0.109 \\
\hline $\mathrm{Fx} 2$ & -912.463776044488 & & & & -912.466209322132 & & & \\
\hline $\mathrm{xF} 2$ & -912.463767854572 & & & & -912.466212076848 & & & \\
\hline FF2 & -1824.92968283165 & -1.363 & -1.342 & -0.021 & -1824.93507707936 & -1.660 & -1.666 & 0.006 \\
\hline \multicolumn{9}{|c|}{ Butanes } \\
\hline $\mathrm{H}$ & -158.230488269785 & & & & -158.230620228219 & & & \\
\hline $\mathrm{F}$ & -1150.08536238358 & & & & -1150.08821681391 & & & \\
\hline Hx1 & -158.230455597828 & & & & -158.230615017062 & & & \\
\hline $\mathrm{xH} 1$ & -158.230468825232 & & & & -158.230613642962 & & & \\
\hline Hн1 & -316.465418788441 & -2.788 & -2.820 & 0.033 & -316.465679348846 & -2.785 & -2.793 & 0.007 \\
\hline $\mathrm{Hx} 2$ & -158.230453964235 & & & & -158.230611156389 & & & \\
\hline $\mathrm{xH} 2$ & -158.23046035736 & & & & -158.230617580791 & & & \\
\hline HH2 & -316.463373653961 & -1.504 & -1.543 & 0.039 & -316.463908297992 & -1.674 & -1.681 & 0.007 \\
\hline $\mathrm{Hx} 1$ & -158.23046944099 & & & & -158.230615513641 & & & \\
\hline $\mathrm{xF} 1$ & -1150.0846351836 & & & & -1150.08771116569 & & & \\
\hline $\mathrm{HF} 1$ & -1308.3181972081 & -1.472 & -1.941 & 0.468 & -1308.32189804079 & -1.921 & -2.241 & 0.320 \\
\hline $\mathrm{Hx} 2$ & -158.23047175533 & & & & -158.230603385692 & & & \\
\hline $\mathrm{xF} 2$ & -1150.08531658225 & & & & -1150.08817328028 & & & \\
\hline $\mathrm{HF} 2$ & -1308.31891191766 & -1.921 & -1.960 & 0.039 & -1308.32227519998 & -2.157 & -2.195 & 0.038 \\
\hline $\mathrm{Hx} 3$ & -158.230450789595 & & & & -158.230589327012 & & & \\
\hline $\mathrm{xF} 3$ & -1150.08517794591 & & & & -1150.08810668018 & & & \\
\hline HF3 & -1308.31868840588 & -1.781 & -1.920 & 0.139 & -1308.32217862525 & -2.097 & -2.185 & 0.089 \\
\hline $\mathrm{Hx} 4$ & -158.230425195218 & & & & -158.230569980284 & & & \\
\hline $\mathrm{xF} 4$ & -1150.08531267617 & & & & -1150.08820129335 & & & \\
\hline $\mathrm{HF} 4$ & -1308.31867097241 & -1.770 & -1.841 & 0.071 & -1308.32220383099 & -2.113 & -2.154 & 0.041 \\
\hline Fx1 & -1150.08522612095 & & & & -1150.08815864686 & & & \\
\hline $\mathrm{xF} 1$ & -1150.08520973919 & & & & -1150.08814110261 & & & \\
\hline FF1 & -2300.17306169007 & -1.466 & -1.648 & 0.181 & -2300.17949081754 & -1.918 & -2.002 & 0.084 \\
\hline $\mathrm{Fx} 2$ & -1150.08504471506 & & & & -1150.08802522914 & & & \\
\hline $\mathrm{xF} 2$ & -1150.08518408051 & & & & -1150.08806632909 & & & \\
\hline FF2 & -2300.17306236078 & -1.467 & -1.778 & 0.311 & -2300.17960669457 & -1.991 & -2.206 & 0.215 \\
\hline
\end{tabular}




\begin{tabular}{|c|c|c|c|c|c|c|c|c|}
\hline & \multicolumn{4}{|c|}{ NormalPNO-DLPNO-CCSD(T)/CBS(34) } & \multicolumn{4}{|c|}{ TightPNO-DLPNO-CCSD(T)/CBS(34) } \\
\hline & $\mathrm{E}[\mathrm{Ha}]$ & $\mathrm{BAE}\left[\mathrm{kcal} \mathrm{mol}^{-1}\right]$ & $\mathrm{BIE}\left[\mathrm{kcal} \mathrm{mol}{ }^{-1}\right]$ & E-def $\left[\mathrm{kcal} \mathrm{mol}^{-1}\right]$ & $\mathrm{E}[\mathrm{Ha}]$ & $\mathrm{BAE}\left[\mathrm{kcal} \mathrm{mol}^{-1}\right]$ & $\mathrm{BIE}\left[\mathrm{kcal} \mathrm{mol}^{-1}\right]$ & E-def $\left[\mathrm{kcal} \mathrm{mol}^{-1}\right]$ \\
\hline \multicolumn{9}{|c|}{ Pentanes } \\
\hline н & -197.488752226916 & & & & -197.489046101804 & & & \\
\hline $\mathrm{F}$ & -1387.70699376063 & & & & -1387.71050735664 & & & \\
\hline Hx1 & -197.488720324413 & & & & -197.489041714705 & & & \\
\hline $\mathrm{xH} 1$ & -197.488720312273 & & & & -197.489041706961 & & & \\
\hline нH1 & -394.983304864186 & -3.640 & -3.680 & 0.040 & -394.983878502499 & -3.631 & -3.636 & 0.006 \\
\hline Hx2 & -197.488718314884 & & & & -197.489036751423 & & & \\
\hline $\mathrm{xH} 2$ & -197.488718287335 & & & & -197.489036726222 & & & \\
\hline нH2 & -394.980697750479 & -2.004 & -2.046 & 0.043 & -394.981591213658 & -2.196 & -2.207 & 0.012 \\
\hline Hx1 & -197.48863896484 & & & & -197.488945867352 & & & \\
\hline $\mathrm{xF} 1$ & -1387.70684319738 & & & & -1387.71034583079 & & & \\
\hline HF1 & -1585.19947836648 & -2.342 & -2.508 & 0.166 & -1585.20381128164 & -2.672 & -2.836 & 0.164 \\
\hline $\mathrm{Hx} 2$ & -197.48871662501 & & & & -197.489013289816 & & & \\
\hline $\mathrm{xF} 2$ & -1387.70658610746 & & & & -1387.71013796355 & & & \\
\hline $\mathrm{HF} 2$ & -1585.19898809687 & -2.034 & -2.313 & 0.278 & -1585.20325589262 & -2.323 & -2.576 & 0.252 \\
\hline Hx3 & -197.488736432724 & & & & -197.48903155831 & & & \\
\hline $\mathrm{xF} 3$ & -1387.7069336869 & & & & -1387.71048612585 & & & \\
\hline HF3 & -1585.19944778121 & -2.323 & -2.371 & 0.048 & -1585.20359800258 & -2.538 & -2.560 & 0.022 \\
\hline $\mathrm{Hx} 4$ & -197.488703868569 & & & & -197.489007214974 & & & \\
\hline $\mathrm{xF} 4$ & -1387.70689166249 & & & & -1387.71049681111 & & & \\
\hline $\mathrm{HF} 4$ & -1585.1997595802 & -2.519 & -2.613 & 0.094 & -1585.20419633452 & -2.913 & -2.944 & 0.031 \\
\hline $\mathrm{Fx} 1$ & -1387.70688247019 & & & & -1387.71049027577 & & & \\
\hline $\mathrm{xF} 1$ & -1387.70692240684 & & & & -1387.71041886084 & & & \\
\hline $\mathrm{FF} 1$ & -2775.41768992575 & -2.323 & -2.438 & 0.115 & -2775.4253988967 & -2.751 & -2.817 & 0.066 \\
\hline $\mathrm{Fx} 2$ & -1387.706958301 & & & & -1387.7104822998 & & & \\
\hline $\mathrm{xF} 2$ & -1387.70684032426 & & & & -1387.71049053408 & & & \\
\hline $\mathrm{FF} 2$ & -2775.41738019717 & -2.129 & -2.247 & 0.119 & -2775.42522895213 & -2.644 & -2.671 & 0.026 \\
\hline \multicolumn{9}{|c|}{ Hexanes } \\
\hline $\mathrm{H}$ & -236.747058132828 & & & & -236.747501883392 & & & \\
\hline $\mathrm{F}$ & -1625.32854816406 & & & & -1625.33277572995 & & & \\
\hline $\mathrm{Hx} 1$ & -236.747001322901 & & & & -236.74749361514 & & & \\
\hline $\mathrm{xH} 1$ & -236.747001155531 & & & & -236.747493470246 & & & \\
\hline HH1 & -473.500874618682 & -4.241 & -4.312 & 0.071 & -473.501816683307 & -4.275 & -4.286 & 0.010 \\
\hline $\mathrm{Hx} 2$ & -236.747007677672 & & & & -236.747494086152 & & & \\
\hline $\mathrm{xH} 2$ & -236.747007622285 & & & & -236.747494034557 & & & \\
\hline нH2 & -473.497979196046 & -2.424 & -2.487 & 0.063 & -473.499304559628 & -2.699 & -2.709 & 0.010 \\
\hline $\mathrm{Hx} 1$ & -236.74688716963 & & & & -236.747315564704 & & & \\
\hline $\mathrm{xF} 1$ & -1625.3279818125 & & & & -1625.33219611172 & & & \\
\hline HF1 & -1862.07988175585 & -2.683 & -3.146 & 0.463 & -1862.0849724539 & -2.946 & -3.427 & 0.481 \\
\hline $\mathrm{Hx} 2$ & -236.746973440593 & & & & -236.747408823833 & & & \\
\hline $\mathrm{xF} 2$ & -1625.32814618926 & & & & -1625.33230949939 & & & \\
\hline $\mathrm{HF} 2$ & -1862.07974344451 & -2.596 & -2.901 & 0.305 & -1862.08486937564 & -2.881 & -3.232 & 0.351 \\
\hline Hx3 & -236.746932240669 & & & & -236.747374558127 & & & \\
\hline $\mathrm{xF} 3$ & -1625.32818213363 & & & & -1625.33247236672 & & & \\
\hline HF3 & -1862.07975632521 & -2.604 & -2.913 & 0.309 & -1862.08516480069 & -3.067 & -3.337 & 0.270 \\
\hline $\mathrm{Hx} 4$ & -236.747013583376 & & & & -236.747476792503 & & & \\
\hline $\mathrm{xF} 4$ & -1625.32856153143 & & & & -1625.33270972626 & & & \\
\hline $\mathrm{HF} 4$ & -1862.0802881985 & -2.938 & -2.958 & 0.020 & -1862.0855392657 & -3.302 & -3.359 & 0.057 \\
\hline $\mathrm{Fx} 1$ & -1625.32848543712 & & & & -1625.33272867229 & & & \\
\hline $\mathrm{xF} 1$ & -1625.32847640981 & & & & -1625.33270905255 & & & \\
\hline FF1 & -3250.6621173447 & -3.151 & -3.235 & 0.084 & -3250.67149979911 & -3.733 & -3.804 & 0.071 \\
\hline $\mathrm{Fx} 2$ & -1625.32839954454 & & & & -1625.33270649309 & & & \\
\hline $\mathrm{xF} 2$ & -1625.32838038054 & & & & -1625.33270736838 & & & \\
\hline $\mathrm{FF} 2$ & -3250.66024838001 & -1.978 & -2.176 & 0.199 & -3250.66966163483 & -2.579 & -2.666 & 0.086 \\
\hline
\end{tabular}




\begin{tabular}{|c|c|c|c|c|c|c|c|c|}
\hline & \multicolumn{4}{|c|}{ VeryTightPNO-DLPNO-CCSD(T)/CBS(34) } & \multicolumn{4}{|c|}{$\mathrm{MP} 2 / \mathrm{CBS}(34)$} \\
\hline & $\mathrm{E}[\mathrm{Ha}]$ & $\mathrm{BAE}\left[\mathrm{kcal} \mathrm{mol}^{-1}\right]$ & BIE $\left[\mathrm{kcal} \mathrm{mol}^{-1}\right]$ & E-def $\left[\mathrm{kcal} \mathrm{mol}^{-1}\right]$ & $\mathrm{E}[\mathrm{Ha}]$ & $\mathrm{BAE}\left[\mathrm{kcal} \mathrm{mol}^{-1}\right]$ & BIE $\left[\mathrm{kcal} \mathrm{mol}^{-1}\right]$ & E-def $\left[\mathrm{kcal} \mathrm{mol}^{-1}\right]$ \\
\hline \multicolumn{9}{|c|}{ Methanes } \\
\hline $\mathrm{H}$ & -40.458829605571 & & & & -40.473039630273 & & & \\
\hline $\mathrm{F}$ & -437.222292901903 & & & & -437.404761018784 & & & \\
\hline Hx1 & -40.458828797064 & & & & -40.473040873783 & & & \\
\hline $\mathrm{xH1}$ & -40.458828804595 & & & & -40.473040735131 & & & \\
\hline HH1 & -80.918462536915 & -0.504 & -0.505 & 0.001 & -80.946816065582 & -0.462 & -0.461 & -0.001 \\
\hline $\mathrm{Hx} 2$ & -40.458829355838 & & & & -40.473039514805 & & & \\
\hline $\mathrm{xH} 2$ & -40.458829382253 & & & & -40.473039419455 & & & \\
\hline $\mathrm{HH} 2$ & -80.918296349692 & -0.400 & -0.400 & 0.000 & -80.946682691332 & -0.379 & -0.379 & 0.000 \\
\hline Hx1 & -40.458829350677 & & & & -40.473040033543 & & & \\
\hline $\mathrm{xF} 1$ & -437.222278372874 & & & & -437.40474154585 & & & \\
\hline HF1 & -477.68231594593 & -0.749 & -0.758 & 0.009 & -477.878852364044 & -0.660 & -0.672 & 0.012 \\
\hline $\mathrm{Hx} 2$ & -40.458828714761 & & & & -40.473040071465 & & & \\
\hline $\mathrm{xF} 2$ & -437.222282187068 & & & & -437.404746621481 & & & \\
\hline $\mathrm{HF} 2$ & -477.68174550036 & -0.391 & -0.398 & 0.007 & -477.8783357398 & -0.336 & -0.345 & 0.009 \\
\hline Fx1 & -437.222279356036 & & & & -437.40474438795 & & & \\
\hline $\mathrm{xF} 1$ & -437.222281940974 & & & & -437.404746562853 & & & \\
\hline FF1 & -874.445721163073 & -0.712 & -0.728 & 0.015 & -874.810534395821 & -0.635 & -0.655 & 0.020 \\
\hline $\mathrm{Fx} 2$ & -437.222281580913 & & & & -437.404745587253 & & & \\
\hline $\mathrm{xF} 2$ & -437.222281240395 & & & & -437.404745861874 & & & \\
\hline $\mathrm{FF} 2$ & -874.445626004777 & -0.653 & -0.667 & 0.014 & -874.810424401864 & -0.566 & -0.585 & 0.019 \\
\hline \multicolumn{9}{|c|}{ Ethanes } \\
\hline $\mathrm{H}$ & -79.713696715595 & & & & -79.749427666505 & & & \\
\hline $\mathrm{F}$ & -674.844748222117 & & & & -675.131824576874 & & & \\
\hline $\mathrm{Hx} 1$ & -79.713696909679 & & & & -79.749425053668 & & & \\
\hline $\mathrm{xH} 1$ & -79.713697027752 & & & & -79.749425373311 & & & \\
\hline HH1 & -159.429547825058 & -1.352 & -1.352 & 0.000 & -159.50112155962 & -1.422 & -1.425 & 0.003 \\
\hline $\mathrm{Hx} 2$ & -79.713697858593 & & & & -79.749427274354 & & & \\
\hline $\mathrm{xH} 2$ & -79.71369788307 & & & & -79.749427151209 & & & \\
\hline $\mathrm{HH} 2$ & -159.429296305 & -1.194 & -1.193 & -0.001 & -159.500802427155 & -1.222 & -1.222 & 0.001 \\
\hline $\mathrm{Hx} 1$ & -79.713693127083 & & & & -79.749425362763 & & & \\
\hline $\mathrm{xF} 1$ & -674.844727527179 & & & & -675.131791138192 & & & \\
\hline HF1 & -754.559997736102 & -0.974 & -0.990 & 0.015 & -754.882709901028 & -0.915 & -0.937 & 0.022 \\
\hline $\mathrm{Hx} 2$ & -79.713696479885 & & & & -79.749428775361 & & & \\
\hline $\mathrm{xF} 2$ & -674.844725764402 & & & & -675.131778944523 & & & \\
\hline $\mathrm{HF} 2$ & -754.560240266736 & -1.127 & -1.141 & 0.014 & -754.882930636669 & -1.053 & -1.081 & 0.028 \\
\hline Hx3 & -79.713696488727 & & & & -79.749428544236 & & & \\
\hline $\mathrm{xF} 3$ & -674.844735472761 & & & & -675.131797431439 & & & \\
\hline HF3 & -754.559803688484 & -0.853 & -0.861 & 0.008 & -754.882520217856 & -0.796 & -0.812 & 0.016 \\
\hline $\mathrm{Hx} 4$ & -79.71369435948 & & & & -79.749427595914 & & & \\
\hline $\mathrm{xF} 4$ & -674.844741687269 & & & & -675.131808410933 & & & \\
\hline $\mathrm{HF} 4$ & -754.560181534136 & -1.090 & -1.095 & 0.006 & -754.882899595339 & -1.034 & -1.044 & 0.010 \\
\hline $\mathrm{Fx} 1$ & -674.844730329606 & & & & -675.131800185421 & & & \\
\hline $\mathrm{xF} 1$ & -674.844694142796 & & & & -675.131771704903 & & & \\
\hline FF1 & -1349.69128091431 & -1.120 & -1.165 & 0.045 & -1350.26528087191 & -1.024 & -1.072 & 0.048 \\
\hline $\mathrm{Fx} 2$ & -674.844722848258 & & & & -675.131780367396 & & & \\
\hline $\mathrm{xF} 2$ & -674.84473794725 & & & & -675.131806232494 & & & \\
\hline FF2 & -1349.69097989253 & -0.931 & -0.953 & 0.022 & -1350.26498223949 & -0.837 & -0.876 & 0.039 \\
\hline
\end{tabular}




\begin{tabular}{|c|c|c|c|c|c|c|c|c|}
\hline & \multicolumn{4}{|c|}{ VeryTightPNO-DLPNO-CCSD(T)/CBS(34) } & \multicolumn{4}{|c|}{$\mathrm{MP} 2 / \mathrm{CBS}(34)$} \\
\hline & $\mathrm{E}[\mathrm{Ha}]$ & $\mathrm{BAE}\left[\mathrm{kcal} \mathrm{mol}^{-1}\right]$ & $\mathrm{BIE}\left[\mathrm{kcal} \mathrm{mol}^{-1}\right]$ & E-def $\left[\mathrm{kcal} \mathrm{mol}^{-1}\right]$ & $\mathrm{E}[\mathrm{Ha}]$ & $\mathrm{BAE}\left[\mathrm{kcal} \mathrm{mol}{ }^{-1}\right]$ & $\mathrm{BIE}\left[\mathrm{kcal} \mathrm{mol}^{-1}\right]$ & E-def $\left[\mathrm{kcal} \mathrm{mol}^{-1}\right]$ \\
\hline \multicolumn{9}{|c|}{ Propanes } \\
\hline H & -118.972022669517 & & & & -119.029774534044 & & & \\
\hline $\mathrm{F}$ & -912.467399702174 & & & & -912.858925862397 & & & \\
\hline Hx1 & -118.97201944583 & & & & -119.029771061025 & & & \\
\hline $\mathrm{xH} 1$ & -118.972019819919 & & & & -119.029771147672 & & & \\
\hline HН1 & -237.947221518947 & -1.993 & -1.997 & 0.004 & -238.062918904442 & -2.115 & -2.119 & 0.004 \\
\hline $\mathrm{Hx} 2$ & -118.972019145367 & & & & -119.029759395825 & & & \\
\hline $\mathrm{xH} 2$ & -118.972020582871 & & & & -119.029774551531 & & & \\
\hline HH2 & -237.945963119191 & -1.203 & -1.207 & 0.004 & -238.061466882786 & -1.203 & -1.213 & 0.009 \\
\hline $\mathrm{Hx} 1$ & -118.972018348951 & & & & -119.029776667231 & & & \\
\hline $\mathrm{xF} 1$ & -912.467328616063 & & & & -912.858844561952 & & & \\
\hline HF1 & -1031.44196843866 & -1.598 & -1.645 & 0.047 & -1031.89118353796 & -1.558 & -1.608 & 0.050 \\
\hline $\mathrm{Hx} 2$ & -118.972020762989 & & & & -119.029771569985 & & & \\
\hline $\mathrm{xF} 2$ & -912.467355375762 & & & & -912.858856805422 & & & \\
\hline $\mathrm{HF} 2$ & -1031.44148288504 & -1.293 & -1.322 & 0.029 & -1031.89068976309 & -1.248 & -1.294 & 0.045 \\
\hline $\mathrm{Hx} 3$ & -118.97202241253 & & & & -119.029748713332 & & & \\
\hline $\mathrm{xF} 3$ & -912.467353911319 & & & & -912.858864466224 & & & \\
\hline HF3 & -1031.44153026236 & -1.323 & -1.352 & 0.029 & -1031.8907011493 & -1.255 & -1.310 & 0.055 \\
\hline $\mathrm{Hx} 4$ & -118.972018370909 & & & & -119.029777076259 & & & \\
\hline $\mathrm{xF} 4$ & -912.46734077082 & & & & -912.858844347636 & & & \\
\hline $\mathrm{HF} 4$ & -1031.44151527512 & -1.313 & -1.353 & 0.040 & -1031.89072660804 & -1.271 & -1.321 & 0.050 \\
\hline Fx1 & -912.467271830224 & & & & -912.858811311382 & & & \\
\hline $\mathrm{xF} 1$ & -912.467366321716 & & & & -912.858891153689 & & & \\
\hline FF1 & -1824.937286182 & -1.560 & -1.662 & 0.101 & -1825.72022386377 & -1.489 & -1.582 & 0.094 \\
\hline $\mathrm{Fx} 2$ & -912.467407755647 & & & & -912.858925679747 & & & \\
\hline $\mathrm{xF} 2$ & -912.467405993299 & & & & -912.85892957805 & & & \\
\hline FF2 & -1824.93774235481 & -1.847 & -1.838 & -0.009 & -1825.72050550751 & -1.665 & -1.663 & -0.002 \\
\hline \multicolumn{9}{|c|}{ Butanes } \\
\hline $\mathrm{H}$ & -158.230471666199 & & & & -158.310243431337 & & & \\
\hline $\mathrm{F}$ & -1150.08978210425 & & & & -1150.5857899199 & & & \\
\hline Hx1 & -158.230467683388 & & & & -158.310242735017 & & & \\
\hline $\mathrm{xH} 1$ & -158.230466661263 & & & & -158.310238832543 & & & \\
\hline Hн1 & -316.465455021217 & -2.831 & -2.837 & 0.006 & -316.625417394412 & -3.094 & -3.097 & 0.003 \\
\hline $\mathrm{Hx} 2$ & -158.230464211975 & & & & -158.310238001219 & & & \\
\hline $\mathrm{xH} 2$ & -158.23046848121 & & & & -158.310234651012 & & & \\
\hline HH2 & -316.463577552471 & -1.653 & -1.660 & 0.007 & -316.623153129918 & -1.673 & -1.682 & 0.009 \\
\hline $\mathrm{Hx} 1$ & -158.23046833367 & & & & -158.310237829722 & & & \\
\hline $\mathrm{xF} 1$ & -1150.08935614244 & & & & -1150.58528336107 & & & \\
\hline $\mathrm{HF} 1$ & -1308.32330003111 & -1.912 & -2.181 & 0.269 & -1308.89911851347 & -1.936 & -2.257 & 0.321 \\
\hline $\mathrm{Hx} 2$ & -158.230456313239 & & & & -158.310226978252 & & & \\
\hline $\mathrm{xF} 2$ & -1150.0897675522 & & & & -1150.5857556517 & & & \\
\hline $\mathrm{HF} 2$ & -1308.32366338457 & -2.140 & -2.158 & 0.019 & -1308.89953734719 & -2.199 & -2.231 & 0.032 \\
\hline $\mathrm{Hx} 3$ & -158.230443028036 & & & & -158.310236274444 & & & \\
\hline $\mathrm{xF} 3$ & -1150.08970423561 & & & & -1150.5856774136 & & & \\
\hline HF3 & -1308.32364876576 & -2.130 & -2.197 & 0.067 & -1308.89938822685 & -2.105 & -2.180 & 0.075 \\
\hline $\mathrm{Hx} 4$ & -158.230424358444 & & & & -158.310205841967 & & & \\
\hline $\mathrm{xF} 4$ & -1150.08978603459 & & & & -1150.58579187308 & & & \\
\hline $\mathrm{HF} 4$ & -1308.3236474947 & -2.130 & -2.157 & 0.027 & -1308.89936664775 & -2.092 & -2.114 & 0.022 \\
\hline Fx1 & -1150.08973688692 & & & & -1150.58572786092 & & & \\
\hline $\mathrm{xF} 1$ & -1150.08971569626 & & & & -1150.58571096713 & & & \\
\hline FF1 & -2300.18280329497 & -2.033 & -2.103 & 0.070 & -2301.17478923392 & -2.014 & -2.102 & 0.088 \\
\hline $\mathrm{Fx} 2$ & -1150.08964160566 & & & & -1150.58558761295 & & & \\
\hline $\mathrm{xF} 2$ & -1150.08967256438 & & & & -1150.58564200617 & & & \\
\hline $\mathrm{FF} 2$ & -2300.18297058111 & -2.138 & -2.294 & 0.157 & -2301.17474134423 & -1.984 & -2.204 & 0.220 \\
\hline
\end{tabular}




\begin{tabular}{|c|c|c|c|c|c|c|c|c|}
\hline & \multicolumn{4}{|c|}{ VeryTightPNO-DLPNO-CCSD(T)/CBS(34) } & \multicolumn{4}{|c|}{$\mathrm{MP} 2 / \mathrm{CBS}(34)$} \\
\hline & $\mathrm{E}[\mathrm{Ha}]$ & $\mathrm{BAE}\left[\mathrm{kcal} \mathrm{mol}^{-1}\right]$ & $\mathrm{BIE}\left[\mathrm{kcal} \mathrm{mol}^{-1}\right]$ & E-def $\left[\mathrm{kcal} \mathrm{mol}^{-1}\right]$ & $\mathrm{E}[\mathrm{Ha}]$ & $\mathrm{BAE}\left[\mathrm{kcal} \mathrm{mol}^{-1}\right]$ & $\mathrm{BIE}\left[\mathrm{kcal} \mathrm{mol}^{-1}\right]$ & E-def $\left[\mathrm{kcal} \mathrm{mol}^{-1}\right]$ \\
\hline \multicolumn{9}{|c|}{ Pentanes } \\
\hline H & -197.488869247624 & & & & -197.590632808594 & & & \\
\hline $\mathrm{F}$ & -1387.71242766832 & & & & -1388.31295287017 & & & \\
\hline $\mathrm{Hx} 1$ & -197.488864109103 & & & & -197.59063744064 & & & \\
\hline $\mathrm{xH} 1$ & -197.488864093842 & & & & -197.590637515538 & & & \\
\hline нH1 & -394.983595378707 & -3.675 & -3.682 & 0.006 & -395.187736422226 & -4.060 & -4.055 & -0.006 \\
\hline $\mathrm{Hx} 2$ & -197.488860150701 & & & & -197.590618761916 & & & \\
\hline $\mathrm{xH} 2$ & -197.488860121898 & & & & -197.590618731269 & & & \\
\hline нH2 & -394.981206562891 & -2.176 & -2.188 & 0.011 & -395.184803119208 & -2.220 & -2.237 & 0.018 \\
\hline Hx1 & -197.48876966214 & & & & -197.590538222972 & & & \\
\hline $\mathrm{xF} 1$ & -1387.71224464374 & & & & -1388.31274431025 & & & \\
\hline HF1 & -1585.20546998969 & -2.619 & -2.796 & 0.177 & -1585.90794462138 & -2.735 & -2.926 & 0.190 \\
\hline $\mathrm{Hx} 2$ & -197.488836289482 & & & & -197.590595633557 & & & \\
\hline $\mathrm{xF} 2$ & -1387.71204256854 & & & & -1388.31254553895 & & & \\
\hline $\mathrm{HF} 2$ & -1585.20497316136 & -2.307 & -2.569 & 0.262 & -1585.90736306987 & -2.370 & -2.649 & 0.279 \\
\hline $\mathrm{Hx} 3$ & -197.488853939243 & & & & -197.590628115447 & & & \\
\hline $\mathrm{xF} 3$ & -1387.71237286413 & & & & -1388.3128896462 & & & \\
\hline HF3 & -1585.20534848053 & -2.542 & -2.586 & 0.044 & -1585.90764798382 & -2.549 & -2.592 & 0.043 \\
\hline $\mathrm{Hx} 4$ & -197.488831405326 & & & & -197.590606824727 & & & \\
\hline $\mathrm{xF} 4$ & -1387.71240260176 & & & & -1388.3129124386 & & & \\
\hline $\mathrm{HF} 4$ & -1585.20598263007 & -2.940 & -2.980 & 0.039 & -1585.90826976091 & -2.939 & -2.981 & 0.042 \\
\hline $\mathrm{Fx} 1$ & -1387.71239215427 & & & & -1388.31291871597 & & & \\
\hline $\mathrm{xF} 1$ & -1387.71230238936 & & & & -1388.31282284606 & & & \\
\hline $\mathrm{FF} 1$ & -2775.42952881838 & -2.933 & -3.034 & 0.101 & -2776.63059909978 & -2.945 & -3.048 & 0.103 \\
\hline $\mathrm{Fx} 2$ & -1387.71237991222 & & & & -1388.31291228651 & & & \\
\hline $\mathrm{xF} 2$ & -1387.71238372849 & & & & -1388.31291311482 & & & \\
\hline $\mathrm{FF} 2$ & -2775.42925194185 & -2.759 & -2.816 & 0.058 & -2776.63016866222 & -2.675 & -2.725 & 0.050 \\
\hline \multicolumn{9}{|c|}{ Hexanes } \\
\hline $\mathrm{H}$ & -236.747299412113 & & & & -236.871048982949 & & & \\
\hline $\mathrm{F}$ & -1625.33502778657 & & & & -1626.04005403153 & & & \\
\hline $\mathrm{Hx} 1$ & -236.747290444749 & & & & -236.871054554354 & & & \\
\hline $\mathrm{xH} 1$ & -236.747290295613 & & & & -236.871054435193 & & & \\
\hline HH1 & -473.501448018185 & -4.298 & -4.309 & 0.011 & -473.749753465073 & -4.804 & -4.797 & -0.007 \\
\hline $\mathrm{Hx} 2$ & -236.747291722787 & & & & -236.871043108598 & & & \\
\hline $\mathrm{xH} 2$ & -236.747291664414 & & & & -236.871043000593 & & & \\
\hline нH2 & -473.498806834847 & -2.641 & -2.650 & 0.010 & -473.746396898488 & -2.698 & -2.705 & 0.007 \\
\hline $\mathrm{Hx} 1$ & -236.747113053312 & & & & -236.870890601574 & & & \\
\hline $\mathrm{xF} 1$ & -1625.33448744958 & & & & -1626.03946006817 & & & \\
\hline HF1 & -1862.08692414107 & -2.885 & -3.341 & 0.456 & -1862.91599873187 & -3.072 & -3.544 & 0.472 \\
\hline $\mathrm{Hx} 2$ & -236.747209098801 & & & & -236.870968561554 & & & \\
\hline $\mathrm{xF} 2$ & -1625.33458032738 & & & & -1626.03956825392 & & & \\
\hline $\mathrm{HF} 2$ & -1862.08688708679 & -2.861 & -3.199 & 0.337 & -1862.91585254711 & -2.980 & -3.336 & 0.355 \\
\hline $\mathrm{Hx} 3$ & -236.747171848673 & & & & -236.870935192859 & & & \\
\hline $\mathrm{xF} 3$ & -1625.33475026021 & & & & -1626.03976029794 & & & \\
\hline HF3 & -1862.08717179529 & -3.040 & -3.294 & 0.254 & -1862.91607419783 & -3.119 & -3.375 & 0.256 \\
\hline $\mathrm{Hx} 4$ & -236.747275187722 & & & & -236.871030273572 & & & \\
\hline $\mathrm{xF} 4$ & -1625.33496500237 & & & & -1626.03998106786 & & & \\
\hline $\mathrm{HF} 4$ & -1862.08759012109 & -3.303 & -3.357 & 0.055 & -1862.91638935568 & -3.317 & -3.375 & 0.058 \\
\hline $\mathrm{Fx} 1$ & -1625.33499435808 & & & & -1626.04002176516 & & & \\
\hline $\mathrm{xF} 1$ & -1625.33497001083 & & & & -1626.03998505914 & & & \\
\hline FF1 & -3250.67627600161 & -3.903 & -3.961 & 0.057 & -3252.0865009415 & -4.012 & -4.075 & 0.064 \\
\hline $\mathrm{Fx} 2$ & -1625.33497440454 & & & & -1626.04004998724 & & & \\
\hline $\mathrm{xF} 2$ & -1625.33497575486 & & & & -1626.04004725869 & & & \\
\hline $\mathrm{FF} 2$ & -3250.67450174373 & -2.790 & -2.856 & 0.066 & -3252.084493053 & -2.752 & -2.758 & 0.007 \\
\hline
\end{tabular}




\begin{tabular}{|c|c|c|c|c|c|c|c|c|}
\hline & \multicolumn{4}{|c|}{ RI-MP2/CBS(34) } & \multicolumn{4}{|c|}{ RI-SCS-MP2/CBS(34) } \\
\hline & $\mathrm{E}[\mathrm{Ha}]$ & $\mathrm{BAE}\left[\mathrm{kcal} \mathrm{mol}^{-1}\right]$ & $\mathrm{BIE}\left[\mathrm{kcal} \mathrm{mol}^{-1}\right]$ & E-def $\left[\mathrm{kcal} \mathrm{mol}^{-1}\right]$ & $\mathrm{E}[\mathrm{Ha}]$ & $\mathrm{BAE}\left[\mathrm{kcal} \mathrm{mol}^{-1}\right]$ & BIE $\left[\mathrm{kcal} \mathrm{mol}^{-1}\right]$ & E-def $\left[\mathrm{kcal} \mathrm{mol}^{-1}\right]$ \\
\hline \multicolumn{9}{|c|}{ Methanes } \\
\hline $\mathrm{H}$ & -40.435492481401 & & & & -40.445986267425 & & & \\
\hline $\mathrm{F}$ & -437.189772434115 & & & & -437.165045632345 & & & \\
\hline Hx1 & -40.435492969586 & & & & -40.445986282521 & & & \\
\hline $\mathrm{xH} 1$ & -40.435492884472 & & & & -40.445986229959 & & & \\
\hline HH1 & -80.871766282232 & -0.490 & -0.490 & -0.001 & -80.892444412396 & -0.296 & -0.296 & 0.000 \\
\hline $\mathrm{Hx} 2$ & -40.435492314922 & & & & -40.445986067793 & & & \\
\hline $\mathrm{xH} 2$ & -40.435492264406 & & & & -40.445986044929 & & & \\
\hline $\mathrm{HH} 2$ & -80.871582920018 & -0.375 & -0.375 & 0.000 & -80.892330101407 & -0.224 & -0.225 & 0.000 \\
\hline Hx1 & -40.43549264231 & & & & -40.445986277061 & & & \\
\hline $\mathrm{xF} 1$ & -437.18976163049 & & & & -437.165037591519 & & & \\
\hline HF1 & -477.626398860948 & -0.712 & -0.718 & 0.007 & -477.611773322418 & -0.465 & -0.470 & 0.005 \\
\hline $\mathrm{Hx} 2$ & -40.435492429291 & & & & -40.445985904851 & & & \\
\hline $\mathrm{xF} 2$ & -437.189764248269 & & & & -437.165039420644 & & & \\
\hline $\mathrm{HF} 2$ & -477.625823413853 & -0.350 & -0.356 & 0.005 & -477.611329772827 & -0.187 & -0.191 & 0.004 \\
\hline Fx1 & -437.189761186263 & & & & -437.165036066588 & & & \\
\hline $\mathrm{xF} 1$ & -437.189763962477 & & & & -437.165039059105 & & & \\
\hline $\mathrm{FF} 1$ & -874.380673358703 & -0.708 & -0.721 & 0.012 & -874.330813181171 & -0.453 & -0.463 & 0.010 \\
\hline $\mathrm{Fx} 2$ & -437.189763775124 & & & & -437.165039133253 & & & \\
\hline $\mathrm{xF} 2$ & -437.18976311495 & & & & -437.165038158121 & & & \\
\hline $\mathrm{FF} 2$ & -874.38046847986 & -0.580 & -0.591 & 0.011 & -874.330631616688 & -0.339 & -0.348 & 0.009 \\
\hline \multicolumn{9}{|c|}{ Ethanes } \\
\hline $\mathrm{H}$ & -79.674742123241 & & & & -79.68836916151 & & & \\
\hline $\mathrm{F}$ & -674.79145751654 & & & & -674.752574362835 & & & \\
\hline Hx1 & -79.674740048867 & & & & -79.688367073939 & & & \\
\hline $\mathrm{xH} 1$ & -79.674740350804 & & & & -79.688367533178 & & & \\
\hline HH1 & -159.351741835024 & -1.417 & -1.419 & 0.002 & -159.378102816602 & -0.856 & -0.859 & 0.002 \\
\hline $\mathrm{Hx} 2$ & -79.674742198032 & & & & -79.688368699852 & & & \\
\hline $\mathrm{xH} 2$ & -79.674742113684 & & & & -79.688368637248 & & & \\
\hline HH2 & -159.351429924236 & -1.221 & -1.221 & 0.000 & -159.37796412311 & -0.769 & -0.770 & 0.001 \\
\hline Hx1 & -79.674739018593 & & & & -79.688365478233 & & & \\
\hline $\mathrm{xF} 1$ & -674.79143655709 & & & & -674.752564517323 & & & \\
\hline HF1 & -754.467725395654 & -0.957 & -0.973 & 0.015 & -754.44186013616 & -0.575 & -0.584 & 0.008 \\
\hline $\mathrm{Hx} 2$ & -79.674742818406 & & & & -79.688369215021 & & & \\
\hline $\mathrm{xF} 2$ & -674.791431613982 & & & & -674.752561013076 & & & \\
\hline $\mathrm{HF} 2$ & -754.467953106209 & -1.100 & -1.116 & 0.016 & -754.441995471855 & -0.660 & -0.668 & 0.008 \\
\hline $\mathrm{Hx} 3$ & -79.674742604006 & & & & -79.688369108877 & & & \\
\hline $\mathrm{xF} 3$ & -674.791438050698 & & & & -674.752567696445 & & & \\
\hline HF3 & -754.467520102061 & -0.829 & -0.841 & 0.012 & -754.441746150483 & -0.504 & -0.508 & 0.004 \\
\hline $\mathrm{Hx} 4$ & -79.674741121841 & & & & -79.688367667324 & & & \\
\hline $\mathrm{xF} 4$ & -674.791446559011 & & & & -674.752572213556 & & & \\
\hline $\mathrm{HF} 4$ & -754.467893735162 & -1.063 & -1.071 & 0.008 & -754.441938747135 & -0.625 & -0.627 & 0.002 \\
\hline $\mathrm{Fx} 1$ & -674.791441432108 & & & & -674.75256196063 & & & \\
\hline $\mathrm{xF} 1$ & -674.791411187472 & & & & -674.752530607489 & & & \\
\hline $\mathrm{FF} 1$ & -1349.58460524901 & -1.061 & -1.100 & 0.039 & -1349.50617392248 & -0.643 & -0.679 & 0.035 \\
\hline $\mathrm{Fx} 2$ & -674.791428576729 & & & & -674.752558707676 & & & \\
\hline $\mathrm{xF} 2$ & -674.791442232505 & & & & -674.752567166265 & & & \\
\hline FF2 & -1349.58430540736 & -0.872 & -0.900 & 0.028 & -1349.50603764072 & -0.558 & -0.572 & 0.014 \\
\hline
\end{tabular}




\begin{tabular}{|c|c|c|c|c|c|c|c|c|}
\hline & \multicolumn{4}{|c|}{ RI-MP2/CBS(34) } & \multicolumn{4}{|c|}{ RI-SCS-MP2/CBS(34) } \\
\hline & $\mathrm{E}[\mathrm{Ha}]$ & $\mathrm{BAE}\left[\mathrm{kcal} \mathrm{mol}^{-1}\right]$ & $\mathrm{BIE}\left[\mathrm{kcal} \mathrm{mol}^{-1}\right]$ & E-def $\left[\mathrm{kcal} \mathrm{mol}^{-1}\right]$ & $\mathrm{E}[\mathrm{Ha}]$ & $\mathrm{BAE}\left[\mathrm{kcal} \mathrm{mol}^{-1}\right]$ & $\mathrm{BIE}\left[\mathrm{kcal} \mathrm{mol}^{-1}\right]$ & E-def $\left[\mathrm{kcal} \mathrm{mol}^{-1}\right]$ \\
\hline \multicolumn{9}{|c|}{ Propanes } \\
\hline $\mathrm{H}$ & -118.917917059007 & & & & -118.933987930226 & & & \\
\hline $\mathrm{F}$ & -912.393212253362 & & & & -912.339665036802 & & & \\
\hline $\mathrm{Hx} 1$ & -118.917911407732 & & & & -118.933985828876 & & & \\
\hline $\mathrm{xH} 1$ & -118.91791163553 & & & & -118.933986370387 & & & \\
\hline HH1 & -237.839242613518 & -2.139 & -2.146 & 0.007 & -237.870011144179 & -1.277 & -1.279 & 0.002 \\
\hline $\mathrm{H} \times 2$ & -118.917903734797 & & & & -118.933984163937 & & & \\
\hline $\mathrm{xH} 2$ & -118.9179143726 & & & & -118.93398666009 & & & \\
\hline HH2 & -237.83778980864 & -1.227 & -1.237 & 0.010 & -237.869137080427 & -0.729 & -0.732 & 0.003 \\
\hline Hx1 & -118.917917177098 & & & & -118.933984506492 & & & \\
\hline $\mathrm{xF} 1$ & -912.393131515729 & & & & -912.33957670393 & & & \\
\hline HF1 & -1031.31370320277 & -1.615 & -1.666 & 0.051 & -1031.27521852806 & -0.982 & -1.040 & 0.058 \\
\hline $\mathrm{Hx} 2$ & -118.917914208475 & & & & -118.933985961423 & & & \\
\hline $\mathrm{xF} 2$ & -912.393163254693 & & & & -912.339616805787 & & & \\
\hline HF2 & -1031.31324610535 & -1.328 & -1.361 & 0.033 & -1031.27497139944 & -0.827 & -0.859 & 0.032 \\
\hline $\mathrm{Hx} 3$ & -118.917899530606 & & & & -118.933978140383 & & & \\
\hline $\mathrm{xF} 3$ & -912.393167716094 & & & & -912.339618284443 & & & \\
\hline HF3 & -1031.31325363174 & -1.333 & -1.372 & 0.039 & -1031.27506561233 & -0.886 & -0.922 & 0.035 \\
\hline $\mathrm{Hx} 4$ & -118.917916648783 & & & & -118.933987568864 & & & \\
\hline $\mathrm{xF} 4$ & -912.393151940683 & & & & -912.339600185458 & & & \\
\hline $\mathrm{HF} 4$ & -1031.31330092862 & -1.363 & -1.401 & 0.038 & -1031.27491074012 & -0.789 & -0.830 & 0.041 \\
\hline Fx1 & -912.393063604665 & & & & -912.339501548281 & & & \\
\hline $\mathrm{xF} 1$ & -912.393179392134 & & & & -912.339613845924 & & & \\
\hline FF1 & -1824.78881466499 & -1.500 & -1.614 & 0.114 & -1824.68070106287 & -0.860 & -0.995 & 0.135 \\
\hline $\mathrm{Fx} 2$ & -912.393212148871 & & & & -912.339664416705 & & & \\
\hline $\mathrm{xF} 2$ & -912.393215641175 & & & & -912.33966745534 & & & \\
\hline FF2 & -1824.7892023759 & -1.743 & -1.741 & -0.002 & -1824.6808973028 & -0.983 & -0.982 & -0.001 \\
\hline \multicolumn{9}{|c|}{ Butanes } \\
\hline $\mathrm{H}$ & -158.161214371129 & & & & -158.179685179575 & & & \\
\hline $\mathrm{F}$ & -1149.99467502283 & & & & -1149.92634565488 & & & \\
\hline $\mathrm{Hx} 1$ & -158.161211184182 & & & & -158.179681801609 & & & \\
\hline $\mathrm{xH} 1$ & -158.161208403364 & & & & -158.179680065527 & & & \\
\hline HH1 & -316.327427388356 & -3.137 & -3.142 & 0.006 & -316.362406870214 & -1.905 & -1.911 & 0.005 \\
\hline $\mathrm{H} \times 2$ & -158.161208558704 & & & & -158.179676459869 & & & \\
\hline $\mathrm{xH} 2$ & -158.16120696587 & & & & -158.179682829791 & & & \\
\hline HH2 & -316.325175210878 & -1.723 & -1.732 & 0.008 & -316.361049469501 & -1.054 & -1.061 & 0.007 \\
\hline $\mathrm{Hx} 1$ & -158.16120951982 & & & & -158.179682840458 & & & \\
\hline $\mathrm{xF} 1$ & -1149.99428134088 & & & & -1149.92600093304 & & & \\
\hline HF1 & -1308.15918358405 & -2.067 & -2.317 & 0.250 & -1308.10806379463 & -1.276 & -1.493 & 0.218 \\
\hline $\mathrm{Hx} 2$ & -158.161197784108 & & & & -158.179671037446 & & & \\
\hline $\mathrm{xF} 2$ & -1149.99465477503 & & & & -1149.92633479001 & & & \\
\hline HF2 & -1308.15952831722 & -2.283 & -2.307 & 0.023 & -1308.10831726004 & -1.435 & -1.450 & 0.016 \\
\hline $\mathrm{Hx} 3$ & -158.16119976441 & & & & -158.179662776496 & & & \\
\hline $\mathrm{xF} 3$ & -1149.99460473627 & & & & -1149.92630267501 & & & \\
\hline HF3 & -1308.1594674361 & -2.245 & -2.299 & 0.053 & -1308.10819478085 & -1.358 & -1.399 & 0.041 \\
\hline $\mathrm{Hx} 4$ & -158.161170552327 & & & & -158.179639514864 & & & \\
\hline $\mathrm{xF} 4$ & -1149.99468844172 & & & & -1149.92636242434 & & & \\
\hline $\mathrm{HF} 4$ & -1308.1593934866 & -2.199 & -2.218 & 0.019 & -1308.10824966733 & -1.392 & -1.410 & 0.018 \\
\hline $\mathrm{Fx1}$ & -1149.99462978799 & & & & -1149.926316129 & & & \\
\hline $\mathrm{xF} 1$ & -1149.9946152374 & & & & -1149.92627851558 & & & \\
\hline $\mathrm{FF} 1$ & -2299.99261428004 & -2.048 & -2.114 & 0.066 & -2299.85491059159 & -1.393 & -1.453 & 0.061 \\
\hline $\mathrm{Fx} 2$ & -1149.99452833668 & & & & -1149.92623692003 & & & \\
\hline $\mathrm{xF} 2$ & -1149.99457012064 & & & & -1149.92625282515 & & & \\
\hline FF2 & -2299.99278332072 & -2.154 & -2.312 & 0.158 & -2299.85489779506 & -1.385 & -1.511 & 0.126 \\
\hline
\end{tabular}




\begin{tabular}{|c|c|c|c|c|c|c|c|c|}
\hline & \multicolumn{4}{|c|}{ RI-MP2/CBS(34) } & \multicolumn{4}{|c|}{ RI-SCS-MP2/CBS(34) } \\
\hline & $\mathrm{E}[\mathrm{Ha}]$ & $\mathrm{BAE}\left[\mathrm{kcal} \mathrm{mol}^{-1}\right]$ & BIE $\left[\mathrm{kcal} \mathrm{mol}^{-1}\right]$ & E-def $\left[\mathrm{kcal} \mathrm{mol}^{-1}\right]$ & $\mathrm{E}[\mathrm{Ha}]$ & $\mathrm{BAE}\left[\mathrm{kcal} \mathrm{mol}^{-1}\right]$ & $\mathrm{BIE}\left[\mathrm{kcal} \mathrm{mol}^{-1}\right]$ & E-def $\left[\mathrm{kcal} \mathrm{mol}^{-1}\right]$ \\
\hline \multicolumn{9}{|c|}{ Pentanes } \\
\hline H & -197.404462162306 & & & & -197.425337724747 & & & \\
\hline $\mathrm{F}$ & -1387.59646061689 & & & & -1387.51332788493 & & & \\
\hline $\mathrm{Hx} 1$ & -197.404463077491 & & & & -197.425334214906 & & & \\
\hline $\mathrm{xH} 1$ & -197.404463123273 & & & & -197.425334213012 & & & \\
\hline нH1 & -394.815475718482 & -4.111 & -4.110 & -0.001 & -394.854663930587 & -2.503 & -2.507 & 0.004 \\
\hline $\mathrm{Hx} 2$ & -197.404448993591 & & & & -197.425325317936 & & & \\
\hline $\mathrm{xH} 2$ & -197.40444897344 & & & & -197.425325292134 & & & \\
\hline нH2 & -394.812540564044 & -2.269 & -2.286 & 0.017 & -394.852813553745 & -1.342 & -1.357 & 0.016 \\
\hline Hx1 & -197.404361090133 & & & & -197.42523983588 & & & \\
\hline $\mathrm{xF} 1$ & -1387.59628966361 & & & & -1387.51318734358 & & & \\
\hline HF1 & -1585.0054873144 & -2.864 & -3.035 & 0.171 & -1584.94152957574 & -1.797 & -1.947 & 0.150 \\
\hline $\mathrm{Hx} 2$ & -197.404427449449 & & & & -197.425306347887 & & & \\
\hline $\mathrm{xF} 2$ & -1387.5961038781 & & & & -1387.51298761459 & & & \\
\hline $\mathrm{HF} 2$ & -1585.00490723059 & -2.500 & -2.746 & 0.246 & -1584.9411915187 & -1.585 & -1.818 & 0.233 \\
\hline $\mathrm{Hx} 3$ & -197.404453656203 & & & & -197.42532658121 & & & \\
\hline $\mathrm{xF} 3$ & -1387.59642608015 & & & & -1387.51332367002 & & & \\
\hline HF3 & -1585.00522889461 & -2.702 & -2.729 & 0.027 & -1584.94144681702 & -1.745 & -1.755 & 0.010 \\
\hline $\mathrm{Hx} 4$ & -197.404429825236 & & & & -197.425304769556 & & & \\
\hline $\mathrm{xF} 4$ & -1387.59643678052 & & & & -1387.51332164591 & & & \\
\hline $\mathrm{HF} 4$ & -1585.00584065694 & -3.086 & -3.121 & 0.035 & -1584.94177947046 & -1.954 & -1.979 & 0.025 \\
\hline $\mathrm{Fx} 1$ & -1387.59642976193 & & & & -1387.51330085803 & & & \\
\hline $\mathrm{xF} 1$ & -1387.5963601874 & & & & -1387.51324484781 & & & \\
\hline $\mathrm{FF} 1$ & -2775.19770855459 & -3.004 & -3.086 & 0.082 & -2775.02976879154 & -1.953 & -2.023 & 0.069 \\
\hline $\mathrm{Fx} 2$ & -1387.5964296109 & & & & -1387.51330161886 & & & \\
\hline $\mathrm{xF} 2$ & -1387.59643112323 & & & & -1387.5133027558 & & & \\
\hline $\mathrm{FF} 2$ & -2775.19736543233 & -2.789 & -2.827 & 0.038 & -2775.0296231026 & -1.862 & -1.894 & 0.032 \\
\hline \multicolumn{9}{|c|}{ Hexanes } \\
\hline $\mathrm{H}$ & -236.647743952463 & & & & -236.671014658316 & & & \\
\hline $\mathrm{F}$ & -1625.1982147814 & & & & -1625.10029601806 & & & \\
\hline $\mathrm{Hx} 1$ & -236.64774368556 & & & & -236.671007251215 & & & \\
\hline $\mathrm{xH} 1$ & -236.647743546025 & & & & -236.671007184506 & & & \\
\hline HH1 & -473.303240272074 & -4.865 & -4.865 & 0.000 & -473.346749513861 & -2.962 & -2.971 & 0.009 \\
\hline $\mathrm{Hx} 2$ & -236.647735652197 & & & & -236.671007256512 & & & \\
\hline $\mathrm{xH} 2$ & -236.647735579256 & & & & -236.671007195554 & & & \\
\hline нH2 & -473.299965399022 & -2.810 & -2.820 & 0.010 & -473.344733159233 & -1.697 & -1.706 & 0.009 \\
\hline $\mathrm{Hx} 1$ & -236.647567121088 & & & & -236.670836191603 & & & \\
\hline $\mathrm{xF} 1$ & -1625.19765538289 & & & & -1625.09977559826 & & & \\
\hline HF1 & -1861.85109980175 & -3.226 & -3.688 & 0.462 & -1861.7742809699 & -1.864 & -2.302 & 0.439 \\
\hline $\mathrm{Hx} 2$ & -236.647657407232 & & & & -236.670924343473 & & & \\
\hline $\mathrm{xF} 2$ & -1625.19776112768 & & & & -1625.09987433242 & & & \\
\hline $\mathrm{HF} 2$ & -1861.8509344224 & -3.122 & -3.461 & 0.339 & -1861.77442790802 & -1.956 & -2.277 & 0.321 \\
\hline $\mathrm{Hx} 3$ & -236.647619640759 & & & & -236.670890983152 & & & \\
\hline $\mathrm{xF} 3$ & -1625.19793075979 & & & & -1625.10001291096 & & & \\
\hline HF3 & -1861.85120433896 & -3.292 & -3.548 & 0.256 & -1861.77446496225 & -1.979 & -2.235 & 0.255 \\
\hline $\mathrm{Hx} 4$ & -236.647720860827 & & & & -236.670993950899 & & & \\
\hline $\mathrm{xF} 4$ & -1625.19815109942 & & & & -1625.10024212975 & & & \\
\hline $\mathrm{HF} 4$ & -1861.8515083028 & -3.482 & -3.537 & 0.054 & -1861.77489373176 & -2.248 & -2.295 & 0.047 \\
\hline $\mathrm{Fx} 1$ & -1625.19816042238 & & & & -1625.10023607984 & & & \\
\hline $\mathrm{xF} 1$ & -1625.19814840912 & & & & -1625.10022541542 & & & \\
\hline FF1 & -3250.40287990912 & -4.048 & -4.123 & 0.076 & -3250.20471563305 & -2.588 & -2.670 & 0.082 \\
\hline $\mathrm{Fx} 2$ & -1625.19818263553 & & & & -1625.10022309199 & & & \\
\hline $\mathrm{xF} 2$ & -1625.19818061266 & & & & -1625.1002218105 & & & \\
\hline $\mathrm{FF} 2$ & -3250.40096728658 & -2.847 & -2.889 & 0.042 & -3250.20328463178 & -1.690 & -1.782 & 0.092 \\
\hline
\end{tabular}




\begin{tabular}{|c|c|c|c|c|}
\hline & \multicolumn{4}{|c|}{ RI-MP3/CBS(34) } \\
\hline & $\mathrm{E}[\mathrm{Ha}]$ & $\mathrm{BAE}\left[\mathrm{kcal} \mathrm{mol}^{-1}\right]$ & BIE $\left[\mathrm{kcal} \mathrm{mol}^{-1}\right]$ & E-def $\left[\mathrm{kcal} \mathrm{mol}^{-1}\right]$ \\
\hline \multicolumn{5}{|c|}{ Methanes } \\
\hline $\mathrm{H}$ & -40.450132125006 & & & \\
\hline $\mathrm{F}$ & -437.17451400149 & & & \\
\hline $\mathrm{Hx} 1$ & -40.450132335882 & & & \\
\hline $\mathrm{xH} 1$ & -40.450132270113 & & & \\
\hline HH1 & -80.901004636956 & -0.465 & -0.464 & 0.000 \\
\hline $\mathrm{Hx} 2$ & -40.45013194056 & & & \\
\hline $\mathrm{xH} 2$ & -40.450131906272 & & & \\
\hline HH2 & -80.900854151209 & -0.370 & -0.370 & 0.000 \\
\hline $\mathrm{Hx} 1$ & -40.45013219751 & & & \\
\hline $\mathrm{xF} 1$ & -437.174471626474 & & & \\
\hline HF1 & -477.625662076919 & -0.638 & -0.664 & 0.027 \\
\hline $\mathrm{Hx} 2$ & -40.450131893386 & & & \\
\hline $\mathrm{xF} 2$ & -437.174483188876 & & & \\
\hline $\mathrm{HF} 2$ & -477.625143108106 & -0.312 & -0.331 & 0.019 \\
\hline $\mathrm{Fx} 1$ & -437.174483057299 & & & \\
\hline $\mathrm{xF} 1$ & -437.174483723941 & & & \\
\hline FF1 & -874.349921058965 & -0.560 & -0.599 & 0.038 \\
\hline $\mathrm{Fx} 2$ & -437.174480664149 & & & \\
\hline $\mathrm{xF} 2$ & -437.174483324126 & & & \\
\hline FF2 & -874.349860161874 & -0.522 & -0.562 & 0.040 \\
\hline
\end{tabular}




\subsection{Rigid Geometry Scans}

The following tables provide the results of the rigid geometry scans using RI-MP2 and DLPNO-CCSD $(\mathrm{T}) \cdot \mathrm{R}_{\mathrm{C}-\mathrm{C}}$ refers to the distance of the carbon chains of the interacting molecules, E refers to the electronic energy of the whole system and BIE refers to the bond interaction energy at the respective distance.

\begin{tabular}{|c|c|c|c|c|c|c|c|c|}
\hline \multicolumn{9}{|c|}{ RI-MP2/CBS(34) } \\
\hline $\mathrm{R}_{\mathrm{C}-\mathrm{C}}[\AA]$ & $\mathrm{E}[\mathrm{Ha}]$ & $\mathrm{BIE}\left[\mathrm{kcal} \mathrm{mol}^{-1}\right]$ & $\mathrm{R}_{\mathrm{C}-\mathrm{C}}[\AA]$ & $\mathrm{E}[\mathrm{Ha}]$ & $\mathrm{BIE}\left[\mathrm{kcal} \mathrm{mol}^{-1}\right]$ & $\mathrm{R}_{\mathrm{C}-\mathrm{C}}[\AA]$ & $\mathrm{E}[\mathrm{Ha}]$ & $\mathrm{BIE}\left[\mathrm{kcal} \mathrm{mol}^{-1}\right]$ \\
\hline 1-HH1 & & & 1-HH2 & & & 1-HF1 & & \\
\hline 3.30371146716 & -80.871314375168 & -0.206096699090305 & 3.5804338401 & -80.871164919099 & -0.113164914840978 & 3.40464005758 & -477.625676691007 & -0.265071426879289 \\
\hline 3.41383518274 & -80.871597872807 & -0.383994153421553 & 3.69978163477 & -80.871426494135 & -0.277305728087989 & 3.5181280595 & -477.626115103494 & -0.540179415973888 \\
\hline 3.52395889831 & -80.871729151672 & -0.466372884943958 & 3.81912942944 & -80.871547069978 & -0.35296821191429 & 3.63161606142 & -477.626324103687 & -0.671329017164381 \\
\hline 3.63408261388 & -80.87176721384 & -0.490257255966371 & 3.93847722411 & -80.871583062571 & -0.375553905014655 & 3.74510406334 & -477.626395230664 & -0.715961869083122 \\
\hline 3.74420632945 & -80.871750641883 & -0.479858195943085 & 4.05782501878 & -80.87157063085 & -0.367752882306686 & 3.85859206526 & -477.626385375171 & -0.7097774538695 \\
\hline 3.85433004502 & -80.871703827028 & -0.450481430908516 & 4.17717281345 & -80.871532862273 & -0.344052742418505 & 3.97208006718 & -477.62632960142 & -0.674778896716017 \\
\hline 3.9644537606 & -80.871642239594 & -0.411834732591534 & 4.29652060812 & -80.871483605481 & -0.31314363878198 & 4.0855680691 & -477.626249854687 & -0.62473706621551 \\
\hline 4.07457747617 & -80.871575555299 & -0.369989705716685 & 4.41586840279 & -80.871430861487 & -0.280046282847654 & 4.19905607102 & -477.626159826906 & -0.568243780733287 \\
\hline 4.18470119174 & -80.871509508202 & -0.328544526614265 & 4.53521619746 & -80.871379053245 & -0.247536120157735 & 4.31254407294 & -477.626068293851 & -0.510805921528554 \\
\hline 4.29482490731 & -80.871447308882 & -0.289513864038841 & 4.65456399213 & -80.871330503495 & -0.217070692078107 & 4.42603207486 & -477.625980579791 & -0.455764517883792 \\
\hline 4.40494862289 & -80.871390531901 & -0.253885770557731 & 4.7739117868 & -80.871286325796 & -0.189348767418662 & 4.53952007678 & -477.625899782159 & -0.405063238330317 \\
\hline 4.51507233846 & -80.871339632057 & -0.22194563622367 & 4.89325958147 & -80.871246927011 & -0.16462565656329 & 4.6530080787 & -477.625827083041 & -0.359443853002518 \\
\hline 4.62519605403 & -80.87129472787 & -0.193767833454524 & 5.01260737614 & -80.871212291518 & -0.142891556572556 & 4.76649608062 & -477.625762480478 & -0.318905132683972 \\
\hline 4.7353197696 & -80.871255339209 & -0.169051075512503 & 5.13195517081 & -80.871182151959 & -0.12397869775697 & 4.87998408254 & -477.625705211272 & -0.282968163370774 \\
\hline 4.84544348517 & -80.871220937473 & -0.147463660249133 & 5.25130296548 & -80.871156105264 & -0.107634149879229 & 4.99347208446 & -477.625654186379 & -0.250949559587473 \\
\hline 4.95556720075 & -80.871190963884 & -0.128654949185662 & 5.37065076015 & -80.871133691272 & -0.093569157549499 & 5.10696008638 & -477.625608333626 & -0.222176522686138 \\
\hline 1-HF2 & & & 1-FF1 & & & 1-FF2 & & \\
\hline 3.82497722955 & -477.62504796232 & 0.130970790454453 & 3.67080659217 & -874.379996195724 & -0.295586445116713 & 3.88048063632 & -874.378909067058 & 0.38768979579546 \\
\hline 3.95247647054 & -477.625494231289 & -0.149067215527506 & 3.79316681191 & -874.380426057967 & -0.565329075173816 & 4.00982999086 & -874.37977942358 & -0.158467167517413 \\
\hline 4.07997571152 & -477.625718009162 & -0.289489950890891 & 3.91552703165 & -874.380618388711 & -0.686018439157131 & 4.13917934541 & -874.38023142074 & -0.442099667628959 \\
\hline 4.20747495251 & -477.625810847935 & -0.347747160511797 & 4.03788725139 & -874.380673966356 & -0.720893937910469 & 4.26852869995 & -874.380435608704 & -0.57022954956144 \\
\hline 4.33497419349 & -477.625830372334 & -0.359998905872243 & 4.16024747113 & -874.380653092282 & -0.707795258761323 & 4.39787805449 & -874.380499010687 & -0.610014894545747 \\
\hline 4.46247343448 & -477.62581113238 & -0.347925652466979 & 4.28260769087 & -874.38059112512 & -0.668910277477654 & 4.52722740904 & -874.380486609748 & -0.602233187837231 \\
\hline 4.58997267546 & -477.62577295038 & -0.323966085714584 & 4.40496791061 & -874.38050848369 & -0.617051997262556 & 4.65657676358 & -874.380436292132 & -0.570658407087547 \\
\hline 4.71747191645 & -477.625726952577 & -0.295102028549269 & 4.52732813035 & -874.38041696968 & -0.55962608896922 & 4.78592611813 & -874.380368917421 & -0.528380137648465 \\
\hline 4.84497115743 & -477.625679340599 & -0.265225061275488 & 4.64968835009 & -874.380323552297 & -0.501005796083257 & 4.91527547267 & -874.380295094996 & -0.482055866526288 \\
\hline 4.97247039842 & -477.625633475402 & -0.236444215652078 & 4.77204856983 & -874.380232455284 & -0.443841557400714 & 5.04462482721 & -874.380219736624 & -0.434767774159215 \\
\hline 5.0999696394 & -477.625591165247 & -0.209894192519202 & 4.89440878957 & -874.380146230332 & -0.389734583098939 & 5.17397418176 & -874.38014501555 & -0.387879592361225 \\
\hline 5.22746888039 & -477.625553027746 & -0.185962549322063 & 5.01676900931 & -874.380066334319 & -0.339599078038824 & 5.3033235363 & -874.380072123443 & -0.342139104621549 \\
\hline 5.35496812137 & -477.62551904461 & -0.164637809521719 & 5.13912922904 & -874.379993496201 & -0.293892468889151 & 5.43267289085 & -874.380002129658 & -0.298217341401261 \\
\hline 5.48246736236 & -477.625488818465 & -0.145670617180479 & 5.26148944878 & -874.379927978382 & -0.252779416789085 & 5.56202224539 & -874.379936225471 & -0.256861839711385 \\
\hline 5.60996660334 & -477.625461807651 & -0.128721075498562 & 5.38384966852 & -874.379869761769 & -0.216247940543583 & 5.69137159993 & -874.379875615966 & -0.218828801038807 \\
\hline 5.73746584433 & -477.625437491047 & -0.113462176132189 & 5.50620988826 & -874.379818653798 & -0.184177204550751 & 5.82072095448 & -874.379821292258 & -0.184740159668724 \\
\hline
\end{tabular}




\begin{tabular}{|c|c|c|c|c|c|c|c|c|c|c|c|}
\hline \multicolumn{12}{|c|}{ RI-MP2/CBS(34) } \\
\hline ] & a] & ] & & a] & {$\left[\mathrm{mol}^{-1}\right]$} & $\mathrm{R}_{\mathrm{C}-\mathrm{C}}$ & a] & IF & & [a] & 1] \\
\hline 2-HH1 & & & 2-HH2 & & & 2-HF1 & & & 2-HF2 & & \\
\hline 3.34273389134 & 59.350949729516 & -0.922018398173698 & 58048380658 & -159.350668726325 & -0.743231388291426 & 3.90237412775 & -754.466415720511 & -0.150693154709938 & 3.77935622887 & -754.466513107231 & -0.212 \\
\hline 3.45415835439 & -159.351483696921 & -1.25708800362146 & 3.6998332668 & -159.351214217536 & -1.08553229117745 & 4.03245326534 & -754.46722702637 & -0.6597 & 3.9053 & -754.4673 & -0.758 \\
\hline 3.56558281743 & -159.351716524255 & -1.40318936151428 & 81918272702 & -159.351431486543 & -1.22187065146477 & 4.16253240293 & -754.467602413971 & -0.895354543593359 & 4.03131331079 & -754.467799578388 & -1.01 \\
\hline 3.67700728048 & -159.3517571959 & -1.42871120407237 & 3.93853218724 & -159.351451338471 & -1.23432792437205 & 4.29261154053 & -754.467721543581 & -0.970109502483782 & 4.15729185176 & -754.467943916397 & -1.11036797970522 \\
\hline 3.78843174352 & -159.351680946545 & -1.38086401142086 & 4.05788164746 & -159.351359553442 & -1.17673194909463 & 4.42269067812 & -754.467698133337 & -0.955419352577752 & 4.28327039272 & -754.467932549948 & -1.1032354253245 \\
\hline 3.89985620657 & 159.351538617298 & 19343 & 17723110768 & -159.351210676546 & 17 & 71 & 4.467602149935 & 17378 & 4.4092 & -754.46 & 347 \\
\hline 4.01128066961 & -159.351363735611 & -1.18181114507059 & 4.29658056789 & -159.351038299307 & -0.975141935818958 & 4.6828489533 & -754.467475245236 & -0.815554957554053 & 4.53522747464 & -754.467704079445 & -0.959868020106714 \\
\hline 4.12270513266 & -159.351177757921 & -1.06510838264619 & 4.41593002811 & -159.3508623665 & -0.864742432641196 & 4.81292809089 & -754.467341108038 & -0.731382595017946 & 4.66120601561 & -754.467556383622 & -0.867 \\
\hline 4.234 & -159 & 194 & 3 & 4815 & 367 & 49 & 179 & 87 & 57 & -754.4 & 48 \\
\hline 4.34555405875 & -159.350819791931 & -0.840481332556879 & 4.65462894855 & -159.350539508614 & -0.66214605041769 & 5.07308636608 & -754.467094288005 & -0.576500685938342 & 4.91316309753 & -754.46727156323 & -0.688459997589334 \\
\hline 4.45697852179 & -159.35065970828 & 24924059 & 10877 & 9.350400932039 & -0.575187936741347 & 20316550367 & -754.466989026833 & 51 & 5.039 & -754.46 & -0. \\
\hline 4.56840298483 & -159.3505146464 & -0.648999620904695 & 4.89332786899 & -159.350278886194 & -0.498603012730381 & 5.33324464126 & -754.466896061924 & -0.452111942110083 & 5.16512017945 & -754.467034615334 & -0.5397 \\
\hline 4.67982744788 & -159.350385523694 & -0.567973899581551 & 5.01267732921 & -159.350172657412 & -0.431943445621594 & 5.46332377885 & -754.466814045371 & -0.400645778116915 & 5.29109872042 & -754.466936475194 & -0.478189080324272 \\
\hline 4.7 & & & & & & & & & & & \\
\hline 4.90267637397 & -159.350171547197 & -0.433701620495597 & 25137624965 & -159.350001949135 & -0.324822384506947 & 5.72348205404 & -754.466676479586 & -0.314321944697857 & 5.54305580234 & -754.466774551942 & -0.376 \\
\hline 5.01410083701 & -159.350084338888 & -0.378977580388251 & 37072570987 & -159.349934154841 & -0.28228082274943 & 5.85356119163 & -754.466618192283 & -0.277746109865832 & 5.6690343433 & -754.466707115693 & -0.3342 \\
\hline 2-HF3 & & & $2-\mathrm{HF} 4$ & & & & & & & & \\
\hline 4.1164580051 & -754.465943261392 & 0.148966552401164 & 3.78617705941 & -754.466356019137 & -0.105633868741545 & 4.18662183349 & -1349.58217947699 & 0.422403352588597 & 4.58731127773 & -1349.5813414665 & 0.959677054642131 \\
\hline 4.25367327193 & -754.466936785684 & -0.474479353459763 & 91238296139 & -754.467275370218 & -0.682535381962415 & 4.3261758946 & -1349.5835459017 & -0.435041098515189 & 4.74022165365 & -1349.58313615688 & 1808554 \\
\hline 4.39088853877 & -754.467377534664 & -0.751053514099607 & .03858886337 & -754.467722182154 & -0.962914104935162 & 4.46572995572 & -1349.5842539442 & -0.879344475282897 & 4.893132 & -1349.583 & -0.6846 \\
\hline 4.52810380561 & -754.46751435537 & -0.836909803329708 & 4.16479476535 & -754.467881858744 & -1.0631126779284 & 4.60528401684 & -1349.58456095936 & -1.07199939680257 & 0460424055 & -1349.5842695924 & 734245 \\
\hline 4.66531907244 & -754.467494946883 & -0.824730793866361 & 29100066733 & -754.467876041844 & -1.05946251803348 & 4.74483807 & -1349.58463511239 & -1.1185311256706 & 143 & -1349.58 & -0.907 \\
\hline 4.80253433928 & -754.467402560086 & -0.76675720350744 & 4.41720656931 & -754.467782122607 & -1.00052730704131 & 4.88439213907 & -1349.58458250581 & -1.08551999827309 & 5.35 & -1349.58424199907 & -0.860 \\
\hline 4.93974960612 & -754.4672 & -0.691211665448513 & 29 & 4.46 & 21655931 & 18 & -1349.584 & -1.0130 & 27 & -1349.584 & -0 . \\
\hline 5.07696487295 & -754.467157190089 & -0.612785205716842 & 4.66961837327 & -754.467501219054 & -0.824257666286135 & .1635002613 & -1349.5843245924 & -0.923676890028927 & 6576839092 & -1349.58398553846 & -0.699503150226163 \\
\hline 5.21418013979 & 754.467039177046 & 0.538730903183184 & 9582427525 & -754.467358163094 & -0.734488696041244 & 5.30305432242 & -1349.58417461646 & -0.829565566759294 & & 1349.58385309267 & -0.616392162298197 \\
\hline 540663 & -754.466933228916 & -0.472247447856241 & 92203017723 & -754.467226396483 & -0.651803899323041 & 8353 & -1349.5 & -0.7 & 05 & 9484 & 0218131 \\
\hline 5.48861067346 & -754.466840851946 & -0.414280024018215 & 04823607921 & -754.467108882831 & -0.578062969323857 & 5.58216244465 & -1349.58388418813 & -0.647319038235104 & 6.11641503697 & -1349.58360844471 & -0.462873249592967 \\
\hline & -754.4667 & 364448944440687 & & & & & & & & & \\
\hline 5.76304120714 & -754.46669317709 & -0.321612652765051 & 0064788317 & -754.466915846803 & -0.456931032925216 & 5.86127056688 & -1349.58362490683 & -0.484617566034023 & 6.42223578882 & -1349.58339702867 & 7681553587 \\
\hline 25647397 & -754.466633760049 & -0.284327896673321 & 2685378515 & -754.466837029369 & -0.407472346390664 & 00824628 & -1349.5835103514 & -0.412732948445053 & 6.57514616474 & -1349.58330754626 & -0.274056621394634 \\
\hline 81 & -754.46 & 68 & & & & & & & & & 26 \\
\hline 6.17468700765 & -754.466533425286 & -0.22136688229853 & 5.67926558911 & -754.466704176365 & -0.324105827764389 & 6.27993275023 & -1349.58331754881 & -0.291747496648453 & 6.88096691659 & -1349.58316650397 & -0.185551248249558 \\
\hline
\end{tabular}




\begin{tabular}{|c|c|c|c|c|c|c|c|c|c|c|c|}
\hline \multicolumn{12}{|c|}{ RI-MP2/CBS(34) } \\
\hline $\mathrm{R}_{\mathrm{C}-\mathrm{C}}[\AA]$ & $\mathrm{E}[\mathrm{Ha}]$ & $\mathrm{BIE}\left[\mathrm{kcal} \mathrm{mol}^{-1}\right]$ & $\mathrm{R}_{\mathrm{C}-\mathrm{C}}[\AA]$ & $\mathrm{E}[\mathrm{Ha}]$ & $\mathrm{BIE}\left[\mathrm{kcal} \mathrm{mol}^{-1}\right]$ & $\mathrm{R}_{\mathrm{C}-\mathrm{C}}[\AA]$ & $\mathrm{E}[\mathrm{Ha}]$ & BIE $\left[\mathrm{kcal} \mathrm{mol}^{-1}\right]$ & $\mathrm{R}_{\mathrm{C}-\mathrm{C}}[\AA]$ & $\mathrm{E}[\mathrm{Ha}]$ & BIE $\left[\mathrm{kcal} \mathrm{mol}^{-1}\right]$ \\
\hline 3-HH1 & & & 3 - $\mathrm{HH} 2$ & & & 3-HF1 & & & $3-\mathrm{HF} 2$ & & \\
\hline 3.43594668895 & -237.837861377554 & -1.27907407939742 & 4.03806907476 & -237.836522602713 & -0.442077485178922 & 4.05575650873 & -1031.3113995789 & -0.220184335009038 & 4.26586656335 & -1031.31130221495 & -0.141033872547684 \\
\hline 3.55047824525 & -237.83874095408 & -1.83101668258137 & 4.17267137725 & -237.837307613826 & -0.934679395787768 & 4.19094839235 & -1031.31285655846 & -1.13445281238934 & 4.40806211546 & -1031.31258788714 & -0.947805352137851 \\
\hline 3.66500980155 & -237.839150172 & -2.0878048042995 & 4.30727367975 & -237.837678630023 & -1.16749557440859 & 4.32614027597 & -1031.3135109562 & -1.54509359396339 & 4.55025766757 & -1031.31312693244 & -1.28606138480416 \\
\hline 3.77954135785 & -237.839254745088 & -2.15342540775082 & 4.44187598224 & -237.837790617196 & -1.23776858643242 & 4.4613321596 & -1031.31370274065 & -1.66544015327481 & 4.69245321968 & -1031.31325615605 & -1.36715042447023 \\
\hline 3.89407291414 & -237.839169394898 & -2.09986735491477 & 4.57647828473 & -237.837749307621 & -1.21184643676427 & 4.59652404322 & -1031.31364340516 & -1.62820657126654 & 4.83464877179 & -1031.31317832977 & -1.31831369633365 \\
\hline 4.00860447044 & -237.838973324662 & -1.97683142425481 & 4.71108058722 & -237.837624452991 & -1.13349897356532 & 4.73171592685 & -1031.31345993928 & -1.51307999333098 & 4.97684432391 & -1031.31300847649 & -1.21172915401553 \\
\hline 4.12313602674 & -237.838719333703 & -1.81744969118365 & 4.84568288971 & -237.837460299879 & -1.03049134060137 & 4.86690781047 & -1031.31322601078 & -1.3662876433813 & 5.11903987602 & -1031.3128076432 & -1.08570436176005 \\
\hline 4.23766758304 & -237.838442099831 & -1.64348280999129 & 4.98028519221 & -237.837283714427 & -0.919682296494421 & 5.00209969409 & -1031.31298287917 & -1.21372025469163 & 5.26123542813 & -1031.31260627994 & -0.959347008409867 \\
\hline 4.35219913934 & -237.83816395024 & -1.46894130643353 & 5.1148874947 & -237.837110149298 & -0.810768533697704 & 5.13729157772 & -1031.31275244341 & -1.06911963218628 & 5.40343098024 & -1031.31241863296 & -0.841596750793424 \\
\hline 4.46673069564 & -237.837898055062 & -1.30208956315436 & 5.24948979719 & -237.836947820504 & -0.708905677556631 & 5.27248346134 & -1031.3125449229 & -0.938898546110254 & 5.54562653235 & -1031.31225064694 & -0.736183931626196 \\
\hline 4.58126225193 & -237.837651986313 & -1.14767909190598 & 5.38409209968 & -237.836800543288 & -0.616487829211417 & 5.40767534497 & -1031.31236339598 & -0.824988683906854 & 5.68782208446 & -1031.312103845 & -0.644064323480366 \\
\hline 4.69579380823 & -237.837429162035 & -1.00785474641623 & 5.51869440218 & -237.836669547111 & -0.534286487082257 & 5.54286722859 & -1031.3122066766 & -0.726645788258997 & 5.83001763658 & -1031.31197713831 & -0.564554675212889 \\
\hline 4.81032536453 & -237.837230414556 & -0.883138820407496 & 5.65329670467 & -237.836554616218 & -0.462166262866155 & 5.67805911222 & -1031.31207134739 & -0.641725426859504 & 5.97221318869 & -1031.31186788429 & -0.495996742516836 \\
\hline 4.92485692083 & -237.83705498468 & -0.773054911207008 & 5.78789900716 & -237.836454635955 & -0.399427700619418 & 5.81325099584 & -1031.31195328892 & -0.567642618461369 & 6.1144087408 & -1031.31177282207 & -0.43634429884862 \\
\hline 5.03938847713 & -237.836901102104 & -0.676492136882983 & 5.92250130965 & -237.836368497742 & -0.345375155893108 & 5.94844287946 & -1031.31184871055 & -0.502018700555898 & 6.25660429291 & -1031.31168888208 & -0.383671159899212 \\
\hline 5.15392003343 & 766974394 & 181 & 6.05710361214 & 94 & -0 & 6.08363476309 & -1031.3117547 & -0.443 & 6.39879984502 & -103 & -0 . \\
\hline 3 -HF3 & & & $3-\mathrm{HF} 4$ & & & 3 -FF1 & & & $3-\mathrm{FF} 2$ & & \\
\hline 4.37726127205 & -1031.31209771694 & -0.646629838228183 & 4.22420781328 & -1031.31058766216 & 0.30178644078492 & 4.44732592776 & -1824.78445056558 & 1.1247675713992 & 4.42086165258 & -1824.78052595176 & 3.70345943850342 \\
\hline 4.52316998112 & -1031.31297410286 & -1.19657030593169 & 4.36501474039 & -1031.31220980401 & -0.716122938275052 & 4.59557012535 & -1824.78702710472 & -0.492035149025557 & 4.56822370767 & -1824.78559165462 & 0.524682901343512 \\
\hline 4.66907869019 & -1031.31328584863 & -1.39219373007186 & 4.5058216675 & -1031.3129888652 & -1.20499121573787 & 4.74381432294 & -1824.7882612566 & -1.26647714607955 & 4.71558576275 & -1824.78802608247 & -1.00294363826856 \\
\hline 4.81498739925 & -1031.31329098054 & -1.39541405223501 & 4.6466285946 & -1031.31327446575 & -1.38420826671427 & 4.89205852053 & -1824.78875098608 & -1.57378703449108 & 4.86294781784 & -1824.7890464368 & -1.6432256471596 \\
\hline 4.96089610832 & -1031.3131452053 & -1.30393870815846 & 4.78743552171 & -1031.31328710823 & -1.39214154268117 & 5.04030271812 & -1824.78884791919 & -1.63461347932528 & 5.01030987293 & -1824.78934412849 & -1.83003000302429 \\
\hline 5.10680481739 & -1031.31293825837 & -1.17407754889265 & 4.92824244882 & -1031.31316088249 & -1.31293369488555 & 5.18854691572 & -1824.78875142358 & -1.57406156983622 & 5.15767192801 & -1824.78929792993 & -1.80103996888578 \\
\hline 5.25271352646 & -1031.3127180476 & -1.03589320439887 & 5.06904937593 & -1031.31297302434 & -1.19505092601954 & 5.33679111331 & -1824.78856912456 & -1.45966720766942 & 5.3050339831 & -1824.78910640865 & -1.68085855125686 \\
\hline 5.39862223553 & -1031.31250807999 & -0.904136539977102 & 5.20985630304 & -1031.31276593365 & -1.06509955615817 & 5.4850353109 & -1824.78835469745 & -1.32511216466507 & 5.45239603818 & -1824.78886681809 & -1.53051320490476 \\
\hline 5.5445309446 & -1031.31231861639 & -0.78524633599342 & 5.35066323015 & -1031.31256212623 & -0.937208469160786 & 5.63327950849 & -1824.78813210007 & -1.18543019989738 & 5.59975809327 & -1824.78862081133 & -1.37614163241176 \\
\hline 5.69043965366 & -1031.31215278152 & -0.681183383866519 & 5.49147015726 & -1031.31237305927 & -0.818567160510878 & 5.78152370608 & -1824.78791128926 & -1.04686932459436 & 5.74712014836 & -1824.78838193633 & -1.22624530685852 \\
\hline 5.83634836273 & -1031.31200985422 & -0.591495149038477 & 5.63227708437 & -1031.31220392045 & -0.71243094856727 & 5.92976790367 & -1824.78769733805 & -0.912612913443614 & 5.89448220344 & -1824.7881525155 & -1.08228156249431 \\
\hline 5.9822570718 & -1031.31188725786 & -0.514564771713646 & 5.77308401148 & -1031.3120562612 & -0.619773370310587 & 6.07801210127 & -1824.78749441437 & -0.785276381722467 & 6.04184425853 & -1824.78793286868 & -0.944451101921318 \\
\hline 6.12816578087 & -1031.31178179961 & -0.44838872065294 & 5.91389093859 & -1031.31192944759 & -0.540196628606443 & 6.22625629886 & -1824.78730661091 & -0.667427931326234 & 6.18920631361 & -1824.78772482796 & -0.813903579109802 \\
\hline 6.27407448994 & -1031.31169039359 & -0.391030577172944 & 6.0546978657 & -1031.31182148696 & -0.472450310477856 & 6.37450049645 & -1824.78713747843 & -0.56129569770496 & 6.3365683687 & -1824.78753178467 & -0.692767085753593 \\
\hline 6.419983199 & -1031.31161042869 & -0.340851844900511 & 6.19550479281 & -1031.31172959288 & -0.414785904664084 & 6.52274469404 & -1824.78698940015 & -0.468375174214675 & 6.48393042379 & -1824.78735736284 & -0.583315735083428 \\
\hline 6.56589190807 & -1031.31153990507 & -0.296597605194244 & 6.33631171992 & -1031.31165070082 & -0.365280389624495 & 6.67098889163 & -1824.78686323827 & -0.389207399138361 & 6.63129247887 & -1824.78720417806 & -0.48719083425142 \\
\hline
\end{tabular}




\begin{tabular}{|c|c|c|c|c|c|c|c|c|c|c|c|}
\hline \multicolumn{12}{|c|}{ RI-MP2/CBS(34) } \\
\hline $\mathrm{R}_{\mathrm{C}-\mathrm{C}}[\AA]$ & $\mathrm{E}[\mathrm{Ha}]$ & $\mathrm{BIE}\left[\mathrm{kcal} \mathrm{mol}^{-1}\right]$ & $\mathrm{R}_{\mathrm{C}-\mathrm{C}}[\AA]$ & $\mathrm{E}[\mathrm{Ha}]$ & $\mathrm{BIE}\left[\mathrm{kcal} \mathrm{mol}^{-1}\right]$ & $\mathrm{R}_{\mathrm{C}-\mathrm{C}}[\AA]$ & $\mathrm{E}[\mathrm{Ha}]$ & BIE $\left[\mathrm{kcal} \mathrm{mol}^{-1}\right]$ & $\mathrm{R}_{\mathrm{C}-\mathrm{C}}[\AA]$ & $\mathrm{E}[\mathrm{Ha}]$ & $\mathrm{BIE}\left[\mathrm{kcal} \mathrm{\textrm {mol } ^ { - 1 } ]}\right.$ \\
\hline 4-HH1 & & & 4-HH2 & & & 4-HF1 & & & 4-HF2 & & \\
\hline 3.45206253651 & -316.32564440408 & -2.0236029269884 & 4.10663636738 & -316.323655396208 & -0.778031196860239 & 4.0391979304 & -1308.15613439667 & -0.403824918087795 & 4.07104188193 & -1308.15630426445 & -0.283449362666392 \\
\hline 3.56713128772 & -316.326814175797 & -2.75764576182457 & 4.24352424629 & -316.324630880772 & -1.3901570025344 & 4.17383786142 & -1308.15806771584 & -1.6170010134617 & 4.206743278 & -1308.15829111768 & -1.53021858789934 \\
\hline 3.68220003894 & -316.327342334374 & -3.08907027268291 & 4.38041212521 & -316.325073551191 & -1.66793688430191 & 4.30847779243 & -1308.15894102419 & -2.16501027683348 & 4.34244467406 & -1308.15922061181 & -2.11348496056763 \\
\hline 3.79726879016 & -316.327453943624 & -3.15910613443404 & 4.51730000412 & -316.325185961149 & -1.73847519790526 & 4.44311772345 & -1308.15918831531 & -2.32018779753475 & 4.47814607013 & -1308.15951830946 & -2.30029305633781 \\
\hline 3.91233754137 & -316.327306557984 & -3.06662024900802 & 4.65418788303 & -316.325104942558 & -1.68763526451575 & 4.57775765446 & -1308.15909063928 & -2.25889516332134 & 4.61384746619 & -1308.15946100554 & -2.26433430355895 \\
\hline 4.02740629259 & -316.327008835124 & -2.87979633372436 & 4.79107576194 & -316.324919958382 & -1.57155594151658 & 4.71239758547 & -1308.15882413314 & -2.09166003548013 & 4.74954886225 & -1308.15922245654 & -2.11464254607102 \\
\hline 4.14247504381 & -316.326633873091 & -2.64450410563114 & 4.92796364086 & -316.324687461667 & -1.42566205018004 & 4.84703751649 & -1308.15849258775 & -1.88361216230285 & 4.88525025832 & -1308.15890683864 & -1.91658932364805 \\
\hline 4.25754379502 & -316.32622991513 & -2.39101665800716 & 5.06485151977 & -316.324441569378 & -1.27136230924812 & 4.9816774475 & -1308.15815260346 & -1.6702687992403 & 5.02095165438 & -1308.15857311715 & -1.70717592696354 \\
\hline 4.37261254624 & -316.325827446367 & -2.13846369622711 & 5.20173939868 & -316.324201776109 & -1.12088976116512 & 5.11631737851 & -1308.1578323836 & -1.4693278033358 & 5.15665305045 & -1308.15825227922 & -1.50584708636506 \\
\hline 4.48768129746 & -316.32544457813 & -1.89821025020886 & 5.33862727759 & -316.32397834577 & -0.980685106651279 & 5.25095730953 & -1308.15754406009 & -1.28840206926593 & 5.29235444651 & -1308.1579588236 & -1.32170090457441 \\
\hline 4.60275004867 & -316.325090964031 & -1.67631405296552 & 5.47551515651 & -316.323775969878 & -0.85369231711096 & 5.38559724054 & -1308.15729098345 & -1.12959407995991 & 5.42805584258 & -1308.15769792257 & -1.15798303643523 \\
\hline 4.71781879989 & -316.324770885697 & -1.47546186594108 & 5.61240303542 & -316.323596084211 & -0.74081235681695 & 5.52023717155 & -1308.15707192951 & -0.992135657372862 & 5.56375723864 & -1308.15746967203 & -1.01475366015454 \\
\hline 4.83288755111 & -316.324485020144 & -1.29607852314568 & 5.74929091433 & -316.323438289386 & -0.641794609190055 & 5.65487710257 & -1308.1568834251 & -0.873847354168304 & 5.69945863471 & -1308.15727156061 & -0.890436867274115 \\
\hline 4.94795630232 & -316.324232086675 & -1.13736037507459 & 5.88617879324 & -316.323301187214 & -0.555761697351135 & 5.78951703358 & -1308.1567210049 & -0.77192713989117 & 5.83516003077 & -1308.15709986937 & -0.782698987581169 \\
\hline 5.06302505354 & -316.324009740335 & -0.997835940200208 & 6.02306667216 & -316.323182885079 & -0.481525986857464 & 5.92415696459 & -1308.15657993674 & -0.683405532999417 & 5.97086142683 & -1308.1569505106 & -0.68897494428149 \\
\hline 5.17809380476 & 66165 & -0.8757 & 6.15995455107 & 95161 & -0.417 & 6.05879689561 & -1308.1 & -0.60 & 6.1065628229 & -1308 & -0.6 \\
\hline 4-HF3 & & & $4-\mathrm{HF} 4$ & & & 4 -FF1 & & & 4-FF2 & & \\
\hline 4.08162960506 & -1308.15519200744 & 0.384345310824318 & 4.17634016747 & -1308.15638707146 & -0.331373579686099 & 4.75115085185 & -2299.98867221467 & 0.359444153805805 & 4.63747496367 & -2299.98657913224 & 1.58090035586702 \\
\hline 4.21768392523 & -1308.15772169597 & -1.20305820794187 & 4.31555150639 & -1308.15828290484 & -1.521026986684 & 4.90952254691 & -2299.991298698 & -1.28869901907521 & 4.79205746246 & -2299.99031882083 & -0.765789664186952 \\
\hline 4.3537382454 & -1308.15895653107 & -1.97792893211324 & 4.4547628453 & -1308.15914304788 & -2.06077489326169 & 5.06789424198 & -2299.99236170672 & -1.95574706206679 & 4.94663996125 & -2299.99206476011 & -1.86138310355788 \\
\hline 4.48979256557 & -1308.159421429 & -2.26965678762324 & 4.59397418422 & -1308.15939429479 & -2.21843470960689 & 5.22626593704 & -2299.9926239729 & -2.12032157473662 & 5.10122246004 & -2299.99271294703 & -2.26812653677373 \\
\hline 4.62584688574 & -1308.15945200837 & -2.28884563193011 & 4.73318552313 & -1308.15931096664 & -2.1661455062285 & 5.3846376321 & -2299.99251293278 & -2.05064284720833 & 5.25580495883 & -2299.99278955683 & -2.31619991184578 \\
\hline 4.76190120591 & -1308.15925682531 & -2.1663664126243 & 4.87239686205 & -1308.15906392204 & -2.01112267910198 & 5.54300932716 & -2299.99225113552 & -1.88636258628532 & 5.41038745762 & -2299.99259634766 & -2.19495932721164 \\
\hline 4.89795552608 & -1308.15895978383 & -1.9799700697201 & 5.01160820097 & -1308.15875343387 & -1.81628841097728 & 5.70138102222 & -2299.99194493412 & -1.69421830697097 & 5.56496995641 & -2299.9922949113 & -2.00580515570587 \\
\hline 5.03400984625 & -1308.1586307797 & -1.77351686117214 & 5.15081953988 & -1308.15843400863 & -1.61584604640579 & 5.85975271728 & -2299.99163773285 & -1.50144659958264 & 5.7195524552 & -2299.99196533771 & -1.79899460555096 \\
\hline 5.17006416641 & -1308.15830727499 & -1.57051459078367 & 5.2900308788 & -1308.15813261128 & -1.42671635407394 & 6.01812441235 & -2299.99134245093 & -1.31615439728859 & 5.87413495398 & -2299.99164178973 & -1.59596518260961 \\
\hline 5.30611848658 & -1308.15800791066 & -1.38266063754606 & 5.42924221771 & -1308.15786050491 & -1.25596702885299 & 6.17649610741 & -2299.99106125625 & -1.13970207145246 & 6.02871745277 & -2299.99133512726 & -1.4035315774029 \\
\hline 5.44217280675 & -1308.15774038252 & -1.21478419509867 & 5.56845355663 & -1308.15762030431 & -1.10523887680595 & 6.33486780247 & -2299.99079739006 & -0.974123537405948 & 6.18329995156 & -2299.99104708878 & -1.22278470233029 \\
\hline 5.57822712692 & -1308.157506039 & -1.06773141620461 & 5.70766489555 & -1308.15741020914 & -0.973402167106314 & 6.49323949753 & -2299.99055208258 & -0.820190769743027 & 6.33788245035 & -2299.9907779907 & -1.05392310767921 \\
\hline 5.71428144709 & -1308.15730269285 & -0.940129780514541 & 5.84687623446 & -1308.15722656343 & -0.858162744242505 & 6.65161119259 & -2299.99033241357 & -0.682346384685167 & 6.49246494914 & -2299.99052950073 & -0.897993297538926 \\
\hline 5.85033576726 & -1308.15712639721 & -0.829502596250264 & 5.98608757338 & -1308.15706527243 & -0.756951113646242 & 6.80998288765 & -2299.99014268995 & -0.563293015838413 & 6.64704744793 & -2299.99030444649 & -0.75676962963825 \\
\hline 5.98639008743 & -1308.15697257382 & -0.732976961661903 & 6.12529891229 & -1308.1569225265 & -0.667376690112234 & 6.96835458272 & -2299.98998434538 & -0.463930297955702 & 6.80162994672 & -2299.99010552769 & -0.63194619812905 \\
\hline 6.1224444076 & -1308.1568368484 & -0.647807974734881 & 6.26451025121 & -1308.15679513315 & -0.58743615613474 & 7.12672627778 & -2299.98985577784 & -0.383252948662157 & 6.95621244551 & -2299.98993418862 & -0.524429308299203 \\
\hline
\end{tabular}




\begin{tabular}{|c|c|c|c|c|c|c|c|c|c|c|c|}
\hline \multicolumn{12}{|c|}{ RI-MP2/CBS(34) } \\
\hline $\mathrm{R}_{\mathrm{C}-\mathrm{C}}[\AA]$ & $\mathrm{E}[\mathrm{Ha}]$ & $\mathrm{BIE}\left[\mathrm{kcal} \mathrm{mol}^{-1}\right]$ & $\mathrm{R}_{\mathrm{C}-\mathrm{C}}[\AA]$ & $\mathrm{E}[\mathrm{Ha}]$ & $\mathrm{BIE}\left[\mathrm{kcal} \mathrm{mol}^{-1}\right]$ & $\mathrm{R}_{\mathrm{C}-\mathrm{C}}[\AA]$ & $\mathrm{E}[\mathrm{Ha}]$ & $\mathrm{BIE}\left[\mathrm{kcal} \mathrm{mol}^{-1}\right]$ & $\mathrm{R}_{\mathrm{C}-\mathrm{C}}[\AA]$ & $\mathrm{E}[\mathrm{Ha}]$ & BIE $\left[\mathrm{kcal} \mathrm{mol}^{-1}\right]$ \\
\hline 5-HH1 & & & 5 - $\mathrm{HH} 2$ & & & 5-HF1 & & & $5-\mathrm{HF} 2$ & & \\
\hline 3.45905710032 & -394.813085628483 & -2.61008030011037 & 4.15692279807 & -394.809603043717 & -0.442442300372499 & 4.10335551576 & -1585.00185437984 & -0.755286779080415 & 4.32605659689 & -1585.00169258799 & -0.728701928505616 \\
\hline 3.57435900366 & -394.814633576371 & -3.58143226509733 & 4.29548689134 & -394.811368359287 & -1.5501945451278 & 4.24013403296 & -1585.00419094514 & -2.22150364135994 & 4.47025848346 & -1585.00388581532 & -2.10497285674995 \\
\hline 3.68966090701 & -394.815342800908 & -4.02647738125208 & 4.43405098461 & -394.81222066863 & -2.08502673265863 & 4.37691255015 & -1585.0052218196 & -2.86838713148758 & 4.61446037002 & -1585.00477682229 & -2.66408817185668 \\
\hline 3.80496281035 & -394.815505454051 & -4.12854376944743 & 4.57261507788 & -394.812520570453 & -2.27321796785117 & 4.51369106734 & -1585.00549862548 & -3.04208544367686 & 4.75866225658 & -1585.00494629473 & -2.77043373347148 \\
\hline 3.9202647137 & -394.815326837432 & -4.01646014881367 & 4.71117917115 & -394.812499360426 & -2.25990847496456 & 4.65046958453 & -1585.00536571698 & -2.95868410071194 & 4.90286414315 & -1585.00474170385 & -2.64205101797572 \\
\hline 4.03556661704 & -394.814948731591 & -3.779195151424 & 4.84974326442 & -394.812302974418 & -2.13667439439266 & 4.78724810173 & -1585.00503125628 & -2.74880684288633 & 5.04706602971 & -1585.00436807736 & -2.4075968557349 \\
\hline 4.15086852038 & -394.814467116977 & -3.4769774183292 & 4.98830735769 & -394.812019659631 & -1.95889168142722 & 4.92402661892 & -1585.00461599273 & -2.48822503094976 & 5.19126791627 & -1585.00394105381 & -2.13963553252256 \\
\hline 4.26617042373 & -394.81394529263 & -3.14952769682034 & 5.12687145096 & -394.811704559238 & -1.76116319957018 & 5.06080513611 & -1585.00418645284 & -2.21868468059866 & 5.33546980283 & -1585.0035210091 & -1.8760534975401 \\
\hline 4.38147232707 & -394.813423420483 & -2.82204798034053 & 5.26543554423 & -394.811388393271 & -1.56276605989907 & 5.1975836533 & -1585.00377719983 & -1.96187453953059 & 5.4796716894 & -1585.00313626399 & -1.63462229589709 \\
\hline 4.49677423042 & -394.812925537337 & -2.50962158927386 & 5.4039996375 & -394.811088331554 & -1.37447448970653 & 5.33436217049 & -1585.00340473237 & -1.72814767961713 & 5.62387357596 & -1585.00279734042 & -1.42194454484144 \\
\hline 4.61207613376 & -394.812464642187 & -2.22040551615564 & 5.54256373077 & -394.810813008687 & -1.201706782239 & 5.47114068769 & -1585.00307510737 & -1.52130486928165 & 5.76807546252 & -1585.00250483238 & -1.23839297849057 \\
\hline 4.7273780371 & -394.812046486195 & -1.95800866956047 & 5.68112782403 & -394.810565947916 & -1.04667380780804 & 5.60791920488 & -1585.0027879402 & -1.3411047495023 & 5.91227734909 & -1585.00225413988 & -1.08108105967767 \\
\hline 4.84267994045 & -394.811672266429 & -1.72318222103633 & 5.8196919173 & -394.810347649498 & -0.909689482341613 & 5.74469772207 & -1585.00253894343 & -1.18485691727122 & 6.05647923565 & -1585.00203864698 & -0.945857223349749 \\
\hline 4.95798184379 & -394.811340517787 & -1.51500680519635 & 5.95825601057 & -394.810156893262 & -0.789988137021487 & 5.88147623926 & -1585.00232198658 & -1.04871443847593 & 6.20068112221 & -1585.0018517505 & -0.828577911464193 \\
\hline 5.07328374714 & -394.811048347524 & -1.33166719711931 & 6.09682010384 & -394.809991531633 & -0.686222148187047 & 6.01825475645 & -1585.00213082188 & -0.928756778114617 & 6.34488300878 & -1585.00168790661 & -0.725764318200819 \\
\hline 18858565048 & 16973 & 81632 & 6.23538419711 & 26 & 01 & 6.15503327365 & 19602 & -0.8216 & 6.48908489534 & -1585 & -0.6 \\
\hline 5 -HF3 & & & 5 -HF4 & & & 5 -FF1 & & & 5 -FF2 & & \\
\hline 4.28282642262 & -1585.00117601367 & -0.185916823298706 & 4.08325326395 & -1585.00156467174 & -0.438043018372701 & 4.5471930552 & -2775.19101248781 & 1.11537394351929 & 4.89471861206 & -2775.19067480704 & 1.37168995849074 \\
\hline 4.42558730337 & -1585.0037138363 & -1.77842456700206 & 4.21936170608 & -1585.00417563905 & -2.07644974165176 & 4.69876615704 & -2775.19509961334 & -1.44933604803141 & 5.05787589912 & -2775.19483264114 & -1.23739033040057 \\
\hline 4.56834818412 & -1585.0048705519 & -2.50427456473713 & 4.35547014821 & -1585.00541528991 & -2.85434240078111 & 4.85033925888 & -2775.19698422899 & -2.63195022321876 & 5.22103318619 & -2775.19670237998 & -2.41066916641524 \\
\hline 4.71110906488 & -1585.00522277345 & -2.72529692429295 & 4.49157859034 & -1585.00582358259 & -3.11054992567687 & 5.00191236072 & -2775.1976487433 & -3.04893924825614 & 5.38419047326 & -2775.19732169336 & -2.79929417999859 \\
\hline 4.85386994563 & -1585.00513440654 & -2.6698458511116 & 4.62768703247 & -1585.00575716769 & -3.06887394674497 & 5.15348546256 & -2775.19767813052 & -3.06738000745009 & 5.54734776033 & -2775.19730548382 & -2.78912253991441 \\
\hline 4.99663082639 & -1585.00482861738 & -2.47796025620382 & 4.7637954746 & -1585.00544556325 & -2.87333920853112 & 5.3050585644 & -2775.19740897956 & -2.89848522991247 & 5.7105050474 & -2775.1970010412 & -2.5980819116781 \\
\hline 5.13939170714 & -1585.00443591668 & -2.2315368464422 & 4.89990391674 & -1585.00502916063 & -2.61204261949776 & 5.45663166624 & -2775.19701638743 & -2.65212994886732 & 5.87366233447 & -2775.19659212646 & -2.34148403821221 \\
\hline 5.28215258789 & -1585.00402819551 & -1.97568794954848 & 5.03601235887 & -1585.00458814091 & -2.33529856695896 & 5.60820476808 & -2775.19659197969 & -2.38581007120587 & 6.03681962154 & -2775.19616659829 & -2.07446108000413 \\
\hline 5.42491346865 & -1585.00364241596 & -1.73360762703843 & 5.172120801 & -1585.0041645749 & -2.06950688274864 & 5.75977786992 & -2775.1961737625 & -2.12337482248571 & 6.1999769086 & -2775.19575963046 & -1.81908491118905 \\
\hline 5.5676743494 & -1585.00329539503 & -1.51584870571103 & 5.30822924313 & -1585.00377776481 & -1.82677988660407 & 5.91135097176 & -2775.19577430191 & -1.87270951779577 & 6.36313419567 & -2775.19538092313 & -1.58144247366034 \\
\hline 5.71043523016 & -1585.00299214504 & -1.32555646406865 & 5.44433768526 & -1585.00343396224 & -1.61104051681445 & 6.0629240736 & -2775.19539654296 & -1.63566219779472 & 6.52629148274 & -2775.1950309865 & -1.36185392313259 \\
\hline 5.85319611091 & -1585.00273080417 & -1.16156259213067 & 5.58044612739 & -1585.00313235281 & -1.42177774208845 & 6.21449717544 & -2775.19504220271 & -1.41331033365139 & 6.68944876981 & -2775.19470948101 & -1.16010618201415 \\
\hline 5.99595699166 & -1585.00250599392 & -1.02049203049938 & 5.71655456953 & -1585.00286878135 & -1.25638415373111 & 6.36607027728 & -2775.19471468778 & -1.20779161237415 & 6.85260605688 & -2775.19441796739 & -0.977178623861053 \\
\hline 6.13871787242 & -1585.00231114745 & -0.898224024539625 & 5.85266301166 & -1585.00263794612 & -1.11153286010746 & 6.51764337912 & -2775.19441860577 & -1.02199734588709 & 7.01576334395 & -2775.19415932317 & -0.814876925328425 \\
\hline 6.28147875317 & -1585.00214007931 & -0.790877146031166 & 5.98877145379 & -1585.00243455607 & -0.983903676785985 & 6.66921648096 & -2775.19415799273 & -0.858460194427516 & 7.17892063102 & -2775.19393594099 & -0.674702490968874 \\
\hline 6.42423963392 & -1585.00198783895 & -0.695344877827 & 6.12487989592 & -1585.00225389129 & -0.870534815626312 & 6.8207895828 & -2775.19393263197 & -0.717044182211092 & 7.34207791808 & -2775.19374824127 & -0.55691913849892 \\
\hline
\end{tabular}




\begin{tabular}{|c|c|c|c|c|c|c|c|c|c|c|c|}
\hline \multicolumn{12}{|c|}{ RI-MP2/CBS(34) } \\
\hline $\mathrm{R}_{\mathrm{C}-\mathrm{C}}[\AA]$ & $\mathrm{E}[\mathrm{Ha}]$ & $\mathrm{BIE}\left[\mathrm{kcal} \mathrm{mol}^{-1}\right]$ & $\mathrm{R}_{\mathrm{C}-\mathrm{C}}[\AA]$ & $\mathrm{E}[\mathrm{Ha}]$ & BIE $\left[\mathrm{kcal} \mathrm{mol}^{-1}\right]$ & $\mathrm{R}_{\mathrm{C}-\mathrm{C}}[\AA]$ & $\mathrm{E}[\mathrm{Ha}]$ & $\mathrm{BIE}\left[\mathrm{kcal} \mathrm{mol}^{-1}\right]$ & $\mathrm{R}_{\mathrm{C}-\mathrm{C}}[\AA]$ & $\mathrm{E}[\mathrm{Ha}]$ & $\mathrm{BIE}\left[\mathrm{kcal} \mathrm{mol}^{-1}\right]$ \\
\hline 6-HH1 & & & 6-HH2 & & & 6-HF1 & & & 6-HF2 & & \\
\hline 3.66066245322 & -473.299921047196 & -2.78226130187702 & 4.10083752819 & -473.297115542932 & -1.0318210312603 & 4.07836427965 & -1861.84505717283 & 0.103746861768915 & 4.2821480345 & -1861.84646193192 & -0.65474150770167 \\
\hline 3.78268453499 & -473.302064635571 & -4.12738331555836 & 4.23753211246 & -473.298853708634 & -2.12253647666118 & 4.21430975564 & -1861.84874646628 & -2.21131973058476 & 4.42488630232 & -1861.84942330538 & -2.51303140992823 \\
\hline 3.90470661677 & -473.303035173339 & -4.73640495986073 & 4.37422669673 & -473.299690756044 & -2.64779165663346 & 4.35025523163 & -1861.85047104451 & -3.29350890848152 & 4.56762457013 & -1861.85067242442 & -3.2968654417069 \\
\hline 4.02672869854 & -473.303270922303 & -4.88433966823978 & 4.51092128101 & -473.299959550278 & -2.8164625850181 & 4.48620070762 & -1861.85106037463 & -3.66331914207303 & 4.71036283795 & -1861.85096468256 & -3.48026019341498 \\
\hline 4.14875078031 & -473.303063236651 & -4.75401495398663 & 4.64761586528 & -473.299888314835 & -2.77176166965859 & 4.6221461836 & -1861.85102680542 & -3.64225414475207 & 4.85310110577 & -1861.85075559058 & -3.34905299504737 \\
\hline 4.27077286209 & -473.30260498797 & -4.46645956523825 & 4.78431044955 & -473.299627253157 & -2.6079429933954 & 4.75809165959 & -1861.85067875421 & -3.42384871315776 & 4.99583937358 & -1861.85031350551 & -3.07164042526913 \\
\hline 4.39279494386 & -473.302022180777 & -4.10074253008972 & 4.92100503383 & -473.299273217238 & -2.38578210010279 & 4.89403713558 & -1861.85019453786 & -3.11999836602954 & 5.1385776414 & -1861.84979073654 & -2.74359794382697 \\
\hline 4.51481702564 & -473.301393483241 & -3.70622886996847 & 5.0576996181 & -473.298886621118 & -2.14318937219434 & 5.02998261157 & -1861.84967264042 & -2.79250277799404 & 5.28131590922 & -1861.84926804331 & -2.41560299008894 \\
\hline 4.63683910741 & -473.300768293732 & -3.31391653003787 & 5.19439420237 & -473.298501022173 & -1.90122238104138 & 5.16592808756 & -1861.84916507911 & -2.47400324727853 & 5.42405417703 & -1861.84878396714 & -2.11184060721597 \\
\hline 4.75886118918 & -473.300174800302 & -2.94149377994536 & 5.33108878664 & -473.298137364226 & -1.673023574009 & 5.30187356355 & -1861.84869748588 & -2.1805840654492 & 5.56679244485 & -1861.84835355296 & -1.84175163147618 \\
\hline 4.88088327096 & -473.299627601647 & -2.59812143977331 & 5.46778337092 & -473.297804606073 & -1.46421468042836 & 5.43781903953 & -1861.84828016435 & -1.91871085167361 & 5.70953071267 & -1861.84797925419 & -1.60687560726726 \\
\hline 5.00290535273 & -473.2991326992 & -2.28756546559147 & 5.60447795519 & -473.297506390366 & -1.27708149898708 & 5.57376451552 & -1861.84791439698 & -1.68918836179048 & 5.85226898048 & -1861.84765688837 & -1.40458800111637 \\
\hline 5.1249274345 & -473.298690881967 & -2.01032096609199 & 5.74117253946 & -473.297242974478 & -1.11178553369175 & 5.70970999151 & -1861.84759639775 & -1.48964083224581 & 5.9950072483 & -1861.84737926743 & -1.23037823102515 \\
\hline 5.24694951628 & -473.29829948655 & -1.76471663383702 & 5.87786712374 & -473.297012694476 & -0.967282650745907 & 5.8456554675 & -1861.84732001195 & -1.31620612418237 & 6.13774551612 & -1861.84713854397 & -1.07932197931729 \\
\hline 5.36897159805 & -473.297955938149 & -1.54913675745529 & 6.01456170801 & -473.296812874207 & -0.841893538840237 & 5.98160094349 & -1861.84707847812 & -1.1646413576151 & 6.28048378393 & -1861.84692769202 & -0.947010383089046 \\
\hline & -473.297655337072 & -1.36050673373376 & & -473.296640384628 & -0.733654693868979 & & -1861.84686555392 & -1.03102940486261 & & -1861.84674121046 & -0.829991437511718 \\
\hline $6-\mathrm{HF} 3$ & & & & & & & & & & & \\
\hline 4.20626486631 & -1861.84465613971 & 0.561157148702416 & 4.20917353341 & -1861.84733034042 & -0.915147375290368 & 4.33323976416 & -3250.39431899719 & 1.24863988110531 & 5.1937903833 & -3250.38387467508 & 7.83669794339467 \\
\hline 4.34647369519 & -1861.84866024618 & -1.95145759605987 & 4.34947931786 & -1861.84996300203 & -2.56716747729046 & 4.47768108963 & -3250.39932794826 & -1.89452437016434 & 5.36691672941 & -3250.39420638939 & 1.35344933103976 \\
\hline 4.48668252407 & -1861.85052048804 & -3.1187769871152 & 4.48978510231 & -1861.85116960267 & -3.32432081036078 & 4.62212241511 & -3250.40178886551 & -3.43877325905846 & 5.54004307552 & -3250.39891420176 & -1.60074753283396 \\
\hline 4.62689135294 & -1861.85115845884 & -3.51910970837523 & 4.63009088675 & -1861.85151536121 & -3.54128756985137 & 4.76656374058 & -3250.4027632384 & -4.05020147883085 & 5.71316942163 & -3250.40072495566 & -2.73701276011462 \\
\hline 4.76710018182 & -1861.85113663334 & -3.50541400036872 & 4.7703966712 & -1861.85137478002 & -3.45307154128961 & 4.91100506605 & -3250.40290814767 & -4.141133418719 & 5.88629576774 & -3250.40113422805 & -2.99383506243881 \\
\hline 4.9073090107 & -1861.85078769241 & -3.28645026089423 & 4.91070245565 & -1861.85098851568 & -3.21068700831444 & 5.05544639152 & -3250.402624422 & -3.96309287264205 & 6.05942211385 & -3250.40092932957 & -2.86525932490142 \\
\hline 5.04751783957 & -1861.85030102682 & -2.98106299252424 & 5.0510082401 & -1861.85050239709 & -2.9056429877064 & 5.19988771699 & -3250.40214608261 & -3.66293037381702 & 6.23254845996 & -3250.400500485 & -2.59615529448057 \\
\hline 5.18772666845 & -1861.84977945004 & -2.65376862159188 & 5.19131402454 & -1861.84999858695 & -2.58949735182845 & 5.34432904247 & -3250.40160164861 & -3.32129288059625 & 6.40567480607 & -3250.40002518241 & -2.29789841619149 \\
\hline 5.32793549733 & -1861.84927606355 & -2.33788883004489 & 5.33161980899 & -1861.84951968893 & -2.28898430720369 & 5.48877036794 & -3250.40105489086 & -2.9781972126034 & 6.57880115218 & -3250.39957050652 & -2.01258498757397 \\
\hline 5.46814432621 & -1861.84881659355 & -2.04956705209131 & 5.47192559344 & -1861.84908487884 & -2.01613685624049 & 5.63321169341 & -3250.40053189351 & -2.65001142067059 & 6.75192749829 & -3250.39915099629 & -1.74933834372686 \\
\hline 5.60835315508 & -1861.8484112345 & -1.79520040778716 & 5.61223137788 & -1861.84869976552 & -1.7744745992921 & 5.77765301888 & -3250.40003943985 & -2.34099208340666 & 6.9250538444 & -3250.39876343966 & -1.50614288675123 \\
\hline 5.74856198396 & -1861.84806062379 & -1.57518886555791 & 5.75253716233 & -1861.84836267356 & -1.56294620086836 & 5.92209434435 & -3250.39957737158 & -2.05103986645607 & 7.09818019051 & -3250.39840501753 & -1.28122960453476 \\
\hline 5.88877081284 & -1861.84775943391 & -1.38618936223002 & 5.89284294678 & -1861.84806863088 & -1.37843163353495 & 6.06653566983 & -3250.39914571985 & -1.78017431628966 & 7.27130653662 & -3250.39807815327 & -1.07611918471522 \\
\hline 6.02897964171 & -1861.84749938082 & -1.22300358467988 & 6.03314873123 & -1861.84781163376 & -1.21716350579867 & 6.2109769953 & -3250.39874724346 & -1.53012660651303 & 7.44443288273 & -3250.39778802378 & -0.894060180948259 \\
\hline 6.16918847059 & -1861.84727176679 & -1.08017362451372 & 6.17345451567 & -1861.84758574059 & -1.07541340154042 & 6.35541832077 & -3250.39838667548 & -1.30386678310688 & 7.61755922884 & -3250.39753544248 & -0.735563022419248 \\
\hline 6.30939729947 & -1861.84706929155 & -0.953118493001637 & 6.31376030012 & -1861.84738558549 & -0.949814179911805 & 6.49985964624 & -3250.39806851767 & -1.10421974285525 & 7.79068557495 & -3250.39732750376 & -0.605079505610587 \\
\hline
\end{tabular}




\begin{tabular}{|c|c|c|c|c|c|c|c|c|c|c|c|c|c|c|c|}
\hline \multicolumn{16}{|c|}{ TightPNO-DLPNO-CCSD(T)/cc-pVQZ } \\
\hline$R_{C, C}[\AA]$ & BIE $\left[\mathrm{kcal} \mathrm{mol}^{-1}\right]$ & $\mathrm{RCCC}_{\mathrm{CC}}[\hat{A}]$ & BIE $\left[\right.$ kcal mol $\left.^{-1}\right]$ & $R_{C C C}[\AA]$ & $\mathrm{BIE}\left[\mathrm{kcal} \mathrm{mol}{ }^{-1}\right]$ & $R_{C \rightarrow C}[\AA]$ & $\operatorname{BIE}\left[\right.$ kcal mol $\left.{ }^{-1}\right]$ & $R_{C-C}[A]$ & $\mathrm{BIE}\left[\mathrm{kcal} \mathrm{mol}{ }^{-1}\right]$ & $\mathrm{R}_{\mathrm{C}-\mathrm{C}}[\AA]$ & $\operatorname{BIE}\left[\right.$ kcal mol $\left.{ }^{-1}\right]$ & $\operatorname{ReCC}_{C \rightarrow}[\AA]$ & $\operatorname{BIE}\left[\mathrm{kcal} \mathrm{mol}^{-1}\right]$ & $\operatorname{RCCC}_{C-C}[\hat{A}]$ & BIE $\left[\mathrm{kcal} \mathrm{mol}^{-1}\right]$ \\
\hline 1-HH1 & & 1-HH2 & & 1-HF1 & & 1-HF2 & & & & & & 1-FF1 & & 1-FF2 & \\
\hline 3.30371146716 & -0.112609 & 3.5804338401 & -0.0419088 & 3.40464005758 & -0.0721955 & 3.82497722955 & 0.2242 & & & & & 3.67000659217 & -0.0907777 & 3.88048063632 & 0.482832 \\
\hline 3.41383518274 & -0.300277 & 3.69978163477 & -0.214587 & 3.5181280595 & -0.347836 & 3.95247647054 & -0.0596355 & & & & & 3.79316681191 & -0.366859 & 4.00982999086 & -0.0434683 \\
\hline 3.52395889831 & -0.389495 & 3.81912942944 & -0.295629 & 3.63161606142 & -0.479656 & 4.07997571152 & -0.196788 & & & & & 3.91552703165 & -0.487365 & 4.13917934541 & -0.306829 \\
\hline 3.63408261388 & -0.419488 & 3.93847722411 & -0.326033 & 3.74510406334 & -0.517481 & 4.20747495251 & -0.252219 & & & & & 4.03788725139 & -0.477106 & 4.26852869995 & -0.422088 \\
\hline 3.74420632945 & -0.414464 & 4.05782501878 & -0.325639 & 3.85859206526 & -0.505145 & 4.33497419349 & -0.264318 & & & & & 4.16024747113 & -0.434117 & 4.39787805449 & -0.447067 \\
\hline 3.85433004502 & -0.388837 & 4.17717281345 & -0.305492 & 3.97208006718 & -0.462119 & 4.46247343448 & -0.248703 & & & & & 4.28260769087 & -0.390042 & 4.52722740904 & -0.415557 \\
\hline 3.964537606 & -0.354816 & 4.29652060812 & -0.279457 & 4.0855680691 & -0.40885 & 4.58997267546 & -0.215904 & & & & & 4.40496791061 & -0.295796 & 4.656576763538 & -0.355531 \\
\hline 4.07457747617 & -0.315621 & 4.41586840279 & -0.253394 & 4.19905607102 & -0.343115 & 4.71747191645 & -0.179351 & & & & & 4.52732813035 & -0.217168 & 4.78592611813 & -0.284486 \\
\hline 4.184770119174 & -0.273674 & 4.53521619746 & -0.22228 & 4.31254407294 & -0.278651 & 4.84497115743 & -0.139164 & & & & & 4.64968835009 & -0.146014 & 4.91527547267 & -0.216121 \\
\hline 4.29482490731 & -0.234539 & 4.65456399213 & -0.191847 & 4.42603207486 & -0.217661 & 4.97247039842 & -0.101815 & & & & & 4.77204856983 & -0.0759355 & 5.04462482721 & -0.15038 \\
\hline 4.40494862289 & -0.202115 & 4.7739117868 & -0.164035 & 4.53952007678 & -0.165643 & 5.0999696394 & -0.0687836 & & & & & 4.89440878957 & -0.0134125 & 5.17397418176 & -0.0907891 \\
\hline 4.51507233846 & -0.172812 & 4.89325958147 & -0.140377 & 4.6530080787 & -0.111975 & 5.22746888039 & -0.0391888 & & & & & 5.01676900931 & 0.0394407 & 5.3033235363 & -0.0368613 \\
\hline 4.62519605403 & -0.145541 & 5.01260737614 & -0.119173 & 4.766496000062 & -0.0640438 & 5.35496812137 & -0.0120497 & & & & & 5.13912922904 & 0.0840676 & 5.432672890085 & 0.00691842 \\
\hline 4.7353197696 & -0.12392 & 5.13195517081 & -0.10222 & 4.87998408254 & -0.0216397 & 5.48246736236 & 0.0115913 & & & & & 5.26148944878 & 0.123147 & 5.56202224539 & 0.0470864 \\
\hline 4.84544348517 & -0.103927 & 5.25130296548 & -0.0862979 & 4.99347208446 & 0.0175056 & 5.60996660334 & 0.0329467 & & & & & 5.38384966852 & 0.158872 & 5.69137159993 & 0.0838928 \\
\hline 4.95556720075 & -0.0899487 & $\begin{array}{l}5.37065076015 \\
2 \text {-HH2 } 2\end{array}$ & -0.0726374 & $\begin{array}{l}5.10696008638 \\
2 \text {-HF1 }\end{array}$ & 0.0514087 & $\begin{array}{c}5.73746584433 \\
2 \text {-HF2 }\end{array}$ & 0.0514417 & & & & & $\begin{array}{l}5.50620988826 \\
\text { 2-FF1 }\end{array}$ & 0.18999 & $\begin{array}{l}5.82072095448 \\
2 \mathrm{FF} 2\end{array}$ & 0.114971 \\
\hline $\begin{array}{c}\text { 2-HH1 } \\
3.34273389134\end{array}$ & -0.507082 & 3.58048380658 & -0.414245 & 3.90237412775 & 0.0969077 & 3.77935622887 & 0.0675529 & 4.1164580051 & 0.35832 & 3.78617705941 & 0.18243 & $\begin{array}{c}\text { 2-FFI } \\
4.18662183349\end{array}$ & 0.713446 & $\begin{array}{c}2 \mathrm{FF} 2 \\
4.58731127773\end{array}$ & 1.21386 \\
\hline 3.45415835439 & -0.897779 & 3.6998332668 & -0.811118 & 4.03245326534 & -0.4305 & 3.90533476983 & -0.50209 & 4.25367327193 & -0.282909 & 3.91238296139 & -0.417481 & 4.3261758946 & -0.123781 & 4.740222165365 & 0.104539 \\
\hline 3.56558281743 & -1.09092 & 3.81918272702 & -0.982045 & 4.16253240293 & -0.672959 & 4.03131331079 & -0.774183 & 4.390888538777 & -0.56882 & 4.038558886337 & -0.724556 & 4.465729955772 & -0.558518 & 4.89313202958 & -0.399381 \\
\hline 3.67700728048 & -1.15075 & 3.93853218724 & -1.02402 & 4.29261154053 & -0.749613 & 4.15729185176 & -0.869884 & 4.528103800661 & -0.653699 & 4.16479476535 & -0.827095 & 4.60528401684 & -0.737665 & 5.0460424055 & -0.564998 \\
\hline 3.78843174352 & -1.13225 & 4.05788164746 & -0.990036 & 4.42269967812 & -0.734984 & 4.28327039272 & -0.867846 & 4.66531907244 & -0.638451 & 4.291000667733 & -0.82778 & 4.74483807795 & -0.758373 & 5.19895278143 & -0.573188 \\
\hline 3.899856220657 & -1.06784 & 4.17723110768 & -0.914908 & 4.55276981571 & -0.678422 & 4.40924893368 & -0.810097 & 4.80253433928 & -0.577486 & 4.41720656931 & -0.773283 & 4.88439213907 & -0.692446 & 5.35186315735 & -0.506162 \\
\hline 4.01128066961 & -0.974643 & 4.29658056789 & -0.828953 & 4.6828489533 & -0.586352 & 4.53522747464 & -0.719285 & 4.93974960612 & -0.492962 & 4.54341247129 & -0.679954 & 5.02394620018 & -0.594191 & 5.50477353327 & -0.407677 \\
\hline 4.12270513266 & -0.874077 & 4.41593002811 & -0.738431 & 4.81292809089 & -0.496506 & 4.66120601561 & -0.616672 & 5.07696487295 & -0.405076 & 4.66961837327 & -0.578967 & 5.1635002613 & -0.469877 & 5.6576839092 & -0.299621 \\
\hline 4.2341295957 & -0.792078 & 4.53527948833 & -0.639432 & 4.94300722849 & -0.404911 & 4.78718455657 & -0.507038 & 5.21418013979 & -0.316983 & 4.79582427525 & -0.475324 & 5.30305432242 & -0.354923 & 5.81059428512 & -0.201467 \\
\hline 4.34555405875 & -0.692637 & 4.65462894855 & -0.547928 & 5.07308636608 & -0.322186 & 4.91316309753 & -0.402337 & 5.35139540663 & -0.235586 & 4.92203017723 & -0.38176 & 5.44260838353 & -0.243377 & 5.96350466105 & -0.114678 \\
\hline 4.45697852179 & -0.611091 & 4.77397840877 & -0.471391 & 5.20316550367 & -0.236508 & 5.03914163849 & -0.306846 & 5.48861067346 & -0.167641 & 5.04823607921 & -0.29298 & 5.58216244465 & -0.137027 & 6.11641503697 & -0.0368178 \\
\hline 4.56840298483 & -0.528576 & 4.89332786899 & -0.39677 & 5.33324464126 & -0.165429 & 5.16512017945 & -0.222755 & 5.6258259403 & -0.107273 & 5.17444198119 & -0.211981 & 5.72171650576 & -0.0491073 & 6.2693254129 & 0.0371114 \\
\hline 4.67982744788 & -0.4575899 & 5.01267732921 & -0.340982 & 5.46332377885 & -0.100063 & 5.29109872042 & -0.15255 & 5.76304120714 & -0.0552279 & 5.30064788317 & -0.141477 & 5.86127056688 & 0.0264806 & 6.42223578882 & 0.0932827 \\
\hline 4.79125191092 & -0.387747 & 5.13202678943 & -0.286831 & 5.59340291644 & -0.0459152 & 5.41707726138 & -0.0840435 & 5.90025647397 & -0.00395252 & 5.42685378515 & -0.078384 & 6.000824628 & 0.0923936 & 6.57514616474 & 0.149055 \\
\hline 4.902676373997 & -0.328123 & 5.25137624965 & -0.239485 & 5.72348205404 & 0.0053974 & 5.54305580234 & -0.0255223 & 6.03747174081 & 0.0362027 & 5.55305968713 & -0.0211873 & 6.14037868911 & 0.153425 & 6.728056554067 & 0.186285 \\
\hline $\begin{array}{l}\text { 5.01410083701 } \\
\text { 3-HH1 }\end{array}$ & -0.276534 & $\begin{array}{c}5.37072570987 \\
3-\mathrm{HH} 2\end{array}$ & -0.204714 & $\begin{array}{c}5.85356119163 \\
\text { 3-HF1 }\end{array}$ & 0.0511367 & $\begin{array}{c}5.6690343433 \\
3 \text {-HF2 }\end{array}$ & 0.026357 & $\begin{array}{c}6.17468700765 \\
3-\mathrm{HF} 3\end{array}$ & 0.0729573 & $\begin{array}{c}5.67926558911 \\
\text { 3-HF4 }\end{array}$ & 0.0289137 & $\begin{array}{c}6.27993275023 \\
3-\mathrm{FF} 1\end{array}$ & 0.201559 & $\begin{array}{c}6.88096696599 \\
3 \text {-FF2 }\end{array}$ & 0.218014 \\
\hline 3.43594668895 & -0.597062 & 4.038069907476 & -0.114213 & 4.055756500873 & 0.228009 & 4.265866563335 & 0.226684 & 4.37726127205 & -0.293392 & 4.22420781328 & 0.798957 & 4.447325992776 & 1.64127 & 4.42086165258 & 4.424246 \\
\hline 3.55047824525 & -1.25341 & 4.17267137725 & -0.658265 & 4.19094839235 & -0.721951 & 4.40806211546 & -0.619477 & 4.52316998112 & -0.866789 & 4.36501474039 & -0.270346 & 4.59557012535 & 0.0122426 & 4.56822370767 & 1.21394 \\
\hline 3.66500980155 & -1.6037 & 4.3072727367975 & -0.941821 & 4.32614027597 & -1.15691 & 4.55025766757 & -0.981608 & 4.66907869019 & -1.08102 & 4.5058216675 & -0.789156 & 4.74381432294 & -0.742174 & 4.71558576275 & -0.353331 \\
\hline 3.77954135785 & -1.72195 & 4.441875988224 & -1.06276 & 4.4613321596 & -1.28733 & 4.69245321968 & -1.06881 & 4.81498739925 & -1.09102 & 4.6466285946 & -0.990677 & 4.89205852053 & -1.05222 & 4.86294781784 & -0.990336 \\
\hline 3.89407291414 & -1.70594 & 4.57647828473 & -1.05557 & 4.59652404322 & -1.27007 & 4.83464877179 & -1.02173 & 4.96089610832 & -1.00969 & 4.78743552171 & -1.01854 & 5.04030271812 & -1.10688 & 5.01030987293 & -1.20215 \\
\hline 4.00860447044 & -1.61299 & 4.71108058722 & -0.988879 & 4.73171592685 & -1.16315 & 4.97684432391 & -0.913895 & 5.10680481739 & -0.889177 & 4.92824244882 & -0.950691 & 5.18854691572 & -1.03835 & 5.15767192801 & -1.18195 \\
\hline 4.12313602674 & -1.48567 & 4.84568288971 & -0.895304 & 4.86690781047 & -1.01086 & 5.11903987602 & -0.781867 & 5.25271352646 & -0.744296 & 5.06904937593 & -0.837481 & 5.33679111331 & -0.893223 & 5.3050339831 & -1.03852 \\
\hline 4.23766758304 & -1.38261 & 4.98028519221 & -0.80586 & 5.00209969409 & -0.844059 & 5.26123542813 & -0.646347 & 5.39862223553 & -0.603626 & 5.2098563030304 & -0.70491 & 5.4850353109 & -0.716528 & 5.45239603818 & -0.829339 \\
\hline 4.35219913934 & -1.23418 & 5.1148874947 & -0.703956 & 5.13729157772 & -0.679288 & 5.40343098024 & -0.518883 & 5.5445309446 & -0.475589 & 5.35066323015 & -0.576705 & 5.63327950849 & -0.546162 & 5.59975809327 & -0.623749 \\
\hline 4.46673069564 & -1.10031 & 5.24948979719 & -0.604706 & 5.27248346134 & -0.533556 & 5.54562653235 & -0.392423 & 5.69043965366 & -0.360389 & 5.49147015726 & -0.445555 & 5.78152370608 & -0.383508 & 5.74712014836 & -0.440116 \\
\hline 4.58126225193 & -0.967273 & 5.38409209968 & -0.510882 & 5.40767534497 & -0.399039 & 5.68782208446 & -0.280126 & 5.83634836273 & -0.261203 & 5.63227708437 & -0.328321 & 5.92976790367 & -0.232854 & 5.89448220344 & -0.264919 \\
\hline 4.69579380823 & -0.829829 & 5.51869440218 & -0.436706 & 5.54286722859 & -0.293142 & 5.83001763658 & -0.183479 & 5.9822570718 & -0.175272 & 5.77308401148 & -0.224751 & 6.07801210127 & -0.116461 & 6.04184425853 & -0.106823 \\
\hline 4.810325354453 & -0.713197 & 5.653296670467 & -0.364543 & 5.67805911222 & -0.186403 & 5.97221318869 & -0.10242 & 6.12816578087 & -0.10574 & 5.91389093859 & -0.144229 & 6.222625629886 & -0.005842007 & 6.18920631361 & 0.0204041 \\
\hline 4.92485692083 & -0.60631 & 5.78789900716 & -0.301609 & 5.81325099584 & -0.0950333 & 6.1144087408 & -0.0350295 & 6.27407448994 & -0.0411108 & 6.0546978657 & -0.0736421 & 6.37450049645 & 0.0940649 & 6.3365683687 & 0.136018 \\
\hline 5.03938847713 & -0.509879 & 5.92250130965 & -0.250749 & 5.94844287946 & -0.0172386 & 6.2566042929291 & 0.0269559 & 6.419983199 & 0.0144938 & 6.19550479281 & -0.0102317 & 6.52274469404 & 0.173002 & 6.483930442379 & 0.233225 \\
\hline 5.153920003343 & -0.443403 & 5710361214 & 0.4221 & 476309 & 0.052602 & 34502 & 0.0790619 & 6.565891908007 & 0.0619782 & 6.33631171992 & 0.0481404 & 6.67098889163 & 0.235852 & 6.63129247887 & 0.313131 \\
\hline
\end{tabular}




\subsection{Energy Decomposition Analysis}

The following tables provide the computational raw data for the SAPT and LED computations. In SAPT, El refers to electrostatics, Ex refers to exchange, In refers to induction, Disp refers to dispersion, BIE refers to the bond interaction energy. In LED, BIE refers to the bond interaction energy, $\mathrm{HF}_{\text {int }}$ refers to the $\mathrm{HF}$ component of the interaction energy, $\mathrm{HF}_{\text {prep }}$ refers to the HF component of the electronic preparation energy, Cor int $_{\text {refers to the corre- }}$ lation energy component of the interaction energy, Cor $_{\text {prep }}$ refers to the correlation energy component of the interaction energy, El refers to electrostatics, Ex refers to exchange, Disp refers to dispersion, CT refers to charge-transfer and (T) refers to the perturbative triples correction component of the interaction energy. 
SSAPT0/jun-cc-pVDZ

El $\left[\mathrm{kcal} \mathrm{mol}^{-1}\right] \quad$ Ex $\left[\mathrm{kcal} \mathrm{mol}^{-1}\right] \quad$ In $\left[\mathrm{kcal} \mathrm{mol}^{-1}\right] \quad$ Disp $\left[\mathrm{kcal} \mathrm{mol}^{-1}\right] \quad$ BIE $\left[\mathrm{kcal} \mathrm{mol}^{-1}\right]$

\begin{tabular}{|c|c|c|c|c|c|}
\hline \multicolumn{6}{|c|}{ Methanes } \\
\hline HH1 & -0.1655 & 0.6412 & -0.0454 & -0.5785 & -0.1481 \\
\hline $\mathrm{HH} 2$ & -0.1462 & 0.5257 & -0.0347 & -0.4368 & -0.0918 \\
\hline HF1 & -0.3017 & 0.7804 & -0.0274 & -0.6775 & -0.2258 \\
\hline HF2 & -0.1219 & 0.5724 & -0.0204 & -0.4542 & -0.0237 \\
\hline FF1 & 0.2219 & 0.3977 & -0.0081 & -0.5809 & 0.0306 \\
\hline FF2 & -0.3626 & 0.7541 & -0.0157 & -0.5896 & -0.2134 \\
\hline \multicolumn{6}{|c|}{ Ethanes } \\
\hline HH1 & -0.5483 & 1.8674 & -0.1497 & -1.7955 & -0.6241 \\
\hline HH2 & -0.4353 & 1.4234 & -0.1084 & -1.4379 & -0.5576 \\
\hline HF1 & -0.2777 & 1.1779 & -0.0506 & -1.1746 & -0.3242 \\
\hline HF2 & -0.4147 & 1.4056 & -0.0604 & -1.3314 & -0.4001 \\
\hline HF3 & -0.2454 & 1.0090 & -0.0469 & -1.0072 & -0.2900 \\
\hline HF4 & -0.3753 & 1.4296 & -0.0620 & -1.3305 & -0.3371 \\
\hline $\mathrm{FF} 1$ & -0.4096 & 1.1732 & -0.0311 & -1.1293 & -0.3963 \\
\hline FF2 & -0.1611 & 0.8058 & -0.0168 & -0.8516 & -0.2234 \\
\hline \multicolumn{6}{|c|}{ Propanes } \\
\hline HH1 & -0.8415 & 2.9705 & -0.2724 & -2.9617 & -1.1029 \\
\hline HH2 & -0.5316 & 1.8318 & -0.1310 & -1.8072 & -0.6356 \\
\hline HF1 & -0.5894 & 1.8992 & -0.0864 & -1.9644 & -0.7395 \\
\hline HF2 & -0.4091 & 1.4736 & -0.0774 & -1.5875 & -0.5995 \\
\hline HF3 & -0.3821 & 1.1785 & -0.0666 & -1.4111 & -0.6805 \\
\hline HF4 & -0.5801 & 1.9893 & -0.1124 & -1.8131 & -0.5141 \\
\hline FF1 & -0.5519 & 1.6868 & -0.0511 & -1.7687 & -0.6843 \\
\hline FF2 & -0.6313 & 2.4109 & -0.0701 & -2.1253 & -0.4148 \\
\hline \multicolumn{6}{|c|}{ Butanes } \\
\hline HH1 & -1.2350 & 4.1487 & -0.3776 & -4.2930 & -1.7530 \\
\hline HH2 & -0.6707 & 2.3040 & -0.1641 & -2.4901 & -1.0174 \\
\hline HF1 & -0.8010 & 2.4715 & -0.1385 & -2.6728 & -1.1391 \\
\hline HF2 & -0.8034 & 2.6421 & -0.1427 & -2.7558 & -1.0578 \\
\hline HF3 & -0.9149 & 3.0342 & -0.1571 & -2.8962 & -0.9310 \\
\hline HF4 & -0.7539 & 2.4869 & -0.1227 & -2.6009 & -0.9885 \\
\hline FF1 & -0.4532 & 1.3455 & -0.0419 & -1.9254 & -1.0746 \\
\hline FF2 & -0.6180 & 2.0733 & -0.0511 & -2.3286 & -0.9236 \\
\hline \multicolumn{6}{|c|}{ Pentanes } \\
\hline HH1 & -1.6127 & 5.3858 & -0.4846 & -5.6800 & -2.3877 \\
\hline HH2 & -0.9523 & 3.3861 & -0.2346 & -3.5262 & -1.3221 \\
\hline HF1 & -0.8901 & 3.1166 & -0.1770 & -3.5828 & -1.5310 \\
\hline HF2 & -0.7447 & 2.4912 & -0.1432 & -3.0342 & -1.4293 \\
\hline HF3 & -0.9255 & 2.9806 & -0.1629 & -3.1994 & -1.3049 \\
\hline HF4 & -1.1477 & 3.6389 & -0.1916 & -3.7320 & -1.4286 \\
\hline FF1 & -0.8443 & 2.6409 & -0.0869 & -3.1834 & -1.4728 \\
\hline FF2 & -0.7482 & 2.2696 & -0.0656 & -2.7247 & -1.2681 \\
\hline \multicolumn{6}{|c|}{ Hexanes } \\
\hline HH1 & -1.9335 & 6.3845 & -0.5775 & -6.7867 & -2.9085 \\
\hline HH2 & -1.1511 & 3.9393 & -0.2797 & -4.2908 & -1.7757 \\
\hline HF1 & -1.2676 & 4.3638 & -0.2421 & -4.6080 & -1.7497 \\
\hline HF2 & -1.0073 & 3.2889 & -0.1952 & -3.9062 & -1.8178 \\
\hline HF3 & -1.2177 & 4.1424 & -0.2232 & -4.3421 & -1.6370 \\
\hline HF4 & -1.1879 & 3.7916 & -0.1979 & -4.1249 & -1.7157 \\
\hline FF1 & -1.2223 & 3.7755 & -0.1188 & -4.4151 & -1.9792 \\
\hline FF2 & -0.9438 & 3.2711 & -0.0952 & -3.2594 & -1.0259 \\
\hline
\end{tabular}


SAPT2+/aug-cc-pVDZ

$\mathrm{El}\left[\mathrm{kcal} \mathrm{mol}^{-1}\right] \quad$ Ex $\left[\mathrm{kcal} \mathrm{mol}^{-1}\right] \quad$ In $\left[\mathrm{kcal} \mathrm{mol}^{-1}\right] \quad$ Disp $\left[\mathrm{kcal} \mathrm{mol}^{-1}\right] \quad$ BIE $\left[\mathrm{kcal} \mathrm{mol}^{-1}\right]$

\begin{tabular}{|c|c|c|c|c|c|}
\hline \multicolumn{6}{|c|}{ Methanes } \\
\hline HH1 & -0.2178 & 0.7190 & -0.0332 & -0.9490 & -0.4810 \\
\hline HH2 & -0.1889 & 0.5757 & -0.0265 & -0.7285 & -0.3681 \\
\hline HF1 & -0.3655 & 0.9508 & -0.0389 & -1.2066 & -0.6601 \\
\hline HF2 & -0.1820 & 0.6794 & -0.0238 & -0.8256 & -0.3520 \\
\hline FF1 & -0.0219 & 0.5850 & -0.0164 & -1.1912 & -0.6445 \\
\hline FF2 & -0.3762 & 1.0425 & -0.0251 & -1.2385 & -0.5973 \\
\hline \multicolumn{6}{|c|}{ Ethanes } \\
\hline HH1 & -0.6603 & 2.0879 & -0.1456 & -2.5149 & -1.2329 \\
\hline $\mathrm{HH} 2$ & -0.5398 & 1.5928 & -0.0983 & -2.0682 & -1.1136 \\
\hline HF1 & -0.4080 & 1.4149 & -0.0517 & -1.9006 & -0.9453 \\
\hline HF2 & -0.5421 & 1.6830 & -0.0615 & -2.1682 & -1.0888 \\
\hline HF3 & -0.3560 & 1.2121 & -0.0474 & -1.6361 & -0.8273 \\
\hline $\mathrm{HF} 4$ & -0.5234 & 1.7117 & -0.0629 & -2.1633 & -1.0378 \\
\hline FF1 & -0.5011 & 1.5888 & -0.0380 & -2.1004 & -1.0507 \\
\hline $\mathrm{FF} 2$ & -0.3240 & 1.1032 & -0.0255 & -1.6084 & -0.8547 \\
\hline \multicolumn{6}{|c|}{ Propanes } \\
\hline HH1 & -1.0134 & 3.3548 & -0.2747 & -3.9636 & -1.8969 \\
\hline HH2 & -0.6837 & 2.1044 & -0.1294 & -2.5143 & -1.2229 \\
\hline HF1 & -0.7669 & 2.2922 & -0.0860 & -3.0472 & -1.6079 \\
\hline HF2 & -0.5547 & 1.7538 & -0.0795 & -2.4207 & -1.3011 \\
\hline HF3 & -0.4724 & 1.4037 & -0.0660 & -2.1720 & -1.3067 \\
\hline HF4 & -0.7464 & 2.3460 & -0.1137 & -2.7717 & -1.2859 \\
\hline FF1 & -0.6796 & 2.2708 & -0.0590 & -3.0945 & -1.5623 \\
\hline FF2 & -1.0800 & 3.2783 & -0.0939 & -3.8724 & -1.7680 \\
\hline \multicolumn{6}{|c|}{ Butanes } \\
\hline HH1 & -1.5017 & 4.7227 & -0.3845 & -5.6107 & -2.7743 \\
\hline HH2 & -0.8830 & 2.6888 & -0.1624 & -3.3967 & -1.7533 \\
\hline HF1 & -0.9912 & 2.9380 & -0.1369 & -3.9650 & -2.1551 \\
\hline HF2 & -1.0355 & 3.1456 & -0.1395 & -4.1051 & -2.1345 \\
\hline HF3 & -1.2138 & 3.6455 & -0.1601 & -4.3913 & -2.1197 \\
\hline $\mathrm{HF} 4$ & -0.9982 & 2.9997 & -0.1266 & -3.9696 & -2.0947 \\
\hline FF1 & -0.5589 & 1.8385 & -0.0456 & -3.2464 & -2.0124 \\
\hline FF2 & -0.9603 & 2.8616 & -0.0641 & -4.0849 & -2.2477 \\
\hline \multicolumn{6}{|c|}{ Pentanes } \\
\hline HH1 & -1.9734 & 6.1493 & -0.4936 & -7.3317 & -3.6494 \\
\hline HH2 & -1.2628 & 3.9647 & -0.2305 & -4.8190 & -2.3475 \\
\hline HF1 & -1.1901 & 3.7200 & -0.1764 & -5.1941 & -2.8406 \\
\hline HF2 & -0.9764 & 2.9917 & -0.1396 & -4.4419 & -2.5663 \\
\hline HF3 & -1.2059 & 3.5841 & -0.1649 & -4.7320 & -2.5187 \\
\hline $\mathrm{HF} 4$ & -1.4824 & 4.3690 & -0.1937 & -5.5860 & -2.8931 \\
\hline FF1 & -1.1151 & 3.5558 & -0.0953 & -5.2701 & -2.9247 \\
\hline FF2 & -1.0010 & 3.1049 & -0.0762 & -4.6981 & -2.6704 \\
\hline \multicolumn{6}{|c|}{ Hexanes } \\
\hline HH1 & -2.3649 & 7.3092 & -0.5928 & -8.6740 & -4.3225 \\
\hline HH2 & -1.5342 & 4.6407 & -0.2760 & -5.7280 & -2.8975 \\
\hline HF1 & -1.6978 & 5.1984 & -0.2405 & -6.6419 & -3.3819 \\
\hline HF2 & -1.2957 & 3.9296 & -0.1860 & -5.6633 & -3.2155 \\
\hline HF3 & -1.6355 & 4.9790 & -0.2202 & -6.4036 & -3.2803 \\
\hline HF4 & -1.5591 & 4.5665 & -0.2048 & -6.0923 & -3.2896 \\
\hline \multicolumn{6}{|l|}{ FF1 } \\
\hline FF2 & & & & & \\
\hline
\end{tabular}




\begin{tabular}{|c|c|c|c|c|c|}
\hline & \multicolumn{5}{|c|}{ 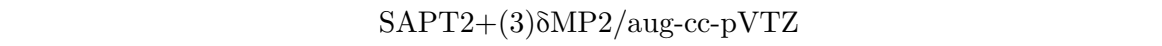 } \\
\hline & $\mathrm{El}\left[\mathrm{kcal} \mathrm{mol}^{-1}\right]$ & Ex $\left[\mathrm{kcal} \mathrm{mol}^{-1}\right]$ & In $\left[\mathrm{kcal} \mathrm{mol}^{-1}\right]$ & Disp $\left[\mathrm{kcal} \mathrm{mol}^{-1}\right]$ & BIE $\left[\mathrm{kcal} \mathrm{mol}^{-1}\right]$ \\
\hline \multicolumn{6}{|c|}{ Methanes } \\
\hline HH1 & -0.1791 & 0.6892 & 0.0131 & -1.0104 & -0.4871 \\
\hline $\mathrm{HH} 2$ & -0.1677 & 0.5564 & 0.0050 & -0.7739 & -0.3802 \\
\hline HF1 & -0.3451 & 0.9132 & 0.0155 & -1.3281 & -0.7445 \\
\hline HF2 & -0.1545 & 0.6526 & 0.0047 & -0.9071 & -0.4043 \\
\hline FF1 & 0.0308 & 0.5667 & 0.0128 & -1.3359 & -0.7256 \\
\hline FF2 & -0.3345 & 0.9961 & 0.0226 & -1.4161 & -0.7319 \\
\hline \multicolumn{6}{|c|}{ Ethanes } \\
\hline HH1 & -0.5997 & 2.0146 & -0.0873 & -2.6366 & -1.3090 \\
\hline $\mathrm{HH} 2$ & -0.4776 & 1.5343 & -0.0449 & -2.1652 & -1.1534 \\
\hline HF1 & -0.3472 & 1.3605 & -0.0087 & -2.0483 & -1.0437 \\
\hline HF2 & -0.4817 & 1.6153 & -0.0134 & -2.3402 & -1.2201 \\
\hline HF3 & -0.3046 & 1.1665 & -0.0126 & -1.7588 & -0.9095 \\
\hline HF4 & -0.4533 & 1.6452 & -0.0168 & -2.3288 & -1.1538 \\
\hline $\mathrm{FF} 1$ & -0.4285 & 1.5217 & 0.0113 & -2.3362 & -1.2317 \\
\hline FF2 & -0.2579 & 1.0556 & 0.0164 & -1.7875 & -0.9735 \\
\hline
\end{tabular}




\begin{tabular}{|c|c|c|c|c|c|}
\hline & \multicolumn{5}{|c|}{ SAPT2+(CCD) $\delta \mathrm{MP} 2 /$ aug-cc-pVTZ } \\
\hline & $\mathrm{El}\left[\mathrm{kcal} \mathrm{mol}^{-1}\right]$ & Ex $\left[\mathrm{kcal} \mathrm{mol}^{-1}\right]$ & In $\left[\mathrm{kcal} \mathrm{mol}^{-1}\right]$ & Disp $\left[\mathrm{kcal} \mathrm{mol}^{-1}\right]$ & $\mathrm{BIE}\left[\mathrm{kcal} \mathrm{mol}^{-1}\right]$ \\
\hline \multicolumn{6}{|c|}{ Methanes } \\
\hline HH1 & -0.1913 & 0.6892 & 0.0131 & -1.0340 & -0.5230 \\
\hline $\mathrm{HH} 2$ & -0.1781 & 0.5564 & 0.0050 & -0.7922 & -0.4088 \\
\hline HF1 & -0.3709 & 0.9132 & 0.0155 & -1.3356 & -0.7777 \\
\hline HF2 & -0.1751 & 0.6526 & 0.0047 & -0.9128 & -0.4305 \\
\hline FF1 & -0.0209 & 0.5667 & 0.0128 & -1.3205 & -0.7619 \\
\hline FF2 & -0.3675 & 0.9961 & 0.0226 & -1.4031 & -0.7520 \\
\hline \multicolumn{6}{|c|}{ Ethanes } \\
\hline HH1 & -0.6321 & 2.0146 & -0.0873 & -2.7030 & -1.4079 \\
\hline HH2 & -0.5082 & 1.5343 & -0.0449 & -2.2152 & -1.2340 \\
\hline HF1 & -0.3890 & 1.3605 & -0.0087 & -2.0472 & -1.0844 \\
\hline HF2 & -0.5270 & 1.6153 & -0.0134 & -2.3406 & -1.2658 \\
\hline HF3 & -0.3396 & 1.1665 & -0.0126 & -1.7581 & -0.9438 \\
\hline HF 4 & -0.5014 & 1.6452 & -0.0168 & -2.3296 & -1.2026 \\
\hline FF1 & -0.4839 & 1.5217 & 0.0113 & -2.2962 & -1.2472 \\
\hline FF2 & -0.3110 & 1.0556 & 0.0165 & -1.7604 & -0.9993 \\
\hline
\end{tabular}




\begin{tabular}{|c|c|c|c|c|c|c|c|c|c|c|}
\hline & \multicolumn{10}{|c|}{ LED-TightPNO-DLPNO-CCSD(T)/cc-pVQZ } \\
\hline & $\mathrm{BIE}\left[\mathrm{kcal} \mathrm{mol}^{-1}\right]$ & $\mathrm{HF}_{\text {int }}\left[\mathrm{kcal} \mathrm{mol}^{-1}\right]$ & $\mathrm{HF}_{\text {prep }}\left[\mathrm{kcal} \mathrm{mol}^{-1}\right]$ & $\mathrm{Cor}_{\text {int }}\left[\mathrm{kcal} \mathrm{mol}^{-1}\right]$ & $\mathrm{Cor}_{\text {prep }}\left[\mathrm{kcal} \mathrm{mol}^{-1}\right]$ & $\mathrm{El}\left[\mathrm{kcal} \mathrm{mol}^{-1}\right]$ & Ex $\left[\mathrm{kcal} \mathrm{mol}^{-1}\right]$ & Disp $\left[\mathrm{kcal} \mathrm{mol}^{-1}\right]$ & $\mathrm{CT}\left[\mathrm{kcal} \mathrm{mol}^{-1}\right]$ & (T) $\left[\mathrm{kcal} \mathrm{mol}^{-1}\right]$ \\
\hline \multicolumn{11}{|c|}{ Methanes } \\
\hline HH1 & -0.4228 & -1.2560 & 1.6863 & -1.1074 & 0.2543 & -0.8259 & -0.4302 & -0.8187 & -0.1601 & -0.1286 \\
\hline $\mathrm{HH} 2$ & -0.3297 & -1.0838 & 1.4214 & -0.8440 & 0.1767 & -0.7351 & -0.3487 & -0.6170 & -0.1283 & -0.0987 \\
\hline HF1 & -0.5185 & -2.4290 & 2.8692 & -1.2994 & 0.3406 & -1.8757 & -0.5532 & -1.0008 & -0.3593 & -0.1555 \\
\hline $\mathrm{HF} 2$ & -0.2619 & -1.6811 & 2.0975 & -0.8740 & 0.1957 & -1.2863 & -0.3949 & -0.6878 & -0.1370 & -0.0982 \\
\hline $\mathrm{FF} 1$ & -0.4643 & -1.3964 & 1.9097 & -1.1205 & 0.1430 & -1.0429 & -0.3535 & -1.0144 & -0.0288 & -0.0774 \\
\hline $\mathrm{FF} 2$ & -0.4332 & -2.6955 & 3.0986 & -1.2149 & 0.3786 & -2.1537 & -0.5418 & -1.0339 & -0.0694 & -0.1117 \\
\hline \multicolumn{11}{|l|}{ Ethanes } \\
\hline HH1 & -1.1517 & -3.6255 & 4.7819 & -2.9893 & 0.6811 & -2.3506 & -1.2749 & -2.1308 & -1.0814 & -0.3656 \\
\hline HH2 & -1.0192 & -2.9478 & 3.8134 & -2.4352 & 0.5504 & -1.9577 & -0.9901 & -1.7526 & -0.4609 & -0.2980 \\
\hline HF1 & -0.7522 & -3.4510 & 4.2879 & -2.0180 & 0.4288 & -2.6373 & -0.8136 & -1.5815 & -0.6836 & -0.2209 \\
\hline $\mathrm{HF} 2$ & -0.8819 & -4.2212 & 5.1375 & -2.3180 & 0.5198 & -3.2486 & -0.9726 & -1.7994 & -0.3167 & -0.2623 \\
\hline HF3 & -0.6621 & -3.0106 & 3.7186 & -1.7161 & 0.3459 & -2.3083 & -0.7022 & -1.3516 & -0.8224 & -0.1755 \\
\hline HF4 & -0.8379 & -4.2216 & 5.1991 & -2.3141 & 0.4986 & -3.2384 & -0.9831 & -1.7782 & -0.9072 & -0.2598 \\
\hline $\mathrm{FF} 1$ & -0.7582 & -4.0169 & 4.7685 & -2.0074 & 0.4976 & -3.2117 & -0.8052 & -1.7281 & -0.1235 & -0.1558 \\
\hline $\mathrm{FF} 2$ & -0.5745 & -2.7525 & 3.3443 & -1.4919 & 0.3256 & -2.1830 & -0.5695 & -1.3087 & -0.0785 & -0.1047 \\
\hline \multicolumn{11}{|c|}{ Propanes } \\
\hline HH1 & -1.7283 & -5.9781 & 7.8295 & -4.6709 & 1.0912 & -3.9172 & -2.0610 & -3.2331 & -1.0388 & -0.5887 \\
\hline HH2 & -1.0701 & -3.5448 & 4.7036 & -2.8678 & 0.6389 & -2.3730 & -1.1717 & -2.1021 & -0.9529 & -0.3469 \\
\hline HF1 & -1.2931 & -5.6153 & 6.8290 & -3.2221 & 0.7152 & -4.3250 & -1.2902 & -2.4638 & -1.3955 & -0.3501 \\
\hline $\mathrm{HF} 2$ & -1.0645 & -4.3570 & 5.3336 & -2.5406 & 0.4995 & -3.3559 & -1.0011 & -2.0141 & -0.8686 & -0.2619 \\
\hline HF3 & -1.0688 & -3.6071 & 4.3367 & -2.2434 & 0.4450 & -2.7964 & -0.8107 & -1.7972 & -0.3503 & -0.2249 \\
\hline $\mathrm{HF} 4$ & -1.0177 & -5.6779 & 6.9699 & -2.9264 & 0.6167 & -4.4056 & -1.2723 & -2.2746 & -1.3889 & -0.3081 \\
\hline $\mathrm{FF} 1$ & -1.0959 & -5.8027 & 6.9031 & -2.9673 & 0.7711 & -4.6354 & -1.1673 & -2.5310 & -0.1811 & -0.2552 \\
\hline $\mathrm{FF} 2$ & -1.0919 & -7.9548 & 9.5825 & -3.7118 & 0.9923 & -6.3266 & -1.6282 & -3.1194 & -0.2646 & -0.3279 \\
\hline \multicolumn{11}{|l|}{ Butanes } \\
\hline HH1 & -2.5133 & -8.2529 & 10.7756 & -6.5687 & 1.5327 & -5.4007 & -2.8522 & -4.5348 & -1.5720 & -0.8308 \\
\hline HH2 & -1.5119 & -4.5493 & 6.0109 & -3.8277 & 0.8542 & -3.0525 & -1.4969 & -2.8106 & -0.8198 & -0.4618 \\
\hline HF1 & -1.8065 & -7.2869 & 8.8276 & -4.1659 & 0.8188 & -5.6628 & -1.6241 & -3.2559 & -1.0812 & -0.4189 \\
\hline $\mathrm{HF} 2$ & -1.7537 & -7.5827 & 9.2840 & -4.3375 & 0.8825 & -5.8714 & -1.7113 & -3.3495 & -1.3807 & -0.4503 \\
\hline HF3 & -1.6842 & -8.5403 & 10.4982 & -4.6658 & 1.0237 & -6.5875 & -1.9528 & -3.5165 & -2.2677 & -0.5072 \\
\hline $\mathrm{HF} 4$ & -1.7015 & -7.2192 & 8.8152 & -4.1794 & 0.8819 & -5.5690 & -1.6502 & -3.2311 & -0.7695 & -0.4494 \\
\hline $\mathrm{FF} 1$ & -1.4874 & -4.8906 & 5.7644 & -3.0415 & 0.6803 & -3.8994 & -0.9911 & -2.6752 & -0.1417 & -0.2245 \\
\hline $\mathrm{FF} 2$ & -1.5431 & -7.0455 & 8.4265 & -3.8582 & 0.9340 & -5.6061 & -1.4394 & -3.3005 & -0.4170 & -0.3120 \\
\hline \multicolumn{11}{|c|}{ Pentanes } \\
\hline HH1 & -3.2890 & -10.7154 & 13.9828 & -8.5458 & 1.9895 & -7.0186 & -3.6968 & -5.9167 & -1.6238 & -1.0800 \\
\hline HH2 & -1.9799 & -6.6387 & 8.8281 & -5.4121 & 1.2428 & -4.4628 & -2.1759 & -3.9376 & -1.1283 & -0.6561 \\
\hline HF1 & -2.3580 & -8.8550 & 10.9050 & -5.4759 & 1.0680 & -6.8193 & -2.0357 & -4.2590 & -2.5971 & -0.5548 \\
\hline $\mathrm{HF} 2$ & -2.1264 & -7.3394 & 8.9512 & -4.6544 & 0.9162 & -5.6642 & -1.6752 & -3.6163 & -1.9033 & -0.4716 \\
\hline HF3 & -2.0523 & -8.5165 & 10.4056 & -4.9817 & 1.0403 & -6.5740 & -1.9426 & -3.7850 & -1.6877 & -0.5215 \\
\hline HF4 & -2.3477 & -10.4022 & 12.6961 & -5.8867 & 1.2451 & -8.0478 & -2.3544 & -4.5378 & -1.5210 & -0.6332 \\
\hline $\mathrm{FF} 1$ & -2.0535 & -9.0827 & 10.8071 & -5.0100 & 1.2320 & -7.2488 & -1.8338 & -4.2965 & -0.3063 & -0.4072 \\
\hline $\mathrm{FF} 2$ & -1.9018 & -7.8007 & 9.2848 & -4.4262 & 1.0402 & -6.2235 & -1.5772 & -3.8356 & -0.2504 & -0.3401 \\
\hline \multicolumn{11}{|l|}{ Hexanes } \\
\hline HH1 & -3.8853 & -12.6854 & 16.5336 & -10.0815 & 2.3480 & -8.3072 & -4.3783 & -6.9774 & -1.9601 & -1.2727 \\
\hline HH2 & -2.4579 & -7.7052 & 10.2079 & -6.4026 & 1.4419 & -5.1818 & -2.5234 & -4.6784 & -1.4741 & -0.7692 \\
\hline HF1 & -2.7893 & -12.0610 & 14.9135 & -7.0105 & 1.3687 & -9.3009 & -2.7601 & -5.4285 & -3.2386 & -0.7015 \\
\hline $\mathrm{HF} 2$ & -2.6772 & -9.5310 & 11.6349 & -5.9277 & 1.1466 & -7.3766 & -2.1543 & -4.6434 & -2.1057 & -0.5937 \\
\hline HF3 & -2.6872 & -11.4912 & 14.1912 & -6.7359 & 1.3488 & -8.8474 & -2.6439 & -5.1350 & -2.7370 & -0.6878 \\
\hline $\mathrm{HF} 4$ & -2.6969 & -10.9335 & 13.3236 & -6.3840 & 1.2970 & -8.4645 & -2.4690 & -4.9660 & -1.0364 & -0.6677 \\
\hline FF1 & -2.7133 & -12.7262 & 15.1942 & -6.8939 & 1.7126 & -10.1625 & -2.5636 & -5.8944 & -0.4389 & -0.5606 \\
\hline $\mathrm{FF} 2$ & -1.7413 & -10.4968 & 12.7335 & -5.2852 & 1.3072 & -8.3808 & -2.1161 & -4.4953 & -0.3793 & -0.4107 \\
\hline
\end{tabular}




\begin{tabular}{|c|c|c|c|c|c|c|c|c|c|}
\hline & \multicolumn{9}{|c|}{ LED-TightPNO-DLPNO-CCSD(T)/cc-pVQZ } \\
\hline & $\mathrm{C} 1-\mathrm{C} 2\left[\mathrm{kcal} \mathrm{mol}^{-1}\right]$ & $\mathrm{C} 1-\mathrm{X} 2\left[\mathrm{kcal} \mathrm{mol}^{-1}\right]$ & $\mathrm{X} 1-\mathrm{C} 2\left[\mathrm{kcal} \mathrm{mol}^{-1}\right]$ & $\mathrm{X} 1-\mathrm{X} 2\left[\mathrm{kcal} \mathrm{mol}^{-1}\right]$ & $\mathrm{C} 1-\mathrm{CX} 2\left[\mathrm{kcal} \mathrm{mol}^{-1}\right]$ & $\mathrm{CX} 1-\mathrm{C} 2\left[\mathrm{kcal} \mathrm{mol}^{-1}\right]$ & $\mathrm{X} 1-\mathrm{CX} 2\left[\mathrm{kcal} \mathrm{mol}^{-1}\right]$ & $\mathrm{CX} 1-\mathrm{X} 2\left[\mathrm{kcal} \mathrm{mol}^{-1}\right]$ & $\mathrm{CX} 1-\mathrm{CX} 2\left[\mathrm{kcal} \mathrm{mol}^{-1}\right]$ \\
\hline \multicolumn{10}{|c|}{ Methanes } \\
\hline нН1 & -0.2706 & 0.0000 & 0.0000 & 0.0000 & -0.1704 & -0.1668 & 0.0000 & 0.0000 & -0.2109 \\
\hline $\mathrm{HH} 2$ & -0.1264 & 0.0000 & 0.0000 & 0.0000 & -0.1643 & -0.1585 & 0.0000 & 0.0000 & -0.1677 \\
\hline HF1 & 0.0000 & -0.3181 & 0.0000 & 0.0000 & -0.1573 & 0.0000 & 0.0000 & -0.2113 & -0.3141 \\
\hline $\mathrm{HF} 2$ & 0.0000 & -0.2481 & 0.0000 & 0.0000 & -0.0612 & 0.0000 & 0.0000 & -0.2390 & -0.1395 \\
\hline FF1 & 0.0000 & 0.0000 & 0.0000 & -0.8033 & 0.0000 & 0.0000 & -0.0833 & -0.0804 & -0.0474 \\
\hline $\mathrm{FF} 2$ & 0.0000 & 0.0000 & 0.0000 & -0.7698 & 0.0000 & 0.0000 & -0.0983 & -0.0995 & -0.0663 \\
\hline \multicolumn{10}{|c|}{ Ethanes } \\
\hline HH1 & -0.5235 & 0.0000 & 0.0000 & 0.0000 & -0.5514 & -0.5281 & 0.0000 & 0.0000 & -0.5278 \\
\hline HH2 & -0.4541 & 0.0000 & 0.0000 & 0.0000 & -0.4152 & -0.4155 & 0.0000 & 0.0000 & -0.4679 \\
\hline HF1 & -0.0325 & -0.6689 & 0.0000 & 0.0000 & -0.1131 & -0.0468 & 0.0000 & -0.3996 & -0.3206 \\
\hline HF2 & -0.0410 & -0.6807 & 0.0000 & 0.0000 & -0.1997 & -0.0501 & 0.0000 & -0.4986 & -0.3294 \\
\hline HF3 & -0.0328 & -0.5701 & 0.0000 & 0.0000 & -0.1065 & -0.0324 & 0.0000 & -0.3206 & -0.2892 \\
\hline $\mathrm{HF} 4$ & -0.0350 & -0.7182 & 0.0000 & 0.0000 & -0.1814 & -0.0553 & 0.0000 & -0.4276 & -0.3606 \\
\hline $\mathrm{FF} 1$ & -0.0041 & -0.0647 & -0.0774 & -1.1472 & -0.0120 & 0.0000 & -0.1757 & -0.1877 & -0.0593 \\
\hline $\mathrm{FF} 2$ & -0.0029 & -0.0540 & -0.0517 & -0.8749 & -0.0027 & -0.0030 & -0.1227 & -0.1349 & -0.0618 \\
\hline \multicolumn{10}{|c|}{ Propanes } \\
\hline HH1 & -0.8321 & 0.0000 & 0.0000 & 0.0000 & -0.8191 & -0.8014 & 0.0000 & 0.0000 & -0.7806 \\
\hline HH2 & -0.6254 & 0.0000 & 0.0000 & 0.0000 & -0.5182 & -0.4588 & 0.0000 & 0.0000 & -0.4997 \\
\hline HF1 & -0.0882 & -0.9498 & 0.0000 & 0.0000 & -0.2729 & -0.0954 & 0.0000 & -0.4897 & -0.5679 \\
\hline $\mathrm{HF} 2$ & -0.0704 & -0.7875 & 0.0000 & 0.0000 & -0.2031 & -0.0823 & 0.0000 & -0.4863 & -0.3845 \\
\hline HF3 & -0.0565 & -0.8134 & 0.0000 & 0.0000 & -0.1432 & -0.0517 & 0.0000 & -0.4645 & -0.2679 \\
\hline $\mathrm{HF} 4$ & -0.0630 & -0.9620 & 0.0000 & 0.0000 & -0.1641 & -0.0658 & 0.0000 & -0.6632 & -0.3565 \\
\hline FF1 & -0.0121 & -0.1233 & -0.1474 & -1.4830 & -0.0160 & -0.0201 & -0.3913 & -0.2191 & -0.1186 \\
\hline FF2 & -0.0109 & -0.1486 & -0.1484 & -1.7565 & -0.0108 & -0.0107 & -0.4198 & -0.4199 & -0.1936 \\
\hline \multicolumn{10}{|c|}{ Butanes } \\
\hline HН1 & -1.2381 & 0.0000 & 0.0000 & 0.0000 & -1.1032 & -1.1108 & 0.0000 & 0.0000 & -1.0827 \\
\hline HH2 & -0.9501 & 0.0000 & 0.0000 & 0.0000 & -0.5990 & -0.6018 & 0.0000 & 0.0000 & -0.6597 \\
\hline HF1 & -0.1387 & -1.3930 & 0.0000 & 0.0000 & -0.3278 & -0.1489 & 0.0000 & -0.6875 & -0.5601 \\
\hline $\mathrm{HF} 2$ & -0.1437 & -1.5151 & 0.0000 & 0.0000 & -0.3282 & -0.1331 & 0.0000 & -0.6633 & -0.5662 \\
\hline HF3 & -0.1548 & -1.4167 & 0.0000 & 0.0000 & -0.3691 & -0.1443 & 0.0000 & -0.7451 & -0.6865 \\
\hline $\mathrm{HF} 4$ & -0.1472 & -1.2261 & 0.0000 & 0.0000 & -0.3592 & -0.1228 & 0.0000 & -0.6787 & -0.6970 \\
\hline $\mathrm{FF} 1$ & -0.0197 & -0.2013 & -0.1742 & -1.5540 & -0.0050 & -0.0116 & -0.2745 & -0.2947 & -0.1402 \\
\hline $\mathrm{FF} 2$ & -0.0234 & -0.2229 & -0.2279 & -1.7060 & -0.0092 & -0.0091 & -0.4586 & -0.4372 & -0.2063 \\
\hline \multicolumn{10}{|c|}{ Pentanes } \\
\hline HH1 & -1.6107 & 0.0000 & 0.0000 & 0.0000 & -1.4902 & -1.4898 & 0.0000 & 0.0000 & -1.3260 \\
\hline $\mathrm{HH} 2$ & -1.4310 & 0.0000 & 0.0000 & 0.0000 & -0.8713 & -0.8699 & 0.0000 & 0.0000 & -0.7653 \\
\hline HF1 & -0.2157 & -1.8426 & 0.0000 & 0.0000 & -0.4046 & -0.1918 & 0.0000 & -0.8317 & -0.7726 \\
\hline $\mathrm{HF} 2$ & -0.1911 & -1.5619 & 0.0000 & 0.0000 & -0.4135 & -0.1505 & 0.0000 & -0.6544 & -0.6449 \\
\hline HF3 & -0.2032 & -1.5620 & 0.0000 & 0.0000 & -0.4087 & -0.1682 & 0.0000 & -0.7561 & -0.6868 \\
\hline $\mathrm{HF} 4$ & -0.2250 & -1.7000 & 0.0000 & 0.0000 & -0.5546 & -0.1659 & 0.0000 & -0.9732 & -0.9191 \\
\hline FF1 & -0.0400 & -0.3166 & -0.3293 & -2.2452 & -0.0265 & -0.0285 & -0.5381 & -0.5037 & -0.2686 \\
\hline $\mathrm{FF} 2$ & -0.0270 & -0.2582 & -0.2582 & -2.1589 & -0.0077 & -0.0078 & -0.4711 & -0.4674 & -0.1792 \\
\hline \multicolumn{10}{|c|}{ Hexanes } \\
\hline HН1 & -2.0050 & 0.0000 & 0.0000 & 0.0000 & -1.6503 & -1.6288 & 0.0000 & 0.0000 & -1.6934 \\
\hline $\mathrm{HH} 2$ & -1.7640 & 0.0000 & 0.0000 & 0.0000 & -0.9824 & -0.9822 & 0.0000 & 0.0000 & -0.9498 \\
\hline HF1 & -0.3184 & -2.4036 & 0.0000 & 0.0000 & -0.4817 & -0.2211 & 0.0000 & -1.0655 & -0.9382 \\
\hline $\mathrm{HF} 2$ & -0.2587 & -2.0403 & 0.0000 & 0.0000 & -0.4899 & -0.1794 & 0.0000 & -0.8557 & -0.8194 \\
\hline HF3 & -0.2867 & -2.1555 & 0.0000 & 0.0000 & -0.5224 & -0.2095 & 0.0000 & -1.0138 & -0.9471 \\
\hline $\mathrm{HF} 4$ & -0.2808 & -1.9099 & 0.0000 & 0.0000 & -0.5101 & -0.1942 & 0.0000 & -1.1094 & -0.9616 \\
\hline FF1 & -0.0604 & -0.4425 & -0.4499 & -3.0478 & -0.0536 & -0.0465 & -0.6475 & -0.6226 & -0.5236 \\
\hline $\mathrm{FF} 2$ & -0.0362 & -0.3166 & -0.3152 & -2.4311 & -0.0163 & -0.0170 & -0.5533 & -0.5451 & -0.2646 \\
\hline
\end{tabular}




\subsection{Simple Interaction Models}

The first table contains the computational raw data for determination of the adiabatic ionization potential, as well as, the molecular polarizabilities, the molecular volumes and the molecular surfaces. $\mathrm{E}(\mathrm{X})$ refers to the electronic energy of the ground-state, $\mathrm{E}\left(\mathrm{X}^{+}\right)$refers to the electronic energy of the ionized state, IP refers to the ionization potential, $\alpha_{\text {iso }}$ refers to the isotropic polarizability. The following tables contain all the data of the London dispersion interaction models. $\mathrm{I}_{\text {eff }}$ refers to the pre-factor in the London dispersion formula derived from the ionization potentials of the two molecules, $\mathrm{r}(\mathrm{C}-\mathrm{C})$ refers to the intermolecular distance of the respective carbon chains, $r$ (Disp) refers to the $\mathrm{r}^{-6}$-weighted average distance of the atom types interacting most strongly by dispersion, $\alpha_{1} \alpha_{2}$ refers to the product of the polarizabilities of the interacting molecules, $\mathrm{E}_{\text {disp }}(\mathrm{C}-\mathrm{C})$ refers to energy derived from the intermolecular London dispersion model using the carbon chain distance, $\mathrm{E}_{\text {disp }}(\mathrm{Disp})$ refers to the energy derived from the intermolecular London dispersion model using the $\mathrm{r}^{-6}$-weighted average distance between the molecules. C-C, C-H, H-H, C-F, F-F and H-F refer to the percentage of the contribution of the interaction between these types of atoms to the overall atom-pairwise intermolecular dispersion, $\mathrm{E}_{\mathrm{disp}}$.

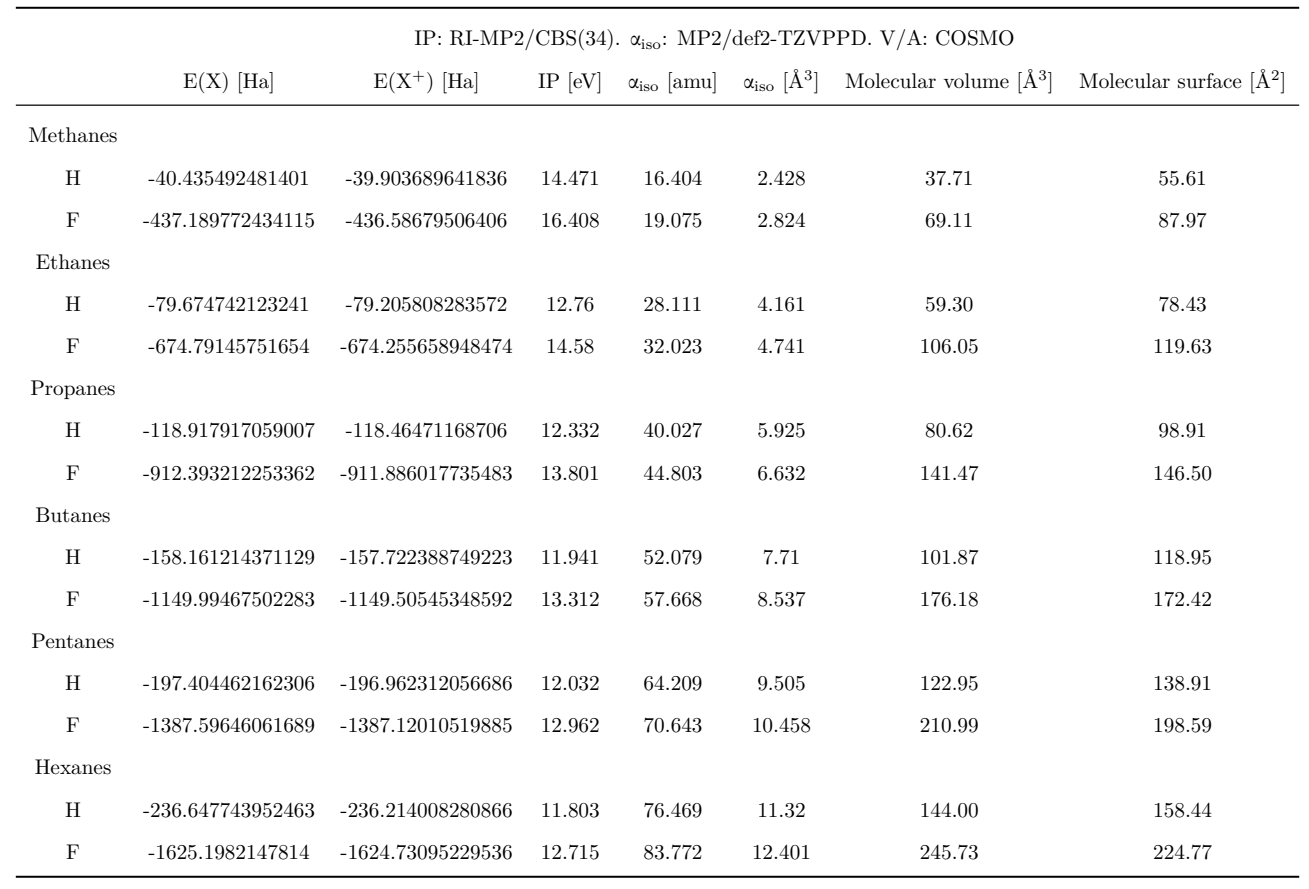




\begin{tabular}{|c|c|c|c|c|c|c|}
\hline & \multicolumn{6}{|c|}{ Intermolecular London Dispersion Model } \\
\hline & $\mathrm{r}(\mathrm{C}-\mathrm{C})[\AA]$ & $\mathrm{r}(\mathrm{Disp})[\AA]$ & $\mathrm{I}_{\mathrm{eff}}[\mathrm{eV}]$ & $\alpha_{1} \alpha_{2}\left[\AA^{6}\right]$ & $\mathrm{E}_{\text {disp }}(\mathrm{C}-\mathrm{C})\left[\mathrm{kcal} \mathrm{mol}^{-1}\right]$ & $\mathrm{E}_{\text {disp }}$ (Disp) $\left[\mathrm{kcal} \mathrm{mol}^{-1}\right]$ \\
\hline \multicolumn{7}{|c|}{ Methanes } \\
\hline HH1 & 3.671 & 3.671 & 7.236 & 5.897 & -0.603 & -0.603 \\
\hline $\mathrm{HH} 2$ & 3.978 & 3.978 & 7.236 & 5.897 & -0.372 & -0.372 \\
\hline $\mathrm{HF} 1$ & 3.783 & 3.717 & 7.689 & 6.857 & -0.622 & -0.692 \\
\hline HF2 & 4.250 & 4.026 & 7.689 & 6.857 & -0.310 & -0.428 \\
\hline $\mathrm{FF} 1$ & 4.079 & 3.874 & 8.204 & 7.974 & -0.492 & -0.670 \\
\hline FF2 & 4.312 & 3.845 & 8.204 & 7.974 & -0.352 & -0.700 \\
\hline \multicolumn{7}{|c|}{ Ethanes } \\
\hline HH1 & 3.714 & 3.857 & 6.380 & 17.318 & -1.456 & -1.160 \\
\hline $\mathrm{HH} 2$ & 3.978 & 3.994 & 6.380 & 17.318 & -0.964 & -0.941 \\
\hline HF1 & 4.336 & 4.118 & 6.805 & 19.727 & -0.699 & -0.953 \\
\hline HF2 & 4.199 & 4.038 & 6.805 & 19.727 & -0.847 & -1.071 \\
\hline HF3 & 4.574 & 4.225 & 6.805 & 19.727 & -0.507 & -0.816 \\
\hline $\mathrm{HF} 4$ & 4.207 & 4.040 & 6.805 & 19.727 & -0.838 & -1.067 \\
\hline $\mathrm{FF} 1$ & 4.652 & 4.112 & 7.290 & 22.473 & -0.559 & -1.172 \\
\hline $\mathrm{FF} 2$ & 5.097 & 4.293 & 7.290 & 22.473 & -0.323 & -0.905 \\
\hline \multicolumn{7}{|c|}{ Propanes } \\
\hline HH1 & 3.818 & 4.064 & 6.166 & 35.111 & -2.419 & -1.661 \\
\hline HH2 & 4.487 & 4.459 & 6.166 & 35.111 & -0.918 & -0.953 \\
\hline HF1 & 4.506 & 4.280 & 6.513 & 39.301 & -1.057 & -1.441 \\
\hline HF2 & 4.740 & 4.451 & 6.513 & 39.301 & -0.781 & -1.138 \\
\hline HF3 & 4.864 & 4.522 & 6.513 & 39.301 & -0.669 & -1.035 \\
\hline $\mathrm{HF} 4$ & 4.694 & 4.350 & 6.513 & 39.301 & -0.828 & -1.307 \\
\hline $\mathrm{FF} 1$ & 4.941 & 4.270 & 6.901 & 43.990 & -0.721 & -1.733 \\
\hline FF2 & 4.912 & 4.113 & 6.901 & 43.990 & -0.748 & -2.168 \\
\hline \multicolumn{7}{|l|}{ Butanes } \\
\hline HH1 & 3.836 & 4.187 & 5.971 & 59.437 & -3.855 & -2.278 \\
\hline HH2 & 4.563 & 4.642 & 5.971 & 59.437 & -1.360 & -1.227 \\
\hline $\mathrm{HF} 1$ & 4.488 & 4.471 & 6.295 & 65.815 & -1.754 & -1.794 \\
\hline HF2 & 4.523 & 4.406 & 6.295 & 65.815 & -1.673 & -1.958 \\
\hline HF3 & 4.535 & 4.368 & 6.295 & 65.815 & -1.647 & -2.062 \\
\hline $\mathrm{HF} 4$ & 4.640 & 4.468 & 6.295 & 65.815 & -1.435 & -1.800 \\
\hline $\mathrm{FF} 1$ & 5.279 & 4.581 & 6.656 & 72.878 & -0.775 & -1.815 \\
\hline FF2 & 5.153 & 4.420 & 6.656 & 72.878 & -0.896 & -2.251 \\
\hline \multicolumn{7}{|c|}{ Pentanes } \\
\hline HH1 & 3.843 & 4.305 & 6.016 & 90.350 & -5.833 & -2.952 \\
\hline $\mathrm{HH} 2$ & 4.619 & 4.737 & 6.016 & 90.350 & -1.936 & -1.663 \\
\hline HF1 & 4.559 & 4.527 & 6.240 & 99.403 & -2.389 & -2.494 \\
\hline HF2 & 4.807 & 4.652 & 6.240 & 99.403 & -1.740 & -2.118 \\
\hline HF3 & 4.759 & 4.617 & 6.240 & 99.403 & -1.848 & -2.215 \\
\hline $\mathrm{HF} 4$ & 4.537 & 4.510 & 6.240 & 99.403 & -2.460 & -2.550 \\
\hline FF1 & 5.052 & 4.514 & 6.481 & 109.363 & -1.474 & -2.899 \\
\hline FF2 & 5.439 & 4.577 & 6.481 & 109.363 & -0.947 & -2.667 \\
\hline \multicolumn{7}{|c|}{ Hexanes } \\
\hline HH1 & 4.067 & 4.439 & 5.901 & 128.146 & -5.777 & -3.421 \\
\hline $\mathrm{HH} 2$ & 4.556 & 4.844 & 5.901 & 128.146 & -2.923 & -2.025 \\
\hline $\mathrm{HF} 1$ & 4.532 & 4.590 & 6.121 & 140.383 & -3.433 & -3.178 \\
\hline HF2 & 4.758 & 4.722 & 6.121 & 140.383 & -2.562 & -2.682 \\
\hline HF3 & 4.674 & 4.636 & 6.121 & 140.383 & -2.852 & -2.995 \\
\hline $\mathrm{HF} 4$ & 4.677 & 4.709 & 6.121 & 140.383 & -2.840 & -2.728 \\
\hline $\mathrm{FF} 1$ & 4.815 & 4.516 & 6.357 & 153.790 & -2.715 & -3.988 \\
\hline FF2 & 5.771 & 4.686 & 6.357 & 153.790 & -0.916 & -3.194 \\
\hline
\end{tabular}




\begin{tabular}{|c|c|c|c|c|c|c|c|}
\hline & $\begin{array}{l}\text { Atom-pa } \\
\text { C-C [\%] }\end{array}$ & $\begin{array}{l}\text { rwise Lonc } \\
\text { C-H [\%] }\end{array}$ & $\begin{array}{l}\text { lon Disper: } \\
\text { H-H [\%] }\end{array}$ & $\begin{array}{l}\text { ion Model } \\
\text { C-F [\%] }\end{array}$ & $\begin{array}{l}\text { 1: Atom-t } \\
\text { F-F [\%] }\end{array}$ & $\begin{array}{r}\text { ype depen } \\
\text { H-F [\%] }\end{array}$ & $\begin{array}{l}\text { ionization potentials } \\
\mathrm{E}_{\text {disp }}\left[\mathrm{kcal} \mathrm{mol}^{-1}\right]\end{array}$ \\
\hline 1-HH1 & 20.89 & 46.01 & 33.1 & 0 & 0 & 0 & 0.461 \\
\hline 1-HH2 & 17.36 & 45.41 & 37.23 & 0 & 0 & 0 & 0.343 \\
\hline 2-HH1 & 22.98 & 46.66 & 30.36 & 0 & 0 & 0 & 1.302 \\
\hline 2-HH2 & 23.27 & 45.96 & 30.77 & 0 & 0 & 0 & 1.043 \\
\hline 3-HH1 & 25.22 & 45.22 & 29.57 & 0 & 0 & 0 & 2.054 \\
\hline 3-HH2 & 24.3 & 46.3 & 29.4 & 0 & 0 & 0 & 1.226 \\
\hline 4-HH1 & 27.37 & 45.38 & 27.25 & 0 & 0 & 0 & 2.967 \\
\hline 4-HH2 & 26.4 & 46.25 & 27.35 & 0 & 0 & 0 & 1.661 \\
\hline 5-HH1 & 28.43 & 45.28 & 26.29 & 0 & 0 & 0 & 3.885 \\
\hline $5-\mathrm{HH} 2$ & 26.61 & 46.11 & 27.28 & 0 & 0 & 0 & 2.308 \\
\hline 6-HH1 & 29.46 & 45.12 & 25.41 & 0 & 0 & 0 & 4.662 \\
\hline 6-HH2 & 28.67 & 45.97 & 25.37 & 0 & 0 & 0 & 2.84 \\
\hline 1-HF1 & 4.06 & 4.45 & 0 & 42.43 & 0 & 49.06 & 0.648 \\
\hline 1-HF2 & 3 & 4.12 & 0 & 39.1 & 0 & 53.78 & 0.435 \\
\hline 2-HF1 & 6.29 & 6.31 & 0 & 43.44 & 0 & 43.96 & 1.054 \\
\hline 2-HF2 & 4.52 & 8.16 & 0 & 29.04 & 0 & 58.28 & 1.332 \\
\hline 2-HF3 & 5.9 & 5.9 & 0 & 43.57 & 0 & 44.64 & 0.901 \\
\hline 2-HF4 & 6.42 & 6.32 & 0 & 43.03 & 0 & 44.23 & 1.193 \\
\hline 3-HF1 & 8.65 & 7.27 & 0 & 43.41 & 0 & 40.67 & 1.693 \\
\hline 3-HF2 & 7.88 & 7.09 & 0 & 43.35 & 0 & 41.69 & 1.341 \\
\hline 3-HF3 & 7.3 & 6.2 & 0 & 43.9 & 0 & 42.6 & 1.188 \\
\hline 3-HF4 & 6.48 & 5.9 & 0 & 43.86 & 0 & 43.75 & 1.499 \\
\hline 4-HF1 & 9.99 & 7.53 & 0 & 43.21 & 0 & 39.27 & 2.19 \\
\hline 4-HF2 & 9.36 & 7.1 & 0 & 44.9 & 0 & 38.64 & 2.296 \\
\hline 4-HF3 & 9.77 & 7.43 & 0 & 44.35 & 0 & 38.46 & 2.45 \\
\hline 4-HF4 & 9.95 & 7.75 & 0 & 43.14 & 0 & 39.16 & 2.2 \\
\hline 5-HF1 & 10.74 & 7.73 & 0 & 45.1 & 0 & 36.43 & 2.923 \\
\hline 5-HF2 & 10.75 & 7.73 & 0 & 44.89 & 0 & 36.63 & 2.506 \\
\hline 5-HF3 & 10.23 & 7.31 & 0 & 45.05 & 0 & 37.41 & 2.623 \\
\hline $5-\mathrm{HF} 4$ & 7.78 & 9.14 & 0 & 34 & 0 & 49.08 & 3.466 \\
\hline 6-HF1 & 10.98 & 7.79 & 0 & 45.67 & 0 & 35.55 & 3.759 \\
\hline 6-HF2 & 10.87 & 7.43 & 0 & 45.48 & 0 & 36.22 & 3.277 \\
\hline 6-HF3 & 11.22 & 7.83 & 0 & 44.9 & 0 & 36.05 & 3.636 \\
\hline 6-HF4 & 11.67 & 8.09 & 0 & 43.51 & 0 & 36.73 & 3.385 \\
\hline 1-FF1 & 0.7 & 0 & 0 & 14.67 & 84.64 & 0 & 0.786 \\
\hline 1-FF2 & 0.49 & 0 & 0 & 12.92 & 86.6 & 0 & 0.803 \\
\hline 2-FF1 & 1.64 & 0 & 0 & 21.61 & 76.75 & 0 & 1.369 \\
\hline 2-FF2 & 1.7 & 0 & 0 & 21.27 & 77.03 & 0 & 1.054 \\
\hline 3-FF1 & 2.33 & 0 & 0 & 24.74 & 72.93 & 0 & 1.97 \\
\hline 3-FF2 & 2.21 & 0 & 0 & 23.67 & 74.12 & 0 & 2.387 \\
\hline 4-FF1 & 3.27 & 0 & 0 & 28.06 & 68.67 & 0 & 2.067 \\
\hline 4-FF2 & 3.34 & 0 & 0 & 28.13 & 68.54 & 0 & 2.61 \\
\hline $5-\mathrm{FF} 1$ & 3.96 & 0 & 0 & 30.03 & 66.01 & 0 & 3.297 \\
\hline $5-\mathrm{FF} 2$ & 3.45 & 0 & 0 & 28.22 & 68.32 & 0 & 2.944 \\
\hline 6-FF1 & 4.26 & 0 & 0 & 30.75 & 65 & 0 & 4.513 \\
\hline 6-FF2 & 3.58 & 0 & 0 & 28.32 & 68.1 & 0 & 3.55 \\
\hline
\end{tabular}




\begin{tabular}{|c|c|c|c|c|c|c|c|}
\hline & \multicolumn{7}{|c|}{ Atom-pairwise London Dispersion Model 2: Atom-type dependent pre-factors A } \\
\hline & $\mathrm{C}-\mathrm{C}[\%]$ & $\mathrm{C}-\mathrm{H}[\%]$ & $\mathrm{H}-\mathrm{H}[\%]$ & $\mathrm{C}-\mathrm{F}[\%]$ & F-F [\%] & $\mathrm{H}-\mathrm{F}[\%]$ & $\mathrm{E}_{\mathrm{disp}}\left[\mathrm{kcal} \mathrm{mol}^{-1}\right]$ \\
\hline 1-HH1 & 21.59 & 48.93 & 29.48 & 0 & 0 & 0 & 0.45 \\
\hline 1-HH2 & 18.04 & 48.59 & 33.36 & 0 & 0 & 0 & 0.333 \\
\hline 2-HH1 & 23.64 & 49.42 & 26.93 & 0 & 0 & 0 & 1.277 \\
\hline 2-HH2 & 23.96 & 48.72 & 27.32 & 0 & 0 & 0 & 1.022 \\
\hline 3-HH1 & 25.93 & 47.86 & 26.21 & 0 & 0 & 0 & 2.014 \\
\hline 3-HH2 & 24.97 & 48.98 & 26.05 & 0 & 0 & 0 & 1.203 \\
\hline 4-HH1 & 28.05 & 47.88 & 24.07 & 0 & 0 & 0 & 2.92 \\
\hline 4-HH2 & 27.05 & 48.78 & 24.16 & 0 & 0 & 0 & 1.635 \\
\hline 5-HH1 & 29.09 & 47.71 & 23.2 & 0 & 0 & 0 & 3.829 \\
\hline 5-HH2 & 27.26 & 48.64 & 24.1 & 0 & 0 & 0 & 2.272 \\
\hline 6-HH1 & 30.12 & 47.49 & 22.4 & 0 & 0 & 0 & 4.6 \\
\hline 6-HH2 & 29.29 & 48.36 & 22.35 & 0 & 0 & 0 & 2.802 \\
\hline 1-HF1 & 3.99 & 4.51 & 0 & 41.03 & 0 & 50.47 & 0.665 \\
\hline 1-HF2 & 2.95 & 4.16 & 0 & 37.71 & 0 & 55.18 & 0.448 \\
\hline 2-HF1 & 6.2 & 6.41 & 0 & 42.08 & 0 & 45.32 & 1.078 \\
\hline 2-HF2 & 4.42 & 8.2 & 0 & 27.88 & 0 & 59.51 & 1.376 \\
\hline 2-HF3 & 5.81 & 5.98 & 0 & 42.21 & 0 & 46 & 0.922 \\
\hline 2-HF4 & 6.32 & 6.41 & 0 & 41.69 & 0 & 45.58 & 1.221 \\
\hline 3-HF1 & 8.54 & 7.39 & 0 & 42.11 & 0 & 41.97 & 1.73 \\
\hline 3-HF2 & 7.77 & 7.2 & 0 & 42.03 & 0 & 43 & 1.371 \\
\hline 3-HF3 & 7.2 & 6.3 & 0 & 42.56 & 0 & 43.95 & 1.215 \\
\hline 3-HF4 & 6.39 & 5.99 & 0 & 42.51 & 0 & 45.11 & 1.534 \\
\hline 4-HF1 & 9.86 & 7.65 & 0 & 41.94 & 0 & 40.55 & 2.237 \\
\hline 4-HF2 & 9.25 & 7.22 & 0 & 43.61 & 0 & 39.92 & 2.344 \\
\hline 4-HF3 & 9.65 & 7.55 & 0 & 43.07 & 0 & 39.73 & 2.501 \\
\hline 4-HF4 & 9.82 & 7.88 & 0 & 41.87 & 0 & 40.43 & 2.247 \\
\hline 5-HF1 & 10.62 & 7.87 & 0 & 43.83 & 0 & 37.68 & 2.982 \\
\hline 5-HF2 & 10.63 & 7.87 & 0 & 43.63 & 0 & 37.88 & 2.557 \\
\hline 5-HF3 & 10.11 & 7.44 & 0 & 43.78 & 0 & 38.67 & 2.676 \\
\hline 5-HF4 & 7.63 & 9.23 & 0 & 32.79 & 0 & 50.35 & 3.563 \\
\hline 6-HF1 & 10.87 & 7.94 & 0 & 44.42 & 0 & 36.78 & 3.832 \\
\hline 6-HF2 & 10.75 & 7.56 & 0 & 44.22 & 0 & 37.46 & 3.342 \\
\hline 6-HF3 & 11.1 & 7.97 & 0 & 43.65 & 0 & 37.28 & 3.708 \\
\hline 6-HF4 & 11.54 & 8.23 & 0 & 42.27 & 0 & 37.96 & 3.454 \\
\hline 1-FF1 & 0.72 & 0 & 0 & 14.95 & 84.32 & 0 & 0.764 \\
\hline 1-FF2 & 0.51 & 0 & 0 & 13.18 & 86.32 & 0 & 0.78 \\
\hline 2-FF1 & 1.7 & 0 & 0 & 21.99 & 76.31 & 0 & 1.334 \\
\hline 2-FF2 & 1.76 & 0 & 0 & 21.64 & 76.6 & 0 & 1.027 \\
\hline 3-FF1 & 2.41 & 0 & 0 & 25.15 & 72.45 & 0 & 1.921 \\
\hline 3-FF2 & 2.28 & 0 & 0 & 24.07 & 73.65 & 0 & 2.328 \\
\hline 4-FF1 & 3.38 & 0 & 0 & 28.49 & 68.13 & 0 & 2.018 \\
\hline 4-FF2 & 3.44 & 0 & 0 & 28.56 & 68 & 0 & 2.548 \\
\hline 5-FF1 & 4.08 & 0 & 0 & 30.47 & 65.45 & 0 & 3.222 \\
\hline 5-FF2 & 3.56 & 0 & 0 & 28.65 & 67.78 & 0 & 2.875 \\
\hline 6-FF1 & 4.39 & 0 & 0 & 31.18 & 64.42 & 0 & 4.412 \\
\hline 6-FF2 & 3.7 & 0 & 0 & 28.75 & 67.55 & 0 & 3.467 \\
\hline
\end{tabular}




\subsection{Interaction Descriptors}

In the following table, $\mathrm{Q}_{\mathrm{atom}}(\mathrm{C})$ refers to the largest $\mathrm{Q}_{\mathrm{atom}}$ of carbon in the respective molecule on its van der Waals surface, $\mathrm{Q}_{\mathrm{atom}}(\mathrm{C})$ refers to the corresponding $\mathrm{Q}_{\mathrm{atom}}$ of either hydrogen or fluorine (depending on the molecule), the same is analogously valid for the $\mathrm{P}_{\text {atom values. }}$ $Q_{\text {tot }}\left(\right.$ ave) refers to the average $Q_{\text {tot }}$ value of the molecule on its van der Waals surface,

$\mathrm{Q}_{\text {tot }}(\max )$ refers to the corresponding maximum on the van der Waals surface, the same is analogously valid for the $\mathrm{P}_{\text {tot }}$ values.

\begin{tabular}{|c|c|c|c|c|c|c|c|c|}
\hline Molecule & $\mathrm{Q}_{\text {atom }}(\mathrm{C})$ & $\mathrm{Q}_{\text {atom }}(\mathrm{X})$ & $\mathrm{P}_{\text {atom }}(\mathrm{C})\left[\mathrm{kcal}^{0.5} \mathrm{~mol}^{-0.5}\right]$ & $\mathrm{P}_{\text {atom }}(\mathrm{X})\left[\mathrm{kcal}^{0.5} \mathrm{~mol}^{-0.5}\right]$ & $\mathrm{Q}_{\text {tot }}$ (ave) & $\mathrm{Q}_{\mathrm{tot}}(\max )$ & $\mathrm{P}_{\text {tot }}$ (ave) $\left[\mathrm{kcal}^{0.5} \mathrm{~mol}^{-0.5}\right]$ & $\mathrm{P}_{\text {tot }}(\max )\left[\mathrm{kcal}^{0.5} \mathrm{~mol}^{-0.5}\right]$ \\
\hline $1-\mathrm{H}$ & 0.0307 & 0.0369 & 0.545 & 0.384 & 0.282 & 0.324 & 3.59 & 4.32 \\
\hline $2-\mathrm{H}$ & 0.0310 & 0.0360 & 0.552 & 0.374 & 0.337 & 0.450 & 4.43 & 6.21 \\
\hline $3-\mathrm{H}$ & 0.0310 & 0.0356 & 0.551 & 0.369 & 0.373 & 0.561 & 4.98 & 7.88 \\
\hline $4-\mathrm{H}$ & 0.0314 & 0.0351 & 0.559 & 0.365 & 0.400 & 0.600 & 5.41 & 8.53 \\
\hline $5-\mathrm{H}$ & 0.0312 & 0.0347 & 0.554 & 0.361 & 0.420 & 0.636 & 5.73 & 9.14 \\
\hline $6-\mathrm{H}$ & 0.0312 & 0.0345 & 0.555 & 0.358 & 0.437 & 0.658 & 6.00 & 9.50 \\
\hline $1-\mathrm{F}$ & 0.0101 & 0.0383 & 0.180 & 0.721 & 0.238 & 0.330 & 4.46 & 6.16 \\
\hline $2-\mathrm{F}$ & 0.0163 & 0.0383 & 0.290 & 0.720 & 0.282 & 0.406 & 5.26 & 7.54 \\
\hline $3-\mathrm{F}$ & 0.0171 & 0.0377 & 0.305 & 0.709 & 0.310 & 0.513 & 5.77 & 9.51 \\
\hline $4-\mathrm{F}$ & 0.0179 & 0.0372 & 0.317 & 0.699 & 0.331 & 0.524 & 6.16 & 9.69 \\
\hline $5-\mathrm{F}$ & 0.0179 & 0.0368 & 0.317 & 0.692 & 0.347 & 0.551 & 6.45 & 10.18 \\
\hline $6-\mathrm{F}$ & 0.0176 & 0.0370 & 0.313 & 0.696 & 0.363 & 0.579 & 6.73 & 10.69 \\
\hline
\end{tabular}




\subsection{Interaction Geometries}

The following table contains volumes, surfaces and the $\mathrm{n}_{\text {contacts }}$ measure of the intermolecular complexes. $\mathrm{V}_{\mathrm{COSMO}}$ refers to the molecular COSMO volume, $\Delta \mathrm{V}_{\mathrm{COSMO}}$ refers to the volume change upon interaction, $\mathrm{S}_{\mathrm{COSMO}}$ refers to the molecular COSMO surface, $\Delta \mathrm{S}_{\mathrm{COSMO}}$ refers to the surface change upon interaction, $\mathrm{n}_{\text {contacts }}$ refers to the newly introduced $\mathrm{n}_{\text {contacts }}$ measure. 


\begin{tabular}{|c|c|c|c|c|c|}
\hline & $\mathrm{V}_{\text {COSMO }}\left[\AA^{3}\right]$ & $\Delta \mathrm{V}_{\mathrm{COSMO}}\left[\AA^{3}\right]$ & $\mathrm{S}_{\mathrm{COSMO}}\left[\AA^{2}\right]$ & $\Delta \mathrm{S}_{\mathrm{COSMO}}\left[\AA^{2}\right]$ & $\mathrm{n}_{\text {contact: }}$ \\
\hline \multicolumn{6}{|c|}{ Methanes } \\
\hline HH1 & 77.77 & 2.357 & 100.72 & -10.50 & 4.973 \\
\hline HH2 & 77.39 & 1.979 & 102.01 & -9.22 & 3.723 \\
\hline HF1 & 109.92 & 3.103 & 126.83 & -16.75 & 6.047 \\
\hline $\mathrm{HF} 2$ & 109.00 & 2.183 & 131.60 & -11.98 & 3.969 \\
\hline $\mathrm{FF} 1$ & 141.29 & 3.074 & 153.64 & -22.30 & 5.571 \\
\hline $\mathrm{FF} 2$ & 139.94 & 1.723 & 156.76 & -19.17 & 5.361 \\
\hline \multicolumn{6}{|l|}{ Ethanes } \\
\hline HH1 & 119.93 & 1.339 & 136.08 & -20.79 & 13.564 \\
\hline $\mathrm{HH} 2$ & 120.65 & 2.055 & 137.73 & -19.14 & 10.852 \\
\hline $\mathrm{HF} 1$ & 167.27 & 1.926 & 174.25 & -23.82 & 9.027 \\
\hline HF2 & 167.30 & 1.953 & 173.50 & -24.56 & 10.304 \\
\hline HF3 & 167.98 & 2.635 & 179.06 & -19.00 & 7.631 \\
\hline $\mathrm{HF} 4$ & 166.94 & 1.595 & 173.84 & -24.23 & 10.235 \\
\hline $\mathrm{FF} 1$ & 214.21 & 2.106 & 209.44 & -29.82 & 8.749 \\
\hline $\mathrm{FF} 2$ & 214.17 & 2.071 & 213.09 & -26.18 & 6.722 \\
\hline \multicolumn{6}{|c|}{ Propanes } \\
\hline HH1 & 163.64 & 2.389 & 169.04 & -28.77 & 20.921 \\
\hline $\mathrm{HH} 2$ & 164.61 & 3.361 & 175.40 & -22.42 & 12.507 \\
\hline $\mathrm{HF} 1$ & 223.24 & 1.147 & 213.32 & -32.09 & 14.288 \\
\hline $\mathrm{HF} 2$ & 224.50 & 2.405 & 220.54 & -24.87 & 11.174 \\
\hline HF3 & 225.97 & 3.879 & 218.70 & -26.71 & 10.059 \\
\hline HF4 & 223.74 & 1.650 & 217.79 & -27.62 & 12.591 \\
\hline $\mathrm{FF} 1$ & 281.81 & -1.132 & 257.26 & -35.75 & 12.486 \\
\hline $\mathrm{FF} 2$ & 280.93 & -2.008 & 250.52 & -42.49 & 15.662 \\
\hline \multicolumn{6}{|c|}{ Butanes } \\
\hline HH1 & 205.46 & 1.717 & 199.06 & -38.84 & 29.619 \\
\hline $\mathrm{HH} 2$ & 207.27 & 3.527 & 209.17 & -28.73 & 16.632 \\
\hline $\mathrm{HF} 1$ & 281.10 & 3.051 & 252.00 & -39.37 & 18.309 \\
\hline $\mathrm{HF} 2$ & 278.49 & 0.434 & 248.90 & -42.46 & 19.075 \\
\hline HF3 & 279.30 & 1.246 & 251.43 & -39.94 & 20.303 \\
\hline $\mathrm{HF} 4$ & 280.42 & 2.371 & 250.39 & -40.98 & 18.450 \\
\hline $\mathrm{FF} 1$ & 352.49 & 0.129 & 304.55 & -40.29 & 13.325 \\
\hline FF2 & 351.13 & -1.226 & 296.80 & -48.04 & 16.643 \\
\hline \multicolumn{6}{|c|}{ Pentanes } \\
\hline HH1 & 248.41 & 2.516 & 230.61 & -47.21 & 38.483 \\
\hline $\mathrm{HH} 2$ & 249.76 & 3.873 & 238.07 & -39.75 & 23.268 \\
\hline $\mathrm{HF} 1$ & 334.17 & 0.233 & 286.31 & -51.19 & 24.154 \\
\hline $\mathrm{HF} 2$ & 335.10 & 1.159 & 291.69 & -45.81 & 20.691 \\
\hline HF3 & 334.55 & 0.614 & 294.84 & -42.66 & 21.835 \\
\hline $\mathrm{HF} 4$ & 335.24 & 1.298 & 284.72 & -52.78 & 25.827 \\
\hline $\mathrm{FF} 1$ & 420.03 & -1.958 & 341.67 & -55.51 & 21.323 \\
\hline $\mathrm{FF} 2$ & 421.83 & -0.157 & 340.53 & -56.65 & 19.185 \\
\hline \multicolumn{6}{|c|}{ Hexanes } \\
\hline HH1 & 289.01 & 1.004 & 262.38 & -54.50 & 45.440 \\
\hline $\mathrm{HH} 2$ & 292.90 & 4.895 & 274.70 & -42.17 & 27.772 \\
\hline $\mathrm{HF} 1$ & 392.17 & 2.434 & 325.93 & -57.28 & 30.733 \\
\hline HF2 & 392.10 & 2.361 & 327.56 & -55.65 & 26.282 \\
\hline HF3 & 390.82 & 1.081 & 325.35 & -57.86 & 29.614 \\
\hline $\mathrm{HF} 4$ & 392.61 & 2.874 & 326.57 & -56.64 & 28.076 \\
\hline $\mathrm{FF} 1$ & 486.80 & -4.663 & 377.79 & -71.76 & 29.102 \\
\hline $\mathrm{FF} 2$ & 489.62 & -1.846 & 390.76 & -58.78 & 22.435 \\
\hline
\end{tabular}




\subsection{Predicted Microscopic and Macroscopic Mixing Behavior}

The following table contains the macroscopic bond association energies obtained on the basis of Flory-Huggins solution theory. $\mathrm{N}_{\text {coord }}$ refers to the average coordination number of one molecule, $\mathrm{BAE}_{\text {macro }}$ refers to the macroscopic bond association energies, the corresponding method used for the respective microscopic bond association energy is provided in parenthesis. For each method, the first column gives the value of the respective complex, the second column gives the average over all complexes of a specific type (i.e. all $\mathbf{1 - H H}$ complexes considered). 


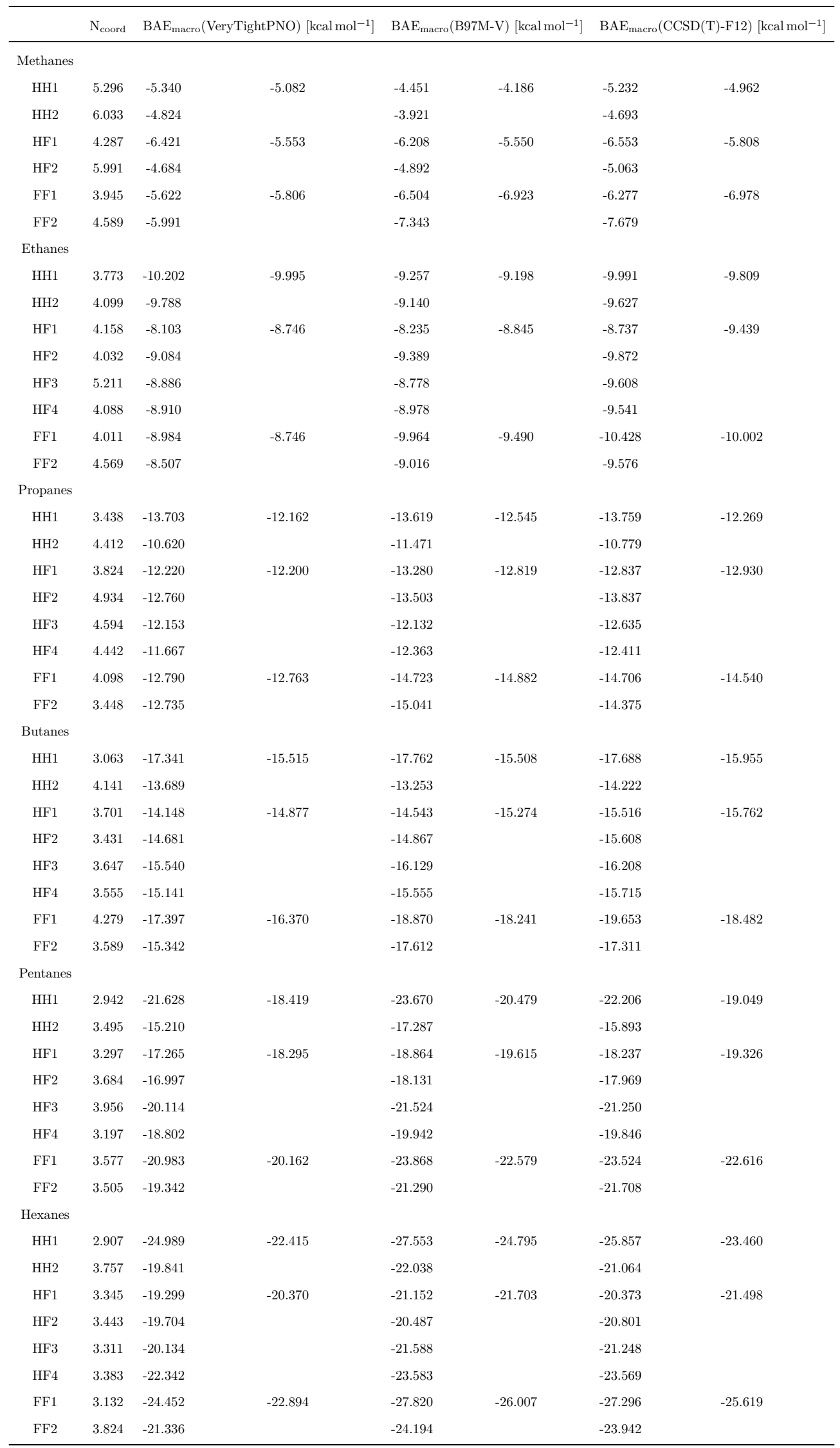




\section{References}

(1) Neese, F. The ORCA program system. WIREs Comput. Mol. Sci. 2012, 2, 73-78.

(2) Neese, F. Software update: The ORCA program system, version 4.0. Wiley Interdiscip. Rev. Comput. Mol. Sci. 2017, 1-6.

(3) Turney, J. M.; Simmonett, A. C.; Parrish, R. M.; Hohenstein, E. G.; Evangelista, F. A.; Fermann, J. T.; Mintz, B. J.; Burns, L. A.; Wilke, J. J.; Abrams, M. L.; Russ, N. J.; Leininger, M. L.; Janssen, C. L.; Seidl, E. T.; Allen, W. D.; Schaefer, H. F.; King, R. A.; Valeev, E. F.; Sherrill, C. D.; Crawford, T. D. Psi4: an open-source ab initio electronic structure program. WIREs Comput. Mol. Sci. 2012, 2, 556-565.

(4) Parrish, R. M.; Burns, L. A.; Smith, D. G.; Simmonett, A. C.; DePrince, A. E.; Hohenstein, E. G.; Bozkaya, U.; Sokolov, A. Y.; Di Remigio, R.; Richard, R. M.; Gonthier, J. F.; James, A. M.; McAlexander, H. R.; Kumar, A.; Saitow, M.; Wang, X.; Pritchard, B. P.; Verma, P.; Schaefer, H. F.; Patkowski, K.; King, R. A.; Valeev, E. F.; Evangelista, F. A.; Turney, J. M.; Crawford, T. D.; Sherrill, C. D. Psi4 1.1: An OpenSource Electronic Structure Program Emphasizing Automation, Advanced Libraries, and Interoperability. J. Chem. Theory Comput. 2017, 13, 3185-3197.

(5) Werner, H. J.; Knowles, P. J.; Knizia, G.; Manby, F. R.; Schütz, M. Molpro: A generalpurpose quantum chemistry program package. Wiley Interdiscip. Rev. Comput. Mol. Sci. 2012, 2, 242-253.

(6) Frisch, M. J.; Trucks, G. W.; Schlegel, H. B.; Scuseria, G. E.; Robb, M. A.; Cheeseman, J. R.; Scalmani, G.; Barone, V.; Mennucci, B.; Petersson, G. A.; Nakatsuji, H.; Caricato, M.; Li, X.; Hratchian, H. P.; Izmaylov, A. F.; Bloino, J.; Zheng, G.; Sonnenberg, J. L.; Hada, M.; Ehara, M.; Toyota, K.; Fukuda, R.; Hasegawa, J.; Ishida, M.; Nakajima, T.; Honda, Y.; Kitao, O.; Nakai, H.; Vreven, T.; Montgomery, J. A., Jr.; Peralta, J. E.; Ogliaro, F.; Bearpark, M.; Heyd, J. J.; Brothers, E.; Kudin, K. N.; 
Staroverov, V. N.; Kobayashi, R.; Normand, J.; Raghavachari, K.; Rendell, A.; Burant, J. C.; Iyengar, S. S.; Tomasi, J.; Cossi, M.; Rega, N.; Millam, J. M.; Klene, M.; Knox, J. E.; Cross, J. B.; Bakken, V.; Adamo, C.; Jaramillo, J.; Gomperts, R.; Stratmann, R. E.; Yazyev, O.; Austin, A. J.; Cammi, R.; Pomelli, C.; Ochterski, J. W.; Martin, R. L.; Morokuma, K.; Zakrzewski, V. G.; Voth, G. A.; Salvador, P.; Dannenberg, J. J.; Dapprich, S.; Daniels, A. D.; Farkas, .; Foresman, J. B.; Ortiz, J. V.; Cioslowski, J.; Fox, D. J. Gaussian 09 Revision D.01. Gaussian Inc. Wallingford CT 2009 .

(7) Baerends, E. J.; Ziegler, T.; Autschbach, J.; Bashford, D.; Berces, A.; Bickelhaupt, F. M.; Bo, C.; Boerrigter, P. M.; Cavallo, L.; Chong, D. P.; Deng, L.; Dickson, R. M.; Ellis, D. E.; van Faassen, M.; Fan, L.; Fischer, T. H.; Fonseca Guerra, C.; Franchini, M.; Ghysels, A.; Giammona, A. ADF2016.

(8) Shao, Y.; Gan, Z.; Epifanovsky, E.; Gilbert, A. T.; Wormit, M.; Kussmann, J.; Lange, A. W.; Behn, A.; Deng, J.; Feng, X.; Ghosh, D.; Goldey, M.; Horn, P. R.; Jacobson, L. D.; Kaliman, I.; Khaliullin, R. Z.; Kus, T.; Landau, A.; Liu, J.; Proynov, E. I.; Rhee, Y. M.; Richard, R. M.; Rohrdanz, M. A.; Steele, R. P.; Sundstrom, E. J.; Woodcock, H. L.; Zimmerman, P. M.; Zuev, D.; Albrecht, B.; Alguire, E.; Austin, B.; Beran, G. J.; Bernard, Y. A.; Berquist, E.; Brandhorst, K.; Bravaya, K. B.; Brown, S. T.; Casanova, D.; Chang, C. M.; Chen, Y.; Chien, S. H.; Closser, K. D.; Crittenden, D. L.; Diedenhofen, M.; Distasio, R. A.; Do, H.; Dutoi, A. D.; Edgar, R. G.; Fatehi, S.; Fusti-Molnar, L.; Ghysels, A.; Golubeva-Zadorozhnaya, A.; Gomes, J.; HansonHeine, M. W.; Harbach, P. H.; Hauser, A. W.; Hohenstein, E. G.; Holden, Z. C.; Jagau, T. C.; Ji, H.; Kaduk, B.; Khistyaev, K.; Kim, J.; Kim, J.; King, R. A.; Klunzinger, P.; Kosenkov, D.; Kowalczyk, T.; Krauter, C. M.; Lao, K. U.; Laurent, A. D.; Lawler, K. V.; Levchenko, S. V.; Lin, C. Y.; Liu, F.; Livshits, E.; Lochan, R. C.; Luenser, A.; Manohar, P.; Manzer, S. F.; Mao, S. P.; Mardirossian, N.; Marenich, A. V.; 
Maurer, S. A.; Mayhall, N. J.; Neuscamman, E.; Oana, C. M.; Olivares-Amaya, R.; Oneill, D. P.; Parkhill, J. A.; Perrine, T. M.; Peverati, R.; Prociuk, A.; Rehn, D. R.; Rosta, E.; Russ, N. J.; Sharada, S. M.; Sharma, S.; Small, D. W.; Sodt, A.; Stein, T.; Stück, D.; Su, Y. C.; Thom, A. J.; Tsuchimochi, T.; Vanovschi, V.; Vogt, L.; Vydrov, O.; Wang, T.; Watson, M. A.; Wenzel, J.; White, A.; Williams, C. F.; Yang, J.; Yeganeh, S.; Yost, S. R.; You, Z. Q.; Zhang, I. Y.; Zhang, X.; Zhao, Y.; Brooks, B. R.; Chan, G. K.; Chipman, D. M.; Cramer, C. J.; Goddard, W. A.; Gordon, M. S.; Hehre, W. J.; Klamt, A.; Schaefer, H. F.; Schmidt, M. W.; Sherrill, C. D.; Truhlar, D. G.; Warshel, A.; Xu, X.; Aspuru-Guzik, A.; Baer, R.; Bell, A. T.; Besley, N. A.; Chai, J. D.; Dreuw, A.; Dunietz, B. D.; Furlani, T. R.; Gwaltney, S. R.; Hsu, C. P.; Jung, Y.; Kong, J.; Lambrecht, D. S.; Liang, W.; Ochsenfeld, C.; Rassolov, V. A.; Slipchenko, L. V.; Subotnik, J. E.; Van Voorhis, T.; Herbert, J. M.; Krylov, A. I.; Gill, P. M.; Head-Gordon, M. Advances in molecular quantum chemistry contained in the Q-Chem 4 program package. Mol. Phys. 2015, 113, 184-215.

(9) Kannemann, F. O.; Becke, A. D. van der Waals Interactions in Density-Functional Theory: Intermolecular Complexes. J. Chem. Theory Comput. 2010, 6, 1081-1088.

(10) Otero-de-la Roza, A.; Johnson, E. R. Non-covalent interactions and thermochemistry using XDM-corrected hybrid and range-separated hybrid density functionals Noncovalent interactions and thermochemistry using XDM-corrected hybrid and rangeseparated hybrid density functionals. J. Chem. Phys. 2013, 138, 204109.

(11) Grimme, S.; Antony, J.; Ehrlich, S.; Krieg, H. A consistent and accurate ab initio parametrization of density functional dispersion correction (DFT-D) for the 94 elements H-Pu. J. Chem. Phys. 2010, 132, 154104.

(12) Weymuth, T.; Proppe, J.; Reiher, M. Statistical Analysis of Semiclassical Dispersion Corrections. 2018, 1-34. 
(13) Grimme, S.; Bannwarth, C.; Shushkov, P. A Robust and Accurate Tight-Binding Quantum Chemical Method for Structures, Vibrational Frequencies, and Noncovalent Interactions of Large Molecular Systems Parametrized for All spd-Block Elements (Z =1-86). J. Chem. Theory Comput. 2017, 13, 1989-2009.

(14) Ahrens, J.; Geveci, B.; Law, C. In ParaView: An End-User Tool for Large Data Visualization; Handbook, V., Ed.; Elsevier, 2005.

(15) Ayachit, U. The ParaView Guide: A Parallel Visualization Application; Kitware, 2015.

(16) Krawczuk, A.; Pérez, D.; MacChi, P. PolaBer: A program to calculate and visualize distributed atomic polarizabilities based on electron density partitioning. J. Appl. Crystallogr. 2014, 47, 1452-1458.

(17) Keith, T. A. AIMAll (Version 17.11.14); TK Gristmill Software, 2017 (aim.tkgristmill.com).

(18) Lu, T.; Chen, F. Multiwfn: A multifunctional wavefunction analyzer. J. Comput. Chem. 2012, 33, 580-592.

(19) Johnson, E. R.; Keinan, S.; Mori-Sánchez, P.; Contreras-Garcia, J.; Cohen, A. J.; Yang, W. Revealing Noncovalent Interactions. J. Am. Chem. Soc. 2010, 132, 64986506 .

(20) Contreras-Garcia, J.; Johnson, E. R.; Keinan, S.; Chaudret, R.; Piquemal, J. P.; Beratan, D. N.; Yang, W. NCIPLOT: A program for plotting noncovalent interaction regions. J. Chem. Theory Comput. 2011, 7, 625-632.

(21) Schrödinger, LLC, The PyMOL Molecular Graphics System, Version 1.8, Schrödinger, LLC. The PyMOL Molecular Graphics System, Version 1.8; 2015.

(22) Chemcraft - graphical software for visualization of quantum chemistry computations.; https://www.chemcraftprog.com. 
(23) Foundation, P. S. Python Language Reference, version 3.6; Available at http://www.python.org.

(24) Kozuch, S.; Martin, J. M. L. Spin-component-scaled double hybrids: An extensive search for the best fifth-rung functionals blending DFT and perturbation theory. $J$. Comput. Chem. 2013, 34, 2327-2344.

(25) Weigend, F.; Ahlrichs, R. Balanced basis sets of split valence, triple zeta valence and quadruple zeta valence quality for $\mathrm{H}$ to Rn: Design and assessment of accuracy. Phys. Chem. Chem. Phys. 2005, 7, 3297-305.

(26) Johnson, E. R.; Otero-de-la Roza, A.; Dale, S. G.; DiLabio, G. A. Efficient basis sets for non-covalent interactions in XDM-corrected density-functional theory. J. Chem. Phys. 2013, 139, 214109.

(27) Neese, F.; Wennmohs, F.; Hansen, A.; Becker, U. Efficient, approximate and parallel Hartree-Fock and hybrid DFT calculations. A 'chain-of-spheres' algorithm for the Hartree-Fock exchange. Chem. Phys. 2009, 356, 98-109.

(28) Kossmann, S.; Neese, F. Efficient structure optimization with second-order many-body perturbation theory: The RIJCOSX-MP2 method. J. Chem. Theory Comput. 2010, 6, 2325-2338.

(29) Weigend, F. Accurate Coulomb-fitting basis sets for H to Rn. Phys. Chem. Chem. Phys. 2006, 8, 1057-1065.

(30) Hellweg, A.; Hättig, C.; Höfener, S.; Klopper, W. Optimized accurate auxiliary basis sets for RI-MP2 and RI-CC2 calculations for the atoms Rb to Rn. Theor. Chem. Acc. 2007, 117, 587-597.

(31) Dunning, T. H. Gaussian basis sets for use in correlated molecular calculations. I. The atoms boron through neon and hydrogen. J. Chem. Phys. 1989, 90, 1007-1023. 
(32) Kendall, R. A.; Dunning, T. H.; Harrison, R. J. Electron affinities of the first-row atoms revisited. Systematic basis sets and wave functions. J. Chem. Phys. 1992, 96, $6796-6806$.

(33) Liakos, D. G.; Neese, F. Is It Possible To Obtain Coupled Cluster Quality Energies at near Density Functional Theory Cost? Domain-Based Local Pair Natural Orbital Coupled Cluster vs Modern Density Functional Theory. J. Chem. Theory Comput. 2015, 11, 4054-4063.

(34) Pavošević, F.; Peng, C.; Pinski, P.; Riplinger, C.; Neese, F.; Valeev, E. F. SparseMaps - A systematic infrastructure for reduced scaling electronic structure methods. V. Linear scaling explicitly correlated coupled-cluster method with pair natural orbitals. J. Chem. Phys. 2017, 146, 174108.

(35) Bannwarth, C.; Ehlert, S.; Grimme, S. GFN2-xTB - an Accurate and Broadly Parametrized Self-Consistent Tight-Binding Quantum Chemical Method with Multipole Electrostatics and Density-Dependent Dispersion Contributions. 2018, 10.26434/chemrxiv.7246238.v2.

(36) Adler, T. B.; Knizia, G.; Werner, H. J. A simple and efficient CCSD(T)-F12 approximation. J. Chem. Phys. 2007, 127, 221106.

(37) Knizia, G.; Adler, T. B.; Werner, H. J. Simplified CCSD(T)-F12 methods: Theory and benchmarks. J. Chem. Phys. 2009, 130, 054104.

(38) Peterson, K. A.; Adler, T. B.; Werner, H. J. Systematically convergent basis sets for explicitly correlated wavefunctions: The atoms H, He, B-Ne, and Al-Ar. J. Chem. Phys. 2008, 128, 084102.

(39) Sinanoglu, O. Many-electron theory of atoms and molecules. I. Shells, electron pairs vs many-electron correlations. J. Chem. Phys. 1962, 36, 706-717. 
(40) Cizek, J. On the Correlation Problem in Atomic and Molecular Systems. Calculation of Wavefunction Components in Ursell-Type Expansion Using Quantum-Field Theoretical Methods. J. Chem. Phys. 1966, 45, 4256.

(41) Vahtras, O.; Almlöf, J.; Feyereisen, M. W. Integral approximations for LCAO-SCF calculations. Chem. Phys. Lett. 1993, 213, 514-518.

(42) Feyereisen, M.; Fitzgerald, G.; Komornicki, A. Use of approximate integrals in ab initio theory. An application in MP2 energy calculations. Chem. Phys. Lett. 1993, 208, 359-363.

(43) Neese, F.; Wennmohs, F.; Hansen, A. Efficient and accurate local approximations to coupled-electron pair approaches: An attempt to revive the pair natural orbital method. J. Chem. Phys. 2009, 130, 114108.

(44) Riplinger, C.; Neese, F. An efficient and near linear scaling pair natural orbital based local coupled cluster method. J. Chem. Phys. 2013, 138, 034106.

(45) Riplinger, C.; Sandhoefer, B.; Hansen, A.; Neese, F. Natural triple excitations in local coupled cluster calculations with pair natural orbitals. J. Chem. Phys. 2013, 139, 134101.

(46) Weigend, F. A fully direct RI-HF algorithm: Implementation, optimised auxiliary basis sets, demonstration of accuracy and efficiency. Phys. Chem. Chem. Phys. 2002, 4, 4285-4291.

(47) Moller, C.; Plesset, M. S. Note on an Approximation Treatment for Many-Electron Systems. Phys. Rev. 1934, 46, 618-622.

(48) Grimme, S. Improved second-order Møller-Plesset perturbation theory by separate scaling of parallel- and antiparallel-spin pair correlation energies. J. Chem. Phys. 2003, 118, 9095-9102. 
(49) Pople, J. A.; Binkley, J. S.; Seeger, R. Theoretical models incorporating electron correlation. Int. J. Quantum Chem. 1976, 10, 1-19.

(50) Pople, J. A.; Seeger, R.; Krishnan, R. Variational configuration interaction methods and comparison with perturbation theory. Int. J. Quantum Chem. 1977, 11, 149-163.

(51) Mardirossian, N.; Head-Gordon, M. Mapping the genome of meta-generalized gradient approximation density functionals: The search for B97M-V. J. Chem. Phys. 2015, 142, 074111.

(52) Newton, M. D.; Ostlund, N. S.; Pople, J. A. Projection of Diatomic Differential Overlap: Least-Squares Projection of Two-Center Distributions onto One-Center Functions. J. Chem. Phys. 1968, 49, 5192-5194.

(53) Baerends, E.; Ellis, D.; Ros, P. Self-consistent molecular Hartree-Fock-Slater calculations I. The computational procedure. Chem. Phys. 1973, 2, $41-51$.

(54) Skylaris, C.-K.; Gagliardi, L.; Handy, N.; Ioannou, A.; Spencer, S.; Willetts, A. On the resolution of identity Coulomb energy approximation in density functional theory. THEOCHEM 2000, 501-502, 229 - 239.

(55) Weigend, F. Hartree-fock exchange fitting basis sets for $\mathrm{H}$ to Rn. J. Comput. Chem. 2008, 29, 167-175.

(56) Mardirossian, N.; Head-Gordon, M. $\omega$ B97M-V: A combinatorially optimized, rangeseparated hybrid, meta-GGA density functional with VV10 nonlocal correlation. J. Chem. Phys. 2016, 144, 214110.

(57) Becke, A. D. Density-functional exchange-energy approximation with correct asymptotic behavior. Phys. Rev. A 1988, 38, 3098-3100.

(58) Lee, C.; Yang, W.; Parr, R. G. Development of the Colle-Salvetti correlation-energy formula into a functional of the electron density. Phys. Rev. B 1988, 37, 785-789. 
(59) Becke, A. D. Density-functional thermochemistry. III. The role of exact exchange. J. Chem. Phys. 1993, 98, 5648-5652.

(60) Becke, A. D.; Johnson, E. R. Exchange-hole dipole moment and the dispersion interaction. J. Chem. Phys. 2005, 122, 154104.

(61) Becke, A. D.; Johnson, E. R. A density-functional model of the dispersion interaction. J. Chem. Phys. 2005, 123, 154101.

(62) Johnson, E. R.; Becke, A. D. A post-Hartree-Fock model of intermolecular interactions. J. Chem. Phys. 2005, 123, 024101.

(63) Johnson, E. R.; Becke, A. D. A post-Hartree-Fock model of intermolecular interactions: Inclusion of higher-order corrections. J. Chem. Phys. 2006, 124, 174104.

(64) Becke, A. D.; Johnson, E. R. Exchange-hole dipole moment and the dispersion interaction revisited. J. Chem. Phys. 2007, 127, 154104.

(65) Perdew, J. P.; Burke, K.; Ernzerhof, M. Generalized gradient approximation made simple. Phys. Rev. Lett. 1996, 7r7, 3865-3868.

(66) Zhao, Y.; Truhlar, D. G. A new local density functional for main-group thermochemistry, transition metal bonding, thermochemical kinetics, and noncovalent interactions. J. Chem. Phys. 2006, 125, 194101.

(67) Vydrov, O. A.; Van Voorhis, T. Nonlocal van der Waals density functional: The simpler the better. J. Chem. Phys. 2010, 133, 244103.

(68) Hujo, W.; Grimme, S. Performance of the van der waals density functional VV10 and (hybrid)GGA variants for thermochemistry and noncovalent interactions. J. Chem. Theory Comput. 2011, 7, 3866-3871.

(69) Grimme, S.; Ehrlich, S.; Goerigk, L. Effect of the damping function in dispersion corrected density functional theory. J. Comput. Chem. 2011, 32, 1456-1465. 
(70) Zhao, Y.; Truhlar, D. G. The M06 suite of density functionals for main group thermochemistry, thermochemical kinetics, noncovalent interactions, excited states, and transition elements: Two new functionals and systematic testing of four M06-class functionals and 12 other function. Theor. Chem. Acc. 2008, 120, 215-241.

(71) Steinmann, S. N.; Corminboeuf, C. A system-dependent density-based dispersion correction. J. Chem. Theory Comput. 2010, 6, 1990-2001.

(72) Steinmann, S. N.; Corminboeuf, C. Comprehensive benchmarking of a densitydependent dispersion correction. J. Chem. Theory Comput. 2011, 7, 3567-3577.

(73) Steinmann, S. N.; Corminboeuf, C. A generalized-gradient approximation exchange hole model for dispersion coefficients. J. Chem. Phys. 2011, 134, 044117.

(74) Van Lenthe, E.; Baerends, E. J. Optimized Slater-type basis sets for the elements 1-118. J. Comput. Chem. 2003, 24, 1142-1156.

(75) Franchini, M.; Philipsen, P. H. T.; Van Lenthe, E.; Visscher, L. Accurate Coulomb potentials for periodic and molecular systems through density fitting. J. Chem. Theory Comput. 2014, 10, 1994-2004.

(76) Grimme, S. Semiempirical hybrid density functional with perturbative second-order correlation. J. Chem. Phys. 2006, 124, 034108.

(77) Tao, J.; Perdew, J. P.; Staroverov, V. N.; Scuseria, G. E. Climbing the density functional ladder: Nonempirical meta-generalized gradient approximation designed for molecules and solids. Phys. Rev. Lett. 2003, 91, 3-6.

(78) Lin, Y. S.; Li, G. D.; Mao, S. P.; Chai, J. D. Long-range corrected hybrid density functionals with improved dispersion corrections. J. Chem. Theory Comput. 2013, 9, $263-272$. 
(79) Lee, K.; Murray, É. D.; Kong, L.; Lundqvist, B. I.; Langreth, D. C. Higher-accuracy van der Waals density functional. Phys. Rev. B - Condens. Matter Mater. Phys. 2010, 82, 3-6.

(80) Jensen, F. Polarization consistent basis sets: Principles. J. Chem. Phys. 2001, 115, 9113-9125.

(81) Jensen, F. Polarization consistent basis sets. II. Estimating the Kohn-Sham basis set limit. J. Chem. Phys. 2002, 116, 7372-7379.

(82) Jensen, F. Polarization consistent basis sets. III. The importance of diffuse functions. J. Chem. Phys. 2002, 117, 9234-9240.

(83) Grimme, S. Semiempirical GGA-type density functional constructed with a long-range dispersion correction. J. Comput. Chem. 2006, 27, 1787-1799.

(84) Parker, T. M.; Burns, L. A.; Parrish, R. M.; Ryno, A. G.; Sherrill, C. D. Levels of symmetry adapted perturbation theory (SAPT). I. Efficiency and performance for interaction energies. J. Chem. Phys. 2014, 140, 094106.

(85) Papajak, E.; Zheng, J.; Xu, X.; Leverentz, H. R.; Truhlar, D. G. Perspectives on Basis Sets Beautiful: Seasonal Plantings of Diffuse Basis Functions. J. Chem. Theory Comput. 2011, 7, 3027-3034.

(86) Papajak, E.; Truhlar, D. G. Convergent Partially Augmented Basis Sets for PostHartree - Fock Calculations of Molecular Properties and Reaction Barrier Heights. J. Chem. Theory Comput. 2011, 10-18.

(87) Gonthier, J. F.; Sherrill, C. D. Density-fitted open-shell symmetry-adapted perturbation theory and application to $\pi$-stacking in benzene dimer cation and ionized DNA base pair steps. J. Chem. Phys. 2016, 145, 134106. 
(88) Schneider, W. B.; Bistoni, G.; Sparta, M.; Saitow, M.; Riplinger, C.; Auer, A. A.; Neese, F. Decomposition of Intermolecular Interaction Energies within the Local Pair Natural Orbital Coupled Cluster Framework. J. Chem. Theory Comput. 2016, 12, $4778-4792$.

(89) Wuttke, A.; Mata, R. A. Visualizing dispersion interactions through the use of local orbital spaces. J. Comput. Chem. 2017, 38, 15-23.

(90) Werner, H. J.; Manby, F. R.; Knowles, P. J. Fast linear scaling second-order MøllerPlesset perturbation theory (MP2) using local and density fitting approximations. $J$. Chem. Phys. 2003, 118, 8149-8160.

(91) Head-Gordon, M.; Pople, J. A.; Frisch, M. J. MP2 energy evaluation by direct methods. Chem. Phys. Lett. 1988, 153, 503-506.

(92) Rappoport, D.; Furche, F. Property-optimized Gaussian basis sets for molecular response calculations. J. Chem. Phys. 2010, 133, 134105.

(93) Klamt, A. Conductor-like Screening Model for Real Solvents: A New Approach to the Quantitative Calculation of Solvation Phenomena. J. Phys. Chem. 1995, 99, 22242235 .

(94) Pye, C. C.; Ziegler, T.; van Lenthe, E. L.; Louwen, J. N. An implementation of the conductor-like screening model of solvation within the Amsterdam density functional package - Part II. COSMO for real solvents. Can. J. Chem. 2009, 87, 790-797.

(95) Rezáč, J.; Riley, K. E.; Hobza, P. Benchmark Calculations of Noncovalent Interactions of Halogenated Molecules. J. Chem. Theory Comput. 2012, 8, 4285-4292.

(96) Burns, L. A.; Marshall, M. S.; Sherrill, C. D. Appointing silver and bronze standards for noncovalent interactions: A comparison of spin-component-scaled (SCS), explicitly 
correlated (F12), and specialized wavefunction approaches. J. Chem. Phys. 2014, 141, 234111.

(97) Friedrich, J. Efficient Calculation of Accurate Reaction Energies - Assessment of Different Models in Electronic Structure Theory. J. Chem. Theory Comput. 2015, 11, 3596-3609.

(98) Sirianni, D. A.; Burns, L. A.; Sherrill, C. D. Comparison of explicitly correlated methods for computing high-accuracy benchmark energies for noncovalent interactions. $J$. Chem. Theory Comput. 2017, 13, 86-99.

(99) Liakos, D. G.; Sparta, M.; Kesharwani, M. K.; Martin, J. M. L.; Neese, F. Exploring the accuracy limits of local pair natural orbital coupled-cluster theory. J. Chem. Theory Comput. 2015, 11, 1525-1539.

(100) Minenkov, Y.; Wang, H.; Wang, Z.; Sarathy, S. M.; Cavallo, L. Heats of Formation of Medium-Sized Organic Compounds from Contemporary Electronic Structure Methods. J. Chem. Theory Comput. 2017, 13, 3537-3560.

(101) Minenkov, Y.; Sliznev, V. V.; Cavallo, L. Accurate Gas Phase Formation Enthalpies of Alloys and Refractories Decomposition Products. Inorg. Chem. 2017, 56, 1386-1401.

(102) Jurecka, P.; Sponer, J.; Cerný, J.; Hobza, P. Benchmark database of accurate (MP2 and $\operatorname{CCSD}(\mathrm{T})$ complete basis set limit) interaction energies of small model complexes, DNA base pairs, and amino acid pairs. Phys. Chem. Chem. Phys. 2006, 8, 1985-1993.

(103) Liakos, D. G.; Neese, F. Improved correlation energy extrapolation schemes based on local pair natural orbital methods. J. Phys. Chem. A 2012, 116, 4801-4816.

(104) Chattoraj, J.; Risthaus, T.; Rubner, O.; Heuer, A.; Grimme, S. A multi-scale approach to characterize pure $\mathrm{CH} 4, \mathrm{CF} 4$, and $\mathrm{CH} 4 / \mathrm{CF} 4$ mixtures. J. Chem. Phys. 2015, 142, 164508. 
(105) Caldeweyher, E.; Bannwarth, C.; Grimme, S. Extension of the D3 dispersion coefficient model. J. Chem. Phys. 2017, 147, 034112.

(106) London, F. Zur Theorie und Systematik der Molekularkräfte. Zeitschrift für Phys. 1930, 63, 245-279.

(107) Eisenschitz, R.; London, F. Über das Verhältnis der van der Waalsschen Kräfte zu den homöopolaren Bindungskräften. Zeitschrift für Phys. 1930, 60, 491-527.

(108) Alvarez, S. A cartography of the van der Waals territories. Dalt. Trans. 2013, 42, 8617.

(109) Huggins, M. L. Thermodynamic Properties of Solutions of Long-chain Compounds. Ann. N.Y. Acad. Sci. 1942, 43, 1-32.

(110) Flory, P. J. Thermodynamics of High Polymer Solutions. 1942, 10, 51-61.

(111) Grosse, A. V.; Cady, G. H. Properties of Fluorocarbons. Ind. Eng. Chem. 1947, 39, 367-374.

(112) Smart, B. E. Fluorine substituent effects (on bioactivity). J. Fluorine Chem. 2001, $109,3-11$.

(113) Acree, W. E., Jr.; Chickos, J. S. In NIST Chemistry WebBook, NIST Standard Reference Database Number 69; Linstrom, P. J., Mallard, W. G., Eds.; National Institute of Standards and Technology: Gaithersburg MD, 20899, (retrieved September 17, 2018); Chapter Boiling Point Data.

(114) Gilmour, J. B.; Zwicker, J. O.; Katz, J.; Scott, R. L. Fluorocarbon solutions at low temperatures. V. The liquid mixtures C2H6 + C2F6, C3H8 + C2F6, CH4 + C3F8, $\mathrm{C} 2 \mathrm{H} 6+\mathrm{C} 3 \mathrm{~F} 8, \mathrm{C} 3 \mathrm{H} 8+\mathrm{C} 3 \mathrm{~F} 8, \mathrm{C} 4 \mathrm{C} 10+\mathrm{C} 3 \mathrm{~F} 8$, iso-C4H10 + C3F8, C3H8 + C4F10, nC6H14 + C4F10, n-C7H16 + C4F10, n-C,H20 + n-C4F10, and n-C10H22 + n-C4F10. J. Phys. Chem. 1967, 71, 3259-3270. 
(115) Weast, R. C., Grasselli, J. G., Eds. CRC Handbook of Data on Organic Compounds; CRC Press, Inc.: Boca Raton, FL, 1989.

(116) Majer, V.; Svoboda, V. Enthalpies of Vaporization of Organic Compounds: A Critical Review and Data Compilation; Blackwell Scientific Publications: Oxford, UK, 1985.

(117) van der Waals, J. D. Nobel Lectures, Physics 1901-1921; Elsevier Publishing Company: Amsterdam, 1967; pp 254-265.

(118) Hill, T. L. An Introduction to Statistical Thermodynamics; Dover Publications: New York, 1986; pp 286-289.

(119) Acree, W. E., Jr.; Chickos, J. S. In NIST Chemistry WebBook, NIST Standard Reference Database Number 69; Linstrom, P. J., Mallard, W. G., Eds.; National Institute of Standards and Technology: Gaithersburg MD, 20899, (retrieved September 17, 2018); Chapter Phase Transition Enthalpy Measurements of Organic and Organometallic Compounds.

(120) Saikawa, K.; Kijima, J.; Uematsu, M.; Watanabe, K. Determination of the critical temperature and density of hexafluoroethane. J. Chem. Eng. Data 1979, 24, 165167.

(121) Leu, A. D.; Robinson, D. B. High-pressure vapor-liquid equilibrium phase properties of the octafluoropropane (K-218)-chlorodifluoromethane (Freon-22) binary system. $J$. Chem. Eng. Data 1992, 37, 7-10.

(122) Vandana, V.; Rosenthal, D. J.; Teja, A. S. The critical properties of perfluoro nalkanes. Fluid Phase Equilib. 1994, 99, 209-218.

(123) Mousa, A. H. N. Study of vapor pressure and critical properties of perfluoro-n-hexane. J. Chem. Eng. Data 1978, 23, 133-134. 
(124) Steele, W. V.; Chirico, R. D.; Knipmeyer, S. E.; Nguyen, A. Vapor Pressure, Heat Capacity, and Density along the Saturation Line, Measurements for Cyclohexanol, 2-Cyclohexen-1-one, 1,2-Dichloropropane, 1,4-Di-tert-butylbenzene, $( \pm)$-2Ethylhexanoic Acid, 2-(Methylamino)ethanol, Perfluoro-n-heptane, and Sulfolane. J. Chem. Eng. Data 1997, 42, 1021-1036.

(125) Stephenson, R. M.; Malanowski, S. Handbook of the Thermodynamics of Organic Compounds; Elsevier: New York, Amsterdam, London, 1987.

(126) Witt, R. K.; Kemp, J. D. The Heat Capacity of Ethane from $15^{\circ} \mathrm{K}$. To the Boiling Point. The Heat of Fusion and the Heat of Vaporization. J. Am. Chem. Soc. 1937, 59, 273-276.

(127) Aston, J. G.; Sagenkahn, M. L.; Szasz, G. J.; Moessen, G. W.; Zuhr, H. F. The Heat Capacity and Entropy, Heats of Fusion and Vaporization and the Vapor Pressure of Trimethylamine. The Entropy from Spectroscopic and Molecular Data. J. Am. Chem. Soc. 1944, 66, 1171-1177.

(128) Simons, J. H.; Mausteller, J. W. The properties of n-butforane and its mixtures with n-butane. J. Chem. Phys. 1952, 20, 1516-1519.

(129) Van Ness, H. C.; Soczek, C. A.; Peloquin, G. L.; Machado, R. L. Thermodynamic Excess Properties Of Three Alcohol-Hydrocarbon Systems. J. Chem. Eng. Data 1967, 12, 217-224.

(130) Michou-Saucet, M. A.; Jose, J.; Michou-Saucet, C.; Merlin, J. C. Pressions de vapeur et enthalpies libres d'exces de systemes binaires: Hexamethylphosphorotriamide (HMPT) + n-hexane; n-heptane; n-octane: A 298,15 K; 303,15 K; 313,15 K; 323,15 K; 333,15 K. Thermochim. Acta 1984, 75, 85-106.

(131) Varushchenko, R.; Bulgakova, L. L.; Minzabekyants, P. S.; Makarov, K. Russ. J. Phys. Chem. 1981, 55, 1480. 
(132) Viton, C.; Chavret, M.; Jose, J. Enthalpies of vaporization of normal alkanes from nonane to pentadecane at temperatures from 298 to 359 K. ELDATA: Int. Electron. J. Phys. Chem. Data 1996, 2, 103.

(133) Lo Nostro, P.; Scalise, L.; Baglioni, P. Phase separation in binary mixtures containing linear perfluoroalkanes. J. Chem. Eng. Data 2005, 50, 1148-1152.

(134) Straty, G. C. Velocity of sound in dense fluid methane. Cryogenics 1974, 14, 367-370.

(135) Tsumura, R.; Straty, G. C. Speed of sound in saturated and compressed fluid ethane. Cryogenics 1977, 17, 195-200.

(136) Younglove, B. A. Velocity of Sound in Liquid Propane. J. Res. Natl. Bur. Stand. 1980, $86,165-170$.

(137) Hwang, C.-a.; Holste, J. C.; Hall, K. R.; Gammon, B. E.; Marsh, K. N.; Parrish, W. R. PVT Properties and Bubble Point Pressures for Compositionally Characterized Commercial Grade Butane, 2-Methylpropane, and Natural Gasoline. J. Chem. Eng. Data 1996, 41, 1517-1521.

(138) Hawary, A. E.; Meier, K. Measurements of the Speed of Sound in Liquid n-Butane. J. Chem. Eng. Data 2016, 61, 3858-3867.

(139) Chávez, M.; Palaclos, J. M.; Tsumura, R.; Palaclos, J. M.; Tsumura, R. Speed of Sound in Saturated Liquid n-Pentane. J. Chem. Eng. Data 1982, 27, 350-351.

(140) Mascato, E.; Mosteiro, L.; Piñeiro, M. M.; García, J.; Iglesias, T. P.; Legido, J. L. Density, speed of sound and refractive index of (n-hexane + cyclohexane + 1-hexanol) at $\mathrm{T}=298.15 \mathrm{~K}$. J. Chem. Thermodyn. 2001, 33, 1081-1096.

(141) Devi, R.; Gahlyan, S.; Rani, M.; Maken, S. Thermodynamic and acoustic properties of binary mixtures of diisopropyl ether, benzene and alkanes at 298.15, 308.15 and 
318.15 K: Prigogine-Flory-Patterson theory and graph theory. J. Mol. Liq. 2019, 275, $364-377$.

(142) Acree, W. E., Jr.; Chickos, J. S. In NIST Chemistry WebBook, NIST Standard Reference Database Number 69; Linstrom, P. J., Mallard, W. G., Eds.; National Institute of Standards and Technology: Gaithersburg MD, 20899, (retrieved December 15, 2018); Chapter REFPROP version 7.

(143) Brown, J. A.; Mears, W. H. Physical properties of n-perfluorobutane. J. Phys. Chem. 1958, 62, 960-962.

(144) Burger, L. L.; Cady, G. H. Physical Properties of Perfluoropentanes. J. Am. Chem. Soc. 1951, 73, 4243-4246.

(145) Cochran, A.; North, M.; Chemistry, A. Ultrasonic Studies of Perfluoro-n-alkanes. J. Chem. Soc., Faraday Trans. 2 1974, 70, 1274-1279.

(146) Hallewell, G.; Vacek, V.; Vinš, V. Properties of saturated fluorocarbons: Experimental data and modeling using perturbed-chain-SAFT. Fluid Phase Equilib. 2010, 292, 6470.

(147) Kayukawa, Y.; Hasumoto, M.; Kano, Y.; Watanabe, K. Liquid-phase thermodynamic properties for propane (1), n-butane (2), and isobutane (3). J. Chem. Eng. Data 2005, $50,556-564$.

(148) Defibaugh, D. R.; Moldover, M. R. Compressed and Saturated Liquid Densities for 18 Halogenated Organic Compounds $<\sup >\dagger</$ sup $>$. J. Chem. Eng. Data 1997, 42, $160-168$.

(149) Campos-Vallette, M.; Campos-Vallette, M.; Diaz-Fleming, G. Some physical and thermodynamic properties of $\mathrm{n}-\mathrm{CnF} 2 \mathrm{n}+2$ compounds with $\mathrm{n}=4-8$. Semina 1983, 4 , 405-409. 
(150) Marcus, Y. Internal pressure of liquids and solutions. Chem. Rev. 2013, 113, 65366551.

(151) Serratrice, G.; Delpuech, J.-J.; Diguet, R. Compressibilites Isothermes des Fluorocarbures. Relation avec la Solubilite des Gaz. Nouv. J. Chim. 1982, 6, 489-493.

(152) Straty, G. C.; Tsumura, R. PVT and vapor pressure measurements on ethane. J. Res. Natl. Bur. Stand. 1976, 80A, 35-39.

(153) Prausnitz, J. M.; Sprow, F. B. Surface tensions of simple liquid mixtures. Trans. Faraday Soc. 1966, 62, 1105-1111.

(154) Cachadiña, I.; Tian, J.; Mulero, A. New corresponding-states correlation model for the surface tension of refrigerants. J. Chem. Thermodyn. 2017, 110, 201-210.

(155) Leadbetter, A. J.; Taylor, D. J.; Vincent, B. THE DENSITIES AND SURFACE TENSIONS OF LIQUID ETHANE AND NITROUS OXIDE. Can. J. Chem. 1964, 42, 2930-2932.

(156) Baidakov, V.; Khotienkova, M. Capillary constant and surface tension of propane (R-290) with helium dissolved in it. Int. J. Refrig. 2018,

(157) Tian, J.; Zheng, M.; Yi, H.; Zhang, L.; Liu, S. Corresponding state-based correlations for the surface tension of saturated fluids. Mod. Phys. Lett. B 2017, 31, 1750110.

(158) Grigoryev, B. A.; Nemzer, B. V.; Kurumov, D. S.; Sengers, J. V. Surface tension of normal pentane, hexane, heptane, and octane. Int. J. Thermophys. 1992, 13, 453-464.

(159) Rohrback, G. H.; Cady, G. H. Surface Tensions and Refractive Indices of the Perfluoropentanes. J. Am. Chem. Soc. 1949, 71, 1938-1940.

(160) Kennan, R. P.; Pollack, G. L. Solubility of xenon in perfluoroalkanes: Temperature dependence and thermodynamics. J. Chem. Phys. 1988, 89, 517-521. 
(161) Smith, J. H.; Pace, E. L. The Thermodynamic Properties of Carbon Tetrafluoride from $12 \mathrm{~K}$ to Its Boiling Point. The Significance of the Parameter nu. J. Phys. Chem. 1969, 73, 4232-4236.

(162) Parks, G. S.; Huffman, H. M. Thermal data on organic compounds. IX. A study of the effect of unsaturation on the heat capacities, entropies and free energies of some hydrocarbons and other compounds. J. Am. Chem. Soc. 1930, 52, 4381-4391.

(163) Pace, E. L.; Aston, J. G.; Pace, E. L. The Thermodynamics of Hexafluoroethane from Calorimetric and Spectroscopic Data. J. Am. Chem. Soc. 1948, 70, 566-570.

(164) Kemp, J. D.; Egan, C. J. Hindered Rotation of the Methyl Groups in Propane. The Heat Capacity, Vapor Pressure, Heats of Fusion and Vaporization of Propane. Entropy and Density of the Gas. J. Am. Chem. Soc. 1938, 60, 1521-1525.

(165) Pace, E. L.; Plaush, A. C. Thermodynamic Properties of Octafluoropropane from $14^{\circ} \mathrm{K}$ to Its Normal Boiling Point. An Estimate of the Barrier to Internal Rotation from the Entropy and Heat Capacity of the Gas. J. Chem. Phys. 1967, 47, 38-43.

(166) Aston, J. G.; Kennedy, R. M.; Schumann, S. C. The Heat Capacity and Entropy, Heats of Fusion and Vaporization and the Vapor Pressure of Isobutane. J. Am. Chem. Soc. 1940, 62, 2059-2063.

(167) Yarrington, R. M.; Kay, W. B. The Liquid Specific Heats of Some Fluorocarbon Compounds. J. Phys. Chem. 1957, 61, 1259-1260.

(168) McCabe, C.; Bedrov, D.; Borodin, O.; Smith, G. D.; Cummings, P. T. Transport Properties of Perfluoroalkanes Using Molecular Dynamics Simulation: Comparison of United- and Explicit-Atom Models. Ind. Eng. Chem. Res. 2003, 42, 6956-6961.

(169) Morgado, P.; Laginhas, C. M.; Lewis, J. B.; McCabe, C.; Martins, L. F.; Filipe, E. J. 
Viscosity of liquid perfluoroalkanes and perfluoroalkylalkane surfactants. J. Phys. Chem. B 2011, 115, 9130-9139.

(170) Dantzler, E. M.; Knobler, C. M.; Windsor, M. L. Interaction Virial Coefficients in Hydrocarbon Mixtures. J. Phys. Chem. 1968, 72, 684.

(171) Dantzler, E. M.; Knobler, C. M. Interaction Virial Coefficients in Fluorocarbon Mixtures. J. Phys. Chem. 1969, 73, 1335-1341.

(172) Dantzler Siebert, E. M.; Knobler, C. M. Interaction virial coefficients in hydrocarbonfluorocarbon mixtures. J. Phys. Chem. 1971, 75, 3863-3870. 